\title{
SCIENTIFIC ABSTRACTS AND SESSIONS
}

\section{Education Council Symposium Internet Tools for Education}

\section{SU-A1-01 \\ Educational Foundations for Electronic Teaching \\ Cupido Daniels, Dalhousie University, Halifax, Nova Scotia, Canada}

The development of computer-aided-instructional (CAI) programs in medicine occurs within the general framework of the emerging field of medical informatics. This field is concerned with organizing and managing information by technology to support patient care, education, research, and administration. Technology-based educational initiatives can form the basis for computer-mediated-instruction (CMI) which is a faculty-guided, learnercentered approach to learning that takes advantage of the strength of the instructor, learner, and multimedia technology to create a personalized learning environment to increase student academic achievement. CMI preserves the core elements of traditional instruction and introduces the new element of CAI. CMI gives students better access to more learning resources, when they need it, and at the level which they require.

It appears that many programs fail to achieve their intended objectives because of one or more of the following factors: (a) narrowly conceptualized, (b) limited in scope, (c) theoretically chaotic, (d) pedagogically confusing, and (e) non-transformative (i.e, a simple transformation of traditional teaching methods and material without taking advantage of the added enhancing features of multimedia authoring).

The teaching of medical physics principles is generally directed at medical and physics students, residents, technologists, and physicians. The principles of adult education should form the theoretical framework for technology-based programs directed at these populations. One of the criteria of adult education is that of self-directed learning. CAI programs are ideally suited to the principle of self-directed learning.

Many CAI programs are being developed without adequate formative evaluation. Formative evaluation (i.e, a rigid process to obtain feedback, both quantitative and qualitative, from learners and experts during development of the program) are essential, and again conforms to two principles of adult education: "adults learn best if they are involved in the planning of their educational experience" and "feedback is a critical component".

This presentation will demonstrate some of these educational principles in CAI programs which the author has developed.

Educational Objectives

1. Discuss the importance of sound educational criteria in developing electronic educational programs.

2. Demonstrate the value of formative evaluation processes during program development.

\section{SU-A1-02}

Teleteaching Medical Physics

Perry Sprawls and Kwan Hoong (1) Emory University, Atlanta, GA (2)

University of Malaya Medical Center, Kuala Lumpur, Malaysia

\section{INTRODUCTION}

Teleteaching is the process of using contemporary computer and communications technology to link students to a teacher who is not in the same physical location. It makes it possible for educational programs to expand the scope and quality of teaching by utilizing highly qualified and experienced faculty who might be located anywhere else in the world. It is an economical and efficient method for institutions to share faculty for the purpose of expanding the scope of the educational experiences provided to their students.
GOALS

The goals for the design and implementation of an effective and efficient tele-teaching program include:

- Expanding the faculty capabilities of an educational program beyond the constraints imposed by economics and geographical location

- Retaining the advantages of traditional classroom teaching by providing for effective student-teacher interactions, communications, and discussion

- Improving the quality of teaching provided to students

- Reducing problems of scheduling student-teacher interactions

- Emphasizing the development and utilization of high-quality educational materials

- Increasing the capability of individual faculty to teach more students regardless of geographic location

\section{SYSTEM DESIGN AND FUNCTION}

A teleteaching system is designed to provide the following specific functions:

- Multimedia presentation and lecture delivery

- Transmission of selected educational materials

- Teacher-to-student transmission of objectives and assignments

- Two-way interaction and discussion

- Two-way data transfer

- Access to web based resources

- Student-to-teacher transmission of completed problem solutions, papers, and tests

\section{CONCLUSIONS}

Teleteaching can be used to expand medical physics educational programs to include faculty from anywhere in the world.

As an example, a very successful teleteaching program has been developed and is used by one of the authors (PS), who is located at Emory University in Atlanta, USA, to teach both graduate medical physics students and radiology residents at the University of Malaya, in Kuala Lumpur, Malaysia.

\section{EDUCATIONAL OBJECTIVES}

1. Identify appropriate applications for teleteaching

2. Evaluate the advantages and limitations of teleteaching

3. Select appropriate equipment and software for teleteaching

4. Design an effective teleteaching program

5. Use teleteaching to improve the scope and quality of medical physics education

\section{SU-A1-03}

Internet-Based Information for Medical Physicists

J. Daniel Bourland, Wake Forest University School of Medicine, Winston-

Salem, North Carolina

Access to electronic networks, collectively termed the "Internet", allows access to a large library of information of relevance to the medical physicist. Sources of information include government agencies, professional organizations, educational institutions, healthcare providers, and individuals, comprising both commercial and non-profit entities. Presentations of information take the form of newsgroups, listservers, interactive forums, and webpages, with a variety of mechanisms for information transfer via command line and menu-driven tools for a variety of file formats. Webbased tools using links and search engines are quite popular, in particular. Major sources of medical physics information are reviewed, including government (NRC, FDA), professional (AAPM, RSNA), basic science (journals, organizations), educational, and other sites. The role of the AAPM's website (www.aapm.org) will be reviewed in this context, as an example of a medical physics information pool. Methods used to reach specific audiences, such as potential medical physicists, are discussed.

Educational Objectives:

1. List classes and sources of electronic (internet) information for medical physicists.

2. List mechanisms for access to electronic information.

3. Review the current status of www.aapm.org, the AAPM's website

4. Postulate the electronic future of the medical physics community. 


\section{SU-A1-04}

Remotely Directed Continuing Education on the Internet

Charles Kelsey, Radiology Department, University of New Mexico Albuquerque NM

The AAPM Education Committee has developed a remotely directed continuing education program to provide AAPM members access to continuing education and an opportunity to earn continuing education credits, comparable with Category I credits for physicians, over the internet. The program is based on the participant reading an article or report, answering at least 8 out of 10 questions correctly, providing some evaluation of the questions. The questions are designed to cover almost all categories of Medical Physics. The 14 categories in the RDCE program are: Bioeffects, Computers, Computed Tomography, Diagnostic Medical Physics, General Medical Physics, Laws and Regulations, Mammography, Magnetic Resonance, Nuclear Medicine, Radiation Protection, Radiation Oncology Physics, Brachytherapy, Linear Accelerators, and Ultrasound.

The sources of the questions are articles in Medical Physics, or other journals readily accessible to AAPM members, AAPM Task Group or government reports. Each The Category has an article question assignor who assigned individuals to write questions based on a particular article, These article question writers and the article question assignor certify that the intellectual effort in reading the article and answering the questions is at least equivalent to attending a one hour lecture at a national meeting. In order to participate in the RDCE program AAPM members must have signed up for, and paid the \$20 RDCE program fee. After logging onto the AAPM web site the RDCE program is selected. The various categories and the number of quizzes are indicated. After selecting a quiz the questions are presented. As part of the continuing education program the participant must provide and evaluation of the questions in order before the answers can be submitted. Participants receive immediate feedback on whether they have correctly answered 8 of the 10 questions. If they have not they must wait until the next day to retake the test. Presumably this time will be spent in rereading the article for a better understanding of its content. At the end of the year each participant receives a certificate from the Commission on Accreditation of Medical Physics Educational Programs (CAMPEP) listing the continuing education credits earned.

Educational Objectives

1. To describe the purpose and extent of the RDCE program.

2. To describe the operational details of the RDCE program.

\section{Professional Council Symposium Room: Pearl CRCPD Overview and Malpractice Insurance Issues}

\section{SU-B1-01}

Postmodern Management: Restructuring Radiation Control Programs Jill A. Lipoti, New Jersey Department of Environmental Protection, Trenton, NJ

The term "postmodernism" is usually reserved for art and architecture, not management style, but it is an emerging trend for governmental managers. There is a deconstruction of the bureaucracy, a careful dismantlement of the hierarchical structure of government. The military model of command and control is being replaced with a more egalitarian approach. There is renewed emphasis on using creativity and not doing things the way they have always been done. A new spirit of cooperation is growing between the regulated community and the regulators. The emphasis is on results that matter - using a risk-based prioritization. Indicators are collected to measure conditions, trends, and results.

What led to this burgeoning partnership? How do our respective professional organizations fit within this model? How can our partnership enhance public health and safety? What are the caveats?

The Conference of Radiation Control Program Directors (CRCPD) is a nonprofit organization made up of individuals in state and local government who regulate and control the use of radiation sources. Affiliate membership is available to individuals, regardless of employer affiliation, who have expressed an interest in radiation protection. The CRCPD's mission statement is "A partnership dedicated to radiation protection".
In this talk, I will describe the structure and function of the CRCPD, our strategic plan, our recent achievements and plans for the future, and how AAPM members can become involved with CRCPD activities. After the "thinking globally" portion of the talk, I will describe how the AAPM members can "act locally" and become a valuable resource for the state radiation control program director in their immediate area. I will stress opportunities for input in rulemaking and policy determination.

Governmental change has not occurred in a vacuum. It has occurred because of a change in attitude in the regulated community and in the general population. As I develop the theme of postmodern management think about how it may help you to achieve your goals. I will use some of the federal government's reengineering processes as examples, but I will talk about what states should do and what we should avoid. This will lead to a discussion of the proper state and federal roles in radiation protection, as well as roles for surrogate regulators.

Medical physicists and state regulators have an important, mutually reinforcing message to deliver. We are trying to protect the public while not restricting the profitability of industry within our borders or restricting the availability of new technological tools for diagnosis and treatment. My vision of postmodern management is: international standards developed jointly with all levels of regulators and regulated community; quality improvement measures enforced by surrogate regulators and economic incentives; prioritized and focused inspections using indicator systems; multidisciplinary participation in regulatory policy; and enhancement of public participation.

I hope that we will be able to explore mutual goals and define concrete actions that each of us can take to improve our working relationship and make our respective roles complementary.

\section{SU-B1-02}

\section{Professional Liability for the New Millennium}

William Roventine, AAPM Insurance Coordinator, Cumberland, RI

As the medical landscape continues to evolve under the mounting pressure of managed care there has been a concurrent growth in the professional liability concerns of less resources. In the past, these issues were of concern primarily to the consulting physicist. Now, however, they are equally important to the non-consulting employed physicist as well. The AAPM Professional Liability Program was established to address some of these concerns and has now been in operation for over five years. This session will present an overview of the current status of the program, review our claims experience to date, address the Y2k liability issue, and enable you to assess your liability coverage requirements.

\section{Harold Johns Memorial Symposium Room: Pearl}

\section{SU-C1-01}

\section{Comments on Harold E. Johns}

John S. Laughlin, Scarsdale, NY

I first became acquainted with Harold during the winter of 1947. Harold and two of his colleagues, Professors Haslam and Katz, all members of the faculty of the University of Saskatchewan, were guests of our betatron and nuclear physics group for nearly a month. They were interested in our beam collimation and dose distribution measurements for different fields, and also in the photodisintegration nuclear reaction studies. These had already resulted in nearly a dozen reaction thresholds suitable for beam energy calibration purposes from about $2 \mathrm{MeV}$ up to over $20 \mathrm{MeV}$. They were a bright and friendly group and popular with our staff. Johns was particularly interested in the applications to this field and a basis of future cooperation came into being. Harold and I collaborated on a chapter on the interactions of x-rays and electrons with matter requested by Gerald Hine and Gordon Brownell for their book on Radiation Dosimetry. Harold also served on the Board of Scientific Consultants of the Memorial Sloan-Kettering Cancer Center for several years. His originality was expressed in many developments. His concept of tumor-air-ratio and its applications was very helpful; he designed the first teletherapy unit for cobalt-60 and initiated its 
use in 1951; his textbook became the medical physics "bible"; and other to be mentioned. He was asked as the unofficial dean of American medical physicists to supply a guest editorial for the Journal Medical Physics, which he did for the July/August issue, 1988. He received many awards, including the Order of Canada and the AAPM Coolidge Award. He was always constructive and original; time permitting I should like to mention some addresses.

\section{SU-C1-02}

Research in Electronic Imaging and Digital Radiography at the University of Toronto

Martin J. Yaffe, Sunnybrook and Women's College Health Sciences Centre, Toronto, Ontario, Canada

Harold Johns made many outstanding contributions in the field of Medical Physics. Although he is mainly known for his work in radiation therapy physics, in the last few years of his professional career his primary efforts were in research in diagnostic imaging. As with any activity in his life, he attacked these problems with great energy and enthusiasm. He was particularly concerned about the high radiation doses received by patients because of the inefficient imaging etector systems available in the early 70 's, especially for mammography.

Following a sabbatical with Professor Jack Boag in England, he became interested in electronic radiography and initiated a strong effort in both the theoretical and experimental development of high pressure gas ionization chambers and liquid ionization methods for imaging. This led to application of ionization detectors for the newly-emerging modality of computed tomography for both conventional and dual energy image formation. His strong commitment to understanding the science underlying the technology with which he was working, led to investigation in his lab of the key factors determining imaging performance as well as the methods for the quantitative analysis of image quality using MTF, noise power spectra and DQE. Eventually, under his guidance and intense stimulation, research efforts in the laboratory moved toward photoelectronic and solid state detectors for application in quantitative vascular imaging, digital radiography and fluoroscopy and digital mammography. This presentation will focus on the character and influence of Harold Johns in building a strong research effort and will look at some of his contributions to digital radiography and particularly, digital mammography.

Educational Objectives:

1. To familiarize the participant with the work of Professor Harold E. Johns in the physics of diagnostic radiology

2. To consider how strong leadership qualities and values can influence success in research

3. To review the progress of research in digital mammography initiated under the guidance of Harold Johns

\section{SU-C1-03}

\section{Harold Johns - In Memoriam}

Ervin B. Podgorsak, McGill University, Montreal, Quebec, Canada

Dr. Johns' illustrious career and tremendous contributions to Medical Physics and Cancer Treatment are well known and were recognized with accolades and numerous honors and awards nationally and internationally. His invention of the cobalt unit, his over 200 scientific publications, his textbook "The Physics of Radiology" which he wrote jointly with Jack Cunningham, his long and successful leadership of Medical Physics at the Princess Margaret Hospital in Toronto, his leadership role in national and international medical physics organizations, his firm dealings with hospital administrators, and his tough yet fair attitude toward students are legendary and clearly qualify him for his recent induction into the Canadian Medical Hall of Fame. If there was a global Medical Physics Hall of Fame, Dr. Johns would be the first inductee. He will forever remain a giant among his peers in the profession of medical physics.

Dr. Johns also made an important and lasting contribution to the Canadian society through his teaching efforts. He had a direct and strong influence on the careers of over 100 graduate students and postdoctoral fellows. Students certainly learned about physics and physics research from Dr. Johns, but the physics knowledge that he passed on to his students was far less important than the values and code of behavior that he instilled in his students. His personality traits and professional ethics had a tremendous influence on his young students. One could not help but admire and learn from Dr. Johns' strong will, his honesty and ethical behavior, his no-nonsense approach to solving problems, his love of his family and respect for his colleagues, as well as his love and respect for his country and his institution.

Dr. Johns' students are spread around the world; however, most of them chose to practice their profession in Canada and a large number of them have a teaching career in their own right. It is safe to say that most contemporary medical physicists in Canada, either directly or indirectly, trace their professional roots to Dr. Johns. And this is the real legacy that Dr. Johns is leaving behind. His enormous positive influence on the medical physics and radiation oncology professions will be felt for years to come.

Dr. Johns has had a full and extremely productive life and his contributions to his chosen field of medical physics are truly outstanding. Those who knew him owe him an enormous gratitude for who he was, for what he did with his talents, and for the way he touched and influenced their lives.

\section{Refresher Course}

\section{Room: Pearl}

\section{MO-A1-01}

TG-64 Prostate Implants - Technique and Dosimetry Review David Mellenberg, University of Iowa, Iowa City, IA

Image guided brachytherapy techniques increase the efficacy and convenience of implant procedures. This is particularly evident with ultrasound guided prostate implants. Several techniques are being used to optimally implant the prostate. After a brief review of the pertinent literature, this refresher course will review the basics of prostate implant physics as contained in the report of Task Group 64. Particular attention will be paid to: equipment selection, source preparation, source calibration, preplanning philosophies, seed insertion methods, post implant dosimetry, time of post implant dosimetry, NIST seed strength definition changes, and prostate implant dose evaluation. The advantages and disadvantages of each philosophy and method will be highlighted. The impact ultrasound guided prostate implant programs have on the medical physicist will be discussed.

Educational Objectives:

1. To learn about the equipment and supplies available for ultrasound guided prostate implants and ways in which they are used.

2. To learn the various philosophies used to plan, perform and evaluate prostate implants.

3. To highlight the impact medical physicists have on the treatment of prostate implant patients with an emphasis on future developments.

\section{Continuing Education Course Room: Mississippi IMRT-1}

\section{MO-A2-01}

IMRT Optimization

R. Mackie, G. Olivera, D. Shepard, P. Reckwerdt, University of Wisconsin, Madison, WI

Optimized radiotherapy planning is the basis of intensity-modulated radiation therapy (IMRT) because this type of planning invariably results in specifying intensity distributions that are non-uniform. Radiotherapy optimization is often called "inverse" planning to imply that the desired dose distribution is input to the calculation and the intensity pattern is the output. In fact, most methods are actually iterative in nature in that the optimal solution is searched for from a set of feasible ones.

The results depend on the objective function guiding the search, the constraints placed on the search and the type of "search engine" used. The objective function is a function, or more generally an operator on the dose distribution and a descriptor of the regions-of-interest, which results in a single scalar value characterizing the quality of the solution. Both physical and biological objective functions have been used. A least-square sum of the deviation between the desired and actual dose distributions is an oftenused physical objective function. The probability of uncomplicated control, using several biological models of tumor control probability and normal tissue complication probability, has been used as a biological objective 
function. Physical objective functions are currently more trusted but biological objective functions may ultimately be a better figure-of-merit for the solution when there are better biological bases for the models.

Constraints specify values of dose or intensity that are allowed. For example, the intensity pattern must have only positive values. The search for the best solution must be confined within the bounds of the constraints. If constraints are too tight no solution may be possible. Instead of constraints, penalty terms may be introduced into objective functions so that unfeasibility does not result. Dose-volume "constraints" have often been implemented as penalty functions.

Search engines are generally classified into stochastic methods, such as simulated annealing or the genetic algorithm, and deterministic methods like the gradient descent or maximum likelihood methods. Stochastic methods are capable of finding a global minimum of an objective function (but are only guaranteed to for infinite calculation times) but deterministic methods may be quicker, and for many problems, find solutions which do not appreciably differ from the global minimum or are not needed because there is only one minimum. On the other hand, problems such as determining the best beam directions to use or some types of biological objective functions have been shown to result in a multitude of local minima. It is possible to have solutions which, in effect, tighten up constraints so that the search space becomes progressively reduced.

The character of the optimized solution depends on many factors. Some objective functions result in higher dose uniformity in the target volume. Increased dose uniformity in the target volume usually results in higher dose to sensitive structures. Increasing the number of beam directions results in better uniformity and lower maximum dose to normal tissue but the improvement becomes asymptotic. Cases that include the presence of a complex target volume along with sensitive structures that are adjacent to the tumor can benefit the most from larger numbers of beam directions and highly-modulated high-resolution intensity patterns. Coplanar beams are usually used for IMRT below the head because the optimization results suggest that there are limited additional benefits resulting from the use of non-coplanar IMRT fields and the selection of optimal field directions expands the solution space substantially. When sufficient numbers of beams are used the beam orientation become less important and an odd number of equally spaced beams are typically used.

The field of radiotherapy optimization is young. As computer speed and memory increases, search parameters such as beam energy, beam type, treatment fractionation, and the impact of setup error and organ motion will be included in the objective function or constraints. Search techniques borrowed from other field such as economics will be implemented. It is likely that optimization and IMRT will have an impact on the field of radiotherapy comparable with that of megavoltage beams.

Educational Objectives:

1. To be introduced to IMRT optimization.

2. To understand the relationship between objective functions, constraints, and solution search methods.

3. To see examples of the capabilities and limitations of IMRT optimization.

4. To look into the future of radiotherapy optimization.

TRM and PJR have financial interest in TomoTherapy Incorporated which is participating in the commercialization of tomotherapy.

\section{Continuing Education Course Room: Sewanee Special Dosimetry Measurements - 1 (Session Dedicated to Hy Glasser)}

\section{MO-A3-01}

The AAPM's TG-51 Protocol for Clinical Reference Dosimetry of HighEnergyBeams

Peter Almond ${ }^{1}$, Peter Biggs ${ }^{2}$, Bert Coursey ${ }^{3}$, William Hanson ${ }^{1}$, M Saiful Huq ${ }^{4}$, Ravinder Nath ${ }^{5}$, D. Rogers ${ }^{6}$, AAPM Task Group 51 (1) UT M.D. Anderson Cancer Center, Houston, TX (2) Massachusetts General Hospital, Boston, MA (3) National Institute of Standards and Technology, Gaithersburg, MD (4) Thomas Jefferson University Hospital, Philadelphia,
PA (5) Yale University School of Medicine, New Haven, CT (6) National Research Council of Canada, Ottawa, Ontario, Canada

This talk describes a new protocol for clinical reference dosimetry of external beam radiation therapy using photon beams with energies between ${ }^{60} \mathrm{Co}$ and $50-\mathrm{MV}$ and electron beams with nominal energies between 4 and $50-\mathrm{MeV}$. The protocol was written by Task Group 51 of the AAPM's Radiation Therapy Committee and has been formally approved by the AAPM for clinical use. The protocol uses ion chambers with absorbed-doseto-water calibration factors, $N_{D, w}^{60} \mathrm{Co}$, which are traceable to national primary standards, and the equation: $D_{w}^{Q}=M k_{Q} N_{D, w}^{60}$ co , where Q is the beam quality of the clinical beam, $D_{w}^{Q}$ is the absorbed dose to water at the point of measurement of the ion chamber placed under reference conditions, $\mathrm{M}$ is the fully corrected ion chamber reading, and $k_{Q}$ is the quality conversion factor which converts the calibration factor for a ${ }^{60} \mathrm{Co}$ beam to that for the beam quality Q. Values of $k_{Q}$ are presented as a function of $\mathrm{Q}$ for many ion chambers. The value of $\mathrm{M}$ is given by $M=P_{\text {ion }} P_{\text {tp }} P_{\text {elec }} P_{\text {pol }} M_{\text {raw }}$ where $M_{\text {raw }}$ is the raw, uncorrected ion chamber reading, $P_{\text {ion }}$ corrects for ion recombination, $P_{t p}$ for temperature and pressure variations, $P_{\text {elec }}$ for inaccuracy of the electrometer if calibrated separately and $P_{p o l}$ for chamber polarity effects. Beam quality, Q, is specified: (i) for photon beams, by $\% d d(10)_{x}$, which is the photon component of the percentage depth dose at $10 \mathrm{~cm}$ depth for a field size of $10 \times 10 \mathrm{~cm}^{2}$ on the surface of a phantom at an SSD of $100 \mathrm{~cm}$; and (ii) for electron beams, by $R_{50}$, the depth at which the absorbed-dose falls to $50 \%$ of the maximum dose in a beam with field size $\geq 10 \times 10$ $\mathrm{cm}^{2}$ on the surface of the phantom ( $\geq 20 \times 20 \mathrm{~cm}^{2}$ for $R_{50}>8.5 \mathrm{~cm}$ ) at an $\mathrm{SSD}$ of $100 \mathrm{~cm}$. $R_{50}$ is determined directly from the measured value of $I_{50}$, the depth at which the ionization falls to $50 \%$ of its maximum. All clinical reference dosimetry is performed in a water phantom. The reference depth for calibration purposes is $10 \mathrm{~cm}$ for photon beams and $0.6 R_{50}-0.1$ $\mathrm{cm}$ for electron beams. For photon beams clinical reference dosimetry is performed in either an SSD or SAD setup with a $10 \times 10 \mathrm{~cm}^{2}$ field size defined on the phantom surface for an SSD setup or at the depth of the detector for an SAD setup. For electron beams clinical reference dosimetry is performed with a field size of $\geq 10 \times 10 \mathrm{~cm}^{2}$ ( $\geq 20 \times 20 \mathrm{~cm}^{2}$ for $R_{50}>8.5 \mathrm{~cm}$ ) at an SSD between 90 and $110 \mathrm{~cm}$. This protocol represents a major simplification compared to the AAPM's TG-21 protocol in the sense that large tables of stopping power ratios and mass-energy absorption coefficients are not needed and the user does not need to calculate any theoretical dosimetry factors. The talk concludes discussing the preparations of the ADCLs for doing absorbed-dose calibrations and the differences expected between doses assigned using TG-51 or TG- 21 .

Educational Objectives:

1. To outline the contents of the new TG-51 protocol.

2. To review the expected differences between doses assigned with TG51 or TG-21.

Refresher Course

Room: Carroll

\section{MO-A4-01}

The Basics of MTF, Wiener spectra, and DQE

Robert Nishikawa, The University of Chicago, Chicago, IL

One role of a medical physicist is to help maximize patient benefit while minimizing the cost of a diagnostic procedure. In many instances, this can be restated as maximizing image quality while minimizing risk to the 
patient. This is a complex task, but a number of concepts have been developed over the years that are useful in quantifying image quality, namely, modulation transfer function (MTF), noise (Wiener) power spectrum (NPS), noise equivalent quanta (NEQ), and detective quantum efficiency (DQE). These concepts are measured in the spatial frequency domain. The MTF describes the spatial resolution properties of the system, the NPS describes the noise properties, and together they can be combined to determine the NEQ, which is related to the signal-to-noise ratio (SNR) of the system. The DQE describes how efficiently information (SNR) is passed through the system. Drawing on examples from x-ray imaging, I will describe what these concepts are, how they are measured and how they can be used.

Educational Objectives

1. Understand the concepts of modulation transfer function (MTF), noise (Wiener) power spectrum (NPS), noise equivalent quanta (NEQ), and detective quantum efficiency (DQE).

2. Understand how measure MTF, NPS, NEQ, and DQE for analog detectors

3. Understand how measure MTF, NPS, NEQ, and DQE for digital detectors

\section{Continuing Education Course \\ Taylor \\ MRI-1}

\section{MO-A5-01}

Jeff Hayden, American College of Radiology, Reston, VA

The ACR MRI Accreditation Program was developed by the ACR due to the concern among imaging specialists about the variation in quality of the performance of MRI in current practice. The MRI Accreditation program was designed to be educational in nature, and evaluates the qualifications of personnel, equipment performance, effectiveness of quality control measures, and quality of clinical images. It is believed that these are the primary factors which impact the quality of clinical images and the quality of patient care. A full term of accreditation for a MRI facility is a three year period. The facility receiving ACR MRI Accreditation is awarded a certificate recognizing its achievement. A confidential final report is sent to the supervising physician of the MRI site at the end of the accreditation process regardless of whether or not it achieves accreditation. This peer review document discusses accomplishments, defines issues which could be improved, and provides recommendations about the performance of magnetic resonance imaging for consideration. This presentation will outline the details about the MRI Accreditation Program requirements and the application process

Educational Objectives:

1. To learn about the ACR MRI Accreditation Program Requirements.

2. To learn how to apply for MRI site Accreditation.

\section{Continuing Education Course Room:Browning A Ultrasound- 1}

\section{MO-A6-01}

Ultrasound Principles and Problems: Communicating with Physicians Christopher Merritt, Thomas Jefferson Hosptal, Philadelphia, PA

Ultrasound is currently the most rapidly growing imaging method, both in clinical applications and technology. Growing use, coupled with the need for accreditation of ultrasound practices, has created a need for active participation of medical physicists in support of clinical ultrasound departments. ACR and AIUM ultrasound accreditation programs have defined an important role for the Medical Physicist in quality assurance and the monitoring the performance of ultrasound imaging equipment. In addition to this task, the Medical Physicist must be prepared to interact closely with clinicians and sonographers in the application of new technology and in the interpretation and understanding of imaging artifacts.

In order to meet these needs of today's sophisticated ultrasound applications and technology, Medical Physicists require a solid understanding of basic acoustics, Doppler principles and applications, and ultrasound bioeffects, as well as newly introduced ultrasound methods including harmonic imaging, and the use of ultrasound contrast agents.

In this presentation, common problems confronting the clinician and sonographer will be presented and the role of the Medical Physicist in addressing these problems will be emphasized. Basic concepts and approaches useful for communicating these concepts to clinicians will be emphasized.

\section{Refresher Course}

\section{Room: Pearl}

\section{MO-B1-01}

Gamma Knife Dosimetry and Treatment Planning

Jürgen Arndt ${ }^{1}$, Steven Goetsch ${ }^{2}$, (1) Elekta Instrument AB, Stockholm, Sweden, (2) San Diego Medical Physics, Inc., LaJolla, CA

All the beam channels of the Gamma Knife are stationary and are manufactured to very narrow mechanical tolerances. If allowance is made for the decay of the ${ }^{60}$ Cobalt, these beam channels may be considered identical and unchanging from a radiophysical point of view. The design of the Gamma Knife is such that all beam channels of the same size are identical, independent of the individual unit or its model. This means that the storage of beam data in the dose-planning system GammaPlan can be greatly simplified. This is a great advantage since it would be difficult, to measure individual beams to the required accuracy inside the unit on site.

Despite the simplicity of pre-storing the beam data, this approach has one disadvantage. It requires that the users, must trust in the reliability of this data at any specific installation.

The justification for pre-storing the beam data will be illustrated by discussing the nature of the Gamma Knife single beam data, how it is obtained and its reliability. In this context, the Out Put Factor (OPF) of the 4 $\mathrm{mm}$ helmet will receive extra attention. The dosimetry of the Gamma Knife is influenced by the unit's technical design. Thus, introductory remarks will outline those aspects of this design which affect dosimetry.

Today, a more selective delivery of the radiation dose to the target volume may be achieved as the result of better imaging techniques, faster computers, improved functionality of the treatment planning software and technical improvements in the irradiation procedure of the Gamma Knife. These developments enable improved radiosurgical procedures. However, an unavoidable consequence of the improvements is that the treatment plans become increasingly complex as more isocenters are used. This greater complexity does not affect the irradiation procedure itself, but it does make it more difficult to assess the appropriateness of a given dose-plan. It should be emphasised that the consequences of increasingly complex dose-plans do not apply to the dose calculation algorithms, since these can be tested under simpler situations. The concern is that with greater complexity there may be a greater risk that human errors or expression of hitherto hidden errors in the software may remain undetected.

An investigation over how different detectors respond to narrow photon beams is made at the Karolinska Institute and will briefly be reported.

Educational Objectives:

1. Introductory remarks outlining those aspects of the Gamma Knife design, which affect dosimetry.

2. Presentation of radiophysical data underlying the dose calculations in GammaPlan with repeat to:

- $\quad$ type of data

- $\quad$ how it is obtained

- $\quad$ its reliability

3. Specific issues:

- Correction of dose rate for its dependence on beam size. The out put factor of the $4 \mathrm{~mm}$ helmet will receive extra attention

- dose normalization

- $\quad$ are there simple and reliable means to validate the calculated treatment time

4. Response of detectors when used to measure properties of narrow photon beams. 


\section{Continuing Education Course IMRT - 2}

\section{MO-B2-01}

Serial Tomotherapy

Daniel A. Low, Mallinckrodt Institute of Radiology, St. Louis, MO

Serial tomotherapy, a rotational application of intensity modulated radiation therapy (IMRT), was originally envisioned by Rock Mackie and has been developed as a commercial modality by the NOMOS Corporation. The commercial system consists of a treatment planning and optimization software and a tertiary dynamic multileaf collimator. IMRT, and specifically tomotherapy, has characteristics that are significantly different from conventional conformal therapy and requires the development of new quality assurance (QA) methods. This talk will describe some of the techniques used for commissioning, routine QA, and patient QA, as well as some of the dosimetric characteristics of tomotherapy.

Many dose measurement methods used to validate traditional treatment planning systems cannot be used with dynamically delivered IMRT. In conventional systems, dosimetric validation is conducted using water phantoms and point dosimeters, such as ionization chambers. The radiation dose distribution is assumed to be static, allowing the dosimeter to be moved throughout the phantom to integrate a linear or planar dose distribution. However, because the entire fluence distribution must be delivered for each measurement point, a traditional real-time scanning system cannot be used to collect dose distribution data. The implications of dosimeter limitations and methods to optimize each available dosimeter to gather the most useful data will be discussed. The independent determination of the spatial location of the measured and calculated dose distributions will be stressed.

The patient treatment verification is also different from conventional treatments. Many centers verify the delivered dose to each patient, either through the use of anthropomorphic or geometrically regular phantoms, or using in-vivo measurements. Developing a robust method for the verification of patient positioning is also critically important. Because IMRT provides complex high gradient dose distributions that often conform tightly and surround critical structures, the patient position and orientation relative to the gantry coordinate system must be accurately verified. Some centers use portal images obtained with the open collimator leaves, yielding a narrow portal (e.g., $3.4 \times 20 \mathrm{~cm}^{2}$ ) which may yield few quantitative anatomical references, so the portal orientations and positions should be selected to maximize anatomical reference data.

Dosimetric tests require the use of target and critical structure volumes to generate treatment plans. Selection criteria for targets and critical structures will be discussed as will phantoms used for dosimetric measurements

Tomotherapy makes inefficient use of monitor units, each slice is only approximately $1.68 \mathrm{~cm}$ long, so the treatment of longer targets requires the use of multiple slices ( $200 \mathrm{cGy} \approx 3000 \mathrm{MU}$ ). The whole-body dose due to leakage radiation is correspondingly greater, and should be considered as part of patient selection. The breakup of treatments into multiple abutted slices requires accurate patient movement between slices as well as good patient immobilization. The accuracy of the patient moving hardware, the dosimetric consequences of inaccurate motion, and intrinsic abutment region dose distributions will be addressed.

This work was sponsored in part by a grant from the NOMOS Corporation.

Educational Objectives:

To describe:

1. Serial tomotherapy treatment delivery techniques

2. Commissioning and QA procedures

3. Clinical implementation

4. Dosimetry features
Refresher Course

Room: Sewanee

\section{MO-B3-01}

Navigating in the Bay of Funding

Paul Strudler, NIH/CSR, Bethesda, MD

The number of NIH grant applications focused on medical physics, and reviewed in Radiation Study Section, mostly submitted by investigators who probably consider this society as their "home", has more than doubled in the last two years. This development is mirrored, $\mathrm{NIH}$-wide, in many sectors of Bioengineering, Technology and Instrument Development research leading to an initiative coordinated by the NIH Center for Scientific Review, to develop a technology-friendly review infrastructure, including more applicable review criteria.

The opportunities for applicants stemming from this initiative will be discussed, as will other new developments including Modular Grants, changes in the scoring system, and tracking grants through the review process. The role of the SBIR/STTR program in supporting technology development will be reviewed.

We will demonstrate the utility of the NIH Website in identifying appropriate study sections, Institutes, and grant mechanisms, as well as searching the grant database for identification of projects with similar foci.

We will discuss the special handling of new investigators, criteria for successful (and unsuccessful) applications, and your rights as an applicant. Appropriate interactions with $\mathrm{NIH}$ review staff and program directors will be illustrated.

This presentation will focus on navigating through a system that receives some 40,000 applications per year. Many of the strategies suggested are not necessarily described in official documents.

\section{Refresher Course}

Room: Carroll

\section{MO-B4-01}

Understanding Imaging-System Performance: Quantum Sinks and the Detective Quantum Efficiency

Ian Cunningham, Robarts Research Institute, Imaging Research

Laboratories, London, Ontario, Canada

An important aspect of imaging science is understanding the fundamental physics and engineering principles that determine image quality. This understanding is particularly important for medical physicists involved with the design, evaluation or testing of $x$-ray imaging systems. In the first of this two-part presentation, metrics developed to describe image quality were introduced, including the modulation transfer function (MTF), Wiener noise-power spectrum (NPS), and detective quantum efficiency (DQE). In this second part, the relationship between system design and image quality (in terms of the DQE) is described using a linear-systems Fourier approach.

While it has been known since the work of Albert Rose in the 1940s that image quality is directly tied to the number of quanta used to create an image, the Rose approach is too simplistic to use in many situations of practical importance. More recently, Fourier-based methods of describing image signal and noise have been adapted from foundations laid out by scientists and engineers studying communications theory and are now widely used. In this presentation, principles of linear-systems theory used in the analysis of medical-imaging systems to describe signal and noise transfer are described. The link is made to metrics of image and system quality including the MTF, noise-equivalent number of quanta (NEQ), quantum sinks, and the DQE. The theoretical bases for the NPS of digital-imaging systems is presented, including a description of noise aliasing. It is shown how noise aliasing can degrade the DQE of digital systems. Examples are given showing how the theoretical principles presented can be applied to the analysis of several types of imaging systems including $\mathrm{x}$-ray image-intensifier based systems and the new active-matrix flat panel digital systems.

Educational Objectives:

1. Understand methods used to predict the DQE for particular system designs. 
2. Understand the effect of primary and secondary quantum sinks as a function of spatial-frequency.

3. Understand the importance of noise aliasing on the DQE of digital imaging systems.

\section{Continuing Education Course Taylor$$
\text { MRI- } 2
$$

Room:

\section{MO-B5-01}

Test Objects/Equipment for Acceptance Testing, Quality Assurance and Compliance Testing

Geoffrey D. Clarke, University of Texas Southwestern Medical Center at Dallas, TX

This course will review test objects and equipment needed to perform clinical medical physics services in magnetic resonance imaging (MRI). The historical development of MRI test objects will be reviewed. The material covered will emphasize the recommendations of the AAPM's Magnetic Resonance Task Groups on MRI Quality Assurance and MRI Acceptance Testing. The rationale for image quality tests, including slice thickness and position accuracy, geometric distortion, signal-to-noise ratio, absolute and low contrast spatial resolution, will be discussed. The performance and analysis of these tests, carried out using the phantom of the American College of Radiology's MRI Accreditation Program, will be reviewed in detail. Test objects for advanced, high-speed imaging methods will also be discussed.

Educational Objectives:

1. Determine appropriate test objects for use in Quality Assurance, ACR Accreditation Compliance Testing, and Acceptance Testing programs for Magnetic Resonance Imaging.

2. Set reasonable acceptance and action criteria for these MRI image quality programs.

3. Produce the appropriate records and reports associated with these programs.

Some of the information in this presentation was developed while the author was a consultant to the American College of Radiology.

\section{President's Symposium Room: Pearl Intravascular Brachytherapy: An Emerging Role for Medical Physicists}

\author{
Introduction \\ Geoffrey S. Ibbott, University of Kentucky, Lexington, KY
}

Coronary artery disease is the most frequent cause of death in the United States. Nearly a million therapeutic procedures are conducted each year to combat the disease. Most procedures are either bypass surgery or percutaneous transluminal balloon angioplasty (PCTA). Angioplasty is less invasive, less morbid, and less expensive, and the initial success rate is very high. However, forty to sixty percent of patients experience failure within six months due to restenosis of the vessel. Restenosis can occur as a result of several processes, including the proliferation of smooth muscle cells into the arterial lumen. This process is called neointimal hyperplasia. Implantation of a stent can combat some causes of restenosis, but does not prevent neointimal hyperplasia, and may in fact exacerbate it.

External or intraluminal irradiation of arteries following balloon angioplasty or stent implantation can reduce the proliferation of smooth muscle cells and consequent neointimal hyperplasia. Prevention of restenosis by irradiation has been demonstrated in coronary and peripheral arteries, in humans and animal models. Long term follow up studies and phase III randomized trials are now being conducted.

The use of intravascular brachytherapy presents a number of new problems of radiation biology, dosimetry and protection. Medical physicists have been called upon to provide estimates of dose distributions as well as to supervise the use of radioactive materials during clinical trials. Some of these practical issues will be discussed during this symposium. In addition, several speakers will describe the procedures currently being investigated and the clinical results to date. The roles of and relationships between medical professionals will be addressed. As these procedures become more widely used, it is important that medical physicists become familiar with the techniques and the special issues involved.

\section{MO-C1-01}

Intravascular Brachytherapy and its Role in the Management of the Cardiac Patient

Jeffrey Moses, Lenox Hill Hospital, New York, NY

Coronary artery disease is still the major cause of death in the industrialized world. Its principal manifestations are sudden death, heart attack and angina pectoris. Over the past 20 years coronary angioplasty (PTCA), non-surgical opening of arteries with balloons under x-ray guidance, has emerged as a principal treatment to relieve anginal symptoms and treat heart attacks. An estimated 600,000 PTCAs procedures will be performed in the US in 1999. A principle limitation of this procedure is an early $(<6$ months) recurrence rate of $30-45 \%$. These recurrences are termed restenosis. Since the mid 1990s the placement of an endovascular prosthesis (coronary stent) has been popularized in an attempt to reduce restenosis. This has been partially effective, and has reduced recurrence rates by about $30-40 \%$. This still leaves a restenosis rate of about $20 \%$, with clinical recurrence occurring at $15 \%$ by $6-9$ months.

Restenosis in stents can be a vexing problem. The cause of this recurrence is the buildup of scar tissue within the stent. If the segment of buildup is short $(<10 \mathrm{~mm})$ it can be easily and effectively treated with another balloon procedure. If it is longer then this ("diffuse") the recurrence rate after balloons can be as high as $60-80 \%$. Some patients must be referred to coronary bypass to resolve their symptoms, while others are not suitable for bypass and remain disabled. It is estimated that about one-half of the stent restenosis fall into this high risk group, about 40,000 patients.

Over the history of angioplasty there have been scores of trials of mechanical and pharmacological strategies to reduce restenosis. Up until now, no drug or mechanical treatment besides stents has been successful in reducing recurrences. While there are a few promising new agents in clinical trials, there is no clinically available drug for this problem.

The emergence of vascular brachytherapy as a potential treatment has excited the cardiologic community. The prospect of the ability to reduce recurrences in these difficult patients by up to $80 \%$ has inspired many centers to participate in clinical trials of vascular brachytherapy. Some have focused on primary prevention (at the time of initial PTCA), but most on their treatment of restenosis after stent implantation.

Such a program requires tight co-ordination between the interventional cardiologist, radiation oncologist, radiation physicist and safety officer. Similar to intraoperative therapy the radiation team cannot apply its therapy until the initial operative procedure is complete. A diffuse restenosis in a stent can be a protracted procedure requiring the ablation of tissue and the placement of more stents. The cardiologist's job is to restore an adequate lumen to the vessel to supply symptom relief. Once, this is achieved (taking about 30'-120') the cardiologist places the treatment system in the appropriate location in the coronary. Some anatomic subsets can make this a challenge. Usually, however, source placement is predictable and quick. It is at that time the physicist and oncologist enter the room.

Treatments with current systems range from 3 to 25 minutes. However, prep time, QA and lab surveys add considerably to the time spent by the oncologist and physicist in the catheterization suite.

Because of vagaries of scheduling, procedure length and technical issues, it is difficult to schedule the entry of the "radiation team" into the treatment room on anything but a "more or less" basis. Thus the coordination of such an effort takes a dedicated team that is available to the patient on an "ad hoc" basis. While we and others try to minimize the disruption of the noncardiologists' schedules by planning a "treatment day", certain patients do not have the luxury of waiting for an elective slot. 
With an estimated 30-40,000 patients a year eligible for endovascular brachytherapy the coordination of these issues amongst the professionals on the team will present a challenge. In our experience, the physicist is the natural coordinator for the team on a daily basis.

Intravascular brachytherapy will be an important therapeutic tool over the next 1-5 years, and it behooves us to address our manpower needs now.

Educational Objectives: At the end of the presentation

1. The participants will be able to describe the magnitude of the problem of coronary restenosis

2. Will understand the pivotal role of intra-coronary brachytherapy in the treatment of this problem

3. Will understand the logistics and manpower needs for the institution of bracytherapy.

\section{MO-C1-02}

Review of Intravascular Brachytherapy Physics for Prevention of Restenosis

Howard Amols, Memorial Sloan Kettering Cancer Center, New York, NY

Intravascular Brachytherapy (IVB) is rapidly gaining acceptance as a new treatment modality for reducing restenosis and improving the success rate of PTCA. Some clinical studies report reductions in restenosis of up to $70 \%$. IVB confronts the medical physicist with an array of new problems including:

- Safely introducing high activity radioactive sources into the cardiac catheterization laboratory.

- Assessing the multitude of radiation delivery systems such as catheter based seeds and wires, radioactive stents, gas and liquid filled radioactive balloons, gamma versus beta isotopes, miniature $\mathrm{x}$-ray tubes, etc.

- Determining dose with sub-millimeter accuracy at distances $\leq 2 \mathrm{~mm}$ from a brachytherapy source.

- Utilizing treatment planning information from 'unfamiliar' imaging systems such as Intra-Vascular Ultra Sound (IVUS) and Angiography.

The dosimetric requirements for IVB can be summarized as follows:

- $\quad$ Single fraction dose of 8-40 Gray to a 2-5 cm length of arterial wall, approximately 2-4 $\mathrm{mm}$ inner diameter, $0.5-3 \mathrm{~mm}$ wall thickness.

- Minimize dose to normal tissues and to cath lab staff.

- Dose rate $>2 \mathrm{~Gy} / \mathrm{min}$ (to keep treatment time $<10$ minutes).

- Radioactive source design suitable for use with cardiac catheters.

We estimate the 'biological dose window' for IVB to be 8-40 Gy; with 8 Gy the minimum curative dose, and $40 \mathrm{~Gy}$ the normal tissue tolerance. Thus, the dose falloff from the radiation source must be no more than $40 \mathrm{~Gy} / 8 \mathrm{~Gy}$ over the thickness of the vessel wall. If the dose falloff is more severe then it will not be possible to deliver 8 Gy of radiation to the adventitia without exceeding 40 Gy to the lumen wall. This biological window (if true) is a key issue for IVB dosimetry.

These dose requirements are best met by a low energy $(<100 \mathrm{keV})$ photon emitter with activity $>1 \mathrm{Ci}$. This would provide the best dose fall off, and is easily shielded by a few millimeters lead. No such source currently exists. Thus, all IVB trials utilize alternative sources:

- $\operatorname{Ir}^{192}$ (7 de-excitation gammas with energies $\left.296-612 \mathrm{keV}\right)$.

- $\quad$ Beta minus emitters, such as $\mathrm{P}^{32}, \mathrm{Sr}^{90}, \mathrm{Y}^{90}, \mathrm{~W}^{188}, \mathrm{Re}^{188}$.

- One of several 'exotic' dose delivery systems.

$\mathrm{Ir}^{192}$ provides an ideal dose distribution, but the high gamma energy presents radiation safety concerns. Beta emitters simplify radiation safety, but may not provide adequate depth dose penetration. In a typical IVB geometry, dose decreases by approximately $35 \%$ per millimeter for $\operatorname{Ir}^{192}$ and by nearly $70 \%$ for $\mathrm{P}^{32}$. Beta safety versus gamma depth penetration defines the great 'gamma vs. beta debate' in IVB. The search for the 'ideal isotope' continues. Other more 'exotic' dose delivery systems are also being tested, such as:

- $\quad$ radioactive stents

- $\quad$ radioactive gas and liquid filled balloons.

- $\quad$ radioactive coated balloons.

- miniature $\mathrm{x}$-ray tube for catheter insertion.
- $\quad$ teletherapy photon beams.

- intravascular laser therapy.

Educational Objectives:

1. Discuss the clinical and biological criteria which determine radiation dose and isotope selection.

2. Discuss and compare different radiation delivery systems and isotopes being used to treat restenosis.

3. Discuss techniques for dose calculations and source calibration.

\section{MO-C1-03}

Treatment Planning for IVBT

Tim Fox, Emory University, Atlanta, Georgia

Prescription of radiation, in the early trials of ICRT, has been done in three distinctly different ways. In some trials, radiation has been prescribed at a fixed distance from the center of the source with the dose or distance being adjusted depending upon the reference vessel diameter. In other studies employing a balloon-centering device, the radiation has been prescribed at the balloon-lumen interface or at some depth from this structure. In addition, two trials have been completed where the investigators attempted to limit the maximum dose delivered to the media based on calculations of the delivered dose to a single near point in the treatment volume. However, none of these methods address whether the prescribed treatment will deliver the desired dose to the entire vessel wall. A goal of intravascular brachytherapy treatment planning is to enable the clinician to make a rapid, pre-treatment evaluation of the radiation dose delivered to the target structure and surrounding tissue and determine whether it is optimal.

Our institution has developed a real-time, three-dimensional (3-D) treatment planning system (iPlan ) for intravascular brachytherapy using intravascular ultrasound (IVUS) data. This systems allows the clinician to prospectively plan and evaluate the treatment delivered to the vessel wall using spatial dose distributions, dose volume histograms and figures of merit. The system allows various source delivery devices such as noncentered or lumen-centered source trains with ${ }^{90} \mathrm{Sr} / \mathrm{Y},{ }^{192} \mathrm{Ir}$ and ${ }^{125} \mathrm{I}$ seeds. Data will be presented which illustrates the use of treatment planning with clinical case examples from various clinical trials. In addition, a retrospective dosimetric comparison of beta versus gamma and centered versus non-centered will be presented using IVUS data from clinical trials. The use of figures of merit for ranking various delivery systems for a specific patient treatment will be described as an example of a rapid clinical decision-making tool. Finally, the usefulness of treatment planning in the clinical environment will also be addressed which correlates dose with treatment outcome. Intravascular treatment planning provides a powerful software system, which may ultimately lead to an improved and efficient treatment plan.

Educational objectives include:

1. Concepts and implementation of treatment planning for intravascular brachytherapy.

2. Dosimetric comparisons of different source delivery systems using patient IVUS-data from IVBT trials.

\section{MO-C1-04}

President's Symposium: Intravascular Brachytherapy: An Emerging Role for the Medical Physicists

Robert Ayers, U. S. Nuclear Regulatory Commission, Washington, DC

The new and escalating interest in investigating the applicability of using ionizing radiation therapy to reduce or eliminate restenosis in coronary and peripheral arteries after angioplasty has led to the continuing development of a varied number of innovative approaches. Most of these involve the use of byproduct material(s) as the source of the ionizing radiation. As such, the use of these materials require licensure by either NRC or an Agreement State. As might be expected, the requirements for the NRC licensure for participation in these varied investigative studies of intravascular brachytherapy are, of necessity, being developed on a case by case basis as each of these evolving intravascular catheter based systems often require unique radiation health and safety considerations. For example, for simple stent systems with microcurie quantities of implanted beta particle emitters, the necessary radiation protection measures required to protect both the health care providers and the patient from unwarranted and potentially 
dangerous radiation exposures are quite basic and easily implemented. On the other hand, high dose rate afterloading systems, particularly those using sources emitting penetrating gamma radiation, can pose very serious radiation hazards to not only the patient and health care providers, but to members of the public if control of this source is lost for even a short time.

Despite the unique characteristics of each of these device based protocols, using sealed sources containing byproduct materials, there are also several uniform requirements required by the NRC for authorization (licensure) to participate in these investigative studies. These are:

1. The requirements of 10 CFR 35.6, "Provisions for research involving human subjects," must be met (For intravascular brachytherapy this requirement is met by obtaining the required Food and Drug Administration investigational device exemption (IDE) application approval);

2. Only those physicians meeting the training and experience requirements set forth in 10 CFR 35.940, "Training for use of brachytherapy sources," can be designated as authorized users for these procedures and, in nearly every study, a medical physicist is a required member of the team; and,

3. The radiation sources and/or devices used in the research must have undergone an appropriate sealed source and/or device review(s) and be listed in the Registry of Radioactive Sealed Sources and Devices as approved for use in intravascular radiotherapy (Note: Medical licensees of broad-scope are exempted from this requirement and can participate in human trails using unreviewed and unregistered sealed sources and devices).

The present NRC requirements for medical physicist participation in these studies will be discussed and compared to the forthcoming requirements in the new 10 CFR Part 35 regulations. NRC's present training and experience requirements for medical physicists will also be presented and contrasted with the new 10 CFR Part 35 requirements.

The presentation will conclude with a brief overview of misadministrations and other events that have occurred during these intravascular brachytherapy trials and NRC response to these events.

\section{MO-C1-05}

Clinical Results in Vascular Brachytherapy

Ian Crocker, Emory University, Atlanta, GA

The potential usefulness of vascular radiotherapy to prevent coronary restenosis has rapidly developed from positive preclinical studies carried out in animals in the 80's and 90's to a large number of clinical trials which are underway in both United States and Europe in 1999. Trials are currently underway which encompass varying treatment techniques (Temporary and Permanent Implants), varying source preparations (sealed and non-sealed sources), various isotopes and a variety of delivery methods. At the time that clinical trials were initiated there was little information available as to the mechanism of radiation in preventing restenosis. This situation unfortunately continues today. In this presentation I will review some of the principals of vascular brachytherapy elaborated in the early pre-clinical trials. Newer preclinical information regarding the effect of radiation on healing, thrombosis, and newer source preparations will be presented.

As mentioned previously there are a large number of clinical trials underway in the United States and Europe which are mostly sponsored by Device Companies. Three double blind randomized trials of catheter based therapy using 192 Iridium have been reported showing 50-70\% reductions in restenosis rates and clinical events. Similarly positive results compared with historical controls have been reported with catheter based beta emitting sources $(90 \mathrm{Sr} / \mathrm{Y}, 32 \mathrm{P})$. In contrast to the positive results of catheter based irradiation we are seeing discouraging results with the radioactive stent with the description of "the candy-wrapper effect" with higher activity stents.

In general radiation has been well accepted by the patients and the cardiology community. The future of brachytherapy looks promising with newer more sophisticated devices coming to the marketplace. Incorporation of treatment planning and increasingly sophisticated treatment techniques may allow further reductions in restenosis. It is my belief that we are in the early phase of the development of vascular brachytherapy much as we were
40 years ago with the first megavoltage treatment machines. Furthermore the possibility that external radiation treatment may come to be used in the prevention of restenosis is certainly within the realm of possibility. I believe that the Radiation Oncologist brings to this field a unique level of expertise and should continue to play a role in the therapy of vascular disease with radiation over the long-term.

Educational Objectives:

1. Familiarize the audience with the benefits of the application of radiation in preventing restenosis in animals models including various devices and treatment techniques.

2. Familiarize the audience with the various clinical studies involving radiation in preventing restenosis

3. Familiarize the audience with new developments in the field of vascular radiotherapy

\section{Young Investigators Symposium Room: Pearl}

\section{MO-D1-01}

In Vitro and In Vivo Investigation of Artifacts in 3D Computed Rotational Angiography

R. Fahrig ${ }^{1,2}$, S. Lownie ${ }^{3,4}$, A.J. Fox ${ }^{3}$, D.W. Holdsworth ${ }^{1,3}$, (1) The J.P. Robarts Research Institute, (2)Dept. of Medical Biophysics, University of Western Ontario (3)Dept. of Diagnostic Radiology, London Health Sciences Center, (4)Dept. of Clinical and Neurological Sciences, London Health Sciences Center, London, Canada

Several groups have recently produced $3 \mathrm{D}$ reconstructions from 2D projections acquired during rotation of a C-arm-mounted XRII for use during interventional procedures (ie. endovascular treatment of aneurysms using platinum coils). We produce reconstructions using a modified Siemens Multistar (130 projections covering $200^{\circ}$ in $4.5 \mathrm{~s}$, [85 kVp, 371 $\mathrm{mA}, 3 \mathrm{~ms}$ ] per projection). Projections are corrected for angle-dependent distortion (within \pm 0.1 pixels), non-idealities in gantry motion (within \pm 0.12 pixels) and pixel-to-pixel sensitivity variation, and reconstructed using modified conebeam backprojection. Vessel signal-to-noise ratio in these volumes is limited by artifact from view aliasing and from time-varying opacification of vessels. We investigated the relative magnitudes of these noise sources using a rigid in vitro pulsatile flow-through model of the intracranial vasculature. The signal-difference-to-noise ratio (SNR) was measured as a function of injection rate $(2-5 \mathrm{ml} / \mathrm{s})$, flow rate $(8-14 \mathrm{ml} / \mathrm{s}$ total), contrast dilution ( 300 and $150 \mathrm{mg} / \mathrm{ml})$, and selective injection site. An injection rate of $3 \mathrm{ml} / \mathrm{s}$ provides SNR $>25$ for all combinations of parameters investigated. Vessel diameters were within $\pm 6 \%$ of the specified phantom values. SNR remains high despite noise due to view-aliasing and time-varying opacification, and geometric accuracy is preserved in 3D CRA reconstructions. In Vivo experiments in a porcine model demonstrated similar SNR even in the presence of respiration and normal cardiac pulsatility.

The research described in this abstract was supported by a grant from Siemens Medical Systems.

\section{MO-D1-02}

Simulation of CT Scanner Geometry and Physics using Monte Carlo Methods

C Cagnon*, M McNitt-Gray, J DeMarco, UCLA Medical Center, Los Angeles, CA

The purpose of this project is to model an X-Ray CT scanner and simulate its operation to investigate the effects of various physical parameters on image quality.

A Monte Carlo-based simulation software package (MCNP4b, Los Alamos $\mathrm{NL}$ ) was modified and utilized to model a polychromatic, phase space sampled X-ray source, a symmetrical test object and a single plane of detectors. Initial investigations were made using a fifth generation CT scanner (ElectronBeam CT- Imatron). A symmetrical model was utilized to reduce computational time necessary by reducing the number of projections required. MCNP was used to statistically model the X-ray production and transport of photons through a series of simulated test objects. A phasespace model of the $\mathrm{x}$-ray source was constructed to improve geometric 
efficiency. Ideal detectors captured all photons reaching them. From simulated air calibration and test object scans, attenuation projection calculations were performed. Exploiting the symmetry of the system, the sinogram was filled and vendor reconstruction software was used to form an image.

Preliminary images of homogeneous and heterogeneous symmetrical phantoms have been obtained examining the effects of beam energy, scatter rejection, and phantom attenuation on reconstruction CT number.

These preliminary results are very encouraging. Further developments will allow us to reduce our simulation times and to investigate non-symmetric geometries. These will allow more detailed investigations into the physical effects of beam filtration, scatter rejection, patient size/thickness effects, etc., on image quality and their quantitative impact on $\mathrm{CT}$ number.

\section{MO-D1-03}

Validation of a Computerized Method for the Diagnosis of Mammographic Lesions

Z Huo*, M Giger, C Vyborny, C Metz, D Wolverton, University Chicago, Chicago, IL

Purpose: Robust performance of a computerized method is crucial for its implementation in a clinical CAD system. This study is to validate the performance of a computerized method for the classification of benign and malignant mammographic lesions.

Materials and Methods: A computerized method was developed on a database consisting of 39 malignant and 26 benign cases. The method includes 1) automated segmentation, 2) automated feature-extraction, and 3) automated classification using an artificial neural network. An observer study using this database showed that the method can perform significantly better than average general radiologists in the task to differentiating between malignant and benign masses. In this study, the performance of the method was independently evaluated on 50 malignant and 60 benign cases in terms of $\mathrm{A}_{\mathrm{Z}}$ and partial $\mathrm{A}_{\mathrm{Z}}$ from ROC analysis. The independent database was digitized with two film scanners. Both the effects of variations in case selection and in film digitization techniques on the performance of the method were assessed.

RESULTS: The method achieved an $\mathrm{A}_{\mathrm{Z}}$ of 0.90 and a partial $\mathrm{A}_{\mathrm{Z}}$ of 0.40 on the training database in a round-robin evaluation, and an $A_{Z}$ of 0.82 and partial $\mathrm{A}_{\mathrm{Z}}$ of 0.43 on the independent database. These differences in the performances were found not to be statistically significant ( $p$-value $\geq 0.1$ ). CONCLUSION: Our method is proved to be robust to both case and filmdigitization variations, and is ready for preclinical evaluation.

\section{MO-D1-04}

A Bo Shift Correction Method Based on Edge RMS Reduction (ERMSR) and Comparison with other Correction Strategies in EPI fMRI.

P Kochunov*, H Liu, J Lancaster, T Andrews, P Fox, J Gao, Research Imaging Center, $\mathrm{U}$ of Texas Health Science Center at San Antonio, San Antonio, TX

fMRI measurements of subtle signal changes are very sensitive to system instabilities. One effect of system instability for EPI fMRI is position shift of the image in the phase encoding direction resulting from a shift of the central frequency. The most common method for solving this problem uses linear interpolation to correct for changes of the center of mass (COM). Navigator echoes have been used to correct signal fluctuations and subject motion for conventional gradient-echo fMRI, and image shift for EPI sequences. Unfortunately, one has to sacrifice the extent of k-space in the phase encoding direction (reduced as much as $10 \%$ ) for navigator echo implementation. A new, efficient shift correction algorithm based on reduction of RMS error is proposed. The purpose of this study was to evaluate the performance of the algorithm and compare its effectiveness to other strategies. We performed stability tests for EPI imaging with a convection free dimethyl silicone fluid phantom and a human brain study targeting superior motor cortex. In all tests ERMSR method shows performance comparable to the navigator echo method, without loss of spatial resolution. Correction by ERMSR method is superior to and faster than COM method with shift performed by Fourier shift theorem. It was shown that COM method with linear interpolation was not acceptable for the fMRI study due to severe interpolation artifacts. 
MO-D1-05

\section{Detection and Correction of Organ Size Variations in Tomography}

W Lu*, M Chen, E Fitchard, G Olivera, K Ruchala, F Dong, T Mackie, University of Wisconsin-Madison, WI

Intra-scanning organ size variations, such as respiratory or cardiac motion, cough etc, result in various artifacts including blurring and streaks in clinical imaging of subjects based on reconstruction from projections. In intensitymodulated radiation therapy, especially in tomotherapy, the interplay of the movement of the beam aperture and variations of the organ size during delivery can create "hot" and "cold" spots throughout the field. Thus detection and correction of organ size variations is essential in tomography imaging and tomotherapy dose reconstruction.

Tomographic sinograms encode not only the patient anatomy information, but also the information of the organ motion during scanning. In this work, we present an algorithm, which detects the organ size variations directly in the sinogram space and reconstruct a motion free image from the corrected sinograms. The organ size variations are modeled as two-directional timevarying scaling. The effect of that motion encoded in sinograms is discussed. Based on the traces of some nodal point(s) in the sinograms, the organ size variations during the scanning are determined. A motion correction algorithm on the sinograms is presented. The corrected sinograms are used for reconstruction by the "filtered backprojection" method.

Some preliminary simulation results are presented. The encoded motion information can be successfully detected from the sinograms. The reconstructed images from the corrected sinograms have greatly reduced artifacts.

The method can be applied to projection data used in CT, SPECT, PET and MRI, as well as in tomotherapy setup verification and dose reconstruction.

\section{MO-D1-06}

Megavoltage CT Imaging Using the Incomplete Data Collected During Modulated-Treatment Delivery

K Ruchala*, G Olivera, T Mackie, University of Wisconsin-Madison, WI

Among the benefits of tomotherapy is that it provides for vast improvements in treatment verification. One such verification processes is megavoltage $\mathrm{CT}$ (MVCT), which is the acquisition of a CT image of the patient using the radiation therapy beam.

Several groups have studied MVCT in the context of pre-treatment patient positioning and anatomical verification, typically by adding a detector system to a C-arm linac. Unfortunately, these systems are typically slow, and require a high delivered dose to the patient. However, the variable dose rate, and faster rotational speed of the UW Tomotherapy machine make it more amenable to fast, efficient, low-dose pre-treatment scanning.

Moreover, since tomotherapy treatments are delivered rotationally in slices, it may be possible to generate MVCT images of the patient DURING the radiotherapy treatment. The benefit is that these images not only can depict the patient at precisely the time of treatment, but that they utilize the much higher doses delivered during radiotherapy, giving them significantly better contrast resolution. A key imaging problem is that the treatment conformally irradiates the tumor while avoiding sensitive structures, meaning that the sinogram data set is fundamentally incomplete.

We have found that it is feasible to use the data collected during radiotherapy to reconstruct MVCT images that have improved contrast relative to standard pre-treatment MVCT imaging. This paper regards the algorithms for reconstructing MVCT images from the limited data sets collected during treatment, and the capabilities of this technique.

\section{MO-D1-07}

Monte Carlo Calculated Beamlets for Photon Beam Inverse Planning T Pawlicki*, S Jiang, J Deng, J Li, C Ma, Stanford University School of Medicine, Stanford, CA

Current dose calculation techniques, like the finite size pencil beam (FSPB) algorithm used in some commercial inverse planning systems, exhibit systematic errors within heterogeneous regions of a patient. Monte Carlo dose calculation based on the fluence map determined using the FSPB approach shows dose differences up to $7 \%$ in the target and as large as $100 \%$ in adjacent critical structures in some "optimized" treatment plans. In this work, we have developed a method to calculate Monte Carlo based beamlets specifically for inverse planning. Beamlet dose distributions that contribute to the PTV are "turned-on" for pre-optimization dose calculation using the EGS4/MCDOSE code. A multiple source model is used to accurately reconstruct the beam phase space. The complete 3D dose deposition coefficient of each beamlet is scored. The CPU time to simulate a single 1 $\mathrm{cm} \times 1 \mathrm{~cm} 4 \mathrm{MV}$ beamlet is about 20 seconds on a Pentium Pro $200 \mathrm{MHz}$ PC. Due to the differences between Monte Carlo and FSPB calculated beamlet profiles (near patient heterogeneities); the weights of those beamlets going through the heterogeneous regions in a patient may differ significantly. Our preliminary results of calculations for head and neck treatments show significant improvement in dose distribution conformity using Monte Carlo calculated beamlets over that using FSPB. It is expected that the accuracy in the dose distribution will be reduced to about $3 \%$ of the maximum dose, anywhere in the phantom.

\section{MO-D1-08}

Independent, Real-Time Verification of Dynamically Shaped Intensity Modulated Radiotherapy (IMRT) using an Amorphous Silicon (a-Si:H) Detector Array

T Paul*, T Solberg ${ }^{1}$, M Leu, P Rosemark ${ }^{2}$, J Smathers ${ }^{1}$, U Mollenhauer ${ }^{3}$, N Agazaryan ${ }^{1}$, (1)Dept. of Radiation Oncology, UCLA Medical Center, Los Angeles, CA, (2)Dept. of Radiation Oncology, Cedars-Sinai Cancer Center, Los Angeles, CA, (3) Wellhöfer Dosimetrie, Schwarzenbruck, Germany

A method to verify dynamic delivery of IMRT has been developed. Leaf positions are verified in radiotherapy images acquired with an amorphous silicon (a-Si:H) detector array during patient treatment. An MLC leaf sequence file is generated from the output of the inverse planning process. The user specifies if the treatment is to be delivered dynamically or in a step-and-shoot fashion. At predetermined intervals (cumulative MU), the prescribed leaf positions are read from the MLC leaf sequence file. Images are acquired using an a-Si:H imaging system at user specified intervals during treatment. Monitor chamber readings are digitized and counted using a 16 bit counter; images are time stamped with the delivered cumulative monitor units. Leaf edge positions are found by application of an approximated 2-D laplacian operator and compared to those prescribed in the leaf sequence file. Finally, audiovisual leaf position feedback is provided when leaves exceed tolerance limits.

\section{MO-D1-09}

A Generalized Phase Space Source Model Incorporating Efficient Sampling Algorithms for Clinical Treatment Planning using the Monte Carlo Method

I Chetty*, J DeMarco, T Solberg, UCLA, Los Angeles, CA

We have developed a phase-space source description of the linear accelerator head for simulating arbitrary intensity distributions, using the Monte Carlo code, MCNP4B. The novelty of the phase space source is its ability to simulate irregular field shapes without the inefficient step of transporting particles through the field defining collimators. This was previously impractical, as earlier source descriptions were adequate only for square field dosimetry studies. The source model has been interfaced with a commercially available treatment planning system to generate a Monte Carlo-based treatment plan. The interface reads in a patient-specific MLC shape and creates a cumulative distribution function for sampling particle fluence. Fluence distributions were acquired by first simulating the components of the linac treatment head using the code MCNP4B. A particle's direction is sampled using an azimuthally symmetric point-source approximation. Extra-focal radiation and electron contamination are also accounted for by sampling EGS4-derived sub-sources to characterize each of these components. Depth dose and profile benchmarks show the phasespace source to be within $2 \%$ of measurement for field sizes from $5 \times 5 \mathrm{~cm}^{2}$ to $15 \times 15 \mathrm{~cm}^{2}$. Irregular field calculations for various gantry, collimator and table angles show that an arbitrarily shaped distribution is accurately sampled. Finally, effective sampling algorithms, including the cut-point method (for sampling a discrete probability distribution) and the deltascattering photon transport scheme, are implemented to increase the efficiency of the Monte Carlo calculation by a factor of five over conventional sampling techniques. 


\section{MO-D1-10}

Matching Photon and Electron Fields with Dynamic Intensity Modulation

J Li*, L Xing, A Boyer ${ }^{1}$, R Hamilton, D Spelbring, J Turian², (1)Stanford University, Stanford, CA, (2)University of Chicago, Chicago, IL

An iterative algorithm was developed to optimize composite plans of conventional electron fields with intensity modulated photon fields. For a given patient, the algorithm generates the optimal weights of the electron fields and the beam profiles of the photon fields. In its simplest application, the method can be used to reduce the size and magnitude of the hot/cold spots in the abutting region of photon and electron fields. The dynamic MLC serves as a photon penumbra generator, effectively broadening the photon penumbra and complementing it with the electron penumbra at a preselected depth or in the whole target volume. Both computer calculations and film measurements for matching a 6-MV photon beam with a 9-MeV electron beam indicated that when an exact match is created at a specified depth, dose uniformity is improved at the specified depth and beyond compared with unmodulated photon beams. A slight increase in dose inhomogeneity is seen at shallower depths. The overall areas of the hot and cold spots were significantly reduced. The method also reduced the sensitivity of dose homogeneity to patient setup errors. The technique was used to optimize an electron/photon combined treatment plan for a follicular lymphoma involving the right pleura. Good tumor coverage was obtained with significantly reduced dose to the critical structures when compared to a regular 7-field IMRT plan. The technique has also been applied to the breast and head and neck treatment with favorable results.

\section{MO-D1-11}

\section{Beam Orientation Optimization in Intensity Modulated Radiation} Therapy

A Pugachev*, L Xing, A Boyer, Stanford University, Stanford, CA

The choice of the beam orientations for intensity modulated radiation therapy (IMRT) influences not only the quality of the treatment plan, but also the number of fields required. An optimization of beam directions allows us to utilize fully the technical capacity of IMRT. The computational difficulty arises from the fact that the beam orientations are strongly correlated with the beam profiles. An effective algorithm for beam orientation optimization has been investigated. This algorithm separates the variables into two groups, the gantry angles and the beamlet weights. The gantry angels are sampled according to a simulated annealing algorithm. For each beam configuration, the beamlet weights are determined using either a fast simulated annealing or a modified filtered backprojection algorithm (the conventional filtered backprojection method is modified to include the importance factors of the target and sensitive structures). Minimization of an objective function with respect to gantry angles and beamlet weights results in the optimal solution. Both methods lead to the same results but backprojection is an order of magnitude faster and thus is more suitable for the application. Several two-dimensional cases were studied. A comparison with equally spaced beams and other beam configurations based on beameye's view suggests that this algorithm can lead to significantly improved dose distributions and has strong potential for clinical IMRT treatment planning.

\section{Symposium Advancements in Breast Imaging}

Room: Carroll

\section{MO-D4-01}

\section{Computer Simulation of Breast Cancer Screening Efficacy}

John Boone, Karen Lindfors, University of California Davis Medical Center, Sacramento, California

Recent controversy over the age at which a women should begin mammographic screening, and at what screening interval, has underlined the need for better understanding in this area. Screening efficacy is traditionally studied using large scale clinical trials, an expensive and time consuming procedure. Furthermore, at a time of rapid technological development as mammography has experienced recently, the results of long clinical trials (such as the Canadian study) are difficult to interpret because the results are averaged over both new and older technologies. To complement clinical trials, we propose the use of computer simulation techniques to better understand screening efficacy, and to identify salient factors which are important in improving efficacy.

Computer simulation of breast cancer screening requires the development of several different sub-components to the model. The principal subcomponents are: (1) the growth rate (and variability in the growth rate) of breast cancer, from a single cell to a macroscopic, detectable tumor, (2) the age-dependent probability of developing breast cancer, (3) the demographics (number of women versus age) of the population being screened, (4) the post-diagnosis prognosis, defining average survival versus time after diagnosis as a function of lesion diameter (etc.) at diagnosis, and (5) the detectability of breast cancer as a function of its size and other variables of the breast (breast density, breast thicknesses, role of calcifications, etc.). Many other factors are involved as well, including death-rate statistics, breast self examination (BSE) efficacy, ethnic variables, radiation risks, etc.

Once all the sub-components of model are mathematically parameterized, they are combined with Monte Carlo techniques to produce the screening model. When run, the model generates the life histories of "women", one by one, and statistics can be developed by running the model over millions of "women". As on overlay onto the model, different breast cancer screening methodologies can be incorporated. Mammography is the focus here, but other breast cancer screening tests such as blood tests, BSE, and MRI could be studied using similar techniques. The screening model can be re-run over and over, studying the effect of screening women starting at ages 50, 45, 40, 35 , for example, and studying the effect of the screening interval as well. Because the exact same study population can be run through numerous lifespans with different screening strategies, the years-of-life-saved for each women can be tallied, and this forms the basis for evaluating screening efficacy.

The talk will discuss the overall approach towards computer modeling epidemiological data, and the details of each of the specific sub-components under development will be presented. Validation issues will be discussed. Results derived from the screening model will be presented which demonstrate the utility of the proposed methodology as a tool in breast cancer research. This work is still preliminary and the results need to be carefully verified; therefore, specific recommendations concerning breast cancer screening strategies will not be made.

Educational Objectives:

1. To introduce participants to an analysis technique which may led to a better understanding of breast cancer screening, and how different parameters influence its efficacy.

\section{MO-D4-02}

Full-Field Digital Mammography and Computer-Aided Diagnosis

Robert Nishikawa, Laura Yarusso, The University of Chicago, Chicago, IL

Two of the most important technologies for improving breast cancer detection and diagnosis are full-field digital mammography and computeraided diagnosis. Over the past 15 years, these 2 technologies have been developed independently, while acknowledging the natural synergy that exist between the two. With one CAD system having received FDA approval and several FFDM systems in the approval process, it will not be long before FFDM with CAD capabilities become a clinical reality. In this talk, I will describe existing FFDM systems and new detector technologies on the horizon. I will also give an overview of the state-of-the-art in CAD systems and active areas of research. I will end by describing the potential of combining these two technologies, including a discussion on pixel size required for a combined system.

Financial Disclosure

Robert M. Nishikawa is a shareholder in R2 Technology, Inc. (Los Altos, CA).

Education Objectives:

1. To learn about the latest advances in full-field digital mammography (FFDM) technology.

2. To learn about the latest advances in computer-aided diagnosis (CAD).

3. To learn about the potential of combining CAD and FFDM. 
MO-D4-03

\section{Diffraction Enhanced Mammography}

Dean Chapman, Illinois Institute of Technology, Chicago, IL

Recently, a x-ray imaging technique has been developed that exploits x-ray refraction and scattering as sources of image contrast in addition to absorption, the conventional source of radiographic contrast. This technique, Diffraction Enhanced Imaging (DEI) ${ }^{1}$, uses perfect crystals of silicon to prepare and "analyze" a highly collimated x-ray beam from a synchrotron source. DEI allows for independent images of the object's $x-$ ray refraction gradient and absorption to be obtained. The refraction gradient images are sensitive to edge structures in the object and give a natural edge enhanced image. The absorption images include an enhanced contrast arising from the very high scatter rejection afforded by the perfect crystal analyzer (extinction contrast).

DEI and conventional radiographs of phantoms, test objects, animals, and tissue samples have been acquired and will be presented as examples of the applications of the technique. The primary focus of the presentation will be on images of breast tissue samples with cancer, a project to optimize DEI parameters, and the prospects of developing a laboratory or clinical source.

Educational Objectives:

The attendee will be instructed on:

1. the experimental setup and parameters to apply DEI.

2. $\mathrm{x}$-ray refraction as a source of radiographic contrast (refraction gradient contrast).

3. ultra-small angle scattering as a source of radiographic contrast (extinction contrast)

4. how to uniquely determine the refraction gradient image from the combined absorption and extinction image.

5. how the DEI images are affected by setting of the analyzer angle, the imaging energy, and the order of the crystal reflection.

6. how DEI images are useful in the possible detection of cancer in breast tissue.

7. how DEI images may be used in other areas of radiography

${ }^{I}$ Diffraction enhanced $x$-ray imaging, D. Chapman, W. Thomlinson, R.E. Johnston, D. Washburn, E. Pisano, N. Gmur, Z. Zhong, R. Menk, F. Arfelli, D. Sayers, Physics in Medicine and Biology 42 (1997) 2015-2025.

\section{MO-D4-04}

Elasticity as a Contrast Mechanism for Breast Imaging

Donald Plewes, Department of Medical Biophysics, University of Toronto, Sunnybrook and Women's College Health Science Centre, Toronto, Ontario, Canada

X-ray attenuation, proton NMR relaxation times, ultrasound attenuation and acoustic impedance generally exhibit only small variations between normal breast tissue and cancer. Accordingly, detection and diagnosis of breast small cancers can be challenging in young women or those with dense breasts. While contrast enhanced MRI has been shown to be a highly sensitive method of breast cancer detection, its specificity is generally less than optimal and widely variable depending on the patient population. Alternatively, a number of researchers are exploring alternative contrast mechanisms which might offer new insights to tissue histology based on physiologic properties or morphology to overcome specificity limitations. One interesting candidate is the use of tissue biomechanical properties. This is motivated by the fact that palpation of breast disease is known to be an effective means of cancer detection for large, superficial lesions. Furthermore, in-vitro studies have shown that the Young's modulus of many soft tissues span several orders of magnitude, and therefore offers the potential for extremely large soft tissue contrast. However, while palpation is simple, it is necessarily qualitative and insensitive to small tumours. Imaging techniques which visualize and quantify the spatial distribution of tissue elasticity are collectively referred to as Elastography. In general, these methods involve the use of some form of subtle mechanical tissue perturbation in conjunction with a method of measuring the resulting tissue movement with motion sensitized imaging. Through an appropriate analysis of this motion, tissue elasticity can be determined. The original research into this area was performed with Ultrasound which continues to be a primary imaging method for Elastography. In this case, motion sensitivity is achieved through Doppler flow methods, speckle-tracking or RF crosscorrelation techniques. Mechanical motions include slow compression and transverse vibrational excitations with frequencies of 50-1000 Hz. Similarly, MRI Elastography has been performed with similar mechanical excitation schemes but using phase contrast or spin tagging methods for motion detection. In the case of slow compression methods, strain imaging has been shown to provide visualization of tissue modulus distribution and shown to be effective for small modulus variations and modest strains. Elastography based on transverse excitations, image the propagation of shear waves throughout tissue which propagate at velocities related to the tissue shear modulus. In general, visualizing tissue modulus requires some form of image processing while quantitative measurement of tissue modulus requires three dimensional inverse methods. In this presentation, we will review the range of tissue biomechanical properties which are seen in breast disease, outline the physics of Elastography techniques, review issues in Elastography inverse problems and summarize clinical experience to date as applied to breast imaging.

\section{Scientific Session Stereotactic Radiotherapy}

Room: Pearl

\section{MO-E1-01}

Dynamic Shaped Beam Radiosurgery: A Dose-Volume Study

R Fogg* ${ }^{1}$, T Solberg ${ }^{2}$, M Selch ${ }^{1}$, A De Salles ${ }^{3}$, (1) Department of Radiation Oncology, University of CA, (2) Dept of Radiation Oncology, UCLA Medical Center, Los Angeles, CA (3) Division of Neurosurgery, University of Califorania, CA

Recently we commissioned a stereotactic radiosurgery system capable of dynamic field shaping combined with arc delivery. The system consists of a micro-multileaf collimator (mMLC) integrated into a dedicated $6 \mathrm{MV}$ linear accelerator. Leaf positions are obtained from the treatment planning system at ten-degree intervals along the arc through beams-eye-view projections of the target volume. Linear interpolation is performed between leaf segments to allow continuous motion of the leaves and gantry. To determine the clinical efficiency of this approach, we compared dose-volume histograms from conventional arc and conformal (stationary gantry) radiosurgery with dynamic arc. The mean normal tissue dose was determined, and a conformity index (CI), defined as the ratio of the whole brain volume enclosed by the prescribed isodose to the planning target volume, was calculated for various irradiation configurations.

Multiple isocenter radiosurgery can minimize peripheral brain dose at the expense of increasing target dose heterogeneity. With conformal radiosurgery, peripheral brain dose decreases as additional beams are added. For irregularly shaped targets, use of dynamic field shaping significantly reduces whole brain dose compared with static shaped fields, while preserving the target dose homogeneity of single isocenter plans. Dosevolume histogram analysis shows that the dynamic arc approach is superior to the other two in terms of minimizing peripheral brain dose while preserving target dose homogeneity. In addition, the treatment planning tools and delivery technique make dynamic field shaping significantly more efficient than conventional means of stereotactic irradiation

\section{MO-E1-02}

Stereotactic Conformal Fixed Field Radiosurgery/therapy: Verification of a Dose Calculation Algorithm

C Audet*, L Watts, B Clark, B.C. Cancer Agency, Vancouver, British Columbia, Canada

The stereotactic irradiation of irregularly shaped intracranial lesions may be performed with conformal fixed fields to provide a high dose volume that conforms to the lesion and spares healthy tissue. In this work a dose calculation algorithm for a commercial conformal stereotactic fixed field system is verified. The verification 1) involves comparing calculated isodose lines and absolute point doses with the ones measured using film, and 2) is done for a complete six field treatment involving slightly irregular field apertures as well as for a six individual fields with apertures of varying shape, size and irregularity. There is good agreement between most of the measured and calculated data, however, the algorithm overestimates the dose in the small protrusions possessed by some of the field aperture shapes (i.e., $\sim 20 \%$ at the tip of a $5 \mathrm{~mm}$ protrusion). The dose at a point within a protrusion is probably overestimated because the algorithm does not factor 
in the effect of the penumbra from adjacent edges of the protrusion. Also, for conformal fixed field systems involving poured blocks, the algorithm underestimates the low dose end of the penumbra by not accounting for the $\sim 6 \%$ transmission of radiation through the Cerrobend. The transmission is misrepresented because the relative dose profiles used in the algorithm were measured for circular titanium alloy collimators that transmit relatively little radiation. Use of the conformal fixed beam system with the limitations in the dose calculation algorithm is discussed.

\section{MO-E1-03}

Radiosurgery Treatment Planning Optimization via Mixed Integer Programming

T Fox ${ }^{1}$, E Lee $^{2}$, L Davis ${ }^{1}$, I Crocker $^{1}$, (1) Emory University, Atlanta, GA, (2) Georgia Tech, Atlanta, GA

An expert system has been developed which automatically generates optimized LINAC radiosurgery treatment plans using a mixed-integer programming (MIP) engine in a fraction of the time typically needed in planning. The physician prescribes dose constraints for both normal tissue structures and tumor volumes. An objective function such as minimizing the dose to normal tissue or maximizing dose to the tumor volume may be selected. The expert system is then capable of automatically (i.e. no human intervention) generating a treatment plan, including the determination of the number and location of isocenters, beam orientation, beam size and beam intensity. The expert system also allows the planner to impose constraints on physical planning parameters such as the maximum number of target points and maximum number of arcs to ensure that the optimal plan will be sufficiently easy to implement in clinical practice. This study evaluates the resulting treatment plans from our expert system versus human expert planners. Two patient cases are presented in this study: 1) tumor volume adjacent to a critical structure and 2) critical structure embedded in a normal tissue structure. Spatial dose evaluation, dose-volume histograms and figures of merit are used to analyze the computer-generated and manual plans. These initial patient cases indicate that the expert system has an excellent potential of becoming an effective component for conventional radiosurgery treatment planning and delivery.

\section{MO-E1-04}

Collimator Output Factors for Leksell Gamma Unit Based on the Measurement Using a Diode Detector and Monte Carlo Simulation Z Zheng ${ }^{1 *}$, D Shearer ${ }^{1}, J_{\text {Shi }}^{2}$, G Norén ${ }^{1}$, P Chougule ${ }^{1}$, M Epstein ${ }^{1}$, Rhode Island Hospital, Providence, RI, (2) Sun Nuclear Corporation, Melbourne, FL

Although $4 \mathrm{~mm}$ collimator has been used in the Gamma Knife radiosurgery since 1983, its output factor relative to the $18 \mathrm{~mm}$ collimator is still a controvertial issue. Measurement in different Gamma Knife centers yields a result ranging from 0.780 to 0.900

In this investigation, we design a miniature diode detector which fits exactly into the standard $16-\mathrm{cm}$ diameter polystyrene phantom. The effective volume of the diode detector is about $0.04 \mathrm{~mm}^{3}$, with the maximum linear dimension no bigger than $1 \mathrm{~mm}$. Its ionization response to the radiation for the 14,8 , and $4 \mathrm{~mm}$ collimators relative to that for the $18 \mathrm{~mm}$ collimator is $0.978,0.944$, and 0.896 , respectively. We did a Monte Carlo simulation of the diode detector to obtain the absorbed dose output factors and the electron spectra traversing the silicon diode. The output factors obtained in the computer simulation agrees with the measured ones within $2 \%$.

The same Monte Carlo simulation codes were applied to an imaginged detector made of water and immensed at the center of a $16-\mathrm{cm}$ diameter water phantom. The output factors obtained are $0.978,0.943$, and 0.847 for the 14,8 , and $4 \mathrm{~mm}$ collimators, respectively, compared with $0.985,0.952$, and 0.800 suggested by the manufacturer.

\section{MO-E1-05}

GammaPlan - Leksell Gamma Knife Radiosurgery Treatment Planning Verification

S M Marcu*, Q J Wu, K Pillai, C H Sibata, T J Kinsella, Case Western Reserve University, Cleveland, $\mathrm{OH}$

Independent check calculations are always used for teletherapy to minimize treatment errors. Such checks have not been reported for Gamma Knife radiosurgery. This work intends to fill this gap.

Localization: First, the skull is reconstructed using measurements from several locations on the patient's skull. Next, a computer model simplifies the skull as a partial sphere and then calculates its radius and center relative to the center of the head frame. Dose calculation: An analytical solution is developed for fast ray tracing of the path length for each of the 201 beamlets of the helmet collimator, in contrast to the GammaPlan which uses an iterative interpolation process. The dose rate at the isocenter of a single shot is adjusted for collimator factor and attenuation, with special plugs being taken into account. Multi-shot treatment: For any arbitrary point, the dose rate is derived from isocenter values by off-axis profile, collimator factor, and relative weighting. This algorithm also accounts for special plugging of individual helmet holes.

Compared to the GammaPlan for spherical phantoms, the calculated dose rates and point doses are within $0.5 \%$. For patients, the dose at any point for multi-shot treatments agrees to within $3 \%$ and agrees up to $5 \%$ for a single shot treatment. The same accuracy is achieved when plugs are used to block some of the 201 beamlets in order to protect critical anatomical structures.

\section{MO-E1-06}

A Technique for Three-Dimensional Dose Verification in Conformal Stereotactic Radiosurgery

J L Robar*, B G Clark, University of British Columbia, BC Cancer Agency, Vancouver, Canada

Conventional convergent-arc stereotactic radiosurgery with circular collimation produces approximately spherical dose distributions. Typical dose verification methods for arc techniques take advantage of this inherent symmetry by characterizing a small number of dose profiles rather than the entire distribution. In contrast, conformal techniques using shaped beams demand an accurate method for measuring complete, integral dose distributions in three-dimensions with high spatial resolution. This work presents the design and evaluation of a system of dose verification for use in conjunction with static or dynamic irregularly-shaped fields and illustrates the application of this system to assess the accuracy of static conformal radiosurgical treatment plans. Monte Carlo simulation results indicate that for small $(<40 \mathrm{~mm}$ diameter) $6 \mathrm{MV}$ photon beams, the arrangement of radiographic film used in the system's anthropomorphic phantom preserves tissue-equivalence to within $<1.0 \%$, and thus the numerical accuracy of the recorded dose is maintained. The spatial resolution in the plane of the film is $0.25 \mathrm{~mm}$, which permits the characterization of small irregularities and regions of rapid dose fall-off. By using multiple views of closely-spaced film layers, the spatial resolution in three dimensions is sufficient to highlight minor spatial discrepancies between planned and delivered threedimensional dose distributions. This comparison is made directly within the treatment planning software by fusing the measured dosimetric information with treatment-planning images. Our results indicate that this system provides a practical and accurate method of validating dose calculations, verifying irregular dose distributions and providing periodic quality assurance.

\section{MO-E1-07}

Output Factors and Dose Profiles for Gamma-Knife Collimators S Goddu*, J Dempsey, R Drzymala, D Low, J Purdy, Mallinckrodt Institute of Radiology, St. Louis, MO

Because the spatial resolution of ionization chambers (primary dosimeters) is limited by their spatial dimensions, the accuracy of small field measurements (e.g. stereotactic radiosurgery fields) can be compromised by volume averaging. An example is the output factor for $4 \mathrm{~mm}$ collimator of the Leksell Gamma-Knife. Based on radiochromic film measurements, Kellerman et al (Phys. Med. Biol. 43, 1998, p2251) reported that the recommended relative output factor for $4 \mathrm{~mm}$ collimator (OF for $4 \mathrm{~mm} / \mathrm{OF}$ 
for $18 \mathrm{~mm}=0.8$ ) is found to be lower than their measured value. During the commissioning of our Leksell Gamma-Knife model B, we paid special attention to the $4 \mathrm{~mm}$ collimator to resolve this controversy. Ionization chambers traceable to NIST, $1 \mathrm{~mm}^{3}$ TLDs and Radiochromic film are used to measure the relative output factors as well as dose profiles. TG-21 calibration protocols were followed for ionization chamber measurements. Dose profiles and absolute doses were measured along X-axis. $6 \mathrm{MV}$ photon beam is used for absolute calibration. Irradiated Radiochromic films were scanned in a He-Ne laser densitometer at $635 \mathrm{~nm}$ wave length. For output measurements of the small fields the ion chamber measurements are inaccurate. Both Radiochromic film measurements and $1 \mathrm{~mm}$ TLD measurements showed good agreement with the Leksell GammaPlan treatment planning system within their experimental uncertainties. The relative output factors measured using radiochromic film were found to be closer to the manufacturer provided values than Kellermann et al.

\section{Scientific Session Radiotherapy Instrumentation I}

\section{Room: Mississippi}

\section{MO-E2-01}

Evaluation of A Passive Infrared Marker System for Radiotherapy Patient Positioning

R Boone*, T Solberg, University of California Los Angeles, Los Angeles, $\mathrm{CA}$

We have evaluated a passive IR system as an alternative to conventional methods of patient positioning. Because positioning of the target at the isocenter depends on marker shift, we first used the system to measure the movement of markers due to breathing. As expected, bony areas only move about $1 \mathrm{~mm}$. Shallow breathing limits movement of the chest area between 2 to $5 \mathrm{~mm}$. Even large movements of some markers, however, displace the calculated target position by only 1 to $3 \mathrm{~mm}$. Between successive exhales, the markers return within $1 \mathrm{~mm}$ of their original positions and the target shifts by only $0.5 \mathrm{~mm}$. Positioning during exhalation thus improves accuracy. A vacuum couch that rigidly holds the initial position of the patient results in further improvement. Under these conditions, the markers shift less than $2 \mathrm{~mm}$ on the skin between reattachments. Reattaching the markers several times on the same day displaces the target by $2 \mathrm{~mm}$ on the rib and $1.5 \mathrm{~mm}$ on the pelvis. Attaching the markers over several days worsens these values by $0.5 \mathrm{~mm}$. Four markers increase the displacement to over $2 \mathrm{~mm}$. Six markers also worsen displacement if the sixth marker attaches to a location that moves considerably. In conclusion, positioning during exhalation and rigidly supporting the patient allow the system to consistently reposition a target on a living subject within $2 \mathrm{~mm}$ of its initial position.

\section{MO-E2-02}

Prospects for Two-Dimensional Brachytherapy Dosimetry using Plastic Scintillator: Signal-to-Noise Ratio and System Design Considerations J Williamson*, A Kirov, S Devic, J Dempsey, W Binns, Washington University, St. Louis, MO

Plastic scintillator (PS) is a promising dosimeter for brachytherapy because of its high sensitivity, approximate tissue equivalence and potential for simultaneous high-resolution dose measurement everywhere in the $2 \mathrm{D}$ detector plane. We have developed a prototype $2 \mathrm{D}$ dosimetry system using a liquid nitrogen-cooled CCD camera with a $512 \times 512$ detector array. For a $1 \mathrm{~mm}$ thick BC-400 scintillator sheet near a model $6702 \mathrm{I}-125$ seed $\left(\mathrm{S}_{\mathrm{K}}=30\right.$ $\mathrm{U})$, repeated measurements were performed to quantify signal-to-noise ratio, $\mathrm{SNR}^{-1}$, as the \%standard deviation, $\sigma$, relative to the mean net counts, $\mu$, for each pixel. By modeling $100 \%(\sigma / \mu)$ 's dependence on count rate and integration time, total single-pixel noise was decomposed into its Poisson photon counting, time-dependent background and stationary background components. For dose rates and data acquisition times above $50 \mathrm{cGy} / \mathrm{h}$ and $60 \mathrm{~s}$, system precision is limited by Poisson counting statistics, showing that $\pm 2 \%$ precision for Cs- 137 LDR sources is possible for integration times under $300 \mathrm{~s}$. By improving sensitivity 100-500-fold (achievable by merging pixels, increasing photon collection efficiency, and using water equivalent scintillator), dose rates of $\cong 0.2 \mathrm{cGy} / \mathrm{h}$ should be measurable in $300 \mathrm{~s}$ with $\pm 2-3 \%$ precision, demonstrating the feasibility of I-125 dose measurements.
Comparison of measured 2D I-125 optical scintillation images with corresponding 2D Monte Carlo calculations, shows good agreement.

This work was supported by NIH Grant R01 CA 57222.

\section{MO-E2-03}

New Design Of Optical CT Microscanner For High-Resolution 3D Dosimetry Using BANG® Gels

M Maryanski*, M Ranade', A Rangarajan ${ }^{2}$, (1) MGS Research, Inc., Madison, CT, MGS Research, Inc., Guilford, CT, (2) Department of Diagnostic Radiology, Yale University, New Haven, CT

Optical CT microimaging of BANG ${ }^{\circledR}$ gels was recently introduced ${ }^{1}$ for $3 \mathrm{D}$ near-field dosimetry of brachytherapy sources. In BANG gels, radiationinduced polymerization produces permanent 3D images of dose distributions.

Initially, the rotating cylindrical gel vial was immersed in a refractive index matching liquid in a tank which was translated across a stationary laser beam. That design, despite producing encouraging performance test results, needed improvement, because even trace amounts of dust or microbubbles in the tank liquid could cause image artifacts, and vibrations from the translation motor contributed to noise.

The new design, which we call "dry scanner", employs neither the tank with the matching liquid nor the translation of the gel. The laser beam is first focused and then reflected by a rotating mirror whose center of rotation coincides with the focal spot of the rotating cylindrical gel vial (for paraxial rays, within about $1 / 3$ of the vial radius). A photodiode located at the opposite focal spot of the gel vial collects the transmitted light. Filtered back projection is used for reconstructing the image of dose distribution in the gel.

We tested the performance of the "dry scanner" for linearity, spatial uniformity, noise, and for reconstructing the images of test patterns. We found the results very satisfactory and promising. The "dry scanner" may find use in many clinical applications.

This was supported by grant HL59813 from NHLBI.

${ }^{1}$ Maryanski et al, Med.Phys.25(7), Part 1, p.A107, 1998

\section{MO-E2-04}

High Precision Absolute Dose Measurement Using Radiochromic Film and a Validated Optical Densitometry System

J Dempsey*, D Low, S Mutic, A Kirov, G Nussbaum, J Williamson, Mallinckrodt Inst of Radiology, Washington University, St. Louis, MO,

We present an evaluation of Gafchromic MD-55-2 radiochromic film (RCF) dosimetry performed with a high-resolution $(100 \mu \mathrm{m}$ pixel size $)$ heliumneon scanning-laser film digitizer which has been modified to eliminate optical densitometry artifacts as large as $30 \%$. An exposure and readout protocol was developed to prevent systematic errors inherent to RCF: temperature-dependent time evolution of RCF response, sensitivity inhomogeneity, and optical-polarization artifacts. RCF precision was evaluated with films given uniform 6MV x-ray doses between 1 and $200 \mathrm{~Gy}$. RCF absolute dose accuracy was evaluated by comparing RCF measurements to ion chamber measurements for conformal external beam and Monte Carlo calculation for ${ }^{125} \mathrm{I}$ and ${ }^{192} \mathrm{Ir}$ brachytherapy sources. Single pixel-to-pixel standard deviations of uniformly irradiated films were less than $1 \%$ for doses between 10-150 Gy; between 1-5\% for lower doses and $1-1.5 \%$ for high doses. Pixel averaging to form $200-800 \mu \mathrm{m}$ pixels reduces these values by $50-80 \%$. Comparison of absolute doses show agreement within $1.5-4 \%$ of dose benchmarks, consistent with a highly accurate dosimeter limited by its observed precision and the precision of the dose standards. These results provide a comprehensive benchmarking of RCF and show a considerable improvement over previously reported precision and accuracy attained by optical densitometry imaging systems.

This work was supported in part by NIH GRANT R01 CA 46640 AND a grant from Proxima Therapeutics Inc. 


\section{MO-E2-05}

In-vitro Evaluation of a Non-invasive Ultrasound Prostate Localization Device

J Markman*, D Low, R Halperin, J Michalski, J Purdy, Mallinckrodt Institute of Radiology, St. Louis, MO

The traditional solution of increasing prostate treatment volumes to account for localization and organ motion uncertainties gives the possibility of increasing normal tissue toxicity. A new ultrasound prostate localization device (BAT, NOMOS corp.) is available which may allow patient setup to be based directly on the prostate position at time of treatment.

A study is presented evaluating the localization precision and accuracy of the BAT using an ultrasound phantom with an embedded hypoechoic and radiopaque spherical target model. The phantom was purposefully misaligned by independently measured precise distances along the three principal axes. The BAT system was used to determine the couch motion required to return the target to its planning position. This was repeated over a series of days to measure reproducibility.

The mean error (accuracy) in locating the phantom varied as much as $3 \mathrm{~mm}$ from one session to another, with a single example of $1 \mathrm{~cm}$, due to relatively large systematic errors from the registration process. The optimal rigid body transformation was determined to correct for each session's systematic offset. Applying the transformation reduced the error (precision) to less than $0.5 \mathrm{~mm}$ in all directions.

The results indicate that the intrinsic precision of the BAT in controlled environments is sufficient to warrant further study in clinical settings, but that the BAT registration method needs to be improved.

This work supported in part by an industrial grant from NOMOS corp.

\section{MO-E2-06}

New Water Equivalent Liquid Scintillation Solutions for 3D Dosimetry A Kirov*, S Shrinivas, J Poblete, Z Piao, J Dempsey, W Binns, C Hurlbut, Washington University, St. Louis, MO; Ludlum Measurements, Sweetwater, TX

Despite recent advances in radiochromic film and gel dosimetry techniques, radiation therapy still lacks an efficient, accurate and convenient dose measurement method capable of measuring the dose simultaneously over a plane or volume (3D). We investigate the possibility of creating a new 3D dose measurement method for brachytherapy by detecting the collimated scintillation light emitted by an irradiated volume at multiple angles and reconstructing the light emission density in the volume with tomographic analysis. The liquid scintillator (LS) which fills the volume plays simultaneously the role of a water equivalent phantom material and dose sensitive media. Through parametric study of the LS attenuation and absorption coefficients, Monte Carlo dose calculations, scintillation efficiency measurements, and point spread function measurements we developed new LS materials. The calculated dose in LS is within 5\% of dose to water for distances up to $3 \mathrm{~cm}$ from $30 \mathrm{keV}$ point photon source and up to $5 \mathrm{~cm}$ for $50 \mathrm{keV}$ and higher energy photons. The new LS solutions are loaded with a $\mathrm{Si}$ containing compound, retain more than $85 \%$ of the scintillation efficiency of the unloaded solutions, and exhibit high localization of the scintillation process. The new LS solutions are superior with respect to efficiency and water equivalence to plastic scintillator materials used in dosimetry and may be used apart from the proposed 3D method.

This work is sponsored by the Whitaker Foundation under grant No. 970336 and Washington University

\section{MO-E2-07}

Tests of a New Three Dimensional Dosimetry Method Using Liquid Scintillator

A Kirov*, Z Piao, S Devic, T Miller, W Binns, J Epstein, Washington University, St. Louis, MO

A new three dimensional (3D) dose measurement method is described which uses liquid scintillation (LS) solution both as a phantom material and as a dose registration medium. The method is designed to measure simultaneously the brachytherapy dose in all points of a volume. The scintillation photons emitted from the volume at multiple angles are detected by using a highly collimated image detector. The scintillation light emission density for each voxel of the solution can be estimated by using a tomographic reconstruction technique. A highly efficient and radiologically water equivalent liquid scintillation solution was developed and used in the measurements. Scintillation light images induced by a rotationally symmetric dose distribution in the vicinity of a therapeutic ${ }^{125}$ I source in a cubical LS volume with a $25.4 \mathrm{~mm}$ side were obtained to estimate the signal to noise ratio and to test the experimental accuracy. We conclude that exposure times of $10 \mathrm{~min} . /$ projection can provide count rate precision better than $5 \%$ per $0.4 \mathrm{~mm}$ image pixel for dose rates $\sim 1 \mathrm{cGy} / \mathrm{h}$ for experimental geometry with resolution better than $2 \mathrm{~mm}$ in the volume center. Simulations of the detector signal for selected sample dose distributions were performed and used to verify the range of reliability for the reconstruction algorithm.

This work is sponsored by the Whitaker Foundation under grant No. 970336 and Washington University.

\section{Symposium \\ Room: Sewanee Dose-Volume Specification for Radiation Oncology}

\section{MO-E3-01}

ICRU Report 50: Prescribing, Recording and Reporting Photon Beam Therapy - Current Clinical Practice Versus New Developments

Ben Mijnheer, The Netherlands Cancer Institute / Antoni van Leeuwenhoekhuis, Amsterdam, The Netherlands

In order to facilitate communication and consistency in radiotherapy it is necessary to define unambiguous procedures for the specification of both target volume and dose delivery. Although such a statement is obvious, still a large diversity has been observed among the procedures applied in the various radiotherapy institutions or even in the same institution. In order to use common procedures more widely, the International Commission on Radiation Units and Measurements, ICRU, updated in Report 50 definitions and recommendations for target volume and dose specification. New volumes have been defined: the Gross Tumor Volume, GTV, the Clinical Target Volume, CTV, the Planning Target Volume, PTV, the Treated Volume and the Irradiated Volume. For dose reporting three levels have been described: level 1: only the dose at a reference point and its variation along a central beam axis are available; level 2: the dose distribution can be computed for plane(s); level 3: the dose distribution can be computed for volumes. At any level the dose at the ICRU Reference Point and the maximum and minimum dose to the PTV should be reported. In addition, this information could be supplemented by, e.g., isodose plans, dosearea/volume histograms, and other information, when available, at levels 2 or 3 .

The recommendations given in ICRU Report 50 are in principle relatively easy to implement and are already in use in a large number of radiotherapy institutions and applied in various protocols of clinical trials. Despite its widespread use, these concepts have limitations. First, according to the ICRU, the dose should be specified at a reference point centrally located in the PTV. From a radiobiological point of view, the mean dose to the cancer cell population is the parameter which is best correlated with tumor response. Although the ICRU also mentions the specification of the mean dose to the PTV, such a procedure is conceptually not correct because the PTV contains margins for setup uncertainties that do not contain tumor cells. Furthermore, no indications are given how to quantify the margin to derive the PTV from the CTV. Consequently it will be difficult to take into account more detailed insight in the 3-D movement of the CTV, or better knowledge of uncertainties in patient positioning. Also for new developments like deliberately chosen non-uniform dose distributions over the target volume brought on by IMRT, the ICRU recommendations are no longer valid and other concepts for the reporting of dose distributions have to be explored.

For the time being, the ICRU Report 50 recommendations seem to be a useful compromise between current clinical practice and new developments in most institutions, although improvements with respect to the incorporation of margins, both in the target volume and in organs at risk are required. In the future the ultimate goal should be to deliver and specify the dose to a moving CTV with well-known variations in position with respect to the treatment beam. These recommendations should include probability 
based dose specification procedures and be extended to reporting doses in a series of patients.

\section{MO-E3-02}

ICRU Report 62: A Review of the New Supplement to ICRU Report 50 James Purdy, Mallinckrodt Institute of Radiology, Washington University School of Medicine, St. Louis, MO

One of the important factors that has contributed to the success of the current 3-D treatment planning process is the standardization of nomenclature published in the International Commission on Radiation Units and Measurements (ICRU) Report 50. This report has given the radiation oncology community a language and a methodology for image-based 3-D planning for defining the volumes of known tumor, suspected microscopic spread, and marginal volumes necessary to account for setup variations and organ and patient motion. However, since publication of Report 50, significant advances in 3-D planning have been made. New conformal irradiation techniques have been introduced and modern imaging procedures provide even more information on the location, shape, and limits of the tumor/target volumes, as well as the organs at risk (OAR). Commercial 3-D treatment planning systems with many advanced features are now widely available. Also, there are some limitations and practical issues when using Report 50 methodology that have spurred vigorous discussions and debates. For these reasons, the ICRU has decided to publish a supplement to Report 50 (ICRU Report 62) to formulate more accurately some of the definitions of concepts and to take into account the consequences of the advances made over the last six years. Perhaps the most criticized limitation of Report 50 is that it does not account for OAR positional uncertainties. In response, Report 62 introduces a new concept, the Planning Organ at Risk Volume $(P R V)$, in which a margin is added to the OAR to account for movements and changes in shape and/or size of the OAR, as well as set-up uncertainties. Thus, the PRV for the OAR is analogous to the PTV for the CTV. Report 50 does not address directly how to combine the different positional uncertainties (e.g., setup margin and internal organ motion margin) that make up the PTV or PRV margins. This is a complicated situation since the margins result from random and systematic uncertainties. Report 62 introduces the concept of the Internal Margin (IM) which is defined to account for variations in size, shape, and position of the CTV in relation to anatomical reference points. Report 62 also introduces the concept of the Set-up Margin (SM) which is defined to account for uncertainties in patient-beam positioning. Report 62 recognizes that simple linear addition of the two margins to account for each of their independent effects may make the PTV inappropriately large and the problem is discussed in some detail. The selection of an overall margin and delineation of the border of the PTV and PRV involve a compromise that requires the experience and the judgment of the radiation oncology team. Other pertinent issues in defining and reporting volumes for 3-D planning will be discussed.

Educational Objectives:

This presentation will provide a review of the new concepts introduced in ICRU Report 62 including:

1. Internal Margin and Set-up Margin

2. Combining margins

3. Planning Organs at Risk Volumes (PRV)

4. Conformity Index

\section{MO-E3-03}

\section{Reporting and Analyzing Dose for IMRT}

Andrzej Niemierko, Massachusetts General Hospital and Harvard Medical School, Boston, MA

Objective: to discuss dose reporting issues emphasizing problems with dose inhomogeneity.

The ICRU Report 50 describes and recommends systematic methods for dose prescribing, recording, and reporting for photon beams. The supplement to ICRU Report 50 - the ICRU Report 62 further explores the problem discussing some weaknesses in the recommendations of the Report 50. Because a dose distribution is intrinsically a multidimensional object (with volumetric and temporal dimensions), the problem of concise but also clinically meaningful reporting is not trivial. Ideally, a dose report of a given treatment plan should be perfectly correlated with the clinical consequences of the plan. After all, one of the essential objectives of dose specification is to provide relevant data for subsequent analyses of the observed clinical results, in order to optimize the treatment strategy. Unfortunately, dose distributions are never exactly uniform, and may often be far from it, especially for normal tissues. It is impossible to quantitatively evaluate, compare, and score two or more non-uniform three-dimensional (3D) dose distributions without reducing them to the corresponding single numbers. It is intuitively sensible to represent a 3D dose distribution for a given structure of interest by a single number - a dose that, when delivered uniformly to that structure, would result in the same relevant effect (say, probability of local control for tumors, or probability of complication for normal tissues). This concept of Equivalent Uniform Dose (EUD) has recently been proposed and investigated $(1,2)$. The EUD takes into account dose inhomogeneity and absolute volume of the irradiated structure, and may also incorporate dose per fraction effects and cell proliferation. The EUD concept can be applied to both tumors and normal structures. It can be shown that for tumors the EUD is always bounded by the minimum target dose and the mean target dose. For normal structures the EUD is bounded by the mean dose and the maximum dose. For a given dose distribution (or a given dose-volume histogram) the value of EUD is organ dependent. For example, the EUD is closer to the maximum organ dose for the spinal cord, and it is closer to the mean organ dose for the lung.

It should be emphasized that the clinical response of a given patient depends not only on the delivered dose distribution but also on other non-dosimetric considerations (for example histology, gender, age, concurrent chemotherapy, genetic predisposition, and stress). In addition, some important functional end-points depend not only on the local dose but also on the dose outside the structure of interest. For example, in lung cancer treatment radiation pneumonitis may develop in the unirradiated lung. Clearly, this effect is not determined by the dose distribution within this lung no matter how we report that dose.

1. Niemierko, A. Reporting and analyzing dose distributions: a concept of equivalent uniform dose. Medical Physics. 24(1): 103-10; 1997.

2. McGary, J. E.; Grant, W., 3rd; Woo, S. Y. Butler, E. B. Comment on "Reporting and analyzing dose distributions: a concept of equivalent uniform dose" [Med. Phys. 24, 103-109 (1997)] [letter; comment]. Medical Physics. 24(8): 1323-4; discussion 1325-7; 1997.

\section{Scientific Session Breast Imaging}

Room: Carroll

\section{MO-E4-01}

A Mean Glandular Dose Patient Survey Of 6,006 Women Undergoing Mammography

R Kruger*, B Schueler, Mayo Clinic, Rochester, MN, Mayo Clinic, Rochester, MN

A survey was conducted to estimate the mean glandular dose (MGD) for women undergoing mammography during a four-month period and report the distribution of doses, tissue glandular content, and mammographic parameters employed. Clinical data was collected and analyzed from 24,471 mammograms, originating from 6,006 women. The survey data included mammograms from seven modern units using a molybdenum (Mo) anode and either Mo or rhodium ( $\mathrm{Rh}$ ) filter. The $\mathrm{Rh}$ filter was used in approximately $7 \%$ of the exposures. The Kodak MinR 2000 screen-film system was used with phototiming set to produce an ACR phantom density of $1.55 \pm 0.20$ O.D. Exposure parameters for each mammogram were automatically recorded onto a floppy disk on each unit. All mammography units were calibrated individually so that based on recorded patient data (mAs, thickness, filter, and tube potential) breast glandular content could be accurately estimated. Phantoms containing $0 \%, 30 \%, 50 \%, 70 \%$, and $100 \%$ simulated glandular equivalent material were measured at thicknesses of 2 , 4,6 , and $8 \mathrm{~cm}$. The data from the phototimed exposures of these phantoms were used to create an algorithm to estimate the patient's breast composition. The MGD was then estimated for each mammogram based on the normalized glandular dose, $\mathrm{D}_{\mathrm{gN}}$, and calculated entrance exposure in air. This survey found a median MGD of $2.62 \mathrm{mGy}$. Results include a median patient glandular content of $27.5 \%$ and a mean breast thickness of $5.1 \mathrm{~cm}$ with a standard deviation of $1.3 \mathrm{~cm}$. 


\section{MO-E4-02}

How Consistently Do MQSA Inspectors Score Phantom Images?

O Suleiman*, S Belella, J Tuttle-Kuhm, FDA, Rockville, MD

The evaluation of phantom image quality depends upon a number of variables such as viewing conditions (ambient light illuminance, viewbox luminance, masking), the phantom film optical density, variability among phantoms, and variability among readers. An evaluation of 235 Mammography Quality Standards Act (MQSA) inspectors in 1997 was performed to address the issue of consistency of their phantom image quality scoring. A set of 5 different phantom images were duplicated and scored by each inspector. The inspectors each received an individual test packet consisting of the 5 films, with each film labeled differently, and were instructed to score the films using two different criteria. Each individual scoring sheet and film packet was returned. The primary difference between the two criteria was that one was independent of artifact subtraction, the other included artifact subtraction. Consistency was measured as the coefficient of variation for each of the sixteen image quality test objects embedded within the standard mammography phantom. Scoring consistency depended upon the difficulty of the individual test object scored, the scoring of partial test objects, and confusion in the scoring criteria. Test objects scored with artifact subtraction accounted for the largest source of variability. Scoring without artifact subtraction resulted in the most consistency, or least variability in phantom scores among the inspectors.

\section{MO-E4-03}

Scatter-to-Primary Measurements in Mammography Using Edge Spread Functions

V Cooper, III*, J Boone, J Seibert, C Pellot, UC Davis, Sacramento, CA

A novel method of quantifying scatter and its spread functions is presented in this work. Data for a range of mammographic imaging parameters including breast thickness $(2,4,6$, and $8 \mathrm{~cm})$, glandular/fat composition $(0$, 43 , and $100 \%$ glandular), and beam quality (Mo/Mo, 24-36 kV by $3 \mathrm{kV}$ increments) are also presented.

Tissue equivalent material (BR-Fat, BR-12, and BR-Gland) was placed on a flat panel imager employing a Lanex Regular screen with the x-ray field defined by lead sheets fixed on an experimental stand. An edge image including scatter was acquired followed by the removal of the tissue equivalent material and the subsequent acquisition of a primary-only edge image. Edge spread functions (ESFs) were acquired from the images and the normalized primary ESF was used to correct the scatter ESF for focal spot blurring and to delineate the primary component in the scatter ESF. The scatter to primary ratio (SPR) was then computed. The ESFs were differentiated to yield the line spread functions (LSFs)

Experiments consisting of five repeated measures were conducted. The data showed expected behaviors and good precision with errors on the order of $5 \%$. SPRs for $43 \%$ glandular breasts were 0.157 $\pm 0.010,0.266 \pm 0.011,0.376 \pm 0.017$, and $0.465 \pm 0.019$, for $2 \mathrm{~cm} / 24 \mathrm{kV}, 4$ $\mathrm{cm} / 27 \mathrm{kV}, 6 \mathrm{~cm} / 30 \mathrm{kV}$, and $8 \mathrm{~cm} / 33 \mathrm{kV}$, respectively. Finally, comparisons were made with the works of others.

\section{MO-E4-04}

Electrical Impedance Spectroscopy (EIS):

Preliminary Experiments Imaging the Human Breast

T Kerner*, K Osterman, D Williams, F Reiss, A Hartov, S Poplack, K Paulsen, Dartmouth Medical School, Nahover, NH

We imaged the breasts of 12 women to investigating the feasibility of routine EIS exams with a system we built. We simultaneously applied spatially varying voltages (1Vpeak, $10 \mathrm{kHz}$ to $1 \mathrm{MHz})$ through $16 \mathrm{Ag} / \mathrm{AgCl}$ surface electrodes equally spaced on a radially translating apparatus. The magnitude and phase of the resulting currents were digitized. Using the measured currents as the boundary conditions, we numerically solved the complex Laplace equation to produce spatial plots of absolute electrical conductivity and permittivity. Imaging experiments consisted of acquiring data at 10 frequencies on both breasts. Participants lay down prone on an examination table with one breast positioned in an electrode array. Exams easily performed and well tolerated, lasting about 10 minutes per breast. The resulting images seem to be quite sensitive but not specific to pathology. For example, of the 3 cases with known tumors, we saw "abnormal" images in all 3 cases, but we could not definitely distinguish these from other abnormalities. While localized near-surface electrode artifacts have been evident, several findings have emerged. The normal mammogram breast appears to have a characteristic EIS permittivity image emerging across subjects. Structural features in the EIS images have generally correlated with tumors, cysts, scarring from recent lumpectomy and follow-up radiation therapy. We can detect lesions on the order of $1 \mathrm{~cm}$ but cannot always precisely locate them. With improved calibration and electrode artifact modeled compensation, we hope to distinguish specific lesions by their absolute conductivity \& permittivity values.

\section{MO-E4-05}

MRI 3D Contrast Enhanced Acquisition And Phase Contrast Flow Analysis for Internal Mammary Artery Evaluation

Y Liu*, J Blechinger, R Breger, Radiology Dept./St. Luke's Medical Center, Milwaukee, WI

Internal mammary artery (IMA) bypass grafting has been widely used for patients with coronary artery disease. However, stenosis and occlusion of IMA grafts is not uncommon. The purpose of this study is to use contrast enhanced 3D MR angiography for visualization of the IMA, and phase contrast flow analysis for functional evaluation of IMA so that the recurrent cardiac symptoms can be monitored or predicted. Contrast enhanced 3dimensional (3D) fast gradient echo acquisition is used for patients post IMA graft operation. Forty cc of Gadodiamide contrast is bolus injected in 20 seconds. Images are displayed using maximum intensity projection and reformatting algorithms. To measure the flow of the internal mammary artery, segmented cine phase contrast flow analysis images are acquired. A torso phased array coil is used for the 3D contrast enhanced MRA acquisition. During the followed phase contrast flow analysis acquisition, only the left anterior coil element from the 4 coil element is activated. This approach provides excellent signal during 3D contrast MRA acquisition, while in flow analysis high signal for the left IMA without aliasing artifacts. The IMA is well visualized from its origin at subclavian to left anterior descending coronary anastomosis. Velocity measured from a grafted IMA without stenosis shows biphasic forward flow pattern which is characteristic for IMA graft flow. Combining the 3D contrast enhanced MRA with the phase contrast flow analysis, both morphological and functional evaluation for IMA flow patency can be achieved, so that recurrent cardiac symptoms could be predicted or monitored.

\section{MO-E4-06}

Preliminary Evaluation of ETACT Scintimammography

F Fahey*, R Webber, E Bayram, B Harkness, Z Mu, P Hemler, Wake Forest University School of Medicine, Winston Salem, NC

We have performed a preliminary evaluation of emission tuned aperture computed tomography (ETACT), a novel approach to scintimammography. In ETACT, a series of tomographic slices of the tracer distribution in the breast can be reconstructed from a small number (5-10) of projection images with the use of fiducial markers. The projection data are acquired with a pinhole collimator to take advantage of its high spatial resolution. Projection data are reconstructed tomosynthetically and an iterative axial deconvolution is applied. Computer simulations were performed to investigate the effect of pinhole size on signal-to-noise ratio (SNR). ETACT projections of a computer model of a breast with a $1 \mathrm{~cm}$ tumor $(5: 1 \mathrm{~T} / \mathrm{NT}$ ratio) were simulated for a 3,4 and $5 \mathrm{~mm}$ pinhole aperture. The use of a $4 \mathrm{~mm}$ pinhole yielded the optimum contrast and SNR for this particular imaging task $(\mathrm{C}=.133, .177, .122$ and $\mathrm{SNR}=1.92,2.20$ and 2.01 for $3,4,5$ $\mathrm{mm}$, respectively). ETACT was then performed on the Data Spectrum breast phantom with a $1 \mathrm{~cm}$ tumor $(10: 1 \mathrm{~T} / \mathrm{NT})$. Eight projections were acquired with a $4 \mathrm{~mm}$ pinhole for $5 \mathrm{~min}$ each about the left lateral of the phantom. The ETACT reconstructed data yielded a significant improvement in contrast ( 0.52 vs 0.20$)$ and SNR ( 1.85 vs 0.54$)$ relative to planar imaging. These preliminary results indicate that ETACT is a promising new approach to scintimammography. 


\section{Refresher Course}

Room: Pearl

\section{TU-A1-01}

Manpower Issues for Radiation Oncology

Michael Mills, Kenneth Hogstrom ${ }^{1}$, Geoffrey Ibbott $^{2}$, University of Louisville, Lousiville, KY (1) UT M.D. Anderson Cancer Center, Houston, TX (2) University of Kentucky, Lexington, KY

The emphasis on cost reduction associated with Managed Care continues to burden medical physicists. Radiation oncology managers need to defend levels of staffing and other resources in order to maintain acceptable levels of clinical quality. New and emerging high technologies require the purchase of specialized equipment. The manager must provide for both an initial commissioning effort and an ongoing clinical effort from physicists and dosimetrists. Two comprehensive national surveys measure physics and dosimetry personnel resources associated with radiation oncology. These are The Abt Study of Medical Physics Work Values for Radiation Oncology Physics Services, and the Survey of Physics Resources for Radiation Oncology Special Procedures. The former examines routine radiation oncology procedures. The latter addresses the following special procedures: total body irradiation, total skin irradiation, electron arc therapy, intraoperative radiotherapy, stereotactic radiosurgery, stereotactic brachytherapy, high-dose rate brachytherapy, and three-dimensional treatment planning. These survey data provide a national profile standard for resource management. Effort, equipment and cost are analyzed as a function of number of patients treated with routine and special technologies. The data defends staffing levels, resource management decisions, and the cost benefits of treatment centers becoming referral centers for routine or high technology procedures.

\section{Continuing Education Course IMRT - 3}

\section{TU-A2-01}

IMRT with Conventional MLC's

Lynn Verhey, University of California, San Francisco, CA

The use of three-dimensional conformal radiotherapy (3DCRT) has now become common practice in radiation oncology departments around the world. Typical applications of 3DCRT involve a number of fixed beams entering the patient from directions which are hand-selected with the aid of beams' eye viewing to avoid the traversal of sensitive normal tissues to the maximal extent possible, even if those directions are out of the axial plane. Plan optimization is accomplished iteratively until a satisfactorily uniform dose to the target is achieved without exceeding the dose tolerance of neighboring sensitive tissues.

There remain situations for which conventional 3DCRT cannot produce a satisfactory treatment plan due to limitations of the method along with the geometry of the problem. Intensity modulated radiotherapy (IMRT) uses modifications in the intensity of the beams across the irradiated field as an additional degree of freedom to enhance the capability of conforming dose distributions in three dimensions. There are a number of different methods of producing these intensity-modulated dose distributions, some of which are relatively simple and others quite elegant and complex

Simple IMRT methods are those which can be planned and iteratively optimized with existing "forward" 3D treatment planning systems. These IMRT plans include fields made up of two or more subfields, shaped with a multileaf collimator (MLC), at least one of which is designed to reduce dose to overlying normal tissues. This planning scheme replaces noncoplanar methods that avoid normal tissue irradiation by complex angle selection alone.

General IMRT methods are those that require the use of inverse treatment planning programs with computerized optimization. These treatments can be accomplished by static "stop and shoot" MLC delivery methods or by dynamic sliding window methods. A significant advantage of the static IMRT method is that each MLC segment is a separate field that can be verified with the accelerator $\mathrm{R} / \mathrm{V}$ system and that portal verification methods can still be used.
For dynamic MLC methods of intensity modulation the gantry is fixed and the leaves are moving across the field while the beam is on. With this approach, any number of intensity levels can be delivered without a significant increase in treatment time using special DMLC accelerator control software. For DMLC methods, verification of the shape and location of the intensity pattern at the time of treatment is either difficult or impossible.

Dosimetric verification of inverse treatment plans requires the use of phantoms with both multiple point and multiple plane dose capabilities. The dosimetric differences of the treatment delivery methods and the MLC designs will be addressed. The resources required to mount an IMRT program and methods of identifying patients with the most potential gain will be discussed.

Educational Objectives:

1. Understand the concept of intensity modulation

2. Learn how to predict the most useful application of IMRT

3. Understand the different methods of delivering IMRT with conventional MLCs

4. Understand planning methodologies

5. Learn about different verification methods

6. Understand dosimetric consequences of MLC design and treatment method

7. Understand the required resources for launching IMRT capabilities

\section{Continuing Education Course Special Dosimetry Measurements - 2}

\section{TU-A3-01}

Dosimetric Pitfall for Relative Dosimetry in Electron Disequilibrium Region

Timothy Zhu, Dept. of Radiation Oncology, University of Pennsylvania, Philadelphia, PA

The purpose of the lecture is to review our current understanding of onedimensional relative dose measurement for therapeutic electron and photon beams. Emphasis will be placed on problematic areas when electron equilibrium is not present, such as small field dosimetry, near-surface depth dose, penumbra, and 1D dosimetry for intensity modulated beams. Thimble and parallel-plate ionization chambers and solid-state detectors will be examined. These detectors are suitable for point measurement or relative dosimetry for static beam if there is electron equilibrium. Their restrictions under electron disequilibrium are examined using the concept of detector response curve. This concept can be correlated to gradient correction, detector displacement correction, and electron fluence correction used in absolute dosimetry. These correction factors can be used to extrapolate dosimetric quantities from measurements under electron disequilibrium. Other factors (such as energy, dose rate, accumulative dose) that affect detector sensitivity, especially diode detector, will be examined. For dosimetry of dynamic intensity modulated beam, detector array made of diodes or ionization chambers is reviewed. Specific dosimetric issues for the detector array, such as relative sensitivity calibration, detector location dependence, and data acquisition electronics for detector array, will be discussed.

Educational Objectives:

1. Understanding the perturbation effects for one-dimensional relative dosimetry.

2. Reviewing the concept of detector response curve and its application to dose profile, near-surface depth dose, and small field output dosimetry.

3. Commissioning of detector array for relative dosimetry of static and dynamic beams. 


\section{Refresher Course}

Room: Carroll

\section{TU-A4-01}

Computed Radiography Physics

J. Anthony Seibert, University California - Davis, Radiology Research, Sacramento, CA

\section{Abstract:}

Computed radiography (CR) systems are becoming prevalent in diagnostic radiology as the availability of lower cost devices and the increased emphasis on digital image networks in radiology occurs. Basic knowledge of the CR system and an understanding of system characteristics by the radiologist, physicist, and technologist are important for the installation and operation of these devices in the clinical environment.

Course content:

The process of photostimulated luminescence, creation of the latent image, and acquisition of the digital image are reviewed. CR detector performance issues are explained, including the characteristic curve and dynamic range response, amplification and scaling of the electronic latent image, spatial resolution / sampling rate relationships, and contrast detectability related to bit depth and image noise. Radiation dose requirements of $\mathrm{CR}$ and "appropriate" exposures for typical imaging procedures are presented. Post processing capabilities, anatomic parameter settings, hardcopy and softcopy displays, image size, and network interfaces are examined. Finally, acceptance testing and quality control protocols to ensure optimal and consistent performance are recommended.

Educational Objectives:

The attendee of this course can expect to learn several key issues regarding computed radiography:

1. Functional aspects of the CR system.

2. Understanding the benefits and limitations of CR compared to screenfilm detectors.

3. Implementation of image processing parameters and acquisition protocols for CR.

4. Optimization of hard and soft-copy images for CR.

5. Determination of incident exposures on the imaging plate in order to identify under/overexposures.

6. Acceptance testing and periodic quality control procedures to ensure long-term operational stability and function.

\section{Refresher Course}

Room: Taylor

\section{TU-A5-01}

Physical Principles of Phase-contrast, Time-of-flight, and Contrastenhanced MR Angiography

Frank Korosec, University of Wisconsin-Madison, Madison, WI

Magnetic resonance angiographic imaging techniques can be categorized as phase contrast, time-of-flight, or contrast-enhanced methods. When properly implemented, all of the MR angiographic methods can yield diagnosticquality images.

Phase contrast techniques derive contrast between flowing blood and stationary tissues by manipulating the phase of the magnetization. Data acquired using phase contrast methods can be used to produce phasedifference, complex-difference, or magnitude images. The phase difference images can be used to determine quantitative information regarding the velocity and volume flow rate of the blood.

Phase contrast methods can be implemented using two-dimensional or threedimensional acquisition. Two-dimension acquisition can be completed rapidly and is effective for localizing. It also can be cardiac gated to provide velocity or volume flow rate information throughout the cardiac cycle.

Time-of-flight techniques derive contrast between flowing blood and stationary tissues by manipulating the magnitude of the magnetization. With these methods, the contrast between blood and stationary tissues is strongly dependent on several imaging parameters, including TR, slice thickness, tip angle, slice orientation, etc., which must be carefully chosen when designing imaging protocols.

Time-of-flight methods can be implemented using two-dimensional or threedimensional acquisition. When three-dimensional acquisition is employed, additional mechanisms must be implemented in order to reduce the signal from stationary tissues, including the use of magnetization transfer, the appropriate echo time, and a ramped tip angle. With both two-dimensional and three-dimensional acquisition methods, a spatial saturation pulse can be applied outside the imaging volume to eliminate signal from unwanted venous blood that is going to flow into the imaging volume.

Contrast-enhanced techniques derive signal differences between blood and stationary tissues by manipulating the magnitude of the magnetization. These signal differences are achieved by using the appropriate acquisition parameters and an intravenous injection of a contrast agent into the vascular system, which selectively, and dramatically, shortens the T1 of the blood. By implementing a T1-weighted imaging sequence during the first pass of the contrast agent, images can be produced that show arteries with striking contrast relative to surrounding stationary tissues and veins. Synchronizing the acquisition with the arrival of the contrast agent is critical to image quality. Several methods have been developed to ensure proper timing of the acquisition relative to the passage of the contrast agent. Contrast enhanced methods provide high quality images with fewer artifacts than the noncontrast-enhanced methods. Large fields-of-view can be imaged to demonstrate large vascular areas in a short acquisition time. The short imaging time permits acquisition in a single breath-hold interval, providing high quality images even in areas affected by respiratory motion.

After attending this Refresher course, the participant will be able to:

1. Describe the characteristics of each of the MRA techniques, and the mechanisms that are employed to achieve contrast in each of the three classes of MRA techniques.

2. Identify artifacts associated with each of the MRA techniques, and suggest modifications to diminish or eliminate the artifacts.

3. Determine which MRA techniques are best suited for given applications.

\section{Continuing Education Course Room: Browning A Nuclear Medicine - 1}

\section{TU-A6-01}

New Scintillators \& New Detectors for PET

R. Nutt, C. Melcher, B Bendriem, CTI, Inc., Knoxville, TN

1. Current Status

The value of positron imaging is becoming widely recognized in nuclear medicine. All major suppliers of gamma cameras are offering coincidence circuitry to address this trend toward positron imaging. There are several factors that have encouraged the trend toward positron imaging, including:

- Fluoro-deoxy glucose (FDG) studies being widely recognized as significant in oncology and as the gold standard in cardiac viability,

- Wider availability of FDG distributed in unit doses,

- Higher sensitivity and better image resolution than is possible with other imaging techniques,

- Higher patient throughput.

There are more and more PET (Positron Emission Tomography) isotopegenerating cyclotrons in the United States, Europe, and in many other countries. FDG is the only radioisotope distributed at this time, but there exist a large number of other fluorine isotopes that may become important, such as Fluoro-Dopa, Fluoro-Tyrosine, F- and others.

The resolution and sensitivity improvement using positron imaging are well known. A ring tomograph such as the ECAT EXACT HR+ has an image resolution of approximately $4 \mathrm{~mm}$ and a sensitivity of $1,000,000$ counts $/ \mathrm{sec} / \mu \mathrm{ci} / \mathrm{ml}$. A typical SPECT (Single Photon Emission Tomography) tomograph has a resolution of $10 \mathrm{~mm}$ and a sensitivity 20 to 100 times less than a 3D PET tomograph. This ratio also applies to the maximum coincidence count rate, which is typically 600,000 counts/sec for PET and less than 20,000 counts/sec for a SPECT operated in PET mode. In the latter 
case much longer time is necessary to permit the accumulation of statistically significant images.

\section{Detector Materials}

Lutetium Oxyorthosilicate (LSO) was discovered in the late 1980 s by a group from Schlumberger led by Charles Melcher. The group was looking for an ideal scintillator for use in "down-hole" oil exploration. As the scintillator was developed and better understood, it became evident that its characteristics were almost ideal for nuclear detection in medical applications and not so ideal for the high temperature and more hazardous environment of oil exploration application.

3. Scintillation Detectors: Past and Future

PET detector development has progressed significantly since the first PET tomographs. The number of elements have increased from a few tens of elements in the first tomograph at St. Louis to more than 100,000 in the new High Resolution Research Tomograph (HRRT) that was delivered to MPI, Koln in February of 1999. The HRRT is the first commercial LSO tomograph and it represents a major change in the technology used in detectors for PET. The ECAT HR+ is presently the highest resolution commercial PET tomograph and it uses BGO for the detector material. The commercial tomograph with the same detector as the ECAT HR+ but having six rows of detectors rather than four was the ECAT HR++ delivered to the MRC Hammersmith Hospital in London in 1997.

\section{Refresher Course Pearl}

\section{TU-B1-01}

Revised U.S. Nuclear Regulatory Commission 10 CFR 35 Regulations for Medical Use of Byproduct Material in Radiation Oncology

Jeffrey F. Williamson, Washington University School of Medicine, St. Louis, MO

In 1997, The U.S. Nuclear Regulatory Commission (NRC) began the process of completely rewriting its regulations (10 CFR 35) governing medical use of by product material, in response to its own Strategic Assessment Initiative (1995-1997) and the Institute of Medicine's (IOM) highly critical 1995 report, "Radiation Medicine: a Need for Regulatory Reform." The final rule is expected to be approved by the NRC Commissioners in June 1999 and implemented in Fall 1999. The new rule, like its predecessor, addresses only byproduct materials, leaving regulation of naturally occurring and accelerator produced radiation and radioactivity to the states. However, the revised rule is more "risk informed" in that "low risk" modalities (diagnostic nuclear medicine) will enjoy some regulatory relief compared to "high risk" therapeutic modalities. In several areas, the new rule is more "performance based" specifying the regulatory endpoint and leaving the detailed compliance methodology to the licensee's discretion. Technical requirements for HDR brachytherapy and stereotactic radiosurgery are explicitly outlined in Part 35 rather than in Regulatory and Licensing Guides. As of mid-March, some of the more striking changes include:

(a) Retains the requirement for a radiation safety committee for licensees practicing two or more modalities but does not specify its role in executing the licensee's mandated administrative and safety responsibilities.

(b) Eliminates QMP requirements, but retains requirements for written directives, and verifying patient identity, treatment calculations and correct implementation of the treatment. Part 20 ALARA requirements are no longer duplicated in Part 35/

(c) Replaces "teletherapy physicist" with "authorized medical physicist" (AMP) and defines AMP duties for all byproduct modalities.

(d) Minor modifications in Training and Experience Requirements for AMP and for authorized user (including retention of three-year residency requirement) of therapeutic modalities.

(e) Technical requirements for gamma stereotactic, teletherapy, remote afterloading and manual brachytherapy that are more consistent with Task Group 40, 56 and 59 recommendations

(f) Exempts end users from verifying brachytherapy source calibrations if vendors can supply calibrations satisfying "nationally recognized protocols." (g) To cover emerging treatment modalities, a new category (35.1000) is added for medical uses not elsewhere defined that can be authorized by license amendment without requesting variances from 10 CFR 35 . Intravascular irradiation is not explicitly addressed.

(h) Establishes a minimum dose threshold $(>0.5 \mathrm{~Sv}$ and $20 \%$ deviation from expected dose) for wrong-site misadministration (called "Medical Event").

With respect to technical details, the revised 10 CFR 35 improves upon its predecessor. However, few, if any, of the basic deficiencies of the overall regulatory system identified by IOM and other critics are addressed.

\section{Continuing Education Course Room: Mississippi IMRT - 4}

\section{TU-B2-01}

\section{Clinical Implementation of IMRT}

Chen-Shou Chui, Memorial Sloan-Kettering Cancer Center, New York, NY

Standard treatment planning process typically starts with CT-image acquisition, followed by contour drawing, beam definition, dose calculation, and plan evaluation. For intensity-modulated radiation therapy (IMRT), two additional steps, optimization and delivery, are needed. Moreover, other considerations specific to IMRT such as QA and radiation protection also need to be addressed.

Optimization is the process by which optimal intensity distribution is determined that would produce the dose distribution closest match the user's desire. The user typically specifies the prescription dose (or range of doses) to the target, dose constraints (or dose/volume constraints) to critical organs, and other optimization parameters. A special problem encountered in certain cases (e.g., breast and neck nodes) is the 'skin-flash' problem. The intensity profile determined by optimization does not extend beyond the skin, where the target ends. This may cause the target to be underdosed due to motion or setup uncertainties. Possible solutions to this problem will be discussed.

Once the intensity distribution is determined, it can be delivered through the use of a physical compensator; a special device such as MIMiC; or a conventional multileaf collimator (MLC), either in dynamic or in step-andshoot mode. For clinical implementations, it is convenient to use MIMiC or MLC. In these approaches, the entire intensity profile is not delivered all at once, but made up of many segments, each covering only a portion of the treatment volume. If the treatment volume moves (e.g. breathing) during a fraction, it moves in and out of these segments, resulting in doses delivered to deviate from those expected. The effects on such deviation can be calculated if the pattern of motion is known. Another restriction on conventional MLC is the maximum field width. If the field width exceeds this limit, the intensity-modulated field must be split into two. Various ways of splitting will be described.

Quality assurance includes those that are normally done for conventional treatment and those that are particular to IMRT. The latter includes recordand-verify (R\&V), flat-phantom dosimetry, and independent MU calculation. R\&V system verifies all leaf positions before the first and after the last segment. Flat-phantom dosimetry compares calculated versus measured data, obtained with chamber and/or film. The independent MU program calculates the absolute dose from leaf sequence, including effects of extended source, rounded leaf-ends and transmission through the leaves.

Another consideration specific to IMRT is the increased total-body dose and room shielding requirement. This is caused by the longer beam-on-time typically required by IMRT than conventional treatments. These issues are examined based on treatment sites and delivery techniques.

IMRT is still an evolving technology. While it produces superior dose distributions than conventional treatment, it also requires additional efforts to ensure its proper use. The issues addressed here are only part of what have been encountered so far. There is no doubt new problems will emerge. As with any other new technique, IMRT must be used with care.

Education Objectives:

1. To provide general understanding of IMRT methodologies and procedures.

2. To address special issues associated with IMRT. 


\section{Refresher Course Taylor}

\section{TU-B5-01}

Fast Imaging Methods in MRI

Wlad Sobol, UAB Health System, Birmingham, AL

Due to the repetitive nature of the data acquisition in MRI, states of nuclear magnetization play an important role in the design of MR imaging protocols. In a standard Spin Echo protocol, the sequence repetition time is long enough that no transverse magnetization remains at the end of the cycle. As a result, the magnetization used to encode the image data is the same from view to view, which allows for a straightforward encoding scheme.

When the acquisition is accelerated to shorten the exam time, a residual transverse magnetization exists at the end of each repetition cycle and the magnetization used to encode the image data may vary from view to view. If the acquisition scheme is not specifically adjusted to minimize the impact of this effect, large artifacts (severe ghosting or blurring) will occur in images.

Two practical approaches emerged to solve this problem. In one approach, known as a k-space reordering, nonstationary states of magnetization are used to encode the image data in a carefully crafted temporal order. As a result, the image is acquired quickly but a trade-off exists between the speed of acquisition that minimizes motion artifacts and the image blurring caused by k-space reordering of nonstationary magnetization states. This method is utilized in fast spin echo, turbo (magnetization prepared) gradient echo, mixed gradient- and spin- echo, and various implementations of echo planar imaging sequences.

In the other approach, a stationary state of magnetization is created by using a series of evenly spaced rf pulses. This stationary state is shown to be a sum of magnetic substates, each with unique contrast characteristics. When more than one substate contributes to an MR image, the substates interfere with each other, producing ghosts and other artifacts. However, appropriately designed gradient protocols can image single substates, producing ghost free, non-blurred images. The contrast of the image depends largely on the choice of the imaged substate. This method is utilized in fast gradient echo protocols that are used in MR angiography, dynamical contrast uptake studies, and functional MRI.

The needs of commercial MR market resulted in a wide range of proprietary protocols identified to clinical users by trademark acronyms. Vendors claim that each protocol is unique and distinctly different from all others. However, when a detailed analysis of physical principles that govern the design of fast MR imaging protocols is performed, a simpler pattern emerges. Since the implementation details of an imaging protocol matter to imaging clinicians only to the extent to which these details affect the image contrast, it becomes possible to group the large number of existing protocols into a few simple classes. Each protocol within a class, although possibly containing unique proprietary implementation elements, produces images with the same contrast when used with the same set of imaging parameters.

Educational Objectives:

1. Review recent advances in fast MRI applications

2. Formulate conditions necessary and sufficient to produce artifact-free images using stationary and non-stationary states of magnetization.

3. Understand commercial implementations of fast MRI protocols.

\section{Continuing Education Course Room: Browning A Ultrasound - 2}

\section{TU-B6-01}

Intravascular Ultrasound Imaging: Today and Beyond

Peter Fitzgerald, Stanford University Medical Center, Stanford, CA

Catheter-based intravascular ultrasound (IVUS) has become a common tool in today's catheterization laboratory. Imaging is increasingly being used to assist in device selection and endpoint assessment, such as guiding the cutting protocol in directional coronary atherectomy, appropriately sizing the rotational atherectomy burr, optimizing stent deployment, and quantitating luminal geometry following balloon angioplasty.

One of the most significant contributions made by ultrasound imaging has been to modify and redefine the concept of restenosis. Recent work suggests that a significant contribution to late lumen narrowing following intervention is overall vessel contracture or "reverse" remodeling. In other words, intimal hyperplasia is not the sole culprit responsible for restenosis. Additionally, intravascular ultrasound studies have provided strong evidence that plaque burden or residual plaque stenosis may play a critical role in determining which lesions are likely to develop restenosis. The GUIDE trial showed a strong correlation between residual plaque burden and both angiographic and clinical restenosis for patients undergoing either balloon angioplasty and/or directional coronary atherectomy. Testing this finding in related trials, aggressive DCA with ultrasound guidance (OARS, ABACAS) has shown that a reduction in plaque residual directly impacts favorably on lowering the restenosis rate without increasing acute complication rates.

The most widely used application of IVUS is to optimize stent deployment. Early on, despite adequate deployment by angiography, ultrasound demonstrated incomplete expansion in the majority of stented segments. This finding lead to the adoption of high-pressure expansion techniques, which markedly improved subacute closure rates, despite a reduction in anticoagulation protocols. Several ongoing trials are designed to determine if the ultrasound findings of incomplete expansion, incomplete apposition, and/or the presence of edge tears in the high-pressure deployment era impact on the short and long-term outcome of patients undergoing coronary stenting. Recently the CRUISE trial showed that IVUS guidance of stent implantation results in a $44 \%$ reduction in target vessel revascularization compared to angiographic guidance alone.

In general, intravascular ultrasound has modified our concept of restenosis, provided detail about plaque/vessel morphology for directing device selection and optimization, and is becoming a standard tool for endpoint assessment in today's interventional cath lab.

The author would like to acknowledge partial support from Boston Scientific, Medtronics, and Endosonics corporations.

Educational Objectives:

1. To review the use of catheter-based intravascular ultrasound as a standard tool for assessment in today's interventional catheterization laboratory.

2. To discuss how intravascular ultrasound has made significant contributions toward modifying and redefining the concept of restenosis.

3. To describe how intravascular ultrasound is widely used to optimize stent deployment.

\section{Scientific Session \\ Pearl \\ Brachytherapy}

\section{TU-C1-01}

Implementation of Intraoperative Source Position Reconstruction in Prostate Brachytherapy

S Sutlief*, K Wallner ${ }^{1,2}$, K Lai ${ }^{2}$, K Thornton, W Katz, T Jackson ${ }^{3}$, (1) VA Puget Sound Health Care System, Seattle, WA, (2) University of Washington, Seattle, WA, (3) Multimedia Medical Systems, Charlottesville, VA

Prostate brachytherapy is an effective therapy for early stages of prostate carcinoma. To enhance quality of implants, we are implementing an intraoperative system to visualize the brachy dose. The dose reconstruction is used to identify under-dosed regions, so that extra seeds can be optimally added. After implanting the planned seeds, three isocentric fluoroscope images are taken at 15 degree increments; seed positions are reconstructed and registered with an edema-adjusted version of the pre-implant volume study. We have identified several factors which affect the accuracy of this technique, including accuracy of the reconstruction, swelling of the prostate during the procedure, and accuracy of the registration with the volume study. To investigate the accuracy of the reconstruction, we used a phantom to measure source position reconstruction accuracy from fluoroscopic images, 
for which the seed position discrepancy averages $2.1 \mathrm{~mm}$ (range: 0.0 to 4.8 $\mathrm{mm}$ ). Swelling of the prostate during the procedure occurs primarily in the anterior-posterior dimension, averaging $29 \%$. To account for this, we use ultrasound to estimate the new anterior-posterior, lateral and base-apex dimensions. To register the reconstructed source positions with the volume study, a wire with radio-opaque markers is placed in the urinary catheter to locate where the urethra enters and exits the prostate. These reference points can be identified to within $3 \mathrm{~mm}$. This dose evaluation technique can be repeated after additional seeds are implanted, eliminating the need for postimplant CT.

\section{TU-C1-02}

Dosimetric Effects of Edema in Permanent Prostate Seed Implants: A Rigorous Solution

Z Chen*, N Yue, R Peschel, R Nath,Yale University School of Medicine, New Haven, CT

By parametrizing the dose fall-off from a radioactive seed as a single inverse power function of distance $\left(\mathrm{D}(\mathrm{r}) \propto \mathrm{r}^{-2.4}\right.$ for ${ }^{125} \mathrm{I}$ and $\propto \mathrm{r}^{-2.8}$ for $\left.{ }^{103} \mathrm{Pd}\right)$, a rigorous solution of the dosimetric effects of edema on a permanent prostate seed implant has been derived. The solution provided an explicit proof that the relative dosimetric effects of edema, as found in the previous numerical studies by Yue et. al. [1], are independent of the size of implant volume, the number of seeds used, and the seeding configuration for a given edema. It showed that the size of relative dosimetric effects caused by an edema is also independent of the dose evaluation point within the target volume. The analytic solution was found in good quantitative agreement with those obtained from the direct numerical simulation [1], which enables a complete tabulation of the relative dosimetric effects of edema as a function of the edema magnitude and edema half-life for both iodine-125 and palladium103 permanent implants. These tables make it easy to assess the impact of edema in preplanning a prostate seed implant and in evaluating the accuracy of conventional post implant dosimetry.

[1] Yue et al, Int. J. Radiat. Oncol. Biol. Phys. 43, 447-454, 1999.

\section{TU-C1-03}

Identification of the Target Tissue by comparison of Studies with Widely Different Dose/Depth Functions

A Li*, J Hausleiter, C Abbey, R Makkar, J Whiting, N Eigler, CSMC, Los Angeles, CA

Background. In the pig model isotopes and intracoronary radiation delivery systems have inhibited neointima thickening after stent placement or balloon injury. We compared several studies with widely disparate depth/dose functions to locate the tissue that is most closely correlates with a beneficial effect.

Methods. We retrospectively compared 22 protocols of 9 studies comprising 170 arteries irradiated with $\operatorname{Ir}{ }^{192}, \mathrm{Sr} / \mathrm{Y}^{90}, \operatorname{Re}^{188}, \mathrm{Re}^{188 / 186}$ and $\operatorname{Re}^{186}$. Studies with balloon overstretch injury and stent placement were included. Depth dose functions were recalculated from 0 to $2.5 \mathrm{~mm}$ radial depth from the arterial surface at $0.25 \mathrm{~mm}$ intervals. Inhibition of neointimal area was calculated relative to the control groups comprising a total of 93 nonradiated arteries. The recalculated dose at different tissue depths was compared with the inhibition of neointima area.

Results. Inhibition of neointima area was dose related irrespective of tissue location. In the model the best agreement between dose and inhibition was found for a tissue depth at $0.75 \mathrm{~mm}$ from the lumen surface. At the lumen surface and at deeper tissue depths the agreement was considerably lower. To achieve a $50 \%$ inhibition of neointima proliferation a dose of about $12 \mathrm{~Gy}$ at 0.75 tissue depth is needed regardless of the isotope.

Conclusions. This analysis supports earlier histochemical data indicating that the adventitia is the target tissue for radiation therapy. The data support that the dose prescription point should be at $0.75 \mathrm{~mm}$ radial to the vessel/lumen interface.

\section{TU-C1-04}

Dosimetry of a New Design 103Palladium Brachytherapy Source

R Wallace*1, J Fan ${ }^{2}$, (1)UCLA Radiation Oncology, Los Angeles, CA, (2)North American Scientific Inc., N. Hollywood, CA
New source designs of encapsulated low-energy gamma emitting isotopes for permanent implant require full dosimetric analysis and calibration standardization before responsible clinical application. The results of such experimental measurement and analysis are reported for a new ${ }^{103}$ Palladium source, model MED3633, for liquid water medium in accord with AAMP Task Group \#43 recommendations. Dose measurements used standard methods employing thermoluminscent dosimeters in a water equivalent plastic phantom. Precision machined bores in the phantom located dosimeters and source(s) in reproducible fixed geometry providing transverse-axis and angular dose profiles over a range of distances from 0.17 to $7 \mathrm{~cm}$. The dose-rate constant, $\Lambda$, was evaluated with reference to a ${ }^{60}$ Cobalt standard, accounting for response variation with isotope energy spectrum. The radial dose function, $\mathrm{g}(\mathrm{r})$, anisotropy function, $\mathrm{F}(\mathrm{r}, \theta)$, anisotropy factor, $\phi_{\mathrm{an}}(\mathrm{r})$, and anisotropy constant, $\bar{\phi}_{\mathrm{an}}$, were derived from dose distribution data measured in the phantom, accounting for finite dosimeter volume and inter-chip effects. The results are compared to TG43 data for ${ }^{103} \mathrm{Pd}$ sources. The new source demonstrates a radial dose function, $g(r)$, that is equivalent to that of the model $200{ }^{103} \mathrm{Pd}$ source design. The MED3633 source appears more isotropic than the model 200 source. The dose-rate constant, $\Lambda=0.70$, of the MED3633 source with NIST strength calibration, while that of the model 200 source is 0.74 , using the manufacturer's strength calibration. A true comparison would require the same calibration standard.

Supported in part by North American Scientific, Inc.

\section{TU-C1-05}

Dosimetry of a New Design 125Iodine Brachytherapy Source

R Wallace*, J Fan, UCLA Radiation Oncology, Los Angeles, CA, North American Scientific Inc., N. Hollywood, CA

Dosimetric measurements were performed to characterize a new ${ }^{125}$ I source that is a variant design of an existing source, designated as MED3631-A/S, and that has application in interstitial brachytherapy. The new source, designated as MED3631-A/M, has centralized radio-opaque markers. In the original MED3631-A/S source, the radio-opaque markers are separated. Thermoluminescent dosimeters were placed in phantom to measure transverse-axis and angular dose profiles over a range of distances from 0.5 to $7 \mathrm{~cm}$. The data were analyzed in terms of parameters recommended by AAPM Task Group \#43. Tabular data evaluated in liquid water are provided for the dose-rate constant, $\Lambda$, radial dose function, $g(r)$, the anisotropy function, $F(r, \theta)$, the anisotropy factor, $\phi_{a n}(r)$, the point-source approximation anisotropy constant, $\bar{\phi}_{\mathrm{an}}$. The dose-rate constant was determined by an absolute method using a Cobalt- 60 reference and by relative measurements using calibrated ${ }^{125}$ I source(s). Values of the doserate constant are provided for both the 1985 and 1999 NIST air-kerma strength standards. The new source is comparable to both the MED3631-A/S and the model $6702{ }^{125}$ I source designs, demonstrating equivalent radial dose function, $\mathrm{g}(\mathrm{r})$. Differences in the value of the doserate constant, $\Lambda$, and the anisotropy of the dose distributions in phantom are discussed in light of the improved isotropy of the new design, the MED3631-A/M source, and the uncertainty involved in the dose measurement using a Cobalt-60 reference.

Supported in part by North American Scientific, Inc.

\section{TU-C1-06}

New 125-I Brachytherapy Source Dosimetric Characteristics

A Nelson*, T Painter, A Meigooni, G Ibbott, University of Kentucky, Lexington, $\mathrm{KY}$

Because prostate brachytherapy implants are growing in popularity, there is a market for new seeds. The dosimetric characteristics (anisotropy function, radial dose function, and dose rate constant) of a new ${ }^{125}$ I seed have been determined experimentally according to the AAPM Task Group 43 recommendations and were related to the 1999 NIST calibration assigned to this source $\left[\mathrm{S}_{\mathrm{k}, 99 \mathrm{std}}\right]$. Phantoms made of solid water were machined to accommodate the $\mathrm{LiF}$ thermoluminescent dosimeters that were used to perform the measurements. Two sizes of chips were used with dimensions $3.1 \times 3.1 \times 0.8 \mathrm{~mm}^{3}$ and $1.0 \times 1.0 \times 1.0 \mathrm{~mm}^{3}$. The TLD chips were 
surrounded by at least $10 \mathrm{~cm}$ of Solid Water ${ }^{\mathrm{TM}}$ phantom material to provide full scattering conditions. MD-55* radiochromic film and BANG gel were also used to take measurements. The anisotropy function, $F(r, \theta)$, of the new ${ }^{125} \mathrm{I}$ source was measured at distances of $2 \mathrm{~cm}, 3 \mathrm{~cm}, 5 \mathrm{~cm}$, and $7 \mathrm{~cm}$ from the source center. These data compared favorably with those from the 6711 and 6702 sources. The radial dose function, $\mathrm{g}(\mathrm{r})$, of the new ${ }^{125} \mathrm{I}$ source was measured at distances ranging from $0.2 \mathrm{~cm}$ to $10 \mathrm{~cm}$. The results indicated a dose rate constant, $\Lambda$, of $1.06 \pm 4 \% \mathrm{cGy}^{-1} \bullet^{-1} \mathrm{U}^{-1}$ for the new ${ }^{125} \mathrm{I}$ source as compared to $0.98 \mathrm{cGy} \mathrm{h}^{-1} \bullet \mathrm{U}^{-1}$ and $1.04 \mathrm{cGy}^{\circ} \mathrm{h}^{-1} \bullet \mathrm{U}^{-1}$ for the 6711 and 6702 seeds, respectively. Complete dosimetric data are described in this manuscript. Research supported by a contract with Imagyn Medical Technologies Inc.

\section{TU-C1-07}

TLD Dosimetry for a New Model of I-125 Seed

N Patel *, S Chiu-Tsao ${ }^{1}$, D Shasha ${ }^{1}$, L Harrison ${ }^{1}$, (1) Beth Israel Medical Center, New York, NY, (2) Beth Israel Medical Center and St. Lukes Roosevelt, New York, NY

As the efficacy of brachytherapy prostrate treatment is becoming realized, new models of ${ }^{125}$ I seeds are being introduced. In this paper we present TLD Dosimetry in solid water phantom for a new design of ${ }^{125}$ I seed (Model I25.S06) from UroMed/Bebig. TLD cubes (LiF TLD-100) with dimension 1 x $1 \mathrm{x} 1 \mathrm{~mm}$ were irradiated at various distances from the seed at angles ranging from $0^{\circ}$ to $90^{\circ}$ in $10^{\circ}$ increments. Sensitivity calibration of the TLD's was achieved by irradiation with a Cobalt Teletherapy unit between exposures of ${ }^{125} \mathrm{I}$. Concurrent with the ${ }^{125} \mathrm{I}$ seed exposures, a group of TLD's was also exposed to ${ }^{60} \mathrm{Co}$ as controls. Bicron Model 5500 Automatic TLD Reader was used to read the cubes. Dose rates per unit air kerma strength were determined based on the NIST traceable standard. Dose data are presented in TG43 format as a function of distance and angle. Values for $\mathrm{F}(\mathrm{r}, \theta), \mathrm{g}(\mathrm{r})$, and the anisotropy constant are obtained for use in treatment planning computer systems. We find that the relative dose distributions of Model I25.S06 are similar to Model 6702. Agreement with Monte Carlo results is discussed.

This work is financially supported by UroMed/ Bebig.

\section{TU-C1-08}

Virtual Simulation and CT-Based Planning of Interstitial GYN Brachytherapy

Z Li*, J Palta, T Mitchell, C Liu, S Chafe, R Marcus, N Bhandare, University of Florida, Gainesville, FL

The pre-planning of interstitial GYN implants using a template and postimplant planning based on planar films is traditionally a tedious and laborious process. A virtual simulation technique has been developed for the pre-planning of these implants that allows the simultaneous visualization of patient anatomy and the potential needle tracks in the patient. A patient is scanned on MRI or CT with a vaginal obturator and template applicator inserted. The applicator set is also scanned separately on CT with needles inserted into the template. This image data set is then registered to the patient image data set through image fusion, using the vaginal obturator for alignment. The fused image data set, with all potential needle tracks in the patient displayed as overlay on the patient anatomy, is used directly for preplanning of the implant. A set of post-implant CT images is obtained of the patient for treatment planning. The needles are localized on CT images and dose distributions are calculated. Image fusion may again be used to overlay the preplanning CT/MRI images on the post-implant CT images to evaluate the accuracy of needle insertion. This process has been found to be significantly more efficient than the traditional process of preplanning and post-implant dosimetry for interstitial GYN implants based on planar films, and allows quantitative evaluation of doses to organs at risk and target coverage.

\section{TU-C1-09}

Comparison of Radiochromic Film Dosimetry to a Theoretical Model of a Beta Emitting Stent in a Realistic Artery With Soft Tissue and Plaque C Janicki ${ }^{1}, D$ Duggan, A Gonzalez*, C Coffey ${ }^{2}$,(1) University of Montreal, Montreal, Quebec, (2)Vanderbilt University Medical Center, Nashville, TN
Radiochromic film dosimetry was performed with stents coated with P-32 and Y-90 in an experimental setup designed to test a model for the description of the dose deposition from a beta-emitting stent in an arterial system consisting of soft tissue and plaque. The model is based on the scaling properties of the dose-point-kernel which are extended to heterogeneous media consisting of a series of layers of different materials. For each point source originating from the stent surface, the kernel for water is scaled consistently along the path through the different layers of material to predict the dose at a given point in the heterogeneous media. Radiochromic film dosimetry on actual P-32 stents is used to validate the new model. The experimental setup consists of a water equivalent phantom in which a stent is deployed with or without a thin layer of teflon to simulate the presence of plaque. Layers of radiochromic films stacked over the phantom are used to measure the dose at distances varying from $0.1 \mathrm{~mm}$ to 3 $\mathrm{mm}$ from the stent surface. The experimental measurements on all films exposed to P-32 stents and are in very close agreement with the model predictions. The dose per decay from a Y-90 stent is higher at large distances as expected. A theoretical model for a Y-90 stent is under development.

Supported by a grant from Isostent, Inc.

\section{TU-C1-10}

Dosimetry of Beta-ray Ophthalmic Aapplicators: Comparison of Different Measurement Methods

C Soares*1, S Vynckier ${ }^{2}$, H Jarvinen ${ }^{3}$, W Cross $^{4}$, J Hokkanen ${ }^{3}$, P Sipila ${ }^{3}$, D Fluehs $^{5}$, B Schaeken ${ }^{6}$, F Mourtada ${ }^{1}$, G Bass ${ }^{7}$, T Williams ${ }^{7}$, (1)NIST, Gaithersburg, MD, (2)Catholic University of Louvain, St-Luc Hospital, Brussels, Belgium, (3)Radiation and Nuclear Safety Authority, Helsinki,Finland, (4)Atomic Energy of Canada Ltd., Chalk River, Canada, (5)University Hospital of Essen, Essen, Germany, (6)A.Z. Middelheim, Antwerp, Belgium, (7)National Physical Laboratory, Teddington, UK

An international intercomparison of the dosimetry of three beta particle emitting ophthalmic applicators was performed, which involved measurements with radiochromic film, thermoluminescence dosimeters (TLDs), alanine pellets, plastic scintillators, an extrapolation ionization chamber, a small fixed-volume ionization chamber, a diode detector and a diamond detector. The sources studied were planar applicators of ${ }^{90} \mathrm{Sr}^{90} \mathrm{Y}$ and ${ }^{106} \mathrm{Ru}-{ }^{106} \mathrm{Rh}$, and a concave applicator of ${ }^{106} \mathrm{Ru}-{ }^{106} \mathrm{Rh}$. Comparisons were made of absolute dosimetry determined at $1 \mathrm{~mm}$ from the source surface in water or water-equivalent plastic, and relative dosimetry along and perpendicular to the source axes. The results of the intercomparison indicate that the various methods yield consistent absolute dosimetry results at the level of $10 \%$ to $14 \%$ (one standard deviation) depending on the source. For relative dosimetry along the source axis at depths of $5 \mathrm{~mm}$ or less, the agreement was $3 \%$ to $9 \%$ (one standard deviation) depending on the source and the depth. Crucial to the proper interpretation of the measurement results is an accurate knowledge of the detector geometry, i.e . sensitive volume and amount of insensitive covering material. From the results of these measurements, functions which describe the relative dose rate along and perpendicular to the source axes are suggested.

\section{Scientific Session IMRT Optimization}

Room: Mississippi

\section{TU-C2-01}

Intensity Modulation in Radiotherapy: Photons versus Protons

A Lomax*, M Goitein, J Adams, Paul Scherrer Institute, Villigen, Aargau, Switzerland

Aims.

To investigate the potential differences between intensity modulated photon and proton therapies through the application of progressively more stringent dose constraints.

Methods.

Intensity Modulated (IM) treatment plans for a para-nasal sinus case have been calculated for both photons and protons using an identical, dose based optimisation algorithm. IM plans (X1 and P1) for both modalities were initially constructed using standard maximum dose constraints. Additional photon plans were then calculated to try to match the DVHs of the orbits 
(plan X2) and all critical structures (plan X3) to the corresponding DVHs of the proton plan.
Results.

Plans X1 and P1 both produced good target coverage, and provided similar compliance of all identified critical structures to the defined dose constraints. On reducing the low dose contribution to both orbits in the photon plan (X2), an increased dose heterogeneity across the target resulted. Similarly, when all normal tissues were more strictly constrained (plan X3), target dose homogeneity was further compromised, as was conformation of the dose to the target volume. Analysis of normal tissue integral dose showed an increasing dose load to non-critical normal tissues when progressing from plan X1 to X3.

Conclusions.

Both IM protons and photons were found to provide comparable target volume conformation and high dose sparing of critical structures. However, the use of IM protons provided the only method by which critical structures could be spared at all dose levels, and still provide an acceptable dose homogeneity within the target volume.

\section{TU-C2-02}

Dose Painting for Prostate Cancer Using IMRT techniques

L Happersett*, M Hunt, C Chui, C Burman, C Ling, M Zelefsky, S Leibel, H Amols, Memorial Sloan Kettering Cancer Center, New York, NY

Dose painting, the ability to identify specific areas of gross disease and normal tissue within a target and sculpt the dose distribution to deliver desired dose to these areas, is becoming possible by incorporating biological imaging into IMRT planning. The purpose of this study was to evaluate the efficacy of IMRT planning to deliver these types of treatments for prostate cancer. Plans using 30 equispaced coplanar fields were designed to treat a small GTV within the PTV (prostate and seminal vesicles plus margin). The goal was to deliver 99 Gy to the GTV and 91 Gy to the PTV, except where the PTV and critical structures overlap. Doses to normal structures were constrained such that the urethral dose was limited to 86 Gy and no more than $30 \%$ of the rectal wall received more than $75.6 \mathrm{~Gy}$.

Treatment plans were generated for 5 patients. The average volume of the PTV excluding critical structures which received at least 86.5 Gy $(95 \%$ of 91 Gy) was $89 \pm 3 \%$. Minimum and average GTV doses were $94 \pm 5$ Gy and $104 \pm 1 \mathrm{~Gy}$, respectively. Maximum urethral dose was $85.6 \pm 0.7 \mathrm{~Gy}$ and the rectal wall volume receiving more than 75.6 Gy was $21 \pm 7 \%$. The effects of GTV position and number of fields on the dose distributions will also be discussed. This study indicates the feasibility of dose painting and IMRT to dose escalate to unprecedented levels while respecting the predetermined normal tissue constraints.

\section{TU-C2-03}

Global and Local Maxima in the Dynamically Penalized Likelihood Method of Inverse Therapy Planning

J Llacer*, EC Engineering Consultants LLC, Los Gatos, CA

It is generally known and well documented that the Simulated Annealing (SA) method of inverse therapy planning for Intensity Modulated Radiation Treatment (IMRT) must have a provision to "jump out" from local minima of its objective function if it is to reach a desired global minimum. In this paper, it is shown that the iterative Dynamically Penalized Likelihood (DPL) method either does not need to jump out of any local maxima in the easier problems or it can do so easily, if needed, in the more difficult optimization problems. The model for DPL IMRT that emerges from this study is one in which the DPL solutions lie on, or very close to, a nearly flat shell in multidimensional parameter space (beam weight space) which has the Maximum Likelihood (ML) solution for the PTV at its maximum. For the IMRT case, maximum likelihood implies minimum least square errors. In the case in which the final DPL solution lies on the ML shell, the DPL solutions exhibit very small rms errors in the PTV. When the final DPL solution is not on the ML shell, a few passes with a Maximum Likelihood Estimator (MLE) will bring the solution to that shell, "jumping out" of an apparent local maximum, with a rapid improvement of the dose distribution for the PTV, all the while maintaining excellent low doses in the OAR's. 


\section{TU-C2-04}

Optimization of Weightings for the Beams Selected Through Forwardplanning Process

Y Xiao*, J Galvin, R Valicenti, Thomas Jefferson University, Philadelphia, PA

Inverse planning (IP) has stirred considerable excitement in the community. Its objective is to make the dose conform to the tumor while sparing other organs. Since IP is time-consuming and does not necessarily produce an ideal plan, we sought to achieve the same goal by an extension of conventional treatment planning techniques. The beam orientation and wedge angles were chosen in the conventional rule-based manner. However, within each beam's-eye view, we used multiple field openings. One field opening conformed to the tumor only, with an appropriate margin. The other field openings were smaller and served to reduce the dose to critical structures in the path of the larger opening. As the number of organs to be spared increases, the number of fields for each beam increases. Fortunately, there are very sound analytical tools for dealing with multiple fields, the Cimmino simultaneous projection method, etc.... We employed the method to obtain the optimized weighting for each field of each beam. We found that if the dose constraint for tumor and critical organ is reasonable, we were able to obtain a plan with a limited number of beams that satisfy the specified dose objective.

\section{TU-C2-05}

Implementation of a Fast Algorithm for 3-D Inverse Treatment Planning for IMRT

Y Xiao*, Thomas Jefferson University, Philadelphia, PA

Deriving accurately and efficiently the optimized beam angle and beam intensity pattern, given certain constraints for the tumor to be treated and the critical organs to be spared, is the key to successful delivery of IMRT (Intensity Modulated Radiation Therapy). The search for the optimized parameters consists of two processes: dose calculation and the adjustment of parameters to achieve the objective. In this study, we have used evenly spaced beam angles. For each beam angle, the beam opening is divided into two-dimensional matrices of beam intensities. Dose calculation is performed for each of these voxels using beam tracing and FFT convolution of electron transport kernels and scatter kernels. A reduced size of the matrices is chosen in order to increase the calculation speed while maintaining the calculation accuracy. Dose to the tumor and critical organ due to each beam voxel is scored. The optimized weighting of beam voxels is obtained by using the Cimmino simultaneous projection method, which solves a system of inequalities without resorting to repetitious dose calculations. The time required for the optimization of beam intensity of five $10 \times 10 \mathrm{~cm}^{2}$ fields $(1 \times 1$ $\mathrm{cm}^{2}$ voxels) with $0.5 \times 0.5 \times 0.5 \mathrm{~cm}^{3}$ dose resolution for the phantom is on the order of 5 minutes on a standard $300 \mathrm{Mhz}$ pentium PC.

\section{TU-C2-06}

Generic Inverse Treatment Planning of Few-field Intensity-modulated Boost Treatments of Prostate Cancer

D. Hristov*1, B.A. Moftah ${ }^{1}$, L. Dyke ${ }^{1}$, L. Souhami ${ }^{1}$, C. Huntzinger ${ }^{2}$, E.B. Podgorsak $^{1}$, (1) Department of Radiation Oncology, McGill University, Montreal, QC, (2) Varian Associates, Palo Alto, CA

In our clinic, conformal prostate boost treatments are delivered by a threefield technique, which employs custom-made shields and physical modifiers. In order to decrease the departmental workload involved in the planning, preparation and delivery of such treatments, we have been exploring the possibility of replacing the current technique by a similar one based on the use of intensity-modulated beams delivered by multiple MLC fields. Such an approach requires routine use of inverse treatment planning (ITP), a process which involves the tuning of several parameters. However, we have demonstrated that a single optimal set of these parameters produces consistent and clinically acceptable plans for prostate boosts. Using the forward planning (FP) beam geometries we replanned fifteen patients with ITP. A single set of dose/weight parameters was used. Compared to FP, the ITP provided better target coverage and consistently reduced the average doses to the rectum and the bladder by, on average, 2 Gy for a prescription target dose of 25.2 Gy. The ITP resulted in "Hot Spots" in the PTV but the dose values of these "Hot Spots" were generally within the ICRU 50 limit $(+7 \%$ of the prescription dose). Our study shows that generic ITP planning of three-field boost in the course of the radiation therapy of prostate cancer is feasible. Generic ITP can provide the basis for routine IMRT, which, once commissioned, can significantly reduce the departmental workload involved in the treatment of prostate cancer.

\section{TU-C2-07}

Designing IMRT Plans for Head and Neck Cancers

Q Wu*, R Mohan, R Schmidt-Ullrich, Medical College of Virginia, Virginia Commonwealth University, Richmond, VA

Conventional 3D Conformal radiotherapy (3DCRT) of head and neck cancers results in fairly good local control, but significant complications are observed. Normal tissue toxicity limits the dose to the tumor and nodes. In addition, 3D conformal treatments are complex and prone to mistakes. Intensity Modulated Radiotherapy (IMRT) has the ability to spare critical organs around the tumor to a greater extent and allow escalation of tumor dose. An IMRT optimization program developed at our institution is interfaced to a commercial $3 \mathrm{D}$ treatment planning system. The program can be used with either dose- or dose-volume-based objective functions. In this study, we applied the program to a group of $H \& N$ patients with tumors of various shapes, sizes and locations. The IMRT plans were compared with conventional plans with the aid of isodose distributions on orthogonal planes and dose-volume histograms. Beam configurations and optimization parameters were adjusted to accommodate the unique requirements of each case. It was found that IMRT plans offer improved target dose conformity and critical structure sparing. The planning and delivery of IMRT plans is, in principle, simpler than the 3DCRT plans. In IMRT plans, it is possible to treat multiple targets and elective nodes with different levels of prescription doses simultaneously in a single plan, thus eliminating field matching problems of conventional treatments. In addition, the use of electrons can be avoided for many cases. IMRT treatments designed in this way may also be biologically advantageous.

\section{TU-C2-08}

CONFORMAL BOOSTS: Should They be Included in One Dose Distribution or as an Aditional Plan?

L Jones*1, P Hoban ${ }^{2}$, M Barton ${ }^{3}$, (1) Department of Radiation Oncology, Liverpool hospital,Sydney,Australia (2) Department of Radiation Oncology, Prince of Wales Hospital, Sydney,Australia, (3) Simpson Centre, Liverpool Hospital, Sydney, Australia

This study investigates options for conformal boosting when IMRT is used. A boost dose can be delivered as part of the standard dose distribution where the dose per fraction to the centre region is greater, or the boost can be delivered after the main treatment as has traditionally occurred.

The effects to the tumour, the acutely reacting normal tissues and the late reacting normal tissues have been assessed in this study using the Linear quadratic model. The effects to the tumour and early reacting normal tissues have been set to be the same as that which would be achieved for a standard treatment of 25,2 Gy fractions and a following boost of another 5,2 Gy fractions. These calculations have been performed with and without a proliferation factor. When proliferation is not included the advantage, to late responding normal tissues with included boosting, results when the total number of fractions to the large volume is extended to that delivered to the central boost volume. This changes when proliferation is included. For short potential doubling times a minimum in the late tissue BED curve results as a larger dose per fraction is necessary for long schedules. For this situation with a potential doubling time of 4 days, 25 fractions is the most advantageous for the late responding normal tissue in the central boost volume, while 20 fractions is the most advantageous for the larger volume.

\section{TU-C2-09}

Should Intensity Modulated Cost Functions be Biologically or Physically Based?

L Jones*1, P Hoban², (1) Department of Radiation Oncology, Liverpool Hospital, Sydney,Australia, (2) Department of Radiation Oncology, Prince of Wales Hospital, Sydney Australia

This study has involved determining Intensity Modulated Radiotherapy (IMRT) plans using physical and biologically-based optimisation 
algorithms. The difference applies to both the cost function and the (deterministic) method of updating fluence elements.

In both cases a ratio based fluence changing method was used in an iterative process to determine the fluence distributions necessary to conform to the tumour volume while avoiding critical structures. For the biological optimisation two parameters were used to assess the distribution compared to an ideal distribution. Effective Uniform Dose was used for the tumour volume and Integral Biological Equivalent Dose was used for the critical and normal structures.

Thirty-five beams, each consisting of 99 pencil beams were used for the optimisation which resulted in highly conformal distributions in both situations. The homogeneity of the distributions was however very different. The biologically based distribution resulted in a very high dose to the centre of the tumour volume with sharp fall off at the edge. The physically optimized distribution resulted in a much flatter distribution but not as dramatic a fall off at the edge of the tumour volume.

The important question is whether a dose distribution with favorable biological indices resulting from biologically-based optimisation should be accepted even though it is less favorable than the result of dose-based optimisation, in terms of the traditional criterion of dose homogeneity in the tumour. The answer obviously depends on the tolerance of normal structures inside the target volume.

\section{TU-C2-10}

Evaluation of a Quantitative Dose Comparison Tool for IMRT and Conformal Therapy

D Low*, J Markman, J Dempsey, S Mutic, J Purdy, Mallinckrodt Institute of Radiology, St. Louis, MO

A quantitative dose comparison tool has recently been introduced that provides a method for comparison between two dose distributions. The method uses a generalized dose-difference and distance-to-agreement metric (termed $\gamma$ ) to compare reference and evaluated distributions, which can be measurements-to-measurements, measurements-to-calculations, or calculations-to-calculations. Values of $\gamma$ range from zero to infinity, and $\gamma>1$ indicates that the distribution fails the specified comparison criteria. Tests of this technique are made using well-described test dose distribution geometries including a beam's-eye-view of a square open megavoltage field with the evaluated distribution modified to provide tests that include varying dosimetric and spatial offsets between the two distributions. Pseudorandom noise is added to both distributions to mimic the effect of experimental noise and evaluate the efficacy of the tool under these conditions. The sensitivity of the tool to variations in the evaluation criteria are also investigated. Because the technique provides a numerical value to the dose distribution comparison, the values are gathered into cumulative histograms for summary presentation. The addition of noise to the reference distribution has significantly larger impact than does noise added to the evaluated distribution, with large variations in $\gamma$ with changes as small as 0.67 the size of the criteria. Adjustments in the criteria have significant impact as expected, with the influence of the criteria principally a function of the type of disagreement between the two distributions. This study provides guidelines for the use of this tool in clinical evaluations.

\section{Symposium CIRMS Measurements for Radiation Therapy Applications}

\section{TU-C3-03}

CIRMS Measurements for Radiation Therapy Applications

Bert Coursey ${ }^{1}$, Chris Soares ${ }^{1}$, Larry DeWerd ${ }^{2}$, Jileen Shobe ${ }^{1}$, Tom Heaton $^{3}$, (1) National Institute of Standards and Technology, Gaithersburg, $\mathrm{MD}$,

(2) University of Wisconsin, Madison, WI, (3) CDRH FDA, Rockville, MD

The Council on Ionizing Radiation Measurements and Standards (CIRMS) represents users of ionizing radiation and radioactive sources engaged in medical radiation and other programs. CIRMS provides a forum for discussing ionizing radiation issues; identifying, defining and prioritizing needed work for standards; disseminating information on standards; and organizing workshops and meetings to advance ionizing radiation technology. The National Institute of Standards and Technology (NIST) is heavily involved with this organization and considers its directives as statements of work to be completed. A presentation of the research and measurements made for standards for Medical Radiation Therapy Applications will be summarized addressing three major topics: Absorbed Dose to Water Calibration, Brachytherapy seed calibrations and calibrations for intravascular applications. Each of these standards is described in Measurement Program Descriptions (MPD), which are statements of requirements and the reasons for the measurements. Discussion of the measurements to determine an absorbed dose to water in-phantom calibration, in support of AAPM's new protocol written by TG-51, will be presented. NIST's role was to establish the U.S. standard linking the Domen water calorimeter to measurements made with ionization chambers. This process will be discussed along with results from recent international comparisons. The transfer of this standard to the ADCLs will also be discussed in line with the AAPM task group for this purpose. Also presented will be the results from the ADCL round-robin proficiency test conducted by NIST. Recently there has been a great deal of activity on low energy brachytherapy seeds for prostate treatments. The recent change in brachytherapy I-125 source calibrations is a result of measurements made with the Wide Angle Free Air Chamber (WAFAC). The design and use of this free air chamber to measure low energy brachytherapy seeds, including Pd-103 will be given. A new area of activity has been for the prevention of restenosis after angioplasty with the use of radioactive sources. The AAPM Task Group 60 has indicated the importance of calibration of such sources. The system for standardized dosimetry for intravascular brachytherapy sources will be discussed, emphasizing the developmental work which has been performed and which continues at NIST. Calibration methods for these sources are evolving almost as fast as the sources are being developed for this application. The challenges are the very close distances to the very small sources at which dosimetry is required, and the large number of isotopes and source geometries now under active investigation. Finally a review of the regulatory issues with sources used in radiation therapy will touch on three areas of regulatory concern from a physics point of view: (1) IDE requirements for studies involving intravascular brachytherapy, (2) brachytherapy sources with emphasis on those low energy sources used to treat prostrate cancer, and (3) well ionization chambers. While this talk will focus on the issues from the FDA/CDRH point of view, the NRC also has concerns in these areas that will be mentioned.

\section{Educational Objective:}

1. An understanding of equipment and methodology involved in the establishment of standards for Radiation Therapy Applications.

\section{Scientific Session Computer-Aided Diagnosis/PACS}

Room: Carroll

\section{TU-C4-01}

Performance Evaluation of an Automated Microcalcification Detection System

H Chan*, L Hadjiiski, N Petrick, M Helvie, M Roubidoux, B Sahiner, University of Michigan, Ann Arbor, MI

We have developed a computerized system to assist radiologists in detection of microcalcifications on mammograms. An input mammogram is digitized by a high-resolution laser scanner. A breast segmentation program detects the breast area on the mammogram. Microcalcification detection is performed only within the breast area. The image is first processed with a difference-image technique which subtracts a signal-suppressed image from a signal-enhanced image to remove the structured background. Potential signal sites are identified on the difference image by global thresholding. An adaptive thresholding technique is then used to segment the signals based on the local signal-to-noise ratios (SNR). Morphological features are extracted from the signals that pass the chosen SNR threshold. Falsepositives are subsequently reduced by using the morphological characteristics of microcalcifications. The remaining signals are further examined by a convolution neural network that has been trained to distinguish true and false microcalcifications. Finally, a clustering technique is used to detect clusters that contain three or more microcalcifications within a 1-cm diameter area on the image. 
We are conducting a pre-clinical evaluation of the performance of the detection system. Mammograms that have not been used for training the detection system are used. In our preliminary study, 143 mammograms were processed. At the chosen SNR threshold, the detection sensitivity was approximately $85 \%$ at a false-positive rate of 0.7 clusters per image. The evaluation of the system will continue with a larger data set and the results will be presented.

\section{TU-C4-02}

Bayesian Artificial Neural Networks in the Computerized Detection of Mass Lesions

M Kupinski*, D Edwards, M Giger, A Baehr, University of Chicago, Chicago, IL

Artificial neural networks (ANNs) are frequently used as classifiers in computer-aided diagnosis methods. In the limit of large sample sizes, an ANN approximates an ideal observer discriminant function which is a monotonic transformation of the likelihood ratio. A disadvantage of ANNs is the over-parameterization of the mapping function which results in a poor approximation of an ideal observer discriminant function for smaller sample sizes. Recently, Bayesian methods have been applied to ANNs in order to regularize training to improve the robustness of the classifier. A Bayesian ANN (BANN) should thus better approximate an ideal observer discriminant function given small sample sizes. We evaluated the accuracy of BANN models of ideal observer discriminant functions by varying the number of hidden units, the signal-to-noise ratio of the data, and the number of features or dimensionality of the data. Results on simulated data show that BANNs can effectively model the ideal observer discriminant function even when excess hidden units and limited sample sizes are present. We have used these results to design BANNs to classify mammographic regions as either malignant or normal. Initial comparisons of BANNs with conventional, early-stopping ANNs suggest that BANNs produce more robust results in the task of distinguishing between malignant lesions and false detections.

Maryellen L. Giger is a shareholder in R2 Technology, Inc. (Los Altos, CA).

\section{TU-C4-03}

Comparing Full Digital Mammography and Digitized Film on Selected Features for Classification

L Yang*, C Kimme-Smith, M Mcnitt-Gray, University of California, Los Angeles, CA

Since Full Field Digital Mammography and digitized sceen/film mammography have different image receptors and methods of digital formation, different image qualities, such as image resolution, contrast and noise will result, which will cause differences in the precision and accuracy of segmentation and extracted features for microcalcification detection and diagnosis.

We obtained digital images of the same patients from digitized film and a TREX whole breast mammography unit with same mean glandular doses. We also obtained a diagnosis from six radiologists and biopsy result for patients with BIRADS 4 or 5 diagnosis. The features are extracted from two domains: Morphological measurements and texture features extracted from the co-occurance matrix. Preliminary tests were done on 6 patients $(12$ digitized images, and 12 full field digital images) with the biopsy results ranging from benign to malignant. Based on the radiologists' diagnosis, we manually choose the Region of Interest as $256 * 256$ with the calcifications at the center. Then the microcalcifications are segmented, and after elimination of the false positive on the segmentation results, morphological measurements are taken. Then the co-ocurance matrix is calculated from the ROI with the microcalcifications removed, and 13 texture features are calculated from it.

The initial results show that the differences in digitization methods affect the morphological measurements much more than the features extracted from co-occurance matrix. Whether these difference will affect the classification result still needs further investigation.

\section{TU-C4-04}

Reducing Radiologists' Variability in the Interpretation of Mammograms with Computer-Aided Diagnosis (CAD)

Y Jiang ${ }^{1}, \mathrm{R}$ Nishikawa ${ }^{1}$, R Schmidt ${ }^{2}$, A Toledano ${ }^{1}, \mathrm{~K} \mathrm{Doi}^{1}$, (1) University of Chicago, Chicago, IL, (2) New York University, New York, NY

Studies have shown that considerable variability can occur among the interpretation of and recommendation for the same mammogram when the diagnoses are made independently by different radiologists. Our purpose is to show that computer-aided diagnosis (CAD) can potentially reduce this variability. We analyzed results from an observer performance study designed to compare radiologists' interpretation of mammograms with and without a computer aid. The diagnostic task was to differentiate malignant from benign clustered microcalcifications in mammograms and to make a clinical-management recommendation of biopsy versus follow-up. The computer aid provided to the observers was an estimate of the likelihood of malignancy which was obtained from a computer analysis using an artificial neural network. Ten radiologists participated as observers and read 104 near-consecutive biopsy-proven cases of microcalcifications. When the computer aid was used, the agreements by all observers increased from $13 \%$ to $32 \%$ of total cases. Kappa increased statistically significantly from 0.19 to 0.41 . In addition, two third of extreme disagreements where biopsy and routine screening were recommended for the same patient by different radiologists were eliminated. Furthermore, the standard deviation in $\mathrm{A}_{\mathrm{Z}}$ was reduced by $47 \%$, which was in agreement with an analysis of several other observer-performance studies. We conclude that computer-aided diagnosis can reduce the variability in radiologists' interpretation of mammograms while being able to improve radiologists' diagnostic accuracy.

Disclosure: RMN, RAS, and KD: shareholders, R2 Technology, Inc.

\section{TU-C4-05}

Comparison of Two-Dimensional and Three-Dimensional Features for Lung Nodule Detection in CT

S Armato, III*, M Giger, K Doi, H MacMahon, The University of Chicago, Chicago, IL

Helical computed tomography (CT) is the most sensitive imaging modality for the diagnosis of lung nodules. Complex normal anatomy, however, makes nodule identification cumbersome for radiologists. Therefore, we have developed a computerized scheme to automatically analyze threedimensional structures within the volumetric image data acquired during CT examinations to identify lung nodules.

Gray-level thresholding and a rolling ball algorithm produce segmented lung regions within each $\mathrm{CT}$ section. The resulting segmented lung volume is iteratively thresholded to eliminate pixels with gray levels less than the particular threshold; remaining pixels at each iteration are grouped into contiguous three-dimensional structures. A volume criterion is imposed to determine a set of nodule candidates, which is then subjected to two- and three-dimensional feature analysis. To distinguish between candidates representing nodule and non-nodule structures, these features are merged through automated classifiers.

In a preliminary database, linear discriminant analysis yielded an area under the receiver operating characteristic curve of 0.93 in the task of distinguishing between nodule and non-nodule structures within the set of nodule candidates. We are investigating the robustness of this method using an enlarged database of $40 \mathrm{CT}$ cases (1:1 pitch with 10-mm collimation and reconstruction interval) in conjunction with an artificial neural network. The overall performance of the method and the performance of an enhanced set of two-dimensional features relative to an enhanced set of three-dimensional features will be discussed.

MLG, HM, and KD are shareholders in R2 Technology (Los Altos, CA). 


\section{TU-C4-06}

Computer-aided Diagnosis Scheme for Pulmonary Nodule Detection Using a Contralateral Subtraction Technique

Q Li*, S Katsuragawa, R Engelman, H MacMahon, K Doi, Chicago, IL

A computer-aided diagnostic (CAD) scheme is being developed to assist radiologists in the detection of pulmonary nodules in chest radiographs. A problem in the current scheme is its high false positive rate, due to ribs and rib crossings. In this study, we used a novel contralateral subtraction technique, by which symmetric skeletal structures such as ribs can be eliminated, to reduce false positives. With our CAD scheme, a chest image is first enhanced using a difference image technique, in order to select initial nodule candidates. A rule-based technique and an ANN method are applied to eliminate some false positives based on various image features. Our CAD scheme can achieve $70 \%$ sensitivity at a false positive rate of 1.7 per image. To further remove false positives, a contralateral subtraction image is obtained by subtraction of a right/left reversed "mirror" image from the original chest image. The contrast and relative standard deviation are determined at the corresponding locations of nodule candidates in the subtraction image, to distinguish between nodules and false positives. In a pilot study, the $\mathrm{CAD}$ scheme was applied to 10 chest radiographs, with 10 detected nodules and 14 false positives. By examining two features from the contralateral subtraction images, thirteen false positives were eliminated without a reduction in true positives. Therefore, the contralateral subtraction technique can significantly improve the performance of the nodule detection scheme.

\section{TU-C4-07}

Markov Random Field Modeling in Posteroanterior Chest Radiograph Segmentation

N Vittitoe*, R Vargas-Voracek, C Floyd, Jr, Duke University Medical Center, Durham, NC

PURPOSE: To develop an image processing algorithm to classify each pixel of a digitized posteroanterior chest radiograph (DCR) as belonging to one of six separate region-types: lung, subdiaphragm, heart, mediastinum, body, or background.

MATERIALS AND METHODS: Denote the set of pixel gray levels in a DCR as $\boldsymbol{y}$, and the set of pixel classifications as $\boldsymbol{x}$. The objective is to obtain the optimal segmentation $\boldsymbol{x}^{\text {OPT }}$ given the set of gray level values $\boldsymbol{y}$. A probabilistic approach is used that defines $\boldsymbol{x}^{O P T}$ as the segmentation $\boldsymbol{x}$ that maximizes the conditional distribution $P(\boldsymbol{x} \mid \boldsymbol{y})$. We model $\boldsymbol{x}$ as a spatially varying Markov random field (MRF) that incorporates spatial and textural information of each possible region-type. MRF modeling provides the form of $P(x \mid y)$, and allows for the use of Iterated Conditional Modes to converge to the distribution maximum of $P(x \mid y)$ thus obtaining the optimal segmentation of a given DCR.

RESULTS: The algorithm correctly classified on average $90.0 \% \pm 3.4 \%$ of the pixels in a DCR as belonging to one of the six possible region-types. Additionally, each region-type was identified with an average accuracy greater than $95.0 \%$

CONCLUSION: The results suggest promise for the use of Markov random field modeling in DCR segmentation. Such an algorithm should prove useful for anatomically specific computer applications in chest radiography such as computer-aided diagnosis and image processing.

\section{TU-C4-08}

Evaluation of Digital Imaging Systems and PACS Networks With A New Digital Imaging Phantom

D Goodenough, J Levy (1), The George Washington University Medical Center, Washington DC, (1) The Phantom Laboratory, Salem NY

PURPOSE: Evolution of Digital Imaging Systems creates a need for new phantoms designed to measure system performance.

METHOD/MATERIALS: This phantom incorporates tests for high and low contrast resolution to assess performance of digital capture station through various image review workstations and filming stations within a digital network.
The Phantom provides image quality tests for computed radiology and other digital x-ray imaging systems. To enable evaluation of a wide range of digital imaging parameters, a modular phantom design was selected. The design enables insertion of test modules inside holders with varied amounts of tissue absorbing material to duplicate large or small body absolution characteristics. The modular design enables flexibility and upgrade ability. This feature will enable development of new modules to meet changing requirements for a rapidly changing technology.

The test modules include spatial resolution tests of two types: point or line spread function; or square resolution patterns that can also be used with Square Wave Resolution Function approaches for evaluation of frequency transfer characteristics; low contrast resolution tests of cylindrical test objects of various diameters and differential depth to offer contrast-detail detectability information.

RESULTS: Examples of the use of the phantom in testing a number of digital image systems will be shown. To facilitate faster and more objective monitoring of these images, automated software will be demonstrated.

CONCLUSIONS: The use of this digital phantom enables comprehensive image evaluation both locally and at remote workstations.

\section{TU-C4-09}

A Workstation-User Interface for Softcopy Reading of Mammograms and CAD

M Kallergi*, M Hersh, J Kim, S Chetelat, B Avants ${ }^{1}$, C Thomas ${ }^{2}$,

(1) Department of Radiology, University of South Florida and Moffitt Cancer Center, Tampa, FL, (2) Uniformed University of the Health Sciences, Bethesda, MD

A workstation-user interface was developed and evaluated for interpreting digital mammograms and computer assisted diagnosis (CAD) on CRTs. Hardware included an UltraSPARC 2200 system (dual processor $200 \mathrm{MHz}$; 512MB RAM; Solaris 2.5.1 operating system), two 20482560, $120 \mathrm{ft}-\mathrm{L}$ DR110 monitors (Data Ray Corp), and two display cards (Dome Imaging Systems Inc.). Software development used ANSI C, SPARCworks Visual $\mathrm{C}++$, Motif toolkit, XIL, and Java. The design aimed at a user-friendly interface that simulates film hanging protocols while overcoming the limitations of current display technology for mammography: spatial resolution, luminance, and speed. The system included CAD presentation, image manipulation tools, and a reporting mechanism. The interface was evaluated qualitatively with a questionnaire and quantitatively with an ROC study. ROC results from two readers and 200 mammograms show no significant difference between film and digital reading of mammograms. The interface successfully overcame the limitations of resolution and luminance by using innovative ways to alternate between low and high resolution images and automatic and manual scaling mechanisms. CAD for masses and calcification clusters was also conveniently displayed for consideration. Speed, however, was not adequate for a clinically useful tool. Further hardware/software optimization is necessary for faster image display.

\section{TU-C4-10}

Web-based Access to DICOM Image Databases: Some Practical Issues R McColl*, T Lane, UT Southwestern, Dallas, TX

In a previous presentation [1], we discussed some of the issues involved in the design and implementation of Web-based access to radiological data, with emphasis on implementation and security. Here we discuss some practical aspects of the use of such interfaces, particularly those issues which physician feedback has shown to be important to wider acceptance.

Two main uses of this system have been found to be practical in our institution. One is for off-campus on-call use, the other is for the generation of teaching file material. In the first case, while push technology may be preferable, if large datasets are to be transferred to the physicans home or off-campus location, then availability of DICOM push makes it quicker to provide for pull technology such as http. Thus the bandwidth bottleneck is contained within the (high bandwidth) institution rather than imposing it on the (low bandwidth) connection to the off-campus location. 
In the second case, a familiar interface to more arcane devices (e.g digitizers) motivates the WWW-DICOM interface for the creation of teaching files. Since it is straightforward to Save a Web page or image, window width and center controls, together with zooming and grayscale inversion perhaps, can be applied until a satisfactory image is presented. This image may be saved at a much reduced capacity compared to the original. Thus, multi-image teaching files may be built without immediate storage problems.

[1] McColl R, Lane T. The DICOM-WWW gateway: implementation, configuration, security and privacy. Medical Physics 25(7):A216, 1998.

\section{Continuing Education Course $M R I-3$}

\section{Room: Taylor}

\section{TU-C5-01}

\section{Acceptance Testing of MRI Systems}

Edward Jackson, U.T. M.D. Anderson Cancer Center, Houston, TX

Suggested elements of a general acceptance testing procedure for MRI systems will be presented. Specific tests of each subsystem, including the static magnetic field, gradient magnetic field subsystem, radiofrequency field subsystem, and computer systems, will be discussed. Available published guidelines for acceptance testing will be reviewed and acceptance criteria from such guidelines will be presented. Additional tests and acceptance criteria required for evaluating the performance of modern highspeed MRI scanners will also be suggested.

While the large diversity in capabilities of scanners from various vendors precludes the presentation of a single comprehensive acceptance test procedure, generalized testing procedures for all major subsystems using phantoms that are easily manufactured or commercially available to clinical medical physicists will be described. Acceptance tests that are difficult, or impossible, for typical practicing clinical medical physicists to independently perform without assistance of the vendor's installation or field service engineers will also be discussed, along with suggested approaches to incorporate vendor tests with independent evaluations.

Educational Objectives:

The clinical medical physicist will understand and be prepared to implement a general acceptance testing procedure that will evaluate all major subsystems of modern MRI scanners.

Upon completion of this course, participants will be prepared to:

1. Understand the tests needed to evaluate the performance of the static magnetic field, gradient magnetic field, radiofrequency field, and computer subsystems.

2. Understand typical acceptance criteria.

3. Develop a set of acceptance tests and acceptance criteria that are specific to the capabilities of the MRI system to be evaluated.

4. Determine the feasibility of measuring specific acceptance test parameters independently as opposed to using evaluation tools provided by the vendor to its installation and/or field service engineers.

\section{Scientific Session Calculations - Treatment Planning}

Room: Pearl

\section{TU-D1-01}

Clinical Validation of a Monte Carlo Dose Calculation Tool for Radiotherapy Treatment Planning

JS Li*, T Pawlicki, J Deng, SB Jiang, A Kapur, E Mok, C-M Ma, Stanford University School of Medicine, Stanford, CA

A Monte Carlo program, MCDOSE, was developed at Stanford for radiotherapy treatment planning (RTP). MCDOSE was designed as a dose calculation module for easy implementation into any RTP system. We have implemented MCDOSE on an existing commercial RTP system for conventional photon/electron beams and intensity modulated radiotherapy (IMRT) dose verification. Features of MCDOSE included a multiple-source model to reconstruct the beam phase space, inclusion of beam modifiers such as jaws, wedges, blocks, electron cutouts and bolus in the patient simulation, and the implementation of several variance reduction techniques. Vigorous testing and clinical commissioning of MCDOSE has been carried out. Photon and electron dose calculations using MCDOSE were tested for two Varian accelerators, a Clinac $2100 \mathrm{C}$ and a $2300 \mathrm{C} / \mathrm{D}$. Comparisons of the dose distributions for 4-15 MV photons and 6-20 MeV electrons calculated by MCDOSE and the well-benchmarked EGS4 user code DOSXYZ showed excellent agreement (within statistical uncertainty of $0.3 \%$ ). The CPU time for a given calculation was generally a factor of 10 less for MCDOSE compared to DOSXYZ. The MCDOSE calculated heterogeneity correction factors for layered-lung or layered-bone phantoms were consistent with results from measurement to within $1 \%$. Specifically designed inhomogeneous phantoms were used to test MCDOSE computed IMRT plans. The results showed that the dose calculated by MCDOSE agreed to within $3 \%$ measured results while the dose to critical structures was underestimated by a commercial inverse planning system by up to $100 \%$.

\section{TU-D1-02}

Beam Characterization and Modeling for Photon Monte Carlo Treatment Planning

J Deng*, S Jiang, A Kapur, J Li, T Pawlicki, C Ma, Stanford University School of Medicine, Stanford, CA

Photon beams of 4, 6, and $15 \mathrm{MV}$ from Varian Clinac 2100C and 2300C/D accelerators were simulated using the EGS4/BEAM system. The accelerator was modeled as a combination of component modules $(\mathrm{CMs})$ consisting of a target, primary collimator, flattening filter, monitor chamber, mirror and jaws. A full phase space file was scored directly above the upper jaws and analyzed using a program, BEAMDP (BEAM Data Processor), to derive the particle planar fluence, angular distribution, energy spectrum and the fractional contributions of each individual CM. A multiple sub-source model was further developed to reconstruct the phase space. Separate sub-sources were used for the target, primary collimator and flattening filter components with accurate source intensity, energy, fluence and angular distributions. Good agreement (within 2\%) was achieved in the dose distributions for field sizes $2 \mathrm{~cm} \times 2 \mathrm{~cm}-40 \mathrm{~cm} \times 40 \mathrm{~cm}$ and source surface distances $80 \mathrm{~cm}-120$ $\mathrm{cm}$ between the Monte Carlo calculations with the source model and those with the original phase space, and measurements. The source model was implemented in an EGS4 user code, MCDOSE, for photon beam Monte Carlo treatment planning, which computes the dose distributions in a $3 \mathrm{D}$ rectilinear phantom built from the patient $\mathrm{CT}$ data and simulates the effect of beam modifiers such as wedges, multileaf collimator and blocks. The source model was also employed in the Monte Carlo beamlet profile calculation for inverse planning for intensity modulated radiotherapy (IMRT)

\section{TU-D1-03}

Experimental Validation of Dose Calculation Algorithms for the GliaSite (TM) RTS, a Novel 125I Liquid-Filled Balloon Brachytherapy Applicator

J Monroe*, J Dempsey, J Dorton, S Mutic, J Markman, J Williamson, Washington University, Mallinckrodt Institute of Radiology, St. Louis, MO

We present the experimental validation of dose calculation algorithms for a novel ${ }^{125}$ I liquid-filled brachytherapy balloon applicator (the GliaSite RTS) designed for the treatment of malignant brain-tumor resection-cavity margins. A solid water phantom machined for catheter conformity (verified by Computed Tomography (CT) imaging) was used to perform absolute low dose-rate radiochromic film (RCF) measurements in coronal planes about the applicator. The results were used to validate Monte Carlo photon transport (MCPT) simulations and a point-source dose-kernel model algorithm. Densitometry of RCFs employed a validated optical densitometry system with an artifact elimination process. The absolute activity of the ${ }^{125}$ I solution was determined by inter-comparing a National Institute of Standards and Technology (NIST) ${ }^{125}$ I standard with a known mass of radiotherapy solution (Iotrex ${ }^{\mathrm{TM}}$ ) in an identical vial and geometry. Agreement between RCF and MCPT was found to be within $\pm 5 \%$. This significantly improves upon earlier reports of absolute RCF film dose measurements about ${ }^{125} \mathrm{I}$ sources. Agreement between the simple semiempirical dose-kernel model and the MCPT and, by extension between the RCF measurements and semi-empirical model, was better than 5\% except very near the applicator surface where $8-10 \%$ discrepancies were observed. 
Measurement also confirmed no dose-distribution deviation from equatorial isotropy as predicted.

This work was supported by a grant from Proxima Therapeutics Inc.

\section{TU-D1-04}

CT-Guided Brachytherapy Using a Correlated 3D Stereotactic Arm $\mathrm{J}$ Hevezi*, J Mira ${ }^{1}$, J Marbach, S Chopra ${ }^{2}$, R Vasquez ${ }^{3},(1)$ Cancer Therapy \& Research Center, San Antonio, Texas, (2)University of Texas Health Science Center, San Antonio, Texas, (3)SW Texas Methodist Hospital, San Antonio, TX

Several patients have been treated on an outpatient basis implanting I-125 seeds under CT guidance for recurrent disease. The coordinates for the seed implantation sites are preplanned using the patient's CT data set. On implant day, the I-125 seeds are inserted through a needle guide with the Mick applicator. The interventional radiologist $(\mathrm{RV}, \mathrm{SC})$ uses a local anesthetic along the needle tracks and requires multiple CT slices to follow the needle trajectories to the preplanned coordinates. Needle tracks are optimized on the preplan to deposit multiple seeds along each track. An average of 10 seeds were deposited in our first 8 patients. Recurrent paraspinal tumors post full-course radiotherapy were the most frequent case encountered. Recently, a 3D stereotactic arm attached to the CT scanner (PQ5000, Picker International) and correlated in space with the CT data set has been used to locate needle trajectories and seed deposit coordinates. The patient must be immobilized in the treatment position for the preplan data set and aligned to this position for the implant procedure. Postplans after seed deposition have correlated well with the preplan dose distribution. Several methods are being evaluated to test the congruence postplan to preplan including interseed distance matrices and dose-volume histograms.

\section{TU-D1-05}

Incorporating Organ Motion Due to Breathing into 3D Dose Calculations: Sensitivity to Variations in Motion

A Lujan*, R Ten Haken, J Balter, E Larsen, University of Michigan, Ann Arbor, MI

We have previously described a method to incorporate the effects of organ motion due to breathing into $3 \mathrm{D}$ dose calculations for treatment of liver disease. This method uses an analytic function to describe inferior-superior liver motion during free breathing. Although periodic, each cycle is generally asymmetric, with the majority of time spent near the exhale position. Our analytic function includes parameters that describe the amplitude (exhale to inhale) of the motion and the degree of asymmetry (time at exhale relative to inhale). Representative parameter values are selected by observation for each patient. However, these parameters may vary from the representative values over the course of treatment (e.g. patient may take a few deep breaths over the course of treatment). This study demonstrates the effects such variations may have on the predicted outcome in dose. Monte Carlo-based direct simulations were used to compute 3D dose distributions that included the effects of liver motion due to breathing. The amplitude and/or shape of the breathing cycle function was changed from the representative values for various fractions of the total treatment. The significance of differences in the liver dose distribution was judged by changes (if any) in our normal tissue-based protocol for prescribing dose. Quantification of these effects will be useful in directing future efforts in describing and controlling organ motion due to breathing.

Work supported by NIH grant no. P01-CA59827 and the Sidney Kimmel Foundation

\section{TU-D1-06}

A Multiple Source Model for Monte Carlo Photon Dose Calculations in Radiotherapy

M Fix*, M Stampanoni, H Keller', E Born, R Mini², P Ruegsegger', (1)Institute for Biomedical Engineering and Medical Informatics, ETH Zurich, Switzerland, (2)Division of Med. Radiation Physics, Clinic of Radio-Oncology, Inselspital-University of Berne, Switzerland

A multiple source model (MSM) for MC dose calculations in radiotherapy was developed for the $6 \mathrm{MV}$ photon beam of a Varian Clinac 2300 accelerator. The MSM consists of 12 planar and linear sources representing the main elements of the beam defining system. The characteristics of the sources for all square fields were extracted from phase space data resulting from MC simulations of the radiation transport through the treatment head for a set of square fields.

Using the MSM, phase space data, depth doses, lateral dose profiles and output factors were calculated and compared to measured data or to the original phase space data, respectively. Comparison of the depth dose curves at the depth of $10 \mathrm{~cm}$ showed deviations of less than $2 \%$ for all field sizes. The lateral dose distributions and the reconstructed phase space data showed good agreement with the measurements or original data, respectively. Also, the deviations between measured and calculated output factors for $10 \mathrm{~cm}$ water depth were less than $2 \%$.

In summary, the MSM showed good agreement between measured and calculated data for square fields. The MSM has the potential to characterize more complex geometries for MC photon dose calculations.

\section{TU-D1-07}

The Generalization of a Photon Pencil Beam Algorithm for Electron Beam Dose Calculations

E Wong*1, G Garvey ${ }^{1}$, J Cunningham ${ }^{2}$, J Sun ${ }^{2}, \mathrm{~J}_{\text {VanDyk }}{ }^{1}$, J Battista ${ }^{1}$,

(1) London Regional Cancer Centre, London, ON, Canada, (2) Theratronics, Kanata, ON, Canada

The semi-empirical pencil beam dose calculation algorithm for photon beams based on Scatter-Air-Ratios (SAR) has become the conceptual basis for dose computation in some modern treatment planning systems. For electron beams, the adoption of the SAR algorithm was first proposed by Dutreix et al. and more recently by re-introduced by Cunningham. This provides the possibility of using the same photon beam codes for electron dose calculations. Perhaps, the most deterring factor in such application is the ambiguity of Peak-Scatter-Factor or zero area Tissue-Air-Ratio concepts for electron beams. In contrast, the most widely used algorithm for electron beams in treatment planning was devised by Hogstrom. It is the solution to a superposition integral in which the kernel describes the spatial spread of dose from a pencil beam incident on the surface of a phantom. Hogstrom's formulation is based on Eyges whose analytical solution had a number of simplifying assumptions. The SAR algorithm on the other hand does not contain such assumptions. In this work, we expressed the SAR algorithm in the nomenclature of Hogstrom's analytical solution and defined the Peak Scatter Factor and zero area Tissue-Air-Ratio. We compared the kernels used in the two formalisms and their dose predictions against measurements made in water and inhomogeneous phantoms at different energies. We conclude that SAR and the Hogstrom formalism are similar in concept and performance, their difference lies in how the kernels are obtained.

\section{TU-D1-08}

Electron Beam Modeling and Commissioning for Monte Carlo Treatment Planning

S. B. Jiang*, A. Kapur, C.-M. Ma, Stanford University School of Medicine, Stanford, CA

A hybrid approach for commissioning electron beam Monte Carlo treatment planning systems is studied. The approach is based on the assumption that accelerators with similar designs have similar electron beam characteristics. For one type of accelerators, a reference machine is selected and simulated with the Monte Carlo method. A beam model, which is based on a multiple source model, is built for this type of accelerators using the Monte Carlo simulated phase space information for the reference machine. When commissioning another accelerator of the same type, the appropriate 
parameters in the beam model are tuned according to the standard measured data such as output factors, depth-dose and dose profiles. A Varian Clinac $2100 \mathrm{C}$ accelerator is chosen as the reference machine and simulated using the EGS4/BEAM code. A four-source beam model is established based on the simulated beam information to reconstruct electron phase space down to the last applicator scraper. The model includes a point electron source for direct electrons and electrons scattered from the primary collimator and jaws, a point photon source for all contaminant bremsstrahlung photons, and two square ring electron sources representing electrons scattered from the two scrapers above the last scraper. A Varian Clinac 2300C/D machine, which is similar in design to the reference machine, is commissioned using this beam model. By tuning the appropriate parameters in the model, accurate dose calculation (within about $2 \%$ ) is achieved using the model, compared to the corresponding measurement.

\section{TU-D1-09}

On Monte Carlo Simulation of Eletron Cut-outs in Treatment Planning C Mubata*, F Verhaegen, A Nahum, The Royal Marsden Hospital, Fulham Rd. London, UK, Institute of Cancer Research, London, UK

In simulation of electron beams for treatment planning, it is possible to simulate and collect the beam characteristics in terms of phase space data at the top of the patient dependent cerrobend cut-outs. Simulation times of these cut-outs can be greatly reduced with variance reduction techniques, such as sampling from particles inside the block aperture only and raising the cut-off energies, and range rejection techniques. We have studied the effect of the cut-out walls and cut-off energies on both the CPU time and the dose distributions. The calculations were carried out for a Varian 2100C using the BEAM/DOSXYZ Monte Carlo codes, for beam energies between 9- and 20- MeV. Below 9-MeV the effect of the scatter from the block is marginal, but significant at higher energies, on both the profiles, and on the central axis depth doses. At $16-\mathrm{MeV}$ the scattered electrons increase the dose on the central axis by up to $5 \%$ for depths at and beyond $d_{\max }$. The shoulders and tails of the profiles can both drop by up to $3 \%$ of the maximum dose, if the scatter is ignored. Minimisation of the transport region in the block improves the computational speed, with CPU times reduced by a factor of 2.0 and 5.0 for $2.0 \mathrm{~mm}$ and $10.0 \mathrm{~mm}$ wall thickness compared to no tracking in block material. Raising the cut-off energies for the charged particle also reduces the fluence of these particles.

\section{TU-D1-10}

A Three-way Comparison of Radiotherapy Dose Distributions using Polymergel Dosimetry, Monte Carlo Simulation and Treatment Planning Systems

P Love*1, D Lewis ${ }^{1}, \mathrm{C}$ Smith $^{1}$, P Haraldsson ${ }^{2}, \mathrm{P}_{\text {Magnusson }}^{2}$, S Back ${ }^{2}, \mathrm{~L}$ Olsson $^{2}$, (1) Department of Medical Physics, Velindre Hospital, Cardiff, UK, (2) Department of Radiation Physics, Malmo University Hospital, Sweden

Three independent methods have been used to compare 3D radiotherapy dose distributions: 1) MRI scanned dosimetric polymer gels, 2) Monte Carlo calculations using the NRCC BEAM code and 3) convolution/superposition calculations using a commercial treatment planning system (TPS). Several large phantoms (with volumes 3-4 litres) of varying geometry and complexity have been prepared then dispensed into containers of Barex, a commercially available plastic. Barex is an easily moulded material which gives excellent oxygen barrier properties. (Oxygen seriously degrades the dosimetric response of the polymer gels). Using molecular bonding to seal the Barex has allowed us to produce complex but rigid phantoms, whilst maintaining a good oxygen seal. The gels have been found to be sensitive at low doses $(<0.1 \mathrm{~Gy})$ indicating minimal exposure of the gel to oxygen during the production process. The phantoms were irradiated with $6 \mathrm{MV}$ photons, then both MRI and CT scanned. The CT data are used as input to both TPS and Monte Carlo calculations. Generally good agreement between the 3 methods (within 3\%) has been found when comparing phantoms without inhomogeneities, whereas significant differences are observed for phantoms containing inhomogeneities.

The combined use of gel dosimetry and Monte Carlo simulations allows a more extensive basis for analysis of 3D TPS dose distributions than has been previously possible.

\section{Scientific Session IMRT Delivery}

\section{Room: Mississippi}

\section{TU-D2-01}

In Vivo Verification of Clinical Intensity Modulated Radiotherapy Delivered Via Dynamic Multileaf Collimation

P Williams* ${ }^{1}$, G Budgell ${ }^{2}$, J Mott ${ }^{2}$, B Perrin ${ }^{2}$, H James, ${ }^{2}$ S Atherton ${ }^{2}$, A Hounsell $^{2}$, (1) North Western Medical Physics, Christie Hospital, (2) North Western Medical Physics, Manchester, UK

The delivery of intensity modulated radiation therapy (IMRT) treatments using fully dynamic multileaf collimation (MLC) has recently commenced at our centre. In order to prove the reliability of this new technique in clinical use it has been necessary to institute a thorough program of verification for the initial clinical implementation. Here we describe the in vivo checks which ensure that every patient has been correctly treated.

At treatment, dynamic deliveries start with the MLC positioned as for a static irradiation. This allows comparison with a paper template placed on the couch to check that the correct prescription is loaded and allows a portal image to be acquired (to check for patient set-up errors) before the leaves move to their start positions for dynamic delivery. During delivery, real-time verification of MLC leaf positions is available via electronic portal imaging. A series of snapshot images are acquired during delivery and immediately displayed on-screen with the required leaf positions overlaid electronically. This allows the operator to monitor the progress of the delivery and to intervene should a gross error occur. Absolute in vivo dosimetry is performed using photon diodes placed on the patient surface. Diode correction factors for focus to surface distance and field size have been measured for dynamic MLC and are applied to the readings. This combination of verification techniques facilitates reliable treatment using the dynamic MLC technique.

This work is supported by Elekta Oncology Systems Ltd.

\section{TU-D2-02}

Modeling Multileaf Collimators with the PEREGRINE Monte Carlo System

D Fujino*, N Albright, D Wieczorek, Lawrence Livermore National Laboratory, Livermore, UCSF, San Francisco, CA

Multileaf collimators (MLCs) are becoming increasingly important for beam shaping and intensity modulated radiation therapy (IMRT). Their unique design can introduce subtle effects in the patient/phantom dose distribution. The PEREGRINE 3D Monte Carlo dose calculation system predicts dose by implementing a full Monte Carlo simulation of the beam delivery and patient/phantom system. As such, it provides a powerful tool to explore dosimetric effects of MLC designs. We have installed a new MLC modeling package into PEREGRINE. This package simulates full photon and electron transport in the MLC and includes tongue-and-groove construction and curved or straight leaf ends in the leaf shape geometry. We tested the accuracy of the PEREGRINE MLC package by comparing PEREGRINE predictions with ion chamber, diode, and photographic film measurements taken with a Varian 2100C using 6 and 18 MV photon beams. Profile and depth dose measurements were made for the MLC configured into annulus and comb patterns. In all cases, PEREGRINE modeled these measurements to within experimental uncertainties. Our results demonstrate PEREGRINE's accuracy for modeling MLC characteristics, and suggest that PEREGRINE would be an ideal tool to explore issues such as 1) underdosing between leaves due to the 'tongue-and-groove' effect when dose from multiple MLC patterns are added together, 2) radiation leakage in the bullnose region, and 3 ) dose under a single leaf due to scatter in the patient. 


\section{TU-D2-03}

Can MLC Leakage and Transmission Be Used to Obtain CT Images During Treatment

K Ruchala*, G Olivera, T Mackie, University of Wisconsin-Madison, Madison, WI

The NOMOS Corporation has developed an early form of axial tomotherapy through the use of its binary, temporally modulated MIMiC collimators. While these collimators do provide for highly conformal intensitymodulated treatments, they do not incorporate full treatment verification possibilities, such as megavoltage CT (MVCT), found in an integrated tomotherapy system. Another problem with the NOMOS implementation of tomotherapy is that the MIMiC collimator has been shown to leak and transmit radiation to the patient.

However, these problems may culminate in megavoltage CT imaging with No Additional Dose. That is, the leakage through the MIMiC during a typical treatment is sufficient to create MVCT images showing contrasts of better than $10 \%$.

Tests of this imaging method were conducted on the University of Wisconsin Tomotherapy Benchtop. It was found that during a $220 \mathrm{cGy}$ treatment, enough leakage is transmitted through the closed leaves to generate MVCT images of the patient. While such images are not of diagnostic caliber, they do show the patient's position and the locations of high-contrast objects without any additional dose.

Ultimately, the goal is to merge the high dose treatment signal with the very low dose signal from leakage and transmission. This will allow for MVCT imaging of the entire patient during a conformally modulated treatment to a limited region of the patient, utilizing the high dose signal inherent to the treatment, but without contributing any additional dose beyond the leakage and transmission.

\section{TU-D2-04}

Dependence of Dose Output on the Switch Rate of an Intensity Modulated Radiation Therapy (IMRT) Collimator

J Tsai*, M Engler, Tufts-New England Medical Center, Boston, MA

Multi-vane intensity modulated collimator (MIMiC) of NOMOS features its tomotherapy, beam intensity modulation and shaping conformal to target, and inverse planning. The MIMiC has 40 vanes switching back and forth independently to define the required field shape and control vane's beamlet intensity to the tumor and simultaneously off the critical structures. However, the switchings of these vanes incur momentary dampings and induce additional transient boundaries to their well defined field edges depending on their switching rates. As a result, actual clinical profiles and output factors of the vane beamlets are slightly different from the ones extracted during their stationary openings. In this study, dose outputs of 7 standard vane patterns were measured with an ionization chamber at different switching periods. Profiles of the vane patterns at various switching periods were measured with film. Furthermore, the dose outputs for 6 IMRT patient treatment plans were measured at different gantry speeds using the same ionization chamber embedded in a cylindrical tissueequivalent phantom. These results indicate the switching vane profiles and their outputs are dependent on the switching rate. A theoretical model of vane opening status is provided to interpret the measured profiles and the dose outputs relative to the switching rate. In summary, the dose output and profile of a vane pattern are a function of the switching rate.

\section{TU-D2-05}

\section{Room-Shielding Requirements for IMRT}

S Mutic*, D Low, E Klein, J Purdy, Mallinckrodt Institute of Radiology, St. Louis, MO

Intensity modulated radiation therapy (IMRT) makes relatively inefficient use of monitor units (MUs) when compared to conventional radiation therapy, thus influencing the assumptions used in room shielding calculations. For the same tumor dose delivered, the total number of MUs for a single IMRT fraction can be an order of magnitude greater than the MUs used for conventional treatments. Therefore, the dose from the linear accelerator head leakage will be significantly greater than with conventional treatments. We propose a model that decouples the concepts of workload, MUs, and target dose when determining barrier thicknesses. The workload for primary barrier calculations for conventional multileaf collimator (MLC) IMRT treatments is determined using patient tumor doses. The same calculation for accelerator-based serial tomotherapy IMRT requires multiplication by the average number of treatment slices, but it's inherent use of rotational therapy yields a relatively small use factor that compensates for this increase. For secondary barrier calculations, the patient-scattered dose is assumed to be the same for all IMRT modalities as for conventional therapy. However, the head leakage contribution is linearly proportional to the number of MUs. Therefore, knowledge of the average number of MUs per patient, which is site dependent, is required to estimate the leakage contribution. Measurements using survey meter and shielding calculations for a $6 \mathrm{MV}$ linear accelerator are used as an example of this technique. Average IMRT workload estimates are made based on our experience with 75 tomotherapy patients and published data for conventional MLC IMRT treatments.

\section{TU-D2-06}

Accuracy and Time Considerations in Intensity Modulation Treatment Planning

R Siochi*, Siemens Medical Systems, Concord, CA

The process of converting intensity modulation patterns into a sequence of leaf positions is a key step in IMRT. Both the accuracy (errors arise from leakage and scatter) and the speed of the treatment are affected by the combinations of choices for segmentation algorithms, correction options (tongue and groove, match line, single aperture, fluence), and various settings which affect the segmentation process. Hence, using only one leaf sequencing algorithm can be limiting. IMFAST ${ }^{\mathrm{TM}}$ is a product that gives the user various algorithms and correction options for leaf sequencing on the Siemens MLC. The results for different settings in the software for a very complex intensity map will be presented. These results lead to the following rules of thumb for IMRT treatment planning: 1) choose treatment ports with minimal critical structure involvement, 2) the number of ports times the number of levels per port should be no more than 60 , and 3) choose segmentation algorithms that decouple segment error contributions (slice, platform, optimal platform). Using these guidelines will reduce treatment time and improve delivery accuracy. For those cases that are an exception to the rule, one needs to explore the various algorithms to produce a clinically useful leaf sequence. One such case will be presented. Siemens Medical Systems supported this research.

\section{TU-D2-07}

Clinical Implementation of Intensity-modulated Radiotherapy Using a Dynamic Multi-leaf Collimator

LS Johnson*, F Kuchnir, C Reft, J Kung, University of Chicago, Chicago, IL

Intensity-modulated radiotherapy (IMRT) was implemented in our clinic using commercial treatment planning software (Corvus 2.01), a record and verify system for dynamic dose delivery (VARiS $1.4 \mathrm{~d}^{2}$ ) and a Varian linear accelerator $(2100 \mathrm{CD})$. The intensity-modulated (IM) fields were delivered using the "step-and-shoot" technique and an 80-leaf MLC. The number of fields varied from $5-9$, but the number of intensity levels was always 10 . In some cases, non-coaxial fields were used. In all cases, IM plans had isodose distributions superior to those obtained using conventional $3 \mathrm{D}$ techniques. Before patient treatment, IM plans were verified using an ion chamber in a cubic, tissue-equivalent phantom provided by the vendor ${ }^{1}$. Calculated and measured dose to the ion chamber always agreed to within $\pm 1 \%$. During phantom irradiation, we simultaneously verified the intensity map of each IM field using film taped to the collimator face. On the first day of treatment, AP and lateral radiographs were used to verify the patient's setup before irradiation. On all other treatment days, live video subtraction was used to correct patient setups online in $1-2$ minutes, before radiation was delivered. Treatment time (including patient setup) typically did not exceed 15 minutes, for single fractions of $150-200 \mathrm{cGy}$. Our experience demonstrates that IMRT is a clinically achievable means of significantly 
improving dose distributions in patients. Data on setup accuracy, organ motion, organ sparing through inverse planning and our quality assurance procedures will be presented.

${ }^{1}$ Nomos Corporation

${ }^{2}$ Varian Oncology Systems

\section{TU-D2-08}

Minimizing the Dosimetric Effects of the Rounded MLC Leaf Ends

L Xing*, B Curran, A Beavis, A Kapur, J Li, Y Chen, A Boyer, Stanford

University, Stanford, CA

The intensity profile near a rounded leaf end (RLE) is not a step function and has a penumbral distribution. The segmented nature of DMLC delivery implies that the transmission through the RLE is more serious than for static MLC treatment. The effect is usually approximated by a small offset $\delta(\sim 0.5 \mathrm{~mm})$ applied to the leaf position. In other words, the added transmission is equivalent to enlarging the field size by $2 \delta$. In practice $\delta$ is determined by visually examining a large number of film measurements with different offsets, which is both time consuming and inaccurate. We report an effective optimization algorithm for determining the optimal offset. Both experimental measurement and computer simulation data were used for the study. The experimental dose profiles were obtained using film and the calculated dose profiles were based on a geometric analysis of transmission through MLC. The calculation involved solving simultaneous equations for intercept points of the photon ray and the MLC surfaces. The dose profiles of two opposed leaves with the penumbra of the RLE were the input to the optimization software. The area difference between the idealized matching situation and the superposition of the two adjacent beams was used to guide the minimization to yield the best $\delta$. The results obtained for different photon energies indicated the method provides a robust tool for clinical application of the DMLC delivery.

\section{TU-D2-09}

Optimisation of the Intensity Map, in Level and Resolution, for the Delivery of IMRT

A Beavis*, P Ganney, V Whitton, L Xing, Princess Royal Hospital, Royal Hull Hospitals and University of Hull, England, UK

To deliver an IMRT treatment a continuous fluence profile, produced by an Inverse planning routine, is commonly divided into a fixed number of equal intensity levels a priori. In this study an algorithm was developed to optimise the number of unconstrained intensity levels required to deliver a single leaf pair (1-D) intensity profile.

Initially the number of intensity levels was determined by discretisation of the continuous intensity profile into $M$ equally spaced bins; Bins of width 1 $\mathrm{cm}$ and intensity levels fixed at $10 \%$ increments were assumed.

In comparison the intensity levels were allowed to start at optimal values. The search criteria for these levels was minimisation of the error in the area under the unconstrained curve; It crudely reflects the error in delivered dose compared to the idealised (continuous fluence) case. Furthermore, the optimisation algorithm was employed to reduce the number of levels by appropriate combination of nearest value pairs. Finally, optimisation of the delivered profile was further investigated by allowing the bin widths to vary unevenly across the field.

An optimised leaf sequence was also computed to deliver these 1D profiles, however the final optimised intensity map can be ported to a generic leaf sequencing algorithm.

We have shown that it is practical to produce optimised intensity maps for the delivery of the IMRT plan whose levels are unconstrained in magnitude and number and whose resolution is patient specific.

\section{TU-D2-10}

The Effects of Intra-fraction Organ Motion on the Delivery of Intensitymodulated Fields with a Multileaf Collimator

C Chui*, Memorial Sloan-Kettering Cancer Center, New York, NY

Intensity-modulated field can be conveniently delivered with a Peacock/MiMIC device or a conventional MLC, the latter can operate either in continuous mode or in step-and-shoot mode. Regardless of which device or mode is used, the entire intensity-modulated field is not delivered at once. Rather, it is composed of many small fields, that is, only a small 'window' of the field is irradiated at any given instant. If the treatment volume is stationary during beam-on, then the total dose is the sum of the doses delivered from these small fields. However, if the volume moves during beam-on, then part of the volume may move in or out of the 'window', resulting in dose delivered to be different from what was planned.

In this work, we describe a method that calculates the effects on dose delivered due to this type of organ motion, for a single fraction as well as for multiple fractions. Using this method, we estimated the effects on a breast treatment with IMRT techniques. We concluded that if the magnitude of motion is less than $\pm 3 \mathrm{~mm}$, the effects are minimal over a typical course of treatment. Beyond that, the effect may be significant. In this case, alternative means must be considered, such as compensator or breath-hold techniques.

\section{Scientific Session \\ Room: Carroll Digital Mammography/Digital Radiography Systems}

\section{TU-D4-01}

Comparison of Automatic Exposure Control Logic of Full Field Digital Mammography and Screen-Film Mammography

P Lin*, Department of Radiology, Northwestern University Medical School, Chicago, IL

The automatic exposure control (AEC) operation logic of a full field digital mammography (FFDM) system has been evaluated against the same makeand-model mammography system programmed for conventional screen-film mammography imaging. Various parameters associated with the AEC operation such as the tube potential "kVp", the "mAS", the anode target material, and the filter selection of the "test pulse", and the actual exposure were monitored as functions of the Plexiglas phantom thickness ranging from $1 \mathrm{~cm}$ to $8 \mathrm{~cm}$

The data obtained have clearly indicated the design principle of the mammography system that the AEC Logic for the FFDM tends to have a higher tube potential setup and is programmed to select the rhodium filter, and the rhodium anode target material at a thinner phantom thickness than the conventional mammography system logic. This is to compensate for the lower sensitivity of the image receptor utilized for the FFDM system.

Evaluation of the mid breast dose (based on two different screen-film combinations and the FFDM) and the image quality were conducted by the use of the ACR Mammography Accreditation Program Phantom (ACR MAP Phantom). Interesting results with respect to the mid breast dose and image quality will also be presented.

\section{TU-D4-02}

Comparative Investigation of Image Quality Between Screen-Film and Digital Mammography

L Yarusso*, I Castro, R Nishikawa, The University of Chicago, Department of Radiology, Chicago, IL

Purpose: To test the hypothesis that a prototype digital mammography unit produces better quality images than conventional screen-film mammograms for equivalent exposures.

Methods: The prototype mammography unit tested was a full-field, flatpanel $\mathrm{x}$-ray detector based on a CsI phosphor screen and an amorphous silicon detector array. Image quality for various exposure techniques were characterized through an observer study using a contrast-detail phantom. The CD-phantom consisted of 8 rows of disks of decreasing size, and 9 
columns with different contrasts. For each contrast level, the observer identified the minimum detectable disk size. The observers viewed images of a CD-phantom taken at 31 different techniques - Mo/Mo $26 \mathrm{kVp}, \mathrm{Mo} / \mathrm{Rh}$ $28 \mathrm{kVp}, \mathrm{Rh} / \mathrm{Rh} 30 \mathrm{kVp}$ at $\mathrm{mAs}$ stations ranging from $4 \mathrm{mAs}$ to $600 \mathrm{mAs}$.

Results: For the 31 different techniques, the observers were able to identify more disks in the digital images over $90 \%$ of the time. An impressive result advocating the advantage of digital mammography was for extreme overexposed and under-exposed films. In these cases, there were no disks visible on films, but approximately half the disks were visible on the digital images.

Conclusions: Digital imaging produces images of superior quality than screen-film mammography for a wide variety of exposure conditions.

Acknowledgments: This work is supported in part by NIH grants CA60187 and T32CA09649.

\section{TU-D4-03}

A Fourier Analysis Method for MTFs Measurement with Bar Patterns in Digital X-Ray Imaging

C Shaw*, X Liu, The University of Texas M.D. Anderson Cancer Center, Houston, TX

Tilted X-ray slit method has often been used to measure the line spread function (LSF) and the modulation transfer function (MTF) in digital $x$-ray imaging. However, this method generally requires substantially higher $\mathrm{x}$-ray technique to obtain reasonable signal size for analysis. The bar patterns are generally available as a quality control tool and can be used to provide square wave response function (SQWRF) at regular exposure levels. However, the MTF is more commonly used to characterize the spatial resolution of the imaging system and is required to compute the frequency dependent detective quantum efficiency (DQE). In this paper, a Fourier analysis method is described and used to analyze the bar pattern signals and measure the MTF. With this method, signal profiles over multiple image lines are combined an array which is then transformed to Fourier components. The ratio of the height of the first peak to the zero frequency component is then computed and corrected to obtain the MTF value at the frequency of the specific bar pattern used. Higher order harmonics can be either ignored or used to compute the MTF at multiples of the base frequency. The theoretical basis for the method is described and used to derive the correction factors. The method is demonstrated with MTF measurements for a small field digital mammography system. The results have been found to agree well with those obtained with the tilted slit method.

\section{TU-D4-04}

Effects of Geometric Magnification on Image $\operatorname{MTF}(f)$ and $D Q E(f)$ in Digital Mammography

X Liu*, C Shaw, M. Lemacks, G. Whitman, The University of Texas M.D. Anderson Cancer Center, Houston, TX

Geometric magnification is often used to improve the visualization of details in mammography. This is especially considered helpful in digital mammography as the spatial resolution of a digital mammography unit is often limited and inferior to those of screen/film combinations. In this paper, the MTF and DQE were measured and studied as a function of the spatial frequency in the object planes corresponding to various magnification factors. When the MTF and DQE of the detector are plotted as a function of the spatial frequency in the object plane, there is an automatic improvement due to the re-scaling of the frequency. However, both the MTF and DQE are also degraded by the focal spot blurring effect, thus limiting and eventually reversing the improvement as the magnification factor increases. It was found that degradation of the DQE presents a more serious problem than degradation of the MTF itself. In this paper, a theoretical model is presented to describe the combined effects of the improvement from geometric magnification and the degradation due to focal spot blurring. Measurements with a small field digital mammography unit (SenoVision by General Electric Medical Systems, Inc.) are presented to study the effects of geometric magnification on the MTF and DQE in the object plane. The frequency dependent DQEs were then used to determine the optimal magnification factor. Implications on exposure requirement and detection of low contrast objects are discussed using contrast detail phantom images as examples.

\section{TU-D4-05}

Temporal Stability of Image Quality Between Screen-Film and Digital Mammography

L Yarusso*, I Castro, R Nishikawa, The University of Chicago, Department of Radiology, Chicago, IL

Purpose: To measure the temporal stability of physical characteristics for a prototype full-field digital mammography unit.

Methods: The prototype digital mammography unit tested was a full-field, flat-panel x-ray detector based on a CsI phosphor screen and an amorphous silicon detector array. The temporal stability of both the image quality and the physical characteristics of the digital system were measured. Image quality tests included ACR phantoms, uniformity measurements, and pixel values under a stepwedge ("speed points.") Physical measurements included electronic noise, sensitometric response, modulation transfer function (MTF,) noise power spectrum (NPS,) and detective quantum efficiency (DQE.) Data were collected over a 4-month period from the time of acceptance-testing.

Results: The temporal stability of the physical characteristics of the digital mammography unit was good. Coefficients of variation for the MTF were between $1-3 \%$ and $6-8 \%$ for the NPS. The electronic noise coefficient of variation was $0.25 \%$. The temporal stability of the image quality measures was also good, especially as measured by the $0 \%$ variation in daily ACR phantom scores. The stability of the sensitometric response had a variance coefficient of $0.8-3 \%$ for various stepwedge speed-points, including the image background.

Conclusions: The prototype digital full-field digital mammography unit system is temporally stable in terms of its physical characteristics and the quality of images it produces.

Acknowledgments: This work is supported in part by NIH grants CA60187 and T32CA09649.

\section{TU-D4-06}

Electronic Noise Properties of Active Matrix Flat-Panel Imagers M Maolinbay*, L Antonuk, Y El-Mohri, K.-W. Jee, S Nassif, X Rong, Q Zhao, University of Michigan, Ann Arbor, MI

A detailed empirical and theoretical investigation of the fundamental limits affecting the dark noise level of active-matrix flat-panel imaging systems has been performed. Models of electronic noise have been developed based upon equivalent noise circuits of array pixels and acquisition electronics. At the level of the pixels, thermal noise associated with TFT-on resistance, shot noise, and $1 / \mathrm{f}$ noise associated with pixel leakage current was modeled. At the level of the system, intrinsic pixel noise, data line thermal noise, and readout charge-amplifier noise was modeled. The TFT noise and the data line thermal noise, generally expressed by $\sigma=\sqrt{k T C}$ (where $k$ is the Boltzmann constant, $T$ the temperature, and $C$ the capacitance), are found to be the dominant noise components at frame rates above $\sim 1$ frame-persecond. However, at lower frame rates, the shot noise and $1 / \mathrm{f}$ noise from the leakage current grow larger and eventually become the dominant noise components. Extensive measurements of the various noise components were carried out on a clinical prototype array whose acquisition system incorporated low noise, application specific integrated circuit preamplifiers. Good agreement was found between model predictions and the measurements. Such studies provide a quantitative understanding of the processes determining the minimum dark noise levels possible for active matrix imagers - which directly affects the lowest exposures for which images containing clinically useful information content can be obtained.

This work is supported by NIH grants R01-CA76505 and R01-CA56135

\section{TU-D4-07}

Comparison of a CR to a DR System

R Gould*, K Andriole, D Luth, University of California, San Francisco, CA 
Two systems for acquiring projection images in digital format, a CR system (Agfa Division, Bayer Corporation) and a large-area flat panel DR system (Canon USA, Inc), were compared using test objects. The DR system has an active area of 43 X43 cm with 2,688 x 2,688 pixels. A $35 \times 43 \mathrm{~cm} \mathrm{CR} \mathrm{plate}$ with readout of 2048 x 2508 pixels was used for comparison. Parameters assessed included limiting spatial resolution, low contrast detectability, and exposure range. Images of test objects were obtained using the same x-ray source and exposure conditions and were viewed at full resolution on a softcopy display system having a $2 \mathrm{~K}$, high brightness monitor. Window and level settings could not be matched exactly; rather presentations were matched subjectively.

Results are that the limiting spatial resolution of the DR system slightly exceeds that of the CR system, perhaps expected given the DR's smaller pixel size (160 micron vs 170 micron). Similarly, the CR system exhibited a larger exposure range than the DR as would be expected from the characteristics of CR, although the DR system did not saturate within a $2^{10}$ exposure variation. The systems performed similarly for low contrast detectability.

While both systems have advantages and disadvantages, they appear to be highly competitive in the areas of image quality investigated in this study.

This research was supported by a grant from Canon USA, Inc.

\section{TU-D4-08}

Variable-Resolution X-ray Detector

F DiBianca*, P Zou, L Jordan, J Laughter, University of Tennessee, Memphis, TN

A new X-ray imaging principle called Variable Resolution X-ray (VRX) detection allows the detector's modulation-transfer function (MTF) to be improved by several orders of magnitude over that normally obtained for computed tomographic (CT) or digital radiographic (DR) imaging. When operating in the CT mode with suitable $\mathrm{x}$-ray focal spot size, the VRX spatial resolution is matched to the subject size. Thus, if a $50 \mathrm{~cm}$ field yields $2 \mathrm{cy} / \mathrm{mm}$ resolution, a $1 \mathrm{~cm}$ field may yield $100 \mathrm{cy} / \mathrm{mm}$ resolution.

The first experimental tests were made with a 16-channel array (United Detector Technologies) comprising individual $\mathrm{CdWO}_{4}$ scintillators interfaced to a photodiode array and read out with a 16-channel MicroDAS (Analogic Corp). MTF measurements made with this array exhibited limiting resolution of $64 \mathrm{cy} / \mathrm{mm}$

The VRX principle was also tested in the high-resolution CT imaging mode. A $750 \mu$-thick fan beam passed through small specimens (typically, $2-8 \mathrm{~cm}$ diameter fields) mounted on a rotating table. The VRX detector employed a storage phosphor screen that was scanned vertically to record the CT sinogram. Projection resolution of $20 \mathrm{lp} / \mathrm{mm}$ was measured with a lead bar pattern.

The storage phosphor was read out by a laser scanner and the sinogram, after corrections were applied, was separated into individual projections (views). The views were further aligned, convolved and back projected to yield the CT image of the subjects. Images have been made of a plasticized human forearm section, an anesthetized hamster, a resected human finger and mechanical objects.

\section{TU-D4-09}

A Cone-Beam CT Scanner Based Upon a Flat-Panel Imager: Effects of Image Lag

J Siewerdsen*, D Jaffray, D Drake, William Beaumont Hospital, Royal Oak, MI

Spatial and temporal imaging characteristics of an amorphous silicon flatpanel imager (FPI) were investigated in terms relevant to application of such devices in cone-beam computed tomography (CBCT) and other imaging modalities. Specifically, issues of image lag (including the magnitude, spatial uniformity, frequency characteristics, and dependence upon exposure and frame time) were investigated. First-frame lag was $2-10 \%$, depending upon incident exposure and was spatially nonuniform to a slight degree; second, third, and fourth-frame lag were $1 \%, 0.5 \%$, and $0.3 \%$, respectively (at $25 \%$ sensor saturation). Image lag was also analyzed in terms of the temporal-frequency-dependent transfer function derived from the Fourier transform of the radiation response, allowing a quantitative description of system components contributing to lag. The contrast of objects as a function of time following an exposure (30 min or longer) was measured in order to examine long-term image persistence ("ghosts"); two techniques of reducing ghost images were tested. These results pose important considerations for application of FPIs in CBCT, since image lag can cause significant artifacts in tomographic reconstructions. The effects of image lag in CBCT were examined in volumetric reconstructions obtained with an FPI for various objects and readout conditions. Two lag artifacts were identified: a "comet" resulting from signal carryover between projections, and a "streak" resulting from signal buildup during the first few projections. Procedural and algorithmic methods of reducing the lag artifacts were examined. (Supported by U.S. Army PCRP-97020 and Elekta Oncology Systems)

\section{Continuing Education Course Room: Taylor $M R I-4$}

\section{TU-D5-01}

\section{Quality Assurance of MRI Systems}

David Hearshen, Henry Ford Health System, Detroit, MI

A model Quality Assurance program with recommendations for standard test procedures, frequency of testing, personnel responsibilities, and performance criteria will be presented. The procedures and tests are not intended to establish absolute performance standards, but to track changes in system performance relative to a baseline established during acceptance testing. Criteria for evaluating such changes will be presented.

Educational Objectives:

Upon completion of this course, participants will be prepared to:

1. Design QA programs for Magnetic Resonance Imaging.

2. Develop the appropriate records and reports associated with these programs.

3. Track and interpret changes in parameters associated with MR system subcomponents

4. Understand and interpret artifacts associated with MR system malfunction

\section{Symposium Room: Pearl Recent Developments in Clinical Dosimetry of Low- Energy Interstitial Brachytherapy Sources}

\section{TU-E1-01}

Primary and Secondary Standards of Air Kerma Strength for Low Energy Brachytherapy Sources

Bert Coursey, National Institute of Standards and Technology, Gaithersburg, MD

NIST has developed a new primary standard for the low-energy brachytherapy sources of iodine-125 and paladium-103. The Wide Angle Free Air Ionization Chamber (WAFAC), developed by Robert Loevinger, realizes the quantity air kerma for photon emissions in the energy region from $10 \mathrm{keV}$ to $50 \mathrm{keV}$. NIST is the only national laboratory to offer standards for these low-energy sources. Since January 1, 1999, NIST has calibrated iodine-125 sources for four manufacturers, and palladium-103 sources for two manufacturers, using the new WAFAC standard. In the case of the prostate therapy seeds, the manufacturers are asked to submit five seeds for measurement on the WAFAC. Additional measurements are then made on each batch with well ionization chambers from two manufacturers. Following these source characterization measurements at NIST, a subset of the seeds from a batch (usually three seeds) are sent to the AAPM Accredited Dosimetry Calibration Laboratories (ADCLs) to allow transfer of the calibration capability to the secondary laboratory network. The uncertainties in the measurement systems (NIST-manufacturer-clinic and NIST-ADCL-clinic) for air kerma strength will be described for the prostate seeds presently marketed in the U.S. 


\section{TU-E1-02}

Dosimetry Parameters for Interstitial Brachytherapy Sources: An Update

Ravinder Nath, Dept. of Therapeutic Radiology, Yale University School of Medicine, New Haven, CT

Since the publication of the AAPM Radiation Therapy Task Group 43 (TG43) report in 1995, a number of new reports dealing with the new dosimetry parameters for palladium-103, iodine-125 and iridium-192 sources presented by TG-43 have appeared in the literature. The air kerma strength standard for low energy photon emitters has been revised by National Institute of Standards and Technology (NIST). The use of the new NIST standard results in a revision of values for dose rate constants reported in TG-43. Furthermore, TG-43 based dosimetry data for several newly designed interstitial brachytherapy sources have been reported recently. The original TG-43 formalism will be briefly reviewed and the dosimetry data required by the TG-43 will be updated.

Educational Objectives:

1. To review the task group 43 report on the dosimetry of interstitial brachytherapy sources

2. To update the data on dosimetry parameters for I-125 and Pd-103 sources.

\section{TU-E1-03}

Clinical Implementation of Task Group 43 Dose Calculations for Revised Source-Strength Standards and New Sources

Jeffrey Williamson, Washington University School of Medicine, St. Louis, $\mathrm{MO}$

Implementation of the Task Group 43 dose-calculation formalism for Pd-103 and I-125 brachytherapy sources is complicated by the (i) implantation of a new primary air-kerma strength $\left(\mathrm{S}_{\mathrm{K}}\right)$ standard at the National Institute of Standards and Technology (NIST) on 1 January 1999 and the appearance of many new source designs not addressed by the original TG-43 report. This talk will describe procedures for safely and accurately adapting to these changes.

For I-125 sources, the procedures are straightforward and well understood (a) Revise the prescribed dose to compensate for the differences between pre-TG43 and TG43 dose calculations:

Adopting TG-43 dose distributions for treatment planning using sources available before 1999 or using critically reviewed dosimetry data for new I125 sources, will result in calculated doses that are $10 \%$ to $18 \%$ smaller than doses calculated in the pre-TG43 era. To avoid increasing dose actually delivered to patients upon acceptance of TG43 dosimetry, the prescribed dose must be lowered by a corresponding factor which depends on the implant geometry, dose calculation algorithm, and pre-TG43 dosimetry data assumed by the accumulated clinical experience guiding the physician. Fortunately, pre-TG43 era dosimetry practices were quite uniform. For TRUS-guided definitive I- 125 brachytherapy of prostate tumors, the revised dose should be lowered by $10 \%$ from 160 Gy to 145 Gy. For new sources, users should insist that TG43 data derived from at least two independent published dosimetry studies be available and that vendor calibrations be traceable to NIST's new $\mathrm{S}_{\mathrm{K}}$ standard.

(b) Adapting NIST's new standard

For I-125 seed products marketed prior to 1 January 1999 (Nycomed Amersham and North American Scientific, Inc. products), the TG43 doserate constants, $\Lambda$, were normalized to NIST's 1985 standard. When these vendors adopt the revised NIST 1999 calibration standard, the user must increase $\Lambda$ (or its equivalent) by $11.5 \%$ but should not modify the prescribed dose. Dosimetry data published for sources introduced to the market after January 1 1999, will be traceable to the 1999 standard and will not require correction.

For Pd-103 interstitial seeds, dose prescription changes will probably be needed for all sources. For the one source available before 1999 (Theragenics Model 200), the $\Lambda$ value published in TG43 report will also require revision following implementation of a NIST-traceable $\mathrm{S}_{\mathrm{K}}$ standard for this source. Measurements and Monte Carlo calculations are underway to define the appropriate dosimetry constants for the model 200 seed. Identifying the magnitude of the prescribed dose change, which affects users of all Pd-103 products, is complicated by the possibility that Theragenics' internal pre-1999 calibration standard (which is not traceable to any $S_{K}$ standard) may not have remained constant throughout its history. As of March 1999, $\mathrm{S}_{\mathrm{K}, \mathrm{Th}}$ as inferred from Theragenics' apparent activity calibration, is only $80 \%$ of NIST's measured values. Hopefully, more definitive recommendations will be available soon.

\section{Symposium Room: Mississippi Analysis of IMRT Planning and Delivery Strategies}

\section{TU-E2-01}

A Comparison of Forward and Inverse Treatment Planning for IMRT James Galvin, Thomas Jefferson University Hospital, Philadelphia, PA

The introduction of inverse treatment planning and IMRT dose delivery has revolutionized the way radiation oncologists, medical physicists, and dosimetrists think about achievable dose distributions. Using this new technology, it is easy to generate plans for complex non-coplanar beam arrangements as well as for treatment of lesions that partially or fully surround critical structures. However, the plans generated with inverse planning are often significantly different than traditional plans, and the steps of plan evaluation and implementation can present problems. For example, relative to past standards, the generally poorer dose homogeneity for inverse planning forces the use lower isodose lines for prescribing dose. Additionally, the intensity maps generated by inverse planning can be complex and, in turn, extend dose delivery times, increase leakage radiation, and complicate treatment verification. Given these difficulties, there is a renewed interest in using forward treatment planning as a possible solution for these problems. There are four major reasons why the forward approach could prove valuable for the treatment planning part of IMRT: First, benchmark plans are needed to illustrate what is possible for complex noncoplanar beam arrangements and for treating targets with invaginations. Second, if these benchmark plans prove to be superior to inverse plans, forward planning may become the method of choice for at least the immediate future. Third, designing new dose optimization techniques that mimic forward methods might lead to improved algorithms that do not show some or all of the limitations of inverse methods. Fourth, the simplicity of the intensity maps produced by forward planning could lead to improved techniques for verification of the delivered dose.

This talk describes two forward treatment planning techniques: one for noncoplanar beam arrangements and another for targets with invaginations surrounding critical structures. Using dose-volume histogram analysis, plans generated with these two methods are compared to plans obtained with inverse planning. Additionally, the problem of increased critical structure dose that results from the practice of prescribing to low isodose levels is discussed. Intensity maps generated with the two forward planning methods are compared to determine their relative complexity. Although a number of methods for checking correct delivery of the intensity distributions for IMRT are available, there is no direct equivalent to imaging the treatment field relative to a patient's anatomy. It will be argued here that the wellbehaved intensity distributions for forward planning allow the use of simple port filming for verification. Finally, an optimization technique that is modeled on the forward planning approach will be discussed, and plans generated using this method will be presented. An advantage of this simple optimization approach as well as the forward treatment planning methods described here is the relative ease with which they can be implemented in small clinics that do not have extensive physics support.

\section{Educational Objectives:}

1. Identify practical limitations for IMRT planning and dose delivery.

2. Offer solutions for these problems.

3. Identify role for forward treatment planning for IMRT.

4. Compare forward and inverse treatment planning. 


\section{TU-E2-03}

Conformal Plan Optimization: Inverse and Forward Planning for Full and Segmental IMRT

Benedick Fraass, Karen Vineberg, John Kim, Marc Kessler, Daniel McShan, Dept of Radiation Oncology, University of Michigan, Ann Arbor MI

Conformal radiation therapy makes use of a three-dimensional anatomical description of the patient, target(s) and normal tissues, and requires optimization of the treatment plan(s) used so that the high dose volume conforms to the shape of the target(s), while minimizing the dose to normal tissues. In recent years, conformal treatment strategies have evolved from the use of multiple shaped fixed fields to various intensity modulated radiation therapy (IMRT) techniques, and optimization of those conformal treatment plans has evolved from the use of 3-D treatment planning techniques ("forward planning") to the use of automated optimization systems ("inverse planning"). In this work, a very general automated optimization system is used to perform quantitative comparisons between various kinds of conformal therapy techniques including static conformal, segmental IMRT (multiple MLC shapes per field) and full IMRT based on generalized checkerboard-type intensity distributions. In addition, optimized IMRT plans are compared against static conformal plans with optimized field shapes generated with an automated dose-based conformal shaping algorithm. These comparisons include plans developed for high dose ( $>90$ Gy) brain tumor treatments, and parotid-sparing head and neck treatments. This work will also illustrate methods for careful and quantitative comparisons of plans representing different optimization or treatment technologies.

\section{EducationalObjectives:}

1. Comparisons of Inverse-planned and Forward-planned IMRT plans.

2. Comparisons of optimized conformal plans based on static conformal fields, segmental IMRT, and full (checkerboard intensity distribution) IMRT.

3. Illustration of rigorous plan comparison techniques.

4. Comparisons of various conformal and/or IMRT techniques for high dose brain treatment and sparing of the parotid during treatment for head and neck cancers.

\section{Symposium Applications}

\section{TU-E3-01}

\section{CIRMS Measurements for Diagnostic Radiology Applications}

Michelle O'Brien ${ }^{1}$, Frank Cerra ${ }^{2}$, Larry DeWerd ${ }^{3}$, Paul Lamperti ${ }^{1}$, (1)National Institute of Standards and Technology, Gaithersburg, MD, (2) CDRH FDA, Rockville, MD, (3) University of Wisconsin, Madison, WI

The Council on Ionizing Radiation Measurements and Standards (CIRMS) represents users of ionizing radiation and radioactive sources engaged in medical radiation and other programs. CIRMS provides a forum for discussing ionizing radiation issues; identifying, defining and prioritizing needed work for standards; disseminating information on standards; and organizing workshops and meetings to advance ionizing radiation technology. The National Institute of Standards and Technology (NIST) is heavily involved with this organization and considers its directives as statements of work to be completed. A presentation of the research and measurements made for standards for Medical Diagnostic Radiology Applications will be summarized addressing two major topics: Molybdenum (Mo) and Rhodium (Rh) anode beams for mammographic chamber calibrations and the establishment of medium filtration x-ray beams for other diagnostic radiographic applications. Each of these standards is described in Measurement Program Descriptions (MPD), which are statements of requirements and the reasons for the measurements. The measurements performed for establishing mammographic calibration x-ray beams will be reviewed. Calibration standards with molybdenum and rhodium anode $\mathrm{x}$-ray beams, with molybdenum and rhodium filters, respectively, were established using a variable length Attix Free air chamber. Results from a comparison of this free air chamber with the Ritz free air chamber will be given. The application of the Attix free air chamber to establish these beams at molybdenum and rhodium will be reviewed. The transfer of these beams to the ADCLs and CDRH will also be discussed. The particular application of the mammography beams at the FDA/CDRH will be reviewed, providing brief background information about the FDA's need for traceability of measurements. The role of the CDRH Calibration Laboratory and the FDA's concerns over the lack of Molybdenum and Rhodium-target national standards in the advent of MQSA regulations will be discussed. The difficulties and successes of the CDRH Calibration lab's experience in matching the NIST beams and accreditation requirements will be reviewed. A clarification of the proficiency test requirement in the MQSA final rule will be presented. Following the establishment of Mo and Rh beams, a comparison was made of the response of mammography ionization chambers to the tungsten anode, aluminum filter beam at $0.36 \mathrm{~mm} \mathrm{Al}$ half value layer. There is also a need for two particular beams for general diagnostic chamber calibrations to be established at NIST. The method of establishing beams appropriate for diagnostic ionization chamber calibrations at $3.0 \mathrm{~mm} \mathrm{Al}$ half value layer and $10.0 \mathrm{~mm} \mathrm{Al}$ half value layer will be reviewed. The M80 beam (HVL of $3.0 \mathrm{~mm} \mathrm{Al}$ ) is appropriate for general diagnostic use. The $\mathrm{M} 120$ beam (HVL of $10.0 \mathrm{~mm} \mathrm{Al}$ ) is appropriate for CT chamber use. These two beams fill in a gap in the spectra needed for diagnostic chambers. The method used by NIST for the establishment of these beams and their agreement with other beams in the series will be discussed.

Educational Objective:

1. An understanding of equipment and methodology involved in the establishment of standards for Diagnostic Radiological Applications.

\section{Symposium Imaging Tumor Physiology}

Room: Carroll

\section{TU-E4-01}

Functional MR Imaging of Cancer Physiology and Anatomy Gregory S. Karczmar, University of Chicago, Chicago, IL

Magnetic Resonance Imaging and Spectroscopy are extremely flexible methods which evaluate tumor anatomy, physiology, and metabolism noninvasively, and with good spatial resolution. Because of its excellent soft tissue contrast, MRI has been widely used since its inception for identifying tumors and defining tumor margins.

With rapid improvements in anatomic imaging, functional information regarding tumor proliferation is available from MR. For example, cancer invasion of the prostate capsule can often be detected, and is a reliable marker for highly malignant tumors. New contrast agents are available which may make it possible to identify tumor infiltration of normal tissue with great accuracy.

MR is extremely useful for evaluating tumor blood supply. This is important because angiogenesis is an important marker for malignant tumors. Most hemodynamic measurements in tumors rely on the use of MR contrast agents. High molecular weight blood pool agents report tumor blood volume - and thus microvessel density, as well as capillary permeability. Volumetric blood flow can also be measured with these large molecules, but low molecular weight highly permeable contrast agents are often better suited for this application. Non-invasive methods which do not require tracers, such as 'diffusion weighted images' can be used to infer microvessel flow, as well as other aspects of hydrodynamics in tumors. A variety of targeted contrast agents are being developed are designed to target receptors on blood vessel walls, to provide markers for angiogenesis.

Measurements of oxygen level are extremely important for characterizing tumors and their response to therapy. Fluorine-19 MR and Electron Spin resonance can be used to measure oxygen levels quantitatively, following injection of oxygen sensitive tracers. Gradient echo images are sensitive to changes in blood oxygenation, due to the paramagnetism of deoxyhemoglobin (i.e. the BOLD effect); this approach has very high spatial resolution and does not require injection of tracers, but is not very quantitative.

A variety of MR methods are used to image metabolic processes such as oxidative phosphorylation, glycolysis, $\mathrm{pH}$, and ion fluxes. These methods include proton and phosphorous MR spectroscopy and use of a variety of 
probe molecules. Dr. Gillies has done outstanding work in this area and his presentation will focus on some of these issues.

MR is likely to play an increasing role in cancer diagnosis and treatment planning. Several areas where there is tremendous growth in clinical application of MR to oncology are:

1. Early identification and staging of breast and prostate cancer.

2. Monitoring changes in hemodynamics during therapy.

3. Use of MR spectroscopy to stage cancer.

It is extremely important to minimize cost of MR exams. A challenge for MR physicists in the next few years will be decreasing the duration of MR exams while at the same time increasing information content. In addition, the development of hardware and software which facilitate inexpensive low field MR scans will be of great importance.

Educational Objectives:

1. Provide overview of MR methods

2. Review current and future applications of MR to cancer diagnosis and treatment

\section{TU-E4-02}

Heterogeneity of Tumor Perfusion and pH Measured by MRI/MRS

Robert Gillies, Arizona Cancer Center, Tuscon, AZ

Dynamic contrast enhanced MRI monitors the time course of signal enhancement following a bolus injection of contrast agent, usually GdDTPA (Magnevist ${ }^{\circledR}$ ). The time-dependent signal enhancement kinetics can be analyzed on a pixel-by-pixel basis using a variety of algorithms to yield values for vascular density and the capillary permeability-surface area product. These analyses illustrate that virtually all solid tumors, in animal models as well as human patients, are heterogeneously perfused. Regions of high perfusion co-exist with regions with virtually no perfusion.

The poorly perfused volumes are important both therapeutically and biologically. Because they are hypoxic and aciditic, they are resistant to radio- and chemotherapies. Under controlled, in vitro, conditions hypoxia and acidity also lead to development of more aggressive tumorigenic phenotypes.

Poorly perfused regions likely correspond to the hypoxic volumes that can be visualized with PET, SPECT or MRI. Hypoxic regions are resistant to ionizing radiotherapy due to the generation of longer lived reactive species. Cells under hypoxic conditions must derive their energy from glycolysis, the end-product of which is lactic acid. The combination of poor perfusion and high glycolysis is the likely major cause for the acidic extracellular $\mathrm{pH}$ seen in tumors.

Acidic extracellular $\mathrm{pH}(\mathrm{pHe})$ in tumors can be measured non-invasively using 31-P MRS. This approach also allows the $\mathrm{pH}$ distribution across tumors to be measured, which shows that some tumor cells exist under very acidic pHe conditions (e.g. 6.2). More recently, regional pHe distributions have been measured using 1-H spectroscopic imaging, which allows spectra from multiple voxels to be obtained simultaneously. These measurements show large regional variations in tumor $\mathrm{pHe}$, which are likely due to perfusion heterogeneity.

Acid pHe in tumors leads to a "physiological" resistance to weakly basic chemotherapeutics, such as anthracyclines. This occurs via a mechanism of "ion trapping" wherein weakly basic molecules are sequestered in relatively acidic compartments. In the case of tumors, ion trapping favors drugs to be retained in the interstitium, and not enter the cells. The extracellular space can be alkalinized with bicarbonate, and this leads to increased sensitivity of tumors to anthracyclines, such as doxorubicin or mitoxantrone. The acidic $\mathrm{pHe}$ of tumors can also affect the process of tumorigenesis itself since, in vitro, acid $\mathrm{pHe}$ causes transformation, mutation and chromosomal rearrangements.

\section{TU-E4-03}

Oncologic Applications of Metabolic Imaging with Positron Emission Tomography

Dominique Delbeke, Department of Radiology and Radiological Sciences, Vanderbilt University Medical Center, Nashville, TN

This presentation is designed for physicians, scientists, and technologists that are new to the positron imaging fields. The learning objectives are the following: describe the principles of metabolic imaging using positron emitting tracers, understand the normal distribution of ${ }^{18} \mathrm{~F}$ fluorodeoxyglucose (FDG) in the body, discuss the clinical uses of FDG PET for oncology patients, understand the pattern and causes of false positive and false negative results. Positron imaging is unique in one respect: Positron emitters allows labeling of radiopharmaceuticals that closely mimic endogenous molecules and there is continuous developments of new biological tracers. FDG, a derivative of glucose, allows the evaluation of glucose metabolism, and is the most commonly used tracer because of the practical half-life of ${ }^{18} \mathrm{~F}$ (110 minutes) compared to the other positron emitters. Most tumor cells demonstrate increased glucose metabolism. This is due, in part, to increased number of glucose transporter proteins and increased intracellular enzyme levels of hexokinase and phosphofructokinase, among others, which promote glycolysis. Although variations in uptake are known to exist among tumor types, elevated uptake of FDG has been demonstrated in various malignant primary tumors. The applications for FDG PET imaging are rapidly growing and accepted in the field of oncology. FDG PET imaging does not replace other imaging modalities such as CT, but appear to very helpful in specific situations where CT has known limitations, such as differentiation of benign from malignant indeterminate lesions on $\mathrm{CT}$, staging malignant lesions, differentiation of benign from malignant lymph nodes, differentiation of post treatment changes versus recurrent tumor, and monitoring therapy. The addition of FDG PET in the evaluation of oncological patients in well defined algorithms including a combination of imaging studies appear to be cost effective by identifying accurately patients that will benefit from invasive procedures and saving unnecessary costly invasive procedures on patients that will not benefit from them. The Health Care Financing Administration (HCFA) which regulates Medicare started reimbursement for solitary pulmonary nodule and staging non small cell lung carcinoma in January 98, and recently announced their approval for reimbursement for colorectal carcinoma, lymphoma and melanoma.

\section{Refresher Course Pearl}

\section{WE-A1-01}

The Multileaf Collimator: Past, Present and Future

James Galvin, Thomas Jefferson University Hospital, Philadelphia, PA

Two of the multileaf collimator (MLC) systems currently in common use in this country were introduced in the early 1990's by Philips (now Elekta) and Varian. These collimators were designed for simple block replacement, but are now also viewed as convenient tools for intensity modulated radiation therapy (IMRT). More recently, another MLC was introduced as an addition to Siemens' product line. Although these collimators share some characteristics, each has a unique combination of design features. The differences have given rise to endless debate about which system is the "best." This talk will compare the different collimator designs and discuss the dosimetric characteristics for each. The convenience of MLC field shaping has had a major impact on the way radiation therapy is currently practiced. Field shaping can be accomplished in a matter of minutes, and changes can be implemented with ease. However, there is some concern about leaf stepping at an MLC defined edge and the importance of the accompanying dose undulation. Dose distributions will be presented to compare divergent block and MLC defined edges. It is possible to remove the dose undulation at a stepped MLC edge by dithering the field using a series of pre-programmed couch movements. Dose distributions for this technique of smoothing the undulating dose pattern will be presented and compared to block-defined edges. Acceptance testing, commissioning, and routine quality control of MLC systems are important issues for the medical physicist. This talk will present procedures for accomplishing each of these tasks. Systems differ in terms of their effect on the calculation of dose to a point within an irregular MLC defined field. A major factor contributing to 
this difference is the distance of the collimator from the source of radiation. Methods of dose calculation when MLC is used will also be discussed. The use of MLC for intensity modulated dose delivery is quickly gaining popularity. This application introduces new dosimetry problems that must be addressed. For example, sliding window IMRT requires very exact leaf positioning. Although all manufacturers can control leaf placement with great precision, exact definition of the leaf edge is difficult when the leading end is rounded so that the point where the intensity falls to one-half is hard to identify. Another consideration for IMRT is the build up of leakage radiation relative to traditional treatment approaches. This presentation will include a discussion of these problems.

Educational Objectives:

1. Compare dosimetric and design characteristics of three multileaf collimator systems in common use in this country.

2. Discuss the problem of dose undulation at stepped MLC edge.

3. Present methods for acceptance testing, commissioning and routine QA for MLC systems.

4. Discuss the effect of MLC on the "collimator factor" when performing dose calculations.

5. Discuss the impact of MLC design features on IMRT dose delivery.

\section{Refresher Course}

Room: Mississippi

\section{WE-A2-01}

The Current Status of the Clinical Use of Electronic Portal Imaging Devices

John W. Wong ${ }^{1}$, Michael G. Herman ${ }^{2}$, James M. Balter ${ }^{3}$, David A. Jaffray 1, (1) William Beaumont Hospital, Royal Oak, MI (2) Mayo Clinic, Rochester, MN (3) University of Michigan, Ann Arbor, MI

In the more than 10 years since the commercial introduction of electronic portal imaging devices, the adaptation of the technology for routine treatment verification remains sporadic. In 1998, an informal survey conducted by several members of the AAPM Task Group 58 indicated that, in the US, less than $30 \%$ of those clinics with these devices used them clinically on a routine basis. The poor utilization stemmed primarily from our lack of understanding of how the digital images might be used, exacerbated by the lack of software tools to manage and process the large volume of data. For a busy clinic, electronic portal imaging became a hindrance, rather than the catalyst that would enhance accurate and efficient treatment. Not withstanding, valuable insights about setup variation and portal imaging were attained by those groups that had embraced the technology clinically. The EPID is first most, a very powerful device for acquiring large number of portal images and for establishing the baseline variation of daily setup for each clinic. Many investigations have shown that setup variation $>5 \mathrm{~mm}$ is not uncommon. It is important for a clinic to establish its baseline setup variation and to adapt the appropriate setup correction model, or models. There are 3 general models of utilization: (1) on-line visual examination for gross errors, (2) off-line quantitative evaluation with subsequent application of the necessary correction, and (3) on-line determination and application of the correction. For each model, the achievable level of setup accuracy in relationship with the time and effort required of the treatment personnel must be well understood. Selection of a particular correction model is best made by considering the treatment goal. Properly implemented, electronic portal imaging generally improves setup accuracy and can be cost-effective. Setup accuracy of the order of 2 to 3 $\mathrm{mm}$ can be achieved, a level deemed necessary for pursuing dose escalation. It should also be cautioned that there is a limit to the improvement in setup accuracy achievable with electronic portal imaging. Treatment setup is inherently a complicated $3 \mathrm{D}$ problem that cannot be totally addressed with the use of $2 \mathrm{D}$ projection radiographic images. Interestingly, despite its lackluster impact in the past, the future for EPID is exciting. With the advent of intensity modulated radiation therapy, the increase in networking capability, the commercial availability of software tools, and the improving image quality with new imaging technology, it seems inevitable that electronic portal imaging will be a integral component of modern radiation therapy.

EducationalObjectives:

1. To discuss issues related to the implementation of electronic portal imaging
2. To elucidate different models to utilize electronic portal imaging clinically

3. To discuss the role of electronic portal imaging in $3 \mathrm{D}$ conformal therapy

Research of J. Wong and D. Jaffray is supported in part by Elekta Oncology Systems and NCI grant R01 CA 66074

\section{Continuing Education Course Special Dosimetry Measurements - 3 \\ Room: Sewanee}

\section{WE-A3-01}

Two Dimensional Radiation Field Mapping using Radiochromic Film Azam Niroomand-Rad ${ }^{1}$, Christopher G. Soares ${ }^{2}$, (1)Georgetown University Medical Center, Washington, DC, (2) National Institute of Standards and Technology, Gaithersburg, MD

Use of radiochromic film for two-dimensional radiation field mapping is increasing rapidly in the medical field. This film largely satisfies the need for a detection medium which is tissue equivalent, offers a high spatial resolution, does not require a special developmental procedure, gives a permanent record of absolute values of absorbed dose, has acceptable sensitivity, accuracy and precision, and offers ease of handling and data acquisition and analysis. To fully realize these advantages, however, takes some knowledge and experience, which it is the object of this course to impart. To this end, the characteristics of the various types of available radiochromic films will be discussed. While the films require no special handling, there are quite a few "tricks of the trade" which should be known to achieve optimal results. Thus, procedures for using radiochromic films will be covered. Crucial to the optimal use of the films is the equipment used to record the irradiated film density pattern, so the characteristics of available film readout systems will also be discussed. Conversion of the measured film densities into absorbed doses is effected through the proper application of calibration functions, so methods to prepare and use such calibration functions will be covered. The use of radiochromic film for dosimetry mapping has been the subject of a recent AAPM report and the recommendations of the Task Group 55 will be presented and discussed. Finally, some examples of past uses of therapy applications of radiochromic film for two-dimensional radiation field mapping will be reviewed.

Educational Objectives:

1. To familiarize the attendee with characteristics of the various available types of radiochromic film.

2. To cover procedures for using radiochromic films for dosimetry.

3. To discuss methods of radiochromic film readout.

4. To cover methods for calibration of radiochromic film.

5. To discuss precautions and the limitations of the use of radiochromic film.

6. To cover the recommendations of AAPM Task Group 55 on radiochromic film dosimetry.

7. To review some applications of radiochromic film for mapping therapy radiation fields.

\section{Refresher Course}

Room: Carroll

\section{WE-A4-01}

Spiral CT: Single and Multiple Detector Systems

Michael McNitt-Gray, UCLA Department of Radiological Sciences, Los Angeles, CA

Spiral CT scanners have seen dramatic developments in the past year. Though twin detectors and rotation times of less than a second were previously available, current systems now offer both multi-detectors systems ( 2 and 4 with pathways to 8,16 and 34 ) and rotation times down to .5 second. These improvements combined with increased X-ray tube heat capacity and faster image reconstruction times demonstrate that Spiral CT continues to undergo rapid changes that are increasing the ability to quickly and continuously acquire a large volume of image data. This has led to significant improvements in diagnostic imaging methods, such as: volumetric acquisition and 3-D display of image data; improved vascular imaging and CT angiography (CTA), single breathhold thoracic scans which 
avoid breathing misregistration; and rapid acquisition of contrast enhancement protocols that allow tissues to be imaged during different phases of enhancement (arterial, venous and parenchymal). Finally, the choice of operational parameters (such as slice thickness, table speed and reconstruction interval) affect image quality and/or patient radiation dose and become even more complex in multiple detector systems. Because of these developments, basic knowledge of spiral CT and an understanding of the design and operational characteristics are becoming essential for physicists, radiologists and technologists.

Educational Objectives:

1. Define Spiral CT and some affiliated terms (pitch, reconstruction interval and interpolation algorithm) for both single and multiple detector systems.

2. Describe the basic differences between conventional axial CT and spiral CT in how data is acquired and how images are formed.

3. Describe some of the recent developments in multi-detector systems with subsecond rotation times and how these concepts affect image formation.

4. Discuss the implications of Spiral CT on clinical usage (volumetric acquisitions, vascular imaging, single breathhold thoracic scans, etc.).

5. Describe the effects of Spiral CT scanning and the selection of operational parameters on image quality and radiation dose; compare and contrast these effects for single and multiple detector systems.

6. Discuss possible future developments in Spiral CT.

\section{Continuing Education Course Room: Taylor MRI - 5}

\section{WE-A5-01}

\section{Annual Compliance Testing}

David O. Hearshen, Henry Ford Health System, Detroit, MI

Annual Compliance Testing is a requirement for ACR accreditation and some regulatory agencies. A standard set of tests and procedures that could satisfy compliance testing will be discussed. A model program for satisfying the requirement of a state regulatory agency will be presented. The American College of Radiology Performance Standards will also be presented and discussed in light of the of the recently initiated ACR MRI accreditation Program.

Educational Objectives:

Upon completion of this course, participants will be prepared to:

1. Design compliance testing programs for Magnetic Resonance Imaging.

2. Develop the appropriate records and reports associated with these programs.

3. Understand the origins and interpret phase stability and uniformity tests

4. Implement a CT program satisfying ACR accreditation requirements.

\section{Continuing Education Course Room: Browning A Ultrasound - 3}

\section{WE-A6-01}

Hands On Ultrasound Quality Control Workshop

Carolyn Kimme-Smith ${ }^{1}$, Mitchell Goodsitt ${ }^{2}$, Evan Boote ${ }^{3}$, Mark Holland ${ }^{4}$, James Zagzebski ${ }^{5}$, Heather Miller ${ }^{6}$, Jim Kofler ${ }^{7}$, (1) UCLA, Los Angeles, CA (2) University of Michigan, Ann Arbor, MI (3) University Missouri Columbia, Columbia, MO (4) Washington University, St. Louis, MO (5) University Wisconsin, Madison, WI (6) Computerized Imaging Reference Systems, Inc., Norfolk, VA (7) Mayo Clinic, Rochester, MN

As in previous years, this workshop will provide attendees with an opportunity to refresh their skills in US quality control procedures for realtime gray mode imaging. This will include depth of visualization, uniformity, vertical and horizontal distance accuracy, lateral and axial resolution, ring down, and slice thickness focal range. In addition, a US prostate treatment planning system and prostate phantom will be available. Medical Physicists who have a theoretical knowledge of Ultrasound procedures will be guided by experienced instructors to scan a variety of QC phantoms. In order to provide sufficient equipment for attendees, small portable ultrasound units will be provided. No Doppler or color flow imaging units will be available. Attendees should be familiar with the AAPM Ultrasound Task Group 1 Report; "Real-time B-mode ultrasound quality control test procedures" in Medical Physics 1998; 25: 1385-1406. Copies of appendix A from that report will be available at the workshop. The QC tests will take about an hour to complete, and participants can attend anytime during the scheduled workshop.

EducationalObjectives:

1. To learn US QC test procedures for Real-time B-mode units.

2. To become acquainted with new US QC phantoms.

3. To learn to calibrate a prostate US treatment planning unit.

\section{Refresher Course}

Room: Pearl

\section{WE-B1-01}

\section{Mini and Micro Multileaf Collimators}

Thomas Bortfeld, Wolfgang Schlegel, Karl-Heinz Höver, Daniela SchulzErtner, German Cancer Research Center (DKFZ), Dept. of Medical Physics, Heidelberg, Germany

The treatment of small and irregularly shaped lesions especially in the head is a challenging problem in radiotherapy. The use of isocentric circular fields does not generally yield the desired degree of dose conformation to the target volume. Multiple shot Gamma knife or multiple isocenter linac treatments give better conformation but may lead to unacceptable dose inhomogeneities in the target volume and may be difficult to plan and treat. It has been shown that much better results can be achieved with multiple individually shaped irregular fields instead of circular fields. Unfortunately, most commercial multileaf collimators are not well suited to perform accurate field shaping for small lesions because their projected leaf width of $1 \mathrm{~cm}$ is too coarse. This holds especially for cases where the target volume is very close to small critical structures such as the optic nerves.

This lecture deals with miniature multileaf collimators (mMLC) with a projected leaf width of less than $5 \mathrm{~mm}$, which are specifically designed for such stereotactic applications and offered by various vendors of equipment for stereotactic radiotherapy (e.g., Brainlab (Heimstetten, Germany), Leibinger (Freiburg, Germany), Radionics (Burlington, MA)). The design characteristics of the mMLCs will be presented and their pros and cons will be evaluated with respect to three different modes of application:

1. multiple static fields,

2. multiple dynamic arc treatments, and

3 . intensity modulated treatments.

The technical requirements on the mMLCs increase in this order.

First of all we will discuss and compare the geometrical design features such as parallel or focused leaf design, leaf end shape (rounded or straight), projected leaf width, maximum field size, travel over the centerline, clearance to isocenter, and tongue and groove construction. Then we will describe basic technical features such as the maximum leaf speed and the accuracy of leaf positioning. In the third part we will talk about dosimetric characteristics such as penumbra, leaf transmission and interleaf leakage. Some aspects of how to perform dosimetry for mMLCs with films and diamond detectors will also be covered. Finally, we will briefly address the techniques and the potential of intensity modulated treatments of small lesions using an mMLC, thereby focusing on the aspect of penumbra sharpening.

\section{Educational Objectives:}

1. To provide useful information to institutions who wish to start stereotactic MLC treatments.

2. To learn the most important features of commercial mMLCs.

3. To understand the significance of these features for various modes of application (static, dynamic, IMRT).

4. To learn about the potential merit of mMLC treatments as compared to conventional treatments with regular fields. 


\section{Refresher Course}

\section{Room: Mississippi}

\section{WE-B2-01}

Information Systems and Networks in Radiation Oncology

Michael Herman, Mayo Clinic, Rochester, MN

Radiation therapy depends critically on organizing and communicating information. Medical informatics is concerned with the organizational management, acquisition, storage, and retrieval of information for problem solving and decision making in support of patient care, medical education and medical research. It also involves understanding evolving technology and relationships and standards by which this information can be managed. An information system (IS) provides the backbone for communication, documentation and quality control and must integrate all necessary data/images in a seamless, reliable, efficient manner.

As radiation oncology technology and technique have advanced, the IS have become electronic and built on computer networks, which provide data communication between servers and clients, comprised of hardware and software configured in a specific topology. Networks can be connected or divided by switches that route data traffic to correct locations. Communications must be performed through standard protocols with specific information formatting.

The IS integrates and manages the interrelationships of information objects in a seamless, effective manner. The information objects in radiation oncology include patient demographics/notes, diagnostic studies, treatment planning, treatment delivery, record and verify, billing, scheduling, quality control. The simplest IS is the paper patient chart where pen, paper and sneakers represent the communication web. Electronic IS were developed to reduce errors, increase efficiency, automate recording and organize large amounts of information. The organization and interrelationship of information objects is critical in the design of a functional IS. The method by which the information model is built, determines its ultimate success or failure and its longevity. The object-oriented approach is intended to make the IS standard, open and modular and is discussed in the context of DICOM and DICOM-RT. Proprietary tools and secret database formats make universal information integration impossible.

Quality assurance and quality improvement of the IS and the underlying network help manage and maintain accurate, complete and accessible patient data. Quality control procedures and test frequencies for software and hardware must be developed, implemented and documented. Backup and failure recovery must be guaranteed if the IS is to be used in human therapy. A comprehensive IS will include at least the following, accessible anywhere on the system:

- $\quad$ Patient- demographics, identification, notes, correspondence

- Diagnostic-images, reports

- $\quad$ Therapy- simulation, planning, delivery, validate, verify, billing

- Interface to RT-PACS, PACS using DICOM and DICOM-RT

- $\quad$ Scheduler for all objects

- Backup, failure recovery and routine QA.

Investment of human and capital resources in IS and networks is inevitable and ongoing. The quality and availability of information in the clinic directly influences the quality of patient care. An integrated IS, based on standards and built on an efficient, well-designed network, represents a key component in radiation therapy treatments. If radiation oncology treatments are to continue to become more complex and be successful, the fully integrated electronic IS will be part of it.

Educational Objectives:

1. Introduce components and concepts in radiation oncology information systems.

2. Review network hardware, software, topology and protocols.

3. Present sample specifications and questions.
Refresher Course

Room: Sewanee

\section{WE-B3-01}

How to Prepare and Deliver Electronic Presentations

Robert Pizzutiello, Upstate Medical Physics, Inc., Victor, NY

Medical Physicists are often called upon to present educational sessions to colleagues and facility staff members. Venues range from small groups of a few people to national meetings with hundreds or thousands of attendees. The familiar method of delivering such presentations employs $35 \mathrm{~mm}$ slides, prepared by Medical Illustration departments or shot from personal $35 \mathrm{~mm}$ cameras and projected from carousel projectors. Technological advances in computers, software, scanners, digital cameras and computer projectors have made electronic presentations a viable, practical and cost-effective alternative to conventional $35 \mathrm{~mm}$ presentations.

This "How To" session will explore the advantages and disadvantages of 35 $\mathrm{mm}$ vs. electronic presentations. We will describe the equipment and techniques used to create and project educational programs that are dynamic and stimulating in ways that are not possible with conventional $35 \mathrm{~mm}$ presentations. The relative costs to equip and produce $35 \mathrm{~mm}$ and electronic programs will be compared.

Educational Objectives:

1. To compare the benefits of presentations using conventional $35 \mathrm{~mm}$ and electronic methods

2. To understand the equipment, materials, methods and costs of preparing and delivering electronic presentations

3. To demonstrate "How To" prepare electronic presentations in Radiology, Radiation Oncology and Medical Physics

4. To suggest future directions for those interesting in developing electronic presentations

\section{Refresher Course}

Room: Carroll

\section{WE-B4-01}

Tomographic Image Reconstruction

Xiaochuan Pan, The University of Chicago, Chicago, IL

Tomographic imaging refers to the non-invasive determination of the internal structure of an object from transmission, emission, or reflection data collected from different positions around the object.

Tomographic imaging techniques have found a number of medical applications, ranging from X-ray CT to nuclear emission tomography to magnetic resonance imaging to ultrasonic diffraction tomography, and have had a revolutionary impact on diagnostic medicine.

The problem of image reconstruction from the collected data has been one of the most active and rapidly evolving areas of research in medical physics. A wide variety of reconstruction techniques have been developed to reconstruct images for different tomographic imaging modalities. The purpose of this paper is to provide a tutorial overview on the subject of tomographic image reconstruction.

We will describe and review in an intuitive way the general mathematical and physical principles and concepts involved in tomographic imaging. We will also provide a basic understanding of some most widely used reconstruction algorithms by focusing on the image reconstruction problem in X-ray CT. In addition, we will discuss the evaluation of image quality and analyze image artifacts that arise in tomographic imaging such as X-ray CT

\section{Continuing Education Course MRI-6}

\section{WE-B5-01}

MRI in Stereotactic Procedures

Edward Jackson, U.T. M.D. Anderson Cancer Center, Houston, TX

Magnetic resonance imaging is the modality of choice for the evaluation of soft tissue lesions, particularly those in the central nervous system. 
Therefore, MRI is being used more and more frequently in the planning stages of stereotactic procedures, including stereotactic biopsy and stereotactic radiosurgery. Furthermore, MRI data is now being used to guide interventional and surgical procedures, sometimes while the patient is in the actual bore of the magnet. Before such treatment planning procedures based on MRI data is undertaken, however, it is critical to understand the large number of intrinsic and extrinsic parameters that affect the spatial accuracy of MR images. An understanding of such parameters then allows the clinical medical physicist to assist in choosing appropriate image acquisition techniques and acquisition parameters that will provide optimal spatial accuracy of the resulting images. This course will review the primary limitations of spatial accuracy in MR imaging, and present techniques for minimizing such sources of error when using MRI data in planning stereotactic procedures.

Educational Objectives:

To review the physical basis of the primary limitations in spatial accuracy in MRI and present acquisition techniques that minimize spatial inaccuracies.

Upon completion of this course, the participant will be prepared to:

1. Understand the basic sources of error in spatial accuracy inherent in common MRI acquisition techniques.

2. Appreciate typical spatial accuracies that are achievable in MR imaging studies, and under what conditions such accuracies can be obtained.

3. Develop MR scan protocols that minimize the inherent spatial inaccuracies and provide optimal information for use in stereotactic procedures.

\section{Scientific Session \\ Pearl \\ Radiotherapy Delivery Systems and Techniques}

\section{WE-C1-01}

Comparative Treatment Planning Using Calculations of Secondary Cancer Incidence

U Schneider*1, A Lomax², N Lombriser ${ }^{1}$, (1)Department of Radiation Oncology and Nuclear Medicine, City Hospital Zuerich, Switzerland, (2)Department of Radiation Medicine, Paul Scherrer Institute, Villigen, Switzerland

Purpose: Probabilities for mortality from secondary cancers have been calculated for a patient with Hodgkin's disease which has been planned with different radiation treatment modalities using photons and protons.

Materials and Methods: The ICRP calculation scheme has been used to calculate mortality from dose distributions. To this purpose target volumes as well as critical structures have been outlined in the CT set of a patient with Hodgkin's disease. Dose distributions have been calculated using conventional as well as intensity modulated treatment techniques using photon and proton radiation. From the mean doses of each organ the mortality has been derived.

Results:

(i) Intensity modulated treatment of Hodgkin's disease using 9 photon fields $(15 \mathrm{MV})$ results in nearly the same mortality as treating with two opposed photon fields (6 MV).

(ii) Intensity modulated treatment using 9 proton fields (maximum energy $177.25 \mathrm{MeV}$ ) results in nearly the same mortality as treating with one proton field $(160 \mathrm{MeV})$

(iii) Irradiation with protons using the spot scanning technique decreases the avoidable mortality compared to photon treatment by a about a factor of two. This result is independent of the number of beams used.

Conclusions: By looking at the incidence of secondary cancer our work suggests that there are radiotherapy indications where intensity modulated treatments will give little or no improvement over conventional treatments. However proton treatment can result in a lower mortality than photon treatment.

\section{WE-C1-02}

A High Resolution Collimating Device - An Affordable Alternative to a Micro-Multileaf Collimator

P Williams*, North Western Medical Physics, Christie Hospital, Withington, Manchester, UK

A secondary collimating device used in conjunction with a standard multileaf collimator has been shown to produce shaped fields with spatial resolution as high as a micro multileaf collimator.

The secondary collimator consists of a series of slits each aligned with one of the pairs of leaves in the standard MLC. Irradiation through both collimators produces a series of narrow strips each receiving a high dose. The length of each strip is determined by the MLC and the width by the slits. To build up a continuous dose distribution the collimators are indexed relative to the patient, by a distance equivalent to the slit width, and the irradiation repeated with a different MLC setting.

Preliminary results will be presented showing that high resolution is achieved without compromising penumbra. The uniformity of the beam depends on the precision of the indexing system, $\pm 5 \%$ has achieved with the experimental model that has been constructed.

This method of collimation is compatible with IMRT and the maximum field size for which high resolution shielding is limited only by the aperture of the MLC to which it is attached.

The device is extremely simple, requiring no moving parts other than the indexing system. The manufacturing cost will be comparable to that of an electron applicator making it affordable to most centres with an MLC.

A Patent has been applied for by Elekta Oncology Systems naming the author as inventor

\section{WE-C1-03}

Virtual Micro-IMRT

R Siochi*, Siemens Medical Systems, Concord, CA

Techniques for smoothing the edges of a field using a multi-leaf collimator (MLC) with a $1 \mathrm{~cm}$ leaf width involve delivering a sequence of fields that are shifted relative to each other. Unfortunately, this technique would be very difficult to implement in the context of intensity modulation. A new technique would allow the delivery of intensity modulated fields with a pencil beam size of $5 \mathrm{~mm} \times 5 \mathrm{~mm}$ using a $1 \mathrm{~cm}$ leaf width double focused MLC. Two $1 \mathrm{~cm} \times 5 \mathrm{~mm}$ intensity modulated fields, whose collimator settings are 90 degrees apart, are combined to produce these smaller pencil beams. This technique does not require the patient to be moved, but higher leaf positioning accuracy is important. Also, since 4 of these pencil beams fit into a $1 \mathrm{~cm} \times 1 \mathrm{~cm}$ pencil beam, the gradients between pairs of pencil beams are constrained to be equal in the leaf motion directions. The clinical use of this technique has yet to be assessed, since no inverse treatment planning system can plan for this technique. However the feasibility of the technique has been demonstrated using film dosimetry for a test case. Siemens Medical Systems supported this research.

\section{WE-C1-04}

Adaptation of an Optically-Guided Stereotactic Localization System for Clinical Use in Conformal Therapy and IMRT

M Phillips*, K Singer, E Miller, University of Washington, Seattle, WA, University of Washington, Seattle, WA, University of Washington, Seattle, WA

A number of emerging radiation therapy modalities, such as stereotactic radiotherapy, conformal therapy and IMRT, require better anatomical localization than has traditionally been achievable. A system for use in the treatment of brain and head \& neck tumors has been adapted from a commercially available stereocamera system designed for stereotactic radiotherapy. An optically guided localization system from Sofamor Danek (Memphis, TN) was installed in a vault equipped with an Elekta SL20 linac with multileaf collimator. A tertiary, circular collimator $(5$ to $50 \mathrm{~mm}$ diameter) could be attached to the gantry if desired. This system uses two infrared emitting sources and cameras to stereotactically locate a biteblockmounted fiducial array and to guide the therapist in positioning the patient so that the target center coincides with the radiation isocenter. With the aid of 
the manufacturer, this system was adapted for direct use with our in-house 3D treatment planning system (Prism) for planning of arbitrary tumor types and shapes. For stereotactic radiotherapy purposes, a manufacturer-supplied treatment planning system was also available. A set of procedures was devised in which no invasive headring was necessary, nor were any specific or proprietary reference systems required. Measurements were performed to determine the effects of target localization, patient positioning, gantry sag, and couch rotation on the accuracy and precision of the system. The overall accuracy was determined to be $2 \mathrm{~mm}$, and the precision $1 \mathrm{~mm}$.

\section{WE-C1-05}

Verification of Transabdominal Ultrasound ( BATR ) as an Effective Localization Modality in Prostate Cancer

S McNeeley*, J Lattanzi, A Hanlon, T Schultheiss, G Hanks, Fox Chase Cancer Center, Philadelphia, PA

Dose escalation in localized prostate carcinoma requires precise target localization to ensure adequate tumor coverage and maintain an acceptable level of complications by protecting surrounding normal tissues. In this study we compare a daily CT localization technique with a newly developed transabdominal ultrasound localization method.

As part of a final conedown treatment, prostate-only fields were created for 35 patients using no margin. For each final conedown treatment, a repeat CT simulation and isocenter comparison was performed.

Immediately following CT simulation, patients also underwent an ultrasound-based prostate localization. The ultrasound system was situated adjacent to the $\mathrm{CT}$ scanner facilitating direct comparison between the modalities. Transverse and sagittal suprapubic ultrasound images were then captured. Using contour and isocenter data from $\mathrm{CT}$, the ultrasound system overlaid the corresponding CT information relative to the isocenter. The CT contours were maneuvered to align with the ultrasound images and couch shifts required to produce field alignment were output.

The directed discrepancies in organ location between the modalities was small; A/P mean -0.09-mm SD 2.8-mm; Lat. mean -0.16-mm SD 2.4-mm; $\mathrm{S} / \mathrm{I}$ mean $-0.3-\mathrm{mm}$ SD $2.36-\mathrm{mm}$.

Ultrasound localization of the prostate, correcting for daily organ motion and set-up variations, has shown to be an efficient method of prostate localization. Therefore, daily localization with transabdominal ultrasound offers the potential to improve the therapeutic ratio in external beam therapy.

\section{WE-C1-06}

Intensity Modulated Radiation Therapy using Very High Energy Electron Beams

C DesRosiers*, L Papiez, Indiana University, Indianapolis, Indiana, Indiana University, Indianapolis, IN

IMRT may be defined as the ability to control beam fluence as a function of direction and position. IMRT with photons may be performed during beam generation by control of incident electrons on the target, or after beam generation by using shielding blocks, compensators or collimators. Some sophisticated methods or beam control post-generation are commercially available and under development (e.g. dynamic multileaf collimation, tomotherapy). Fluence of charged particle beams may be easily controlled electromagnetically.

The most commonly used electron energies $(6-25 \mathrm{MeV})$, have a clinical range of less than $6 \mathrm{~cm}$ with large penumbra, large angular spread and cannot be practically used for deeper tissues due to unacceptable normal tissue dose. There has been relatively recent development in higher energy electron therapy $(30-50 \mathrm{MeV})$ from a racetrack microtron with useful range of 8 $10 \mathrm{~cm}$. For electron energies $100-200 \mathrm{MeV} \mathrm{d}$ max occurs at $10-20 \mathrm{~cm}$ in tissue and the practical range extends beyond a typical patient body, thereby eliminating the large lateral spread and reducing normal tissue dose. The use of a pencil beam scanning device allows for extremely fast and efficient software fluence control as well as eliminates the need for collimators, blocks and beam modifying devices. The advantages of this proposed treatment can be evaluated through treatment simulations by Monte Carlo calculations. Preliminary studies also indicate that technology for building necessary devices for this therapy exists and that demands of radiation protection can be satisfied.

\section{WE-C1-07}

Development of a Comprehensive Compensator System

E Mok*1, C Ma, A Boyer ${ }^{2}$, (1)UCSF Stanford Health Care, Stanford, CA, (2)Stanford University, Stanford, CA

A compensator system for our clinic has been developed, which consists of a computer program being used as an interface between different patient data sources for input, and generates physical compensators or dynamic MultiLeaf (DMLC) leaf-sequencing files as end product. Tissue deficit at the surface of patient contours are measured by a Moire camera or calculated from CT scans. The compensator or DMLC files generated could be just for missing tissue compensation, or could include dose optimization. Thickness of the compensator generated by our commercial treatment planning computer could be imported for the purpose of calculating an intensity map. From the calculated intensity map, leaf-sequence file is generated, which could be used by the Dynamic MLC in place of the physical compensator. We have two Varian machines, one is equipped with a 40-pair MLC and the other is equipped with only a 26-pair MLC. With 26-pair MLC, the size of the compensated treatment field is limited to $26 \mathrm{~cm}$ wide at the center of the treatment field. Due to the finite width of each leaf $(1 \mathrm{~cm})$, the physical compensator is found to be superior in term of spatial resolution. This is important for treatment sites, such as head and neck, which have highly irregular shaped contours. The Pro and con between using physical compensator and dynamic MLC will be discussed.

\section{WE-C1-08}

Physical and Dosimetric Characteristics of a Non-focused Miniature Multileaf Collimator

K Farrey*, F Kuchnir, J Roeske, University of Chicago, Chicago, IL

The physical and dosimetric characteristics of a commercially available, axially non-focused miniature multileaf collimator (mMLC) are investigated. The mMLC is attached to a customized accessory mount of a Siemens MXE-2 linear accelerator and has a source to aperture distance of $67 \mathrm{~cm}$. The maximum field size is $10 \times 12 \mathrm{~cm}$ and the leaf width projected at isocenter is $4 \mathrm{~mm}$. Our investigation consisted of measuring TMRs, relative output factors, beam profiles, leaf leakage and leaf positioning precision. Measured TMRs increased with increasing field size at all depths. Output factors increased rapidly for small field sizes $(<4 \mathrm{~cm})$ and less rapidly for larger field sizes. The distance between the $80 \%$ and $20 \%$ dose levels of the beam profile measured at a depth of $5 \mathrm{~cm}$ was $3.5 \mathrm{~mm}$ which is comparable to that of the photon jaws and blocks. Interleaf leakage was observed to be on average $1.3 \%$ of the primary beam intensity with a maximum of $1.6 \%$. Leaf positioning precision was verified by examining the matchlines of abutting rectangular fields and was found to be better than $1 \mathrm{~mm}$ for all leaves. Our data indicate that the characteristics of this mMLC are consistent with previously reported studies on MLCs.

\section{WE-C1-09}

Chest Wall Irradiation with MLC-shaped Photon and Electron Fields M Essers*, D Binnekamp, M Eggen, C Creutzberg, C Griep, B Heijmen, University Hospital Rotterdam / Dr. Daniel den Hoed Cancer Center, Rotterdam, The Netherlands

In the DDHCC chest wall irradiation after mastectomy is performed with a medial AP electron field matched to a cranial/lateral AP photon field. On conventional treatment units the photon field consists of two segments, shaped with customized blocks, to generate a broadened penumbra. The electron field is produced with an insert in the standard electron applicator. The treatment couch is temporarily rotated in between delivery of the electron and photon fields for safe removal of the electron applicator.

An improved treatment technique was developed for the MM50 Racetrack Microtron. Due to the scanning beam principle and the helium in the treatment head of the MM50, the MLC can be used to shape both photon and electron fields for treatments with the applied source-to-surface distance of $100 \mathrm{~cm}$. All fields are set up and delivered under computer control. 
The use of three MLC-defined photon beam segments (instead of two) and of computer controlled (small) treatment table movements in between segments, resulted in improved dose homogeneity at all depths in both the cranial and the lateral junction areas. In-vivo dosimetry in the junction areas showed that systematic over- and underdosages could be reduced from more than $20 \%$ to less than $10 \%$. The day-to-day variations could be reduced from $22 \%(1 \mathrm{SD})$ to $10 \%$. Cerrobend blocks, inserts, and table rotation to remove the electron applicator are no longer needed. The treatment time is reduced from 10 to 4.5 minutes.

\section{WE-C1-10}

Improved Beam Edge Conformity Using an MLC SHift Technique T Steinberg*, Siemens Medical Systems / Oncology Care Systems, Concord, CA

With the use of a MLC with $1 \mathrm{~cm}$ leaf resolution the treatment port can be somewhat shaped conformally although not as conformal as a custom cerrobend block. If the leaf design were made so that the leaf width were much smaller (i.e. - $5 \mathrm{~mm}$ ) then the amount of conformity to the tumor contour would be much greater. This can be achieved by two methods. The first method would be to design a new MLC with much smaller leaves. This would add significant complexity to the MLC design. Additional motors, drive trains, electronics, and control systems would be needed to support these added number of leaves. In addition, the amount of leakage would need to be considered because of the increased number of interleaf spaces. The second method for a higher resolution would be using the existing hardware and to divide the treatment port dose into segments as a fractional amount of the leaf width. In between each segment the field, with respect to the beam, would be translated, and the leaf positions would be adjusted to maintain the tumor volume. This is the approach that is being proposed by Siemens for High Definition Intensity (HDI).

This work was partially supported by Siemens Medical Systems / Oncology Care Systems

\section{Scientific Session Treatment Planning Optimization \\ Room: Mississippi}

\section{WE-C2-01}

Dose Wall Histograms for the Rectum

G Meijer*, M Hoogeman, J Lebesque, The Netherlands Cancer Institute, Amsterdam, The Netherlands

A mathematical model for the rectum wall is developed, incorporating its stretching due to variable rectum filling and neighboring structures. The model is based on the assumption that the amount of cross-sected rectum wall tissue perpendicular to the central axis of the rectum is constant throughout the entire rectum. The model is used to create a set of inner contours out of the outer contours of the rectum. Both sets of contours are used to derive a dose-wall histogram (DWH). The model is verified using 20 sets of CT data (5 patients $\mathrm{x} 4$ scans). The DWHs are compared with DVHs of the rectum wall, which require contouring of the outer and inner surfaces of the rectum wall and with DVHs of the total rectum. Results:(A) The local wall thickness of the rectum outlined on CT data is in conformity with the described rectum model. (B) The amount of rectum wall tissue per unit length rectum varied considerably between patients $(27 \%$, 1SD). (C) The DWHs correspond well to the DVHs of the rectum wall. (D) Large discrepancies are observed between the DVHs of the total rectum and the DVHs of the rectum wall. The model yields accurate descriptions of the dose distribution of the rectum wall, without delineating the inner surface of the rectum. This reduces both the work load and variations due to inaccurate delineation of the rectum wall. The DWH is an effective tool to estimate the complication probability of the rectum.

\section{WE-C2-02}

Radiation Treatment Uncertainties: Issues and Controversies

J Van Dyk*, T Craig, E Wong, V Moiseenko, J Kempe, J Battista, London Regional Cancer Centre, University of Western Ontario, London, ON, Canada

The radiation treatment process consists of multiple steps, with each step containing inherent uncertainties and assumptions. Our research program involves the evaluation of these uncertainties and the development of models to predict the corresponding clinical outcome using this information. This analysis has highlighted the following issues. 1. Target volume definition: (a) Physicians have a large variability in target volume definition. (b) ICRU definitions of target volume are ambiguous. 2. Dose-volume histograms (DVH): (a) Uncertainties in dose, especially if no inhomogeneity corrections are made. (b) Substantial uncertainty in volume determination dependent on the calculation used in treatment planning systems. (c) DVH reduction schemes make assumptions about biological response, which are invalid for some organs. 3. Optimization concerns: Different treatment techniques will be chosen on the basis of the optimization routine, dependent on whether or not uncertainties are included. 4. Biological modeling: The calculation of TCP and NTCP makes major assumptions about (a) availability of reasonable response data, and (b) accuracy of models used to predict TCP and NTCP for different dose-volume-fractionation schemes. In this overview, one example will be shown of each issue/controversy and specific conclusions will be highlighted.

\section{WE-C2-03}

Registration of Serial CT Images in the Presence of Soft-tissue Deformation and Displacement: Application to Dose Planning for Locally-advanced Carcinoma of the Cervix

J Williamson*, J Dempsey, H Bennet, C Chao, P Grigsby, G Christensen, P Yin, M Vannier, University of Iowa, Iowa City, IA

Integration of three dimensional (3D) imaging into intracavitary brachytherapy treatment planning has been hindered by large, localized deformations and displacements of the pelvic soft tissue structures caused by applicator insertion and tumor regression. We have adapted a novel and fundamental image registration approach, the method of deformable anatomical templates (MDAT; IEEE Trans.Imag.Proc. 5: 1435, 1996), for registration of serial 3D CT pelvic studies obtained at fixed points during definitive radiation therapy for cervix cancer patients. For example, to register pre- and post-applicator insertion CT studies, MDAT identifies the high-dimensional ( $10^{7}$ unknowns), voxel-to-voxel transformation that best preserves coincidence of the contoured anatomical structures (bladder, rectum, sigmoid, vagina, uterus, bony anatomy) and uncontoured gray-scale anatomy on the two studies. This warping process is guided by the laws of continuum mechanics: each anatomical structure is modeled as a deformable elastic solid or viscous fluid. Experience with our first few study patients is encouraging: MDAT is able to model dramatic changes in shape, position, orientation and size of the soft-tissue anatomy within the bony pelvis while retaining excellent alignment of the bony anatomy and peripheral gray-scale anatomy. By transforming CT studies obtained at different times in the patient's treatment course to a common reference frame, 3D dose distributions describing different intracavitary and external beam treatment components can be meaningfully summed.

Supported in part by NIH grant RO1 CA 75371

\section{WE-C2-04}

A New Method of Dosimetrically Setting Multileaf Collimators for Shaping a Radiation Field

L Ma*, C Yu, M Sarfaraz, Department of Radiation Oncology, University of Maryland Medical Center, Baltimore, MD

A new method is developed for dosimetrically setting Multileaf Collimators (MLC) to shape a radiation field. The method eliminates the MLC scalloping effects and could be used for any conventional three-dimensional treatment planning system freeing the vendor and the user from developing a detailed dose calculation model for a specific MLC system. For the current method, the maximum isodose line (MIL) covering the entire planning target volume (PTV) was first determined from a physician prescribed smooth cerrobend block contour in the beam's eye view (BEV). An iterative 
optimization method is developed to position the MLC leaves to best match the MLC field with the MIL contour at the same isodose level. Comparison was carried out between the current dosimetric leaf-setting method and conventional leaf-setting methods using geometric criteria. Phantom measurements were performed to validate the results of the comparison. Significant differences up to $0.5 \mathrm{~cm}$ in MLC leaf positions and isodose coverage were found between the current method and the conventional methods. Therefore, we have demonstrated that it is important to set the MLC leaves dosimetrically rather than geometrically to achieve optimum dose conformation for a treatment field. This becomes essential in using the MLC-shaped fields for conformal radiotherapy where dose conformity plays an important role in improving the therapeutic index.

\section{WE-C2-05}

Retrospective Examination of the Efficacy of the RTOG PTV Definition for Prostate Treatment

D Lockman*, D Yan, D Brabbins, L Tyburski, A Martinez, William Beaumont Hospital, Royal Oak, MI

The RTOG protocol for 3D conformal radiation therapy of prostate cancer provides guidelines for margin selection in forming a planning target volume (PTV) to compensate for expected patient setup error and internal organ motion. Specifically, treatment margins from 0.5 to $1 \mathrm{~cm}$ are recommended. The concern of rectal toxicity in late-phase dose escalation has commonly led clinicians to select the minimal margin allowed in the protocol, without clear knowledge of the resulting dose loss suffered by the clinical target volume (CTV). The goal of this study was to analyze the relationship between margin selection and dose loss, so as to provide clinicians with more complete information in their application of the RTOG PTV definition. Measurements of internal organ motion and patient setup error, obtained from multiple daily CT datasets and portal images of 35 prostate patients at our clinic, were obtained. These daily measurements were used to mimic the actual treatment of the individuals assuming the RTOG PTV definition was applied. The maximum dose reduction in the CTV was then evaluated for a range of treatment margins. The study was first performed assuming conventional conformal delivery, then repeated assuming intensity modulated beam delivery.

Our study indicates that tremendous dose reduction occurs when the minimal margin is applied. Furthermore, even the maximum RTOG margin is inadequate for the IMRT treatment technique, due to its steep dose profile outside the target boundary.

Supported in part by NCI-CA71785

\section{WE-C2-06}

Treatment Planning with Functional MRI Information: A Feasibility Study

W Liu*, M Schulder, A Kalnin, V Narra, A Holodny, C Cathcart, K Mosier, A Gary, G Lange, University of Medicine and Dentistry of New Jersey, Newark, NJ

The purpose of this research is to assess the feasibility of reducing the dose at the eloquent cortices by fMRI aided treatment planning. Eleven brain tumor or AVM patients for stereotactic radiosurgery were studied using BOLD fMRI. Functional MRI paradigms were used to identify the eloquent cortex adjacent to the tumor. Based on the pathology location, several different tasks such as finger-tapping, visual, language, and brainstem paradigms were designed to provide the cortical identification. All paradigms used 3 cycles of $30 \mathrm{sec} \mathrm{ON}$ and $30 \mathrm{sec} \mathrm{OFF}$ with $30 \mathrm{sec}$ baseline. The data was acquired with $\mathrm{TR} / \mathrm{TE}=4000 / 60, \mathrm{FOV}=30 \mathrm{~cm}$, matrix size $=$ $64 \times 64$, slice thickness $=5 \mathrm{~mm}, 28$ slices, flip angle $=90$ on a GE 1.5 Tesla Echospeed Horizon scanner.

The raw data was reconstructed off-line and post-processed for the activation map using cross-correlation method written in IDL. The activation maps were downloaded to a treatment planning workstation, Xknife (Radionics). 3D activation maps were generated and co-registered to a 3D CT anatomical data.

Functional brain maps and structural images were displayed and used for treatment planning. The average maximum dose at the tumor was calculated to be 24.6 Gy (range $15.8-40 \mathrm{~Gy}$ ) with an average 16.5 Gy dose delivered to the immediate surrounding tissue. The eloquent cortices received an average dose of $4.1 \mathrm{~Gy}$ (from $0-8 \mathrm{~Gy}$ ). It is suggested that fMRI aided treatment planning can reliably provide dose reduction in the eloquent cortices.

\section{WE-C2-07}

Effect of Noise on Monte Carlo-based Inverse Treatment Planning R Jeraj*1, P Keall², (1)Jozef Stefan Institute, Slovenia, (2)Medical College of Virginia, Richmond, VA

When using Monte Carlo, the calculated dose distributions are always burdened with statistical noise. The noise introduces two types of error. One is the well-known statistical error because of the finite number of simulated particle histories. In addition the convergence error occurs because the optimisation algorithm converges to the optimum for the noisy data, which is different from the optimum for the noise-free data.

A 2D Monte Carlo-based inverse treatment planning algorithm MCI was used for this study. This algorithm combines Monte Carlo dose calculation with simulated annealing optimisation to obtain the optimal solution for a given objective function. The narrow beam dose distributions (corresponding to narrow beams from multileaf collimators) were calculated to different precision for this purpose.

The narrow beam dose distributions (corresponding to narrow beams from multileaf collimators) were calculated to different precision for this purpose.

For the statistical error it was established, that the absolute error was larger in the target volume as outside, because of the higher total dose delivered to the tumour. Initially acceptable dose volume histograms revealed significant deviations when the almost noise-free (less than $0.1 \%$ error) dose calculation was used. For the convergence error, the absolute error was larger outside the tumour volume, because of the large variations in the calculated intensity distribution of the narrow beams. Dose outside the target volume, delivered from a small number of narrow beams, is affected more than dose inside the tumour volume, which is delivered from a large number of narrow beams. Levels of acceptability of the plans for both types of errors were determined.

\section{WE-C2-08}

Mixed Integer Programming Models for Prostate Brachytherapy Treatment Optimization

W D'Souza*, R Meyer, M Ferris, B Thomadsen, University of Wisconsin, Madison, WI

Classical interstitial brachytherapy optimization is generally a manual iterative process where improved treatment plans can be obtained by changing the configuration of radioactive sources within the target volume. Mathematically, the goal of interstitial treatment planning to find the optimal spatial distribution of seeds within the target volume that will result in the best tumor control with minimal damage to normal tissues. Previous optimization methods based on sampling techniques have been developed. The purpose of this work is to describe mixed integer programming (MIP) approaches using the General Algebraic Modeling language and the CPLEX solver. We considered three objective functions: minimizing the (mean) dose to the urethra, minimizing total underdose to the target and maximizing homogeneity within the target respectively. Optimization models using multiple source strengths were also investigated.

Two important criteria for evaluating this MIP approach are the quality of the solution obtained and the solution time. It was found that minimizing the (mean) dose to the urethra results in longer solution times but results in more a favorable dose distribution in the urethra. However, solution times of only a few minutes are needed in all cases. A composite objective function provided treatment plans of good quality but required somewhat higher solution times. Multiple source strengths resulted in solutions that were marginally better than treatment plans involving a single source strength. 


\section{WE-C2-09}

A generalized concept of Equivalent Uniform Dose (EUD)

A Niemierko*, Massachusetts General Hospital, Boston, MA

Dose distributions are inherently non-uniform, especially for normal structures. Although IMRT is capable of delivering superior dose distributions tailored to the geometry of irradiated structures; it often produces inhomogeneous target dose distributions. To quantitatively evaluate a treatment plan one needs to know the consequences of dose inhomogeneity for all structures of interest. A concept of Equivalent Uniform Dose (EUD) for tumors, based on models of clonogen survival, has recently been developed and investigated in several clinical settings. Here we report on generalization of the EUD concept that applies to both tumors and normal tissues. Based on the analysis of outcomes for several clinical studies providing volumetric information for tumors and normal organs we propose that EUD for a structure of interest be estimated as:

$E U D=\left|\frac{1}{N}{ }_{i=1}^{N} D_{i}^{a}\right|^{\frac{1}{a}}$ or, using a differential doserolume histogranE $U D=\left(\left.{ }_{i=1}^{N} v_{i} D_{i}^{a}\right|^{\frac{1}{a}}\right.$,

where $\left\{\mathrm{v}_{\mathrm{i}}, \mathrm{D}_{\mathrm{i}}\right\}$ are bins of the histogram and "a" is a tissue-specific parameter. It is easy to see that EUD is bounded by the minimum and by the maximum dose, and is equal to the mean dose for "a" equal to one. The parameter "a" is negative for all tumors and it is positive for all normal structures. We estimated the maximum likelihood values of the parameter "a" for several tumors and normal structures and they range from -13.1 for local control of chordoma tumors to 17.7 for perforation of esophagus. We will discuss the rationale for this generalized EUD concept and we will discuss the estimates of the parameter "a" for several important structures and end-points.

\section{WE-C2-10}

A Unified Model of Tissue Response to Radiation

A Niemierko*, Massachusetts General Hospital, Boston, MA

We propose a generalized unified model of tissue response to radiation based on two fundamental assumptions. 1) A structure of interest (tumor or normal tissue) exhibits a specific end-point (local control for tumors or complication for normal tissues) if tolerance of the structure for this endpoint is exceeded. 2) The Equivalent Uniform Dose (EUD) is an index associated with tolerance. If tolerance is log-normally distributed in a population than the probability of a specific end-point for a given case characterized by EUD is $p=\Phi\left|\frac{\ln E U D-\ln D_{50}}{\sigma}\right|$ where $\Phi$ is the standard normal distribution function, $\sigma$ is the standard deviation of logtolerance in a population, and $D_{50}$ is the median "tolerance dose". The formula can be rewritten using slope $\gamma_{50}$ :

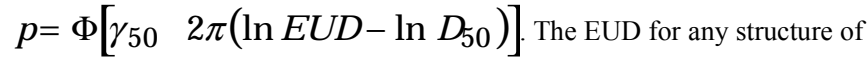
interest (tumor or normal tissue) is estimated as $E U D=\left|{ }_{i=1}^{N} v_{i} D_{i}{ }^{a}\right|^{\frac{1}{a}}$, where $\left\{\mathrm{v}_{\mathrm{i}}, \mathrm{D}_{\mathrm{i}}\right\}$ are bins of a histogram and "a" is a tissue-specific parameter. The parameter "a" is negative for tumors and it is positive for normal structures. The model has only three parameters (a, $\left.\mathrm{D}_{50}, \gamma_{50}\right)$. We fitted the model to several tumor and normal tissue data sets. The fit was better or at least as good as fits of other more complicated models proposed in the literature. We attribute this to the fact that the model captures the fundamental dose-volume-response relationship in the most flexible form. We will discuss the rationale of the model and we will present the results of fitting the model to tumor and normal tissue data.

\section{Scientific Session Computer-Aided Diagnosis (Session Dedicated to Joseph Windham)}

Room: Carroll

\section{WE-C4-01}

Computational Model For "Native Tissue Harmonic Imaging"

J Zagzebski*, Y Li, University of Wisconsin, Madison, WI

"Tissue Harmonic Imaging" is a new echo signal processing and display modality on ultrasound scanners. It detects 2 nd harmonic echo signals that result from nonlinear distortion of ultrasound waves propagating through tissue. We are implementing a computational model to simulate finite amplitude pulse transmission and harmonic echo signal production from pulse-echo transducers. The goal is to more fully understand the information content of tissue harmonic images and the dependence of echo intensity on transmit beam forming parameters as well as on the acoustical properties of the insonified region.

A frequency-domain numerical method described by Aanonsen was applied to

solve the nonlinear model KZK equation for pulsed ultrasonic transmission by a circular disk transducer. This combines effects of diffraction, nonlinear propagation and attenuation. Linear theory is used during echo reception since the amplitudes of backscattered waves are weak compared to transmitted waves. Echoes from both discrete reflectors and randomly positioned scatterers are integrated over the surface of the transducer to obtain the fundamental and the harmonic signals. Pulse-echo responses at different field positions exhibit the details of harmonic build-up and dissipation with depth shown by previous authors. Harmonic images obtained for $2 \mathrm{MHz}$ transmission and $4 \mathrm{MHz}$ reception exhibit improved visualization of spherical voids over images obtained with fundamental 2 $\mathrm{MHz}$ beams, also similar to results with clinical imagers. Migrating the model to array transducers requires large increases in computational capabilities, and is being explored.

\section{WE-C4-02}

Computerized analysis of benign and malignant lesions on breast ultrasound

K Horsch ${ }^{1}$ *, M Giger ${ }^{1}$, L Venta ${ }^{2}$, Z Huo ${ }^{1}$, C Vyborny ${ }^{1}$, M Chinander ${ }^{1}, \mathrm{~K}$ Horsch, (1) University of Chicago, Chicago, IL, (2) Northwestern University, Chicago, IL

Ultrasound is currently used to diagnosis simple benign breast cysts with a reported accuracy of 96 to $100 \%$. However, because of substantial overlap in the sonographic characteristics of benign and malignant solid lesions, it has been argued that ultrasound should not be routinely used to determine whether a particular solid lesion is benign or malignant. Our objective is to explore the diagnosis capability of breast sonography through quantitative, computer extracted features. Our preliminary studies with digitized ultrasound film yield promising results. We are now retrospectively collecting another database of 300 ultrasound cases from an electronic archive. All of these cases have either gone to biopsy or aspiration. To date, we have analyzed 235 images from 88 patients with 115 lesions, of which 95 lesions were benign and 20 were malignant. Of the 235 images, 170 are of benign lesions, and 65 are of malignant lesions.

In the analysis, each lesion is manually extracted by a radiologist. Then, features related to lesion margin, shape, echogenicity (texture) and posterior acoustic attenuation are automatically extracted. Linear discriminant analysis is used to merge the features into an estimate of the likelihood of malignancy. Consistency and round-robin analyses yield Az values of .80 and .77 , respectively. Inclusion of all 300 cases is expected to improve the training of the LDA in the task of distinguishing benign from malignant lesions.

Research supported in parts by USPHS Grants RR11459 and T32 CA 09649, and U.S. Army grant 972445. M. Giger is a shareholder in R2 Technolgy, Inc 


\section{WE-C4-03}

Multiscale Texture Analysis for Determination of Likelihood of Malignancy on Focal Liver Lesions in Sonograms

H Yoshida*, D Casalino, B Keserci, K Doi, The University of Chicago, Chicago, IL

Accurate sonographic characterization of focal liver lesions can reduce the number of unnecessary, expensive imaging (CT, MRI, and scintigraphy) or avoid a biopsy. The purpose of this study is to distinguish between benign and malignant lesions in ultrasound images based on multiscale texture analysis using wavelet packets. In this method, ROIs extracted from within the lesions are decomposed into subimages by wavelet packets. Multiscale texture features are calculated from these subimages based upon a singlescale feature defined on the original ROIs. An artificial neural network (ANN) is used for combining these multiscale features for classification of lesions, and its performance is measured by the area under the ROC curve (Az). A subset of the multiscale features that yields the highest performance is selected in a step-wise manner as the wavelet packet decomposition is performed. Three single-scale features, i.e., entropy, root mean square, and first moment of the power spectrum, are used to generate the multiscale texture features. In an analysis of 193 ROIs consisting of 50 hemangiomas (benign lesions), 69 hepatocellular carcinomas, and 74 metastases (both malignant lesions), the multiscale features yielded a high $A_{z}$ value of 0.92 in distinguishing benign from malignant lesions, whereas the single-scale features yielded only 0.70 . Our multiscale texture analysis method provides accurate differentiation between malignant and benign lesions, and thus can increase the accuracy of diagnosis of focal liver lesions in sonography.

FINANCIAL DISCLOSURE: K. Doi is a shareholder of R2 technology, Inc., Los Altos, CA.

\section{WE-C4-04}

Determination of Cerebral Tumor Infiltration Using a Multispectral Segmentation Method

D Peck*, T Mikkelsen, L Scarpace, R Van Hulle, Henry Ford Health System, Detroit, MI

Ideally, determining tumor infiltration using MRI will allow treatment planning and follow-up to be more accurate. An image analysis method has been developed (Eigenimage Filter) which segments tumor infiltration from normal tissue. The segmentation maintains partial volume information and therefore allows areas with little contrast to be segmented. We applied this method to cerebral tumor patients to determine infiltration into normal tissue and validated based on image directed biopsy.

Twenty tumor patients had MRI prior to surgical resection. MRI consisted of 2-T2 FSE, 1-flair, and 2-T1 SE (pre- and post-Gd). Following MRI regions of interest (ROI) were created within lesion and normal tissue. The average signal under these ROI is used in the Eigenimage Filter. The resulting segmented images are combined with post-Gd SE for display in surgery. In surgery biopsy sites are chosen from the images using an ISG/Elekta Viewing Wand. The number of biopsy samples taken was 2-5 tumor and 0-4 normal samples/patient. A Receiver Operating Characteristics (ROC) test was done to validate the segmentation. Biopsy samples were scored on a scale of 1-10 for presence of tumor with a score of 1 being $100 \%$ confidence.

The sensitivity was $91 \%$, specificity was $44 \%$, and accuracy $71 \%$. The area under the ROC curve was 0.848 . These results demonstrate that the segmentation is accurate and sensitive at determining lesion infiltration.

\section{WE-C4-05}

Diagnostic Interpretation of Perfusion Lung Scans Using Multifractal Texture Analysis

G.D. Tourassi*, E.D. Frederick, C.E. Floyd, Jr., R.E. Coleman, Duke University Medical Center, Durham, NC,

We investigated the feasibility of using multifractal texture analysis for detection and characterization of lung diseases from perfusion lung scans. Forty-five perfusion scans were acquired from patients with clinical suspicion of acute pulmonary embolism (PE). They were all part of complete ventilation-perfusion (V-P) lung studies which were considered indeterminate for $\mathrm{PE}$ and therefore the patients were referred to pulmonary angiography for final diagnosis. An experienced nuclear medicine physician extracted $24 \times 24$ pixel regions of interest (ROIs) from the posterior view of the perfusion scans. There were 270 ROIs in total: 94 normally perfused ROIs and 176 abnormal ROIs representing lung diseases including PE, obstructive lung disease, atelectasis, pleural effusion, and opacity. The true diagnosis was established based on the patients' complete radiographic studies and pulmonary angiograms. Multifractal analysis was then performed on each ROI using the circularly averaged power spectrum technique. Subsequently, a feed-forward artificial neural network (ANN) was developed based on multifractal parameters to predict the presence and potentially type of disease present in each ROI. The diagnostic performance of the ANN was evaluated using Receiver Operating Characteristics (ROC) analysis and the leave-one out sampling method. Based on four multifractal parameters, the ANN achieved a statistically significantly better ROC area index $(A z=0.92 \pm 0.02)$ than the single fractal dimension $(A z=0.87 \pm 0.02)$ in predicting the presence of lung disease. The ANN also showed promise in identifying specifically the presence of PE in ROIs without additional information from ventilation scans and chest radiographs.

\section{WE-C4-06}

Automated Segmentation of the Pulmonary Vascular Tree in Spiral CT Angiography for Computerized Detection of Pulmonary Embolism

Y Masutani*, K Hoffmann, H MacMahon, K Doi, Kurt Rossmann Laboratories, Department of Radiology, The University of Chicago, Chicago

Spiral CT-Angiography (CTA) is increasingly used for the diagnosis of pulmonary embolism. However, radiologists must view more than 50 images in order to detect thrombi in the arteries and distinguish them from artifacts caused by partial volume, heart motion, etc. In this study, we present a new method for automated segmentation of the pulmonary vascular tree in spiral CTA data sets for $3 \mathrm{D}$ visualization and computerized detection of pulmonary embolism. Our segmentation method is based on the combination of several 3D operations. First, the lung volume is segmented by thresholding and math-morphological operations. Next, large vascular structures such as lobar branches are extracted using a hysteresis thresholding technique and connectivity analysis. Finally, region-growing is performed in the segmented lung volume using extracted major vascular structures as a seed. Three-dimensional visualization is using the marchingcube algorithm. Automated segmentation was successfully performed on several clinical cases by adjusting a few parameters. In comparison with simple thresholding methods, our technique significantly improved the visualization quality of the pulmonary vessels as a result of the automated removal of bone structures and extraneous soft tissues. Segmented volumes are about 3-4 percent of the total volume data. Therefore, our segmentation method makes the subsequent analysis for detection of pulmonary embolism extremely efficient obviating the need for searching the entire data. Method for detection of the pulmonary embolism using the segmented volume are currently being developed.

\section{WE-C4-07}

Three-dimensional Localization of Narrow Point Distributions Using a Hybrid Optimization Technique

J Esthappan*, M Kupinski, K Hoffmann, University of Chicago, Chicago, IL

The spatial correlation of three-dimensional (3D) objects with twodimensional (2D) images of those objects is important for medical applications such as catheter navigation and patient positioning. Previously, we presented a technique that determines accurately the $3 \mathrm{D}$ orientation and position of a distribution of points using a single projection image, given the $3 \mathrm{D}$ distances between points. However, as the point distribution narrows to approximately one millimeter, the SPT becomes increasingly sensitive to local solutions, thereby, impairing its accuracy.

To improve the reliability and accuracy of the SPT for application to narrow point distributions, a genetic algorithm (GA) was used to search for a global estimate of the $3 \mathrm{D}$ orientation and position of the points based on 2D reprojection error. Subsequently, the SPT iteratively aligns the reprojected points with their corresponding image points to refine the initial estimate provided by the GA. 
In simulation studies, this hybrid optimization technique (GA-SPT) was applied to points distributed along $5 \mathrm{fr}$ catheters placed at 100 random orientations. Preliminary results indicate that, on the average, the GA-SPT computed orientations that were 38 times more accurate, and positions that were 5 times more accurate, than those computed by the SPT alone. Similarly, the reliability of the GA-SPT exceeded that of the SPT alone.

These results indicate that accurate and reliable orientations and positions can be obtained for objects as small as a catheter.

\section{WE-C4-08}

Merging of Computer-extracted Radiographic Features with Clinical Data in the Prediction of Bone Strength

M Chinander*, M Giger, C Jiang, J Martell, University of Chicago, Chicago, IL

The purpose of this study is to develop models for predicting bone strength from computer-extract radiographic texture features, BMD measures, and patient clinical data.

A direct exposure technique was used to radiograph femoral neck specimens using a high resolution film. Films were digitized with $0.121-\mathrm{mm}$ resolution and 10-bit quantization. Bone mineral densitometry (BMD) measurements were performed on the specimens using a Lunar DPX scanner. Specimens underwent compressive strength testing to determine their actual strength. Texture analysis was performed on regions of interest (ROI) selected from the medial portion of the femoral neck. Texture analysis methods included Fourier-based methods in which the RMS variation and first moment of the power spectrum (FMP) were calculated. Texture features, BMD measurements, and patient clinical information were combined using linear discriminant analysis. ROC analysis was used to evaluate the ability of the individual features and the output from the linear discriminant analysis to discriminate between strong and weak bone.

Linear discriminant analysis with three features (BMD, FMP, and age) achieved an area under the ROC curve (Az) of 0.83 while BMD achieved an Az of 0.75 in the task of distinguishing between strong and weak bone Texture analysis of radiographic bone patterns, combined with BMD and patient information using linear discriminant analysis may aid in predicting bone strength.

\section{M.L. Giger is a shareholder in R2 Technology, Inc. (Los Altos, CA).}

\section{WE-C4-09}

Information Theoretic Criteria for Bayesian Image Segmentation

L Hibbard*, Computerized Medical Systems, Inc., St. Louis, MO

The need for an accurate model of patient anatomy for 3-D RTP motivates the development of automated contouring methods. Our approach, based on the integrated maximum a posteriori (MAP) analysis of region texture, flexible modeling of gradient-edge coincidences, and prior shape (Chakraborty, et al., IEEE TMI, 15:859, 1996), adapted for 3-D RTP (Hibbard, AAPM Abstract WE-D4-10, 1998), assumes stationary Gaussian distributions for voxel textures and contour shape parameters. Voxel and shape data may both deviate from the Gaussian model, so robust MAP inference of organ and tumor boundaries without relying on explicit statistical models would be advantageous. Cover and Thomas (1991) demonstrated that MAP inference via the likelihood ratio test has an equivalent expression in terms of relative entropies of the observed data distributions. This makes possible hypothesis testing on observed data without dependence on any underlying statistical model. An optimal contour that best matches a true object boundary is one for which voxel intensity distributions inside and outside the contour correspond to a maximum in their relative entropy; a single object interior is certainly likely to have a different distribution than the object exterior (the rest of the patient) to which it is being compared. Likewise, shape parameter distributions are most like prior distributions when their relative entropies are minimum. The use of these entropy measures in an automated contouring tool will be demonstrated for defined-content, synthetic images and for actual CT images.

\section{Continuing Education Course Mammography -1}

Room: Taylor

\section{WE-C5-01}

The New NCRP Report on Mammography (NCRP 85 Rewrite)

Lawrence N. Rothenberg, Memorial Sloan-Kettering Cancer Center, New York, NT

Scientific Committee 72 (SC-72) of the National Council on Radiation Protection and Measurements (NCRP) prepared a report entitled "Mammography -- A User's Guide" which was issued in 1986 as NCRP Report No. 85. Since then there have been numerous significant developments related to breast imaging: improvements in $x$-ray imaging equipment, in screen-film and other image receptors, in education and training of radiologists, technologists, and medical physicists with regard to the acquisition, display, and interpretation of breast images, and in the specification of quality assurance programs recommended by accrediting bodies such as the American College of Radiology and required by the federal government through the Mammography Quality Standards Act. Furthermore, new data and methods to evaluate the data for radiation dose and radiation risk to the breast, and of the benefits from mammography breast screening programs have also been published. NCRP SC-72 is preparing a new document on breast imaging to incorporate this new information and to provide recommendations for those involved in mammography. An overview of the current draft version of the SC-72 report will be presented. There will be brief reviews of sections on proper clinical imaging techniques, optimum choice of equipment, factors which affect image quality, radiation dose evaluation, quality assurance programs, and non-mammographic breast imaging techniques. The presentation will conclude with a detailed discussion of benefits and risks of mammography and a review of the Committee's draft recommendations.

Educational Objectives:

1. Describe the members and activities of NCRP Scientific Committee 72.

2. Review the various sections of the current draft of the NCRP SC-72 report on mammography and breast imaging.

3. Provide detailed information on the benefits and risks of mammography.

4. Discuss the draft recommendations of NCRP SC-72 with regard to mammography.

\section{Continuing Education Course Room: Taylor Mammography - 2}

\section{WE-C5-02}

The New MQSA Rules \& New ACR Recommendations

Priscilla Butler, American College of Radiology, Reston, VA

The Mammography Quality Standards Act (MQSA) has changed the way we do and think about mammography in the United States. The Government Accounting Office has published several analyses documenting improvements in mammography throughout the US as a result of the implementation of the Food and Drug Administration's (FDA) Interim Rules for Mammography. The FDA clarified and expanded many of their regulations in their Final Rules for Mammography that went into effect on April 28, 1999. The quality assurance section of the rules specify the minimum level of tests that must be conducted and their performance to ensure good clinical image quality. In order to help facilities prepare and comply with the new rules, the American College of Radiology's Committee on Quality Assurance in Mammography revised their Mammography Quality Control Manual in March of 1999. The revised manual also includes testing and recommended performance criteria that go beyond the MQSA minimums to encourage further improvements in image quality. This presentation will outline the most significant changes in the new rules compared with the old and discuss those that have the greatest impact on medical physicists and they way they practice. The use of the 1999 Quality Control Manual in complying with these rules will be emphasized.

*Supported by Computerized Medical Systems, Inc. 
Educational Objectives:

1. To learn about MQSA Final Rules

2. To learn about the revised 1999 ACR Mammography Quality Control Manual

\section{Continuing Education Course Room: Browning A Ultrasound - 4}

\section{WE-C6-01}

Hands On Ultrasound Quality Control Workshop

Carolyn Kimme-Smith ${ }^{1}$, Mitchell Goodsitt ${ }^{2}$, Evan Boote ${ }^{3}$, Mark Holland ${ }^{4}$, James Zagzebski ${ }^{5}$, Heather Miller ${ }^{6}$, Jim Kofler ${ }^{7}$, (1) UCLA, Los Angeles, CA (2) University of Michigan, Ann Arbor, MI (3) University Missouri Columbia, Columbia, MO (4) Washington University, St. Louis, MO (5) University Wisconsin, Madison, WI (6) Computerized Imaging Reference Systems, Inc., Norfolk, VA (7) Mayo Clinic, Rochester, MN

As in previous years, this workshop will provide attendees with an opportunity to refresh their skills in US quality control procedures for realtime gray mode imaging. This will include depth of visualization, uniformity, vertical and horizontal distance accuracy, lateral and axial resolution, ring down, and slice thickness focal range. In addition, a US prostate treatment planning system and prostate phantom will be available. Medical Physicists who have a theoretical knowledge of Ultrasound procedures will be guided by experienced instructors to scan a variety of QC phantoms. In order to provide sufficient equipment for attendees, small portable ultrasound units will be provided. No Doppler or color flow imaging units will be available. Attendees should be familiar with the AAPM Ultrasound Task Group 1 Report; "Real-time B-mode ultrasound quality control test procedures" in Medical Physics 1998; 25: 1385-1406. Copies of appendix A from that report will be available at the workshop. The QC tests will take about an hour to complete, and participants can attend anytime during the scheduled workshop.

EducationalObjectives:

4. To learn US QC test procedures for Real-time B-mode units.

5. To become acquainted with new US QC phantoms.

6. To learn to calibrate a prostate US treatment planning unit.

\section{Scientific Session Radiotherapy Measurements \\ Room: Pearl}

\section{WE-D1-01}

Beam Quality Specification and Absorbed Dose Beam Quality Correction Factors kQ for High Energy Photons

J Seuntjens*, C Ross, K Shortt, Ionizing Radiation Standards, NRC, Canada

Selection of the parameter to specify radiation quality of clinical photon beams plays an essential role in the final accuracy of protocols based on absorbed dose calibrations such as the AAPM-TG51 or IAEA codes of practice. We compared the adequacy of two beam quality specifiers i.e. TPR and $\% \mathrm{dd}(10)_{\mathrm{x}}$ in their ability to specify experimental $\mathrm{k}_{\mathrm{Q}}$ factors for cylindrical chambers in high energy photon beams of different types. We base our assessment on our water calorimeter based $\mathrm{k}_{\mathrm{Q}}$ data as well as high quality experimental $\mathrm{k}_{\mathrm{Q}}$ factors and beam quality data available from other laboratories. For the NE2571 chambers investigated, there is a systematic improvement in the specification of the beam when using \%dd(10) , i.e. the maximum range of differences between various measured values of $k_{Q}$ amount to $0.4 \%$ over $58<\% \mathrm{dd}(10)_{\mathrm{x}}<88$, whereas differences are up to $1.2 \%$ when using TPR. Similarly over the same range, for the NE2611 or NE2561 chamber, differences are up to $0.4 \%$ when using $\%$ dd $(10)_{\mathrm{x}}$ and up to $0.7 \%$ when using TPR. For the PR06C chamber, the improvement is less obvious but less experimental $\mathrm{k}_{\mathrm{Q}}$ data are available. In addition, the agreement between measured and calculated $\mathrm{k}_{\mathrm{Q}}$ factors is slightly better when beam quality specification is with \%dd(10) maximum difference reduces from $1.2 \%$ to $0.8 \%$, for the NE2611 from $1.2 \%$ to $1.0 \%$ whereas we see no significant improvement for the PR06C chamber.
WE-D1-02

Comparison of dose standards for linac photon beams at NRC Canada and LPRI France

K Shortt*, C Ross, J Seuntjens ${ }^{1}$, F Delaunay, P Gross, E Leroy, A Ostrowsky $^{2}$, (1)Ionizing Radiation Standards, NRC, Canada, (2)Laboratoire Primaire Des Rayonnements Ionisants, CEA, France

The NRC Canada has declared a new absorbed-dose-to-water standard for ${ }^{60} \mathrm{Co}$ based on its sealed water calorimeter. This device was also used with higher energy photon beams for measurements of $\mathrm{k}_{\mathrm{Q}}$ and the energy dependence of G. Three NE 2571 graphite ionisation chambers calibrated at NRC were taken to the Laboratoire Primaire des Rayonnements Ionisants (LPRI), France for a comparison with their dose standard. The LPRI standard was based on measurements of dose to water at ${ }^{60} \mathrm{Co}$ using a graphite calorimeter. For higher energy beams at LPRI, the dose was determined by averaging ionisation chamber and Fricke dosimetry measurements. The change in ionisation chamber sensitivity as a function of beam quality was estimated using the new IAEA dosimetry code of practice, which is similar in principle to TG-51. For Fricke, the energy dependence of $\mathrm{G}$ was based on measurements done previously at NRC. If the comparison uses $\% \mathrm{dd}_{\mathrm{x}}(10)$ to specify beam quality, the LPRI dose is not significantly different than NRC's value at both 12 MV and 20 MV and certainly well within estimated uncertainties. However, these differences would be exacerbated if the comparison were done using TPR20/10. The discrepancy between the NRC measured values and TG-51 calculated values of $\mathrm{k}_{\mathrm{Q}}$ for the NE 2571 increases with increasing energy, reaching about $0.6 \%$ at 30 MV.

\section{WE-D1-03}

Gradient influence on Pfl of Plane Parallel Electron Chambers: Monte Carlo Simulations and Measurements

J Lehmann*1,2, M Lauterbach ${ }^{2}$, U Rosenow ${ }^{2}, \mathrm{C}-\mathrm{M} \mathrm{Ma}{ }^{1}$, (1) Stanford University School of Medicine, Stanford, CA, (2) University of Goettingen, Germany

Using EGS4 with an improved electron algorithm, the pertubation correction factor $\mathrm{P}_{\text {repl }}$ was calculated for the Markus chamber, the Roos chamber, and other, optimized chamber models and was compared to phantom measurements. $\mathrm{P}_{\text {repl }}$ is the product of the gradient correction factor $\mathrm{P}_{\mathrm{gr}}$ and the fluence correction factor $\mathrm{P}_{\mathrm{fl}}$, caused by the differences in stopping power and scattering power, respectively. In this work the influence of the gradient on $\mathrm{P}_{\mathrm{fl}}$ was investigated. It was found that $\mathrm{P}_{\text {repl }}$ (Markus) drops from 0.99 to 0.90 for a mean electron energy at depth of $2 \mathrm{MeV}$ when the chamber is moved upstream from $\mathrm{d}_{\max }\left(0.75 \mathrm{~cm}, \mathrm{E}_{0}=4 \mathrm{MeV}\right)$ to $0.186 \mathrm{~mm}$ $\left(\mathrm{E}_{0}=2.5 \mathrm{MeV}\right)$ due to $\mathrm{P}_{\mathrm{fl}} . \mathrm{P}_{\text {repl }}($ Roos $)$ changes from 1 to 0.965 for the same case. For negative gradients, as in case of $d_{\max }$ measurements for low incident electron energies and for measurements beyond $d_{\max }, P_{\text {repl }}$ raises above unity. The overall dependence of $\mathrm{P}_{\text {repl }}$ on the percentage change of depth between chamber entrance window and backwall is approximately linear with $\mathrm{P}_{\text {repl }}=0.97-0.005 \mathrm{pdd}\left[\% \mathrm{~mm}^{-1}\right]$ for the Markus chamber and $\mathrm{P}_{\text {repl }}=1-0.001 \mathrm{pdd}\left[\% \mathrm{~mm}^{-1}\right]$ for the Roos chamber for energies at depth below $3 \mathrm{MeV}$. Optimized chambers show a smaller dependence. Fricke dosimetry measurements using clinical electron beams were used to confirm the simulation results.

\section{WE-D1-04}

Dependence of Wedge Factors on Source-to-Wedge Distance

J Gibbons*, D Mihailidis, H Alkhatib, Palmetto Richland Memorial Hospital, Columbia, SC

The current volume of data demonstrates a large variation in the field size dependence of wedge factors. Heukelom et al., ${ }^{1}$ developed a general equation which predicted the field size dependence within a few percent for a variety of accelerators equipped with internal wedges. However, recent reports ${ }^{2,3}$ indicate that the Heukelom equation fails for wedges placed beyond the collimating jaws.

The intention of this paper is to develop a consistent approach to determine the field size dependence of external wedge factors as a function of sourceto-wedge distance (SWD). Measurements have been made at $5 \mathrm{~cm}$ depth as a function of field size and SWD for 4-, 6- and 18-MV photon beams. Wedge factors were taken at a depth of $5 \mathrm{~cm}$ with conventional physical wedges placed on a specially constructed adjustable support assembly. 
SWDs were varied from $50-90 \%$ of the nominal SAD. Readings were also taken as a function of wedge angle and material. Additional data were obtained at extended SSDs to simulate typical internal wedge geometries.

Measured data showed deviation from the Heukelom approach. Better agreement was obtained using an inverse square correction ${ }^{2}$ to account for changes in SWD. Quantitative comparisons are made with wedge factor predictions based on different scattering coefficients.

${ }^{1}$ Heukelom et al., Radiother. Oncol. 30: 66-73, 1994.

${ }^{2}$ Thomas, S.J., Radiother. Oncol. 32: 271-273, 1994.

${ }^{3}$ Cozzi, A.F. et al., Radiother. Oncol. 39: 31-34, 1996.

\section{WE-D1-05}

Experimental Determination of the Overall Perturbation Correction Factor for the NACP Chamber in Electron Beams at Depths Greater Than dmax

C. Reft*, F. Kuchnir, University of Chicago, Department of Radiation Oncology, Chicago, IL

Current dosimetry protocols recommend using parallel-plate ionization chambers for electron beam output calibrations at $d_{\max }$. The new TG-51 protocol uses a new reference depth for electron beam calibrations that is beyond $\mathrm{d}_{\max }$ which is in a region of dose gradient for energies greater than 12 $\mathrm{MeV}$. The overall perturbation correction factor, $\mathrm{p}_{\mathrm{q}}$, is defined as the product of the replacement correction factor, $\mathrm{P}_{\text {repl }}$, and the wall correction factor, $P_{\text {wall }}$. Although $\mathrm{p}_{\mathrm{q}}$ is known at $\mathrm{d}_{\max }$, there are little data available for it at depths beyond $\mathrm{d}_{\max }$. This correction factor was obtained for the NACP parallel-plate ionization chamber by intercomparing measurements made with this chamber and with a diamond detector ${ }^{1}$ at incident electron energies of 12,16 and $20 \mathrm{MeV}$ at depths near the 90,80 and $50 \%$ of dose maximum. Our results show that $\mathrm{p}_{\mathrm{q}}$ is unity within the experimental uncertainties for mean energies at depths from 2 to $18 \mathrm{MeV}$ and percentage depth-dose gradients ranging from 0 to $3.5 \% / \mathrm{mm}$. We recently published measurements of $\mathrm{p}_{\mathrm{q}}$ at similar depths for the Markus, Attix and Farmer chambers relative to the NACP chamber for which we assumed $p_{\mathrm{q}}$ to be unity. ${ }^{2}$ This work confirms our assumption. Our results along with an error analysis will be presented.

${ }^{1}$ PTW Type 60003

${ }^{2}$ C.S. Reft and F.T. Kuchnir, Med. Phys. 26, 208 (1999)

\section{WE-D1-06}

\section{A New Method to Measure the Lateral Scatter of Electron Beams}

D Wilson*, University of Louisville Brown Cancer Center, Louisville, KY

Measured lateral electron standard deviations (sigma-x) are often used as input data for electron beam treatment planning, calculation of electron output, verification of electron beam algorithm performance, and in the evaluation of Monte Carlo simulation of clinical beams. The current methods described in the literature involve fitting the distance from two points at the edge of a broad electron beam (i.e. $20 \%$ to $80 \%$ of the maximum as described by Hogstrom et al, Phys. Med. Biol. 1981), or measuring the slope of the dose gradient at the inflection point (normalized to the maximum dose as described by Sandison et al Med. Phys. 1989). In the method described here, the dose distribution profile is differentiated around the edge of the beam. This differential can then be fit to a pure gaussian distribution, or a function describing large angle scattering separately. No normalization to the flat portion of the profile is required. A process for rapidly generating sigma-x values vs. depth was developed by automating this procedure into a computer program which converts film density to dose, differentiates those values, then performs a non-linear least squares regression to a gaussian function.

\section{WE-D1-07}

Heterogeneity Effect of Small Fields for Intensity Modulation with 6MV Photons

J Sohn*, R Drzymala, E Klein, D Low, J Purdy, Radiation Oncology Center, Mallinckrodt, School of Medicine, Washington University, St. Louis, MO

Narrow, elongated or very small beams are typically required for intensity modulated radiation therapy (IMRT) with devices such as the NOMOS
Mimic and dynamic multileaf collimation. Inhomogeneity corrections (IC) for small $6 \mathrm{MV}$ photon beams were investigated for several geometries including: (1) field size, (2) lung thicknesses $(2,4,6 \mathrm{~cm})$, and (3) lung and bone location. Capintec PR-05P $\left(0.07 \mathrm{~cm}^{3}\right)$ and Exradin A14 $\left(0.009 \mathrm{~cm}^{3}\right)$ ionization chambers were used for measurements. The measurement point was at $2 \mathrm{~cm}$ beyond the distal interface of media relative to the source. The ionization readings for various field sizes were compared to those measured in a homogenous water phantom including additional measurements of small-field TARs at 6 and $10 \mathrm{~cm}$ depths. A maximum IC of 1.235 was found for a $1 \mathrm{~cm} \times 1 \mathrm{~cm}$ field size. The RTAR method underpredicted the IC by $7 \%$ for $1 \mathrm{~cm} \mathrm{x} 1 \mathrm{~cm}$ and $1 \mathrm{~cm} \times 10 \mathrm{~cm}$ fields. The ETAR method for this geometry generated the same IC factor as the RTAR method. Without IC, a 9\% error resulted with $2 \mathrm{~cm}$ air gap or $2 \mathrm{~cm}$ bone for a $1 \mathrm{~cm} \times 1 \mathrm{~cm}$ field size. The differences of IC depending on lung and bone location were within $3 \%$. We conclude that IC for small fields can be significant. Benchmark data such as these are needed for IMRT dose calculation algorithm verification.

\section{WE-D1-08}

Evaluation of Wedge Angles in Half-blocked Fields

L Leybovich*, G Glasgow, A Sethi,G Simon-Mumper, N Dogan, Loyola University Medical Center, Maywood, IL

Half-blocked fields produced by linear accelerators are characterized with isodose curves that are tilted toward the blocked edge of the field. The degree of tilt is noticeable although not large (according to our measurements, about $7^{0}$ to $9^{0}$, depending on the beam energy). It was reported in the literature that the effect of asymmetric beam configuration on the wedge angle was minimal. Our measurements, however, only partially supported the aforementioned statement. When a wedge was inserted with the heel toward the blocked area, the isodose tilt was about $5^{0}$ greater than the nominal wedge angle (for a $30^{\circ}$ wedge, $18 \mathrm{MV}$ beam, at $10 \mathrm{~cm}$ depth the tilt was $35^{\circ}$ ). When the wedge orientation was reversed, a significant reduction of the isodose tilt was observed (for example, for the $30^{\circ}$ wedge, $18 \mathrm{MV}$ beam, the degree of isodose tilt became $17^{0}$ ). A similar behavior was observed for enhanced dynamic wedges. On the other hand, no significant change of the wedge angle was found for a Cobalt- 60 beam. Therefore, the observed phenomenon may be attributed to variation of the linear accelerator beam energy with the distance from the central axis. Detailed analysis of the experimental data and evaluation of the clinical significance of the observed phenomenon will be given during presentation of this study.

\section{WE-D1-09}

\section{Dose Reconstruction in Tomotherapy}

J Kapatoes*, G Olivera, H Keller, P Reckwerdt, E Fitchard, K Ruchala, T Mackie, University of Wisconsin, Madison, WI, University of Wisconsin, Madison, WI, Institute for Biomedical Engineering ETHZ, Zurich, University of Wisconsin, Madison, WI, University of Wisconsin, Madison, WI

Dose reconstruction may not be possible in general radiotherapy but is effective for quality assurance of tomotherapy treatments involving steep dose gradients. This process can be accomplished by calculation, measurement, or a mixture of the two. We utilize a hybrid method in which the incident energy fluence emitted from the NOMOS MIMiC multileaf collimator (MLC) is found using measured data and the dose is then computed using convolution/superposition.

The procedure has been applied to three different treatments involving phantoms. It was found that the process is a sensitive measure of the following:

-correct positioning of the phantom

-accelerator variations and corresponding normalization

-sampling of the measured data with respect to the number of beam angles -leakage and transmission through the jaws and MLC -mechanical behavior of the leaves.

The method is most sensitive to the positioning of the phantom, normalization of accelerator output, and leaf behavior. Problems with positioning cause systematic errors related to the magnitude of the positional changes. It is therefore essential that a $\mathrm{CT}$ at the time of treatment be used for dose reconstruction. Possible accelerator output variations cause delivery errors dependent upon the extent of the variations. As this method utilizes measured data, a normalization system must be chosen judiciously. 
Finally, mechanical aspects of the leaves such as leaf latency are problematic since they are variable in the NOMOS MIMiC. The ability to reveal such delivery errors makes dose reconstruction a very attractive tool for radiotherapeutic verification.

\section{WE-D1-10}

A Method for Routine Quality Assurance for Dynamic Multi-leaf Collimators

J Ting*, E Butker, E Elder, M Mitchum, S Ghavidel, L Davis, Emory University School of Medicine, Atlanta, GA

Purpose: To develop a user friendly method for routine quality assurance for Intensity Modulated Radiation Therapy (IMRT) using dynamic multileaf collimators (DMLC).

Methods and Materials: A "stop and shoot" algorithm is used to generate a matrix of $1.0 \times 1.0 \mathrm{~cm}$ beamlets covering the maximum IMRT field of $14 \mathrm{X}$ $40 \mathrm{~cm}$. These beamlets have alternating intensities of 0.0 and $0.0 \mathrm{cGy}$. A Kodak RP/V2 film, located at depths of $1.5 \mathrm{~cm}$ in a solid water phantom for $6 \mathrm{MV}$ and at $4.0 \mathrm{~cm}$ for $18 \mathrm{MV} \mathrm{X}$-rays, is exposed. This procedure takes 1.5 minutes. This film is scanned with an automatic film densitometer and data are collected for analysis.

Results: This test method yields a matrix of beamlet intensities which are sensitive to $0.5 \mathrm{~mm}$ variation of intended leaf positions and $3 \%$ of dose variations. This method is easily implemented for routine quality assurance. Output from the film densitometer is fed into a spread sheet program for analysis.

This quality assurance method has been implemented in our Department since August, 1998. The quality assurance results show that our IMRT program and the DMLC hardware perform well within specifications.

Conclusion: A method has been developed for routine quality assurance of the IMRT program and the DMLC hardware. This method produces a hard copy film for record keeping and a matrix of data points for analysis. This method has adequate sensitivity to detect "out of specification" variations of IMRT and DMLC.

\section{Scientific Session Imaging and Feedback Radiotherapy}

\section{WE-D2-01}

Correlation of a Transabdominal Ultrasound System and Implated Marker Seeds for Prostate Localization

$\mathrm{T}$ Willoughby*1, R Lalonde ${ }^{2}$, D Mohan, M Weinhous, P Kupelian ${ }^{1}$, (1)Cleveland Clinic Foundation, Cleveland, OH, (2)NOMOS Corporation, Sewickley, PA

Accurate localization is crucial with conformal therapy and IMRT, especially as we try to escalate target doses. Localization is of particular concern for target areas such as prostate, which are subject to motion due to bladder and rectal filling. Much work has been done to establish an easy method of prostate localization. We have implemented a new technique that uses daily transabdominal ultrasound along with $\mathrm{CT}$ contours to localize the prostate. This study evaluates the accuracy of the ultrasound alignment relative to the prostate location on orthogonal portal films. Marker seeds were placed in the prostates of six patients undergoing radiotherapy. The expected locations of the seeds were determined from the treatment planning CT. Patients were setup for treatment based on skin marks with minimal immobilization. The ultrasound device was then used to align the patient so the prostate was in the correct location relative to the contours of the treatment plan. After alignment, orthogonal portal films were taken to determine the actual prostate location relative to the expected location. The root mean squared values of the median difference for each patient were LAT $1.59 \mathrm{~mm}$, AP $2.55 \mathrm{~mm}$, and SI $4.78 \mathrm{~mm}$. These results indicate that it is possible to localize the prostate to within $5-\mathrm{mm}$ accuracy using a transabdominal ultrasound system. The greatest difference in location was in the superior/inferior direction and was attributed to uncertainty of CT anatomy at the bladder and prostate interface as well as to CT resolution.

\section{WE-D2-02}

A Cone-Beam CT Scanner Based Upon a Flat-Panel Imager: Evaluation for Image-Guided Radiotherapy

D Jaffray*, J Siewerdsen, D Drake, William Beaumont Hospital, Royal Oak, MI

Organ motion and setup errors in radiotherapy limit the coverage dose applied to the clinical target volume. We have proposed the installation of a cone-beam computed tomography (CBCT) system on a conventional accelerator for kilovoltage image-guided radiotherapy for guiding field placement and minimizing geometric uncertainty. A prototype CBCT scanner has been constructed to study the logistics of performing CBCT with a flat panel and to evaluate its tomographic imaging performance. The main components in the system are an x-ray tube, a rotation stage, and a $20.5 \times 20.5 \mathrm{~cm}^{2}$ flat panel imager $\left(133 \mathrm{mg} / \mathrm{cm}^{2} \mathrm{Gd}_{2} \mathrm{O}_{2} \mathrm{~S}: \mathrm{Tb}\right.$ converter, $512 \times 512$ matrix of photodiodes+TFTs, $400 \mu \mathrm{m}$ pixel pitch). All components operate under computer control and are mounted on an optical bench. The Feldkamp algorithm reconstructs a volumetric CBCT data set from 300 radiographic projections acquired over $360^{\circ}$. Parameters examining scanner performance include uniformity of response $( \pm 2 \%)$ and linearity $( \pm 0.6 \%)$. MTF measurements indicate that the system can pass spatial frequencies up to $1.51 \mathrm{p} / \mathrm{mm}[\mathrm{MTF}(1.51 \mathrm{p} / \mathrm{mm}) \cong 10 \%]$. The noise in the CBCT images follows an inverse-root-exposure dependence, ranging from 54 to $13 \mathrm{HU}$ over an exposure range of 100 to $2200 \mathrm{mR}$. These values are comparable to those generated on a conventional CT scanner. Finally, CBCT images of small animals were found to be comparable in terms of detail and contrast to those produced on the conventional scanner at equivalent technique and exposure. Supported in part by the U.S. Army Prostate Cancer Research Program (PCRP-970201),National Cancer Institute (CA66074) and Elekta Oncology Systems.

\section{WE-D2-03}

Radiographic Image-Guidance Algorithm for Three-Dimensional Patient Localization Using a Non-Rigid Imaging System

M Moreau*, L J Pisani, D A Jaffray, D Yan, J W Wong, William Beaumont Hospital, Royal Oak, MI

We developed an algorithm to perform patient localization in three dimensions (3D) using a priori 3D information of the patient (a prescription $\mathrm{CT}$ dataset) and a series of $\mathrm{N}$ two-dimensional (2D) projection radiographs (where $\mathrm{N} \geq 1$ ) obtained at treatment time. Patient and site-specific anatomic features in the prescription CT dataset are selected and identified in the projection radiographs. The 3D coordinates of these features, their corresponding 2D projected coordinates, along with the motion characteristics of the non-rigid imaging system, are used in an optimization algorithm to determine the proper rigid-body transformation (translation and rotation) between the actual patient position at treatment time and the patient position in the prescription CT dataset. This transformation can then be used to apply a patient setup error correction and/or adjust treatment delivery parameters. Results obtained from ball bearing and humanoid phantoms show that localization can be determined with a precision of $0.4 \mathrm{~mm}$ (along each axis) for the translations and $0.1^{\circ}$ (about each axis) for the rotations when the non-rigid motion characteristics of the imaging system are taken into account. When the imaging system is assumed to be rigid and ideal, localization precision is $1.3 \mathrm{~mm}$ for the translations and $0.2^{\circ}$ for the rotations. However, localization inaccuracy (where the non-rigid imaging system is the truth) is as large as $7.1 \mathrm{~mm}$ for the translations and $0.4^{\circ}$ for the rotations, when using open-field projection radiographs (free of treatment port information).

\section{WE-D2-04}

Study of the Behavior of Focused Ultrasound Lesions with MRI and Histology

Lili Chen*, D Bouley, E Yuh, H D'Arceuil, K Butts, Stanford University, Stanford, CA

This work was aimed at investigating the behavior of focused ultrasound (FUS) lesions with MRI and comparing lesion size measured on MRI with the actual tissue damage measured on histological sections. Experiments were carried out on 5 rabbit brains in vivo under general anesthesia. An approximately $25 \mathrm{~mm} \times 20 \mathrm{~mm}$ of skull was removed surgically, leaving the dura mater intact. Two separate FUS lesions were created on the same 
imaging plane (sagital) in the brain. T1-, T2-weighted fast spin echo and FLAIR sequences were used to detect the ultrasonic lesions after the treatment. Imaging was performed for 4-8 $\mathrm{h}$ after treatment, after which the animals were immediately sacrificed. Ultrasonic lesion diameter was measured on MRI and histological sections after correction for tissue shrinkage during the histological processing. It was found that T1-weighted images showed lesion formation poorly while both T2-weighted and FLAIR images showed the lesion clearly. The lesion diameters on both T2 and FLAIR images correlated well with measurements from histology. The difference between the lesion diameter measured from MRI and that from histology is $0.1-0.7 \mathrm{~mm}$ which is approximately one MRI pixel value $(0.625$ $\mathrm{mm}$ ) for this study. The time delay before lesions appeared on T2-weighted was $0.25-1.0 \mathrm{~h}$. There was a strong correlation between time delay for lesion formation on $\mathrm{T} 2$ and exposure location in the brain.

This investigation was supported by Lucas Foundation.

\section{WE-D2-05}

Radiation Induced Cardiomyopathy as a Function of Radiation Beam Gating to the Cardiac Cycle

D Gladstone*, P Hoopes, M Flanagan, J Southworth, V Hadley, M Wei, Dartmouth Hitchcock Medical Center, Lebanon, NH

Recent studies indicate the oxygen content of the rat heart may decrease from the end of diastole to the end of systole by as much as a factor of 20 . This observation prompted us to look for a method of sparing the heart from radiation damage by irradiating with a pulsed radiation beam gated to the heart cycle.

Rats received 20 Gy to the heart using a $2 \mathrm{~cm}$ diameter, $6 \mathrm{MeV}$ electron beam. Radiation was delivered in $30 \mathrm{~ms}$ pulses centered either at the end of systole or at the end of diastole. Heart function was assessed by in-vivo measurement of the cardiac shortening fraction 60 days post irradiation using ultrasound imaging. Hearts were then excised and studied histologically to assess the amount of collagen formation secondary to radiation damage. Rats receiving gated radiation were compared to controls and to rats receiving $20 \mathrm{~Gy}$ radiation with no gating applied to the beam.

Significantly decreased cardiac function was observed in rats irradiated at the end of systole. Rats irradiated without beam gating and rats irradiated with the beam gated to the end of diastole were indistinguishable from the control group with regard to cardiac function. The histology results show increased damage when radiation is delivered at the end of diastole (high oxygen) and decreased damage when radiation is delivered at the end of systole (low oxygen).

Partially funded by ACS grant \#IRG82-003016IRG IRG-157M.

\section{WE-D2-06}

An Improved Active Breathing Control (ABC) Aapparatus to Immobilize Breathing Motion

M Sharpe*1, L Kim ${ }^{1}$, J Musselwhite ${ }^{2}$, J Wong ${ }^{1}$, (1)William Beaumont Hospital, Royal Oak, MI, (2)Elekta Oncology Systems Inc., Norcross, GA

Organ motion associated with breathing is problematic for dose escalation. The approach of active breathing control $(\mathrm{ABC})$ can be used to temporarily immobilize breathing motion by sealing off airflow with a computercontrolled valve. $\mathrm{ABC}$ can be applied repeatedly at any pre-determined phase of the breathing cycle. The concept was tested successfully with a modified servo-ventilator. Organ position was immobilized reproducibly to within $\pm 3 \mathrm{~mm}$. As the ventilator was unsuitable for practical use, a prototype single valve $\mathrm{ABC}$ apparatus was constructed. Initial patient studies showed that treatment under $\mathrm{ABC}$ allowed significant reduction of treatment margin as compared with free-breathing treatment. However, several areas of improvement were identified. (1) The analog flow meter responded non-linearly to flow, resulting in undesirable variation in lung volume determination. Reproducibility of organ immobilization was thus dependent on patient's performance. (2) The system exhibited temperature dependent signal drift that required reset during operation. (3) The use of a pediatric valve increased flow resistance that was annoying to some patients. In a new $\mathrm{ABC}$ apparatus, a digital flow transducer effectively solves the first two problems. A large bore balloon valve greatly reduces flow resistance.
Laboratory studies show that volume measurements were reproducible to within $4 \%$ at normal breathing flow rates. The improved performance would improve reproducibility of breathing motion immobilization.

This work is supported in part by NCI grant RO1-CA76182 and Elekta Oncology Systems, Inc.

\section{WE-D2-07}

Breathing Synchronized Radiotherapy Program at the University of California Davis Cancer Center

H Kubo* ${ }^{1}$, P Len ${ }^{1}$, S Minohara ${ }^{2}$, H Mostafavi ${ }^{3}$, (1) UC Davis, Sacramento, CA, (2) NIRS, Chiba, Japan, (3) Varian, Palo Alto, CA

This paper presents a complete description of the breathing synchronized radiotherapy (BSRT) system which has been jointly developed between the University of California Davis Cancer Center and Varian Associates. BSRT is a description of an emerging radiation oncology procedure where simulation, CT scan, treatment planning and radiation treatment are synchronized with the patient breathing by undergoing voluntary breathhold, forced breath-hold, or breathing gating. The BSRT system consists of a breathing monitoring system (BMOS) and a linear accelerator gating hardware and software package. Two methods, a video camera-based method and the use of wrap-around inductive plethysmography (RespiTrace), generate the BMOS signals. The BMOS signals and the synchronized fluoroscopic images are simultaneously recorded in the simulation room and are analyzed later to define the ideal treatment point (ITP) where the tumor is completely covered and the critical normal tissue is maximally spared. The BMOS signals at ITP can be used to gate a CT scanner or a Varian "Clinac" series linear accelerator to maintain the same organ configuration as in the simulation. The BSRT system allows the use of breath-hold and gating. This dual role allows the system to be applicable for a variety of patients, i.e., the breath-hold method for those patients who can maintain and reproduce the ITP, and the forced breath-hold or gating method for those who are not ideal for voluntary breath-hold.

Support: this work is supported by the UC Davis Health System Research Award and Varian grant.

\section{WE-D2-08}

Clinical Performance of an Infrared CCD Based Respiratory Gating System

K Spencer, C Ramsey*, I Cordrey, A Oliver, Thompson Cancer Survival Center, Knoxville, TN

In order to increase the effectiveness of $3 \mathrm{D}$ treatment planning for abdominal targets, a method must be developed to compensate for tumor motion during respiration. Various respiratory gating systems are under development that will allow the selective delivery of absorbed dose to moving target volumes during time intervals when the target volume is within the intended region. One such system is an infrared CCD based respiratory gating system under development for use with Varian linear accelerators. This system correlates the location of the target volume with a signal from an infrared CCD video camera that tracks the position of passive markers placed on the patient. During simulation, fluoroscopic images are displayed and digitally recorded in conjunction with the patient's respiration cycle. Video playback of the recorded fluoroscopic images displays only those frames located within the gating threshold. The spatial position of the target during dose delivery can be fixed by interactively adjusting the gating thresholds until organ motion is minimized in the fluoroscopic playback. During treatment, these thresholds are used by a computer control system to gate the delivery of absorbed dose by enabling and disabling the linear accelerator's gridded electron gun delay. The performance of the infrared CCD video camera, passive retro-reflective markers, simulator interface, linac gating circuit, linac electron gun delay, and linac software interface will be presented. 


\section{WE-D2-09}

Interactive 6-DOF 2D/3D Registration Using Transmission Imaging

D LaRose*, J Bayouth, T Kanade, Carnegie Mellon University, UPMC, Carnegie Mellon University, Pittsburgh, PA

This study assesses the accuracy of a new patient registration technique which is based on 2D X-ray images and runs at interactive speeds. Pose estimates in 6 degrees of freedom are recovered by comparing X-ray images with digitally reconstructed radiographs (DRRs). The DRRs are generated using a new data structure called a Transgraph, which is based on recent computer graphics results. The contents of the Transgraph are derived from pre-operative CT data, and the structure permits DRRs to be generated in a fraction of a second without further reference to the original CT volume. The synthesized DRRs reflect the full CT structure, including soft tissues, and permit the full field of view of the X-ray imager to be used in registration.

An anamorphic phantom head was fitted with an LED marker, which was placed outside of the field of view of a pair of X-ray imagers. This marker was tracked through approximately 450 poses using an active infrared optical tracking system having accuracy on the order of $0.1 \mathrm{~mm}$. Data from the optical tracking system were used to evaluate the new registration algorithm. RMS rotation errors of $0.554^{\circ}$, vs. $0.240^{\circ}$ and $0.140^{\circ}$ were observed in the transverse, sagital, and coronal planes respectively. RMS registration error over a $6 \mathrm{~cm} \times 6 \mathrm{~cm} \times 6 \mathrm{~cm}$ volume centered in the cranium was $0.802 \mathrm{~mm}$

\section{WE-D2-10}

Determination of Two Parameters of a Liquid Filled Ionisation Chamber to Predict Portal Dose Images of Dynamically Modulated Treatment Beams

H Keller*, M Fix, P Ruegsegger, Institute for Biomedical Engineering ETHZ, Zurich, Switzerland

IMRT treatments have triggered a growing interest in the verification of intensity-modulated beams with electronic portal imaging systems (EPIDs), such as scanning-liquid-ionisation chambers (SLICs).

The ion concentration in the liquid of a SLIC-EPID and therefore the absorbed dose rate in the chamber is determined by two constants describing the creation and recombination rates of the ions. These two parameters were determined for an incident open $6 \mathrm{MV}$ photon beam.

The creation rate of the ions is proportional to the incoming photon fluence rate and the chamber electron yield. The latter was computed with Monte Carlo calculations. In order to obtain an absolute photon fluence value, an absolute dose was measured in a water phantom and related to a Monte Carlo computed dose. The recombination constant was determined from the ion transit time.

The parameter values were tested for simple beam intensity modulations generated by switching the beam on and off. The parameters reproduced the measured temporal behavior of the ion concentration in the liquid to within a few percent for the beam switch-on experiment. The decrease of the ion concentration in the beam-switch off experiment, however, was too slow, indicating, that the measured transit time may be inaccurate. However, the ratio between calculated and measured ion concentration was nearly constant. Therefore, the results are promising to predict portal dose images for more complicated cases, such as dynamically collimated treatment beams.

\section{Scientific Session \\ Room: Sewanee Diagnostic Imaging: Ultrasound, MRI, \& Nuclear Medicine}

\section{WE-D3-01}

Perfluorocarbon Droplets in a Preserved Tissue Phantom for Doppler Flow Evaluation

J Fowlkes*, K Osman, O Kripfgans, P Carson, University of Michigan, Ann Arbor, MI

In order to examine the performance of ultrasound Doppler systems under conditions more representative of the in vivo state, a phantom has been developed to better simulate physiologic small vessel flow. This phantom is a preserved kidney phantom (Holmes et al., 1984 Advances in Bioengineering. R. L. Spiker. New York, Amer. Soc. Mech. Eng.: 9-10) that we have previously used in ultrasound imaging with contrast agents. Identifying appropriate scatterers for use in a perfusate with this phantom has been a challenge due to the small vessels and the need for blood equivalent scattering. Recent experiments have explored the use of albumin stabilized perfluorocarbon droplets in water. Trichlorotrifluoroethane (boiling point $=48^{\circ} \mathrm{C}$ ) was prepared in the high speed shaking process to form droplets that are 10 microns and smaller in diameter with substantial numbers of the smaller size range. In initial tests in a simple flow tube phantom and a Diasonics Spectra VST scanner using $6 \mathrm{MHz}$ Doppler, Doppler power was shown to be linear $\left(\mathrm{R}^{2}=0.995\right)$ as a function of droplet concentration. Mean Doppler power in comparison to that in the human carotid artery required a droplet concentration of $3.6 \times 10^{5} \mathrm{ml}^{-1}$. These droplets were used in measurements with the fixed kidney phantom for comparison of the Diasonics Gateway 2D and the GE Logiq 700. The slow flows generated in this phantom were a very effective test of the lower detection limits of each system, demonstrating the effects of the lower PRF settings available on the Diasonics scanner.

\section{WE-D3-02}

Therapeutic and Diagnostic Potential of Ultrasonically-transitioned Perfluorocarbon Droplets

O Kripfgans, J Fowlkes, P Carson*, D Miller, University of Michigan, Ann Arbor, MI

Droplets for transitioning to bubbles larger than transpulmonary sizes were generated by mixing albumin (bovine with saline and a low boiling point liquid (dodecafluoropentane). The resulting emulsions contained droplets whose size ranges were determined by the mixing process. The lowest size range was on the order of a few microns diameter. The albumin, as a surface active agent, is assumed to build a shell around the droplets and prevents coalescence. Increases in temperature of the resting host fluid showed that droplets could be superheated well above their natural boiling point. The superheated state could be overcome by the application of ultrasonic pulses. Intravenously-administered droplets did traverse the lung in the rabbit.

The relatively large bubbles produced by these droplets show promise as direct therapeutic agents. Intra-arterial injections caused occlusions or no apparent effects in the leporine brain, depending on the concentration injected.

Acoustically-induced vaporization was observed in three frequency ranges: $\sim 50(\mathrm{kHz}), 2 \mathrm{MHz}$ (in the presence of contrast agent (CA)) and at $7 \mathrm{MHz}$ (with and without CA). Conversions could be made at a mechanical index as low as $0.3(\mathrm{p}=0.8 \mathrm{MPa}$ at $7.5 \mathrm{MHz})$. A diagnostic ultrasound system, using color Doppler mode as well as grayscale, was also able to phase transition the droplets in a flow tube.

[Work supported in part by PHS Grant No. R01 HL54201 from the National Heart, Lung and Blood Institute] 
WE-D3-03

Ultrasound Quality Control Program of Twenty-One Units Over Four Year Experiences

Z Lu*, Columbia University, New York, NY

This paper will present summarized results of the current ultrasound QC program in our institute over past four years. A tissue-mimicking multipurpose QC phantom supplemented with a low contrast phantom was used for performance testing of Gray-scale mode and a Doppler phantom/flow control system for Doppler mode. The Gray-scale tests included distance accuracy, system sensitivity and penetration, axial and lateral high contrast resolution, low contrast detectability, void perception, image uniformity, dead zone and image display evaluation. The Doppler tests included the maximum sensitivity of Doppler signals, Doppler angle accuracy, directional discrimination, range-gate accuracy and peak velocity readout accuracy. In addition, physical inspection checked the probe integrity and system electrical-mechanical cleanliness. These QC tests have been performed annually. Of all the deficiencies revealed during QC testing over the past four years, $23 \%$ was directly related to broken probes or system mechanical integrity revealed from simple physical inspection. $69 \%$ was found in Grayscale mode, of which majority was related to image uniformity, image display and storage devices. $6 \%$ of the deficiencies was associated with software settings. Only $2 \%$ of the deficiencies was found in Doppler mode. The result of this analysis recommends that some QC tests such as image uniformity, image display evaluation and physical inspections should be performed more frequently in order to reveal problems at their earlier stages. The small number of problems revealed in Doppler mode suggests the limitation of the current Doppler QC program.

\section{WE-D3-04}

Coherent Integration of Cross Relaxation and Magnetization Transfer in a Coupled Two-Spin Model of a Paramagnetic Complex in Water Solution.

J Montgomery*, M Dennis ${ }^{1}$, R Deck ${ }^{2}$, (1) Medical College of Ohio, Toledo, $\mathrm{OH},(2)$ University of Toledo, Toledo, $\mathrm{OH}$

Challenges exist in the development of MR contrast materials that may be facilitated by accurate modeling of the relaxation process. Conventional mathematical models, such as by Meiboom, consider the $T_{1}$ relaxation from proton-proton interactions and from proton-electron coupling separately, and incoherently combine them in a weighted summation. Analysis of the spin coupling or coherence effects between these interactions, however, is appropriate.

The developed model is similar to that of Koenig (Mag. Reson. Med., $30: 685$, 1993), however it considers proton-electron coupling with the contrast media, rather than proton coupling with protons bound within proteins. In this model, dipolar relaxation of a paramagnetic complex in water solution is described by a set of coupled macroscopic equations of motion for the net magnetic moment of water protons and unpaired electrons. These are formed by a coherent sum of interfacial and bulk contributions from both of these spin reservoirs. Only after these derivative contributions are in place does the model perform the integration that describes the dephasing and realignment characteristics of relaxation.

Preliminary results of a phantom study are presented comparing this model with conventional methods. Included in the discussion are meaningful parameters related to chemical exchange and the effective transition probability rate associated with internal unpaired electron reservoir coupling.

\section{WE-D3-05}

\section{Functional MRI of Acupuncture Stimulation}

W Liu ${ }^{1}$, D Hung ${ }^{1}$, B Komisaruk ${ }^{2}, \mathrm{H} \mathrm{Lee}^{1}$, A Holodny ${ }^{1}$, Department of Radiology, University of Medicine and Dentistry of New Jersey, Newark, NJ (2) Department of Psychology, Rutgers University, Newark, NJ

The purpose of the present study is to use functional MRI to study brainstem activation produced from acupuncture of the Hegu point. Five normal healthy volunteers participated in the study. Prior to and after acupuncture stimulation, pain stimulation was applied to the subjects by touching the upper gum with the sharp edge of a plastic tube from the acupuncture needle. A visual analog scale was used to record the subjects' perceived intensity of pain. Next, the acupuncture stimulation was applied. An acupuncture needle with diameter of $0.18 \mathrm{~mm}$ was inserted in the Hegu point, (LI 4) of the right hand during the ON period with manual twisting using $1 \mathrm{~Hz}$ low frequency stimulation and was withdrawn from the hand during the OFF period. Each run consisted of four cycles of $30 \mathrm{sec} \mathrm{ON}$ and OFF periods followed a $32 \mathrm{sec}$ baseline scan. The entire study consisted of five acupuncture runs with four 2-minute breaks

The functional MRI was performed on GE 1.5 T Echospeed Horizon. Statistical analysis was performed using SPM96. The fMRI maps of acupuncture showed activation of periaqueductal grey matter in four out of five subjects and midline raphe area in two of those four subjects. Two subjects had a significant decrease in perceived pain intensity after the acupuncture. Through the use of functional MRI in humans, the study provides evidence that acupuncture stimulation of the hegu point of the hand activates the periaqueductal gray and raphe regions of the brainstem.

\section{WE-D3-06}

Tissue-Mimicking Phantoms For Performance Testing of 1H Magnetic Resonance Spectroscopy Of Brain

R Milbrandt*, E Madsen, University of Wisconsin-Madison, Madison, WI

Two 1H MRS head phantoms have been recently constructed using improved tissue-mimicking materials and form the basis of the author's $\mathrm{PhD}$ thesis. Both simulate the human head in terms of size, magnetic inhomogeneities, and MR coil loading. One phantom has been designed to simulate regions of various pathologies (Alzheimer's, multiple sclerosis, astrocytoma), with the remainder of the phantom containing material which mimics the metabolite spectra and relaxation times of normal brain as well as subcutaneous fat. The second phantom has a simulated normal brain background and contains regions which are designed to test the spatial localization, sensitivity and resolution of the MRS aspect of the MR scanner. The tissue-mimicking materials are made from a gelatin-agar base with appropriate amounts of metabolites added as well as $\mathrm{NaCl}$ for coil loading and thimerosal for preservation. Relaxation times of metabolic subgroups in the materials have been measured on a $1.9 \mathrm{~T}$ spectrometer and match those reported for in vivo tissue better than previous phantom materials or aqueous solutions. The phantoms have been scanned in clinical MR units and spectra match those of in vivo brain well. These phantoms will be of use for development of future MRS techniques and as a reference for quantitation of in vivo metabolite concentrations. Their anthropomorphic configuration and realistic magnetic properties make them superior to previously developed phantoms. They are currently being used in a study comparing MRS performance of different scanners.

\section{WE-D3-07}

Application of Square Wave Response Function (SWRF) Measurements for Automated Analysis of SPECT Spatial Resolution Measurements S Dyer*, D Goodenough, Iris, Inc, Frederick, MD, The George Washington University Medical Center, Washington DC

PURPOSE: To perform SWRF measurements using the high contrast resolution gauge in the SpecPhan phantom for quantification of spatial resolution in single photon emission computed tomography (SPECT) imaging.

METHOD/MATERIALS: This investigation will evaluate spatial resolution of a SPECT system using the SWRF method as an alternative to the modulation transfer function (MTF)of the point spread function (PSF). The SpecPhan phantom contains high contrast resolution gauges with spacing of $2 \mathrm{~mm}, 4 \mathrm{~mm}, 6 \mathrm{~mm}, 8 \mathrm{~mm}$ in the axial orientation. This section of the phantom offers a visual determination of spatial resolution in addition to the PSF sections with and without scatter. The position of these gauges will allow measurements in the sagittal orientation as well. This same pattern can be used to apply a SWRF determination from the numerical data in the SWRF pattern. Likewise published methods reveal how to intercompare SWRF and MTF approaches.

CONCLUSIONS: The implementation of the SWRF can be used as an alternative to the MTF of the PSF for spatial resolution evaluation of a SPECT imaging system. 


\section{WE-D3-08}

Optimizing Singles Count Rate for Brain FDG Imaging with an ADAC Scintillation Camera

F Daffin*, M Yester, University Ala. at Birmingham, Birmingham, AL

With the increase in the use of Scintillation Cameras for Coincidence Imaging (CI), questions arise concerning testing Of particular importance is the optimization of the singles count rate to minimize the random fraction and, yet, have the maximum true coincidence rate possible. To aid in assessing the optimum singles count rate, the Noise Equivalent Count Rate (NECR, [true coincidence rate] ${ }^{2} /$ total coincidences) was measured over a count range for an ADAC Vertex Plus camera operating in the coincidence mode. To simulate brain imaging, a circular cylinder with fillable sphere (concentration of 5:1 relative to the phantom) was imaged over a singles count rate of 1.5 Million cps per detector to $100 \mathrm{kcps}$. An additional phantom with equal activity was placed where a patient's chest would be in an actual scan to simulate events outside of the FOV. The low count rate image was used to determine the scatter fraction so that the true and random fractions could be determined for each measurement. The NECR was found to peak at 1.0 Mcps with an approximate $10 \%$ drop at 1.2 Mcps and 0.8 Mcps. The hot spheres were analyzed for image quality and resolution as a function of count rate, also. Based on these results, patient images for brain CI are obtained for singles count rate over a range of 0.9 to 1.1 Mcps/detector.

\section{WE-D3-09}

Performance Characteristics of a Siemens E.CAM+ Dual-Camera Coincidence Imaging System

B Tsui*, C Tocharoenchai, X Zhao, University of North Carolina at Chapel Hill, Chapel Hill, NC

The performance characteristics of a Siemens E.CAM+ dual-camera coincidence imaging system, including spatial resolution, energy resolution and coincidence detection sensitivity, were measured at different energy windows, counting rates and different combinations of coincidence pairs. Measurements were made using point and line sources of Ge-68 and F-18, and a NEMA standard phantom. Experimental data were acquired in listmode, rebinned using the single-slice rebinning (SSRB) method and reconstructed using the filtered backprojection algorithms. The default acquisition mode accepts mostly peak-peak counts and rejects a substantial amount of peak-Compton and Compton-Compton counts for improved image quality at the expense of lower detected counts. Using the default acquisition mode, the energy resolutions for $511 \mathrm{keV}$ are $\sim 7.5 \%$ and $\sim 9 \%$ at low and high dead times. Using a $20 \%$ energy window for the peak-peak counts, the radial and tangential spatial resolutions are $\sim 5 \mathrm{~mm}$ at the centerof-rotation (COR) and increases only slightly as function of distance from the COR. The noise-equivalent count-rate peaks at $\sim 640,690$ and $720 \mathrm{cps}$ (with a $\sim 0.2 \mu \mathrm{Ci} / \mathrm{ml}$ source) and the detection sensitivities are 8.8, 9 and 9.7 $\mathrm{kcps} /(\mu \mathrm{Ci} / \mathrm{ml}$ ) (with a $\sim 8.1 \mathrm{nCi} / \mathrm{ml}$ source) for $20 \%$ peak-peak, $30 \%$ peakpeak and $30 \%$ peak-peak plus scatter, respectively. The performance characteristics vary with different acquisition parameters. In conclusion, results from our study will be useful in determining the optimum acquisition parameters for specific clinical applications. The research described in this abstract was supported in part by a research contract from Siemens Medical Systems.

\section{Scientific Session Diagnostic Image Quality and Quality Assurance}

\section{WE-D4-01}

The Transfer Function of an MTF Calculation Algorithm

P Greer*, T van Doorn, Royal Adelaide Hospital, Adelaide, Australia

The algorithm used to calculate the modulation transfer function (MTF) from an angled edge image has an inherent transfer function. Factors such as angle of the sampling aperture to the edge, registration of profiles using the determined edge angle, differentiation, smoothing and folding all combine to produce the frequency response of the algorithm. Characterization of the transfer function of an MTF algorithm has been undertaken. The description includes the effect of an error in the edge angle determination when registering profiles and calculating the sampling interval. Expressions for the value of the transfer function of each stage of the algorithm at the cutoff frequency were derived. The error in the edge angle determination was found to be an major influence on the MTF of the algorithm. For example, registering 50 profiles sampled at 8 times the cutoff frequency, a $0.01^{\circ}$ error in a $4^{\circ}$ edge angle will produce a $15 \%$ error in the true MTF at the cutoff frequency. The results underline the importance of quantifying the transfer function of the algorithm used when attempting to determine an imaging system modulation transfer function.

\section{WE-D4-02}

New Light Sensitometer for Film Performance and Processing Evaluation

M West*, D Spelic, Mentor Technologies, Inc., Lanham, MD, Food and Drug Administration

A new reference sensitometer has been developed at the Food and Drug Administration's (FDA) Center for Devices and Radiological Health (CDRH) for the calibration of light sensitometers used for Mammography Quality Standards Act (MQSA) inspections. The new device employs a novel light source to sensitize control film. Most commercially available light sensitometers use electroluminescent panel technology in which light emission from a crystal is stimulated by an oscillating electric field. Under clinical conditions, mammography film is exposed to a Gadolinium-OxySulfide intensifying screen inside a film cassette. Intensifying screens produce an emission spectrum peaking at $550 \mathrm{~nm}$, which closely matches the peak spectral sensitivity of orthochromatic mammography film. Emission spectra from commercially available light sensitometers are broad band in structure, peaking at a wavelength of 510 nanometers $(\mathrm{nm})$. This in no way resembles the spectrum of a Gadolinium-Oxy-Sulfide intensifying screen. The new FDA sensitometer, however, faithfully simulates the spectral structure of light produced by intensifying screens. This unique feature would enable researchers to evaluate the relative performance of various types of mammography films without having to perform inverse-square sensitometry. Results of performance and comparison tests between the new sensitometer, commercial sensitometers, and inverse-square sensitometry will be presented.

\section{WE-D4-03}

Bayesian Methods for Salvaging Uninformative Studies

D. Herbert, Dept. of Radiology, K. Ramsey, Dept. of Medicine, V. Parthiban, Dept. of Infectious Diseases, W. Pofahl, Dept. of Surgery, University of South Alabama, Mobile, AL

Most clinical studies, including clinical trials, are famously uninformative, usually a result of poor design and/or execution; in particular, most studies do not have sufficient statistical power to detect clinically useful effects. A random effects meta-analysis of an ensemble of such trials is usually proposed as one remedy. However, the random effects grand mean, which assumes that the between-studies variation is not zero, can be irrelevant to the individual patient. Moreover, investigators often make inappropriate inferences from those under-powered studies in which the point estimate suggests a clinically useful effect but the null hypothesis cannot be rejected. Finally, clinical trials and meta-analyses are currently designed and interpreted on the Neyman-Pearson model, which permits neither inclusion of prior information nor estimation of the predictive probabilities of future observations (e.g., the probability that a given patient will respond to a given treatment).

We present two Bayesian models that solve these, as well as several other refractory problems in design, estimation, and inference. These are the Hierarchical Bayes and the Empirical Bayes models of meta-analysis. The former provides for incorporation of prior information in parameter estimates and for estimation of predictive probabilities in the individual case. The latter takes advantage of the Stein effect to produce more accurate and precise study-specific parameter estimates.

The methods are illustrated with a small set of clinical data in infectious disease, but can obviously be generalized to almost any sort of data. 


\section{WE-D4-04}

ACCR Accreditation Program and the AAPM

J Martensen*, Toledo, $\mathrm{OH}$

The American Chiropractic College of Radiology (ACCR) has initiated an accreditation program for private office $\mathrm{x}$-ray facilities, primarily aimed at Doctors of Chiropractic but not excluding any other private medical office setting. The program is concerned with quality assurance and quality control in the private office and requires multiple steps of compliance before the office is accredited. One of the steps requires a medical physicist to complete a series of standard tests on the equipment as prescribed by the ACCR. This submission is to briefly acquaint the members of the AAPM with the program, its requirements, who to contact and how to be approved to perform services in the program.

\section{Continuing Education Course Mammography - 3}

\section{Room: Taylor}

\section{WE-D5-01}

The Design of Full-field Digital Mammography Systems

Martin Yaffe, Sunnybrook \& Women's College Health Sciences Centre, University of Toronto, Toronto, Ontario, Canada

Full-field digital mammography offers the potential for improved detection and diagnosis of breast cancer and makes possible the use of ancillary techniques which may increase accuracy or provide additional useful information from the examination. The availability of mammograms in digital format can also facilitate image storage and retrieval and remote consultation. In order for digital mammography to be successful, certain key elements must be in place. These include high performance in image acquisition, a practical and effective means of displaying the digital mammograms and an efficient and reliable archiving and retrieval system. In this presentation, the important requirements for each of these processes will be discussed. The designs of detector systems for digital mammography will be described with emphasis on factors determining spatial resolution, noise, dynamic range and image uniformity and both current and future technology will be reviewed. Challenges in image display will be reviewed and approaches to address them through image processing will be suggested. The impact of digital mammography on requirements for image storage and PACS will be discussed. Finally, some of the future applications enabled by digital mammography will be considered.

Educational Objectives:

1. To familiarize the participant with the principles and requirements of digital mammography

2. To describe the current state of the art of detector and display technology

3. To be met for digital mammography to be accepted clinically

4. To suggest future directions for this modality

\section{Continuing Education Course Room: Carroll Mammography - 4}

\section{WE-D5-02}

Current Clinical Status of Full-field Digital Mammography

Etta D. Pisano, MD, The University of North Carolina at Chapel Hill, NC

The previous and ongoing clinical trials in digital mammography will be described in detail, including the studies performed to date for Food and Drug Administration approval purposes.

In addition, federally funded studies involving the diagnostic and screening mammography populations will be described in detail. The effect of display parameters, i.e. printed film vs. softcopy display, and appropriate image processing will be discussed. Recommendations regarding future clinical studies in digital mammography will be described.

Educational Objectives:

1. To understand the ongoing clinical studies on full field digital mammography vs. screen-film mammography;
2. To understand the advantages and disadvantages of softcopy display of digital mammograms;

5. To understand the use of image processing for digital mammography.

Disclosure Statement:

I certify that there is a financial relationship between the University of North Carolina and Fischer Imaging. UNC is being paid by Fischer to run their FDA trial.

\section{Symposium \\ Pearl \\ Monte Carlo Methods in Radiation Treatment Plannin:}

\section{WE-E1-01}

Current Status and Future Directions of Monte Carlo Methods in Radiation Treatment Planning, An Overview

Radhe Mohan, Medical College of Virginia, Virginia Commonwealth University, Richmond, VA

Current status and future directions of Monte Carlo methods in radiation treatment planning, an overview

With the use of Monte Carlo methods imminent in routine clinical practice, it is important for the radiation oncology community to be aware of current developments in the field. Over the last several decades, considerable effort has been expended in the development of approximate empirical and semiempirical dose computation models. In the evolution of these models approximate correction factors and patches were applied continually to account for observed discrepancies between measurements and calculations or to incorporate new treatment methods and accessories. Monte Carlo methods, being based on basic principles of physics, are universally accurate, i.e., apply equally well to photons, electrons, brachytherapy, protons, neutrons and all devices and accessories. Their introduction will obviate the need for incessant refinement of models. In addition to accuracy, there are numerous other advantages of Monte Carlo methods. For instance, they should require considerably less effort to commission and implement. Monte Carlo methods can provide monitor unit settings directly regardless of the modality, device or accessory being used, and will eliminate this source of treatment errors. In the long run, clinical doseresponse data accumulated based on accurate dose distributions will allow improvements in treatment designs.

Current heightened interest in Monte Carlo is a result of the demonstration by the group at Lawrence Livermore National Laboratory of the feasibility of Monte Carlo methods in routine clinical work. This group is the creator of the Monte Carlo code known as Peregrine. Other institutions involved in Monte Carlo efforts include Stanford, NRCC, UCLA, Institute of Cancer Research in UK, Medical College of Virginia and Memorial-Sloan Kettering Cancer Center. Stanford is already using Monte Carlo for electron beam treatment planning. Others are likely to follow suit in the near future. Widespread use may take some time, the main impediments being integrating Monte Carlo codes with existing treatment planning systems, generating and commissioning phase space data for various models of treatment machines, validating results, and complying with FDA regulations. Initially, the add-on cost of Monte Carlo dose computation systems may be another factor.

While a limited use of Monte Carlo in clinical setting has begun, many areas need to be investigated. For example, it is necessary to compare Monte Carlo generated dose distributions with those produced using empirical and semi-empirical methods and estimate whether such differences are clinically significant. Further, assuming that Monte Carlo methods reveal deficiencies in treatment plans which were not apparent in conventional dose distributions, it is important to develop means to rectify such deficiencies, perhaps with intensity-modulated radiotherapy. A related issue is to explore ways of utilizing Monte Carlo dose distributions for optimizing intensity-modulated treatment plans. Monte Carlo methods may also be used for predicting accurate dose images for dosimetric verification of dynamic IMRT.

Educational Objectives:

1. Update the audience on the current status of Monte Carlo methods

2. Describe potential advantages of Monte Carlo 


\section{Identify areas of further research}

\section{WE-E1-02}

Why Monte-Carlo Based Treatment Planning is an Exciting Prospect Alan Nahum, Physics Dept., Royal Marsden HNS Trust and ICR, Sutton, UK

Analytical methods for computing the dose distribution in an inhomogeneous medium (i.e. the patient) from high-energy photon or electron beams involve approximations. For megavoltage $\mathrm{x}$-ray beams, 3D convolution of the pointspread function is currently the most advanced method; however, these watergenerated kernels must be "scaled" for inhomogeneities, involving crude approximations, especially for electron transport over different densities. For electron beams, pencil-beam algorithms can only accurately account for electron scatter if the inhomogeneities are layered perpendicular to the pencil direction. Monte-Carlo simulation avoids approximations in the transport of photons and electrons through arbitrary media. The CPU time on affordable hardware (e.g. a dozen or so Pentium $400 \mathrm{MHz}$ PCs) to generate the required number of histories $\left(\approx 2 \times 10^{8}\right.$ for a photon beam plan, for $\pm 1-2 \%$ in $1-2 \mathrm{~mm}$ voxels $)$ is around 1 hour. Input data for $\mathrm{MC}$ are no longer measured dose distributions in water, but instead a full description of the incident particles i.e. type, energy, position and direction. This is obtained from an initial MC simulation starting at the vacuum window of the linear accelerator. The individual patient simulation starts just above the beam-defining devices, using a cartesian, voxelised geometry "filled" by the data from CT.

Within 2 to 3 years the first commercial MCTP system can be expected. This will open up a number of exciting possibilities. Of particular interest is the interaction between MCTP, DVHs and so-called biological models (TCP, NTCP). Dose-volume histograms (DVHs) summarize 3D dose information at the expense of spatial information; one can therefore expect that MC-generated DVHs will show less statistical noise than the dose distributions themselves. For the target volume, these statistical fluctuations are equivalent to hot and cold spots and could yield underestimates of the Tumour Control Probability if very small scoring volumes are chosen; on the other hand, choosing relatively large MC scoring volumes will yield very precise estimates of the mean tumour dose. There will thus be a fascinating interplay between voxel size, number of histories and TCP. Models for both TCP and Normal-tissue complication probability (NTCP) for the tumour and organs at risk respectively can be used to evaluate the likely clinical impact of the differences between dose distributions generated by $\mathrm{MC}$ and by the various analytical algorithms. It will be possible to compute not just doses but also (electron and photon) fluence spectra at all positions of interest in the phantom/patient, making possible the computation of dosimetric quantities such as water/air stopping-power ratios or $\left(\mu_{\mathrm{en}} / \rho\right)$ ratios for the user's actual clinical beam quality. Additionally the visualization of particle tracks overlayed onto the patient anatomy will have considerable pedagogical value as well as verifying that the beams are in the right place.

The research described in this abstract was supported by a grant from the DOSIGRAY corporation.

Educational Objectives:

1. To summarize the limitations of analytical dose calculation algorithms.

2. To summarize the advantages of Monte-Carlo based treatment planning (MCTP)

3. To predict interesting developments flowing from the use of MCTP

\section{WE-E1-03}

Monte Carlo Based Techniques for Photon Dose Calculations

Jeffrey Siebers, Medical College of Virginia at Virginia Commonwealth University, Richmond, VA

The application of Monte Carlo dose calculation methodology for photon radiation therapy is imminent due to its ability to accurately predict dose deposition in any media and advances in high speed computing technology. This presentation relates experiences gathered from integration and implementation of two Monte Carlo based dose algorithms into a commercial treatment-planning program. The two Monte Carlo based dose calculation algorithms are Monte Carlo Vista (MCV), a local adaptation of the SLAC/NRC EGS4 code with integration into the Pinnacle treatment planning system, and the LLNL developed PEREGRINE Monte Carlo code. Both algorithms were interfaced, acceptance tested, commissioned, and inter-compared with each other, measurements, and with other dose calculation algorithms.

Commissioning tests required for Monte Carlo based algorithms are divided into four categories: 1) ensuring beam orientations and devices used are as specified by the treatment planning system, 2) adjusting phase space data by matching Monte Carlo generated dose distributions to measurements, 3) determining the Monte Carlo particle-to-measured dose calibration (output factor calibration), and 4) comparing measured and calculated dose distributions for a variety of beam conditions. Application of these tests to the two Monte Carlo codes will be presented.

Prior to comparing Monte Carlo dose distributions with other algorithms, dose values must be converted from absorbed-dose-to-material to absorbeddose-to water. In phantom dose distributions computed with $\mathrm{MCV}$, PEREGRINE, and Pinnacles' collapsed cone convolution algorithm will be compared with measurements. Monte Carlo dose distributions for clinical lung, breast, and head and neck treatment plans will be inter-compared with those computed with the collapsed cone convolution algorithm. All plan evaluation is performed using a single treatment-planning platform to ensure uniform evaluation of parameters. For clinical cases, the clearest way to see the dose difference is to use dose difference histograms and dose difference plots.

Due to the superior accuracy of Monte Carlo algorithms, they will routinely be used for dose computation in the $21^{\text {st }}$ century. The improved knowledge of patient dose distributions should reduce treatment dose uncertainty and improve evaluation of patient response data, hence, improve patient outcomes.

\section{WE-E1-04}

Monte Carlo Methods in Electron Beam Treatment Planning Chang-Ming Charlie Ma, Stanford University School of Medicine, Stanford, CA

This presentation outlines recent advances in clinical implementation of the Monte Carlo method for electron beam radiotherapy dose calculation. The results presented are based, in part, on our experience at Stanford with Monte Carlo electron beam treatment planning. Systematic investigation of clinical electron beam characteristics have been carried out by various research groups using specially developed Monte Carlo programs such as the EGS4/BEAM code. As a result of this work, accurate and detailed electron beam phase space data are now available for patient dose calculation. Comparing results of Monte Carlo calculations and measurements of electron beam depth doses, lateral dose profiles and beam output factors for various electron energies, field shapes and sizes, and source to surface distances demonstrate excellent agreement (generally within $3 \%)$. Significant discrepancies $(10 \%$ or more in dose or greater than 1 $\mathrm{cm}$ in the $70 \%$ isodose line) between the dose distributions calculated by Monte Carlo and by analytical algorithms are reported in the literature. The analytical algorithms, like those available in commercial treatment planning systems, show significant uncertainties when applied to clinical cases involving heterogeneities such as air cavities and bones. The Monte Carlo method is clinically implemented at Stanford for patient specific dose calculation. Electron beams of nominal energies $6-20 \mathrm{MeV}$ from three Varian accelerators (Clinac 1800, 2100C and 2300C/D) were simulated using the EGS4/BEAM code. However, a full Monte Carlo phase space is not a realistic clinical tool for electron beam dose calculation. Thus, accurate models are being developed to represent the beam characteristics and reconstruct the beam phase space. This is required to: 1) improve the efficiency of accelerator simulation, 2) reduce data storage requirements and 3) allow for a quick commissioning procedure that can be routinely used by non-research oriented clinical departments. At this time, a model consisting of multiple sub-sources works well to satisfy these objectives. The source model parameters are derived from a reference accelerator's electron beam and are subsequently adjusted so that the simulation results match the measured results from the user's own beam. Additional focus of current research toward clinical implementation is that of calculation speed and the inclusion of all beam modifiers. An EGS4 user code, MCDOSE, is used to perform dose calculations in a $3 \mathrm{D}$ rectilinear phantom that can contain patient specific data from routine CT scans. Beam modifiers such as electron cutouts and bolus are also simulated using MCDOSE. By applying variance reduction techniques, the computation time for an electron beam plan have 
been reduced to $0.1-1.0 \mathrm{~h}$ on a Pentium II $400 \mathrm{MHz}$ PC depending on the beam energy, field size and voxel size. Electron beam plans calculated using Monte Carlo for head and neck, breast/chestwall, and other treatment sites are presented and compared with those calculated by a commercial treatment planning system. Recent developments in Monte Carlo based inverse planning for electron beam IMRT are also discussed.

\section{Symposium Feedback Radiotherapy}

\section{Room: Mississippi}

\section{WE-E2-01}

Feedback Systems for Daily Prostate Localization

James Balter, University of Michigan, Ann Arbor, MI

The past decade has witnessed a renaissance in understanding the nature of prostate movement over the course of radiotherapy. Studies have demonstrated that this movement is anisotropic, and of significant magnitude to cause concern for the delivery of conformal radiotherapy. Studies characterizing the time course of prostate movement have indicated population trends, however significant individual variances hint at difficulty in prospectively predicting prostate position.

In order to address these concerns and improve the precision of prostate radiotherapy, a number of approaches have been developed. These include the placement of stents in the prostate to reproduce position via pressure exerted on the anterior rectal wall, as well as variations of prone or supine positioning with recommended states of bladder filling for treatment. Such attempts seek to reproduce prostate position relative to other pelvic anatomy, but do not resolve the position of the prostate in the treatment room (due to the added variance in patient position).

Recently, a few techniques have been investigated to localize the prostate daily, in order to permit prostate positioning based on the location of the "target of the day". These techniques include radiographic localization of markers placed around the prostate as well as ultrasound localization of the prostate. Developments in tomotherapy imaging as well as cone beam reconstruction may lead to daily volumetric imaging of the prostate.

This talk will focus primarily on the details of systems associated with prostate localization and setup adjustment via radiographic imaging of implanted fiduciuals. The components of a prototype system integrated with a computerized table expanded to include tilt and roll adjustment capability will be discussed. The operating parameters of this system, including speed and final precision, will be summarized. Finally, overviews of other systems based on ultrasound as well as tomography from other institutions will be presented.

\section{WE-E2-02}

High Precision Patient Tracking of Intracranial and Head and Neck Targets

Frank Bova, Sanford Meeks, William Friedman, John Buatti, University of Florida, Brain Institute, Gainesville, FL

The ability to perform high precision repeat location for fractionated radiotherapy treatment has been an ongoing struggle for oncologist, physicist, and therapist. Combination reference and immobilization systems has made repeat localization a major area of clinical research. As radiation therapy formulates more complex dose delivery systems, such as electronic compensation, intensity modulation and expanded use of non-coplanar beams, the ability to solve the repeat localization problem becomes more important

It was not uncommon for the radiosurgical procedure to be abandoned because of an insufficient margin between the high does region and a critical structure. There were other occasions when single dose therapy was not a consideration but increased accuracy, in order to achieve significant volume reduction, was desired. For these reasons a method of repeat stereotactic fixation, applicable to stereotactic radiotherapy, was developed.

The initial design was restricted to intracranial targets. There were two primary design criteria. The first was to be able to detect and adjust the target's alignment in 6 degrees of freedom, 3 translational and 3 rotational.
The system has to be able to adjust the vector alignment of the target center to the unit's isocenter to within $+/-0.5-\mathrm{mm}$. The second was to be able to setup and treat the patient, consisting of 4 to 5 non-coplanar beams or arcs in the same time frame that routine non-stereotactic patients are treated, approximately 15 minutes.

To achieve this goal a system that separated the functions of immobilization and localization was developed. This system is based upon a bite plate and infrared technology. The basic infrared camera system is linked to a PC that sits in the radiation vault and produces a set of alignment vectors that the therapist uses for daily setup. Attention was paid to the refresh rate of the system so that smooth manual adjustment of the patient was possible. Standard vacuum head holders and thermal plastic masks systems are used for immobilization. The immobilization system is totally separate from the biteplate irled system.

The design goal contained a level of accuracy that could not be validated using port films. A system was therefore developed that allowed the new bite plate system to be tested against the commonly employed rigid stereotactic ring. 200 patients underwent testing. Each test consisted of repeated insertions and reading of the absolute location of the biteplate irled array. The average relocation of the system has been $0.13 \mathrm{~mm}+/-0.1 \mathrm{~mm}$. The system is now being expanded to head and neck use

The University of Florida Research Institute has licensed the patents and rights derived from this work. Frank Bova is a paid consultant to Sofamor Danek.

Educational Objectives:

1. The pervious methods of combination immobilization and localization have limited accuracy.

2. In order to prove submillimeter accuracy new experimental systems must be utilized and if one is sub millimeter precision is possible.

\section{WE-E2-03}

Respiratory Gated Radiotherapy

Marc Sontag, St. Jude Children's Research Hospital, Memphis, TN

Conformal therapy techniques combine good patient immobilization to minimize the PTV margin around the CTV coupled with the use of multiple, intensity modulated, non-coplanar beams to reduce treated volume margin beyond the PTV. In the thorax and abdomen, the PTV remains relatively large, largely due to respiratory induced organ motion. The reduction in the PTV margin, achieved by minimizing the effects of organ motion due to respiration, may be as great as the reduction in the treated volume margin gained by using conformal therapy techniques.

Determination of the respiratory cycle or diaphragm position may be inferred by employing an apparatus that measures changes in chest or abdomen position (impedance plethysmography) or pressure (pneumotachometry) using a sensor such as a belt attached to the patient. This approach may have calibration problems caused variation of the tightness of the belt between treatments or slippage that occurs during treatment. If the device is in the beam, radiation damage to the device or loss of skin sparing may occur. Video techniques, employing multiple cameras, allow measurement of chest position while overcoming the calibration and skin sparing problems of the belt systems. Monitors may be used to characterize a patient's respiratory mechanics by placing a sensor in the patient's mouth, clamping the nose and instructing the patient to breathe normally through the mouth. One such device collects data at a rate of 100 times per second, employing a capnograph which measures $\mathrm{CO}_{2}$ using an infrared sensor capnograph and measures airway pressure using a differential pressure pneumotachometer.

Gating at full exhalation is the most effective for eliminating respiratory induced motion because the diaphragm position is most reproducible at this point. Investigators have reported elimination of $80-90 \%$ of diaphragmatic movement. Gating at full inhalation, although less reproducible, may prove to be advantageous because there is maximal separation of the target volume from other normal structures. Reproducibility of the diaphragm position may be improved by using biofeedback circuitry. 
Gating involves a trade-off between elimination of respiratory motion and duty cycle (i.e. fraction of time that the radiation beam is on). Duty cycle may be improved by breath holding. One system forcibly prevents a patient from breathing for short periods at a time. An alternate method relies on the patient to hold his breath

A linear accelerator must be capable of turning on and off rapidly in response to gating signals. One approach is to modify the accelerator's trigger circuit so that during "beam off" sequences electrons are injected out of phase into the wave guide, suppressing x-ray production. Attention must be paid to beam dosimetry because degradation in beam flatness, symmetry and dosimeter calibration is possible. CT, MRI and simulator images obtained for treatment planning must be acquired at the same point in the respiratory cycle at which radiation treatments are delivered.

This work is supported, in part, by Siemens Medical Systems.

Educational Objectives:

1. Understand the role of respiratory gating in conformal radiotherapy.

2. Identify the optimal gating technique.

3. Understand the linear accelerator and treatment planning image acquisition requirements.

\section{Symposium \\ Members' Guide to the AAPM}

\section{Room: Sewanee}

\section{WE-E3-01}

Members' Guide to the AAPM

Michael Gillin, Medical College of Wisconsin, Milwaukee, WI

Even long-time AAPM members are sometimes overwhelmed by the size and complexity of the Association. As a consequence, both new and longtime members may be discouraged from taking full advantage of its benefits or from participating in AAPM activities. This presentation will explain the organization of the Association and help remove barriers to participation that some members may feel. The AAPM needs the active participation of all elements of its membership. The roles of the Board of Directors and the Executive Committee will be described, along with the council and committee structure. Examples will be given of the roles of the Association's liaisons, and the activities carried out by the Headquarters Office will be discussed. The mechanisms by which members participate in the decision-making and policy-setting activities of the Association will be described.

Educational Objectives:

1. The attendee will gain knowledge of the organization of the AAPM.

2. The attendee will learn about the council and committee structure of the AAPM.

3. The attendee will become familiar with the officers and council chairs of the AAPM.

\section{Symposium \\ Advancements in Visualization}

Room: Carroll

\section{WE-E4-01}

Visualization and Electronic Anatomic Atlases for Medical Imaging M. Vannier and G. Christensen, University of Iowa, Iowa City, IA

Visualization has central importance in radiology for diagnosis and therapy. The detection, localization, and characterization of abnormalities depends on their conspicuity and differentiation from normal structures and their variations. Conventional x-ray radiography and motion tomography has been augmented by medical imaging modalities, such as CT and MRI, that exploit physical and functional differences in normal and pathologic structures to create images with better contrast, spatial resolution and fewer artifacts. Subjective analysis of medical images obtained using computer graphics visualization methods has been studied extensively, and the limitations of commonly used medical imaging methods are well known and understood. Fusion of images obtained with multiple medical imaging modalities presents a new challenge for visualization systems that must handle 3,4 or higher dimensional image data.
In this presentation, the fundamental principles of visualization systems applied to medicine will be defined and illustrated. Basic principles of image formation, subjective interpretation and application needs will be delineated, and limitations of current technology will be described. Multidimensional imaging, especially multispectral, multimodality and multitemporal visualization and analysis will be described in qualitatively and quantitatively. The use of visualization systems for image guidance in minimally invasive diagnostic and therapeutic procedures will be described. The incorporation of a priori information in the reconstruction of images from measurements and their visualization will be defined.

Electronic atlases of the body are spatial arrays linked to a hierarchical knowledge base of anatomic nomenclature, functional and vascular territories, and related textual or symbolic reference data. Electronic atlases are used for teaching anatomy, image reconstruction, morphometry and medical image analysis.

Individualized electronic atlases are synthesized by pairwise matching of a normative atlas to superimpose on a target (e.g., patient) volumetric imaging study. This is accomplished by defining the original atlas as a deformable template that is transformed to a target anatomy.

The methods for forming anatomic atlases, labeling them, and synthesizing individualized versions will be explained and illustrated. Global pattern matching, an application of Grenander's global pattern theory, will be introduced as the mathematical basis for determining the transformation needed to deform the original atlas to a target shape under rigorously defined topological constraints.

The application of electronic atlases to automated segmentation, probabilistic generalization to test for group differences, radiotherapy treatment planning and evaluation, and others will be described.

Educational Objectives:

1. Introduce electronic atlases and their application

2. Define the mathematical basis of global pattern matching

3. Describe the use of deformable probabilistic atlases in medical imaging analysis.

\section{WE-E4-02}

A Vision for Visualization in Medicine

Richard Robb, Mayo Foundation/Clinic, Rochester, MN

The practice of medicine has always relied upon visualizations to study the relationship of anatomic structure to biologic function and to detect and treat disease and trauma which disturb or threaten normal life processes. Traditionally, these visualizations have either been direct, via surgery or biopsy, or indirect, requiring extensive mental reconstruction. The revolutionary capabilities of new 3-D and 4-D medical imaging modalities (CT, MRI, PET, US, etc.) along with computer reconstruction and rendering of multidimensional medical and histologic volume image data, obviate the need for physical dissection or abstract assembly of anatomy, and provide powerful new opportunities for medical diagnosis and treatment. Minimally-invasive or non-invasive interventions are now within reach which effectively increase physician performance in arresting or curing disease, which reduce risk, pain, complications and re-occurrence for the patient, and which decrease healthcare costs.

Most current medical interventional procedures (e.g., surgery, biopsy, catheterization) still require "blind" approaches (i.e., the clinicians cannot directly see the target and/or pathway to the target during the procedure), or if visualizations are available, they are limited to 2-D, slow and/or off-line displays. These procedures also often depend on gross approximations and estimates of target position and orientation based on indirect measurements (e.g., EKG). Interactive visualization and Virtual Reality (VR) technology open new realms in the practice of medicine by permitting the images obtained from modern medical imaging systems to be directly displayed and manipulated with intuitive immediacy and with sufficient detail and speed so as to evoke sensorial experience similar to that of real experience. VR allows physicians to "enter" the visualizations, to take up any viewpoint, to see dynamic functional processes, as well as detailed anatomy, to make accurate on-line measurements, and to manipulate and control interventional processes. The ultimate value of VR in medicine will derive 
more from the sensory enhancement of real experience than from the simulation of normally-sensed reality. Visualized objects extend across a vast range of scale from individual molecules and cells through the varieties of tissue and interstitial interfaces to complete organs, organ systems, and body parts, and include functional attributes $f$ these systems, such as biophysical and physiological properties. Medical applications include accurate anatomy and function mapping, enhanced diagnosis, and accurate treatment planning and rehearsal. However, the greatest potential for revolutionary innovation in the practice of medicine lies in direct, fully immersive, real-time multisensory fusion of real and virtual information data streams during an actual clinical procedure. Such technology is under development in our laboratory, but not yet available to the medical practitioner. We plan to further develop, validate and disseminate products based on this technology. The effort will benefit from advanced computer image processing research which has recently facilitated major progress toward fully interactive visualization and simulation. With these advances in hand, there are several important clinical applications possible to deliver now that will have a significant impact on medicine.

Some of the most complex and challenging applications, those which show the greatest promise of significantly changing the practice of medical diagnosis and treatment, have begun to be explored in our laboratory. These include advanced procedures for coronary stent placement and evaluation of cardiac plaques, cardiac ablation therapy, neurosurgery rehearsal and intra-operative guidance, prostate biopsy guidance and analysis, bronchoscopy and colonoscopy, and anesthesia delivery. Preliminary results suggest that in these applications virtual procedures can provide accurate, reproducible and clinically useful visualizations and minimally invasive interventions. They demonstrate significant promise for improving physician performance, minimizing patient risk and morbidity, and reducing health care costs.

\section{WE-E4-03}

Volumetric Visualization of Medical Imagery: A New Paradigm for Radiology

Sandy Napel, Stanford University School of Medicine, Stanford, CA

The past several years have seen an explosion in the detail and amount of medical imaging data that can be routinely produced during the course a cross-sectional imaging examination. Ten years ago a typical CT examination generated 30-50 images; today's helical CT scanners generate hundreds of overlapping slices for interpretation. Spatial resolution and sampling, particularly in the through-plane direction, have also improved. Similar trends are evident in MR and ultrasound. While all of these and other modalities have become more sophisticated, the dominant method for radiological interpretation, that is, visual assessment of each of the crosssectional images generated by the modality, has not changed. And as the number of images increases, so does the time required for interpretation.

While radiologists may be keeping up in 1999, it is doubtful that the current paradigm will even be possible in the near future. As evidence of this, consider the introduction of multiple-detector ring helical CT which, for example, can image a contrast bolus as it travels from above the renal arteries to the toes in one minute, and can generate over $10002.5 \mathrm{~mm}$-thick slices spaced every $1.25 \mathrm{~mm}$. Not only must we worry that the time and, therefore, the cost of interpretation will be significantly increased, but we must consider fatigue and other factors that might compromise diagnostic accuracy.

The new paradigm of radiological interpretation will be based upon treating the acquired image data as a volume to be explored, and from which to extract images and quantitative data that document the condition of the patient. Note that while several volume visualization techniques, such as maximum intensity projection, surface rendering, volume rendering, flat and curved reformatted planes, thin slab renderings, etc., have been available for several years, these have largely been used to supplement the diagnosis made by assessment of the primary source images, and for conveying findings to referring physicians. In the new paradigm of radiological interpretation, these and other techniques, including segmentation and computer-assisted diagnosis, exist as choices that can be made as part of the exploration process. However, the concept of diagnosis based on these methods without, perhaps, ever viewing the primary source images, is new and must be validated for each and every possible diagnosis. Nevertheless, in the new world of $1000+$ images per examination, diagnosis based on review of source images is similarly not yet validated and may, indeed, not even be possible.

This presentation focuses on the motivation and requirements for volumetric visualization of medical imagery as a primary mode of diagnostic interpretation. Examples of our current clinical practice and research in the 3D Medical Imaging Lab at Stanford will be given to illustrate the challenges and potential solutions.

Educational Objectives:

1. To motivate the necessity for changing the diagnostic interpretation paradigm from one of cross-sectional image assessment to volumetric visualization and analysis.

2. To define the requirements for diagnosis by volumetric visualization and analysis.

4. To provide examples of present-day and potential future approaches to this concept.

Travel Funds provided by GE Medical Systems

\section{Symposium Evaluation of Perfusion by MRI}

Room: Taylor

\section{WE-E5-01}

Tissue Perfusion Measurements with MRI, an Overview and Comparision with SPECT, PET and CT

Daryl Bohning, Med. University of SC, Charleston, SC

Tissue perfusion, the rate of exchange of blood in a volume of tissue is quantified by physiologists in milliliters of blood per minute per $100 \mathrm{~g}$ of tissue. It is a measure of the delivery of oxygen and nutrients to the tissue and the simultaneous removal of waste materials, and intimately related to tissue viability. In general, imaging techniques quantify perfusion by monitoring the passage or exchange of some "tracer". Using the principle of conservation of mass, an assumed or measured "tracer" input function, and external measurements (imaging) of the exchange or passage of the tracer, perfusion can be quantified. There are two kinds of tracers, "diffusible" tracers, and intravascular (blood-pool) tracers; both types must be detectable outside the body so they can be imaged. This means that in nuclear medicine techniques, such as single photon emission computed tomography (SPECT) and positron emission tomography (PET), the tracers must be tagged with radioactive isotopes. In contrast-enhanced $\mathrm{CT}$, a nondiffusible contrast agent is used as the tracer, but $\mathrm{CT}$, itself, involves substantial radiation exposure.

With MRI, there are two ways to measure tissue, and neither involves the use of ionizing radiation. The first is by quantifying the exchange of an exogenous blood-pool tracer like Gd-DTPA which has been introduced by injection and the second is by quantifying the local exchange between blood protons which have been "labeled" by RF and gradient manipulation and "unlabeled" blood.

This presentation will describe and compare these methodologies for quantifying tissue perfusion.

Educational Objectives:

1. To understand the distinction between MR measurements of perfusion, diffusion and blood volume.

2. To understand the various methods used for obtaining perfusion maps of the human brain.

3. To understand the differences and similarities of measurements of perfusion with MR, SPECT, PET, and CT.

4. Identify equipment requirements for the performance of MRI perfusion measurements. 
WE-E5-02

The Cerebral MRI Perfusion Study: Promise, Limitations, and Current Relevance in Neuroradiology

Theodore Carl Larson, III, Vanderbilt University Medical Center, Nashville, TN

Cerebral MRI perfusion is based upon rapid imaging of contrast changes, intravoxel, incoherent motion differentiation of diffusion from perfusion, and arterial water spin labeling, both single slice and multislice, using one of several algorithms. The intent of these efforts is to provide relative cerebral blood flow, relative cerebral blood volume, time to peak, and tissue mean transit time. The calculation of milliliters of blood per 100 grams of cerebral tissue per minute can be difficult to precisely quantitate and has led to the use of the term "hemodynamically weighted MRI". MR bolus tracking methods are used to most often generate qualitative data similar to nuclear medicine SPECT. Arterial spin labeling methods under some implied assumptions can generate quantitative perfusion data. Zenon CT and oxygen 15 PET are currently recognized standards for brain perfusion values. Limitations of MR perfusion technology focus upon artifacts, limited signal to noise, and lack of efficiency. Other deficiencies include unwanted magnetization transfer contrast, vascular (transit time) effects, and imprecise arterial inversion. The primary use for cerebral MRI perfusion is in acute stroke, the suggestion being made that an area of diminished cerebral perfusion greater than an area of abnormal brain diffusion indicates an ischemic penumbra prompting clinical consideration of intravenous or intraarterial emergent thrombolysis. The cerebral MRI perfusion results are not always this specific however, and may confound image interpretation and be falsely negative. Reproducibility of noninvasive cerebral MRI perfusion could eventually be applied to cerebral trauma, chronic and degenerative disorders including Alzheimer's disease, metabolic derangements, infectious and inflammatory etiologies, and further extending work that has already been performed with neoplasms.

Reference: Jezzard, P. Advances in Perfusion MR Imaging. Radiology 1998; 208: 296-299.

Educational Objectives:

1. Recognize available methodology used in and data parameters produced by cerebral perfusion MRI examinations.

2. Understand the current limitations of cerebral perfusion MRI technology.

3. List several indications for cerebral perfusion MRI examinations.

\section{WE-E5-03}

Quantitative Perfusion MRI Using Arterial Spin Labeling; Methods and Clinical Applications

John Detre, University of Pennsylvania, Philadelphia PA

Tissue perfusion has classically been measured using exogenously administered tracers. The measurement of true perfusion requires a diffusible tracer and results in a measure of blood flow per unit tissue per unit time, though non-diffusible tracers can be used to measure flow related parameters. The dynamic measurement of the first passage of iv GadDPTA or its static distribution provided the earliest means of determining perfusion effects in humans using MRI. More recently, the use of magnetically labeled proximal arterial water as an endogenous diffusible tracer has allowed true tissue perfusion to be measured totally noninvasively using MRI. Several technical advances in arterial spin labeling now allow perfusion to be reliably imaged in a clinical setting. Clinical applications of perfusion imaging of the brain include assessment of cerebrovascular disease, degenerative disease, and epilepsy as well as the detection of functional or pharmacological changes in regional cerebral blood flow.

Educational Objectives:

1. Contrast measurements of classical perfusion with other hemodynamic parameter

2. Review recent technical advances in perfusion imaging with MRI

3. Understand the rationale for common clinical applications of brain MRI perfusion
Refresher Course

Room: Pearl

\section{TH-A1-01}

Modern Day Linac Acceptance Testing and Commissioning Jatinder Palta, Dept. of Radiation Oncology, University of Florida College of Medicine, Gainesville, FL

The process of purchasing an accelerator starts with evaluation of specifications, performance requirements, and clinical needs by the facility physicist, radiation oncologists, and clinical engineers. Manufacturer and vendor information and performance standards established by national and international agencies (as well as information from other users of the equipment) are used to create written specifications for acceptance testing of the equipment. Site visits may also be needed in order to evaluate equipment performance. Those involved with evaluating equipment and establishing performance requirements should collectively recommend which equipment is selected.

The process of acceptance testing should proceed along the lines of the written specifications and the purchase agreement with the manufacturer. The process must include evaluation of radiation leakage, safety interlocks, mechanical and radiation isocenter tolerance limits, dosimetric characteristics, and software functions. The acceptance testing data are subsequently used to establish a baseline for the tolerance limits and action levels for routine quality assurance procedures.

Commissioning of the linear accelerator includes comprehensive measurements of dosimetric parameters needed to validate the treatmentplanning system(s) for all clinical applications that are planned for that machine. The model-based dose-calculation algorithms (convolution/superposition) require much less measured data than correction-based algorithms (equivalent TPR/TAR etc.). Irrespective of the dose-calculation algorithm, it is essential to have a minimum dataset that includes percentage depth dose, isodose distribution, and output characterization for a series of field sizes. The transmission factor for all treatment accessories and dose calibration for all clinical modes is also required for meterset calculations. It is imperative that the measured dosimetric characteristics of the commissioned linear accelerator are compared with published data on the same make and model, if available. AAPM has several task group reports on special topics such as Total Body Irradiation, Intra Operative Radiation Therapy, Total Skin Electron Therapy, Accelerator Safety, Stereotactic Radiosurgery, and Accelerator Code of Practice that are helpful in commissioning special procedures.

Educational Objectives:

1. Learn to develop equipment specifications, the acceptance testing protocol, and the purchase agreement.

2. Learn to interpret acceptance testing results.

3. Data collection for dosimetric commissioning of the accelerator

\section{Continuing Education Course Special Dosimetry Measurements - 4 \\ Room: Sewanee}

\section{TH-A3-01}

Three Dimensional BANG Polymer Gel Dosimeters

Marek Maryanski, MGS Research, Inc., Guilford, CT

The primary advantage of 3D conformal radiation therapy (3D CRT) is the dose escalation within the target volume that theoretically should increase the therapeutic ratio. The clinical success of this strategy depends however on the precision with which the planned dose distributions are delivered to the patient in three dimensional space. This requires adequate quality assurance methods, including 3D dosimetry using phantoms. The measurement of dynamically delivered dose distributions having very high dose gradients calls for a cumulative 3D dosimetry with millimeter resolution. $1 \mathrm{D}$ or $2 \mathrm{D}$ dosimeters such as ion chambers, diodes or TLD's, film, or plastic scintillators do not fulfill these requirements.

In gel dosimetry, a tissue-equivalent, rigid gel phantom undergoes some measurable chemical change when irradiated, usually as a result of progressive radiolysis of its components. For three dimensional interrogation 
of dose information stored in the gels, MRI or optical transmission CT scanning have been used. Gel dosimeters are used as phantoms for which a 3D treatment plan can be written based on initial CT and/or MRI imaging. The irradiated gels are then imaged optically or by MRI, and the resultant dose maps are compared with the plan, using image correlation and fusion techniques. Various dose mapping programs have been developed for analyzing the data derived from the gels, and more are under development.

Many different types of gel dosimeters have been developed over the past fifty years. Recently, gel dosimeters of a new type, so-called BANG ${ }^{\circledR}$ polymer gels, have been introduced. In these gels, radiation-induced freeradical chain polymerization of various acrylic or vinyl monomers that are dissolved in the gel produces sub-micron sized polymer particles whose concentration increases in proportion to the absorbed dose. Because the particles remain entrapped in the gel matrix and because they scatter light, a permanent 3D image of the dose distribution is formed that can be seen as an immobilized white cloud of dose-dependent density in the transparent gel. Both the optical transmission CT scanning and the MRI provide quantitative measurement of dose distributions in the gel, with accuracy of $3 \%$ or better.

BANG gels are muscle tissue equivalent in both the density and atomic composition. The sensitivity of the gel can be chemically modified for specific applications, so that the maximum dose can be as low as $1 \mathrm{~Gy}$ or as high as 50 Gy.

BANG gel dosimeters have been applied to stereotactic radiosurgery, brachytherapy and IMRT. It is anticipated that with the advent of dynamic 3D CRT and with further development of software for gel-derived data analysis the use of BANG polymer gel dosimeters for quality assurance will become more widespread.

Educational Objectives:

1. Describe the need for 3D, high-resolution dosimetry in 3D CRT.

2. Explain the physics and chemistry of gel dosimeters in general, and BANG polymer gels in particular.

3. List the radiological properties of polymer gel dosimeters.

4. Explain how to use gel dosimeters.

5. Review the clinical applications (3D CRT; SRS; IMRT; Brachytherapy -interstitial, intracavitary, intravascular).

\section{Refresher Course}

Room: Carroll

\section{TH-A4-01}

\section{QA of Teleradiology}

Andrew Maidment, Thomas Jefferson University, Philadelphia, PA

A successful quality assurance program requires four steps: acceptance testing, development of performance standards, diagnosis of performance problems, and verification of corrective action. Medical physicists are familiar with these concepts for image acquisition devices, but these steps also apply to data storage, retrieval, transmission and display in PACS. In this lecture, these concepts will be explored in detail, covering both the design and operation of a PACS.

In designing a PACS, the requirements for redundancy, uptime, fault and failure tolerance, timeliness and appropriateness of service, and their implications on overall system operation must be considered for each component in the system. System designs that are dependent upon single points of failure should be avoided. Such failures can be anticipated by examining the flow of data in a PACS/HIS/RIS environment and determining which hardware and software components are used in each step from admission of patients to the facility until study completion or patient discharge. In the event of failure, contingency plans must exist to allow studies to continue to be produced and read.

Once a system is installed, one must design and use metrics by which to measure performance. In the operation of a PACS, one ultimately wants to deliver a digital study to a physician's review workstation, present that study in the correct layout, orientation, window/level, etc. with the correct demographics, necessary supporting studies and old reports, all with the minimum amount of interaction by the radiologist or other personnel. Each time the PACS fails in one of these tasks the radiologist will be required to take time away from reading images to make corrections. While most often this is only an annoying and inconvenient matter for the radiologist, more serious issues arise when for example the demographics are incorrect or the display is suboptimal, hiding subtle pathology. Both the PACS and the institution must have policies and procedures for dealing with such instances, including the interpretation of the performance metrics. Examples of such metrics will be provided.

Ultimately, it has been our experience that PACS can reduce the workload of $\mathrm{x}$-ray technologists and fileroom personnel by automating the more mundane aspects of their jobs. Film printing, for example, is unnecessary if softcopy review is used. The result is an increase in patient throughput. Unfortunately, facilities do not usually allocate workstations for technologist's preparation and review of the electronic study record. The result is that when errors occur, the radiologist is the one who must respond. This is an inefficient use of radiologist's time. Thus, measures, either electronic or procedural, must be instituted which correct or circumvent as many errors as possible. In designing, purchasing, and operating a PACS one must forever be vigilant of how the PACS will integrate into the department's operation, and ensure the PACS conforms to the methods by which the department operates, rather than the converse.

Educational Objectives:

1. Develop acceptance testing and operating performance standards

2. Develop a QA program for PACS.

3. Develop a policy and procedure manual for PACS

\section{Continuing Education Course Room: Browning A Nuclear Medicine - 2}

\section{TH-A6-01}

Radiation Safety Audits In Nuclear Medicine

Ralph Lieto, Henry Ford Hospital, Detroit, MI

The medical physicist may be given responsibility for the radiation safety of the nuclear medicine department or facility because he/she has primary work obligations in that area or is designated as the Radiation Safety Officer (RSO) on the Nuclear Regulatory Commission (NRC) or Agreement State license.

As background, the NRC strongly emphasizes the current concept of the "management triangle". This concept emphasizes that there are three primary entities that are equally responsible and accountable for the radiation safety program: executive management, the RSO, and the Radiation Safety Committee (RSC). In those small programs without a $\mathrm{RSC}$, the involvement of executive management in routine operations may actually be greater.

The purpose of an audit is to provide the RSO, Radiation Safety Committee (RSC), and management with specific information on the status of compliance with the regulations and facility standards, and the strengths and weaknesses of the program. Early identification of problems and immediate corrective action will be viewed much more favorably than discovery by the regulatory agency.

There are two types of audits that involve the nuclear medicine facility. One is a program audit that reviews the overall radiation safety program which includes all areas using ionizing radiation and reports to executive management. The program audit is done annually. The second type of audit is specific to the nuclear medicine operation and reports to the nuclear medicine director, manager, and/or authorized user, depending on the size of the program. These audits are usually done more frequently to identify problems in a timely manner, but not more often than quarterly. The nuclear medicine area frequency may be specified in the license application or condition.

This presentation will reflect NRC regulated licensees. It must be recognized that the NRC regulations for the medical use of byproduct material (10 CFR 35) are undergoing revision and that some requirements may be modified or eliminated in the future revision. If the final revision is available, the impact of the revised regulations on the audit process will be addressed. 
Educational Objectives:

1. To describe the components for an audit of a nuclear medicine facility or department.

2. To describe the components for an audit of the radiation safety program involving radioactive materials.

3. To provide an audit guide to the medical physicist that will result in confidence that the daily use of diagnostic and therapeutic amounts of radioactivity is done properly.

\section{Refresher Course}

\section{Room: Pearl}

\section{TH-B1-01}

Clinical Implementation of Non-physical Wedges

JohnP. Gibbons and Sha Chang ', Palmetto Richland Memorial Hospital, Columbia, SC (1) UNC School of Medicine, Chapel Hill, NC

Both the enhanced dynamic wedge (EDW) and virtual wedge (VW) treatment modalities produce a profile similar to that of a physical wedge by varying the jaw position and/or output rate during treatment. When compared with physical wedges, these non-physical wedges have several clinically relevant advantages including reduction of treatment time, less scatter dose to peripheral areas and, in many cases, extended field size capabilities. Non-physical wedges also have potential roles in intensity modulated radiation therapy.

The current version of EDW allows for seven possible wedge angles using segmented treatment tables (STTs) which govern the output rate at each position of the moving jaw during treatment. In contrast, the VW offers a continuous selection of wedge angles, produced using an exponential variation of the accelerator output rate based on an analytic equation.

For monitor unit calculations, the inclusion of a single VW or EDW factor is required. In contrast to the wedge factors determined for physical wedges, the EDW factor is strongly dependent on field size, varying by up to a factor of two for the $60^{\circ} \mathrm{EDW}$. The VW factor is very close to unity for symmetric fields. For asymmetric fields, the VW factor demonstrates a much greater variation with off-axis distance than that of EDW.

Most treatment planning systems (TPS) are not originally designed to include non-physical wedge modalities. However, one can incorporate VW/EDW into most of the current systems using TPS-specific methods. Possible methods include photon fluence modeling, physical wedge emulation, and synthesis of two basic wedges. Source modeling plays a more important role in dose computation accuracy for narrow off-axis fields, which are rarely used in static treatments but heavily used in VW/EDW treatment. The dynamic nature of these treatments also post some unique concerns in commissioning and treatment. Because of the considerable differences between physical and non-physical wedges in both their dosimetry and operation, it is vital that clinical physicists gain a thorough understanding of non-physical wedges for their safe and accurate clinical application.

\section{Education Objectives:}

1. Principles and operations of Enhanced Dynamic Wedge (EDW) and Virtual Wedge (VW)

2. Wedge factor characteristics of EDW and VW for MU calculations in symmetric and asymmetric fields.

3. Non-physical wedge beam profile measurements and QA.

4. Commissioning of non-physical wedges in treatment planning systems.

5. Issues and concerns with routine clinical implementation of EDW and VW.

\section{Refresher Course (Session Dedicated to Lionel Cohen)}

Room: Mississippi

\section{TH-B2-01}

Biological Indices in Treatment Planning

Mary Martel, University of Michigan Medical Center, Ann Arbor, MI

Evaluation of 3-D treatment plans is often limited to inspection of dose distributions in 2-D, or at most, to analysis of dose-volume histogram (DVH) statistics. In the last several decades, biological models have been developed that use 3-D dose-volume information as input. The purpose of the models is to predict the normal tissue complication risk or tumor control probability and, so, can be used to "score" plans based on an index of risk versus cure. First, the predictive power of the models is assessed through analysis of complication or control data before they can be considered reliable for clinical treatment planning. Consequently, model parameters may need to be adjusted. Aspects of the models may be used in the design of dose escalation trials to gather normal organ tolerance data in a safe and systematic fashion. Finally, biological indices can be used in cost functions in inverse planning systems to aid in the determination of the "optimal" treatment plan.

This refresher course will attempt to elucidate the:

1. Analysis and correlation of 3D dose distributions with complication or tumor control data;

2. Description and use of biological models to design and evaluate treatment plans;

3. Development of cost or score functions (with biological indices) for treatment plan optimization.

\section{Refresher Course}

\section{Room: Carroll}

\section{TH-B4-01}

PACS, CAD and Informatics Standards

Fred Behlen, The University of Chicago, Chicago, IL

Medical Physicists are frequently called upon to participate in the acquisition of imaging information systems and the implementation of those systems in clinical practice. Physicists involved in research must also be aware of how the fruits of their labors can be integrated into practice in the increasingly electronic imaging enterprise. This tutorial aims to provide foundation and practical knowledge for physicists in these roles.

The successful implementation of an imaging information system - such as a Radiology Information System (RIS) or a Picture Archiving and Communications System (PACS) - involves cultural, business and technical factors which must be brought together into an effective solution. In implementing a solution made from commercial components, one spends one's integration efforts (and funds) on interfaces. When a set of components is purchased as an integrated system, it is not necessary to specify how the components within that proprietary boundary interact. It is between proprietary domains that interfaces must be specified, and if the interfaces are not all to be custom-made, there must be standards to which those interfaces conform. In enterprise-wide systems, a multi-vendor environment is inevitable, and standards are an essential tool for multivendor implementations. But like any tool, skill in their use may be as important as their intrinsic power.

The DICOM standard is a comprehensive specification for defining and communicating information used in digital imaging. Significantly, DICOM also specifies a format for a vendor's claim of conformance to the DICOM standard. Other standards, particularly Health Level Seven (HL7) provide a framework for communicating text-based patient data in the health care enterprise of which imaging is a part. The principal value of these standards in acquiring commercial solutions is as a method of expressing requirements to vendors. Real-world examples and scenarios will be presented.

The extension of digital information infrastructure to support Computer Aided Diagnosis (CAD) will be illustrated. CAD needs to fit into the operations of the digital radiology department if it is to achieve wide clinical use. The question remains of where CAD shall be placed, whether in source devices, display workstations or special $\mathrm{CAD}$ computers. Integrating $\mathrm{CAD}$ into digital imaging infrastructure presents a choice: We must either have standards for interfaces between proprietary domains, or we must bind functionality into a single domain and thereby obviate such interface standards. New draft standards supporting both CAD and general-purpose reporting will be described.

Whether for clinical care improvement or translational research, interface standards remain a valuable tool. An understanding of the organizational, political and business context in which they operate enables these tools to be employed profitably. 
Educational Objectives:

1. Prepare attendee for participation in institutional RIS/PACS procurements.

2. Describe purpose and scope of DICOM and HL7 standards.

3. Show how standards are used in procurement and implementation of systems.

4. Illustrate implementation of CAD in departmental image management systems.

Conflict of interest statement:

Support for the speaker's travel to this conference was provided by ADAC Healthcare Information Systems.

\section{Continuing Education Course Mammography - 5}

Room: Taylor

\section{TH-B5-01}

Digital Stereo Breast Biopsy Systems: Design and Testing

Robert Pizzutiello, Upstate Medical Physics, Inc., Victor, NY

Approximately 3,000 - 4,000 Digital Stereotactic Breast Biopsy (SBB) systems are installed in medical imaging and surgical centers across the United States. In April, 1996 the American College of Radiology introduced its Stereotactic Breast Biopsy Accreditation Program (ACR-SBBAP). This session will review the design considerations of the SBB equipment. The personnel, image quality, dose, $\mathrm{QC}$ and equipment requirements for facilities desiring Stereotactic Breast Biopsy Accreditation, as well as the process to submit the required materials. A status report summarizing the activity and latest changes in the ACR-SBBAP will also be presented. The Medical Physicist's QC testing requirements of the ACR-SBBAP will be reviewed.

Educational Objectives:

1. To state the purpose of the ACR-SBBAP.

2. To clarify the requirements for accreditation.

3. To review the status of ACR-SBBAP.

4. To review the Medical Physicist's QC Tests, per the ACR-SBBAP

\section{Continuing Education Course Room: Browning A Nuclear Medicine - 3}

\section{TH-B6-01}

Quality Control of Scintillation Cameras (Planar and SPECT)

Michael O'Connor, Mayo Clinic, Rochester, MN

Quality control of scintillation cameras starts with acceptance testing of the system. The results from acceptance testing become the foundation stone upon which routine quality control is based. A review of planar QC will focus primarily on intrinsic resolution and uniformity for the range of isotopes in clinical use, with periodic checks on the integrity of the collimators and gantry. Checks should also include evaluation of multienergy registration and count-rate performance. In addition we will discuss the potential pitfalls in planar QC due not to equipment malfunction, but due to errors in the performance of the QC procedures. Such errors include incorrect use of point sources and problems with flood sources.

For SPECT systems, the two most important QC parameters are extrinsic uniformity and center of rotation correction. The review of SPECT QC will focus on system uniformity, how it should be measured, how it relates to the creation of ring artifacts, and how good is good enough for clinical use. We will discuss the required statistical accuracy of the uniformity correction and how it affects both clinical and phantom studies. In addition the special considerations for multi-head systems will be discussed. Center of rotation corrections will be reviewed as they relate to collimator hole angulation, gantry alignment and inter-head alignment and we will discuss the special cases of $90^{\circ}$ dual-head systems and fan-beam collimators. We will review additional SPECT QC procedures that should be performed periodically (1-2 / year) to evaluate overall tomographic performance and changes in system uniformity with rotation.
Educational Objectives

1. To understand the basic QC procedures to be performed on planar gamma camera systems, and the potential pitfalls in QC procedures

2. To understand the requirements for uniformity and center of rotation corrections on single and multi-head tomographic systems.

3. To understand the need for additional SPECT QC procedures, such as measurement of tomographic resolution / uniformity and assessment of rotational uniformity.

\section{Scientific Session Radiotherapy Instrumention II}

Room: Pearl

\section{TH-C1-01}

Motion Detection and Prediction with Fluoroscopy and Visible-light Video to Improve Lung Tumor Radiotherapy

Q Chen*, M Weinhous, R Crownover, J Ciezki, J Greskovich, L Hong, R Macklis, The Cleveland Clinic Foundation, Cleveland, $\mathrm{OH}$

In radiotherapy, target motion is a major obstacle to optimization of some lung tumor treatments. Different breathing monitoring techniques, e.g., airflow, chest motion detection, etc., have been employed in lung tumor treatments. Since those techniques are indirect measurements, the accuracy and reliability remain an issue. In this abstract, we report the development of a tumor motion monitoring system. Tumor locations and motion properties were determined from fluoroscopy. The fluoroscopic videos were then converted to a set of template frames representing a single breathing cycle and providing a mathematical model of the motion. In both the simulation and treatment phases, a visible-light video camera was set to monitor the motion of skin markers placed on patient's chest. Being correlated with the fluoroscopic video, the visible-light video was used to determine the tumor location during the treatment. Since the motion detection process has an inherent time delay, a motion prediction procedure relying on the motion properties extracted from the fluoroscopy was developed to predict the future target position, so as to guide the treatment. Over 20 patients' data have been acquired. Tumor motion has been quantitatively determined. Significant phase discrepancies between fluoroscopic video and visible-light videos have been detected. Phantom simulations demonstrated that the motion monitoring system with prediction capability can minimize the errors introduced by the phase discrepancy and by the time delay of the motion detection process, and allow more conformal treatment fields.

\section{TH-C1-02}

A Small-volume Electron Chamber with Flat Energy Response at all Phantom Depths: Result of an Analysis by Monte-Carlo Simulation and Experiment

U Rosenow*, M Lauterbach, J Lehmann, University of Goettingen, Goettingen, Germany

Plane-parallel electron chambers show a replacement correction factor, $\mathrm{P}_{\text {repl }}$, different from unity at low energies and/or phantom depths other than the depth of dose maximum, $\mathrm{d}_{\max }$. We analyse this behaviour with Monte-Carlo simulation (EGS4) and ionization dosimetry. Simulations are performed for nominal energies of 6,4 , and $2 \mathrm{MeV}$, respectively, and in-phantom depths smaller, equal, and larger (e.g. $\mathrm{R}_{50}$ ) than $\mathrm{d}_{\max }$, and thus cover mean energies at depth, $E_{z}$, down to $1 \mathrm{MeV}$. We vary plate-separation from 1 to $2 \mathrm{~mm}$ and guard-ring width from 0 to $3 \mathrm{~mm}$, a range found in typical commercial electron chambers, such as the Markus, Roos, NACP, and Attix chambers. At the small energies considered the chamber volume extends over a larger portion of the depth-dose curve, leading to dose gradients in the air volume which differ strongly from those in the chamber wall. One effect of this is, with $4 \mathrm{MeV}$ at $\mathrm{R}_{50}$, a $40 \%(10 \%)$ drop in dose due to backscatter in a chamber with $3 \mathrm{~mm}$ guard-ring and of $2 \mathrm{~mm}(1 \mathrm{~mm})$ plate separation, resulting in $\mathrm{P}_{\text {repl }}$ of $1.08(1.02)$. For guard-ring widths of zero to $<2 \mathrm{~mm}$ the in-scatter from the side-wall has also a small effect below $\mathrm{E}_{\mathrm{z}}=6 \mathrm{MeV}$. From these results and dosimetric measurements of a variety of chamber modifications a new chamber was designed with a $1 \mathrm{~mm}$ plate separation, 2 $\mathrm{mm}$ guard-ring and a sensitive volume of $20 \mathrm{~mm}^{3}$ which shows a flat energy response at all depths and relevant energies and high resolution. 


\section{TH-C1-03}

Quality Control Tests of a Commercial Ultrasound Localization System for Image-Guided Radiation Therapy

$\mathrm{R}$ Lalonde*, S McKee, C Campbell, R Riker, E Sternick, NOMOS Corporation, Sewickley, PA

A quality control study was performed for a mobile system for ultrasound soft tissue localization for radiation therapy $\left(\mathrm{BAT}^{\mathrm{TM}}\right)$ with a prototype QA ultrasound phantom. BAT combines an ultrasound imaging system with a digitizing arm for tracking the location of the image planes. The purpose of this test was to determine the consistency of the BAT system in repositioning a test object.

Tests were done in a simulated treatment room containing a therapy couch and wall mounted lasers for alignment. Measurements were taken from scales on the phantom, and from a table positioning device $\left(\mathrm{CRANE}^{\mathrm{TM}}\right)$, accurate to $\pm 0.10 \mathrm{~mm}$. Starting from isocenter, the phantom was moved a random distance in $\mathrm{x}, \mathrm{y}$, and $\mathrm{z}$ directions. BAT was then used to image and align the phantom based upon contours of the phantom contrast objects from a treatment planning system. Tests were repeated using lower resolution contours in order to determine the effect of contour resolution on alignment accuracy.

The mean vector distance between measured and BAT offsets was 1.341 $\mathrm{mm}$ and the standard deviation was $0.686 \mathrm{~mm}$. The difference between the mean measured and BAT-determined isocenter position was $(\mathrm{x}, \mathrm{y}, \mathrm{z})=$ $(0.181,0.098,0.030) \mathrm{mm}$. There was little variation of alignment accuracy with contour resolution. This may be due to the regular geometry of the phantom contrast objects.

This work was supported by NOMOS Corporation.

\section{TH-C1-04}

Saturation Current and Collection Efficiency for Ionization Chambers in Pulsed Beams

F DeBlois*, C Zankowski, E Podgorsak, McGill University Health Centre, Montreal, Canada

Saturation currents $I_{\text {sat }}$ and collection efficiencies $f(V)$ in ionization chambers exposed to pulsed radiation beams are determined assuming a linear relationship between $1 / I$ and $1 / V$ in the near-saturation region, with $I$ and $V$ the chamber current and voltage, respectively. This assumption of linearity enables the determination of $I_{\text {sat }}$ and $f(V)$ from currents measured at only two points in the near saturation region ("twovoltage" technique). However, careful measurements of ionization chamber currents with increasing voltage in the extreme near-saturation region reveal a current rising faster than that predicted by the linear relationship. This excess current, combined with conventional techniques for determination of collection efficiency, results in up to a $0.5 \%$ overestimate of the saturation current. The measured excess current is attributed to a non-dosimetric charge multiplication in the chamber air-volume and may be accounted for by an exponential term $\exp (\gamma W)$ used in conjunction with Boag's equation for collection efficiency in pulsed beams. The relationship between $I$ and $V$ is thus given by $1 / I=\left[1 / I_{\text {sat }}+\lambda_{p} / V\right] \exp (-\gamma V)$, with $\lambda_{p}$ a constant, which depends on chamber as well as air parameters and is proportional to the initial charge density per pulse, and $\gamma$ is the charge multiplication parameter. This semi-empirical model, which accounts for both the dosimetric charge recombination and non-dosimetric charge multiplication effects, follows the experimental data well, and yields values for the saturation current which exclude the non-dosimetric effect of charge multiplication in the ionization chamber

\section{TH-C1-05}

Experimental Versus Calculated Perturbation Correction Factors for Ionization Chambers in Clinical Proton Beams with Energies Below 100 $\mathrm{MeV}$

H Palmans*1, F Verhaegen ${ }^{1}$, J-M Denis ${ }^{2}$, S Vynckier ${ }^{2}$, H Thierens ${ }^{1}$, Department of Biomedical Physics, University of Gent, Gent, Belgium, (2) Cliniques Universitaires St-Luc, Université Catholique de Louvain

In dosimetry of proton therapy beams, potentially systematic errors do still show up, due to uncertainties on $\left(\mathrm{W}_{\mathrm{air}} / \mathrm{e}\right)_{\mathrm{p}}$, water to air stopping power ratios and the lack of information on chamber dependent perturbation correction factors. Especially for the latter, only very little experimental information is currently available. Perturbation correction factors are generally assumed to be equal to one.

In the present investigation, the relative response of in total 18 ionization chambers is evaluated at three proton beam energies below $100 \mathrm{MeV}$. The majority of these chambers have a Farmer-type geometry but consist of different combinations of wall and central electrode materials. All chambers have been calibrated in terms of air kerma as well as in terms of absorbed dose to water in a ${ }^{60} \mathrm{Co}$ beam. This allows the comparison of an air kerma based dosimetry formalism and an absorbed dose to water based formalism. The relative response of the ionization chambers is compared with results of Monte Carlo simulations using PTRAN for proton transport and EGS4 for secondary electron transport.

The measurements show that both approaches for absorbed dose to water determination do not give important relative differences when using the same physical data (IAEA, TRS-277) to calculate conversion and correction factors, but small systematic differences appear when using different wall and electrode materials. Preliminary results of electron calculations indicate that the observed experimental differences might be caused by secondary electron effects.

\section{TH-C1-06}

Analysis of Noise in Calibration of BANG Gel Dosimetry Using Magnetic Resonance Imaging

D Low*, J Markman, J Dempsey, R Venkatesan, E Haacke, J Purdy, Mallinckrodt Institute of Radiology, St. Louis, MO

With the development of conformal radiotherapy, particularly intensity modulated radiation therapy (IMRT), there is a clear need for multidimensional dosimeters. A commercial polymerizing gel, BANG, has recently been developed that shows great potential as a dosimeter. This study investigated the characterization of the signal-to-noise ratio, and analysis of the artifacts from magnetic resonance (MR) imaging.

Seven cylindrical vials (4 cm diameter, $20 \mathrm{~cm}$ length) were irradiated end on. The $R_{2}$ values were combined and fit against the corresponding doses in water. A gel calibration sequence was applied which fit a depth-dose curve in water against the change in solvent-proton $R_{2}$ relaxivity of the gel. A larger vial $(13 \mathrm{~cm}$ diameter, $14 \mathrm{~cm}$ length) was also irradiated to test the calibration. $\mathrm{MR}$ scans $\left(\mathrm{B}_{0}=1.5 \mathrm{~T}, \mathrm{TE}=20 \mathrm{~ms} / 100 \mathrm{~ms}, \mathrm{TR}=3000 \mathrm{~ms}\right)$ were acquired over a series of days to measure the time dependence of the gel response. The calibration proved accurate to within $2.5 \%$ in determining the depth dose measured by the larger vial.

Pixel-to-pixel standard deviation ranged from $20 \mathrm{cGy}$ for unirradiated gel to $70 \mathrm{cGy}$ at $2000 \mathrm{cGy}$. Slice-to-slice deviations were seen that provided 20 cGy systematic variations in dose determinations. These variations limited the overall accuracy of the gel depth-dose determination and warrant an investigation of more accurate MR readout sequences. Noise-power spectrum analysis showed the noise was due to the readout sequence rather than intrinsic gel response fluctuations. 


\section{TH-C1-07}

Spatial Resolution of a Plastic Scintillator Sheet Dosimetry System for Brachytherapy: Measurement and Effect on Dose Linearity

S Devic*, A Kirov, J Dempsey, J Williamson, Washington University, School of Medicine, St.Louis, MO

Plastic scintillator (PS) is a promising 2D dosimeter for brachy-therapy since it possesses a high sensitivity and approximate tissue equivalence. Our 2D dosimetry system consists of a $1 \mathrm{~mm}$ thick PS sheet placed near the brachytherapy source, producing a $2 \mathrm{D}$ optical scintillation image which is captured by a liquid nitrogen-cooled CCD camera with a $512 \times 512$ pixels array. The purpose of this study is to evaluate the 2D spatial resolution of our system by measuring its point-spread function (PSF). PS light profile, produced by a low energy X-ray beam collimated to a $1 \mathrm{~cm}$ wide slit, was deconvolved from radiographic film (RGF) data in order to obtain the line spread function (LSF). The rotationally symmetric PSF was analytically derived from the LSF. The FWHM of the PSF is $4 \mathrm{~mm}$ with slowly decaying tails and is caused by transport, scattering and internal reflection of scintillation photons within the sheet. To assess its dosimetric significance, we convolved the PSF with simulated dose distributions $\left(\mathrm{D} \sim 1 / \mathrm{r}^{2}\right)$ in the PS plane as a function of source-to-sheet distance. The transverse-axis PS signal deviates from relative dose by as much as $50 \%$ due to imaging of light photons arising from ionizing radiation interactions occurring far from the measurement point. Accordingly, deconvolution of the light distributions, measured with the PS, is needed in order to obtain accurate dose measurements.

This work was supported by NIH Grant R01 CA 57222.

\section{TH-C1-08}

Influence of Statistical Fluctuations in Monte-Carlo Dose Calculations on Radiobiological Modelling and Dose Volume Histograms

F Buffa*, A Nahum, C Mubata, Institute of Cancer Research and Royal Marsden NHS Trust, Sutton, Surrey, UK

Monte-Carlo (M-C) dose calculations in radiotherapy can potentially yield highly accurate values for the delivered dose.

Unlike analytical algorithms for dose calculations, M-C provides a mean dose $(d)$ and a statistical variance $\left(\sigma_{d}^{2}\right)$ for each scoring region, i.e. the phantom voxel.

The aim of this work is to analyze the statistical nature of M-C dose distributions by investigating the influence of the statistical fluctuations of $d$ on the dose volume histograms (DVH) and radiobiological models outcome. Whilst the quantities $d$ and $\sigma_{d}$ depend on many statistical and physical parameters; it is assumed here that they only depend on the phantom voxel size and the number of histories.

We have analyzed high-energy photon and electron beams. We have found that when considering nominally uniform dose distributions the $\mathrm{M}-\mathrm{C}$ statistical fluctuations do not affect so heavily the outcome of radiobiological models and $\mathrm{DVH}$; in fact even up to values of $\sigma_{d}$ of about 5$6 \%$ the model answers were varying less than $10 \%$. In contrast when dealing with non-uniform dose distributions the outcomes could be strongly dependent on $\sigma_{d}$

To give an example, we consider here the tumor control probability model when the dose to the target is not homogeneous and contains cold spots. The outcome of the model varies by approximately $10 \%$ when running $10^{7}$ and $10^{8}$ histories respectively; this variation reaches values of about $40 \%$ when decreasing the voxel size from $0.5 \times 0.5 \times 0.5$ to $0.25 \times 0.25 \times 0.25 \mathrm{~cm}^{3}$.

\section{TH-C1-09}

Miniature Proportional Counter Microdosimetry Measurements in Clinical BNCT Beams

J Burmeister*, C Kota, R Maughan, Gershenson ROC, Karmanos Cancer Institute, Harper Hospital, and Wayne State University Detroit, MI

Interpretation of the macroscopic absorbed dose in BNCT is difficult due to variations in $\mathrm{RBE}$ within the beam and due to the microscopic ranges of the products of the boron neutron capture reaction. Proportional counter microdosimetry addresses these problems by providing the single event spectrum of charged particles depositing the absorbed dose in a simulated microscopic site in tissue. A dual tissue-equivalent proportional counter (TEPC) system has been developed utilizing miniature TEPCs, thus permitting measurements in high flux epithermal neutron beams. Loading one of the TEPCs with $200 \mu \mathrm{g} / \mathrm{g}{ }^{10} \mathrm{~B}$ allows the boron neutron capture dose to be obtained through subtraction of the two spectra. This dual counter technique facilitates an assessment of the RBE based on the shape of the charged particle spectrum, as well as a direct determination of the boron neutron capture dose in a subcellular site. Measurements have been made at the MITR-II reactor at the Massachusetts Institute of Technology and the BMRR reactor at the Brookhaven National Laboratory. TEPC measured gamma and neutron doses show good agreement with conventional macrodosimetry methods. However, boron neutron capture doses from TEPC measurements differ significantly from those calculated from foil activation measurements.

This work was supported in part by the U.S. Department of Energy, grant \# DE-FG02-96ER62217.

\section{TH-C1-10}

\section{A Portable PET Camera for Tumor Perfusion Imaging}

J Wong*1, M Oldham ${ }^{1}$, W Worstell ${ }^{2}$, S Dolinsky ${ }^{2}$, V Zavarzin ${ }^{3}$, (1)William Beaumont Hospital, Royal Oak, MI,(2)Boston University, Boston, MA, (3)Tomotronics Inc., Boston, MA

Measurement of the washout of in situ activated O-15 provides a noninvasive approach to study tumor perfusion. At $200 \mathrm{cGy}$, a $29 \mathrm{MV}$ beam from a Elekta medical accelerator produces about 0.2 to $0.4 \mu \mathrm{Ci}$ of $\mathrm{O}-15$ per cc of water. Previous washout studies of in situ activated animal tumors were limited to the determination of decay constants due to the low activity and short ( $2 \mathrm{~min}$.) half-life of O-15. Spatial information was not available. The difficulty can be overcome using a new portable PET camera capable of proximity imaging. The camera consists of a parallel-opposed pair of position-sensitive planar coincidence detectors. Three-dimensional (3D) tomography is produced by resolving the depth of field information through parallax imaging. The detection efficiency is greater than 10 times that of conventional PET scanners. A reconstructed spatial resolution of $3 \mathrm{~mm}$ FWHM has been obtained for a high activity line source. Using a pair of 6 $\mathrm{cm} \times 6 \mathrm{~cm}$ detector modules, 3D PET images were obtained for plastic spheres containing radioactive $\mathrm{H}_{2} \mathrm{O}-15$ in a $14 \mathrm{~cm}$-cubed water phantom. A $1 \mathrm{cc}$ sphere with an initial activity of $0.4 \mu \mathrm{Ci}$ per cc was readily visualized. Work is on going to optimize the spatial and temporal resolution of the system for 3D imaging of O-15 washout.

\section{TH-C1-11}

\section{Photon Activation and Perfusion Measurement}

M Oldham*1, S Sapareto ${ }^{2}$, W Worstell ${ }^{3}$, J Allen ${ }^{4}$, L Kim ${ }^{1}$, J Wong ${ }^{1}$, (1) William Beaumont Hospital, Royal Oak, MI, (2) University Ariz. Health Sci. Cntr., Tuscon, AZ, (3) Boston University, Boston, MA, (4) Elekta Oncology Systems, Crawley, UK

We report preliminary investigations into a new method of measuring tumour perfusion via in-situ activation of oxygen using a tuned Elekta SL25 accelerator. High-energy photons generate radioactive ${ }^{15} \mathrm{O}$ by the 'gamma, neutron' reaction. As most in-vivo oxygen is bound in water molecules, such activation is effectively a method of labeling water. Imaging of the ${ }^{15} \mathrm{O}$ distribution using a highly-sensitive positron-emission-tomography (PET) device can yield a 3D perfusion map. The aim of this investigation was to determine whether clinically useful amounts of ${ }^{15} \mathrm{O}$ could be generated by the Elekta SL25 after tuning the beam to give high energy photons. To be clinically useful, enough ${ }^{15} \mathrm{O}$ must be generated to enable measurement of washout. Our results define the performance limits of such imaging devices.

The activity produced per-unit-dose was measured by irradiating spheres of water to known dose, and then placing the sphere in coincidence-counting apparatus. Calibration data from counting measurements of solutions of known activity, in the same spheres, were used to calculate the activity generated by the tuned accelerator beam. A correction for the 'background' counts signal from the walls of the sphere was made to yield the counts signal arising solely from the activated water in the sphere. The tuned beam generated activity of $0.2-0.3 \mu \mathrm{Ci} / \mathrm{cc} / 2 \mathrm{~Gy}$. Full results will be shown, including the effects of different linac tuning strategies, and images from a new portable PET device. 


\section{Scientific Session Radiotherapy Dose Calculations \\ Room: Mississippi}

\section{TH-C2-01}

Accuracy Tests of the New EGSnrc Monte Carlo System in the Simulation of Ion Chamber Response in Low Energy Photon Beams J Seuntjens*, I Kawrakow, D Rogers, Ionizing Radiation Standards, NRC, Canada

Many applications involving ionization chambers require accurate calculation of ion-chamber response. Since cavity theories are fairly restrictive in their applicability especially at lower energies, accurate Monte Carlo calculation of chamber response is important. We tested the performance of the newly developed EGSnrc Monte Carlo system to calculate ion chamber response in low energy photon beams $(10 \mathrm{keV}-1250$ $\mathrm{keV}$ ) by comparison against the Fano theorem. The response of a cavity with wall and gas of the same material, but only differing in density, is equal to the collision kerma in the wall material, provided corrections are made for photon attenuation and scattering, or provided the fluence is unweigthed for photon attenuation and scattering in the chamber. We compared the performance of EGSnrc for a pancake cavity $(2 \mathrm{~mm}$ deep, $1 \mathrm{~cm}$ diameter, materials: carbon, aluminum, copper) irradiated from end to the Fanopredicted result and to EGS4/PRESTA under similar conditions. The results show that EGSnrc produces step size independent doses, consistent with the Fano theorem at the $\pm 0.2 \%$ level for the materials investigated, and for default parameter settings. EGS4/PRESTA showed its expected step-size dependent behaviour of $5 \%$ for Carbon and worse for other higher $\mathrm{Z}$ materials. The calculated cavity dose converged typically to doses which were up to $2 \%$ wrong, depending on the material, transport parameter settings and photon energy.

\section{TH-C2-02}

Photon Dose Calculations with Kernel Scaling Accounting for Electron Transport

P Charland*, E El-Khatib, BC Cancer Agency, Vancouver, Canada

Most clinical photon dose computations ignore the electron transport perturbations arising at the vicinity of a low density or high atomic number medium. To correct for such heterogeneities, the electron density scaling approach has been modified to incorporate the effect of a change in both stopping and scattering properties of the material. This modified scaling was applied to the energy deposition kernel in a convolution dose model and calculated dose distributions were compared to the corresponding Monte Carlo generated data. The convolution dose predictions including both the stopping and scattering properties of the medium are shown to give better agreement with the Monte Carlo simulation data than the ones obtained with the simple electron density scaling for the high atomic number heterogeneity. There is only a minor improvement with the proposed scaling over the electron density scaling when a low density slab with an atomic number close to that of water was tested. The method is still applicable for media containing heterogeneities of different electron densities and/or different atomic numbers. The proposed scaling method is simple to apply since only a mean energy value was used to obtain the stopping power and the scattering power to scale the convolution kernel.

\section{TH-C2-03}

An Analytical Proton Dose Algorithm

P Zygmanski*1, K P Gall2 , M S Z Rabin ${ }^{3}$, (1) Massachusetts General Hospital, Boston, MA, (2) University of Texas South Western Medical Center, Dallas, TX, University of Massachusetts at Amherst, Amherst, MA

Efficient employment of the superior dose localization properties of proton beams requires fast and accurate dose calculation algorithms. (Hong L, et al, Sandinson, et al) This is especially true in the case of proton Intensity Modulated Radiotherapy. (Deasy) We have developed a macroscopic analytical proton transport algorithm. It uses a simplified model of proton transport, derived from the Fokker-Planck equation, which allows an analytical calculation of dose in an inhomogeneous medium for different beam parameters. The algorithm explicitly accounts for multiple Coulomb scattering effects which are responsible for the formation of cold and hot spots downstream of tissue inhomogeneities. Energy loss is modeled by using experimental energy-range tables for specific materials in a continuous-slowing-down approximation, while energy deposition is modeled by using a measured dependence of dose on depth in water (the Bragg curve) and the concept of water equivalent thickness. Nuclear collisions are accounted for by the inclusion of the experimental Bragg curve data in water. Eyges' solution to Fermi's equation is applied in the treatment of the multiple Coulomb scattering. The simplified model of proton transport is suited to study various numerical implementations and approximations in pencil beam dose calculation algorithms. It is also useful as a mathematical tool to examine techniques of proton inverse treatment planning.

References

Hong L, et al, Phys. Med. Biol. 41, 1305-1330 (1996)

Sandinson, G A, et al, Med.Phys.24, $841-849$ (1997)

Deasy J O, Med. Phys. 25, 476-514 (1998)

\section{TH-C2-04}

Three-Dimensional Treatment Planning for Transrectal UltrasoundGuided Cytolytic Adeboviral Gene Therapy, Part I

$\mathrm{S} \mathrm{Li}^{* 1}$, T DeWeese ${ }^{1}, \mathrm{~N}$ Ramakrishna ${ }^{1}$, N Detorie ${ }^{1}, \mathrm{U}$ Hamper $^{2}$, J Simons ${ }^{3}$, (1) Radiation Oncology, (2) Radiology, (3) Medical Oncology, Johns Hopkins Oncology Center, Baltimore, MD

The primary technical challenge in an interstitial cytolytic adenoviral gene therapy program is the delivery of the virus to the tumor in a safe, efficient, and practical way. A technique similar to the implant of radioactive sources by transrectal ultrasound-guided approach is used but the "dosimetric" considerations include viral diffusion, viral replication, and cell lysis. Ideally, the planning system must also consider the treatment side effects resulting from uptake of virus by other normal tissues. Based on our initial clinic data, we have implemented a simple diffusion dosimetric model into the treatment planning system. Conceptually different from the standard drug or radiation doses, the first-generation virus concentration is defined as the dose in the planning system. The dose distribution following diffusion from a single injection will be used for calculation of the total viral dose distribution from multiple injections. By measuring the first generation dose and the tumor response, we can predict the therapeutic outcome resulting from a uniform gene distribution with no requirement for the monitoring of viral replication. This technique has been applied to our FDA approved protocol, "A Phase I Dose Escalation Trial of the Intraprostatic Injection of CN706, a Prostate-Specific Antigen Gene-Regulated Cytolytic Adenovirus, in Patients with Locally Recurrent Prostate Cancer Following Definitive Radiotherapy."

\section{TH-C2-05}

Three-Dimensional Treatment Planning for Transrectal UltrasoundGuided Cytolytic Adeboviral Gene Therapy, Part II

$\mathrm{S} \mathrm{Li}^{*}$, T DeWeese, M Hamper ${ }^{1}$ J Simons ${ }^{2}$, Radiation Oncology, (1) Radiology, (2) Medical Oncology, Johns Hopkins Oncology Center, Baltimore, MD

The first generation virus is not uniformly distributed inside the target volume due the limited number of injection sites and a limited diffusion range. The structure in a tumor or an organ likely influences the viral distribution. With special attention to the rapid falloff of the doses at the limits of diffusion, a topological optimization is introduced to maximize the volume covered by the prescribed dose, to reduce high dose to the nearby tissue, and to minimize the numbers of injections and needles. The high dose region is determined by the injection uncertainty $(5-\mathrm{mm}$ diameter sphere from a single injection) and any cavity connected to the injection point. The cavities in prostate injection include the urethra, the bladder, and the needle punctures. The specific high dose outside the target volume indicates the wrong deposition or possible lost of the virus through the cavity. The prescribed $100 \%$ dose is the required viral concentration to "kill" the cancer cells. A convolution of the viral injections and the diffusion kernel determines the covered volume by the $100 \%$ dose. Ultimately, this topological optimization will allow us to automatically design an optimal pattern of injections and reduce the planning time such that the pre-plan imaging and the gene therapy delivery can be performed within the same system setup. This rapid dosimetry, planning, and treatment system is desired for gene therapy. 


\section{TH-C2-06}

Dose Perturbation Near Air-Water Interfaces: A Potential Clinical Application

X Allen Li*, C Yu, T Holmes, University of Maryland, Baltimore, MD

The purpose of this work is to study the dose perturbation near air-water interfaces, and to explore its potential clinical application to reduce dose to rectal mucosa during external beam treatment of prostate cancers. EGS4 Monte Carlo was used to calculate the perturbations on beam spectra produced by air cavities and effects of photon-beam quality, size and position of air cavity, and the air pressure in cavity. Three types of cavity shapes (planar slab, disk and cylinder) were studied. Significant perturbation on both photon and electron fluence was observed due to the presence of the air cavity. The calculations show that the dose perturbation near the interface depends on energy of photon beam, size and location of air cavity and field size. The dose reduction near interface increases with the energy of photon beam and cavity size, and can be as high as $35 \%$. The EGS4 results were compared with the published data for an air slab. A general agreement was observed except very near the interface. A potential clinical application of dose reduction near a tissue-air interface is proposed to reduce the rectal mucosal dose if patient rectum is ballooned up during the external beam treatment of prostate cancer. For example, the dose reduction at 0.1 and 0.5 $\mathrm{mm}$ away from the interface in the forward region are $20 \%$ and $10 \%$, respectively, for a $15 \mathrm{MV}$ photon beam. Further pathological and clinical study is required to utilize this approach

\section{TH-C2-07}

\section{Peak Scatter Factors for High Energy Photon Beams}

X Allen Li*, University of Maryland, Baltimore, MD and Rush University, Chicago, IL

The peak scatter factor (PSF) for a photon beam is defined as the ratio of the total dose and the primary dose at the depth of dose maximum in phantom. The values of PSF for photon beams ranging from ${ }^{60} \mathrm{Co}$ to $24 \mathrm{MV}$ are calculated using the EGS4 Monte Carlo technique, to avoid measurement limitations. For ${ }^{60} \mathrm{Co}$ beam, the calculation agrees with the data tabulated on BJR Supplement 25 to within $0.8 \%$. The BJR value (1.054) of $10 \times 10 \mathrm{~cm}^{2}$ for ${ }^{60} \mathrm{Co}$ is $0.6 \%$ lower than the present value due to the underestimation of scatters from the source capsule and collimators. For a given field size, PSF is varied by up to $2 \%$ when beam quality changes from ${ }^{60} \mathrm{Co}$ to $24 \mathrm{MV}$. For normalized PSF, the values of BJR Supplement 25 (which are assumed to be the same for beams ranging from ${ }^{60} \mathrm{Co}$ to $50 \mathrm{MV}$ ) agree well with the present calculation for small field sizes, but are higher than our data by up to $2 \%$ for large field sizes. It is found that the effect of SSD on PSF for high energy photon beams is not significant for small fields, but can be as high as $1 \%$ for large fields. The presently calculated PSFs are related to field size $(s)$ by an empirical expression, $P S F=1+m s /(s+n)$, where $m$ and $n$ are the fitting parameters. This equation describes the PSFs within $0.4 \%(0.15 \%$ on average).

\section{TH-C2-08}

Modeling the Extrafocal Source for IMRT Dose Calculation

S Jiang*, A Boyer, C Ma, Stanford University School of Medicine, Stanford, $\mathrm{CA}$

Accurate calculation of monitor units and dose distributions for intensity modulated radiation therapy requires a precise description of the extrafocal source. An analytical dual source model has been developed to estimate the dose contribution from the focal and extrafocal sources. The extrafocal source is assumed isotropic with the intensity distribution represented by a series of Gaussian functions. The monitor chamber backscatter effect is also taken into account. The model is commissioned by fitting measured head scatter factors. It has been tested extensively for $6 \mathrm{MV}$ and $15 \mathrm{MV}$ photon beams from a Varian Clinac 2300C/D accelerator. For fields defined by the movable jaws, the model can accurately predict the $8 \%$ variation in head scatter factors with field size and the 3\% variation of the jaw exchange effect (consistent with measured values to within $0.3 \%$ ), for source-to-surface distances (SSD) from 80 to $120 \mathrm{~cm}$. For fields defined with the multileaf collimator, the agreement between calculation and measurement is within about $0.5 \%$ for jaws at the recommended positions. This extrafocal source model can be easily incorporated into the conventional or Monte Carlo dose algorithms to predict monitor units and dose distributions accurately for intensity modulated radiation therapy.

\section{TH-C2-09}

Fits to Michigan liver data by the $\mathrm{CV}$ model. The Problem of Correlation Between the Model Parameters.

P Stavrev*, A Niemierko, N Stavreva, M Goitein, Massachusetts General Hospital, Boston, MA

The Michigan liver data were fitted by Jackson's and Niemierko's modifications of the Critical Volume model. The minimum of the logLikelyhood function found by us differs from the one found by Jackson. Considerable correlation between two of the parameters in both model modifications, namely the relative critical volume, $v_{50}$ (or $\mu_{\mathrm{cr}}$ ) on one hand and $\mathrm{D}_{50}$ of an FSU, on the other hand is observed.

When Jackson's modification is used, the correlation plots between $v_{50}$ and $\mathrm{D}_{50}$ show that if we accept all sets of parameters values for which $l<l_{\text {Jacksonat }}$ al then all $\mathrm{v}_{50}$ in the range of $[0.12,0.55]$ and $\mathrm{D}_{50}$ in the range of $[38,340] \mathrm{Gy}$ describe equally well the data (the proper Monte-Carlo investigation may prove it) and one cannot distinguish between them.

Hence, we make the conclusion that either

the data set (DVH,Response) is not full enough in order to decrease the correlation between the parameters

or

parameters correlation is inherent to the model(s) which does not allow the determination of the exact values of $v_{50}$ and $D_{50}$ no matter how full the initial data set $\left(D V H\right.$, Response) is. But for each set of $\left(v_{50}, D_{50}\right)$ the theoretical NTCP values fit and predict well enough the experiment.

\section{TH-C2-10}

Monte Carlo Simulated Data for Dose Perturbation at High-z Interfaces Irradiated by Kilovoltage Photon Beams

I Das*, V Moskvin, F Verhaegen, T Tabata, Fox Chase Cancer Center, Philadelphia, PA, Kharkov State University, Kharkov, Ukraine, Institute of Cancer Research, London, UK, Osaka Prefecture University, Osaka, Japan

Dose perturbations (backscatter dose perturbation factor, BSDF, and forward dose perturbation factor, FDPF) near high $\mathrm{Z}$ material interfaces have been reported to be intense and significant in photon beams for biological and clinical applications. In kilovoltage beams, BSDF and FDPF have been measured up to 20 -fold at $5 \mu \mathrm{m}$ from a $\mathrm{Pb}$ interface that falls off rapidly suggesting that low energy electrons are responsible for the interface effect. The magnitude of dose perturbation has been debated due to limitations in the measuring devices (mainly window thickness). Monte Carlo (MC) simulations have been proposed for interface effects but poor statistics in small bins $(1 \mu \mathrm{m})$ near interface makes such data questionable even with a well designed MC code. The PENELOPE MC code was used for simulation at a $0.1 \mathrm{~mm} \mathrm{~Pb}$ interface with $6 \times 10^{5}$ photons traced to the secondary electrons down to $1 \mathrm{keV}$. A moving window least squares polynomial fit method is used to smooth simulated data. Results of smoothing MC data for BSDF and FDPF are extremely positive. The near agreement of smooth MC data beyond $5 \mu \mathrm{m}$ from interfaces with ion chamber suggests that interpolation of data near interface can be reasonably accepted. Such estimation of dose perturbations is important in the estimation of biological effects of low energy electrons from high $\mathrm{Z}$ interfaces. It is concluded that reasonable accuracy can be acquired to the statistically poor $\mathrm{MC}$ data by moving window least squares polynomial fit to reduce statistical fluctuations and make $\mathrm{MC}$ simulation in a reasonable computation time.

\section{TH-C2-11}

Modification of Input Data for Electron Beam Monte Carlo Calculations

M Bieda*, K Hogstrom, J Antolak, The University of Texas M.D. Anderson Cancer Center, Houston, TX

We are currently investigating whether Monte Carlo simulations can be used to reduce the amount of electron dosimetry data measured as a part of the commissioning process of a medical linear accelerator. One of the most difficult parts of this process is determining whether the description of the 
linac treatment head, as provided by the manufacturer, and the incident electron beam description allow the computer simulation to reproduce the measured data. The EGS4/BEAM system was used to simulate a $20 \mathrm{MeV}$ electron beam from a commercially available linac. For the largest open applicator $\left(25 \times 25 \mathrm{~cm}^{2}\right)$, calculated and measured percentage depth dose matched, but calculated off-axis ratios were up to $10 \%$ lower than the measured values near the edge of the beam. To match measured data, we simultaneously adjusted electron energy and either the primary or secondary foil thickness. We found that doubling the thickness of the secondary foil in the Monte Carlo simulation resulted in significantly better agreement for the cross-beam profile with little effect on the percentage depth dose. In conclusion, it may be necessary to vary some of the treatment head geometry prior to using Monte Carlo calculations for electron beams, and the agreement for the largest field size is a good indicator for whether or not linac treatment head description may be adequate.

This work is supported in part by Varian Oncology Systems sponsored research agreement SR97-015.

\section{Scientific Session Radiation Protection, Health Physics, \& Dosimetry}

\section{TH-C4-01}

Broad Beam Aattenuation Measurements in Kilovoltage X-ray Beams

I Das*, S Akber, Fox Chase Cancer Center, Philadelphia, PA, Marshfield Clinic, Marshfield, WI

Radiation protection barrier calculation for public and radiation worker is directly related to the accuracy of available data that need periodic revision in the context of measurement procedure and the choice of measuring devices. Broad beam attenuation, $\mathrm{BBA}(\mathrm{E}, \mathrm{t}, \mathrm{x}, \mathrm{Z}, \mathrm{V})$ is dependent on the beam energy, $E$, thickness of attenuator, $t$, distance between chamber and material, $x$, atomic number, $Z$ and the ion chamber volume, $V$. This study was undertaken to investigate the effect of $\mathrm{x}$ and $\mathrm{V}$ on the BBA. A Philips Super $80 \mathrm{CP}$ unit with $50 \mathrm{kVp}-125 \mathrm{kVp}$ stations was used. Lead sheet $\left(30 \times 30 \mathrm{~cm}^{2}\right)$ of high purity and precision thickness was used. Six different chambers $\left(6 \mathrm{~cm}^{3}, 15 \mathrm{~cm}^{3}, 60 \mathrm{~cm}^{3}, 150 \mathrm{~cm}^{3}, 180 \mathrm{~cm}^{3}\right.$ and $\left.600 \mathrm{~cm}^{3}\right)$ from various manufacturers were used. The source to chamber distance was kept constant (1 meter) and the distance between chamber and $\mathrm{Pb}$ sheet was varied in the distance range of $0-20 \mathrm{~cm}$. Results show that BBA depends on distance, $\mathrm{x}$ for each station and ion chamber. Smaller distances provide higher transmission. The differences are more pronounced at lower transmission values $\left(>10^{-3}\right)$. The choice of detector was shown not to be critical except for the $600 \mathrm{~cm}^{3}$ volume chamber. In conclusions, broad beam attenuation measurements do not depend on the measuring devices except for a very large ion chamber. However, the distance between attenuator and chamber is critical for the accuracy of attenuation measurements for smaller transmission values where public exposure is most critical.

\section{TH-C4-02}

Radiation Transmission through Lead Equivalent Aprons Used in Cardiac Catheterization Laboratories

J Satti*, M Goodsitt, E Christodoulou, H Chan, University of Michigan, Ann Arbor, MI

A study was performed to investigate the radiation transmission through lead equivalent aprons used in Cardiac Catheterization Laboratories. Real conditions were simulated by using "bad geometry" (beam much larger than ionization chamber) and by placing the chamber on top of a 7" thick Lucite slab to account for backscatter. The typical aprons used in Cardiology have $0.5-\mathrm{mm}$ lead equivalent fronts and sides and $0.25-\mathrm{mm}$ lead equivalent backs. 39 aprons were evaluated. Transmission measurements were made through the $0.25-\mathrm{mm}$ and $0.5-\mathrm{mm}$ sections of each apron at $70 \mathrm{kVp}$ and $100 \mathrm{kVp}$. Transmission through $0.508-\mathrm{mm}$ pure lead was also measured and was found to be $1.1 \%$ at $70 \mathrm{kVp}$ and $5.4 \%$ at $100 \mathrm{kVp}$. At $70 \mathrm{kVp}$ the range of measured $\mathrm{x}$-ray transmission through the $0.5 \mathrm{~mm}$ lead equivalent apron sections was $0.6 \%$ to $8.5 \%$ with a mean value of $2.8 \%$ and a standard deviation (s) of $2.8 \%$. Corresponding values for the $0.25-\mathrm{mm}$ sections were $0.5 \%$ to $8.8 \%$, mean $5.2 \%$ and $\mathrm{s} 2.0 \%$. At $100 \mathrm{kVp}$, the transmission range for the $0.5-\mathrm{mm}$ apron sections was $3.8 \%$ to $7.7 \%$, mean $5.2 \%$, and s $1.2 \%$. Corresponding values for nominal $0.25 \mathrm{~mm}$ sections were $6.7 \%$ to $21.2 \%$, mean $15.0 \%$ and $\mathrm{s} 4.5 \%$. In conclusion there is a wide range of radiation transmission through aprons with the same nominal lead equivalence. Therefore, there is a need for the development of a standardized method for measuring the lead equivalence of aprons, and the specification of acceptable transmission values.

\section{TH-C4-03}

Radiation Safety Program Documentation by Database

R Reiman*, T Yoshizumi, Duke University Medical Center, Durham, NC

Personal computer database management systems (DBMS) have been used to archive and report radiation safety-related licensing information, personnel dosimetry records and radioisotope inventories. We describe a technique in which a DBMS (Microsoft Access) employs stored information to produce Internet-ready documents that are more customizable than those created using standard queries, reports and macros. The method is as follows: (1) Query results or data stored in tables are accessed using textbox controls on a standard form. (2) Modules written in Visual Basic for Applications (VBA) are attached to the form's event procedures. Because VBA is a full-featured programming language, arbitrarily complex logical and mathematical operations can be applied to the numerical or textual data displayed in the controls to compute useful parameters. (3) These parameters are concatenated into character strings containing embedded HTML tags and "boilerplate" document text. Formatting elements (bold/italic typefaces, block quotes, tables, etc.) can be readily incorporated. (4) The character strings are written to HTML text files which can be viewed and printed using any Internet "browser" program. One application of this technique is creating documents required for compliance with the radioactive patient release criteria outlined in NUREG 8.39. From stored data on each patient's discharge exposure rate and home situation, the DBMS estimates doses to the general public and composes a set of post-discharge instructions for the patient, as well as the required dosimetry documentation.

\section{TH-C4-04}

Evaluation of Shielding and Function of a Dedicated HDR Room

M Gillin*, R Zhu, D Grimm, J Rownd, F Lopez, Medical College of Wisconsin, Milwaukee, WI

A dedicated HDR treatment suite has been designed and built on the third floor as part of a Radiation Oncology Clinic. The space above and below the suite is occupied non-controlled offices. The third floor is a controlled area, which is part of the clinic. The entrance is a swinging 1.6 inch lead door with approximately $1 / 8$ inch clearance on the floor and $1 / 4$ inch clearance on the leaded door jam. Radiation surveys have been performed using both G-M and area monitoring devices. The attenuation of the door has been measured directly and found to be approximately 3 TVL's. As expected, the room is over designed and is well within regulatory limits.

Concern has been expressed regarding both the medical emergency situation and the worst case source exposure. An average measured dose acquired by entering the HDR room during the 4 second source transit time is less than $10^{-3} \mathrm{cGy}$. Exposure rates greater than $300 \mathrm{mR} / \mathrm{hr}$ were measured near the door with the source on the floor and the door closed. A procedure has been instituted to limit exposure from any procedure in which the source would be exposed within 12 inches of the floor and 6 feet of the door.

An area monitoring program has been initiated in the offices above and below the HDR suite in an effort to reassure the occupants of those offices.

\section{TH-C4-05}

Evaluation of the Contribution of Capture Gamma Rays, X-ray Leakage, and Scatter to the Photon Dose at the Maze Door of High Energy Medical Accelerators

P McGinley*, A Dhaba'an', C Reft ${ }^{2}$, (1) Emory University School of Medicine, Atlanta, GA, (2) University of Chicago, Chicago, IL

The photon dose in the entrance maze of an $18 \mathrm{MV}$ accelerator room was investigated by means of Monte Carlo simulations and experimental measurements. The Monte Carlo simulations were carried out using MCNP version 4B. Based on the Monte Carlo evaluation and measurements, it was found that the photon dose can be represented by the sum of two exponential functions of distance along the maze centerline. The dose due to head leakage and scattered $\mathrm{x}$-rays was found to be insignificant compared to the 
dose due to capture gamma rays for mazes with length greater than 3 meters. It was concluded that the photon shielding requirements for the maze door are dictated primarily by capture gamma rays produced by thermal neutron interactions with the materials used to construct the maze.

\section{TH-C4-06}

Comissionning of the Isotropic Light Dosimetry System for Photodynamic Therapy

M Solonenko*, T Zhu, T Vulcan, University of Pennsylvania, Philadelphia, PA

Scatter-tip spherical isotropic detectors are used for PDT dosimetry because they are small, account for the tissue back scattered light, and have other advantages. We examined a new isotropic light dosimetry system made of 8 fibers $(250 \mu$ core) with the scatter tip, 8 photodiodes, and 8 preamplifiers connected to a PC. For $730 \mathrm{~nm}$ light, the dosimetry system was found to be linear with accuracy of $5 \%$ for the light fluence rates from $0 \mathrm{~mW} / \mathrm{cm}^{2}$ to $60 \mathrm{~mW} / \mathrm{cm}^{2}$. The response correction factor for water relative to air was found to be $1.6 \pm 0.07$. The wavelength dependence of sensitivity was shown to be non-linear, and corresponding quadratic fit parameters were determined. The angular dependence of the isotropic probe response in air and in water was shown to be approximately flat $( \pm 5 \%)$ in the range of $50^{\circ}$ to $300^{\circ}$. However, it increased by $\sim 30-50 \%$ as the angle between the incident light and the fiber approached $30^{\circ}\left( \pm 2^{\circ}\right)$. The effect was caused by the additional contribution to the signal of the light entering the fiber from the side. Blackening of the fiber $2 \mathrm{~mm}$ away from the tip was shown to correct this nonuniformity of the sensor response.

\section{TH-C4-07}

Patient Radiation Exposures in Cardiac Catheterization Procedures M Pina*, J Deye, INOVA Fairfax Hospital, Falls Church, VA

In recent years, several papers have been published which document skin injuries resulting from radiation to which patients are exposed during cardiac catheterization procedures. FDA Advisories recommend that each institution monitor the radiation used during these procedures and develop protocols to allow the estimation of the entrance skin exposure delivered to each patient. At our institution, we have been using two devices to monitor these doses. The Pemnet system uses real-time measurements of generator technique factors as well as x-ray tube angles, source-to-skin distance and radiation mode to calculate the patient entrance skin dose. The dose-area product (DAP) meter uses an ionization chamber to measure the radiation exiting the collimator of the x-ray tube. By multiplying this value by the area of the beam the DAP meter assigns a number which is independent of the distance of the patient from the source of the x-rays. We will present data gathered in our cardiac catheterization laboratories using these two methods. We will also discuss the effects of such complications as the presence of grafts and multiple lesions on entrance skin doses. The overall goal in our program is to deliver the minimum amount of radiation possible to each patient to achieve the success of the procedure. Besides building a database of entrance doses or dose-area products for each type of procedure, we are also providing the cardiologists with a tool to enable them to remember to use radiation only when absolutely necessary.

\section{TH-C4-08}

Preliminary Results of Nationwide 1998 Pediatric Survey

D Spelic, A Moyal, R Kaczmarek, O Suleiman*, FDA, Rockville, MD

The Nationwide Evaluation of X-ray Trends (NEXT) recently completed a national survey of pediatric radiography. The survey used a phantom that approximates a nominal 18-month-old infant. Several hundred facilities were surveyed including facilities that specialize in pediatric care. This presentation will discuss preliminary results for mean entrance air kerma, radiographic technique factors, use of grids, types of screen and film used, phantom film image quality, film processing quality, and darkroom fog.

\section{TH-C4-09}

Comparison of Two Dosimetry Systems for PDT Light Dosimetry

T Vulcan*, A Yodh, T Zhu, D Fraker, S Hahn, Dept. of Radiation Oncology, University of Pennsylvania, Philadelphia, PA

On-line monitoring of light delivery is essential in PDT to ensure proper dosing to critical structures. Earlier light dosimetry for PDT human clinical trials was performed with a flat photodiode detector system that measured the light flux coming directly from the source. Recently, attempts have been made to use spherical (isotropic) fiber optic detectors that measure the light fluence rate. Decreased angular dependence, much smaller probe size, and easier handling, also favor the use of spherical detectors. The comparison of doses measured simultaneously by spherical and flat detectors, both in tissue phantoms, and in PDT human clinical trials is analyzed here. Patients undergoing PDT were given Photofrin ${ }^{\circledR} 48$ hours prior to surgery. After the debulking surgery, flat photodiodes and spherical detectors were placed side by side in representative locations, and simultaneous in vivo dosimetry was performed with both systems. The readings of the spherical detectors were between 1 and 3 times larger than the readings of the flat detectors, with an overall average of $1.75 \pm 0.23$, independent of location. Thus, for a set value of the dose measured with the flat detectors, considerable variability was found among the measurements made with the spherical probes. Phantom experiments and theoretical considerations indicate that the observed variation in signal can be explained by changes in optical properties of the underlying tissues. The observed heterogeneity provides a measure of in vivo tissue optical property variation, with deeper implications for light penetration and light dosimetry.

\section{TH-C4-10}

Radiosensitivity of Duck Embryo Brains to Arrays of Parallel X-Ray Microbeams and to Broad X-Ray Beams

F A Dilmanian*1, G LeDuc ${ }^{2}$, B Ren ${ }^{1}$, X Y Wu ${ }^{1}, X_{\text {Huang }}{ }^{1}, \mathrm{~J}$ A Laissue ${ }^{3}$, L A Pena ${ }^{4}$, I Orion ${ }^{1}$, P Sathe ${ }^{1}$, W C Thomlinson ${ }^{1}, K_{\text {Trandem }}{ }^{1}$, Z Zhong ${ }^{1}$, (1) Brookhaven National Laboratory, Upton, New York (2) European Synchrotron Research Facility, Grenoble, France (3) Institue of Pathology, University of Bern, Bern, Switzerland (4) Memorial Sloan-Kettering Cancer Center, New York, NY

Microbeam Radiation Therapy (MRT) uses arrays of parallel, synchrotrongenerated x-ray microplanar beam slices (microbeams), each typically 30 $\mu \mathrm{m}$ wide, centimeters long, spaced $\sim 100 \mu \mathrm{m}$ on center, with a $50-70 \mathrm{keV}$ half-power energy. Two effects were observed. First, there is sparing of normal tissues at doses that exceed by many folds the broad beams' tissuenecrosis thresholds. It is assumed that lethally injured endothelial cells lying in the direct paths of microbeams are replaced by endothelial cells that survive between microbeams. Second, single-fraction unidirectional MRT of 9LGS rat brain tumor eliminates the tumor or slows its growth. We used duck embryos to study microbeams' radiotoxicity on growing and developing CNS tissues. Duck eggs were irradiated with single-fraction unidirectional microbeams and broad beams 3-4 days before hatching (i.e., incubation days 24-25). With the eggs upright, x-ray fluorescence radiography was used to position the head, and irradiation over $10 \mathrm{~mm} \times 11$ $\mathrm{mm}$ envelope was administered; angle of the embryo's head to the beam was unknown. Microbeam-irradiated embryos given 78, 156 and 312 Gy in-slice entrance doses and $100-\mu \mathrm{m}$ beam spacing hatched and developed normally to 8 months at almost the same success rate to that of unirradiated ducks. Broad-beam-irradiated embryos given 45 Gy had a median lifespan of 32 days, while those given 15 and 30 Gy developed close to normal. Research supported by the Children's Brain Tumor Foundation and the U.S. Department of Energy.

\section{TH-C4-11}

Polyvinyl Alcohol Hydrogel: A New Non-Toxic Gel Matrix for Optical and MR Fricke Dosimetry

K Chu ${ }^{* 1,2}$, K Jordan ${ }^{1,3}$, J Battista ${ }^{1,3}$, J Van Dyk ${ }^{1,3}$, B Rutt ${ }^{2,3}$, (1)London Regional Cancer Centre, (2)The John P. Robarts Research Institute, and (3)The University of Western Ontario, London, Ontario, Canada

Recent developments in 3D conformal radiation therapy can be validated using Fricke gel dosimetry. The accuracy of these gels is limited because of $\mathrm{Fe}^{+3}$ diffusion after irradiation. Hence, we have developed a low-diffusion Fricke dosimeter hydrogel consisting of $20 \%$ (by wt.) polyvinyl alcohol 
(PVA) in a $50 \mathrm{mM} \mathrm{H}_{2} \mathrm{SO}_{4}$ solution with $0.4 \mathrm{mM}$ ferrous ammonium sulphate and xylenol orange. This optically transparent gel can be used with both $3 \mathrm{D}$ optical CT and MR imaging detection methods. The optical density and $1 / \mathrm{T}_{1}$ dose responses are linear $\left(\mathrm{r}^{2}=0.99\right.$ for both) from 0 to 1000 cGy with a sensitivity of $0.046 \mathrm{~cm}^{-1} \mathrm{~Gy}^{-1}$ (at $543 \mathrm{~nm}$ ), and $0.020 \mathrm{~s}^{-1} \mathrm{~Gy}^{-1}$ (at 1.5T), respectively. The $\mathrm{Fe}^{+3}$ diffusion constant, D, was determined from both optical and MR methods in which an irradiated edge spread function was followed as a function of time. Both these methods revealed that at $25^{\circ} \mathrm{C}, \mathrm{D}$ was $0.14 \mathrm{~mm}^{2} \mathrm{hr}^{-1}$. Several variations of gel recipes were made by varying the PVA concentration from 15 to $25 \%$ (by weight) and the ferrous ammonium sulphate and xylenol orange concentrations from 0.2 to $0.8 \mathrm{mM}$. D was found to be inversely proportional to PVA concentration and was significantly lower by a factor of 2 to 5 times when compared to similar preparations in gelatin or agarose. In addition, because of PVA's purity meeting stringent optical film requirements, it is an ideal dosimetric gel matrix for optical CT and MR detection.

\section{Electronic Poster Displays Ryman Exhibit Hall}

\section{EP-01}

Dosimetric Characteristics of Partially Shielded Rectal Applicators for Clinical Application with VariSource HDR Remote Afterloading Device N Brown*, A Meigooni, L Johnson, University of Kentucky, Department of Radiation Medicine, Lexington, KY

In treating early stage rectal carcinomas, endocavitary radiation using the Papillion Technique has been used as an alternative to traditional treatment with external beam radiation or surgery. The limited volume treated with this system makes it possible to give large doses of radiation per fraction. However, the short distance contact therapy units are no longer manufactured, and the long-term availability of spare parts for existing units is a concern. In addition, the treatment of posterior rectal wall lesions is problematic with these systems. Another problem is that most radiation oncology departments don't treat enough patients with rectal cancers to benefit in the cost of operating a unit.

Alternatively, a newly partially shielded rectal applicator has been designed for use with commonly available high dose rate afterloading systems for endocavitary therapy. The applicators are designed to use $90^{\circ}, 180^{\circ}$, and $270^{\circ}$ annular-segmented tungsten shields or can be used without shielding. These applicators are available in $5 \mathrm{~cm}$ and $10 \mathrm{~cm}$ lengths with diameters of $2.5 \mathrm{~cm}$ and $3.0 \mathrm{~cm}$.

The goal of this project was to experimentally determine the dose distribution around these applicators using a commercial available HDR remote afterloading system. The measurements were performed in Solid Water phantom material using LiF TLD and radiochromic film dosimetry techniques according to AAPM recommendations (TG-43). The results will then be compared with Monte Carlo calculated values. Complete dosimetric data about the applicators will be presented.

\section{EP-02}

Automated Image Based Quality Assurance for Medical Linear Accelerators

L Reinstein, K Welsh*, J Xu, SUNY at Stony Brook, Stony Brook, NY

Regular quality assurance measurements recommended by the AAPM TG40 are an important component of the radiation oncology department's overall quality assurance program. The increased availability of dual photon beam multi-modality treatment units creates a time consuming and exacting task for the medical physicist who performs these measurements.

We have developed an innovative system for computerized image based quality assurance (IBQA) composed of a software program with a corresponding imaging phantom. The phantom is designed for use with radiographic film or EPID and contains a unique set of markers, which establish the coordinate system, $x-y$ magnification factors and the positions of the localizing light-field edges. The IBQA system is capable of automatically determining the required radiation beam QA parameters such as field-symmetry, field-flatness, field-width, field-length, penumbra-width, field-edge orthogonality, as well as the congruence of lightlocalizer/radiation-field and cross-hair centricity. The software is user friendly, based on client-server technology, and is integrated with a database with report writing functionality.
The image processing and registration algorithms used by IBQA software automatically detect fiducial markers, adjust $\mathrm{x}$-y magnifications, correct for image warping and rotations, use fitting and smoothing techniques to improve SNR and rapidly computes all field parameters for QA analysis. The IBQA system was tested with a set of images created under range of accelerator field conditions. It successfully determined beam uniformity, field-size and light/radiation-field coincidence within $\pm 0.5 \%, \pm 0.25 \mathrm{~mm}$ and $\pm 0.25 \mathrm{~mm}$ respectively.

Partially supported by a grant from Nuclear Associates.

\section{EP-03}

PMRelax: An Image Processing Ssoftware Designed for Complete 3Devaluation in MRI Gel Dosimetry

P Magnusson*, L Olsson, Dep. of Radiation Physics, Malmo University Hospital, Malmo, Sweden

Gel dosimetry for radiation therapy using MRI is a new and promising technique. One of the obstacles when bringing this method into clinical practice is the lack of a specially designed software able to convert MRIimages into absorbed dose data and to perform comparisons with dose data from other sources.

Numerous image processing tasks are required for an complete 3D evaluation. A thorough comparison with $3 \mathrm{D}$ dose distributions from dose planning systems requires additional image processing. Commercially available standard image processing software may solve any image processing task. However, for general gel dosimetry purposes such systems involves an extensive effort in programming and/or a non-flexible user interface. These systems are thus not suitable for general dosimeter gel use by medical physicists not initiated in advanced image processing. Therefore, there is a need for a flexible and user-friendly stand-alone software, specifically designed for dosimeter gel evaluation.

In this work such image processing software was developed on a PC-based computer and the Windows95/NT operating system. The software comprises several methods for voxel-by-voxel calculation of T1/T2 relaxation time images for different MRI pulse sequences, using unlimited number of curve fitting points and arbitrary image volume size. The program supports further numerous medical image formats including e.g. DICOM-standard and RTOG-standard used by dose planning systems. Moreover, spatial conversions as translations, rotations and sizing enables complete $3 \mathrm{D}-$ matching and comparison of 3D dose distributions from dosimeter gel measurements and dose planning systems. The software has proven to be a powerful 3D gel dosimetry evaluation tool.

\section{Poster Presentations: Therapy Posters}

\section{Ryman Exhibit Hall}

\section{PO-T-01}

Experimental Evidence of a Wall Effect in Proton Beam Ionization Chamber Measurements

K Gall*1, W Newhauser ${ }^{2}$, (1)UT Southwestern Medical Center, Dallas, TX, (2)Massachusetts General Hospital, Boston, MA

Protocols for Ion Chamber (IC) calibration of Proton Therapy Beams do not take into account perturbation effects from the chamber wall. Several authors have proposed that secondary electrons from the wall of an IC would make an appreciable contribution to the total signal and that this contribution may be energy and material dependent. A recent publication predicts the magnitude of these effects for a variety of materials. (Casnati, et al) We have taken proton depth dose data in an approximately $150 \mathrm{MeV}$ proton beam with two ICs that were geometrically identical but were made of different material. The mass of gas contained in the chambers constructed of A150 tissue equivalent plastic and C552 air equivalent plastic, calculated following TG21, differed by less than 1 part in 5000. Our data show an approximately $2 \%$ difference in the ratio of the signals in the Bragg Peak and plateau region. The C552 chamber accumulates approximately $1 \%$ less signal at low energies and approximately $1 \%$ more signal at high energies when compared to the A150 chamber. This is approximately the same magnitude of the signal perturbation predicted by 
Casnati, et al but is in the opposite direction. These data have direct implications on the total uncertainty of measured absorbed dose in proton beams following current protocols. Reference Casnati, et al, Phys Med Biol, 43,3 1998, 547-558.

\section{PO-T-02}

\section{A Shutter Assembly Design for a Reactor Based Boron Neutron Capture Therapy Facility \\ K Turnbull, A Oliver ${ }^{1}$, C Ramsey*2, (1) Thompson Cancer Survival Center, Knoxville, TN, (2) The University of Tennessee, Knoxville, TN}

Plans are under development to modify the Tower Shielding Reactor at the Oak Ridge National Laboratory into a neutron source for clinical BNCT. Because the treatment field is generated by a reactor, a shutter must be designed to turn the beam on and off. In this work, a mixed neutron/photon shutter and mechanical assembly were designed that minimize the treatment SSD.

The combined neutron and gamma dose rate must be reduced to acceptable limits between treatments, but the thickness of the shutter is limited due to the treatment SSD. Photon and neutron shielding calculations were performed using discrete-ordinates radiation transport codes. A compact laminated shield composed of tungsten and borated polyethylene was designed that minimizes both occupational expose and treatment SSD.

In order to further minimize the SSD, the shutter was positioned above the neutron filter in a vertical shutter/filter assembly. By using this configuration, the space between the reactor and the patient is always occupied by either the shutter or filter. Two steel crossbars were used to support the shutter in the beam off position. When the shutter is lowered into closed position, the crossbars support the shutter section of the assembly, even in case of accidental hydraulics failure. Because the weight of the shutter is supported by the crossbars, the neutron filter can be separated from the assembly and easily replaced with other filters.

\section{PO-T- 03}

Absence of Dose Rate Effects on Optical Response of BANG Polymer Gels at Low and Intermediate Dose Rates

K Shahnazi*, J. Yue, P. Bongiorni, R. Nath, M. Maryanski ${ }^{1}$, Department of Therapeutic Radiology, Yale University, (1) MGS Research, Inc., Guilford, CT

Recently, microscopic optical laser CT scanning of irradiated BANG ${ }^{\circledR}$ gels was proposed as a high-resolution 3D dosimetry for endovascular brachytherapy sources. Absence of dose rate effects is a prerequisite for any 3D dosimeter of brachytherapy sources. In BANG gels, radiation-induced free radical chain polymerization of acrylic monomers dissolved in aqueous gelatin, produces sub-micron sized polymer particles which remain entrapped in the gel, and whose spatial distribution corresponds to the dose distribution. Since each particle scatters light, the optical attenuation increases in proportion to dose. To investigate, two sets of measurements using these gels were done. We irradiated a series of BANG gels $(5 \%$ gelatin, $3 \%$ acrylamide, $3 \%$ bisacrylamide, $89 \%$ water) in glass vials to graded doses up to a dose of 2 Gy using a Cesium-137 irradiator (J. L. Shepard Inc, Mark I), at dose rates ranging from 0.0092 up to $1.53 \mathrm{~Gy} / \mathrm{min}$, and at a dose rate of $3 \mathrm{~Gy} / \mathrm{min}$ using $6 \mathrm{MV}$ and $18 \mathrm{MV}$ x-rays from a linac. Optical density per $\mathrm{cm}$ of irradiated gels was measured in a spectrophotometer, at the wavelength of $633 \mathrm{~nm}$, and we found no dose rate dependence. This result supports the feasibility of using optical CT scanning of gels for measuring dose distributions generated by conventional brachytherapy sources up to a dose rate of $3 \mathrm{~Gy} / \mathrm{min}$.

\section{PO-T- 04}

\section{Evaluation of a p-type Scanning Diode Detector}

A Saini*, T Zhu, I Rebo, J Shi, Hackensack University Center, Hackensack, NJ, University of Pennsylvania, Philadelphia, PA, Hackensack University Medical Center, Hackensack, NJ, Sun Nuclear Corporation, Melbourne, FL

Dosimetrical characteristics of a new p-type scanning diode detector from Sun Nuclear was evaluated by intercomparison with the Scanditronix scanning diode and ionization chamber in a water tank. For electron beams, the new diode agrees with the Scanditronix electron diode to within $0.5 \mathrm{~mm}$ for all electron energies studied $(6 \mathrm{MeV}-22 \mathrm{MeV})$. For open and wedged photon beams, PDD measured with the new diode agrees with the ion chamber and Scanditronix photon diode for small field sizes $\left(\leq 10 \times 10 \mathrm{~cm}^{2}\right)$ under 6 and $15 \mathrm{MV}$, but is different from ionization chamber results for square field sizes larger than $20 \times 20 \mathrm{~cm}^{2}$ and for depths larger than $10 \mathrm{~cm}$ (for $6 \mathrm{MV} \sim(3.1 \pm 1.1) \%$, for $15 \mathrm{MV} \sim(1.85 \pm 0.2) \%$ at depth of $10 \mathrm{~cm}$ ). The scanning diode detector exhibits energy dependence, changing $10 \%$ over the photon energy range between Co-60 and $20 \mathrm{MV}$. The energy dependence is smaller $(\sim 4 \%)$ for electron nominal energy range between 6 and $20 \mathrm{MeV}$. Open and wedge photon profiles measured with the new diode agrees with the ionization chamber and the Scanidironix diode for both 6 and $15 \mathrm{MV}$. However, the wedge photon profiles measured by the new scanning diode are little higher outside of beam collimation. We will also present result for output factors measured with the new diode in water for both photon and electron beams.

\section{PO-T-05}

Monte Carlo Simulations of Miniphantom Measurements

J McDonough*, B Bjarngard, University of Pennsylvania, Philadelphia, PA

Miniphantoms are becoming increasingly important for determining characteristics of photon beams (e.g. head-scatter, beam quality, transmission factors, and beam profiles). The relationship between the dose in a phantom and the ionization collected from a detector in a miniphantom is complex so these beam characteristics are usually described as relative measurements. The implicit assumption made is that the relative response of the detector in the miniphantom does not change during the sequence of measurements. Because of small changes in the photon energy spectrum, that assumption is not strictly valid. The questions addressed in this work are (1) how much does the change in the energy spectrum affect the final result and (2) is there a miniphantom design that minimizes the error. Monte Carlo techniques (BEAM98 and ITS) are used to simulate the photons and contaminating electrons exiting the accelerator. Then simulated experiments of head-scatter and narrow-beam attenuation are performed to determine the energy deposited in miniphantoms. The results for different miniphantom materials and dimensions are examined to isolate the effects of attenuation, lateral and longitudinal electron equilibrium, and phantom scatter in the miniphantom. These results are compared to experiments for $6 \mathrm{MV}$ and $15 \mathrm{MV}$ photon beams.

\section{PO-T-06}

Dose Correlation Of A MOSFET Dosimeter For In-vivo Skin Dosimetry In Dynamic And Omni Wedged 6MV/18MV Photon Beams

L Tao*, A Kalend, University of Pittsburgh Medical Center, Pittsburgh, PA

A MOSFET is an excellent dosimter for in-vivo skin dose determination, but the dosimeter's responses are unknown in dynamic or Omni wedged beams and are here measured and evaluated.

MOSFET dose responses were measured in 6/18 MV beams filtered by 15 60 degree dynamic compared to static Omni wedges and rising oblique incident beams. A solid water phantom was irradiated at various SSD, beam sizes and off-axis distances using a Varian 2100CD and an Elekta SL-20 accelerators. MOSFET readings were correlated to skin doses from a parallel plate chamber.

Results showed MOSFET dose responses were within $2 \%$ of the chamber dose in normal incident beams using metal wedges, and within 3-4\% using low angle dynamic wedges. In highly modulated wedges $\left(60^{\circ}\right)$, the correlation dropped to 5-6\%. MOSFET dose responses appeared linear rather than in inverse squared relations with SSD. Great beam obliquity produced correlation deviations of $5-10 \%$. Skin points at off-axis in oblique beams had largest deviations of $15 \%$ for points lying in the open STT modulation segment.

The present skin dose study shows that MOSFET is a dosimeter suitable for in-vivo dosimetry in dynamic as well as universal wedge photon beams. But specific dose-response calibration of the MOSFET should be made when using large angle oblique incident beams or highly modulated dynamic wedges. 


\section{PO-T- 07}

The Spectral Dependence of the Polarity Effect in Electron Dosimetry

S Szendy ${ }^{1}$, C Ramsey*, A Oliver ${ }^{2}$, (1) The University of Tennessee,

Knoxville, TN, (2) Thompson Cancer Survival Center, Knoxville, TN

Parallel-plate ionization chambers are the instrument of choice for use in electron calibration and dosimetry, but these chambers may exhibit large polarity effects. The polarity effect is a phenomenon encountered when using ionization chambers for electron measurements in which the measured readings vary significantly depending upon whether the bias applied to the chamber is positive or negative.

In this work, the spectral dependence of the polarity effect was evaluated by taking measurements using the same parallel-plate ionization chamber and electrometer combination on various Varian, Mitsubishi, and Siemens linear accelerators. Measurements were also taken on the same Varian 2100C, first with a mica, and then with a kapton beam monitoring chamber.

The polarity effect was evaluated using the "polarity error" formalism, where the correct reading (true ionization charge) is defined as the average of the magnitudes of the positive and negative voltage readings. The "polarity error" is defined as the fractional error between the reading taken at positive bias and the correct reading

The magnitude of the polarity error at low energy varied for each linac due to differences in the electron energy spectrum. For the Varian $2100 \mathrm{C}$, the maximum polarity error was $4 \%$ with mica chamber and $2 \%$ with the Kapton chamber. However, the maximum polarity error was less than $1 \%$ for the Mitsubishi EXL-17DP. Differences in the dose monitoring chambers, energy defining slits or electron collimators can result in differing polarity errors for the same electrometer and chamber combination.

\section{PO-T- 08}

Effects of Buildup Thickness and Material to Diode Detector SSD

\section{Dependence}

J Shi*, W Simon, Sun Nuclear Corporation, Melbourne, FL, L Ding, D

Saini, S Rose, Cancer Care Center, Melbourne, FL,

The change of SSD brings two variations to the detector: a) instantaneous dose rate of the beam; $b$ ) character of the beam scatter and contamination. A theoretical model has been established to analyze the instantaneous dose rate dependence of the diode. ${ }^{1}$ This paper studies the effects of the second variation part on the diode response.

Detectors using the same buildup material (brass) with different equivalent area densities were tested. The percentage difference in detector response at $70 \mathrm{~cm}$ relative to $100 \mathrm{~cm} \mathrm{SSD}$ is: a) $+8.8 \%$ with $0.1 \mathrm{~g} / \mathrm{cm}^{2},+4.0 \%$ with 0.5 $\mathrm{g} / \mathrm{cm}^{2},+1.3 \%$ with $1.5 \mathrm{~g} / \mathrm{cm}^{2}$ and $+1.2 \%$ with $2.5 \mathrm{~g} / \mathrm{cm}^{2}$ for $6 \mathrm{MV}$ beam; b) $+20 \%$ with $0.1 \mathrm{~g} / \mathrm{cm}^{2},+4.6 \%$ with $1.5 \mathrm{~g} / \mathrm{cm}^{2},+3.0 \%$ with $2.5 \mathrm{~g} / \mathrm{cm}^{2}$ and $+2.4 \%$ with $3.5 \mathrm{~g} / \mathrm{cm}^{2}$ for $18 \mathrm{MV}$ beam. The data clearly show that lack of buildup could significantly increase the detector SSD dependence due to the beam scatter and contamination.

Detectors with buildup materials of different atomic numbers (13, 29, 42 and 73 ) but the same equivalent thickness were also tested for both $6 \mathrm{MV}$ and 18 MV beam. The results demonstrate that SSD dependence of the diode detector is almost independent of the mass attenuation coefficient $(\mu / \rho)$. However, with the same equivalent thickness, the sensitivity of the diode detector increases with an increase in $\mu / \rho$ of the buildup material at a specified beam energy.

1. J. Shi et al, Med. Phys., Vol. 23, No. 6, p. 1072, 1996.

\section{PO-T- 09}

Detecting Bending Magnet Drifts on Electron Beams with a

Commercially Available Energy-monitor

B Moftah*, M Evans, M Olivares, E Podgorsak, McGill University,

Montreal, Quebec, Canada

Electron beam quality assurance for radiotherapy requires a routine procedure for monitoring the stability of the energy. Subtle changes in the electron beam energy characteristics equivalent to $2 \mathrm{~mm}$ or less in the electron beam depth parameters may be produced by small drifts in the electron beam bending magnet assembly, and current guidelines recommend a technique which can detect this shift. A commercially available electron beam energy-monitor which uses a linear array of ionization chambers is available for routine electron beam quality assurance. The ability of this device to detect small changes in electron beam energy due to bending magnet drift is evaluated.

High energy electron beams are the most problematic for detecting energy changes when using this device, since the slope of the depth dose curve is shallow, and the relative change in integrated charge is smallest. Hence, high energy electron beams with small energy changes were produced by varying the bending magnet voltage for a nominal $22 \mathrm{MeV}$ electron beam from a CL $2300 \mathrm{C} / \mathrm{D}$ linac. In this manner a family of electron beams was produced with nominal energies ranging from 19.5 to $22.5 \mathrm{MeV}$, and the sensitivity of the energy-monitor was evaluated.

The device produces a 3\% change in signal for a shift of $2 \mathrm{~mm}$ in the electron beam depth characteristics showing that the energy-monitor is suitable for detecting energy shifts due to bending magnet drift even for high energy electron beams.

\section{PO-T- 10}

Sensitivity Of BANG® Polymer Gels To Light, Ultrasound And Shock Waves: Possible New Applications In Medicine

M Maryanski*, MGS Research, Inc., Madison, CT

BANG polymer gels ${ }^{1}$ are increasingly being used for $3 \mathrm{D}$ dosimetry in conformal radiation therapy. In these gels, free radical products of water radiolysis initiate free radical chain polymerization of acrylic monomers which are dissolved in a hydrogel. This results in the formation of submicron size polymer microparticles which remain entrapped in the gel. As their number is proportional to dose, and as they scatter visible light as well as increase the water proton NMR relaxation rates, both MRI and optical CT scanning can be applied to imaging 3D radiation dose distributions in this class of gels.

This presentation reports preliminary results showing that at least two other types of physical interactions promote a similar response in the gel+monomer system. First, it is demonstrated that the gel responds gradually to the blue and ultraviolet components of light, and second, that exposure to $100 \mathrm{kHz}$ ultrasound as well as to acoustic shock waves such as those produced by a lithotripter, also results in the formation of the polymer particles in the gel, in proportion to the dose.

During the presentation the above results will be demonstrated and potential applications in photodynamic therapy (PDT) light dosimetry as well as in measurements of acoustic fields generated by lithotripters and ultrasound scalpels will be discussed.

${ }^{1}$ MGS Research, Inc., Guilford, CT

\section{PO-T- 11}

Dwell Time Effects of Laser Scanner Induced Polymerization of GafChromic Film

B Hasson*, P Sullivan, C Grossman, L Simpson, Temple University Hospital, Philadelphia PA, Hahnemann University Hospital, Philadelphia, PA, Swarthmore College, Swarthmore, PA, Hahnemann University Hospital, Philadelphia, PA

Gaf-chromic film is becoming a standard dosimetry media for the measurement of complex radiation fields. Gaf-chromic MD-55 film is composed of thin colorless polycrystalline substituted diacetylene monomer layers in a polyester base. When exposed to radiation the monomer undergoes a solid state polymerization event that renders a blue coloration of the film. The polymerization can also be initiated with ultraviolet, visible light, or heat. The spectra of irradiated gaf-chromic film exhibits two large absorption peaks at $675 \mathrm{~nm}$ and $610 \mathrm{~nm}$. Optical density (OD) measurements taken at these wavelengths provides a sensitive form of dosimetry. Many dosimetry systems used for precise measurements utilize diode lasers $(\lambda=670 \mathrm{~nm})$ or HeNe lasers $(\lambda=633 \mathrm{~nm})$. 
Using a custom built film scanning apparatus to provide accurate OD measurements, this project has investigated the red light induced polymerization of gaf-chromic film. Two laser sources were chosen to match the large absorption peaks of the MD-55 film $(\lambda=671 \mathrm{~nm})$ and a $\mathrm{HeNe}$ laser $(\lambda=633 \mathrm{~nm})$. A Macintosh computer running Labview ${ }^{\mathrm{TM}}$ was used to record the optical density and set the dwell time.

This project has measured a time variation of the polymerization kinetics resulting in an increased coloration rate as the OD measurement dwell time increases. The change in OD per unit time ranges from $2.0 \mathrm{OD} / \mathrm{min}$. to 0.06 $\mathrm{OD} / \mathrm{min}$. This change in OD effects the slope of the calibration curve and dose measurements made with gaf-chromic film. The slope can change by as much as $10 \%$. The non-linear OD changes with dwell time can introduce considerable uncertainties.

\section{PO-T- 12}

Radiotherapy Characterization of Pelletized TLD-100

F Deibel*, M Weinhous, Cleveland Clinic Foundation, Cleveland, $\mathrm{OH}$

A new physical form thermoluminescent dosimeter, manufactured by pelletizing and annealing TLD-100 powder, was investigated as a disposable dosimeter for radiotherapy applications. Since these $3.6 \mathrm{~mm}$ diameter by $0.6 \mathrm{~mm}$ thick pellets are intended to have greater batch uniformity than TLD chips, we studied the precision and linearity of relative pellet sensitivity. Dosimeters from the same batch were exposed to $1,2,5$, $10,20,50,100,200,500,1000$, and 2000 cGy $6 \mathrm{MV}$ radiation and read after 18-24 hours. The average percent standard deviation was $2.5 \%$. Superlinearity was $12 \%$ at $1000 \mathrm{cGy}$ and $6 \%$ at $500 \mathrm{cGy}$, relative to the quasi-linear 20-200 cGy region. The advantage of these pellets over powder capsules are smaller size, similar sensitivity, similar if not better relative sensitivity (precision) without dependence on powder dispensing technique. The advantage over similar-size reuseable TLD chips includes similar relative sensitivity (precision) without a need to determine and use a perchip calibration factor, and no annealing required. Thus pellets appear superior to chips and powder. We intend to use these dosimeters in parallel with powder capsules to monitor total body (TBI) and total skin (TSET) irradiation in the clinic and report this comparison.

\section{PO-T- 13}

Positioning Accuracy and Reproducibility of a Passive Infrared Marker System

R Boone*, T Solberg, University of California Los Angeles, Los Angeles, CA

We have assessed the accuracy of a novel system for positioning targets at the isocenter of the linear accelerator. With two infrared cameras, the system determines the 3D coordinates of IR reflective markers through stereophotogrammetry. Locating a target relative to the markers, the system positions it at the isocenter of the linear accelerator. Displacement of the target from the intersection of the lasers determines positioning accuracy and reproducibility. For stationary markers attached to a torso phantom, the system consistently repositions the target within $0.5 \mathrm{~mm}$ with no dependence on either number or location of markers. Additional errors from CT localization of the markers and target increase this displacement to $1.0 \mathrm{~mm}$. The $3 \mathrm{D}$ coordinates of the stationary markers do fluctuate slightly; shifting the markers on the surface of the phantom by an even larger amount increases mean displacement depending on both number and location of markers. For shifts of $2.5 \mathrm{~mm}$, displacement ranges from 1.2 to $1.5 \mathrm{~mm}$ for any number of markers. For shifts of $5.0 \mathrm{~mm}$, accuracy improves from a mean displacement of $3.5 \mathrm{~mm}$ with four markers to $1.8 \mathrm{~mm}$ with seven. Mean displacement also improves slightly with markers near the target. Collinear arrangements, however, significantly worsen accuracy. Shifts on graph paper produce similar results in positioning with number and location of markers. These results suggest that the system can consistently position the target within $2 \mathrm{~mm}$ of the isocenter even with marker shifts as large as 5 $\mathrm{mm}$.

\section{PO-T- 14}

Air Kerma Calibration Factors and kch Values for PTW Soft X-ray, NACP and Roos Ionization Chambers at Very Low X-ray Energies (0.035 mm- 1.0 mm Al HVL)

N Ipe*, Stanford Linear Accelerator Center, Stanford, CA, K Rosser, C Moretti, J Manning, M Palmer, Centre for Ionizing Radiation Metrology, National Physical Laboratory, Teddington, UK

Several national and international protocols have been established for the dosimetry of $\mathrm{x}$-ray beams used in radiotherapy. For the very low energy $\mathrm{x}$ rays $(0.035 \mathrm{~mm}-1.0 \mathrm{~mm} \mathrm{Al} \mathrm{HVL})$ only two codes are available: the UK IPEMB Code of Practice and the German standard, DIN 6809 Part 4. The measurement of very low energy x-ray beams is normally performed with parallel plate ionization chambers calibrated at a standards laboratory and characterized by an air kerma calibration factor $\mathrm{N}_{\mathrm{k}}$. According to the IPEMB Code of Practice the absorbed dose in the user's beam should be determined by taking measurements with the parallel plate chamber positioned such that its entrance window is at the surface of a full-scatter water equivalent phantom. The absorbed dose to water can then be determined using an equation which includes a factor, $\mathrm{k}_{\mathrm{ch}}$, which accounts for the change in response of the ionization chamber between the calibration in air and measurement at the surface of the phantom. Measurements were performed at the National Physical Laboratory, UK, to determine $\mathrm{N}_{\mathrm{k}}$ and $\mathrm{k}_{\mathrm{ch}}$ for the PTW soft X-ray, NACP and Roos ionization chambers. A maximum value of 1.09 was obtained for $\mathrm{k}_{\mathrm{ch}}$, for the large soft $\mathrm{x}$-ray chamber at a HVL of $1 \mathrm{~mm}$ of $\mathrm{Al}$, using a solid water equivalent phantom.

\section{PO-T- 15}

CVD Diamonds as Dosimeter for Radiotherapy - An Assessment R Iyer ${ }^{1,2}, \mathrm{C}$ Buttar $^{2}$, (1) Department of Medical Physics, Weston Park Hospital, Sheffield, UK,(2) Department of Physics, Sheffield S3 7RH, UK

Detectors based on polycrystalline Diamond grown by Chemical Vapour Deposition ( CVD ) are being studied for use in Radiotherapy Dosimetry. The tissue equivalence of diamond allows the measurement to be directly used for patient dosimetry and a single diamond detector to be used for both photon and electron dosimetry. The principal problem in using diamond detectors is the dose dependence of its response, known as 'Priming'.

The electrical contacts were made using the thermal evaporation of Gold/Chromium and the leakage current measured using a Keithley Source Measure Unit, varied from pico amperes to nano amperes depending upon the sample and applied voltage.

The radiation sensitivity of the diamond samples was measured in an Orthovoltage x-ray beam. The maximum amplification of the signal after subtracting the background was 1400 , demonstrating a good radiation sensitivity of around $7 \mathrm{nC} / \mathrm{cGy}$. The samples were tested for dose dependent effects by irradiating them to a maximum dose of $48 \mathrm{~Gy}$ in steps of $8 \mathrm{~Gy}$ at a dose rate of $90 \mathrm{cGy} / \mathrm{min}$. For each $8 \mathrm{~Gy}$ step, the diamond took around 4 minutes to stabilise but the long time level was stable after $16 \mathrm{~Gy}$. This is possibly due to traps of various depths filling and emptying. The current also increased with time and stabilised after about 4 minutes. The current was measured as a function of dose rate and $\sigma \alpha \mathrm{D}^{0.9}$ was obtained, also indicating the presence of traps.

\section{PO-T- 16}

\section{Design Of An Attenuating Source Holder For HDR 192Ir} Brachytherapy Calibrations.

S Pai*, L.E. Reinstein, Department of Radiation Oncology, University Hospital, SUNY Stony Brook, NY

A new re-entrant chamber $(\mathrm{SS})^{1}$ with excellent spatial characteristic $(4.2 \mathrm{~cm}$ long plateau of $0.1 \%$ dose variation) and sensitivity has recently become available for HDR and LDR brachytherapy source calibrations. The improved sensitivity of the chamber presents a problem for several commonly used electrometers whose narrow dynamic range $(<199 \mathrm{nA})$ prevents their use in the calibration of $10 \mathrm{Ci}^{192} \mathrm{Ir}$ source. These electrometers require that the SS chamber response $(255 \mathrm{nA} / 10 \mathrm{Ci})$ be reduced by at least $25 \%$. We resolved this dilemma by designing an attenuating source holder using appropriate HVL and mass attenuation coefficient computations. Brass was chosen as the attenuator material due to its high density and ease of 
machining. We calculated $5 \mathrm{~mm}$ thick brass to be sufficient to attenuate the ion-current signal. The length of the attenuator was calculated to be $3.5 \mathrm{~cm}$ based on the HDR source size, length of the active chamber volume, the distances of chamber wall and the attenuator surface from the center of the source. These calculations were verified with in-air transmission measurements using a farmer ion chamber in "good geometry". In the final design, brass cylinder is soldered to the hollow brass tube. Axial response of the chamber is measured by the precise movement of the source attenuator in steps of $0.25 \mathrm{~cm}$, with the source programmed in the center of the attenuator each time. Measurements were repeated and the results were found to be within $0.1 \%$.

(1) Sweet-Spot" (SS) chamber, Model\#34-070-5, Nuclear Associates, Carle Place, NY

\section{PO-T- 17}

Investigation of HU-Electron Density Relationship for 3D Conformal Treatment Planning

J Wang*1, A Osian ${ }^{2}$, N Ikoro ${ }^{1}$, D Nori ${ }^{2}$, (1)New York Methodist Hospital, Brooklyn, NY, (2)New York Hospital Med Ctr of Queens, Flushing, NY

While commissioning a 3D treatment planning system, we have encountered a task to establish the conversion of Hounsfield Units (HU) and relative electron densities (RED). To achieve this, an electron density phantom (Computerized Imaging Reference Systems, Model-62) was used for the study. The phantom consists of 17 holes to accommodate segments of different tissue types, ranging from lung to hard-bone, all of known electron densities. The scans were repeatedly performed in our two GE HiSpeed CT/i scanners under different $\mathrm{kVp}, \mathrm{mA}$, thickness, DFOV, and protocols. Two images were selected from each scan. The average of $\mathrm{HU}$ and its standard deviation within each segment were computed. Calculations were done for all segments in all selected images from different scans.

Our results show that the variation in $\mathrm{HU}$ from different scans using different $\mathrm{mA}$, slice-thickness, DFOV for the same tissue type is minimal. Hence, HUs in all scans under the same $\mathrm{kVp}$ are further averaged across different scanns to derive the final HU-RED conversion, which is best represented by a poly-line in each CT scanner. In addition, it is found that the HU-RED conversion of hard-bone is $18 \%$ higher if scanned at $100 \mathrm{kVp}$, and $9 \%$ lower at $140 \mathrm{kVp}$, compared to that at $120 \mathrm{kVp}$. This is understandable, as photoelectric interaction becomes more dominant at lower $\mathrm{kVp}$ in high $\mathrm{Z}$ material. Therefore, we suggest that the $\mathrm{kVp}$ setting for $\mathrm{CT}$ scans used in $3 \mathrm{D}$ treatment planning shall be kept consistent.

\section{PO-T- 18}

Evaluation of a High Contrast Film/Cassette Verification System AK Ho*, FF Yin, AE Ratkewicz, Henry Ford Hospital, Detroit, MI.

A new high-contrast film/cassette verification system (EC-V) has been introduced recently for the purpose of improving the film contrast in patient treatment verification. The purpose of this study is to perform a clinical evaluation of this system, which is comprised of Kodak EC-L film in combination with either a Kodak EC-V Verification Cassette or a Kodak EC-V Verification Fast Cassette. The EC-V has one phosphor-intensifying screen and is supposedly appropriate for treatment ports with a tumor dose of $90 \mathrm{cGy}$ or higher. The EC-V Verification Fast Cassette has two phosphorintensifying screens and is supposedly appropriate for treatment ports with a tumor dose of about $45 \mathrm{cGy}$. The 2-cassette, Kodak EC-V Verification system was designed to accommodate patients with commonly prescribed curative dose. We first studied the basic characteristics curves of the system and compared with the Kodak Ready Pack V film. In the clinical application, the sites which are most appropriate to use with this 2-cassette system were determined. We also look into the possibilities of using this system for dose verification purposes. The effects of patient thickness, SSD, air gap, and field size on the dose response are investigated. Results indicated that high-contrast system predicts doses more accurately than lowcontrast system. However, short latitude associated with high contrast dictates the proper placement of the film from the source. It requires time and effort to set up the proper technique for clinical use in order to generate an acceptable verification image.

\section{PO-T- 19}

An 8-channel Detector for an Ir-192 Brachytherapy Source-Based Computed Tomography Scanner

A Berndt*, S Rathee, DW Rickey, J Bews, MCTRF, Winnipeg, MB

High dose rate (HDR) brachytherapy (BT) is a well-established cancer treatment modality that requires accurate localization of source holders with respect to the tumor and nearby critical structures. Accuracy of localization is significantly improved with computed tomography (CT). In this presentation we describe a solid state 8-channel detector designed for use in a novel CT scanner that utilizes an ${ }^{192}$ Ir HDR BT source to provide the photons needed to form a image of the patient instead of an $x$-ray tube. Each channel consists of an $0.8 \times 0.275 \times 1.0 \mathrm{~cm}^{3} \mathrm{CdWO}_{4}$ scintillating crystal coupled to a pair of photodiodes, a switched-capacitor integrator, a lowpass-filter-gain stage (gain $=47, f_{c}=339 \mathrm{~Hz}$ ), a sample-and-hold circuit and an 8:1 analogue multiplexer.

The detector response is linear over a range of about $10^{3}$ and the electronic noise floor is $115 \mu \mathrm{V}$. Using a $5.3 \mathrm{Ci}^{192} \mathrm{Ir}$ source located $82.5 \mathrm{~cm}$ from the detector, the signal-to-noise ratio (SNR) with no attenuation in the path of the beam is 118; with $41 \mathrm{~cm}$ of Plexiglas placed between the source and the detector, the SNR is 12. The detector signal is much larger than the electronic noise floor up to at least $43 \mathrm{~cm}$ of Plexiglas, demonstrating that this detector is well-suited for use in an ${ }^{192}$ Ir-based CT scanner. Preliminary images of biological specimens obtained using the detector in a first generation $\mathrm{CT}$ geometry show bones and soft tissue. Images of phantoms show BT needles without beam hardening artifacts.

\section{PO-T- 20 \\ ABSTRACT WITHDRAWN}

\section{PO-T- 21}

Depth Dose Characterization of Narrow Proton Beams

S Vatnitsky*, D Miller, M Moyers, Loma Linda University Medical Center, Loma Linda, CA

Narrow proton beams with sizes down to several millimeters are used for stereotactic radiosurgery of intracranial targets. Depth dose characterization of these narrow beams is complicated by the detector alignment and the effects of proton disequilibrium. In the measurements described the possibility of obtaining depth dose distributions for narrow proton beams has been investigated using a large area parallel plate ionization chamber technique. A waterproof $10 \mathrm{~cm}$ collector size parallel plate ionization chamber with an air gap of $3 \mathrm{~mm}$ was made by Exradin. Chamber was operated at a bias of $1000 \mathrm{~V}$ providing $100 \%$ collection efficiency. Polarity effects were found to be negligible at this bias at different depths within the water phantom. Ionization depth dose curves from the large detector were combined with beam profiles measured at different depths with film to produce point-detector depth dose curves. Converted distributions were compared to data from diode, diamond detectors, and radiochromic film. An analysis of applicability of these detectors for depth dose measurements in narrow proton beams is presented.

\section{PO-T- 22}

Comparisons of Dosimetry for Boron Neutron Capture Therapy M Khan ', L MIller* ${ }^{1}$, G Kabalka ${ }^{1}$, T Nichols ${ }^{2}$, K Hubner ${ }^{2}$, G Smith ${ }^{2}$, (1) The University of Tennessee, Knoxville, TN, (2) The University of Tennessee Medical Center, Knoxville, TN

Boron Neutron Capture Therapy is in Phase I/II clinical trials at Brookhaven National Laboratory and at the Massachusetts Institute of Technology. Several patients with glioblastoma multiforme have been diagnosed with Magnetic Resonance Imaging (MRI) and Positron Emission Tomography (PET) and have received primary care at The University of Tennessee Medical Center at Knoxville (UTMCK).

Doses received by the UTMCK patients from B-10 reaction products were calculated by a Monte Carlo neutron particle transport code (BNCT rpte) where the tumor location and beam positioning specifications were based on MRI scans. Boron concentrations are estimated based on histological information and on the tumor location as determined from the MRI information. These doses were adjusted using a boron agent F-18/BPA in 
PET scans registered with the MRI data used to specify the input to BNCT_rpte. In particular, the calculated doses were scaled based on the relative intensity of the PET images. It is apparent that PET scans define tumor location better than MRI images, and the adjusted doses frequently differ significantly from the calculated doses on the tumor boundaries. This information is correlated with post treatment PET scans and with tumor regrowth

\section{PO-T- 23}

\section{General Tissue Response Model}

P Stavrev*, A Niemierko, N Stavreva, M Goitein, MGH, Boston, MA, MGH, Boston, MA

The purpose of the presented work is to develop a general biological model of tissue response to radiation. In respect to their response to radiation three different types of behavior have been observed in the radiation practice, namely tumor, critical element and critical volume ones. Based on the idea that an organ is comprised of functional subunits several investigators have developed models describing these behaviors for an individual. It is clear from these works that actually there is only one general model of tissue response at individual level. However the only observable quantity is the population response. Bearing in mind that:

- the population response is an average over the response of all individuals comprising the population

- the individual response of an organ is based on FSU survival binomial statistics we explore in a series of numerical experiments the behavior of the population response (multi-parametric multi-dimensional integral) for different values of the model parameters

It is shown that :

- the population multi-dimentional integral could be reduced to simpler forms. However in the process of simplification the biological meaning of the parameters is often lost

- $\quad$ in the case of single partial or whole organ homogeneous irradiation a two parametric $\Phi\left(D_{50}, \sigma_{50}\right)$ function approximates sufficiently well the population integral in the case of heterogeneous irradiation the Lyman model is a good 3-parametric approximation of the population integral

\section{PO-T- 24}

Changes in Proton Radiosurgery Dosimetry at the Harvard Cyclotron Laboratory

K Myers*, W Newhauser, A Smith, Massachusetts General Hospital, Boston, MA

The need for consistency in multi-institutional clinical trials has motivated this study of proton therapy dosimetry. Since 1961, Massachusetts General Hospital (MGH) has treated approximately 3400 patients with brain tumors (arteriovenous malformations, pituitary tumors, metastases) using the 160 $\mathrm{MeV}$ proton beam at the Harvard Cyclotron Laboratory (HCL). The absolute dosimeter used in the HCL beam is a diode, which has submillimeter spatial resolution and is calibrated against a Faraday cup. The Faraday cup methodology has been re-examined in light of discrepancies $(\sim 8 \%)$ revealed in a dosimetry intercomparison (Vatnisky, et al. 1996) A second intercomparison found that the difference between the highest and lowest absorbed dose determinations from 11 participating proton centers decreased to $\sim 3 \%$ when the ICRU proton dosimetry protocol was implemented. (Vatnisky, et al. in press). In this work, we report on our implementation of the ICRU dosimetry methodology for our radiosurgery beams, including calibration of the diode against ionization chambers that are calibrated based on ${ }^{60} \mathrm{Co}$ (NIST-traceable) and the ICRU dosimetry protocol.

\section{PO-T- 25}

Calibration of Gammacell Irradiator for Irradiation of Radiotherapy Polymer Dosimetry Gel

C. Baldock, R. Fitchew, P. Murry, M. Murry, P. Back, T. Kron, Centre for Medical and Health Physics, Brisbane, Australia

A Gammacell is a facility used in research for irradiating specimens. It consists of a central chamber surrounded by a number of Co-60 rods. Such a facility has potential use in the irradiation of radiotherapy polymer dosimetry gels for the purposes of investigating their dose responses. A methodology was developed to calibrate a Gammacell using a PTW 0.125 $\mathrm{cm}^{3}$ Semiflex ion chamber (Type M31002) and IQ4 electrometer. The absorbed dose was measured with the ion chamber for eight different irradiation times. Seven measurements were made at each irradiation time. The relationship between absorbed dose and irradiation time was linear with an $\mathrm{R}$ value approximating 1 , a $\mathrm{P}$ value of $10^{-13}$ and a standard error of estimate in the absorbed dose of $0.03 \mathrm{~Gy}$ for an absorbed dose range up to 20 Gy. This demonstrates that a Gammacell may be used to irradiate polymer dosimetry gels to known

absorbed doses with a high accuracy. The three-dimensional absorbed dose distribution in the chamber of the Gammacell was subsequently determined using a polymer dosimetry gel filled phantom manufactured according to the method published previously (Baldock, 1998).

\section{PO-T- 26}

Effects of Glass and Backscatter on Measurement of Absorbed Dose in Radiotherapy Polymer Gel Dosimetry

C. Baldock, G. Michael, C. Henderson, P. Murry, K. Nitschke, Centre for Medical and Health Physics, Brisbane, Australia

In MRI radiotherapy gel dosimetry a method used to produce a calibration curve of relaxation rate against absorbed dose consists of injecting glass vials, pre-filled with nitrogen, with polyacrylamide gel (PAG) (Baldock 1998). The PAG filled vials are then irradiated using ionising radiation before imaging with MRI. Due to the vials being pre-filled with nitrogen, a nitrogen 'gap' remains in the vial of PAG during irradiation. The pertubation effects on the radiation field due to the glass vial and the lack of scatter due to the nitrogen gap were quantified. Monte Carlo modelling of three geometries was undertaken using MCNP4B for $1 \times 10^{7}$ photon histories. The geometries were (1) a glass vial half-filled with gel with a nitrogen gap, (2) a gel with a nitrogen gap but with the glass vial removed and (3) a gel with both nitrogen gap and glass vial removed. In each case the gel was at a depth of $5 \mathrm{~cm}$ water with an SSD of $100 \mathrm{~cm}$ and a field of diameter $10 \mathrm{~cm}$. The maximum energy was $6 \mathrm{MeV}$. Within an uncertainty of $0.8 \%$ there was no difference in the three calculated absorbed doses indicating the influence of the glass and lack of scatter was negligible.

\section{PO-T- 27}

Radiobiology in the Modern Treatment Planning System: the Fourth Dimension

B Nelms*, T Wolf, Computerized Medical Systems, St. Louis, MO

The development of treatment planning systems has always emphasized increasing the accuracy and speed of dose calculation and, more recently, optimizing the delivery of highly conformal dose distributions. As these advances begin to approach their asymptotic limits, the role of biological modeling becomes increasingly important as the "fourth dimension" of radiation treatment planning. The general predictive models of Tumor Control Probability (TCP) and Normal Tissue Complication Probability (NTCP) have long been utilized to assess treatment plans. In addition, clinical research on the effects of fractionation and dose-rate has introduced "Biological Effective Dose" (BED) as a real consideration in treatment optimization. In this work, we introduce a collection of planning tools to incorporate biological considerations into everyday clinical planning. The TCP tool estimates the tumor control probability based upon the heterogeneous 3-D dose distributions calculated for target structures, and optionally models the effects of inter-patient variability. The NTCP model incorporates the volume dependence and dose tolerance of critical structures in estimating normal tissue complication. Both the TCP and NTCP tools allow the interactive assessment of dose escalation. Fractionation schedules (external beam) and dose-rate dependence (Brachytherapy) are analyzed using the predicted response of early- and late-responding tissues described by BED. Incorporating these and other radiobiology tools into the clinical treatment planning system will hopefully raise awareness of biological effects, and perhaps facilitate more clinical research in this area. The work was funded by Computerized Medical Systems. 


\section{PO-T- 28}

\section{An Absorbed Dose Model for Range-Modulated Proton Beams and} Applications in Quality Assurance

W Newhauser*, H Paganetti, A Smith, S Rosenthal, H Kooy ${ }^{1}$, S Schmidt ${ }^{2}$, Massachusetts General Hospital, Massachusetts General Hospital, Boston, MA, Massachusetts General Hospital, Massachusetts General Hospital, Boston, MA, (1) Massachusetts General Hospital, Boston, MA, Ion Beam Applications, Louvain-La-Neuve, Belgium

The consistency of absolute proton dosimetry has been significantly improved (Vatnitsky, et al. Radiother Oncol, in press) with the adoption of standard methods for reference dosimetry, i.e., at single reference point in a phantom. However, the interpretation of proton dose distributions in depth is, in many cases, difficult due to structure in the peak and/or to limited experimental data. In order to overcome these problems, we developed a model for absorbed dose as a function of depth in a spread out Bragg peak (SOBP). This model accurately describes a wide variety of ideal and imperfect SOBPs. In a fitting procedure, several parameters in the model are allowed to vary simultaneously in order to obtain an optimal fit to the entire measured SOBP. The model parameters obtained from the fitting procedure provide clinically relevant information such as the proximal and distal depths of the SOBP, field flatness, distal falloff width, and skin dose. Measured depth dose data from the Northeast Proton Therapy Center were fitted to the model, including SOBPs that ranged from 4 to $31 \mathrm{~cm}$ in water equivalent depth, and with corresponding modulation widths ranging from 1 $\mathrm{cm}$ to full depth. Monte Carlo simulations revealed how proton scattering and non-elastic nuclear reactions influence the model parameters that predict dose at shallow depths. We propose standard methods for characterizing SOBPs in depth, with the aim of achieving consistency in spatial dosimetry of approximately $1 \%(1$ standard deviation) at $\mathrm{MGH}$ and $3 \%(1 \mathrm{s.d}$.) with other proton therapy centers.

\section{PO-T- 29}

\section{4mm Helmet Factor for Elekta Gamma Knife}

S Goetsch*, San Diego Medical Physics, San Diego, CA

Elekta Instruments, A.B. of Stockholm has recently recommended increasing the helmet factor for the $4 \mathrm{~mm}$ helmet (relative to the $18 \mathrm{~mm}$ helmet) from 0.80 to 0.87 for the 100 plus Leksell Gamma Knife users world-wide. This recommendation was based on recent work with liquid ionization chambers as well as revised Monte Carlo calculations. A corresponding reduction in prescribed dose of $9 \%$ was also recommended for single shot plans with the $4 \mathrm{~mm}$ helmet, in order that the actual dose delivered remain constant. North American users were surveyed to discover how many had attempted to measure the $4 \mathrm{~mm}$ helmet factor and what their results were. A total of 14 of the 45 North American centers reported measurement results. Only 3 centers chose to use their own results clinically, all other centers relying instead on the old helmet factor, which dates from the first Elekta Gamma Knife delivered in 1987. Nine radiochromic film measurements yielded an average helmet factor of $0.830+/-0.024$. Three measurements with X-ray film yielded an average factor of $0.782+/-0.024$. Eleven TLD measurements yielded an average helmet factor of $0.829+/$ 0.021 . Four ion chamber measurements yielded an average helmet factor of $0.644+/-0.020$. The sole MOSFET measurement gave 0.750. Low results for the ionization chamber are probably attributable to partial volume effect. North American results average about 4.5\% lower than the new manufacturers factor. Measurement of small high energy photon fields used in stereotactic radiosurgery remains a challenging problem.

\section{PO-T- 30}

Quantification of Intraoperative Displacement of Deep Structures in Neurosurgery Using 3D Ultrasound

A Hartov*, S Eisner, D Roberts, K Paulsen, L Platenik, M Miga, Dartmouth Medical School, Dartmouth College, Hanover, NH

Image-guided neurosurgery directed by a preoperative imaging study, such as MRI or CT, can be very accurate provided no significant changes occur during surgery. A variety of factors are known to affect brain tissue movement and are not reflected in the preoperative images used for guidance. In a previous study we have quantified the amount of displacement that can be expected during neurosurgery for cortical features 1 and found it to be on the order of $1 \mathrm{~cm}$, predominantly in the direction of gravity. In this study we investigated the displacement of deep structures using a hand-held ultrasound scanhead whose position and attitude in space were monitored with a 3D magnetic tracker2. In another study3 we have found that using a stylus rigidly coupled to our $3 \mathrm{D}$ tracker we were able to locate a point with an overall error of $1.36 \mathrm{~mm} \pm 1.67 \mathrm{~mm}(\mathrm{n}=39)$. When coupling our tracker to an ultrasound scanhead, we found that we could locate features appearing on ultrasound images with an error of $2.96 \mathrm{~mm} \pm$ $1.85 \mathrm{~mm}(\mathrm{n}=58)$. To conduct this study we reconstructed features appearing in sequences of $2 \mathrm{D}$ ultrasound images in $3 \mathrm{D}$. By using image sequences acquired at the beginning and end of surgery, and which encompassed the same features, we were able to determine the amount of shift taking place subcortically based on the relative position of the same features.

\section{PO-T- 31}

Automated Repositioning for Body Stereotactic Radiosurgery

S Benedict*, R Cardinale, R Zwicker, E Bump, R Mohan, Virginia Commonwealth University, Medical College of Virginia Hospitals, Richmond, VA

A new system utilizing reflective markers placed on the patient and visible by IR and video cameras mounted in the treatment room has been developed by BrainLab, Inc. for automated repositioning for extracranial stereotactic radiosurgery (Body-SRS). The reflective markers are strategically placed during CT simulation on the patient immobilized on a carbon fiber table insert with vacuum-lock styrofoam. The markers are localized by the planning system to identify the treatment isocenter. The system is relatively easy to install and may utilize up to 7 markers for improved accuracy agreement. Calibration procedures have been developed to insure that the cameras accurately identify the treatment isocenter $(<1 \mathrm{~mm})$ using jigs designed for spatial and isocenter measurements. Patient repositioning in the treatment room is performed using an automated computer controlled pendant, which moves the couch into position with input from the infra-red cameras in the room. A live image may be captured from the video camera which can confirm the correct treatment position, and perform comparisons and image subtractions with subsequent treatments. Modifications of the system in development include a custom belt for shallow breathing, and techniques to insure accurate relocalization of the markers. An IRB approved protocol has been established for fractionated Body-SRS at our institution which utilizes the system clinically. The study tests delivery of three fractions of Body-SRS using the micro-MLC for conformal treatments. This presentation will discuss the problems and approaches to immobilization, repositioning, and dosimetry that will be undertaken in order to provide accurate and safe treatments.

\section{PO-T- 32}

Angular Measurement of the Emitted Radiation Spectrum from the Leksell Gamma Knife

R Drzymala*1, J Sohn ${ }^{1}, \mathrm{C} \mathrm{Guo}^{1}$, L Sobotka ${ }^{2}$, (1)Washington University School of Medicine and (2)Washington University, St. Louis, MO.

The photon radiation spectrum emanating from a Leksell Gamma Knife, model $\mathrm{B}$, was measured between $80 \mathrm{keV}$ and $3.5 \mathrm{MeV}$ with the sources exposed. In order to make the measurements, a $2 \times 2$ inch NaI detector was enclosed in a shielded apparatus having a $1 / 4$ inch measurement aperture, thereby reducing the amount of radiation received by the crystal. All measurements were made 1 meter above the floor within a quadrant toward one side of the Gamma Knife couch. Spectra displayed a doublet of photon peaks with both maxima being greater than $1.0 \mathrm{MeV}$, but shifted to lower energies than the cobalt-60 doublet. These peaks appeared in spectra beginning at angle around 54 to 59 degrees as one proceeds from a point directly lateral to the source enclosure ( 0 degrees) toward the foot of the couch (90 degrees). The average photon energy of the spectrum shifts to lower values and the doublet decreases in magnitude with increasing angle until almost vanishing at an angle equal to 90 degrees. Inserting the $16 \mathrm{~cm}$ sphere phantom, provided with the Gamma Knife, into the radiation beams increases the low energy photon emissions appearing in the spectrum somewhat, especially for measurements at the foot of the couch. Our results impact on the design of the shielding of a room containing the Gamma Knife, Model B. The data is also useful when estimating the radiation exposure to personnel during an emergency procedure in the treatment room with the sources exposed. 


\section{PO-T- 33}

Automatic Verification of the Isocenter in Stereotactic Radiosurgery E Bolla*, C Cauzzo, G Gennaro, L Riccardi, F Simonato, R Zandonà, R Fabbris, Medical Physics Department, Azienda Ospedaliera di Padova, Padua, Italy

A standard portal film-based procedure is commonly used to verify the isocenter position in linac stereotactic radiosurgery. Images of a radiopaque spherical pointer, produced on the same verification film for different gantry positions, are digitized by a high resolution CCD camera. A computer routine was developed in order to recognize the pointer image within the radiation field image. The algorithm is based on the analysis of the gray level histogram and calculates the pointer center position by means of a second order polynomial fit. The radiation field center can also be determined from its penumbra by extracting a circular profile. Pointer misalignment is obtained as the shift between the pointer and the field centers with respect to an orthogonal frame. Our method was compared to the proposed standard, which is based on visual inspection. Several pointer images were taken and the related precision was evaluated. We determined a $0.03 \mathrm{~mm}$ accuracy for our method. As the automatic analysis excludes the human error, it was also possible to find the magnitude of the setup reproducibility, which does not depend on the chosen method and was calculated to be $0.15 \mathrm{~mm}$. From the error propagation formula a $0.14 \mathrm{~mm}$ reproducibility was obtained for the standard procedure.

\section{PO-T- 34}

Clinical Implementation and Verification of a Fractionated Stereotactic Cranial Immobilization System

M Plunkett*, W Shea, J Lukens, G Gade, R Mackintosh, Hoag Cancer

Center, Newport Beach, CA,

Purpose: To implement and measure treatment setup error of a relocatable head frame (BrainLAB) used for multifraction linac-based stereotactic radiotherapy (SRT) of intracranial lesions.

Methods: 32 patients were treated with a total of 639 SRT fractions. A custom multi-layer thermoplastic mask was formed and attached to a stereotactic head frame. External and internal fiducials were identified by the placement of BB's at tattoo points on the patients' skull, and clips placed at the time of surgery respectively. Diagnostic and megavoltage orthogonal film pairs were obtained with a localizer box to define the fiducial points in 3D stereotactic coordinate space. These data were compared against 25 external Depth Helmet ${ }^{\mathrm{TM}}$ (Radionics) measurements obtained at each fraction.

The observed deviations from 3D film localization were: Internal Clips, $\mathrm{AP}=0.6 \pm 0.8 \mathrm{~mm}($ mean $\pm \mathrm{SD})$, Lateral $=0.4 \pm 0.7 \mathrm{~mm}$, Vertical $=0.9 \pm 1.1 \mathrm{~mm}$ and External BB's, $A P=0.8 \pm 0.6 \mathrm{~mm}, \quad$ Lateral $=0.6 \pm 0.9 \mathrm{~mm}$, Vertical $=1.0 \pm 1.3 \mathrm{~mm}$. The Depth Helmet $(\mathrm{DH})$ setup measurement errors were $\mathrm{AP}=0.3 \pm 0.1 \mathrm{~mm}$, Lateral $=0.3 \pm 0.1 \mathrm{~mm}$, and Vertical $=0.5 \pm 0.1 \mathrm{~mm}$. Conclusions: This system was used to deliver precise conformal fractionated radiotherapy while reducing treatment morbidity associated with single fraction radiosurgery. The system has been well tolerated by conscious patients as young as 6 years old as well as patients with dentures. Setup and treatment time is less than 20 minutes. The setup error measured with orthogonal films is larger than the Depth Helmet method most likely due to limited resolution of the DH rod as compared with film scanning and geometric projection methods to analyze the BB positions.

\section{PO-T- 35}

Comparison of an In-house Developed Treatment Planning System, a Commercially Available System and a BETA-version for Linac Based Radiosurgery

D Verellen*, N Linthout, AZ-VUB, Brussels, Belgium

Linac based stereotactic radiosurgery (SRS) has been introduced in our department in 1992 and more than 200 patients have been treated since. An in-house developed algorithm for target localization and dose calculation has been replaced with a commercially available system (BrainSCAN v3.1). Both systems as well as a BETA-version (v3.6) from the same company have been compared, and positional accuracy and dose calculation have been verified experimentally. The full SRS procedure applied to an anthropomorphic phantom has been used as a comprehensive method to assess the uncertainties involved in dose delivery and target positioning. The dose calculation algorithms have been verified against manual calculations and measurements with the anthropomorphic phantom applying ionization chamber, thermoluminescent detectors as well as radiographic film. The image correlation of CT and MRI has also been verified experimentally and mutually compared in clinical situations. Phantom studies have shown that a target, localized by CT, can be irradiated with a positional accuracy of 0.08 $\mathrm{cm}$ in any direction with $95 \%$ confidence. Neglecting the influence of dose perturbation when the beam passes through bone tissue or air cavities the calculated dose values obtained from the 3 systems agreed within $1 \%$ (SD: $1 \%$ ). The application of a 1 dimensional path length correction for tissue heterogeneity influences the treatment prescription with $4 \%$ on average (SD: $1 \%$ ) which is in compliance with the theoretical predictions.

\section{PO-T- 36}

I. Setting Standards for Relocatable Stereotactic Head Frames: Proposals

A Lightstone*, R Ramani, Department of Medical Physics, TorontoSunnybrook, Toronto, Ontario, Canada

I. Setting standards for relocatable stereotactic head frames: Proposals

The head frame system used to immobilize and reproducibly relocate a patient is critical to fractionated high precision conformal stereotactic radiotherapy of intra-cranial lesions. Unfortunately, there are no widely accepted standards of performance, safety and documentation. Organizations such as the AAPM are now considering setting guidelines. The authors propose the following items be quantitatively and qualitatively assessed:

1. Design Concept: method of immobilization and method of relocalization, long-term mechanical rigidity, problem areas, restraining forces, alignment of the neck, frame attachment to various couches, and compatibility with fiducial co-ordinate hardware.

2. Patient Comfort and Safety: comfort, a quick release mechanism, patient hair, use of dentists for bite-blocks, and a formalized list of problem patient characteristics.

3. Compatibility with MRI, CT and Radiotherapy Equipment: MRI artifacts and distortion, frame use in an MRI head coil, CT artifacts, documentation of use in other radiotherapy installations, portal imaging accessories, collision risks, collision interlocks, and beam attenuation factors.

4. Positioning Accuracy: measurements should describe results taken on different days over many areas of the head (including the posterior and superior), using standardized methodology and terminology.

5. Quality Assurance: QA technique, QA technique validation, time required, portal images.

6. Therapist Training: mandatory training program, staff consistency, daily QA, checklists, and a head frame system which is robust and requires few subjective decisions.

\section{PO-T- 37}

A Method for Accurate Conversion of PDD to TMR Values

M Essers*, L van Battum, P Storchi, University Hospital Rotterdam / Dr. Daniel den Hoed Cancer Center, Rotterdam, The Netherlands

Tissue maximum ratios (TMR) are required as input for many treatment planning systems. However, some water phantom systems are not equipped with a TMR measurement option. Therefore, measured percentage depth doses (PDD) need to be converted to TMR.

Several existing methods express the TMR in terms of PDD, phantom scatter factor $\left(\mathrm{S}_{\mathrm{p}}\right)$, and inverse square law. However, the collimator scatter factor needs to be measured to obtain $S_{p}$. For field sizes smaller than the build-up material required for lateral electronic equilibrium, e.g., necessary for stereotactic treatments, such measurements are not reliable and can cause errors in determined TMR values.

To avoid this problem we have developed an alternative method to obtain TMR from PDD. The absolute doses are expressed in terms of PDD, total scatter factor and inverse square law. For each depth, the dose is fitted to a double exponential as a function of field size. The TMR is calculated by taking the ratio of this function at the depth of interest and the reference depth for the correct field sizes. 
The method was applied to obtain TMR values for our stereotactic treatment planning system. For small square and circular fields, calculations were compared with diode measurements. The agreement was always within $1 \%$, which proves that this relatively simple conversion method is very accurate and can be used routinely.

\section{PO-T- 38}

Setup Verification in Linac-Based Radiosurgery

T Falco*, M Lachaine, B Poffenbarger, E Podgorsak, G Fallone, McGill

University Health Centre, Montreal, Canada

A semi-automatic technique has been developed for direct setup alignment to treatment room laser cross-hairs of radiosurgical circular fields from an isocentric linac. Alignment without an intermediate step is achieved by acquiring an image of the treatment room positioning laser cross-hairs superimposed on the radiosurgical circular field image. Two types of detectors, each sensitive to both the laser light and ionizing radiation, may be used to acquire the laser/radiation images. The first detector consists of a $0.3 \mathrm{~mm}$ thick layer of photoconducting amorphous selenium deposited on a copper plate and the second is film. An algorithm uses the laser/radiation image to calculate the center of the radiosurgical field image as well as the intersection of the laser cross-hairs. This determines any alignment deviations, and the information is then used to translate the radiosurgical collimator to its correct aligned position. The algorithm and detector system can detect deviations with a precision of $\sim 0.04 \mathrm{~mm}$.

Once the radiosurgical field is aligned to the ceiling laser, the alignment technique can be extended to determine deviations of the gantry and couch axis-of-rotation from the nominal linac isocenter. A device, resembling a gyroscope, was built in our machine shop for this purpose. It is attached to the treatment couch and holds a piece of radiographic film perpendicularly to the beam axis at the linac nominal isocenter for all possible gantry and couch angles. The device allows us to determine with relative ease the effective diameter of a linac nominal isocenter.

\section{PO-T- 39}

The Use of Laitinen Stereoadapter and Glad-X Relocatable Frame in Stereotactic Radiotherapy

N Ikoro*, H Ashamalla, D Gideo-Addeo, M Cosma, P Ross, NY Methodist Hospital, Brooklyn, NY

A population of our SRT patient with dentures treated with GTC relocatable frame show reproducibility problems and greater degree of inaccuracy. Some of these patients found the GTC intolerable. We have investigated the use of a combination of the Laitinen Stereoadapter and Galdenberg frame (Gald-X) for these patients. This study evaluated reproducibility in scanning, treatment plan parameters and treatment delivery.

The Stereoadapter relies on nasal bridge and auditory canal for fixation. Patient fitted with the Stereoadapter is secured inside the Glad-X with its lateral holders, Occipital headrest and forehead probe. A Rando head phantom is used in this study. A plastic target with placement positions for TLDs was implanted in the phantom. The Glad-X fiducials and subsequent XYZ coordinates are of BRW coordinates. Treatment plan was generated with our commercial stereotactic system. A total of 13 patients (19 isocenters) have been treated with the Stereoadapter/Glad-X system.

CT scan reproducibility based on XYZ coordinates was within $1.0 \mathrm{~mm}$. The difference between the cone center and implanted target center based on orthogonal films calculations was $1.2+0.3 \mathrm{~mm}$. The TLD doses compared favorably with expected values. The isocenter variation for the 19 isocenters treated based on angio port film study was $1.5+0.6 \mathrm{~mm}$.

The Stereoadapter/Glad-X system seems well tolerated with satisfactory reproducibility and accuracy for SRT. At least in patients with dentures who cannot tolerate the GTC, this system offers an alternative. Patient results and phantom efficacy study will be presented.

\section{PO-T- 40}

Iterative Least Squares Approach to Optimizing Large-Scale Treatment Plans

D Shepard*, G Olivera, P Reckerdt, J Aldridge, E Fitchard, T Mackie, University of Wisconsin, Madison, WI

An iterative optimization approach has been developed that minimizes a quadratic objective function without computing gradient functions. This optimization technique, referred to as Iterative Least Squares Minimization, includes a non-negativity constraint that is applied to all of the beam weights. Another important feature of this algorithm is that underdosage and overdosage can be assigned separate penalties. With the addition of dose-volume histogram based penalty functions and weighting factors assigned to each region of the patient, this optimization technique is easily adaptable to a wide variety of treatment situations.

Iterative Least Squares Minimization has been used in the development of numerous tomotherapy treatment plans. Inverse treatment planning for tomotherapy represents a particularly difficult challenge because of the large number of pencil beams of radiation. As an example, a treatment plan will be presented for a nasopharyngeal carcinoma that involved over 300,000 pencil beams. Each of these pencil beams had a corresponding dose matrix that was 128 by 128 by 52 voxels. The success of Iterative Least Squares Minimization is in part due to its ability to work efficiently in conjunction with a convolution/superposition based dose engine. This algorithm can optimize a treatment plan using a limited number of convolution operations without sacrificing the accuracy of the dose computation.

\section{PO-T- 41}

Mathematical Programming Approaches to Tomotherapy Optimization D Shepard*, M Ferris, G Olivera, T Mackie, University of Wisconsin, Madison, WI

A number of mathematical programming approaches have been investigated in search of an optimization technique that is suitable for tomotherapy treatment planning. The mathematical programming techniques that have been investigated include linear, nonlinear, and mixed integer programming.

GAMS, the General Algebraic Modeling System, was employed in each optimization. GAMS is a modeling environment that was chosen because of its strength as a tool for efficiently performing large scale optimizations. One important capability of GAMS is that the user can choose between linear, non-linear, and mixed integer models. GAMS also provides the ability to switch between a number of state of the art commercial solvers including OSL, CPLEX, CONOPT, and MINOS. In the development of each treatment plan, the specifications of the tomotherapy prototype were used along with a convolution/superposition based dose computation.

A case involving a squamous cell carcinoma of the nasopharynx will be used to present the advantages and disadvantages of a number of objective functions and constraints. The constraints that have been tested include two implementations of dose volume constraints that include a new approach using a ramped penalty function. In summary, mathematical programming techniques can provide a robust and flexible approach to optimizing treatment plans. The clinical applicability of these optimization techniques hinges upon the ability to accurately compute the pencil beam dose distributions in an acceptable amount of time and the ability to maintain the data in the computer's memory.

\section{PO-T- 42}

Reduction of Small Bowel, Bladder and Rectal Doses using IMRT in Patients Receiving Whole Pelvic Radiation Therapy

J Roeske*, J Kung, A Mundt, University of Chicago, Chicago, IL

The purpose of this study was to demonstrate that IMRT could reduce the volume of small bowel, bladder and rectum irradiated in patients receiving whole pelvic radiotherapy (WPRT). Ten cervical cancer patients undergoing WPRT were selected for this analysis. A treatment planning CT scan of each patient was obtained using oral, intravenous, and rectal contrast. The common iliac, right and left internal and external iliac lymph node regions, bladder, rectum, vagina and small bowel were contoured on axial slices. The target volume consisted of all contoured lymph node regions, upper vagina, and parametrial tissues. Two plans were created. The first was a standard "4-field box" with apertures shaped to the target in each beam's eye 
view. The second was a 9-field, coplanar, IMRT plan designed to conform to the target while minimizing the volume of normal tissues irradiated. Both plans were normalized to deliver 45 Gy to the target volume. DVH analysis indicated that the volume of small bowel irradiated in the IMRT plans was on average $20 \%$ and $60 \%$ lower than the conventional plans at 20 and $40 \mathrm{~Gy}$, respectively. Additionally, there was significant reduction of the bladder and rectal volumes irradiated at doses greater than $40 \mathrm{~Gy}$, relative to the standard plans. These results demonstrate that normal tissue sparing can be achieved using IMRT in patients receiving WPRT, and offers the potential of higher dose delivery to pelvic lymph nodes.

\section{PO-T- 43}

Stochastic Beam Weight Optimization: The Differential Evolution Algorithm

J Deasy*, University of Louisville, Louisville, KY

Some problems in automated radiotherapy treatment planning are expected to have multiple-local minima, e.g. beam weight optimization using dosevolume constraints or some types of radiobiological objective functions. Recent advances in stochastic global optimization programming techniques which are potentially capable of optimization in the presence of multiple local minima include the differential evolution algorithm (Price, K., and Storn, R. Differential Evolution: A Simple Evolution Strategy for Fast Optimization. Dr. Dobb's Journal, (1997) 264, 18-24). This algorithm evolves a 'population' of feasible beam-weight vectors between iterations. The primary process for evolving the population is to generate trial vectors which are a linear combination of a randomly chosen beam weight vector and the difference between two other randomly chosen vectors. A trial vector is accepted into the new population if it results in a lower objective function than another randomly chosen vector from the old population. In this way the algorithm is 'self-tuning' as the optimization process progresses, which is potentially an advantage over other stochastic methods such as simulated annealing. It is important to note that any stochastic algorithm is likely to provide results which can be improved upon (if only slightly) by finishing with local search methods. We have used this algorithm to optimize IMRT dose distributions for Soederstroem and Brahme's 2D cervical example (Int J Radiat Oncol Biol Phys, (1995) 33:1, 151-9), and achieved good dose distributions (i.e. with minimum gross target volume doses of about $90 \mathrm{~Gy}$ ) for just 3 fields.

\section{PO-T- 44}

Comparison of Algorithms for Multileaf Collimator Field Segmentation W Que*, Toronto Sunnybrook Regional Cancer Centre, Toronto, Ontario, Canada

Several algorithms for multileaf collimator (MLC) field segmentation intended for the "stop and shoot" method of intensity modulated radiation therapy are investigated for their performance, with emphasis on the ability to deliver an intensity distribution in a small number of MLC field segments. The algorithm of Xia and Verhey* and one of its variations are found to be the best algorithms, while others are slightly less efficient. It is pointed out that when the background intensity level is not zero, it is not always efficient to deliver a segment to bring the background level to zero, and a criterion to decide whether it is worthwhile to do so is proposed.

*Med. Phys. 25, 1424-1434 (1998).

\section{PO-T- 45}

Dose Measurement-based Tomographic Intensity Modulated Radiation Therapy: Theory and Numerical Results

M Braunstein, R Levine*, Y Lo, M Urie, Spectral Sciences, Inc., Burlington, MA, University of Massachusetts Memorial Medical Center, Worchester, MA

After a review of the theory of tomographic IMRT with a convolutional dose model, a tomographic IMRT algorithm incorporating phantom dose measurements is presented. Tomographic projection is replaced with a compensated summation using \% depth-dose measurements, and backprojection is replaced with dose computed by interpolation from fixed field dose profile measurements. In this way, dose penumbra and fluence attenuation (\% depth dose) effects are incorporated into tomographic IMRT inversion using special-purpose projection, backprojection, and beam front filtering. It is shown that penumbra deconvolution and geometric beam front filters often require unrealistic high spatial frequencies in beam front modulation. Penumbra effects and low spatial frequency modulation result in a reduction of the tumor dose edge or increased dose heterogeneity. Also, fewer beams are required to achieve optimum results under the constraint of low spatial frequencies in the dose and beam fronts.

The measurement-based inversion algorithm is coupled with a realistic beam front leaf-setting algorithm to demonstrate the trade-offs in tomographic IMRT resulting from dose effects. Comparisons between tomographic IMRT with and without the incorporation of real dose measurements are shown for a series of numerical targets to demonstrate these effects. Techniques are suggested for the removal of dose heterogeneity and normal tissue exposure by augmentation of the dose-based tomographic IMRT algorithm with dose constraints.

\section{PO-T- 46}

Estimation Theory for Therapeutic Treatment Plan Optimization

L Xing, J Li, A Pugachev*, A Boyer, Stanford University, Stanford, CA

In inverse planning in radiotherapy, an optimized solution to a prescribed dose distribution (or prescribed DVHs) is realized under the guidance of an objective function. In this work, the treatment plan optimization is formulated as an estimation problem of discrete and possibly non-convex system. The concept of the preference function of the treatment plans is introduced. Instead of prescribing a dose to a structure (or a set of voxels), the approach allows us to prioritize doses with different preference levels and reduces the problem into selecting a solution using a suitable decision estimator. The approach is quite general and makes it possible to incorporate empirical judgement (or expert knowledge) into the preference function. It is shown that the commonly used quadratic objective function is a special case of the formalism and the importance factors in the quadratic objective function are simply the Gaussian parameters of a Gaussian preference function. A general method for using a computer to determine the values of the model parameters is proposed. The method is illustrated using a simplified two-pixel system as well as two clinical cases. The generality of the approach, coupled with promising demonstrations, indicated that the method has broad implications for radiotherapy treatment plan optimization. Some implications of the formalism to therapeutic plan optimization are addressed.

\section{PO-T- 47}

Intensity Modulated Radiation Therapy with Tomographic Reconstruction: MLC Leaf Width and Beam Number Influence on Dose Conformation to Cranial Targets

M Braunstein*, R Levine, Y Lo, M Urie, Spectral Sciences, Inc., Burlington, MA, Spectral Sciences, Inc., Burlington, MA, University of Massachusetts Memorial Medical Center, Worchester, MA

Dose intensities for IMRT are calculated with a tomographic reconstruction algorithm. Measured dose distributions are used in both the inverse and forward calculations. The delivery of dose is modeled and the conformation of dose-to-target for $1.5 \mathrm{~cm}, 1 \mathrm{~cm}$, and $0.5 \mathrm{~cm}$ width beams, representing different MLC leaf widths, is investigated. Three geometric targets are studied and the number of IMRT beams varied from 4 to 32. Dose volume histograms compare target volume dose uniformity and dose conformation, as measured by the dose volume histogram of surrounding normal tissue (SNT).

The targets, each in a $16 \mathrm{~cm}$ diameter water cylinder, are: (a) an elongated ellipse $(4 \mathrm{~cm} \times 0.5 \mathrm{~cm})$, (b) an $\sim 2 \mathrm{~cm}$ peanut, and (c) a torus $(4 \mathrm{~cm} \times 1.5 \mathrm{~cm}$ ellipse surrounding a $3 \mathrm{~cm} \times 0.5 \mathrm{~cm}$ critical structure). In each case the SNT is a structure $2 \mathrm{~cm}$ larger than the target. All results were computed in two dimensions, although the algorithms are applicable to full three-dimensional treatments. The delivered dose contours are shown for 32 equally spaced beams with beam resolution of (b) $0.5 \mathrm{~cm}$, (c) $1.0 \mathrm{~cm}$, and (d) $1.5 \mathrm{~cm}$.

The improvement in dose conformation with narrower leaf widths is significant for these targets, which are representative of cranial targets. This will be quantified and discussed. The conformation of dose improves from 4 to 8 beams; improvement is much reduced beyond 12 beams, indicating that clinically $8-12$ beams will be sufficient in most cases. 


\section{PO-T- 48}

An Analysis of Speed and Precision in IMRT Delivery

P Geis*, R Hill, S Kalnicki, A Wu, Nomos Corporation, Sewickley, PA, Allegheny General Hospital, Pittsburgh, PA

The multileaf collimator (MLC) has become the most common tool providing the modulation for intensity modulated radiotherapy (IMRT). One requirement of these delivery systems is the translation of intensity modulated fields into a series of static or dynamic field shapes, a process often called 'leaf-sequencing.' For many commercial MLC's, tradeoffs between the accuracy of field delivery and delivery time must be made. A comparison of two alternate leaf-sequencing algorithms was carried out for a commercial IMRT delivery system. Plans from previously treated patients were re-sequenced using both a sliding window algorithm stressing dose accuracy and reduction of tongue-and-groove effects, and an algorithm primarily optimizing treatment delivery time. Both algorithms were used to generate treatments which were then delivered using a Siemen's IMRT delivery system. The precision with which the desired dose distribution was delivered was evaluated for each approach, as well as relative treatment time. An analysis of total treatment times, including time for patient setup and intra-treatment positioning checks, addresses the feasibility of using IMRT on a large fraction of a clinic's patient population.

\section{PO-T- 49}

Evaluation of a Prototype Inverse Treatment Planning System K Spencer, C Ramsey*, A Oliver, Thompson Cancer Survival Center, Knoxville, TN

Over the past several years, three-dimensional radiation treatment planning (3D-RTP) vendors have begun developing inverse treatment planning capabilities for generating intensity modulated radiotherapy (IMRT) treatment plans. However, all of these systems are still in the development phase and are not available for general release. One such system is the prototype inverse planning software under development for use with the ADAC Pinnacle ${ }^{3}$ treatment planning system. This software uses an iterative algorithm with a user defined objective function to solve the inverse problem.

This system is currently able to optimize step-and-shoot intensity modulated treatment plans. The IMRT optimization process starts by calculating dose distributions for 3D conformal treatment fields with blocks, but without any additional modifiers, using the standard convolution/superposition models. The desired optimization parameters, such as the minimum allowable target dose and the maximum allowable normal tissue doses, are defined for the contoured regions of interest. During optimization, isodose lines, isodose clouds, dose volume histograms (DVHs) and normal tissue complication probabilities (NTCPs) are calculated and updated in real-time. After optimization, a leaf sequencing algorithm can be used to generate MLC treatment files.

The capability of the inverse treatment planning software was evaluated using a variety of specially designed test objects, where the solution to the inverse problem was known. After optimization, the results were compared to the known solutions and an excellent agreement was observed in all cases.

\section{PO-T- 50}

A Modified Clarkson Integration (MCI) for IMRT

J Kung *, G Chen, Department of Radiation and Cellular Oncology,

University of Chicago, Chicago, IL

A typical IMRT fluence map resembles a mosaic. We propose a simple and accurate dose calculation algorithm that can be used to determine dose for a given non-uniform fluence map. Briefly, we first exploit the rotational symmetry of scattered dose. We than introduce a variation of the Clarkson integration in which scatter contribution is summed over annular sectors, instead of over the traditional pie sectors. Because of its simplicity, the algorithm can be implemented on a desktop computer.

Method:

A code using MCI was written to calculate doses from an IMRT fluence. As a test of the algorithm, CORVUS IMRT plans were generated for five cases. Doses to target volumes calculated with the MCI algorithm was compared with doses calculated by CORVUS, which uses $1 \mathrm{cmx} 1 \mathrm{~cm}$ pencil beam model. As an approximation skin contour variations and inhomogeneities were neglected. The code reads in MLC sequence files and utilizes beam data (TPR, Sc, and Sp). To calculate a dose to a point, the code requires user input information for a depth in tissue, SSD, and photon jaw sizes.

Result:

The doses calculated with the algorithm agreed within $+/-3 \%$ with the doses calculated with a pencil beam model. We discuss improvements of the MCI algorithm for off axis and skin contour variations.

\section{PO-T- 51}

Conformal Field Margins in Multifield, Axial, Coplanar Treatment Planning

T Grigereit*, E Klein, W Harms, S Goddu, S Mutic, D Low, J Purdy, Washington University School of Medicine, St. Louis, MO

Conformal dose delivery for multi-field axial coplanar treatments using multileaf collimation (MLC) typically involves setting an additional 7 to 8 $\mathrm{mm}$ margin around the planning target volume (PTV) in order that a specific isodose surface encloses the entire PTV. However, the cranial and caudal ends of the PTV lie within overlapping penumbras from all beams, and additionally may lie within narrow leaf separation regions. Opening additional leaf pairs at the field ends to compensate results in a "stovepipe" appearance of the MLC portal and increased irradiation of adjacent structures. Film measurements on single field and composite treatments were performed to assess the dose delivered in these narrow areas of overlapping penumbras, and to confirm three-dimensional treatment plan calculations. Results from film dosimetry and calculation show that when an additional leaf pair was opened the $97 \%-50 \%$ isodose separation increased from an average of $1.5 \mathrm{~cm}$ to $2.0 \mathrm{~cm}$ due to the increasingly restricted geometry in the upper stovepipe region. Only half of the increase in the $50 \%$ isodose area was therefore realized for higher isodose coverage in this process. The $95 \%-50 \%$ isodose separations showed approximately the same percentage change but with absolute values $3-5 \mathrm{~mm}$ less. Protocols that require a strict dose coverage may thus inadvertently sacrifice true conformality when critical structure doses are considered, unless more careful tailoring of the end leaf pairs is used to avoid very narrow field ends.

\section{PO-T- 52}

Dosimetric Capabilities of an Electronic Portal Imaging Device

R Zwicker*, B Libby, R Mohan, Medical College of Virginia

Hospitals/Virginia Commonwealth University, Richmond, VA

Quality assurance for dynamic MLC-based intensity modulated radiotherapy treatment planning is complicated by the fact that the complex motions of the collimator leaves make it essentially impossible to confirm computer generated monitor units by independent calculations. Correct reproduction of the calculated dose distributions by the leaf motions is also difficult to verify. For these and other reasons, transmission dosimetry has been proposed as a possible method of verification of IMRT treatments. This work examines the dosimetric capabilities of a commercial portal imager currently in clinical use. The system features a variety of acquisition modes corresponding to different beam energies, dose rates, scan speeds and scan trigger delays. To establish its utility as a dosimeter, characteristic dosesignal curves were generated and compared with theoretical expectations. To this end, independent ion chamber measurements were carried out and compared with the corresponding detector pixel values in the same geometry. The results confirm the validity of the theory predicting a simple dependence of the detector response on exposure rate. Other dose-response characteristics of the system were also examined to establish detector uniformity and signal reproducibility. Integration of the EPID dose signal can be achieved by means of programmed sequential imaging. The resulting summed images can provide a reasonable qualitative representation of an integrated IMRT field in cases of slow scan speeds. Idealized and real cases will be shown and discussed. 


\section{PO-T- 53}

\section{Optimization Techniques for Intensity Modulated Stereotactic} Radiotherapy

S Simons*, J Figura, Western Pennsylvania Hospital, Pittsburgh, PA

Simulated Annealing, in conjunction with back-projected beam weighting, has proved to be an effective optimization technique for intensity modulated stereotactic radiotherapy. We have developed additional tools that enhance the optimizer's performance and reduce time for radiotherapy planning.

The optimization model's goal is to deliver a plan that fulfills constraints imposed by the physician and physicist. A cost function determines a plan's superiority to other calculated plans. Each target is given a minimum dose goal and each critical structure is assigned maximum dose limits. A hierarchical importance scale (weight) rank each target and sensitive neighboring tissues, which, determines the structures' relative importance. Normal tissues with low dose limits and high relative weights will be highly protected by the algorithm.

To further improve dose distributions, we have developed a 'moat' technique that completely surrounds the tumor with a $5 \mathrm{~mm}$ normal tissue zone followed by a $10 \mathrm{~mm}$ moat of 'highly sensitive tissue.' The moat attempts to drive the dose into the target while limiting dose elsewhere.

The added benefit of the moat technique is seen for a brain metastasis measuring $3.81 \mathrm{cc}$ located directly superior to the brain stem. Using the moat technique decreases each isodose volume by approximately $42 \%$ while delivering a more conformal plan. The $90 \%$ isodose volume for the moat technique measured $3.85 \mathrm{cc}$ compared to $6.72 \mathrm{cc}$ or nearly twice the metastasis volume.

The reduced isodose volume and five-fold planning time decrease proves the moat technique is a valuable planning tool.

\section{PO-T- 54}

Dynamic Multileaf-Diaphragm Sequencing with Adjacency Gap Constraints

H Kuterdem*, P Cho, R Marks II, University of Washington, Seattle, WA

Several methods of beam intensity modulation are currently available. One of the more versatile of these is the dynamic multileaf collimation in which fluence gradients are generated by independently controlled leaf velocity. A chief advantage of this method is the speed as well as the continuously conformal modulation. However, a certain design feature of a particular class of MLC's, namely the minimum gap requirement between opposing and adjacent leaves, has hampered implementation of the dynamic delivery concept. If neglected during conversion of the prescribed fluence to leaf sequence, the errors introduced by the gap constraints could lead to severe overdosage or underdosage. In order to overcome this problem we have developed a leaf sequencing algorithm which respects the gap constraints. In this algorithm the backup diaphragms parallel to the MLC are used to block the beam in place of leaves which may otherwise violate the minimum gap rules. In addition, the diaphragms perpendicular to the MLC are moved during delivery in order to define the horizontal field edges more accurately. The combined use of the orthogonal diaphragms can minimize the dose spillage around the field border. This feature is especially useful when the field shape is convex. The algorithm also permits a hybrid mode of delivery by inserting beam pauses to fine tune the modulation. With these techniques, it is possible to deliver difficult prescriptions such as those with zero regions or adjacent tracks with opposite slopes along the same vertical.

\section{PO-T- 55}

Optimal Constraint Dose of an Overlap Region Between Target and Critical Structures for Inverse Treatment Planning

Y Watanabe*, Columbia University, New York, NY

The aim of inverse treatment planning is to design a plan to deliver a prescribed dose to target volume and minimize the dose to critical structures. Since the planning target volume (PTV) includes a spatial margin, usually PTV overlaps the volumes of one or more critical structures. A question is what dose we should use as a goal of optimization for this overlap region. To determine an optimal constraint dose to the overlap regions we propose to use biological indices of target and critical structures: tumor control probability (TCP) and normal tissue complication probability (NTCP). We assume a simple geometry consisting of a PTV and a critical structure. We include the effect of the organ motion by defining the probability that PTV is completely outside this overlap region. TCP is given by a logistic function. NTCP is calculated from the Lyman model. A cost function, which is defined as a product of TCP and 1-NTCP, is calculated. We did detailed analyses by varying the model parameters. For example, a prostate treatment plan with a prescription dose of 80 Gy involves a PTV overlapping with a rectum volume. The analysis indicates that the cost function is the maximum when the constraint dose in the overlap region is $80 \%$ of the prescription dose. The method is applied to other sites for which there is overlap between PTV and critical structures.

\section{PO-T- 56}

A Leaf Sequence QA Tool for IMRT Beam Delivery

Y Chen*, C Ma, Stanford University School of Medicine, Stanford, CA

We have developed a tool to calculate leaf leakage and tongue and groove effect for beam delivery verification in intensity modulated radiotherapy (IMRT) using multileaf collimator (MLC). A ray tracing method was used to derive the fluence transmission map of an MLC field. The intercept length of a ray is the sum of the lengths intercepted by the leaves in the ray path. The intercept lengths were pre-calculated for a set of fixed leaf positions using a dual extended source model for a Varian Clinac $2100 \mathrm{C}$ accelerator. For an arbitrary leaf position, the intercept lengths can be obtained using the data for the nearest pre-calculated position. For photons with incident directions deviated from a ray line within small angles $(<$ $0.2^{\circ}$ ), the intercept lengths follow a translational transformation. Therefore we could speed up the calculation by convolving a point source fluence map with an extended source representing photons from the target. The calculated fluence maps were used for inverse planning monitor unit calculation and for Monte Carlo IMRT dose verification as a simplified beam modifier. Comparisons of the fluence maps with measurements using film and a beam imaging system (BIS) showed excellent agreement. The calculated fluence map for multi-segment MLC field was used as a reference image to compare with the measured image from the BIS. The difference between the two images and the correlation coefficient for each field segment were evaluated quantitatively for IMRT leaf sequence QA.

\section{PO-T- 57}

Verification of Field Placement for Rotational IMRT

M Huq, G Bednarz*, M Hossain, J Galvin, W Curran, Jr., Thomas Jefferson University Hospital, Philadelphia, PA

Techniques for guaranteeing that intensity patterns are properly positioned relative to a patient's anatomy have not been developed for rotational IMRT. This paper points out the importance of at least assuring that the "slit" opening covers the treatment volume. Using the NOMOS MIMiC collimator, it is possible, and sometimes necessary, to position the treatment unit isocenter so that the collimator opening does not provide full target coverage as the gantry rotates. This report demonstrates that missing the target compromises the dose distribution by forcing normalization to relatively low isodose lines and increasing the dose to critical healthy structures. A simple check of the NOMOS output can detect potential problems. Caution is advised when the beam elements at the field edge are used in the modulation process. As a more complete check, a doubleexposure film technique using the standard jaws is recommended to document field coverage. The technique uses both an anterior and one lateral film to show the extent of the collimator opening. The field size for these films is set to agree with the length of the beam slit (approximately 20 $\mathrm{cm}$ ) in one direction and the slit width in the other direction. A doubleexposure over-flash is used to show this slit relative to the patient's anatomy. Simulation of these fields is also recommended. Since CT scans must be available for any IMRT treatment, the use of CT-simulation simplifies this part of the process. 


\section{PO-T- 58}

Whole-Body Equivalent Dose Estimates in Tomotherapy

D Verellen*, N Linthout, AZ-VUB, Brussels, Belgium

Tomotherapy (the Peacock System $(\circledR)$ has been introduced in our department (first patient June 1995) for treatment of the head and neck region to reduce complications without compromizing treatment outcome. This technique requires an increase in monitor units per target dose yielding an increased risk of inducing secondary malignancies. In vivo measurements of the whole-body equivalent dose have been performed for both the conventional (using parallel opposed wedged treatment fields) and tomotherapy for head and neck lesions with $6 \mathrm{MV}$ photon beams. Thermoluminescent badges and neutron bubble detectors have been applied to obtain the estimated whole-body equivalent dose (WBED) for each treatment technique. The nominal probability coefficient for a lifetime risk of excess fatal cancer (ICRP60) has been used for risk estimates. An estimated WBED per monitor unit equal to $1.2 \times 10^{-2} \mathrm{mSv} / \mathrm{MU}$ and $1.6 \times 10^{-2}$ $\mathrm{mSv} / \mathrm{MU}$ have been obtained with conventional therapy and tomotherapy respectively. Applying the average amount of MU necessary to realize a 70 Gy target dose the estimated WBED becomes $242 \mathrm{mSv}$ (conventional) and $1969 \mathrm{mSv}$ (tomotherapy), yielding an increase in probability coefficients for secondary malignancies with a factor 8 when applying tomotherapy. The results will be discussed against similar reports from literature. The risk of secondary malignancies has been accepted to take advantage of the possible benefits of improved local control and treatment outcome. Introducing sophisticated treatment techniques will change the risk of radiation induced malignancies and risk estimates become important to assess whether the benefits of the treatment technique outweigh the possible risks.

\section{PO-T- 59}

A Leaf Sequencing Algorithm for Dynamic Multileaf Collimators

J Ting*, E Butker, E Elder, M Mitchum, S Ghavidel, L Davis, Emory University School of Medicine, Atlanta, GA

\section{PURPOSE:}

To develop a leaf sequencing algorithm for IMRT field delivery. This algorithm allows visual verification and port filming of treatment setup before the treatment. And, the last segment remains open for another visual inspection of final positions of leaves after treatment.

\section{METHODS AND MATERIALS:}

A "stop and shoot" algorithm was developed using a "zero intensity" seeking method. Total monitor unit computation is accomplished by summing monitor units required for each leaf setting. Though this algorithm has not been optimized for the least number of beam segments, the number of beam segments are comparable to those algorithms which have been optimized for the least number of beam segments.

Outputs using this algorithm were validated with measurements using ionization chambers in water phantom and solid phantom. TLDs were placed inside of a human-like phantom and passed through the entire system as a patient would.

\section{RESULTS:}

Excellent agreements were found between computed values and measurements using DMLC. TLD measurements were also acceptable. This algorithm has been in clinical use since July, 1998. More then 40 patients have been treated using this leaf sequencing algorithm.

\section{CONCLUSION:}

A new leaf sequencing algorithm has been developed for IMRT implementation. This algorithm starts with the largest treatment field and allows visual inspection of the treatment setups and port films before the treatment. The last segment remains open for another inspection of final positions of leaves. This algorithm has comparable number of beam segments with other leaf sequencing algorithms.

\section{PO-T- 60}

A Method of Generating Multiple Static Fields and Delivering Intensity Modulated Radiotherapy

Y Wu*, D Yan, M Sharpe, B Miller, J Wong, William Beaumont Hospital, Royal Oak, MI

A process for delivering intensity-modulated radiotherapy treatments using a sequence of fixed beam directions and a multi-leaf collimator (MLC) has been implemented in our clinic. Non-uniform intensity profiles are delivered for each direction using a stop-and-shoot approach. One aspect of this process deals with generating a series of discrete MLC fields that will create the non-uniform intensity profile derived by the inverse planning. Beam segments are formed by first grouping all nonzero intensity values into a minimum number of levels based on a user specified error tolerance. The Kmeans clustering algorithm is then employed to find the optimal intensity increments to minimize discrepancies between the desired and delivered profiles. Finally, the discrete intensities are decomposed into a sequence of shapes to be formed with the MLC and independent jaws. Apart from the first segment for each gantry angle, all other segments are arranged to minimize the total travel distance of the leaves. The first segment covers the largest treatment area, and is used for patient setup verification in conjunction with electronic portal imaging. This algorithm has been in clinical use for prostate cancer patients since April of 1998. The results have demonstrated that the approach is clinically feasible in terms of accuracy and overall treatment time. The K-means clustering algorithm has the capability to reduce the discrepancy between the desired and deliverable profiles, especially for a larger error tolerance.

Supported in part by Elekta Oncology Systems.

\section{PO-T- 61}

Non-Parallel Plane Intensity Modulated RadioSurgery (IMRS)

B Salter Jr.*, J Hevezi, UT Health Science Center at San Antonio, Cancer Therapy \& Research Center, San Antonio, TX

Intensity Modulated Radiation Therapy (IMRT) delivered via an arc based, tomotherapy style of approach (e.g. Nomos Mimic) has recently begun to find clinical acceptance for delivering highly conformal, fractionated radiotherapy treatments. Previously, a few users have attempted to use the system on stereotactic radio-surgical targets employing only one couch angle. Intuition suggests that steeper 3 dimensional dose gradients, and increased sparing of normal brain tissue might be achievable when multiple, non-parallel plane IMRT arcs are utilized. As originally designed, the only currently available, commercial system for delivering such treatments has not fully supported the accurate delivery of such treatments due to couch indexation problems when multiple couch angles are utilized. We have developed a method to enable the accurate delivery of multiple, non-parallel plane IMRT/IMRS arcs. This methodology has been evaluated in conjunction with the commercial system's prototype post collimation device which was designed to further restrict the physical dimensions of the existing pencil beams.

The couch indexation system developed here has been shown to provide exceptionally accurate target alignment at multiple couch angles, and has yielded significant improvements in the volume of normal brain spared. Lastly, the combination of non-parallel plane arc delivery capability with the reduction in pencil beam dimension afforded by the commercial system's post collimation device resulted in significant improvements in 3 dimensional target conformality. Patient dose distributions, analysis, and hardware involved will be described. The couch indexation technology developed here has been licensed for distribution by Nomos.

\section{PO-T- 62}

Automated Verification of 3D-Conformal and IMRT Treatment Planning Using BANG-gel

G Gluckman*1, J Yang ${ }^{1}$, A Meek ${ }^{1}$, M Maryanski ${ }^{2}$, L Reinstein ${ }^{1}$, (1)SUNY at Stony Brook, New York, (2)MGS Research, Guilford, CT

A major stumbling block to the growth of high-tech modalities such as 3Dconformal radiotherapy and IMRT is the lack of an acceptable methodology to test the agreement between planned and delivered patient dose distributions. Conventional 1D or 2D radiation dosimetry systems that are 
used to verify treatment planning calculations are not capable of efficiently providing the integrated dose information throughout a clinically relevant volume with adequate spatial resolution. Recently BANG-gels in combination with MRI have been shown to record and accurately map 3D dose distributions with high spatial resolution and precision. However, to utilize the full potential of BANG-gel technique, we have developed a software package, which, together with specially designed gel phantoms, provide completely automated verification. Our software is DICOM compatible, enabling it to compare gel/MRI dosimetry to the 3D-RTP calculations. The dose distribution is calculated from $\mathrm{R}_{2}$ maps derived from spin-echo images and automatically registered. Several color-graphic tools (e.g., dose difference histograms with color-wash) are included to facilitate validation of the plan calculations.

Effectiveness of our software is demonstrated using four BANG-gel phantoms irradiated to different multi-beam configurations. The dosimetric and spatial uncertainties (as well as its efficiency and ease of use) have been evaluated and found to conform to the recommended tolerances for RTP QA expressed by AAPM TG40 and TG53.

Supported in part by NIH-SBIR, CA82026

\section{PO-T- 62}

Automated Verification of 3D-Conformal and IMRT Treatment Planning Using BANG-gel

G Gluckman*1, J Yang ${ }^{1}$, A Meek ${ }^{1}$, M Maryanski ${ }^{2}$, L Reinstein ${ }^{1}$, (1)SUNY at Stony Brook, New York, (2)MGS Research, Guilford, CT

A major stumbling block to the growth of high-tech modalities such as 3Dconformal radiotherapy and IMRT is the lack of an acceptable methodology to test the agreement between planned and delivered patient dose distributions. Conventional $1 \mathrm{D}$ or $2 \mathrm{D}$ radiation dosimetry systems that are used to verify treatment planning calculations are not capable of efficiently providing the integrated dose information throughout a clinically relevant volume with adequate spatial resolution. Recently BANG-gels in combination with MRI have been shown to record and accurately map 3D dose distributions with high spatial resolution and precision. However, to utilize the full potential of BANG-gel technique, we have developed a software package, which, together with specially designed gel phantoms, provide completely automated verification. Our software is DICOM compatible, enabling it to compare gel/MRI dosimetry to the 3D-RTP calculations. The dose distribution is calculated from $\mathrm{R}_{2}$ maps derived from spin-echo images and automatically registered. Several color-graphic tools (e.g., dose difference histograms with color-wash) are included to facilitate validation of the plan calculations.

Effectiveness of our software is demonstrated using four BANG-gel phantoms irradiated to different multi-beam configurations. The dosimetric and spatial uncertainties (as well as its efficiency and ease of use) have been evaluated and found to conform to the recommended tolerances for RTP QA expressed by AAPM TG40 and TG53.

\section{Supported in part by NIH-SBIR, CA82026}

\section{PO-T- 63}

Feasibility of IMRT with a Miniature MLC for Small Brain Tumors M Bues*, H Kooy, J Beatty, N Tarbell, P Chapman, J Loeffler, Massachusetts General Hospital, Boston, MA

IMRT using conventional MLCs with a projected leaf width of one centimeter at isocenter is being routinely practiced at various cancer centers. In this paper we study the benefit and the feasibility of treating small intracranial lesions with intensity modulated $4 \mathrm{MV}$ x-ray beams, where the infield modulation is achieved using a miniature $\mathrm{MLC}^{1}$, which has a projected leaf width of four millimeters at isocenter.

We studied a population of eleven patients with intracranial lesions ranging in size from 2.1 to 22.1 cubic centimeters. Patients were planned using the $\mathrm{KONRAD}^{1}$ treatment planning software and the resulting plan was compared to a conformal rotational arc therapy plan and a conventional IMRT plan. Evaluation criteria were tumor coverage, uniformity of dose within the tumor and sparing of healthy tissue. Our results indicate a significant potential benefit of IMRT with a miniature MLC with an average reduction in healthy tissue involvement at prescription dose level of $80 \%$ and $70 \%$ respectively.

We present a segmentation algorithm suitable for delivering intensitymodulated beams in the multiple static approach. This algorithm takes into account finite leaf transmission and penumbra effects, which are especially pronounced for the small apertures necessary to deliver IMRT with the miniature MLC. It also allows for an iterative optimization of the sequence of leaf settings. The predicted deliverable beam fluence patterns are compared to the desired beam fluence patterns as well as film measurements.

${ }^{3}$ Radionics Inc., Burlington, Massachusetts

${ }^{4} \mathrm{MRC}$ Systems GmbH, Heidelberg, Germany

\section{PO-T- 64}

IMRT Dose Delivery Error from Radiation Field Offset (RFO) Inaccuracy

J Kung *, G Chen, Department of Radiation and Cellular Oncology, University of Chicago, Chicago, IL

The Radiation Field Offset (RFO) is the difference between X(radiation) and $\mathrm{X}$ (light) for a MLC. In the conventional use of a MLC as a block substitute, a RFO affects only the dose to a target volume boundary. A block margin is chosen large enough to accommodate such a delivery error. In contrast, leaf edges in IMRT are projected into the interior of a target volume, and no block margin can accommodate such a delivery error.

An IMRT system must incorporate a RFO by decreasing all leaf gaps, e.g., $\mathrm{X}$ (right)-X(left). We analyze dosimetric consequences of incorporating an inaccurate RFO into an IMRT system.

Method:

We derive an analytic method of estimating a dose error from a RFO inaccuracy. To test the theory, we deliberately incorporate an inaccurate RFO into MLC sequence files. A phantom is irradiated with these fields. Dose errors are measured with ion chamber and compared with the theory.

Results:

A dose error from an RFO inaccuracy increases for a field with small MLC segments, i.e., $X($ right $)-X($ left $)$ small. For an IMRT plan comprised of many fields, each with small MLC segments, a target volume dose error is approximately uniform. For Varian $6 \mathrm{MV}$, Varian Mark II MLC, and CORVUS IMRT plans, a fractional target dose error can be as large as $3.5 \%$ per $\mathrm{mm}$ in RFO inaccuracy.

\section{PO-T- 65}

IMRT Dose Verification Using MOSFET Dosimeters

M Kleiman, ${ }^{1}$ S McGinley ${ }^{2}$, A Jones ${ }^{* 1}$, (1)Sacred Heart Hospital, Allentown, PA, (2)Cedar Crest College, Allentown, PA

Traditionally, dose verification with the Nomos Peacock IMRT system has been done with film and TLD. Film has a high spatial resolution, which handles the large dose gradients often found in IMRT, and is readily available in any radiation oncology department. It suffers, however, from being non-tissue equivalent, its need for calibration, and in its need for postprocessing developing and scanning. TLD has good point dose resolution, but the drawbacks include technique dependence, its need for calibration, and pre- and post-processing, i.e. annealing, and reading. Ion chambers are good for real-time readout and can be absolute dosimeters, but suffer from a larger collection volume, verification at only one point, dose gradient averaging, and the need for some post-processing corrections, such as temperature, pressure, and chamber corrections. MOSFET dosimeters combine the spatial resolution of TLD with the convenience of an ion chamber. They are small (200 microns), and give an instant readout in cGy. Additionally, several dosimeters can be implanted in the phantom to verify dose across the distribution. Previous dosimeters have had an angular dependence, which effects the readout in DMLC arc therapy. We have evaluated the MOSFET detectors for angular dependence and compared their readouts to TLD in the Nomos phantom for test plans and clinically delivered IMRT treatment plans. The preliminary results show good 
agreement between TLD and dosimeter. This shows the utility of using MOSFET dosimeters for convenient, real time verification of IMRT dose delivery across the target distribution.

\section{PO-T- 66}

Leaf Sequencing For Fluence Modulated Radiation Therapy

N Agazaryan*, T Solberg, A Arellano, T Paul, Department of Radiation Oncology, UCLA School of Medicine, Los Angeles, CA

In Fluence Modulated Radiation Therapy (FMRT), the radiation field is shaped using a multileaf collimator (MLC) by varying the exposure time throughout the field while maintaining constant beam intensity. Exposure time variation results in fluence and dose modulations throughout the field We have developed a new algorithm for constructing the leaf sequences necessary for FMRT delivery using a multileaf collimator. The algorithm is an enhancement of the sliding window technique and supports several accelerator/MLC combinations. The input format is the output of our inhome developed inverse planning system, which can be modified to support other desired input formats. Several factors are addressed in this application. The user specifies the RMS difference between the desired profile and the profile obtained with translated MLC motion before transmission correction. Tolerance above which the accelerator will turn the beam down allowing leaves to catch-up is also user input. Transmission through MLC leaves is being corrected using an iterative method. Leakage between opposing and neighboring leaves is minimized. Individual leaves are synchronized by varying their relative starting and ending times reducing tongue and groove effect. The application supports Fixed Gantry Step-and-Shoot, Fixed Gantry Dynamic, and Fixed Gantry Optimized modes. During Fixed Gantry Optimized mode, the delivery is dynamic but switches to step-and-shoot in instances where MLC leaves cannot meet tolerance specification. Verifications have been performed using film dosimetry and amorphous Silicon (a-Si:H) 2D array detector acquisitions.

\section{PO-T- 67}

\section{The Effects of Beamlet Sizes on IMRT Optimization}

B Yi*, K Forster, A Boyer, Stanford University,Stanford,CA

Intensity modulated radiation therapy (IMRT) has been implemented using multileaf collimators (MLCs) whose leaves project to a $1-\mathrm{cm}$ width at isocenter using a 1-cm step size for a "step-and-shoot" leaf sequence. This study explored the clinical usefulness of smaller beamlet sizes for IMRT produced by smaller step sizes and thinner leaf widths. IMRT treatment plans were computed for two clinical cases. The first case was in the cranium close to the orbits, the optic nerves, the optic chiasm, and the brain stem. A prostate cancer site was chosen for the second case, for which bladder and rectum were regarded as the critical organs. Nine equally spaced beam directions were used for these plans. The effects of step sizes of $10 \mathrm{~mm}, 5 \mathrm{~mm}$, and $2 \mathrm{~mm}$ for a $10-\mathrm{mm}$ wide MLC leaf were analyzed, while no changes to the input parameters were made. The computation was repeated for 15-MV, 6-MV and 4-MV photon beams in order to investigate energy dependencies. Dose volume histogram (DVH) analysis showed that smaller beamlets generated superior target coverage for both the cranial and the prostate cases. There were no distinct energy dependencies. However, the protection of normal tissue exposure appeared to depend on other factors as well. These results support the intuitive notion that smaller beamlets associated with thinner MLC leaf widths and smaller step sizes will for the most part produce superior optimized IMRT treatment planning results. This investigation was supported in part by NCI grant CA43840.

\section{PO-T- 68}

Use of Dynamic Multileaf Collimator as a Dose Compensator

J Park*, C Chen, C Liu, S Anghaie, J R Palta, Department of Radiation Oncology, Univesity of Florida, Gainesville, FL

Use of dynamic multileaf collimator (DMLC) as a dose compensator for the spine field in the treatment of medulloblastoma is studied. The spine is often treated with an elongated photon field which covers the whole spine axis. The dose variation along the spine can vary by as much as $20 \%$ due to its variable depths under the skin. The method devised here accounts for the physical limitations of DMLC and independent jaws available on the Varian $^{\mathrm{TM}} 2100 \mathrm{CD}$ in creating a one-dimensional intensity modulated beam to deliver the uniform prescribed dose of $120 \mathrm{cGy}$ along the spinal cord.
The intensity distribution necessary to deliver such modulated fields is designed, at $0.5 \mathrm{~cm}$ increments, based on measured beam parameters, such as TMR, OAR, $\mathrm{S}_{\mathrm{cp}}$, etc., using a forward planning system. Preliminary calculations are based on the secondary collimator settings obtained after dividing the $6 \times 40$ field into four $6 \times 10$ field segments. Nominal base line dose, which does not impose too much constraint on generation of leafmotion is subtracted in order to improve both the total treatment time involved as well as the errors introduced at the field junctions. The actual arrangement used to deliver the actual treatment is used to account for transmission through the DMLC leaves. The agreement between the planned and measured intensity profiles is within $2.0 \%$ for the shape of the profile except at the field junctions where it is up to $3 \%$. This is regardless of variations in $\mathrm{S}_{\mathrm{cp}}$ for small temporal field sizes involved in intensity modulation. For comparison purposes, the beam delivery is done using both step-and-shoot and dynamic dose delivery methods. Both methodologies produce comparable results in this implementation.

\section{PO-T- 69}

A Dosimetric Issue for Intensity Modulated Radiation Therapy M Hossain*, M. Saiful Huq, J Galvin, Thomas Jefferson University Hospital, Philadelphia, PA

Rotational IMRT offers the possibility of dose conformality for irregularly shaped targets combined with rapid peripheral dose fall-off. Dose delivery is accomplished with a series of small beam elements whose orientations and intensities are determined by inverse planning. This new treatment modality presents some difficult dosimetry issues. The final dose is the sum of many tiny beams that do not achieve lateral electronic equilibrium. This situation causes a discrepancy between film and ion-chamber measurements when many such beams are superimposed. This difference is evident when the film is calibrated against the chamber for larger $10 \times 10 \mathrm{~cm}$ fields. The extended size of the ion chamber will overestimate the dose in regions of positive curvature at the "low dose" region of the profile for a small beam and underestimate the dose in the central region where the curvature is negative. However, the magnitude of the overestimation does not equal the underestimation when the effect of area (or volume) is considered. The exact amount of net overestimation of the chamber-determined dose reading depends on the details of each delivery, but typically ranges from $5-20 \%$. Film dosimetry does not suffer such anomaly because the grain size is sufficiently small. This paper discusses two simple cases of intensity modulated delivery using the NOMOS MIMiC collimator which demonstrate the delivery dependent anomaly. This phenomenon could lead to reconsideration of the use of ion chamber dosimetry for IMRT with many small beams.

\section{PO-T- 70}

A Modified Method of Planning and Delivery for IMRT Treatments

N Dogan*, L Leybovich, A Sethi, M Krasin, B Emami, Loyola University Medical Center, Maywood, IL

NOMOS-Corvus intensity modulated radiation treatment (IMRT) system generates treatment plans that are delivered in tomographic fashion. This technique requires precise indexing of the treatment couch. Since tomographic method uses multiple abutting fields, it may introduce multiple dose nonuniformity regions if the couch indexing is not accurate or the motion of the couch is not parallel to the multileaf collimator. Even relatively small couch positioning errors of $0.5 \mathrm{~mm}$ to $1 \mathrm{~mm}$ result in dose nonuniformity $\sim 15 \%-30 \%$. To reduce the dose nonuniformity, we developed and experimentally verified a modified treatment planning and delivery technique. This technique was based on examining the dependence of number and positions of treatment arcs on target dimensions. We found that the arc positioning was approximately a cyclical function of the target length (dimension in the direction of couch indexing). The period of this function was $\sim 8 \mathrm{~mm}$. Within each periodic interval, every $2 \mathrm{~mm}$ increase in target length resulted in $\sim 1 \mathrm{~mm}$ shift in the arc positions, and at the end of each interval, the shift was $\sim 5 \mathrm{~mm}$, which was large enough to produce a clinically significant shift in abutment regions. Our technique delivers two plans on alternate days, one with the original target and the second with a slightly increased target length, resulting in a periodic $\sim 5 \mathrm{~mm}$ shift in abutment regions. The experimental verification of this method confirmed that the dose nonuniformity in the target region was reduced by at least half when inaccuracy in indexing was $1 \mathrm{~mm}$. 


\section{PO-T- 71}

Clinical Implementation of Simple Intensity Modulation without Inverse Treatment Planning Software

C Ramsey*, K Spencer, I Cordrey, A Oliver, Thompson Cancer Survival Center, Knoxville, TN

Although intensity modulated radiotherapy (IMRT) promises to improve tumor coverage while sparing normal tissue, IMRT treatment programs generally require inverse treatment planning software. Unfortunately, most 3D treatment-planning systems do not have clinical releases of their inverse planning software. A technique has been clinically implemented that allows step-and-shoot IMRT treatments to be generated using the ADAC Pinnacle ${ }^{3}$ treatment planning system without special inverse planning software.

Dose distributions for a conformal plan with blocks are calculated first, and an optimization plane perpendicular to the beam axis is chosen. Isodose lines in the optimization plane, such as the $<80 \%, 80-90 \%, 90-95 \%$, 95 $100 \%, 100-105 \%$ and $>105 \%$ lines, are then contoured. The first segment in the IMRT plan is the non-optimized 3D conformal field. This segment is irradiated until the maximum contoured isodose (i.e. $>105 \%$ ) has received $100 \%$ of the prescribed dose. In the next segment, this area is blocked and the next isodose contour continues receiving dose until it reaches the prescribed dose. This process is repeated until all isodose contours are irradiated to the prescribed dose, which results in a uniform dose distribution in the optimization plane.

IMRT plan dosimetry was verified for each patient using a linear diode array and an ionization chamber. The measured and predicted doses agree within $\pm 3 \%$ at all points except for high gradient regions. These results indicate that clinical IMRT treatment plans can be generated and clinically implemented without the use of inverse planning software.

\section{PO-T- 72}

Dosimetric Apsects of Intensity Modulation in Serial Tomotherapy

P Xia*1, G Wong ${ }^{1}$, B Curran ${ }^{2}$, L Verhey ${ }^{1}$, (1)University of California at San Francisco, San Francisco, (2)NOMOS Corporation, Sewickley, PA

Because of its unique characteristics, intensity modulated serial tomotherapy has special dosimetric concerns, such as radiation leakage, scattering at different depths and between treatment slices and dose inhomogeneity in the abutment regions. Three series of plans were created to systematically examine several of these issues for an early version of the PeacockPlan system and a later version of the CORVUS system (NOMOS). To investigate radiation leakage and scattering from neighboring treatment slices, a series of plans was designed with targets of varying dimensions, requiring different number of couch indices, and different numbers of intensity profiles. To evaluate the scattering model at different depths, a second series of plans was designed with the target at different depths. To study the dose inhomogeneity in the abutment region away from the isocenter, a third series of plans was designed with targets at different distances from the iso-center. Verification measurements for these plans were conducted using ionization chamber and film dosimetry. The measurement results showed that the radiation leakage and scattering from neighboring treatment slices were modeled more accurately in the CORVUS system than in the PeacockPlan system. In both systems, doses predicted for the target at different depths were in excellent agreement with the measured results. For a $270^{\circ}$ arc delivery, if the location of the target is within $\pm 5.0 \mathrm{~cm}$ of the isocenter, the additional dose non-uniformity due to uncompensated beam divergence, is insignificant.

This research is partially supported By NOMOS Corporation, Sewickley, PA

\section{PO-T- 73}

Enhanced Dynamic Wedge Beam Delivery with Dynamic Multileaf Collimator System

C Chen*, J Park, C Liu, S Anghaie, J R Palta, Department of Radiation Oncology, University of Florida, Gainesville, FL

The dynamic multileaf collimator (DMLC) provides the versatility of generating intensity modulated photon beams while providing field-shaping capabilities. The DMLC on Varian 2300C/D was programmed to deliver wedge beam of $15^{\circ}, 30^{\circ}, 45^{\circ}$ and $60^{\circ}$ EDW using Golden Segmented Treatment Table (GSTT) data of Enhanced Dynamic Wedge. The cross beam profiles and the output factors for the same size EDW and DMLC generated wedge fields compare within $0.5 \%$ except in the penumbra region. Minimum difference in the penumbra region is attributed to round leaf design of Varian MLC. Inter-leaf and intra-leaf transmission contributes minimally to the output and therefore can be ignored in this simple case of intensity modulation. A limit of $14.5 \mathrm{~cm}$ in maximum leaf spread can be easily overcome by using two match fields. The maximum variation at the match point of two fields is less than $4 \%$. This approach allows a maximum symmetric wedge field size of $29 \mathrm{~cm}$, which is $9 \mathrm{~cm}$ larger than maximum symmetric current EDW field. Current treatment planning systems can be easily adapted to simulate dynamic wedge with DMLC without additional effort. Both discrete and dynamic approach of beam delivery gives similar results in this study.

\section{PO-T- 74}

Evaluation of the Matchline Region in Tomotherapy

N Linthout*, D Verellen, AZ-VUB, Brussels, Belgium

The Peacock System ${ }^{\circledR}$, appropriately dubbed "tomotherapy", was introduced in our department in 1994. This technique creates a matchline region between adjacent treatment slices. The inhomogeneity in the dose distribution in this matchline region was verified experimentally.

The matchline problem is twofold: a) the dose calculated in the matchline region depends on the slice thickness obtained during commissioning of the system; b) the delivered dose distribution depends on the accuracy of the longitudinal couch movement between consecutive treatment slices. To evaluate the precision of the dose calculation algorithm, treatment plans calculated with varying slice thickness were compared. The delivered dose inhomogeneity in the matchline region was verified using a homogeneous cubic phantom. Absolute dosimetry with thermoluminiscent (TL) and alanine detectors as well as relative dosimetry with radiographic film has been performed to determine the dose profile in the matchline region.

The treatment planning system uses a default slice thickness, which results in the creation of small erroneous inhomogeneities in the matchline region when introducing the actual slice thickness. The absolute measurements showed a dose escalation up to $15 \%$ dependent on the distance to the gantry rotation axis, while the measurements with radiographic film showed a good agreement between the calculated and the measured dose in the matchline region. A dose reduction of $14 \%$ or a dose escalation of $20 \%$ can be introduced when the treatment couch is indexed respectively $1 \mathrm{~mm}$ more or $1 \mathrm{~mm}$ less than the prescribed indexing between two consecutive slices.

\section{PO-T- 75}

Modulation of Multi leaf Collimator Dose Undulation

I Das*, R Mitra, Fox Chase Cancer Center, Philadelphia, PA, Fox Chase Cancer Center, Philadelphia, PA

With the emergence of dynamic conformal and intensity-modulated therapy, the multileaf collimator (MLC) is expected to play a major role in patient treatment. Unfortunately, the jagged MLC dose profile (undulation) is a major distraction and psychological impediment for full acceptance of such technology. The undulation index (UI) can be minimized or eliminated to a near closeness of a blocked beam-edge dose distribution by changing the MLC pattern or by translating the treatment table. Such study was performed for a Siemens MLC where UI is maximum for the fields at $45^{\circ}$ MLC leaves. The treatment table was incremented in one-millimeter step from the original position to a maximum of $8 \mathrm{~mm}$ in the $\mathrm{x}, \mathrm{y}$, and diagonal perpendicular directions. Results show that the pattern of UI with respect to the table increment has a parabolic shape with a minimum UI at nearly 5 $\mathrm{mm}$ step. The table translations in the $\mathrm{x}$ and $\mathrm{y}$ directions do not provide improvements in UI with MLC. However, when table is increased perpendicular to the leaves, the UI improved significantly. With a perpendicular translational step of $5 \mathrm{~mm}$ the dose distribution of MLC is dramatically close to a blocked field. The table translation perpendicular to the MLC leaves improves the dose distribution and maintains nearly the same penumbra for the $20 \%-80 \%$ isodose lines. However, there is slight increase in $10 \%-90 \%$ isodose penumbra with this method. The table 
translation technique can be easily achieved with modern treatment table with digital interface and record and verify system.

\section{PO-T- 76}

Copper-62 an Alternative Isotope for Intravascular Brachytherapy

R Chan*, N Yang, Washington Hospital Center, Washington, DC, Washington Hospital Center, Washington, DC

Clinical trials have shown that intravascular brachytherapy is effective in reducing restenosis. Beta source is preferred because of shielding issue and liquid or gas filled balloon seems to give the best dose distribution. Major concerns with liquid filled balloon are organ dose and contamination. The purpose of this study is to evaluate the possible use of Copper-62, a PET agent with a half-life of 9.7 minutes as an alternative agent for liquid filled balloon brachytherapy.

As model, a $3 \mathrm{~mm} \times 30 \mathrm{~mm}$ cylinder filled with liquid Rhenium-188 and Copper-62 is used. Monte Carlo (MCNP4B) codes is used to generate the dose rate distribution for the two isotopes in water. With a specific activity of $100 \mathrm{mCi} / \mathrm{ml}$ of $\mathrm{Cu}-62$ can delivers a dose of 24 Gy to a point $0.5 \mathrm{~mm}$ from the surface of a $3 \mathrm{~mm} \times 30 \mathrm{~mm}$ balloon in 5.25 minutes. Surface dose is $38.4 \mathrm{~Gy}$ and decreases to $17 \mathrm{~Gy}$ at $1 \mathrm{~mm}$ depth.

Compare to $\mathrm{Re}-188, \mathrm{Cu}-62$ results in similar treatment time. Moreover, surface dose is lower by about $12.5 \%$ and dose to $1 \mathrm{~mm}$ depth is higher by about $15 \%$. The two $0.511 \mathrm{MeV}$ photons give off by $\mathrm{Cu}-62$ constitute only $0.2 \%$ of the dose. Since the amount of activity needed for treatment is similar to that used for PET scan, the total dose that will be deposited in case of a balloon rupture is safe. With its short half-life, decontamination time would be less than 3.5 hours

\section{PO-T- 77}

\section{A Moving-Frame Method in HDR Brachytherapy}

S Li*, T DeWeese, D Lee, M Wharam, T Frankel, J Dicello, Radiation Oncology, Johns Hopkins Oncology Center, Baltimore, MD

The primary requirement in using HDR brachytherapy for the post-surgical treatment of microscopic soft tissue sarcoma is to adequately encompass the planning target volume, defined as a fixed distance from the surgical bed, with a prescribed dose by adjusting source dwell times in catheters. However, cold spots may exist when using the geometrical optimizations in a commercial planning system (Plato, Nucletron International Corp., Netherlands). The moving frame method described here allows us to determine the lowest dose points and to verify the dose distributions in the treatment depth. The treatment depth is a super-plane in a moving-frame. The moving-frame is defined by the surgical bed or 3D-implant surface. Any low dose point in the depth, $d$, is easily determined by

$$
\left\{X_{i, j}+X_{k, 1}+X_{k, l+1}, Y_{i, j}+Y_{k, 1}+Y_{k, l+1}, Z_{i, j}+Z_{k, 1}+Z_{k, l+1}\right\} / 3+d\left\{N_{x}, N_{y}, N_{z}\right\}
$$

where the subscripts $i$ and $\mathrm{k}$ are the labels of catheters; the $\mathrm{j}, 1$ and $1+1$ indicate the source dwell positions in the two catheters; $\left\{\mathrm{N}_{\mathrm{x}}, \mathrm{N}_{\mathrm{y}}, \mathrm{N}_{\mathrm{z}}\right\}$ is the normal vector of the implanted surface at the point. Using the moving frame method, we have found cold spots of 80-percent or lower at the treatment depth for implants with unevenly spaced catheters and for implants with several catheters. By minimizing the dose variation in the treatment depth, we can eliminate the cold spots and provide an optimum spatial and temporal source distribution.

\section{PO-T- 78}

Multigroup Discrete Ordinates Modeling of I125 6702 Seed Dose Distributions Using a Broad Energy-Group, Cross-Section Representation

G Daskalov*1, R Baker ${ }^{2}$, J Williamson ${ }^{3}$, D Rogers ${ }^{1}$, (1) National Research Council of Canada, IRS/INMS, Ottawa, Ontario, (2)LANL, Los Alamos, New Mexico, (3)Washington University, St. Louis, MO

The Discrete Ordinates Method (DOM) is a deterministic numerical solution of the Boltzmann transport equation. Our previous studies clearly demonstrated that 2D DOM dose calculations, as implemented by the code DANTSYS, accurately ( $\pm 2-3 \%)$ reproduce Monte Carlo photon transport (MCPT) simulations for encapsulated sources throughout the brachytherapy energy range. However, those simulations used very fine discretizations of the energy variable, i.e., 85 energy bins or "groups" below $35 \mathrm{keV}$, which limited the efficiency gains achieved for I-125. We now seek to optimize DOM computational efficiency by minimizing the number of energy groups. A key step is the development of geometry independent, source oriented weighting functions, i.e., approximations to the I-125 photon spectra encountered. The weighting functions developed in this study were used to derive a broad 3-group (G-3) cross-section library. Dose distributions in 2D cylindrical geometry around the model 6702 I-125 seed were calculated by DANTSYS using the G-3 library and were compared to corresponding MCPT simulations. The G-3 DANTSYS simulations retain 5\% accuracy while increasing computational speed by a factor of 9 relative to the 85 group DANTSYS results. The G-3 DANTSYS calculations are a factor of 80 faster then the MCPT ones. These results indicate that the development of accurate source-specific broad group libraries is an important and feasible step opening the opportunity of direct applications of transport calculations to brachytherapy treatment planning.

This work was supported by NIH Grant R01 CA 46640.

\section{PO-T- 79}

\section{Calculation of Beta-ray Dose Distributions from Ophthalmic} Applicators

W Cross ${ }^{* 1}$, J Hokkanen ${ }^{2}$, H Jarvinen ${ }^{2}$, F Mourtada ${ }^{3}$, P Sipila ${ }^{2}$, C Soares ${ }^{3}$, S Vynckier $^{4}$, (1)Atomic Energy of Canada Ltd., Chalk River, Canada, (2)Radiation and Nuclear Safety Authority, Helsinki,Finland, (3)National Institute of Standards and Technology, Gaithersburg, MD, (4)Catholic University of Louvain, St-Luc Hospital, Brussels, Belgium

Beta-ray dose distributions throughout the eye, from three types of ophthalmic applicator, have been calculated using the ACCEPT 3.0, EGS4 and other Monte Carlo codes, for comparison with recent measurements ${ }^{1}$. The beta applicators were those used in an international intercomparison ${ }^{1}-$ planar applicators of ${ }^{106} \mathrm{Ru} / \mathrm{Rh}$ and ${ }^{90} \mathrm{Sr} / \mathrm{Y}$ and a concave $\mathrm{Ru} / \mathrm{Rh}$ applicator. For the $\mathrm{Ru} / \mathrm{Rh}$ planar source, calculated and experimental results agree within the experimental errors (4-10\%) out to a depth of $7 \mathrm{~mm}$. Agreement is significantly better for the concave $\mathrm{Ru} / \mathrm{Rh}$ source, but somewhat poorer for the $\mathrm{Sr} / \mathrm{Y}$ planar source.

Past attempts have been made to derive dose distributions simply by integrating the appropriate point source dose function over the source. Here, we have investigated the accuracy of this procedure for encapsulated sources, by comparing the results with Monte Carlo calculations. We attempted to allow for the effects of the source window, but no corrections were made for scattering from the source backing. In these circumstances, at $6 \mathrm{~mm}$ depth the difference in the two calculations is $14-18 \%$ for a planar $\mathrm{Ru} / \mathrm{Rh}$ applicator and up to $30 \%$ for the concave $\mathrm{Ru} / \mathrm{Rh}$ source. It becomes worse at greater depths. These errors are attributed to changes in the beta spectrum and angular distribution produced by the source encapsulation.

${ }^{1}$ C.G. Soares et al, to be published.

\section{PO-T- 80}

Improved Dose Uniformity for Radioactive Stents in Endovascular Brachytherapy

H Amols*, Memorial Sloan Kettering Cancer Center, New York, NY

Endovascular brachytherapy has rapidly gained acceptance as a new treatment modality for reducing restenosis and improving the success rate of Percutaneous Transluminal Coronary Angioplasty (PTCA). Recent clinical results on patients treated with beta emitting P-32 stents suggest that radiation reduces 'in stent' restenosis but may exacerbate neointimal growth at the edges of the stents. This has been referred to as 'candy wrapper effect'. It is well known that radioactive stents yield extremely inhomogeneous dose distributions, with low doses being delivered to tissues in between stent struts, at the ends of the stent, and also at depth. Some animal model studies suggest that low doses of radiation may stimulate rather than inhibit neointimal growth in an injured vessel, and it is hypothesized that dose inhomogeneity at the ends of a stent may be a cause of 'candy wrapper effect'. We present comparisons of dose distributions for;

a. beta stents vs. gamma stents;

b. 'dumbbell' radioactive loaded stents vs. uniformly loaded stents;

c. stents with alternate strut design. 
It is demonstrated that dose inhomogenieties around a stent- particularly in regions between stent struts and at the ends of stents can be reduced by better stent design and isotope selection. Radiobiological modeling suggests that improved dose distributions could reduce 'candy wrapper effect'.

\section{PO-T- 81}

Calculation of Dose Distribution near an Innovative Concentric Balloon Cather for Endovascular Brachytherapy

Z Xu*, L Reinstein, G Yang, ${ }^{1}$, P Cole, ${ }^{2}$, (1) SUNY at Stony Brook, Stony Brook, NY, (2) Yale University School of Medicine, New Haven, CT

One of the possible intravascular irradiation techniques is to use a radioactive solution filled catheters. This method has the advantage of accurate source position and a uniform dose to the vessel wall. However it has a potential problem of chemical and radiological toxicity of the radioactive liquid due to the risk of balloon rupture. In order to minimize the risk of the toxicity in case of balloon rupture, an innovate concentric balloon catheter was designed to increase the dose per volume of radioactive solution with inner balloon and the outer balloon filled with saline and radioactive solution, respectively. The dose rate versus the radial distance from the center of the radioactive-filled balloon was calculated by numerical integration for P-32, Re-188, and Y-90 with various inner and outer radii. The results showed that for a balloon with outer radius of $1.5 \mathrm{~mm}$, there is no advantage of double balloon. For a balloon with outer radius of $3.0 \mathrm{~mm}$ and the inner radius of $2 \mathrm{~mm}$, the dose rate reduction is $30 \%$ whereas the volume reduction is as big as $45 \%$ at the point of $0.5 \mathrm{~mm}$ away from the outer surface (prescription point). The optimal inner radius is $1.5 \mathrm{~mm}$ since by further increasing the inner radius the reduction of the dose rate is at the same pace as the reduction of the volume. A similar calculation was done for balloon with outer radius of $5 \mathrm{~mm}$. The results showed that in this case $3 \mathrm{~mm}$ inner radius is the optimal radius.

\section{PO-T- 82}

Dose Uniformity Along and Away From the Axis of a Vascular Lumen Undergoing Intravascular Brachytherapy Using Various Radioactive Sources

N Yue*, R Nath, K Roberts, Department of Therapeutic Radiology, Yale University School of Medicine, New Haven,CT

In intravascular brachytherapy, either photon or electron emitters are used in a linear arrangement so that blood vessels of $2-4 \mathrm{~cm}$ lengths can be treated. To investigate the dose uniformity and the range of doses various components of the blood vessel receive, dose distributions were calculated on a $2 \mathrm{~cm}$ long cylindrical blood vessel of $2 \mathrm{~mm}$ radius. The radioactive sources of ${ }^{192} \mathrm{Ir},{ }^{125} \mathrm{I},{ }^{103} \mathrm{Pd},{ }^{188} \mathrm{Re},{ }^{32} \mathrm{P}$, and ${ }^{90} \mathrm{Y} / \mathrm{Sr}$ were studied. All the sources were assumed to be in the form of line and $3 \mathrm{~cm}$ long. It was found that electron emitters provided more uniform dose longitudinally than photon emitters. Uniformity, defined as the ratio of minimum and maximum dose, ranged from 0.99 to 1.00 for the three electron emitters and ranged from 0.93 to 0.94 for the three photon emitters when the source was placed isocentrically. On the other hand, if the source was off-centered during treatment, the uniformity around the blood vessel became increasingly worse as the off-center distance increased. The uniformity was worse for offcentered electron emitters than the photon emitters. For example, if the offcenter distance was $1 \mathrm{~mm}$, the uniformity in the central plane was $0.30,0.30$, $0.28,0.14,0.10$, and 0.16 for ${ }^{192} \mathrm{Ir},{ }^{125} \mathrm{I},{ }^{103} \mathrm{Pd},{ }^{188} \mathrm{Re},{ }^{32} \mathrm{P}$, and ${ }^{90} \mathrm{Y} / \mathrm{Sr}$ sources, respectively; and the maximum dose could be over $300 \%$ of the prescription dose while the minimum dose could be as low as $32 \%$ of the prescription dose.

\section{PO-T- 83}

Effects of Applicator Spatial Placement Variations on Cumulative Dose to Point $A$ in the Treatment of Cervical Cancer with 3 HDR Intracavitary Brachytherapy Treatments

J Rahimian*, Y Qian, R Kagan, Kaiser Permanente Medical Center, Department of Radiation Oncology, Los Angeles, CA.

We evaluated the effects of tandem and ovoids applicator spatial placement variations on the total dose received to points A when treating cervical cancer with 3 fractions of HDR one week apart. A Fletcher-Suit applicator, with a Nucletron Ir-192 remote afterloading HDR unit, and a CMS treatment planning computer were used. We evaluated seven patients with stage I, \& II cervical cancer treated with 3 fractions of HDR. The prescribed dose was 700 cGy per fraction to point A for all except one patient with $770 \mathrm{cGy}$ perfraction. The third fraction's orthogonal AP, and lateral films were used as reference images. The corresponding films from the first, and second implants were superimposed on the reference filmsmatching at the bony landmarks. The Manchester defined point A's, and the source dwell positions from the 3 tandems, and ovoids were digitized. Point A dose variations of as much as $33 \%$ were observed for applicators positioned $2.3 \mathrm{~cm}$ superior to the reference fraction on Y-axis. The spatial displacement of the applicator from midline on $\mathrm{X}$-axis resulted in dose asymmetry between right and left $\mathrm{A}$. Comparisons between fractions should be done prior to second and third treatments. If applicator position varied more than $1.5 \mathrm{~cm}$, its placement should be reevaluated.

\section{PO-T- 84}

In-vivo Dosimetry for Quality Control in Conformal Brachytherapy for Cervical Carcinoma.

A Riet*, L Elders, C Hoekstra, V Althof, E Vonk, A Mak, RISO, Deventer, The Netherlands.

The aim of this study was to demonstrate the suitability of in-vivo dosimetry for on-line quality control in HDR-brachytherapy for cervical carcinoma. The dose distribution delivered by a Fletcher type applicator with standardized source loading is well-known, tables and atlas dosedistributions can be employed for quality control.

Nowadays anatomical data is obtained from MRI making it possible to conform the dosedistribution to the target volume using a stepping source. The inversely planned dosedistribution is difficult to verify by pre-calulated dosetables. For this reason in-vivo dosimetry was introduced.

In our institute the Rotterdam applicator is modified with a channel through each ovoid allowing for implant of needles into the cervix. After insertion, a calibrated semiconductor dosimeter (Scanditronix) is connected to the applicator at a pre-defined position making it possible to pre-calculate the dose.

Since April 1998, 89 measurements have been recorded in 22 patients. On an average the ratio between the dose measured and that computed amounted to $0.88(n=44, \sigma=0.04)$ for the ovoids, $0.95(n=22, \sigma=0.03)$ for the intrauterine tube and $0.91(\mathrm{n}=23, \sigma=0.06)$ for the needles respectively. A systematic discrepancy between computed and measured dose is registered. The calibration factor of the dosimeter should be adjusted accordingly.

The relatively small standard deviations demonstrate the suitability of confirmatory in-vivo dosimetry for on-line quality control. In present-day a difference of $10 \%$ (about $2 \sigma$ ) between measured and pre-computed dose is regarded as tolerance level.

\section{PO-T- 85}

Enhancement of Tumor Coverage by Interstitial Support to the Intracavitary HDR Brachytherapy for Cervical Carcinoma.

A Riet*, C Hoekstra, T Kuipers, L Elders, E Vonk, A Mak, Institute for Radiotherapy and Curietherapy, RISO, Deventer, The Netherlands.

In brachytherapy for cervical carcinoma, generally a Fletcher type applicator is applied. The Gross Tumor Volume (GTV), however, often expands laterally and, in consequence, may not be fully covered by the pear-shaped reference isodose. This study concerns a change of the conventional dosedistribution by combining endocavitary and interstitial irradiation.

To widen the dosedistribution each ovoid was provided with a channel for implant of a needle into the cervix. In order to determine the necessity for needle implant, anatomical data was obtained from pre-treatment MRI. Since April 1994, combined intracavitary and interstitial treatment was indicated in 44 out of 61 patients. Dose-volume histograms were calculated with an without needles respectively and related to the GTVs as identified on the MRI.

The combination with needles and dose optimisation made it possible to achieve a substantial increase of the Covering Index (CI), when compared with endocavitary treatment only. In patients with a relatively small GTV $(<$ $30 \mathrm{cc}$ ) an increase in CI from 0.87 to 0.94 was found while in patients with a 
median GTV $(70 \mathrm{cc})$ an increase in CI from 0.57 to 0.73 was registered. Tolerance dose of bladder or rectum was not exceeded.

In HDR brachytherapy applied in the treatment of cervical carcinoma, the combined method of endocavitary and interstitial irradiation seems to be a feasible method. An increase of the tumor coverage is achieved in association with a decrease of the total volume treated.

\section{PO-T- 86}

Treatment-plan Optimization for Soft-tissue Sarcomas Using a Genetic Algorithm

A Fung ${ }^{* 1}, \mathrm{~K}$ Alektiar ${ }^{1}$, D Silvern ${ }^{2}$, M Zaider $^{1}$, (1) Memorial Sloan-Kettering Cancer Center, New York, (2) Rabin Medical Center, Petah-Tikva, Israel

The treatment of soft-tissue sarcomas using interstitial brachytherapy entails placing catheters at regular intervals in the target and then loading them with ribbons containing Ir-192 or I-125 seeds. The prescription (typically 45 Gy) is given on the hull that surrounds the catheters approximately $0.5-1-\mathrm{cm}$ away. The catheter surface has an irregular shape; thus the single-plane approximation is inapplicable. Furthermore, employing single-strength sources would result in unsuitable plans. The solution adopted at MSKCC is to use combinations of several seed strengths. If the plan is designed manually it may take up to 6 hours to complete. This is inadequate because a manual plan is suboptimal, and because a 24-hour treatment delay is likely (ribbons ordered after $4 \mathrm{pm}$ cannot be delivered next day). As an alternative, we have designed software that selects the distribution of seeds needed for optimal coverage of the target. The input consists of seed coordinates (reconstructed from isocentric films), prescription points, and a list of available seed activities. The optimization is performed using a genetic algorithm. A typical plan obtained in an hour produces better than $80 \%$ coverage (against 50\% in manual planning). If the prescription hull is more than $7.5-\mathrm{mm}$ away from catheters $95 \%$ coverage can be routinely achieved.

\section{PO-T- 87}

Effects of Vessel Curvature on Dose Distribution in Intravascular Brachytherapy For Various Radionuclides

N Yue*, K Roberts, R Nath, Department of Therapeutic Radiology, Yale University School of Medicine, New Haven, CT

When radioactive sources are used to treat restenosis in intravascular brachytherapy, in most cases the blood vessels are curved even though the sources are usually manufactured in a linear arrangement. To investigate the effects of the curvature on the dose distribution in the blood vessels, calculations were performed on a $2 \mathrm{~cm}$ long cylindrical blood vessel of $2 \mathrm{~mm}$ radius of different curvatures. The radioactive source of ${ }^{192} \mathrm{Ir},{ }^{125} \mathrm{I},{ }^{103} \mathrm{Pd}$, ${ }^{188} \mathrm{Re},{ }^{32} \mathrm{P}$, and ${ }^{90} \mathrm{Y}$ were studied. All the sources were assumed to be in the form of line and had a length of $3 \mathrm{~cm}$. It was found that curvature changed the dose uniformity and dose distribution. In general, curvature caused an increase in dose in the inner surface (toward the curvature) of blood vessels and a decrease in dose in the outer surface (away from the curvature), and the changes increased with the increase of curvature. Depending upon radionuclide, if the source was placed at the central axis of blood vessels, the maximum increase was in the range of $20 \%$ to $30 \%$ and the maximum decrease was in the range of $10 \%$ to $15 \%$ when the curvature was less than 45 degree. It seemed that the curvature had similar effects for all the radionuclides studied, no matter whether or not it was a photon emitter or electron emitter.

\section{PO-T- 88}

Radiochromic Film Dosimetry of a High Dose Rate Beta Source for Intravascular Brachytherapy

D Duggan*, C Coffey ${ }^{1}$, J Lobdell ${ }^{2}$, M Schell ${ }^{3}$, (1)Vanderbilt University Medical Center, Nashville, TN, (2)Novoste Corp., Atlanta, GA,(3)University of Rochester, Rochester, NY

Good clinical physics practice requires that dose rates of brachytherapy sources be checked by the institution using them, as recommended AAPM Task Group 56 and the A.C.R. For intravascular brachytherapy with catheter-based systems, AAPM Task Group 60 recommends that the dose rate be measured at a reference point at a radial distance of $2 \mathrm{~mm}$ from the center of the catheter axis. That task group also recommends that: The dose rate along the catheter axis at a radial distance of $2 \mathrm{~mm}$ should be uniform to within $\pm 10 \%$ in the center two-thirds of the treated length. The relative dose rate in the plane perpendicular to the catheter axis through the center of the source should be measured at distances from $0.5 \mathrm{~mm}$ to $\mathrm{R}_{90}$ at intervals of $0.5 \mathrm{~mm}$. Radiochromic film dosimetry has been used to measure the dose distribution in a plane parallel to and at a radial distance of $2 \mathrm{~mm}$ from the axis of a novel, catheter-based, beta source for intravascular brachytherapy. The minimum, maximum and average dose rates on a line parallel to the catheter axis in that plane, in the centered $24.5 \mathrm{~mm}$ of the treated length, were calculated. Four each of four different devices: The average agreed with the dose rate measured with a well ionization chamber by the replacement method, using source trains calibrated with an extrapolation chamber at NIST. The maximum and minimum were within $+10 \%$ of the average.

\section{PO-T- 89}

Stent Effect on Beta Dose Distributions for Intravascular Brachytherapy

P Fan*, S-T Chiu-Tsao, H.S. Tsao ${ }^{2}$,J Pisch, L Harrison, Beth Israel Medical Center and St. Lukes Roosevelt Hospital Center, (2) Sun Microsystems, Hackensack, NJ

In the treatment of in-stent restenosis with intravascular brachytherapy, the influence of stent on the doses delivered to target tissues may be clinically significant. We have performed Monte Carlo dose calculations with and without stent for three beta isotopes, Y-90 $(2.3 \mathrm{MeV}), \mathrm{Rh}-106(3.5 \mathrm{MeV})$ and Pr-144 $(3 \mathrm{MeV})$, in the perpendicular bisector of each source. The stent struts $\left(63.5 \times 100\right.$ microns) are at $1.5 \mathrm{~mm}$ from the source and $18^{\circ}$ apart. Three stent materials, steel, nitinol and tantalum, are studied. The stent factor, $s(r, \phi)$, defined as the ratio of dose with stent strut to that in the homogeneous water, was obtained as a function of radial distance, $r$, and azimuthal angle, $\phi$. The scoring voxels were $0.1 \mathrm{~mm}$ in $\mathrm{r}$ and $2^{\circ}$ in $\phi$.

A dose reduction was seen behind the stent. For all three isotopes, the reduction right behind a strut was $15 \%$ for steel and nitinol, and $40 \%$ for tantalum. We also found dose enhancement right in front of a strut. The stent factors are 1.05 (steel and nitinol) and 1.2 (tantalum) for Rh-106 and Pr-144, and 1.1 (steel and nitinol) and 1.3 (tantalum) for Y-90. In the regions between two neighboring struts, dose enhancement is also noted, with $\mathrm{s}=1.1$ for tantalum and 1.07 for steel and nitinol.

In conclusion, the dose reduction and enhancement due to the presence of stent are significant. We will discuss the stent effect for different strut spacing.

\section{PO-T- 90}

Assessment of EGS4 for Sr-Y 90 Intravascular Brachytherapy Dosimetry

R Wang*, X Allen Li, C Yu, University of Maryland, Baltimore, MD

The purpose of this work is to examine the accuracy and applicability of EGS4/PRESTA system for dose calculation in intravascular brachytherapy (IVBT) using Sr-Y 90 source. Such an examination is necessary because of the potential limitation of the multiple-scattering algorithm used in EGS4 for electron transport when applied to very small geometric region (small source size and scoring regions). We examined the effects of each of the EGS4 transport parameters on the accuracy and efficiency. (1) For dose spatial resolution of $0.1 \mathrm{~mm}$ or coarser, good accuracy can be achieved when the cutoff kinetic energy of electrons and photons is set to $0.01 \mathrm{MeV}$. (2) Limiting the energy loss per electron step (ESTEPE) did not affect significantly on accuracy. Thus, one may use larger ESTEPE or no ESTEPE control to gain efficiency without sacrificing accuracy as long as PRESTA is turned on. (3) Similar independence of AE (the lowest energy for secondary electrons) is also observed for $\mathrm{AE}$ in the range of 0.521- 0.661 MeV. An attempt is also being made to examine the adequacy of the multiplescattering algorithm for smaller region and interface encountered in IVBT by using a single scattering scheme. More results will be presented. 


\section{PO-T- 91}

Distributions of Tissue Volume Dose Resulting from Small Compared to Large Dwell Source Stepping in the Rings and Tandems of Gynecological HDR Brachytherapy

G King*, S Nagappan, R Mogus, A Kalend, University of Pittsburgh Medical Center, Pittsburgh, PA

A RING IS IDEAL FOR CERVIX HDR BRACHYTHERAPY BUT, UNLIKE TRADITIONAL OVOIDS, IS UNSHIELDED HENCE PRODUCES CIRCULAR DOSE DISTRIBUTIONS NEEDING OPTIMIZATION TO LOWER NORMAL TISSUE DOSES. STANDARD SINGLE-PARAMETER OPTIMIZATION TECHNIQUE ADJUSTS DWELL TIMES $\Delta \mathrm{T}$ AT FIXED SPACING, $\Delta \mathrm{S}_{0}$. A DOUBLEPARAMETER $[\Delta \mathrm{S}, \Delta \mathrm{T}]$ OPTIMIZATION IS STUDIED IN TERMS OF VARIOUS TYPES OF DOSE-VOLUME-HISTOGRAMS WITH GRADIENT INDICES.

3D dose-volume matrices were computed for Rings different in degrees of tandem bend and diameters. $\Delta \mathrm{S}$ was incremented $2.5-10 \mathrm{~mm}$ and $\Delta \mathrm{T}$ renormalized to constant tumor doses. Comparative merits rested in natural $[\mathrm{nDVH}]$, cumulative $[\mathrm{cDVH}]$ and differential $[\delta \mathrm{DVH}]$ histogram indices in cc/cGy.

RESULTS SHOWED SMALLER DWELLS PRODUCED SUPERIOR DOSE-VOLUME UNIFORMITY. DECREASED $\triangle$ S LOWERED DOSEVOLUME GRADIENTS. AT 2.5, 5.0 AND 10.0MM $\Delta$ S, DIFFERENTIAL HISTOGRAM RATIOS AVERAGED 0.110, 0.166 AND 0.20 CC/CGY, OR CUMULATIVE VALUES OF 19, 21 AND 24 CGY/CC. NATURAL HISTOGRAMS WERE SHOULDER-LESS AT BACKGROUNDS RISING FROM 9.5, 10.1, TO 12 CC/CGY.

We conclude two-parameter optimization produces dose volume gradient generally flatter with shorter spacing $\Delta \mathrm{S}$. Clinically, it is desirable to sharpen the gradient falls around the bladder and rectum, yet maintain the gradient flat throughout the cervix volume. Dual parameter $[\Delta \mathrm{S}$ plus $\Delta \mathrm{T}]$ optimization may be a desirable HDR optimization technique, particularly for the Ring applicator

\section{PO-T- 92}

Dosimetric Analysis of Permanent Prostate Implant with I-125 and Pd103

Y Cao*1, X Wang ${ }^{1}$, L Potters ${ }^{1}$, C Ling ${ }^{2}$, (1) Memorial Sloan-Kettering at Mercy, Rockville Centre, NY, (2) Memorial Sloan-Kettering, New York, NY

This study was undertaken to assess CT based dosimetry for patients undergo transperineal interstitial permanent prostate brachytherapy (TIPPB) using Pd-103 or I-125 for over 500 patients. TIPPB was performed using a peripherally weighted placement of isotope via an interstitial gun. Postimplant CT images were obtained on 2 weeks after the implant. Preliminary analysis of 100 patients showed that there is no difference in the mean V100\% (target volume covered by prescription dose) between I-125 and Pd103. The mean minimum peripheral dose was $60 \%$ of prescribed dose for I- 125 and $40 \%$ for Pd-103. Analysis of minimum point dose (cold spot) was found more commonly in implants with Pd-103. A difference between the pre-implant ultrasound prostate volume and post-implant CT volume was also apparent. In conclusion, good dose coverage of the prostate is attainable with TIPPB. Prostate volume changes post implant likely represent edema. Differences in minimum dose points between I-125 and Pd-103 may require different implant techniques. The impact of these findings on TIBBP will be discussed in detail.

\section{PO-T- 93}

Permanent 125I Prostate Implants: Post-implant Dosimetry Based on Images Acquired on a Single Day Can Only be Approximate R Taschereau*, A Martin, J Pouliot, CHUQ, Quebec, Canada

It has been recently suggested that for ${ }^{125} I$ permanent prostate implant postimplant dosimetry should be performed with images acquired about 30 days post-implantation. The rationale is that by that time, prostate edema is most likely resolved and a stable volume and seed distribution are available for dosimetry. The objective of this study is to compare post-implant dosimetry performed with the fixed volume obtained at 30 days with dosimetry considering a volume varying with respect to time.

Information on edema has been gathered on ten patients who have been followed for a period of 30 to 180 days post-implant. Based on these observations, a few edema scenarios were elaborated. The scenarios were applied to a real case along with simulated migration. A special dosimetry taking into account the volume variation with time was calculated and compared to the usual fixed-volume post-implant dosimetry. Differences observed between computing methods ranged from +5 to -15 points.

Unless by coincidence, it is not possible to perform an exact post-implant dosimetry based on a fixed volume when in fact the volume varies with time. If edema has resolved by the time images are acquired, then the fixedvolume method will overestimate coverage. If edema has not resolved an underestimation is likely to be attained. A more accurate but less practical post-implant dosimetry could be obtained by using a series of images spread over a period of time

\section{PO-T- 94}

An Organ DVH Based Optimization Algorithm for Ultrasound Guided Prostate Implants

Y Chen*, L Xing, Stanford University School of Medicine, Stanford, CA

A clinical concern with prostate implant using I-125 or Pd-103 seeds is how to limit the rectal and urethra dose. Because the volume of prostactic urethra is also part of the target, the dose-volume histogram $(\mathrm{DVH})$ of the target alone could not provide adequate information for "goodness" of treatment plans. Based on an objective function sensitive to organ DVHs, we developed an inverse planning method that considers the urethra and rectal wall as critical structures in optimization of source locations. The objective function was constructed using different importance factors for the target and critical structures with an additional term to restrict the number of needles involved for source loading. The objective function is minimized by an iteration algorithm. During an iteration each source is moved to a neighboring location if the objective function is decreased. With every source migration the source strength is rescaled to match the minimum target dose to the prescribed dose. The organ DVHs are controlled by an appropriate set of importance factors and the pre-specified organ tolerance doses. To avoid being trapped into an undesired local minimum state, a series of trials with different numbers of sources and randomized initial source positions are generated. Each trial takes less than two minute on a $233 \mathrm{MHz}$ PC. The system selects the final plan by automatically evaluating the "optimal" solutions resulting from these trials. Our system also has the flexibility to adapt the source strength available.

\section{PO-T- 95}

New Nomogram for Peripheral Loading of Prostate Brachytherapy Using Iodine-125 Seeds

A Wu*1, M Johnson ${ }^{1}$, C Lee ${ }^{2}$, D Brown ${ }^{1}, \mathrm{P} \mathrm{Geis}^{1}$, S Kalnicki ${ }^{1}$ (1) Allegheny General Hospital, Pittsburgh, PA (2) Chang-Cung Memorial Hospital, Shaing Taoyuen Hsien, Tiawan

In recent years, brachytherapy has become one of the important primary treatment modalities for early stage prostate cancer patients using iodine radioactive seeds. To determine the total activity required for the implant of uniform seed distributions, the traditional approach has been based on the use of a nomogram. Various nomograms have been developed and used by many other centers. However, for preferential seed loading around the periphery of the prostate gland, a new sphero-cylincrial dose model has been developed. In this presentation, for given sizes of the gland, a special peripheral loading of the seeds is configured. The accumulated isodose surface encompassing the target volume is calculated according to TG-43. As a result, the total activities of the iodine- 125 seeds and the geometrical average dimensions calculated from the various target volumes are plotted on a $\log -\log$ scale. The equation of a nomogram, namely $\mathrm{A}=2.00 \mathrm{~d}^{2.12}$, is an especially formulated power function for peripheral loading. Furthermore, by using this specific nomogram approach, forty (4) implanted patients with iodine seeds were analyzed for their post-implant dosimetry based on CT images. The results including dose coverage, dose homogeneity, dose to the urethra and the overall urinary complications are presented for evaluation. 


\section{PO-T- 96}

The Relationship Between Volume and Dose Conformity Quantifiers in Prostate Brachytherapy

W Bice ${ }^{*}$, J Prete $^{1}$, D Dubois ${ }^{1}$, B Prestidge ${ }^{2}$, (1) UTHSCSA, San Antonio, TX, (2)Texas Prostate Brachytherapy Services, San Antonio, TX

Conformity quantifiers are used in permanent prostate brachytherapy as a measure of implant quality. They can be organized into two distinct groups, volume conformity quantifiers and dose conformity quantifiers. Both types are used to describe the sufficiency of the dose delivered to the clinical target volume, usually the prostate gland. Volume quantifiers delineate the volume of the target (or percentage of the target) that received a specified dose (or percent of the reference dose) or greater. Thus $\mathrm{V}_{100}$ refers the percentage of the target which received $100 \%$ of the reference dose or greater. The dose conformity quantifiers on the other hand refer to the dose at which the specified percentage of the target volume received that dose or greater. $D_{90}$ therefore refers to the dose at which $90 \%$ of the target received that dose or greater. Although both conformity quantifier types are used in the literature, the sole publication that relates implant quality to outcome uses only the dose conformity quantifier, suggesting a $\mathrm{D}_{90}$ of at least $140 \mathrm{~Gy}$ for iodine monotherapy.

In analyzing the results of post implant dosimetry from 150 patients, we have determined the relationship between the volume and dose conformity quantifiers in the clinical environment. Our results illustrate the relationship's dependence upon isotope, implant design, and reference dose. Based upon this analysis, achieving a $\mathrm{V}_{100}$ of $87 \%$ may be assumed to be equivalent to achieving a $\mathrm{D}_{90}$ of 140 for iodine monotherapy.

\section{PO-T- 97}

Analysis of the Quality of Prostate Implants Using Dose Volume Histograms and Homogeneity Indexes

N Dogan*, N Mohideen, L Leybovich, A Sethi, Loyola University Medical Center, Maywood, IL

In this work, we assessed the quality of prostate brachytherapy by calculating post implant 3D dose distributions. For 40 patients who underwent prostate brachytherapy using I125 or Pd103 and ultrasound-based treatment preplanning, CT scans were done prior to implant, 1-day, 7-day, and 28-day post implant. The prostate, rectum and bladder were contoured on all scans. Post treatment dose distributions were generated using FOCUS brachytherapy planning software. Dose calculation was based on TG43 formalism that included anisotropy of the sources. The minimum peripheral dose of 144Gy for I125 and 115Gy for Pd103 was chosen. DVHs for target, rectum, and bladder were analyzed for all patients using the conformity and uniformity quantifiers (CT-based TVR1 and TVR2, dose homogeneity indexes, DHI1, DHI2, and DNR, dose coverage indexes, rectal and bladder doses). All these indexes are described in the literature. The average TVR1, TVR2, and target coverage index were $1.98,2.13$, and 1.04 respectively. The average DVH1, DHI2, and DNR were $0.468,0.399$, and 0.531 respectively. All of these values were within the limits of published report based on the centralized multiinstitutional postimplant analysis. This study differs from the similar studies reported by other institutions in that the analysis was based on the sequential post implant 3-D dose distributions. Therefore, our values reflect evolution of the indexes with time. Results of this study may lead to improved methodology of prostate brachytherapy.

\section{PO-T- 98}

Use of Nomogram vs. Pre-planning to Determine Seed Activities for Prostate Seed Implants

X Wang*, J Zhang, L Potters, C Ling, Memorial Sloan-Kettering Cancer Center at Mercy, Rockville Centre, NY, Memorial Sloan-Kettering Cancer Center, New York, NY

This study was undertaken to assess the use of a nomogram for isotope activity used for transperineal interstitial permanent brachytherapy (TPIPB). One thousand patients have been treated with TPIPB using a pre-implant volume study and the Momorial nomogram to determine the total activity and number of seeds required for each patient. TPIPB was performed using peripherally weighted seed placement with an interstitial gun. Six hundred post implant CT scans were evaluated. Calculations were made to assess the prostate volume receiving $100 \%$ of the prescribed dose (V100), the dose covering $90 \%$ of the volume (D90), the matched peripheral dose and the patient volume encompassed by $100 \%$ of the prescribed dose (Vg100). For pd-103 implants the MPD is $152 \%$ (132-182\%) (mean and range), Vg100 $156 \%(112-181 \%)$, V100 92\% (82-97\%) and D90 108\% (81-127\%). For I125 implants they are, 129\% (121-136\%), 147\% (104-189\%), 90\% (77$99 \%$ ) and $107 \%(83-137 \%)$. To obtain a desired implant with a Vg100 and MPD of $100 \%$ each, a reduction in the total activity calculated by the nomogram, of $50 \%$ (Pd-103) and 30\% (I-125), is required. Manual preplanning to obtain desired activities is performed on two patients with both Pd-103 and I-125, an average reduction of activities by $29 \%$ were achieved compared to the nomogram. In conclusion, use of Memorial nomogram for I-125 and Pd-103 over-estimates the total activity required, individualized pre-planning may obviate this over-estimation. Clinical data, mathematical analysis and computer modeling will be presented.

\section{PO-T- 99}

The Dependence of Prostate Post-implant Dosimetric Quality on CT Volume Determination

W Butler*, G Merrick, J Lief, A Dorsey, Schiffler Oncology Center, Wheeling Hospital, Wheeling, WV

This work investigates the magnitude of the effect that various methods of treatment volume delineation have on dosimetric quality parameters.

8 consecutive prostate brachytherapy patients with a prescribed dose of 145 Gy from I-125 as monotherapy comprised the study population. The mean pretreatment parameters were $30.3 \mathrm{~cm}^{3}$ ultrasound volume, $51.8 \mathrm{~cm}^{3}$ planning volume, 131 seeds per patient and $42.9 \mathrm{mCi}$ total activity. On CT study sets obtained less than 2 hours post operatively, target volumes were drawn using three methods: prostate plus a periprostatic margin, prostate only, and transfer of the preplanning ultrasound of the prostate to the post-implant CT scan. Three sets of 5 dosimetric quality parameters corresponding to the different volumetric approaches were calculated: V100, V150 and V200 (fraction of the target volume covered by 100, 150, and $200 \%$ of the prescribed dose) and D90 and D100 (the minimal dose covering 90 and $100 \%$ of the target volume).

The CT volumes drawn with the prostate plus margin technique were comparable to the initial planning volumes (mean $55.5 \mathrm{~cm}^{3} v s .51 .8 \mathrm{~cm}^{3}$ ), while those determined via superimposing the preplan ultrasound resulted in volumes nearly identical to the initial ultrasound evaluation. The prostate only approach resulted in volumes approximately $25 \%$ larger than the ultrasound volume approach. Despite the volume determinations being markedly different, no significant differences in quality parameters between the approaches were appreciated for these patients in which the prostate plus margin was implanted.

\section{PO-T- 100}

The Effect of Prostate Swelling on the Dosimetric Parameters Proposed by TG-64

D Mellenberg*, G Weidner, S Hedican, University of Iowa, Iowa City, IA

Several objective measurements of prostate implant dosimetry are potential predictors of outcome and are helpful to improving implant technique. TG64 proposes several figures, D100, D90, D80, V200, V100, V90 and V80 for the objective prostate implant evaluation. Other quantities (i.e. V10 and V25 for bladder and rectum) may indicate changes in organ dose over time. By obtaining CT scans on the day of implant (DOI) and three weeks post, we found that swelling decreases by an average $34 \%$ for implant only patients (IMP) and $11 \%$ for external beam plus implant patients (XRTIMP). This agrees with published data. D100, D90 and D80 increase 27\%, 14\% and $12 \%$ respectively for averaged IMP and XRTIMP patients. These increases indicate an increase in the measures of implant quality between the DOI and three weeks. V200 decreases by $12 \%$ for IMP patients and increases by $20 \%$ for XRTIMP patients. The decrease in V200 for IMP patients indicates that smaller hot spots exist three weeks post implant, an increase in the implant quality measurement. V100, V90 and V80 decrease by $23 \%, 14 \%$ and $28 \%$ indicating implant dosimetry changes with the prostate volume during the 3 weeks following the implant. Other quantities such as bladder and rectum doses are somewhat worsened or inconclusive between DOI and three weeks post implant. 


\section{PO-T- 101}

Determination of the Urethral Dose in Prostate Brachytherapy when the Urethra is not Visualized in the Post-Implant CT Scan

F Waterman*, A Dicker, Thomas Jefferson University, Philadelphia, PA

A urinary catheter is often not utilized when the post-implant CT scan is obtained and the urethra cannot be visualized to calculate the urethral dose. This study investigates the feasibility of locating a surrogate urethra at the geometric center of the prostate as a solution to this problem. Twenty I-125 prostate implants which were scanned utilizing a urinary catheter were randomly selected. In each implant, the I-125 seeds were implanted at the periphery of the prostate producing a dose distribution characterized a broad dose minimum in the central region of the prostate containing the urethra. The urethra was outlined from the base to the apex of the prostate using the image of the urinary catheter. In addition, a surrogate urethra was outlined at the geometric center of the prostate. Dose-volume histograms were compiled and the D10, D25, and D50 doses of the actual and surrogate urethras were compared. The difference between surrogate urethra dose and actual urethra dose was $3.3 \pm 5.5 \%$ (mean \pm S.D. $)$ at D10, $1.0 \pm 6.0 \%$ at D25, and $2.3 \pm$ $7.1 \%$ at D50. These results show that urethra doses from D10 to D50 can determined to within $\pm 10 \%$ by a surrogate urethra located at the geometric center of the prostate when the I- 125 seeds are peripherally loaded and the urethra is not visualized in the post-implant CT scan

\section{PO-T- 102}

Seed Stability in the Prostate/Periprostatic Region Following Brachytherapy

W Butler*, G Merrick, M Benson, A Dorsey, J Lief, Schiffler Oncology Center, Wheeling Hospital, Wheeling, WV

This study evaluates the stability of seeds implanted in the prostate gland and periprostatic tissue and the time dependency of seed embolization to the lungs.

175 consecutive patients were implanted with about 130 seeds each of either I- 125 or Pd- 103 . $42 \%$ of I- 125 seeds $(71 \%$ of the total seeds were Rapid Strand) and $40 \%$ of Pd-103 seeds were intra-operatively placed in periprostatic tissue. Serial orthogonal films obtained from day 0 through 15 months evaluated seed stability in the target area. Post-implant chest x-rays were obtained from day 1 through 15 months.

The average seed loss per patient at 30 days was 2.2 seeds and 3.0 seeds for I-125 and Pd-103, respectively. After 30 days, an additional $0.5 \%$ seed loss was noted in each group. The seed pulmonary embolization rate for the entire group was $21 \%$ (range 1-3 seeds per patient). The actual percent of implanted seeds embolizing to the lungs was $0.24 \%$. Chest $\mathrm{x}$-rays obtained less than 30 days following implantation resulted in $0 \%$ of patients being detected with pulmonary seeds. Of patients who experienced local seed loss, approximately $50 \%$ of those seeds were identified in the lungs and the other $50 \%$ were lost to other sites.

A significant number of I-125 Rapid Strand or Pd-103 seeds can safely be placed in the periprostatic tissue with a high probability of prostate bed seed stability and a low incidence of seed embolization to the lung.

\section{PO-T- 103}

Concurrent Optimization of Seed Loading and Minimization of Number of Needles in Prostate Seed Implant by Simulated Annealing

A de la Zerda*, Henry Ford Hospital, Detroit, MI

Trauma to the prostate from multiple needle insertions in interstitial brachytherapy results in considerable swelling of the gland for up to two weeks. It may also contribute to side effects e.g., urethral constriction. Such change in target volume introduces significant uncertainties in dose coverage. Simulated annealing has been implemented here to obtain a plan delivering the prescribed dose to the prostate, while minimizing needle number. The objective function includes five additive components with weight factors that can be adjusted based on specific clinical needs. One component represents the deviation between prescribed and calculated dose at the prostate periphery. Another component is proportional to the current number of needles. A third component reflects the degree of dose uniformity within the prostate. Two additional terms are included in the objective function which impose a penalty if the calculated dose to the rectum or urethra exceed tolerance. An annealing scheme and weights were determined by extensive numerical experimentation. Temperature is defined as $0.999^{\mathbf{k}}$, where $\mathrm{k}$ is the iteration number. Starting with a random seed loading, the program randomly turns seeds on or off. Search terminates if 200 consecutive iterations do not decrease the objective function. This happens after about 5000 tries. Running time is about 20 seconds with an HP PII/300 computer. Comparison is made with a dosimetrist's manual plan in terms of coverage, uniformity, number of needles and dose to critical structures.

\section{PO-T- 104}

Automated Seed Recognition for Intraoperative Prostate Brachytherapy Dosimetry

P Cho*, B Adams, University of Washington, Seattle, WA

Post-implant dose computation of prostate brachytherapy requires seed coordinates. Commonly, the seeds are radiographically captured on film and their positions manually entered by digitizing stylus or mouse. However, for the purpose of intraoperative assessment and modification of dose distribution, manual seed recognition is too slow. To accelerate the process, we have developed an automated seed recognition algorithm based on thresholding, morphological processing, and connected component analysis. The program input is the digital implant image obtained with film or fluoroscopy. Output is the end-point coordinates of the seeds.

A method of extracting high contrast markers in relatively uniform background has been previously reported. However, segmentation of implant seeds is complicated by the presence of other high contrast objects such as balloon catheter, radio-opaque markers, and bony structures. The proposed method begins with grayscale thresholding. Since the balloonocculded region may contain seeds, this solid region is processed separately. To accomplish this, the thresholded image is morphologically processed to generate a mask which is subsequently multiplied by the original image to isolate the seeds within the balloon. Regions inside and outside the balloon are then combined. Residual high frequency pixel fragments are removed by the despeckling operation. Finally, the segmented pixels are logically connected and analyzed for shape and size for the determination of object class and coordinates. The technique was compared with other image segmentation methods including background subtraction and adaptive thresholding and was found to be more robust.

\section{PO-T- 105}

Measurement and Monte Carlo Calculation of Dosimetric Parameters on the Transverse Axis of a New Pd-103 Source

$\mathrm{Z} \mathrm{Li}^{1 *}$, J Fan ${ }^{2}, \mathrm{~J} \mathrm{Palta}^{1}$, (1) University of Florida, Gainesville, FL, (2) North American Scientific, Inc., North Hollywood, CA

Experimental measurements and Monte Carlo Photon Transport (MCPT) calculations are performed to estimate the transverse-axis dosimetric parameters of a new design Pd-103 seed source. The measurements were performed in a large scanning water phantom, using a diode detector. The positioning of the source and detector was achieved by a computercontrolled positioning mechanism in the scanning water phantom. The dose rate constant in water for the new Pd-103 source was measured in comparison with existing I-125 sources. The radial dose function values for the source is measured using the diode detector. An accurate model of the source design was used in MCPT calculations. The measured and calculated results are compared with the dose distribution parameters for the Pd-103 source discussed in the AAPM TG43 report. The measurement results appear to be in good agreement with the existing TP-200 Pd-103 source, with a measured value of $0.711 \mathrm{cGy} / \mathrm{U}-\mathrm{hr}$ for the dose rate constant in water of the new source. The radial dose function values agree quite well with those recommended by the TG43 report as well. This work was partially supported by North American Scientific, Inc. 


\section{PO-T- 106}

Experimental Measurement of Dosimetric Parameters on the Transverse Axis of a New I-125 Source

$\mathrm{Z} \mathrm{Li}^{1}$ *, J Fan ${ }^{2}, \mathrm{~J}$ Palta ${ }^{1}$, (1) University of Florida, Gainesville, FL, (2) North American Scientific, Inc., North Hollywood, CA

As sources of new designs are developed and marketed for application in permanent prostate implant, it is paramount that their dosimetric characteristics are carefully determined, in order to maintain the accuracy of patient treatment. This report presents the results of experimental measurements of dosimetric parameters performed for a newly available I125 seed source. The measurements were performed in a large scanning water phantom, using a diode detector. Positioning of the source and detector was achieved by a computer-controlled positioning mechanism in the scanning water phantom. The dose rate constant in water for the new I125 source was measured in comparison with an existing I-125 source of similar design, and verified using TLD measurement. The radial dose function values were measured using the diode detector. The measurement results are comparable with the dose distribution parameters for the I-125 sources discussed in the AAPM TG43 report. It is recommended that a value of $0.950 \mathrm{cGy} / \mathrm{U}-\mathrm{hr}$ be used for the dose rate constant in water of the new source, based on the NIST

1985 air-kerma strength calibration standard, or $1.060 \mathrm{cGy} / \mathrm{U}-\mathrm{hr}$ based on the NIST 1999 air-kerma strength standard. It is further recommended that the radial dose function values for the model 6702 I-125 source, as recommended by the AAPM TG43 report, be adopted for the new source as well.

This work is partially supported by North American Scientific, Inc.

\section{PO-T- 107}

Experimental Verification of XVMC -- A Fast Monte Carlo Code for Photon Beam Treatment Planning

M Fippel*, W Laub, F Nuesslin, Radiologische Universitaetsklinik Tuebingen, Germany

An experimental verification of the recently developed XVMC code, a fast Monte Carlo algorithm to calculate dose distributions of photon beams in treatment planning is presented. The code is an extension of the VoxelMonte-Carlo (VMC) model for electron beams. Photon transport is taken into account by exponential attenuation, ray tracing, and photon interaction cross sections (Compton scattering, pair production). The required material properties (electron stopping and scattering powers, electron density, attenuation coefficients) are determined directly from a given density distribution. The treatment head is modelled by a point source with energy distribution (primary photons) and an additional head scatter contribution.

XVMC has been compared to EGS4 and experimental data. It is demonstrated that the new algorithm is able to reproduce dose distributions with an accuracy of better than $2 \%$ in a water phantom with and without various slab inhomogeneities embedded. These measurements have been performed by a diamond detector. Furthermore, dose distributions in various solid state phantoms have been measured by film and thermo luminescent dosimetry (TLD). Also for these cases, measured and calculated dose distributions agree by taking into account experimental uncertainties. A beam parameter generation utility and an interface to commercial planning systems will allow the implementation of the code for routine treatment planning of clinical electron and photon beams.

Financial support for the work presented here was provided by the Deutsche Forschungsgemeinschaft under contract number FI 757.

\section{PO-T- 108}

Implementation of Enhanced Dynamic Wedge Calculations in a Treatment Planning System Based on Primary and Scatter Components M Czerminska*, L Chin, Joint Center for Radiation Therapy, Department of Radiation Oncology, Harvard Medical School, Boston, MA

Although dynamic wedges are available on most modern machines, it is not obvious how best to implement such option in a planning system. For the Varian enhanced dynamic wedges, the dose vs. collimator position relationship is contained in the segmented treatment table (STT). We modeled the enhanced dynamic wedge beams as a superposition of 20 static beams using STT tables to generate field sizes and beam weighting. We tested this summation technique on our in-house treatment planning system (Dplan), which uses the primary and scatter dose model. Wedge factors, absolute doses, profiles and depth doses were measured ( ion chamber and film) and compared with the calculated results. Because our model calculates dynamic wedge fields exactly the way they are delivered, it predicts the absolute dose and dose distribution very accurately. The wedge factors agreed to within $0.5 \%$. The profiles were also in a good agreement, with discrepancies of less then $2 \%$ in a low gradient regions and about $4 \%$ in the penumbra region. Depth doses agreed to less than $1.5 \%$. This method of generating dynamic wedges uses only STTs as input data and does not require any additional measurements for a treatment planning software. The measurements are needed only for verification. This approach is direct, fast ( calculation time for a $20 \times 20$ EDW field is below $1 \mathrm{~min}$.) and does not contain approximations inherent to algorithms based on beam libraries.

\section{PO-T- 109}

Effects of CT Number Conversion on Monte Carlo Dose Calculations

T Pawlicki*, K Ayyangar, C Ma, Stanford University School of Medicine, Stanford, CA

Forthcoming improvements in clinical dose calculation models will incorporate some form of the Monte Carlo method. The main benefit of using the Monte Carlo method as a clinical dose calculation engine is that it can accurately recover dose perturbations caused by heterogeneities within a patient's body. Such patient specific heterogeneities are specified from computed tomography (CT) scans of the anatomical region where dose needs to be calculated. The purpose of this work is to investigate the effects of converting patient specific CT data sets to relevant data for the purpose of Monte Carlo dose calculation. In particular, we consider the problems of material identification and conversion to mass density. It has been found that combining pixels of a CT scan to reduce its size for Monte Carlo simulation has the effect of systematically reducing the largest $\mathrm{CT}$ numbers and creating new, lower, CT numbers. This leads to incorrect material identification for CT number conversion to mass density for simulation. Depending on the materials used to determine the CT calibration curve of CT number to mass density, significant errors can result. We have found errors as large as $20 \%$ for high mass density material like bone in the conversion process. It is found that these conversion processes can produce calculated dose discrepancies of $9 \%$ (or $2 \mathrm{~mm}$ ) in the final dose distribution under some circumstances.

\section{PO-T- 110}

Photon Dose Calculation Performances of a 3D Treatment Planning System Compared to the Monte Carlo Code

P Francescon, C Cavedon*, S Reccanello, S Cora, Medical Physics Department, "S.Bortolo" Hospital, Vicenza, Italy

The purpose of this work is the comparison of the photon dose calculation performances of a commercially available 3D treatment planning system (ADAC Pinnacle ${ }^{3}$ v. 4.0e) against BEAM, a Monte Carlo reference code that allows a detailed simulation of a radiotherapy accelerator. The first part of the work is devoted to the benchmarking of BEAM for $6 \mathrm{MV}$ photons and to the optimization of the linac description (SIEMENS Mevatron M6700) to fit the experimental data. The second part takes into account the calculation in virtual phantoms built to simulate electronic non-equilibrium conditions. The third part shows the comparison between the codes working on real patient's CT data sets: a mediastinal treatment and a breast treatment have been simulated. All dose results are given in absolute values; with BEAM, these were obtained reproducing the linac calibration setup.

The results on virtual phantoms show that the $3 \mathrm{D}$ algorithm approaches the $\mathrm{MC}$ computations in situations of electronic non-equilibrium along the beam direction, while it can fail in situations of lateral electronic non-equilibrium. This is probably due to the approximation used in the collapsed cone convolution algorithm.

For the real patients comparison, differences between Pinnacle ${ }^{3}$ and BEAM up to $5.7 \%$ are observed for the total dose calculated at the prescription point. Single field analysis shows that the differences increase with increasing depths and where the effective depth is significantly shorter than the geometrical one. 


\section{PO-T- 111}

Implementation of the Electron Pencil-Beam Redefinition Algorithm into a 3D Treatment Planning System

R Boyd*, G Starkschall, K Hogstrom, The University of Texas M.D. Anderson Cancer Center, Houston, TX

The electron pencil-beam redefinition algorithm (PBRA) has been implemented into COPPER-Plan, M.D. Anderson Cancer Center's 3D treatment planning system. The implementation of the code, previously used for research, included changes in data structures and subroutines, improvements to the transport process from the collimator to the patient, and optimizations to decrease execution time. Minor changes and new routines were made in the PBRA code so that it could interface with existing data structures in COPPER-Plan. Electron transport from the collimators to the proximal patient surface, where the pencil beams are first redefined, has been modified to explicitly account for beam divergence. The PBRA code was modified to decrease execution time by (1) optimizing calculation cutoff values, (2) optimizing energy-loss spread function, and (3) incorporating a small-angle approximation into the electron transport equation. Accuracy of the implementation was verified using the Electron Collaborative Working Group Electron Dose Algorithm Verification Data Set. Results showed the accuracy of the PBRA-calculated dose distributions in the 3D treatment planning system to be similar to those of previous PBRA calculations with a reduction in calculation times of approximately $75 \%$. With successful implementation of the PBRA into COPPER-Plan more rigorous patient tests can be performed to determine the feasibility of using the PBRA for clinical patient treatment planning.

This work is supported by the P.H. and Fay Etta Robinson Professorship in Cancer Research and by a fellowship from the American Legion Auxiliary, Unit 569

\section{PO-T- 112}

A "Multigrid" Method for Accelerating Three-Dimensional Photon Dose Calculations

M Wiesmeyer*, M Miften, Computerized Medical Systems, Inc., St. Louis, $\mathrm{MO}$

The FOCUS superposition algorithm utilizes a number of optimizations to accelerate dose calculations. One of these, the use of mixed-resolution representations, has general applicability to other calculation methods, including Monte Carlo. The key to the approach is automatic identification of regions where high dose gradients are likely. Dose in potential highgradient regions, which exist at beam edges and at tissue interfaces, is calculated using superposition on a fine grid. In low-gradient regions, superposition calculations are applied on a coarse grid and then interpolated onto the fine grid. Hence the term "multigrid." This is a highly effective method of acceleration, since superposition computation times are directly related to the number of points that must be calculated. Finding beam edges is fairly trivial, while identifying tissue interfaces can present a technical challenge: Tissue interfaces must be found in a way that is both accurate and inexpensive computationally. We have adapted Laplacian operators, a standard tool used in image processing for identifying edges in pictures, to locate tissue interfaces in computed tomography data. Since we use Fast Fourier Transform methods to apply the Laplacian operator in three dimensions, calculation of the gradient map is rapid---only about $4 \%$ of total calculation time. A speed up of almost 4 times with a decrease in accuracy of less than $0.5 \%$ is typically observed using this method.

*Supported by Computerized Medical Systems, Inc.

\section{PO-T- 113}

MCDOSE - A Dose Calculation Tool for Radiotherapy Treatment Planning

C Ma*, T Pawlicki, J Li, S Jiang, J Deng, A Kapur, E Mok, G Luxton, A Boyer, Stanford University School of Medicine, Stanford, CA

An EGS4 usercode, MCDOSE, has been developed for radiotherapy treatment planning (RTP). MCDOSE is designed as a dose calculation module suitable for adaptation to host RTP systems. MCDOSE can be used for both conventional photon/electron beam calculation and intensity modulated radiotherapy (IMRT) treatment planning. MCDOSE uses a multiple-source model to reconstruct the treatment beam phase space. Based on measured beam data acquired during commissioning, source-model parameters are adjusted through an automated procedure. Beam modifiers such as jaws, physical and dynamic wedges, blocks, electron cutouts and bolus are simulated in MCDOSE together with a $3 \mathrm{D}$ rectilinear patient geometry model built from CT data. Comparisons of dose distributions calculated using MCDOSE show excellent agreement with those calculated by the EGS4/DOSXYZ code. Heterogeneity correction factors for layeredlung or layered-bone phantoms as calculated by both codes agree with measured data to within $1 \%$. Variance reduction techniques are implemented in MCDOSE to achieve a speedup factor of $10-20$ compared to DOSXYZ. CPU time required for clinical electron beam plans with $0.1-1.0 \mathrm{~cm}^{3}$ dose grids is $0.1-1 \mathrm{~h}$ and $0.5-5 \mathrm{~h}$ for clinical photon beam plans on a PentiumPro $200 \mathrm{MHz}$ PC. Discrepancies up to $10 \%$ (or a $1 \mathrm{~cm}$ shift in the $90 \%$ isodose line) are observed between the dose distributions calculated using MCDOSE and conventional dose algorithms. MCDOSE has also been used to calculate beamlets for an IMRT planning study and for retrospective dose verification of clinical IMRT patient treatments.

\section{PO-T- 114}

Monte Carlo Dose Calculations for Dynamically Wedged Photon Beams A Kapur*, C Ma, A Boyer, T Pawlicki, Stanford University, Stanford, CA

Dynamically wedged photon beams of nominal energies 4-15 MV from Varian Clinac $2100 \mathrm{C}$ and $2300 \mathrm{C} / \mathrm{D}$ linear accelerators were simulated using the Monte Carlo method. The wedged profiles were obtained using segmented treatment tables (STTs) provided by the vendor for 15,30,45 and 60 degree dynamic wedges. Enhanced dynamic wedges were also simulated using the golden STTs for user-defined field sizes and arbitrary isodoseangulations for these energies. Field sizes studied ranged from $4 \times 4 \mathrm{~cm}^{2}$ to $40 \times 40 \mathrm{~cm}^{2}$ at an SSD of $100 \mathrm{~cm}$. The Monte Carlo code EGS4/BEAM was modified to incorporate the monitor unit settings for specified jaw positions. The backscatter of photons and electrons from the jaws into the transmission monitor-chamber of the linacs was accounted for during the Monte Carlo simulation. This way the monitor units delivered for each instance of the jaw settings incorporated backscatter effects automatically. The phase-space files obtained from the simulations were used for beam characterization and dose calculations in homogeneous and heterogeneous phantoms using the Monte Carlo code DOSXYZ. Dose conversion factors for the measured data such as stopping-power ratios for various detector materials for these beams were also computed using DOSXYZ. Calculations for surface dose, relative central-axis dose profiles, dynamic-wedge factors and transverse dose distributions in the wedged and non-wedged directions for both homogeneous and heterogeneous phantoms will be discussed for these beams.

\section{PO-T- 115}

The Effect of Monte Carlo Noise on Radiotherapy Treatment Plan Evaluation

P Keall*, J Siebers, B Libby, R Mohan, R Jeraj ${ }^{1}$, Medical College of Virginia, Richmond, VA, (1) Jozef Stefan Institute, Slovenia

Routine clinical Monte Carlo treatment planning is on the horizon. However, some new challenges need to be addressed. One such challenge is the statistical uncertainty inherent in all Monte Carlo calculations. Precision is traded for accuracy. Hence we need to determine the noise, or uncertainty level, at which dose distributions are not significantly affected, in order to calculate sufficiently precise dose in the minimum time.

To study the effect of noise on radiotherapy treatment plan evaluation, a 'noise free' plan was necessary. A noise free plan calculated using Monte Carlo would take $\infty$ time, and hence it was necessary to determine the relationship between noise and dose. We assumed that dose is proportional to the number of interactions, and hence expect the noise level to have a

$\sqrt{\text { dose }}$ dependence. This relationship was applied to a collapsed cone convolution calculated plan, creating dose distributions with noise levels of $0,1,2,4,8$ and $16 \%$ at the maximum dose point.

The noise function was found to be proportional to $\sqrt{\text { dose }}$, validating the proportionality of dose and interaction number. Critical structure DVHs 
and biological indices are less sensitive to calculation noise than those of the target. Systematic errors affect biological indices significantly more than random noise. A random noise level of $<2 \%$ does not significantly affect isodose lines, DVHs or biological indices.

\section{PO-T- 116}

Integration of a Standard Monte Carlo Code into a Commercial Treatment Planning System

M Woo, B Faddegon*, Toronto-Sunnybrook Regional Cancer Center, Toronto, Canada

The Monte Carlo code EGS4 has been integrated into the commercial treatment planning system Theraplan Plus. This step is part of our program to implement clinical MC electron beam treatment planning. The Theraplan Plus source code was made available to us from the vendor, so the MC functionality was incorporated as an added feature to the standard system, while utilizing the inherent input/output features of the standard system, such as the capturing of patient CT information, the setting-up of beam properties, and the display of EGS4 dose distributions. The MC simulation could be run in batch mode and over multiple computers on the same network (non-Theraplan Plus systems), with the results from different history batches combined automatically. Two users codes have been implemented. One is the standard DOSXYZ code, for benchmarking purposes, and the other, MCRTP, is a custom user code specifically tailored for clinical use, and includes features such as shielding. The beam data input is based on phase space files generated using the BEAM code, but will be replaced in the future by a beam model currently under development. The implementation will be used initially in parallel with Theraplan Plus for evaluation purposes, to pave the way for a fully clinical $\mathrm{MC}$ treatment planning program for electron beams in the near future.

The research described in this abstract was supported by a grant from Theratronics corporation.

\section{PO-T- 117}

Photon Beam Commissioning Methodology for a Commercial 3-D Radiation Treatment Planning System

G Starkschall*, R Steadham, S Ahmad, I Rosen, The University of Texas M.D. Anderson Cancer Center, Houston, TX

Commissioning beam data for the convolution/superposition photon dose calculation algorithm on a commercial three-dimensional radiation treatment planning (3-D RTP) system (ADAC Pinnacle ${ }^{3}$ ) can be a difficult and timeconsuming task. Sixteen parameters, along with spectral weights representing a discrete energy spectrum must be fit to sets of central-axis depth doses and off-axis profiles for a large number of field sizes. The present paper proposes a beam-commissioning methodology that is relatively rapid and provides a set of beam parameters that generates an accurate beam model in addition to being physically reasonable. The methodology begins by automatically generating a single set of beam parameters that gives an approximate fit to relative dose distributions for all beams, open and wedged, in a data set. A limited number of parameters are adjusted small amounts to give accurate beam models for four open-beam field sizes and three wedged-beam field sizes. Beam parameters for other field sizes are interpolated and validated against measured beam data. A complete set of parameters can be generated in approximately a week's time. The parameters yield calculated relative doses that match measured relative doses in a water phantom to within $0.5-1.0 \%$ along the central axis and $2 \%$ along beam profiles for a range of field sizes from $4 \times 4$ to the largest field size available. While the methodology is specific to the particular treatment planning system, the approach may be generalizable to other implementations of the dose model in other treatment planning systems.

\section{PO-T- 118}

Limitations of Convolution Methods for Modeling Geometric Uncertainties in Radiation Treatment

T Craig ${ }^{1,2}$, J Van Dyk ${ }^{1,2}$, E Wong*1, V Moiseenko ${ }^{1}$, J Kempe ${ }^{1,2}$, J Battista ${ }^{1,2}$, (1)London Regional Cancer Centre and (2)The University of Western Ontario, London, ON, Canada

Convolution methods have been incorporated into dose calculations to model the effect of geometric positioning uncertainties. These methods assume shift invariance of the dose distribution, and ignore the stochastic nature of the finite number of fractions. The magnitudes of the resulting errors are not well documented. We specifically address the issues of tissue inhomogeneities, surface contours, and the finite number of treatment fractions by using two approaches. First, the uncertainty in beam positioning was modeled with a Gaussian distribution. A static dose distribution (with surface and inhomogeneity corrections) was calculated and was convolved with the Gaussian to yield a 'blurred' dose distribution incorporating the uncertainties. Second, the dose was calculated using a finite number of laterally displaced individual beams (each with surface and inhomogeneity corrections) weighted by the same Gaussian. The difference between the results of the two methods indicates the error in the convolution method. This analysis was performed for phantoms as well as some clinical situations. Similarly, the effect of the finite number of treatment fractions was modeled by randomly sampling beam positions for each fraction. Significant differences $(>5 \%)$ have been observed

for some typical clinical situations. We conclude that for these algorithms to be of clinical use, they must be augmented to account for these sources of error.

\section{PO-T- 119}

Designing Compensators Using the Pinnacle3 Treatment Planning System Accounting for Scatter from Compensators

H Liu*, C Baird, R Popple, N Zacharopoulos, I Rosen, UT M. D. Anderson Cancer Center, Houston, TX

Dose in photon fields using compensators or other physical attenuators can be affected by many factors including beam energy, field size, depth of interest, compensator location, and construction details of compensators. In this work, we investigated the variation in compensator transmission for these parameters and the accuracy of the Pinnacle ${ }^{3}$ treatment planning system (PTPS, ADAC Laboratory) in calculating doses for compensators. Our compensators were constructed of fine steel balls solidified with wax in molds of styrofoam cut with an automatic milling machine (HEK AUTIMO 2.5D). Transmission factors of sheets of this material at various thickness were measured and calculated using a separate non-clinical convolution/superposition algorithm that accounts for both the scatter and the beam hardening effects from the compensators. The change of compensator transmission with beam geometry and compensator thickness was thoroughly evaluated. Based on these results, density of the compensator materials input to the PTPS was determined and modified for various treatment conditions to obtain accurate transmission calculations from the PTPS. Our results showed that the PTPS calculation accounts for only the beam hardening effect of the compensators. Yet scatter from the compensators can be significant and can cause a change of more than $5 \%$ in the compensator transmission. By appropriately adjusting the density of the compensator material in the PTPS, the accuracy of the PTPS calculation can be improved significantly to agree with measured data.

\section{PO-T- 120}

An Algorithm for Calculating Isodose Distributions and Wedge Factors for Enhanced Dynamic Wedge Symmetric and Asymmetric Fields

M Miften*, M Wiesmeyer, K Takahashi, S Broad, Computerized Medical Systems, Inc., St. Louis, MO

The FOCUS implementation of Varian's Enhanced Dynamic Wedge (EDW) is presented. Calculations of both dose distributions and wedge factors (WFs) are based on Segmented Treatment Tables (STTs), which are energydependent tables that describe dose delivery and jaw position "segments" required to deliver wedged dose. Calculating dose requires a "transmission matrix" derived from an STT to model the modified fluence from the source. The dose calculation is then performed using either the Clarkson or Convolution/Superposition algorithms. An initial "primary dose/MU fraction" WF estimate at the center of symmetric and asymmetric fields is calculated from the STT as the ratio of relative dose delivered on the axis of the weight point divided by total relative dose delivered for the treatment. The novelty in our approach is that we go beyond this initial estimate with a "scatter dose" correction. This correction requires measured 60 degree WFs for five fields. Scatter corrections derived from measured WFs are interpolated for other wedge angles and field sizes in much the same way as arbitrary wedge angle STTs are derived from a "Golden STT" using the "ratio of tangents" formalism. Dose comparisons with measured 
distributions show good agreement for 6 and 18 MV beams for all EDW angles. Agreement with measurements to within $1 \%$ is obtained for WFs in all symmetric and asymmetric fields for 6,10 , and $18 \mathrm{MV}$ beams. For large wedge angles and field sizes, this represents a significant improvement over the $3-4 \%$ errors often observed using the MU fraction model alone.

\section{PO-T- 121}

Study of the efficiency of Auto-Sequencing Technique in Treating the Prostate Cancer Using the Siemens TM; Primus TM; Multi-leaf collimators

C Yang*, Monmouth Medical Center, Department of Radiation Oncology, Long Branch, NJ

The Siemens Primus LINAC with Multi-leaf collimators was installed in our facility during September 1998. Since then we have applied the autosequencing technique from the Primus LINAC to treat the prostate cancer. We utilized the six-field prone patient setup to achieve better tumor confomality and dose uniformity. In order to study the efficiency of treating the prostate cancer, 20 patients were analyzed for the treatment time period. From the conventional blocking fields, it usually takes about 10 minutes or more to finish one treatment course. It involves the changing blocks, setting gantry angles, verify the SSDs, ...etc. From our experience, if the patient were set up correctly, it only takes less than 4 minutes for completely finishing the daily treatment. The efficiency comes from the auto blocking of the MLC, dynamic gantry angle changes, and the high dose rate for delivery. The verification process was also performed between each port. The average treatment time for the 20 patients is 3.75 minutes for the $180 \mathrm{cGy}$ per fraction. Compared to the conventional technique, it provides clinical benefit with geometrical accuracy.

\section{PO-T- 122}

\section{A Modified Rotational Hemi-body Electron Therapy Technique}

A Sethi*, L Leybovich, G Glasgow, I Ouyang, N Dogan, H Forgione, Loyola University Medical Center, Maywood, IL

We have developed a rotational technique to treat patients with mycosis fungoides confined to upper-half of the body. The patient sits in a chair attached to a platform that rotates at $1 \mathrm{rpm}$. A $40 \times 40 \mathrm{~cm}^{2}, 6 \mathrm{MeV}$ electron beam was used at a nominal distance of $3 \mathrm{~m}$. To prevent self-shielding in the underarm area, the patient's arms were raised upright and supported by a rotating horizontal bar. Patient's eyes were protected with eye shields. To reduce bremstrahlung radiation, the gantry was rotated to $96^{\circ}\left(6^{\circ}\right.$ off horizontal), directing the beam central axis above the patient's head. Dose rate, depth dose distribution, and beam profiles were measured, using a parallel plate chamber, film and TLDs in polystyrene and humanoid phantoms. Beam energy at patient's skin was $4.5 \mathrm{MeV}$. The maximum dose was at skin surface, with the $90 \%$ and $10 \%$ doses at $10 \mathrm{~mm}$ and $25 \mathrm{~mm}$ respectively. X-ray contamination was $4 \%$, in agreement with published data. Dose uniformity throughout most of the treatment area, excluding scalp, was within $\pm 7 \%$. The dose to scalp varied $\pm 15-20 \%$ of the prescribed dose, which was judged clinically acceptable. Indeed, a complete response in the patient's condition was observed. The use of rotational technique simplifies patient setup, resulting in shorter treatment times relative to fixed field methods. At a measured dose rate of $15 \mathrm{cGy} / \mathrm{min}$ on patients' skin, delivery of customary $100 \mathrm{cGy} / \mathrm{fx}$ takes approximately 7 minutes. This technique also eliminates field junctions, thereby improving dose uniformity.

\section{PO-T- 123}

\section{A Method of Verification for Craniospinal Irradiation}

Y Lo*, W Lutz, M Anderson, Mount Sinai, New York, NY, Memorial Sloan-Kettering Cancer Center, New York, NY, Mount Sinai, New York, NY

Treatments with craniospinal fields require accurate patient setup and effective verification methods. We have developed a system to verify the setup, for both simulation and treatment, of a standard technique: rotation of the collimator for the cranial fields to match the the spinal field, and rotation of the couch to eliminate the divergence from the cranial fields.

To increase the visibility of the field junction between cranial and spinal fields on the films, we mark, on the skin, around the neck, the superior border of the spinal field as indicated by the light field. Then we place BB's along the curve drawn. If the fields are junctioned properly, the BB's projection should appear as a straight line located in the middle of penumbra of all three relevant field edges: the superior border of the spinal field and the inferior border of lateral cranial fields. When a gap between the cranial and spinal fields is needed as a safety margin, the BB's are placed at the middle of the gap. This BB's system is particular useful for verification of treatment fields when it is nearly impossible to confirm a proper junction anatomically from the port films.

For treatments when port films are not taken, an alternative setup verification is suggested. Since there is a constant relationship between the couch positions and collimator settings (CS), we can verify the patient's setup by checking a patient-specific constant value routinely.

Patient-Specific Constant $=[($ Spinal couch Reading $)-($ Cranial couch Reading $)]-0.5 \times$ [(Spinal CS $)$ - (Cranial CS $)]$

\section{PO-T- 124}

Additional TBI Patient Dose from Neutrons Due to the Photon Beam Intercepting Steel As Opposed to Concrete

M Gillin*, R Zhu, D Grimm, F Lopez, J Rownd, Medical College of Wisconsin, Milwaukee, WI

A new treatment room was built, which included an extended treatment distance for TBI irradiation. While the plans showed a 16.4 inch steel plate centered in 32 inches of concrete, the steel plate was placed on the inside of the TBI room for ease of construction. Concern was then expressed over a potential increase in dose to TBI patients from the neutrons produced in the steel from the $23 \mathrm{MV} x$-ray beam.

Measurements were made with bubble detectors in the old treatment room with its concrete wall as the primary barrier and in the new treatment room with the steel/concrete primary barrier. The in-beam measurements at the extended distance with the steel primary barrier show an increase by approximately a factor of two, from $7 \mathrm{mSv}$ to $12 \mathrm{mSv}$ per photon Gy over the concrete only wall. Measurements at the TBI distances with a phantom again resulted in a factor of two increase in dose on the surface of the phantom close to the wall, $13 \mathrm{mSv}$ per photon Gray as opposed to $7 \mathrm{mSv}$, when comparing the steel wall to the concrete wall. A two inch thick sheet of polyethylene was added in front of the steel. Measurements were repeated which indicated that the measured dose on the surface of the phantom close to the wall is the same as with the concrete wall.

\section{PO-T- 125}

Beam Compensation for Total Body Irradiation by Dynamic Delivery A Kassaee*, J McDonough, M Altschuler, University of Pennsylvania Medical Center, Philadelphia, PA

The feasibility of intensity modulation for TBI treatments has been investigated with the use of phantom studies and treatment planning. In total body irradiation, the beam compensator, bolus or complex beam arrangements are currently used to obtain homogeneous dose distribution. However, modern linear accelerators with independent jaws furnish the capability to modulate intensity along the direction of jaw motion and create any arbitrary beam intensity in that dimension. This one dimensional intensity modulation is suitable for use in TBI treatment where the dominant variation in patient thickness is from superior to inferior. Our Varian 2100 $\mathrm{C} / \mathrm{D}$ linear accelerator is equipped with the dynamic delivery research software which permits us to input parameters such as jaw positions and amount of dose per jaw position. The input parameters to achieve a uniform dose distribution were calculated with our in-house treatment planning system. The calculation is based on 3D treatment planning of a humanoid phantom with CT scans. The calculation and dynamic delivery was performed for AP/PA and lateral beam arrangement. TLD measurements show the dose inhomogeneity of less than $5 \%$ between the prescription point, umbilicus, and the head and neck region. 


\section{PO-T- 126}

A Conformal Dynamic Arc Technique for the Treatment of Pleural Mesothelioma with On-Line Epid Verification.

B Lachance*, R Blais, J Roy, É Vigneault, J Pouliot, CHUQ, Quebec, Quebec, Canada

A conformal external beam technique for the treatment of pleural mesothelioma is presented. Oblique opposed $23 \mathrm{MV}$ beams are used to treat the mediastinal and diaphragmatic pleura; while an arcing $6 \mathrm{MV}$ beam with conformal shielding is used to irradiate the parietal pleura. During a preliminary treatment simulation, the patient is positioned on a tilted board in order to align the thoracic wall with the linac rotation axis. A CT-scan covering the entire thorax and abdominal extension of the disease is obtained. All beams are treated with the same isocentre that is placed using the treatment planning system at the centre of the arc segment defined by the parietal pleura. The rotating BEV of the photon arc is used to optimise the shape of the treatment field in order to obtain good target coverage while minimising the volume of lung irradiated.

EPID is used to assess the positioning reproducibility of the conformal arcing and static fields. The treatment involves a $160^{\circ}$ to $180^{\circ}$ arc and the VEPID detector allows for the clearance needed for the gantry rotation. All fields can be verified on-line by observation of the movie portal images. This ensures that the alignment of the thoracic wall within the arc is in accordance with the calculated plan. This technique allows the delivery of a high dose to the tumour while sparing a substantial portion of the lung and with a good confidence level.

\section{PO-T- 127}

Seven-Field Breast Irradiation Using Mixed Electron-Photon Beams to Treat the Internal Mammary Chain.

B Lachance*, M Larochelle, N Varfalvy, D Tremblay, CHUQ, Quebec, Quebec, Canada

Recently published trials report a beneficial effect of treatment of the internal mammary chain (IMC). The suggested benefits may have been previously overshadowed by the added cardiovascular morbidity resulting from inadequate irradiation techniques. We present the modifications made to our breast technique to include the IMC while minimising myocardial and lung irradiation.

Our standard technique uses half-beam tangential fields with couch angle, rotated collimator and a corner block to define the match-plane with the APPA-axillary and supraclavicular fields. The external collimator angle is adjusted to make the tangential beams coplanar.

The IMC is irradiated by mixed photon and oblique electron beams. The photon component reduces surface dose and prevents acute skin reactions. The photon field uses the same set-up as the internal tangent. The ipsilateral jaw is closed creating a perfect match with the tangential fields while the contralateral jaw is opened $1.5 \mathrm{~cm}$ past midline. To have a sufficiently wide electron field and allow good IMC coverage, the entry point of the internal tangential field is displaced from midline (non-IMC technique) to $2.5 \mathrm{~cm}$ ipsilateral. The electron field is $4 \mathrm{~cm}$ wide (in the central plane) with a gantry angle optimised to obtain a homogeneous dose across the electronphoton joint. A $9^{\circ}$-hinge angle between the 12 or $15 \mathrm{MeV}$ electron beam and the tangential plane was found to be adequate. Clinical integration was easy and the technique involves simple modifications relative to our non-IMC breast technique.

\section{PO-T- 128}

Improved dose homogeneity in Scalp Irradiation using a Single Setup Point and Different Energy Electron Beams

R Yaparpalvi*, D Fontenla, J Beitler, Montefiore Medical Center and the Albert Einstein, Bronx, NY

Homogeneous irradiation of the entire or a large portion of the superficial scalp poses both technical and dosimetric challenges. Some techniques will irradiate too much of underlying normal brain while other techniques are either complex and involve field matching problems or may require sophisticated linac add-ons such as intensity modulated radiation therapy /electron multi-leaf collimator. We propose a practical treatment technique that can be delivered with a standard linear accelerator capable of producing high-energy electrons. The proposed technique offers a simple alternative way of achieving results equivalent to intensity modulated radiation therapy. Dose homogeneity throughout the treatment volume is achieved by aiming different energy electron beams at differential areas of the treatment surface to achieve improved dosimetry and rapid treatment delivery, while using a single set-up point. Idealistically, the treatment surface is envisioned as a set of concentric circles. The lower of the electron energies used treats the treatment surface represented by the innermost circle and the concentric rings outward are treated by higher energy electrons. Depending on clinical indications and target volume different energy electron beams are combined. Typically, an electron applicator large enough to encompass the entire treatment surface plus a margin is used and applicator-inserts defining each beam's treatment surface are changed between beams. Using a 3-D RTP system, the optimal beam weights were selected to minimize the dose variation in radial direction across the entire target volume. We introduced this treatment modality in our institution to treat superficial cancers of the scalp and other irregular surfaces.

\section{PO-T- 129}

TBI - A Review of Techniques

M Urie*1, L Tisch ${ }^{1}$, A Nevelsky ${ }^{2}$, F Laurie $^{1}$, T FitzGerald ${ }^{1}$, ${ }^{1}$ Quality Assurance Review Center, The University of Massachusetts Medical Center, Worcester, MA, ${ }^{2}$ Department of Oncology, Rambam Medical Center, Haifa, Israel

Material: This report summarizes the physical techniques for TBI for institutions that belong to the Pediatric Oncology Group (POG), as reported to QARC. Data are reviewed for 30 institutions representing 33 techniques (three institutions reported multiple techniques based on patient size).

Results: The beam energy of choice for treatment ranges from Co-60 to 24 MV with 20 institutions using a beam spoiler to achieve adequate surface dose. To achieve dose uniformity, 21 of the 33 techniques use tissue compensators. The dose rate at the treatment distance varied from 5 to 22 $\mathrm{cGy} / \mathrm{min}$. The treatment distance, at patient midplane, varied from 1.83 to 6.46 meters. The majority of institutions treat at a distance of at least 3 meters and about $20 \%$ use $>5$ meters. Of the 27 institutions with only one irradiation technique, 13 use an AP/PA technique and 14 use opposed laterals. There are a variety of patient positions for treatment; only 5 of the 33 techniques reported use AP/PA with the patient standing. Further details of the techniques will be presented, and variations in fractionation, dose, and normal tissue doses will be summarized.

Conclusions: TBI techniques remain varied among these 30 POG institutions. These data may be representative of the wide diversity of treatment techniques that exist.

\section{PO-T- 130}

Virtual Wedge Factors and Clinical Implementation of the Siemens Virtual Wedge

X Zhu*, M Gillin, F Lopez, D Grimm, J Rownd, Medical College of Wisconsin, Milwaukee, WI

The virtual wedge factor (VWF) for the Siemens virtual wedge (VW) is designed to be approximately 1.00 . The purpose of this work is to confirm that statement and to identify conditions in which the VWF is not 1.00 and thus should be included in monitor unit calculations. VWF's have been measured for four x-ray beams as a function of wedge angle, field size, depth and SSD. Our measurements show that the VWF can be as large as 1.052 for a $6 \mathrm{MV}$ beam at a depth of $10 \mathrm{~cm}$ for a field size of $20 \times 20 \mathrm{~cm}^{2}$. We have observed the dependence of VWF on field size and depth, but not on SSD. In contrast to a previous report, we could not confirm the dependence of VWF on calibrations (cGy/MU) for either high or low energy beams. The Siemens VW allows a continuous selection of wedge angles, typically from $15^{\circ}$ to $60^{\circ}$. Assuming $\pm 3 \%$ criteria for the dose profile of the wedged beam, we have selected a limited number of wedge angles from measured data for clinical use. This limits the commissioning and QA programs for this valued clinical tool. We have not commissioned the physical wedges. 


\section{PO-T- 131}

Elimination of Field Size Dependence of Enhanced Dynamic Wedge Factors

A Sethi*, L Leybovich, N Dogan, G Glasgow, Loyola University Medial Center, Maywood, IL

Enhanced dynamic wedges are characterized by a strong dependence of wedge factors (EDWF) on field size. Our measurements show, for example, that the variation in $6 \mathrm{MV}, 60^{\circ} \mathrm{EDWF}$ is more than $50 \%$ for fields ranging from $4 \times 4$ to $20 \times 20 \mathrm{~cm}^{2}$. However, for field sizes from $20 \times 20$ to $40 \times 30 \mathrm{~cm}^{2}$, EDWF remain virtually constant for all wedge angles. A method that practically eliminates the field size dependence of EDWF was developed and verified. In this method, MLC defines fields $\leq 20 \mathrm{~cm}$ in the wedged direction and the $\mathrm{Y}$ jaws are set to a $20 \mathrm{~cm}$ opening. The $\mathrm{X}$ jaws enclose the MLC field. If possible, the abutting line of MLC leaves is moved off central axis such that the collimator absorbs radiation leakage through leaf-ends. Using this setup, the wedge factors were measured for field sizes $4 \times 4 \mathrm{~cm}^{2}$ and larger. No dependence on field size and shape was observed for both symmetric and asymmetric fields. Measurements also demonstrated that the degree of isodose tilt did not change, but the magnitude of hot spot under the toe region was reduced by 4 to $8 \%$, depending on the wedge angle. For fields larger than $20 \times 20 \mathrm{~cm}^{2}$, no modification in EDW use is recommended because the overall variation of wedge factors remains $\leq 1 \%$. The elimination of large variation of wedge factors should reduce the likelihood of potential errors in dose delivery and may lead to a wider acceptance of the EDW technology.

\section{PO-T- 132}

A Function Relating Portal Film Dose Distribution to Compensator Thickness Map for Parallel Opposed Fields

M Loyd*, Cancer Care Specialists, P.C., Indianapolis, IN

Render-Plan 3D version $2.72 \mathrm{~b}$ includes a utility for designing physical compensators from information on port films (Program imgcomp). This requires a function to convert dose received by the film to compensator material thickness throughout the field. Literature suggests this function is determined by equating film dose ratio (film dose at a reference point in the field divided by film dose at a point to be compensated) to compensator material transmission. Data presented will show this assumption is incorrect for the circumstances examined. Generally, lead or copper screen cassettes are employed with verification film to obtain the image. The cassette, as designed, eliminates almost-all patient scatter radiation from reaching the film. One desires to make the dose to the patient at some 'compensation' plane uniform within certain limits. A simple method for deriving the function relating port film dose distribution and the compensator thickness map needed to produce a uniform dose in the patient compensation plane will be presented. The derived function will be presented for lipowitz metal and lead for $6 \mathrm{MV}$ x-rays. Limitations in the use of this function will be discussed. Measured dose distributions in water, solid water, and polystyrene phantoms for compensators produced using these functions will be presented. Comparison to dose distributions calculated by the RenderPlan 3-D planning system which include the compensator effects will be made.

\section{PO-T- 133}

The Use of Multiple Static Fields to Smooth MLC Field Edges

M Svatos*1, L Verhey ${ }^{1}$, T Steinberg ${ }^{2}$, (1) University of California at San Francisco, San Francisco, CA, (2)Siemens Medical Systems, Concord, CA

Recently there has been increasing interest in micro- or mini- MLCs to produce fields that are more conformal than those that can be achieved with standard MLCs, which commonly have a projected leaf width of $1 \mathrm{~cm}$. However in this presentation we will demonstrate that is also possible to increase the resolution of a standard MLC without any hardware modification. As originally proposed by Galvin et al [1], this method uses several static segments or subfields to modulate MLC field contours. For each subfield the patient couch is shifted spatially some fraction of the $1 \mathrm{~cm}$ MLC leaf thickness. The MLC leaves are then refitted to the desired contour for each subfield to maintain the same treatment volume. The summation of the shifted subfields results in a field that is shown to better conform to the desired contour than the scalloped edges of an unshifted MLC field. This technique has the added benefit of reducing the peak leakage outside of the field, which can often be made worse by micro- or mini- MLCs due to the increased number of leaves. A convenient implementation of this method is now available which makes this clinically practical. Dosimetric results will be compared with standard MLCs and an MMLC.

[1] Galvin, J.M., et al. Int J Rad Onc Biol Phys, Vol 35, No. 1, pp 89-94, 1996.

This work was partially supported by Siemens Medical Systems, Concord, CA.

\section{PO-T- 134}

The Application of Simple Techniques makes the Elekta $40 \times 40 \mathrm{~cm}$ Multileaf Collimator as accurate but more versatile than a Mini Multileaf Collimator.

M Welch*, Royal Free Hospital Pond Street, Hampstead, London NW3 2QG ENGLAND

The Multileaf Collimator has been available for many years but its versatility and flexibility has only become universally accepted over the last 10. Mainly due to new types planning systems and the need minimise the cost of conformal therapy. The main disadvantages to these devices is the individual leaf width typically $1.0 \mathrm{~cm}$, and the leakage between leaves being applied to the patient. As the leaf tips of any one bank are seldom aligned in exactly the same position it is difficult to accept field joins as hot spots are difficult to avoid. Also the use of this technique increases the treatment time as each field requires the total daily applied dose. A method to artificially reduce the leaf width is required, with a technique to fit the MLC to a contour that is not equivalent to a number of whole leaves. The leaf width may be varied from 1-10mm using a technique called "Phased Fields". This involves a minimum of two fields. Field 1 is created using the original field centre. Field 2 moves the centre $0.5 \mathrm{~cm}$ perpendicular to the direction of leaf movement. There is no increase in treatment time as each field is half the total daily dose. A technique called "Time Share" resolves the problem of contour fitting to whole leaves. The "Phased Field" technique has been used to create clinical shapes smaller than $3.0 \mathrm{~cm}$.

\section{PO-T- 135}

Implementation of a Siemens Virtual Wedge on RenderPlan Planning System

M Bank*, Indiana University Medical Center, Indianapolis, IN

A Siemens virtual wedge creates a wedged isodose distribution by moving one of the asymmetric jaws across the field during treatment. Any wedge angle is potentially possible with a maximum of about 60 degrees. The virtual wedge output factors are the same as the open field values since no physical wedge is in the beam. To use the virtual wedge clinically, the VW was implemented on our RenderPlan planning computer. RenderPlan uses two types of files to store the wedge data - measured profiles, and correction tables to account for beam hardening. New wedge files were created for the virtual wedges by copying the profiles of the hard wedges at $15,30,45$, \& 60 degrees. No correction files were created resulting in computer generated isodose distributions uncorrected for beam hardening. Any wedge angle could then be generated by combining two wedges and varying the beam weighting.

The RenderPlan generated isodose distributions for various wedge angles were then compared with isodose distributions measured with film in a SW cassette.

The Virtual Wedge isodose distributions were obtained with Kodak V-film in a SW film cassette and then scanned in a Scanditronix film scanner with RFA Plus V5.2 software. The film measurements were compared with the RenderPlan generated isodose curves. Good agreement was observed. Problems observed with defining the virtual wedge orientations and limitations will be discussed. 


\section{PO-T- 136}

Comparison of MLC (VARIAN) vs. Cerrobend Block in Clinical Setup

Y Qian*, P Chow, L Maslack, L Lin, S She, R Kagan, Kaiser Permanente

Hospital, Radiation Oncology Department, Los Angeles, CA

Multileaf Collimator (MLC) is often a replacement for custom made cerrobend blocks used in radiation oncology departments. Because the finite width of individual leaves, a step effect (visually distorted to the physician) replaced the smooth cerrobend block curvature. The dosimetry of this step effect has been well documented in literature. Clinically, this step effect is washed out by the day to day variation in the patient setup. In this study, we used a VARIAN 80 leaf MLC system with $1 \mathrm{~cm}$ leaf width on a typical 4 field box prostate technique to demonstrate there is no clinically significant step effect after 15 treatments, assuming the daily setup variation of $5 \mathrm{~mm}$ standard deviation from baseline. Of equal significance, the dose distribution differences between MLC fields and cerrobend block fields after 15 treatments is minimal (dose line $<2 \mathrm{~mm}$ spatial difference). In conclusion, the step effect disappears for a multiple fraction treatment with random setup variations on the order of $5 \mathrm{~mm}$ standard deviation, and the $1 \mathrm{~cm}$ leaf width MLC can effectively replace cerrobend block with no clinical significant step effect.

\section{PO-T- 137}

Comparison of 3D Compensation versus Conventional Wedge Techniques : Potential of a New Compensating Device

C Bletscher*, R Wallace, J DeMarco, C Kimme-Smith, UCLA, Los Angeles, CA

PURPOSE: To compare treatment plans using 3D compensation to the same cases planned with conventional wedge techniques, advocating the utility of a new compensating device.

METHODS AND MATERIALS: Cases were chosen that demonstrated sufficient irregularity of external surface to require compensation for adequate target dose uniformity. Two treatment plans were produced for each case, one using wedges and one compensators. All plans were produced on the Theratronics, Theraplan Plus system, version 3. Plans were compared on the basis of planning time and dose statistics.

RESULTS: A brain case demonstrated a significant decrease in planning time when $3 \mathrm{D}$ compensation was used. The resulting target dose distributions for both plans were almost identical, however, improvement of target dose uniformity in the compensator plan would lower the maximum dose by approximately $1 \%$ for a given prescription, thus producing less intense hot spots. A breast case was planned with comparable ease using either compensators or wedges. A striking difference, however, was demonstrated in target dose uniformity. The $87 \%$ isodose would be used for prescription in the wedge plan, versus $93 \%$ with compensators. Extensive hot spots found with wedges are thus virtually eliminated and maximum lung dose lowered considerably when compensators are used.

CONCLUSIONS: These results demonstrate the efficacy of radiotherapy with compensators, improving target dose homogeneity while requiring less planning time. This project thus advocates the future efforts of this group to develop a new, technologically advanced compensating device.

\section{PO-T- 138}

\section{EDW Fields- A New Tecnnique for Data Collection}

D Bhaduri*, B Anderson, C Bockelman, St.Francis Hospital, CRS, Vero Beach, FL, Saint Francis Hospital, Topeka, KS

In Enhanced dynamic wedge (EDW) mode, the beam intensity is modulated by moving one of the independent collimator jaws as well as by varying the dose rate. Data collection for treatment planning poses special challenge for EDW due the dynamic nature of beam delivery. Two techniques used so far for data collection are film densitometry and linear detector array, each having its special advantages as well as disadvantages.

We used a new technique in which a single ion chamber is moved rapidly back and forth across the radiation field in a water phantom. The software in a CRS Beam Scanner was modified so the system can collect and integrate data at every quarter of a millimeter as the probe moves right and left across the field during multiple passes. A reference probe is positioned in air near the edge of the fixed jaw, at a point that remains unobstructed during the run. The reference signal is used to correct for changes in the dose rate as well as to eliminate some of the noise.

Scanned profiles for the EDW were generated at multiple depths using maximum dose rate and relatively large monitor units. These profiles were incorporated in a ROCS treatment planning computer. Computed isodose distribution shows that the angles of tilt are consistent with the nominal wedge angles and the hot spots compare well with the actual measurement.

\section{PO-T- 139}

Optical Density Changes of Gafchromic Film Resulting From Low Power Laser Sources

P Sullivan*, B Hasson, C Grossman, L Simpson, MCP Hahnemann University, Temple University, Philadelphia, PA, Swarthmore College, Swarthmore, PA, MCP Hahnemann University, Philadelphia, PA

Gafchromic film is becoming the Gold Standard for complex radiation dosimetry measurements. The near tissue equivalence, dose rate independence, and high spatial resolution make the film an ideal dosimeter. As applications for Gafchromic film increase, new densitometry systems continue to emerge. Recently, the AAPM presented TG-55 which included recommendations and information on commercial and custom built Gafchromic film scanners. Though the report is comprehensive it did not explore the induction of polymerization that can result from red laser light scanners during film analysis.

The authors have developed a custom built laser scanning system to investigate the red light polymerization of Gafchromic MD-55 film as a function of input laser power. The scanner utilized a laser diode $(\lambda=671$ $\mathrm{nm})$ or a HeNe laser $(\lambda=633 \mathrm{~nm})$ source operating at variable power settings. The results demonstrate for the first time a non-linear polymerization reaction of MD-55 film induced by these laser sources. The polymerization increases the film's optical density (OD) and is optically indistinguishable from x-ray and ultraviolet light exposures. For laser powers of $0.5 \mathrm{~mW}$ the change in OD is approximately $0.15 \mathrm{OD} / \mathrm{min}$ and $0.244 \mathrm{OD} / \mathrm{min}$ for laser powers of $1.5 \mathrm{~mW}$. This increase in OD is dependent on input laser power as well as the initial absorbed dose of the film being scanned. These findings were not included in the TG-55 report and if ignored could have a significant impact on dose measurements using Gafchromic film.

\section{PO-T- 140}

Calculate Effective Wedge Factors of Any Wedge Angles of Enhanced Dynamic Wedge from a Measured 60 Degree Wedge Factor

M Lii*, L Dong, A Shiu, Department of Radiation Physics, The University of Texas M.D. Anderson Cancer Center, Houston, TX

An equation is derived to calculate wedge factors of any wedge angles for Varian enhanced dynamic wedge (EDW) from a measured 60-degree wedge factor. This method is based on the concept of "ratio of tangents method" which is used to generate "Segmented Treatment Tables" for wedge fields from a single Golden STT. The effective wedge factor for both 6 and $18 \mathrm{MV}$ with wedge factors for $15^{\circ}, 30^{\circ}, 45^{\circ}$ and $60^{\circ}$ degree are measured and compared to calculated values. Agreement are better than $0.2 \%$ for $18 \mathrm{MV}$ and $0.5 \%$ for $6 \mathrm{MV}$.

\section{PO-T- 141}

A Comparison of the AAPM TG51 Protocol and the IAEA AbsorbedDose-to Water Based Code of Practice for Dosimetry Calibration of High Energy Photon Beams

M Huq*, M Hossain, P Andreo (1), Radiation Oncology, Jefferson Medical College of Thomas Jefferson University, Philadelphia, PA (1) International Atomic Energy Agency, Vienna, Austria

Both the AAPM and the IAEA have recently developed a new generation of protocols that are based on standards of absorbed dose to water. The recommended dosimetry procedures in these protocols are based upon the use of an ionization chamber that has been calibrated in terms of absorbed dose to water in a standards laboratory's ${ }^{60} \mathrm{Co}$ beam. This is different from the recommendations given in the earlier generation of protocols, which were based upon either an exposure or air-kerma calibration factor of an ionization chamber. The purpose of this work is to compare the 
recommendations of the two protocols using two widely used Farmer type ionization chambers: PTW N30001 (PMMA wall) and NEL 2571 (graphite wall). This comparison was accomplished by determining the absorbed dose-to-water with both chambers irradiated in a water phantom using 6 and $25 \mathrm{MV}$ photon beams. Beam quality correction factors $k_{Q, Q o}$, determined by following the recommendations of the two protocols, were found to differ by up to $0.2 \%$ at the highest energy. Despite significant differences in the method of beam quality specification, the overall agreement between the two protocols was found to be within $0.1 \%$ for the chambers and energies investigated in this study.

\section{PO-T- 142}

Film Dosimetry for Enhanced Dynamic Wedges

L Dong*, J Lii, A Shiu, H Liu, University of Texas M.D. Anderson Cancer Center, Houston, TX

Radiographic film is a convenient tool for measuring two-dimensional integrated dose distributions with high spatial resolution. However, large differences were found when film was used to measure profiles of the enhanced dynamic wedge (EDW). When the profiles are normalized to $100 \%$ on the central axis, differences as high as $10 \%$ at Dmax and $30 \%$ at $35 \mathrm{~cm}$ depth were found near the "toe" (or high dose) region of a wedged profile between film measurements and direct ion-chamber measurements for $6 \mathrm{MV}, 20 \mathrm{~cm} \times 20 \mathrm{~cm}$ EDW fields. Smaller differences were found for smaller EDW fields or a higher photon energy (18MV). Extensive film measurements and Monte Carlo simulations show that film sensitivity changes along off-axis positions, at deeper depths and for different field sizes. Correction to film measurements is necessary and can be done with only a few single ion-chamber measurements near the toe region of an EDW profile. The method provides an effective way to combine the efficiency of film measurement and the accuracy of ion chamber measurement for EDW commissioning.

\section{PO-T- 143}

A Comparison of Single Focused vs Double Focused MLC Field Blocking

J Killoran*1, J Giraud, ${ }^{2}$, L Chin ${ }^{1},(1)$ Joint Center for Radiation Therapy, Boston, MA (2) CHU de Grenoble, Grenoble, France

Despite extensive literature devoted to MLC dosimetry, uncertainty persists among clinicians as to the dosimetric consequences of MLC usage in specific treatment situations. This is compounded by differences in multileaf collimator design. We preformed measurements to compare the dosimetric properties of two MLC designs: a "single-focused" MLC (SFMLC) mounted below the jaws, and a "double-focused" MLC (DF-MLC) which is a complete replacement for the lower jaws. The ability of each MLC to conform isodose lines to a prescribed field edge (PFE) was evaluated using film dosimetry. Circular fields, centered on-axis and offaxis, were used because they produce a range of "angles of approach" between the MLC leaves and the PFE. They also have the advantage that for an ideal field shaping system the resulting isodoses are concentric perfect circles, a well defined basis for evaluation. The amplitude of the oscillations of the $50 \%$ isodose line about the PFE and the penumbra width as determined by the $20 \%, 80 \%$ and $90 \%$ isodose lines were examined. We observe that the $50 \%$ isodose line oscillates around the PFE with a greater amplitude for SF-MLC. We attribute this, at least in part, to the rounded ends of the SF-MLC leaves. However, the SF-MLC has a noticeably sharper penumbra, which we attribute to its position further from the source. We conclude that these results are relevant for accurate dosimetric modeling of these devices, and guidelines for treatment planning are device dependent.

\section{PO-T- 144}

An Evaluation of the Recommendations of the AAPM TG51 Protocol: Photon Beam Calibration and Comparison with the TG21 Protocol M Huq*, Philadelphia, PA

Task Group 51 of the AAPM Radiation Therapy Committee has recently developed a new protocol for the calibration of high-energy photon and electron beams based on standards of absorbed dose to water. The recommended dosimetry procedures in this protocol are based on the use of an ionization chamber calibrated in terms of absorbed dose to water in a standards laboratory's ${ }^{60} \mathrm{Co}$ beam. This is different from the recommendations given in the TG21 protocol, which are based upon an exposure calibration factor of an ionization chamber in a ${ }^{60} \mathrm{Co}$ beam. The purpose of this work is to compare the recommendations of the two protocols using two widely used Farmer type ionization chambers: PTW N30001 (PMMA wall) and NEL 2571 (Graphite wall). This was accomplished by determining absorbed-dose-to-water with both chambers irradiated in a water phantom using 6 and $25 \mathrm{MV}$ photon beams. Ratios of TG51 to TG21 doses were found to be 1.006 and 1.007 at 6 and 25 MV respectively when the PTW chamber is used; 1.010 and 1.011 when the NEL chamber is used. When the recommendations of TG51 are followed the dose determined by the NEL chamber is found to be higher than that of the PTW chamber by $1.6 \%$ at $6 \mathrm{MV}$ and $1.8 \%$ at $25 \mathrm{MV}$. The corresponding results for the TG21 protocol are $1.2 \%$ at $6 \mathrm{MV}$ and $1.4 \%$ at $25 \mathrm{MV}$ respectively.

\section{PO-T- 145}

Comparison between TG-51 and TG-21: The Results of TG-51 Beta Testing

J Lowenstein*, S Cho, P Balter, W Hanson, U.T. M.D. Anderson Cancer Center, Houston, TX

A new protocol for clinical reference dosimetry of external beam radiation therapy, has been developed by the AAPM Task Group 51 (TG-51) to replace the previous protocol (TG-21). The TG-51 protocol is based on an absorbed dose to water calibration factor $\left(\mathrm{N}_{\mathrm{D}, \mathrm{w}}\right)$ and an energy-dependent correction factor, $\mathrm{k}_{\mathrm{Q}}$. The TG-21 protocol is based on an exposure (air kerma) standard and multiple energy-dependent correction factors. Because of these dosimetry differences, and the incorporation of updated physical data, the results of clinical reference dosimetry based on TG-51 are expected to be somewhat different from those based on TG-21. The Radiological Physics Center (RPC) has conducted a systematic comparison between these two protocols, in which photon and electron outputs following both protocols are compared under identical conditions. Multiple chamber types (cylindrical) used in this study were selected from the list given in the TG-51 report, covering the majority of current manufacturers. Comparison shows discrepancies somewhat larger than expected, $1 \%$ or more difference for all beams for some chambers. The publishing of TG-51 has been delayed while these results are reconsidered. Comparison with any revisions will be included, if available.

This work is supported in part by PHS grant CA10953 awarded by NCI, DHHS.

\section{PO-T- 146}

A Single Diode Detector for In Vivo Dosimetry of Photon Beams

V Colussi*, C Sibata, A Beddar, Q Wu, T Kinsella, Case Western Reserve University, Cleveland, $\mathrm{OH}$

Diode in vivo dosimetry has been recommended to improve the quality of patient care in radiation therapy. We have characterized the response of the Sun Nuclear QED diode detectors with respect to SSD, field size, wedge, temperature, and detector-beam orientation. Photons beam with energies of 4, 6, 10 and $18 \mathrm{MV}$ were used. The measurements were normalized to the ionization chamber reading at dmax. Our empirical response curves show that the total variation of the diode response to all these factors are within $\pm 4 \%$. This response seems to be less dependent on the SSD, field size and wedge than the Isorad Nuclear Associates diode detectors used by Meiler and Podgorsak (1997) which showed a variation in response larger than $15 \%$.

Usually, each diode is calibrated for a specific nominal beam energy and used clinically with that energy only. We have tested our diodes and have concluded that the high energy diodes (18MV) can be used for all photon energies. We are proposing therefore that only diodes with high energy buildup (18MV) need to be bought for any photon energy. All response curves are still within the range of $\pm 4 \%$.

Using the high energy buildup diode will have the added benefit of amplifying the response of the diode when the wrong energy is used in the patient treatment. 


\section{PO-T- 147}

Profiles of Head-Scatter Photons and Contaminating Electrons Measured with Radiographic Film

B Arjomandy*, B Bjangard, Department of Radiation Oncology, University of Pennsylvania, Philadelphia, PA

We have evaluated a technique to measure the profile of the dose contribution from head-scattered photons in high-energy photon beams. Radiographic films, covered by acrylic buildup plates, were exposed with the same monitor-unit setting with a large field and a narrow strip field. They were scanned along corresponding lines. The measured difference between the doses in the large and the narrow fields was assumed to be due to the head-scattered photons. The same technique, without the buildup plate, was used to determine the profile of contaminating electrons in the beams. Experiments were made for 6 and $10 \mathrm{MV}$ x-ray beams, with and without a 60-degree wedge. - The results of the film measurements agreed well with results of point measurements with ionization chambers. The advantage of film for this purpose is that many data can be collected and evaluated easily. The distributions showed that the directional spread of the head-scattered radiation, although large, does not obliterate the dependence on the fluence variation in the primary beam

\section{PO-T- 148}

Transmission and Dose Perturbations with High-Z Materials in Clinical Electron Beams

I Das*, R Mitra, C Cheng, Fox Chase Cancer Center, Philadelphia, PA, Fox Chase Cancer Center, Philadelphia, PA, University of Arizona, Tucson, AZ

High atomic number $(\mathrm{Z})$ materials $(\mathrm{Ti}, \mathrm{Cr}, \mathrm{V}, \mathrm{Fe})$ used in various prostheses and $\mathrm{Pb}$ used for shielding electron beams produce dose perturbations. An accurate knowledge of dose perturbation and transmission through these materials is required for curative and reirradiation planning which is not available for all clinical electron beams. Measurements were taken with thin-window parallel plate ion chambers for $\mathrm{Ti}, \mathrm{Al}, \mathrm{Cu}$, Steel, and $\mathrm{Pb}$ on Varian and Siemens units in the energy range of 5-20 MeV. Two sets of data were acquired, one with the detector at the surface and the other at $d_{\max }$ of the electron beams. It is observed that for all high- $Z$ materials, there are dose enhancements on both sides of thin plates. The dose enhancement on both sides of the plate is due to increased electron fluence that is dependent on the beam energy. In the transmission side, the magnitude depends on the thickness of the plate that reduces with increasing thickness. The bremsstrahlung component increases with beam energy and $\mathrm{Z}$ of the medium. The thickness of material to reduce $100 \%, 50 \%$ and $1 \%$ transmission is linear with the beam energy. For the $50 \%$ and full attenuation, the slope of curves are $0.95 \mathrm{~mm} / \mathrm{MeV}, 1.2 \mathrm{~mm} / \mathrm{MeV}$ and 0.24 $\mathrm{mm} / \mathrm{MeV}$ and $0.4 \mathrm{~mm} / \mathrm{MeV}$ for the $\mathrm{Ti}$ and $\mathrm{Pb}$, respectively. An analytical function is provided for the atomic number and the energy for a given transmission, which may be used for dosimetric planning with metallic plates in electron beams.

\section{PO-T- 149}

Analysis of Dose Distributions in Six Dual Field Total Skin Electron Therapy

K Yenice*, A Sabbas, Memorial Sloan Kettering Cancer Center, New York, NY, New York Presbyterian Hospital, New York, NY

Clinical implementation of a multiple field total skin electron therapy involves the absolute dose calibration in a circular phantom and dose uniformity measurements in the geometry selected. Dose uniformity is generally assessed in an idealized treatment using a Rando phantom. The published data for depth dose distributions using the Rando phantom are usually limited to the mid-transverse plane along a single direction. There is also insufficient data on the relative contribution of single dual fields to the composite treatment dose for different planes. We have done a detailed study of dose distributions in multiple planes with a Rando phantom using film and TLD dosimetry.

V-films were sandwiched between Rando slices and the phantom was irradiated with a six dual beam arrangement in a $6 \mathrm{MeV}$ nominal TSEB degraded by 3.2 and $6.0 \mathrm{~mm}$ thick scatterers. Depth doses were measured by scanning the films along several radii of the phantom slices. The depth dose distributions varied among various scanning directions by $3-6 \mathrm{~mm}$ for all the slices studied. This information is useful when lesions of different thickness are being treated.

We also determined the relative contribution of single dual fields to composite treatment for different body parts to be between 2.25 and 2.65 . In general, any particular site will get contribution from at least two but not more than three fields. When combined with patient TLD measurements, these results are important in assessing sources of non-uniformities.

\section{PO-T- 150}

Design and Implementation of a New Device for The Integral Measurement of Total Source-on Time for a High Dose Rate (HDR) Remote Afterloading Treatment

E Parsai*, K Brown, K Ayyangar, J Feldmeier, Medical College of Ohio, Toledo, $\mathrm{OH}$

Design and implementation of a new device used to manually measure the total integral time that a HDR Ir-192 source spends outside the safe will be presented. Most HDR units are equipped with a timer that measures the source-on interval, however, verification of the accuracy of this internal timer for each treatment has not been addressed. Most radiation therapy rooms have installed a radiation detector unit operating independent of the radiation producing device. One wall mounted sample of such device is simple Geiger Muller (GM) counter known as the "Primalert-35" made by Nuclear Associates ${ }^{\mathrm{TM}}$ that will illuminate when placed in radiation area. Using a digital timer known as "Veeder Root Timer ${ }^{\mathrm{TM}}$ " connected to the auxiliary output of a Primalert-35, we were able to visualize the signal and determine that is was a step function. We also found that auxiliary output is a 200 milli-second pulse when in alarm condition, and the timer could not track the pulse because of the oscillation of the signal. A circuit was then designed and added to the Primalert-35 unit to stretch the $200 \mathrm{~ms}$ pulse so that it appears to be a non-pulsing signal at a constant DC level. This allowed us to measure the time interval for as long as the Primalert-35 was active. This is an equivalent time for the source being out of the safe.

\section{PO-T- 151}

A 16-field Technique for Total Skin Electron Irradiation

C Cheng*, R Wallace, J Gorman, University of Arizona, Tucson, AZ

Total skin treatment for mycosis fungoides with a $5 \mathrm{MeV}$ electron beam (Siemens MD2) was evaluated for uniformity $(<5 \%$ through $200 \mathrm{~cm}$ vertical height), low bremsstrahlung ( $4 \%)$, and clinically significant depths of penetration $(\mathrm{dmax}=1 \mathrm{~mm}, 90 \%=3 \mathrm{~mm}$ and $50 \%=9 \mathrm{~mm})$. The 16 field technique consisted of the patient standing AP or PA at $400 \mathrm{~cm} \mathrm{SSD} \mathrm{on} \mathrm{a}$ platform $25 \mathrm{~cm}$ above the floor and rotated through 4 different angles $\left(20^{\circ}\right.$ and $60^{\circ}$ both clockwise and counterclockwise) and two angled beams $\left(68^{\circ}\right.$ or $112^{\circ}$ ). A $0.6 \mathrm{~cm}$ lucite plate located at the collimator degraded the beam $\left(E_{0}=2.1 \mathrm{MeV}\right)$. The composite treatment effects of self shielding, obliquity, and uniformity were evaluated with films in a Rando phantom at the surface and at $5 \mathrm{~mm}$ depth. At the surface, the $20^{\circ}$ and the $60^{\circ}$ fields differed by about $5 \%$ in dose delivered. At $5 \mathrm{~mm}$, the $20^{\circ}$ fields contributed about 1.9 times more than that of the $60^{\circ}$ fields due to large obliquity effect at $60^{\circ}$. Uniform relative dose in the abdomen was contrasted with small but clinically acceptable hot spots in the neck and head due to the smaller anatomical cross sections when prescribed to the surface. Absolute dose measured with a CPPC chamber or TLDs placed in rectangular and Rando phantoms was delivered $90 \%$ from the 8 anterior fields and $10 \%$ from the remaining posterior fields. The 16 -field complete treatment cycle is delivered in two days for a per treatment prescribed dose of $200 \mathrm{cGy}$.

\section{PO-T- 152}

Evaluation of Clinical Use of Siemens Variable Electron Applicator

A Thavendrarajah*, The Ohio State University, Columbus, $\mathrm{OH}$

The electron beam therapy is an important component of radiation therapy. The use of Siemens Variable Electron Applicator makes it possible to choose any electron field size between $4 \times 4 \mathrm{~cm}^{2}$ and $25 \times 25 \mathrm{~cm}^{2}$. Since this applicator can produce square and rectangular electron fields the use of the electron cutouts can be eliminated in many cases. The output factor, \%DD and profiles were measured for square fields from $5 \times 5 \mathrm{~cm}^{2}$ to $25 \times 25 \mathrm{~cm}^{2}$ using a plane parallel ion chamber (PTW $0.3 \mathrm{cc}$ ) for $6,9,12,15$ and 18 $\mathrm{MeV}$. The output factor is fitted with a fourth order polynomial equation and 
the output factor for any other square field can be readily determined using this equation. The output factor for any elongated field is determined using Clarksons sector integration method with the polynomial as the radial function.

The measured output factors for the elongated fields show significant (as much as $2.9 \%$ difference) exchange effect. However, the calculated output factors agree within $0.5 \%$ with the measured output for the elongated fields when the upper jaws represent the long side and for all square fields. This observation could be used to our advantage by keeping long side on the upper jaws and rotating the collimator to match the field on the patient. The dosimetric characteristics such as $\% \mathrm{DD}, \mathrm{d}_{\max }, \mathrm{R}_{\mathrm{P}}$, penumbra etc. remains nearly the same as for the conventional electron applicator.

\section{PO-T- 153}

Investigation of Patient Position Comparing Two Immobilization Devices with the Use of Port Film/Digitally Reconstructed Radiograph for Brain Tumors

L Butler*, Y Zhu, W Gaber, T Merchant, St. Jude Children's Research Hospital, Memphis, TN

The purpose of this study was to analyze the accuracy of patient position by comparing two immobilization devices for pediatric brain tumor patients. We analyzed the data for 99 treatments involving 8 patients. Patients were immobilized with either a relocateable head frame with a dental impression or vacuum bag with or without sedation. Orthogonal portal films were taken once weekly. These verification films were compared to the Digitally Reconstructed Radiographs (DRRs) that were generated by a 3D treatment planning system. Deviations of the patient position from the original planning references were measured using a program called Portal Image Processing System (PIPS). The over-all systematic and random errors were analyzed. The head frame had an average (systematic) deviation ranging from $0.1 \mathrm{~mm}$ to $1.1 \mathrm{~mm}$, with a standard (random) deviation from $1.2 \mathrm{~mm}$ to $2.3 \mathrm{~mm}$. The vacuum bag had an average (systematic) deviation ranging from $-0.4 \mathrm{~mm}$ to $0.3 \mathrm{~mm}$, with a standard (random) deviation from $1.6 \mathrm{~mm}$ to $2.3 \mathrm{~mm}$. The

effect of sedation on the patient position will be reported. This project will be a useful reference for the radiation oncologist to determine the appropriate planning target volume in the context of pediatric oncology.

\section{PO-T- 154}

\section{Partial Bolus Verification of Patient Dose via MOSFET Dosimeters}

D Lightfoot*, Medical College of Pennsylvania Hospital, Philadelphia, PA

MOSFET dosimeters are useful for a variety of patient dose measurements due to very small size and minimal build up. MOSFET dosimeter use for patient $D_{\max }$ dose verification requires the use bolus for $\mathrm{x}$-ray beams. Use of partial $(1 \mathrm{~cm}$ dosimeter bolus for $6 \mathrm{MV}$ and $2 \mathrm{~cm}$ for $15 \mathrm{MV}$ ) rather than full bolus over the MOSFET minimizes perturbation of the patient dose. Measurements have been done to establish the relationship of partial bolus readings to the dose at $D_{\max }$ which indicate shallower $D_{\max }$ depth at shorter SSD and hence nearly full build-up at the partial bolus depth, presumably due to block tray scatter. Correction factors ranging from about 0.94 to 1.00 for $\mathrm{D}_{\max }$ verification measurements with $15 \mathrm{MV}$ and $6 \mathrm{MV}$ x-ray beams and equal to 1.015 for point dose evaluation due to the method of calibration have been determined. The MOSFET dosimeters have performed well in both $\mathrm{x}$-ray and electron beams with moderate precision.

\section{PO-T- 155}

Mixed-beam 3-D conformal therapy: Dosimetric verification

P Halvorsen*, S Parker, University North Carolina, Chapel Hill, NC

For certain treatment scenarios, a significant dosimetric improvement may be realized by the use of an en-face electron beam combined with intensitymodulated photon beams at angles nearly orthogonal to the electron beam. This combination produces the desired dose uniformity within the target, while significantly reducing exit dose to tissues below the target. All the beams are jointly designed in a 3-D planning system with IMRT capability, and we call the result 'mixed-beam 3-D conformal therapy.'

Implementation of this approach presents some practical concerns often not associated with electron-beam therapy. Immobilization is critical for accurate dose delivery, yet clearance of the electron applicator system from the patient and immobilization device must be assured. Both photon and electron dose calculation models may have increased uncertainty near the skin surface, possibly affecting the design of the mixed-beam technique. For these reasons, a system for reliable and efficient in-vivo dosimetry is crucial to implementing quality mixed-beam conformal therapy.

We have evaluated the accuracy and reproducibility of MOSFET detectors (Thomson\&Nielsen) for this purpose. The detectors' energy, modality, and angular dependence have been investigated. Extensive comparisons to other detector systems have been completed in phantoms, with and without the presence of heterogeneities. Accuracy of dose readings near the surface have also been evaluated.

The results of these evaluations have established the MOSFET system as very well suited to mixed-beam in-vivo dosimetry.

We will show the range of clinical scenarios modeled in these evaluations, and discuss the comparison test findings.

\section{PO-T- 156}

Assessment of the Accuracy of Monitor Unit Calculations in ThreeDimensional Treatment Planning

M Vossler*, J Bayouth, UPMC Shadyside, Pittsburgh, PA

The purpose of this study was to investigate the sources and magnitude of the discrepancies in the monitor unit calculations between a threedimensional treatment planning system and traditional hand calculation techniques. The convolution superposition algorithm is far less dependent on measured data than previous computational methods, and accounts for physical attributes that are roughly approximated or ignored by traditional hand calculation methods. Measurements were carried out in a water phantom and spherical plastic phantom to assess the accuracy of these two calculational methods. Several open and blocked fields with various points of interest were studied, including fields which employ oblique or curved surfaces, tangential geometry, extensive blocking (e.g. mantle fields), and compensators. For each point of interest the monitor units computed by the planning system and hand calculations are compared with those determined by in-phantom measurements. Additionally, a retrospective patient study was performed in which the sources and magnitude of disagreement between computed monitor unit calculations and traditional calculations were quantitatively analyzed for several specific patient cases. The planning system agreed well with the measured data for simple field shapes. Greater disagreement resulted when examining more complex scenarios such as heavily blocked fields, curved surfaces, and points near the heel or toe of wedges. Some discrepancies were unrelated to the accuracy of the algorithm used, such as disparities in patient thickness and SSD measured on CT versus on the simulator.

\section{PO-T- 157}

New Treatment Table Panel with Reduced Skin Dose

F Wittkamper*, M Dwarswaard, W Balk, The Netherlands Cancer Institute, Amsterdam, The Netherlands

The C-arm table-top of the Elekta SL accelerator is provided with a tennis racquet. The sag of the tennis racquet with a normal patient load is larger than for the carbon or wooden table-top at the simulator or CT scanner. Therefore the treatment position of the patient on the treatment table is not the same. At the Netherlands Cancer Institute a carbon fiber panel was developed with minimal sag; less than $2 \mathrm{~mm}$ in the middle with $75 \mathrm{~kg}$ equally distributed load. As a pay-off the dose to the skin increased from 30 $\%$ with the tennis racquet to $70 \%$ with the carbon panel (data are for a $6 \mathrm{MV}$ photon beam). With a maximum tolerable dose to the skin of $30 \mathrm{~Gy}$, the maximum dose midplane for an anterior-posterior treatment is restricted to $40 \mathrm{~Gy}$. If the prescribed dose midplane is higher the tennis racquet has to be used. Recently we started a project to reduce the dose to the skin by looking at the construction and choice of materials. First measurements with a honey cell structure showed a reduction from $70 \%$ to $48 \%$. Additional results will be shown together with the final results of the newly developed panel. It is the intention that this new panel will be available for the C-arm table top of the Elekta SL series accelerators. 


\section{PO-T- 158}

Measurement of Filtered Percentage Depth Dose for Higher Energy Photon Beams

J Dawson, W Kopecky, N Nelson, F Abrath, J Gu*, R McDonald, J Dawson, Saint Louis University, St. Louis, MO

Electron contamination corrections are necessary when the Percentage Depth Dose (PDD) (@ 10cm depth in water, 10x10 $\mathrm{cm}^{2}$ field, SSD=100 $\mathrm{cm}$ ) is used as the beam quality indicator for photon energies higher than $10 \mathrm{MV}$.

Published results indicate that a $0.1 \mathrm{~cm}$ lead sheet placed immediately below the accelerator head can be used to obtain a filtered PDD (PDDF). The measured PDDF

$\left(10 \mathrm{~cm}\right.$ depth in water, $10 \times 10 \mathrm{~cm}^{2}$ field, $\left.\mathrm{SSD}=100 \mathrm{~cm}\right)$ is used to calculate the electron contamination correction factor (ECCF). PDDF were measured for

energies $>=10 \mathrm{MV}$ for several models of linear accelerator. Scanners were used for data acquisition. Different thicknesses of lead foil (0.015" thick) and a lead vinyl sheet $(1 \mathrm{~mm}$ lead eq., $1 / 8$ " thick) were used as filter materials. The effect on PDDF of the position of the filter in the beam was evaluated. Measured PDDF at $10 \mathrm{~cm}$ were within $.5 \%$ (18 MV) and .7\% (15 $\mathrm{MV}$ ) for lead thicknesses between $.4 \mathrm{~mm}$ and $1.6 \mathrm{~mm}$. For $18 \mathrm{MV}$ the measured PDDF at $10 \mathrm{~cm}$ were within $.5 \%$ when the lead filters were placed at $46 \mathrm{~cm}$ and $62 \mathrm{~cm}$ from the source respectively. Commercially available lead foil or lead Vinyl sheets can be taped immediately below the head or the tray of an accelerator to measure the PDDF value used to calculate the ECCF.

\section{PO-T- 159}

\section{The Dose Distribution of Stomal Boosts}

E Yorke*, A Kassaee, T Doyle, M D. Altschuler, D Rosenthal, University of Pennsylvania Medical Center, Philadelphia, PA

An en face electron boost of approximately $10 \mathrm{~Gy}$ is often given to stomal and peristomal tissues. This region is usually assumed to be water equivalent but because the stoma is an air cavity, the dose distribution is more complex. We made two lucite stoma phantoms: a $2.5 \mathrm{~cm}$ diameter circular hole of depth $2 \mathrm{~cm}$ and an elliptical hole of dimensions equal to the average stoma length, width and depth $(2 \mathrm{~cm} \times 1.7 \mathrm{~cm} \times 3.5 \mathrm{~cm})$ determined from CT scans of 20 patients. Film and diode measurements were made with the phantom on standard polystyrene slabs with the hole centered in a $7 \mathrm{~cm}$ circular field at. SSD of $100 \mathrm{~cm}$ for 9 and $12 \mathrm{MeV}$ electrons. Measurements were made at shallow depths below the bottom of the hole and also at approximately $6 \mathrm{~cm}$ from the top of the phantom (to represent the cord). The combined effects of lateral scatter from the lucite surrounding the hole and the reduced equivalent thickness for electrons passing directly through the hole increase doses at both shallow and cord-like depths. The effect is more pronounced for $12 \mathrm{MeV}$ electrons and may lead to clinically significant normal tissue effects, especially for patients with large stomas. Measured dose distributions and dose distributions calculated for a patient CT scan with a pencil beam dose deposition kernel algorithm will be presented.

\section{PO-T- 160}

Characterize Patient-specific Target Motion During The External Beam Treatment of Prostate Cancer

K Kota*,D Yan ${ }^{1}$, J Lebesque, M VanHerk², J Wong ${ }^{1},(1)$ William Beaumont Hospital, Royal Oak, MI, USA,(2)Netherlands Cancer Institute, Amsterdam, The Netherlands

Effective compensation for internal organ motion in the radiotherapy process requires better knowledge of motion characteristics. To do so, clinical target volumes (CTV) of 15 prostate cancer patients who had repeat daily CT scans were analyzed retrospectively. For the individual patient, the displacement of the center of mass (COM) of the CTV was calculated with respect to the initial reference position. The corresponding distribution was characterized and examined using the $\chi^{2}$ good-fit test. In addition, the displacement was also analyzed as a function of treatment time and size of the CTV. The results demonstrate that distribution of the systematic displacement in the patient's anterior-posterior direction has variation significantly larger than the average of the random displacement. This suggests that clinical procedures in the pre-treatment $\mathrm{CT}$ imaging introduce extra variability in the target position. The patients were also divided into four separate groups based on the value of their random displacement. For each of the four groups, there is strong evidence that the Gaussian distribution could be applied to fit the distributions of the COM displacement. Furthermore, variability of the displacement did not systematically reduced as commonly expected as the treatment progressed. However, this could be due to the CTV shrinking during the treatment course as observed in our study. Our result demonstrates that the CTV shrinkage may cause large variation in the displacement of its COM.

\section{Supported in part by NCI grant \#CA71785.}

\section{PO-T- 161}

Optimization of Treatment Planning Using a Fuzzy Weight Function

F Yin*, R Li, Henry Ford Hospital, Detroit, MI

Development of a clinical feasible inverse-planning algorithm involves several important aspects such as the selection of objective function, iteration approach, prescription and dose calculation methods, etc. In this study, we developed a fully analytical solution for optimizing beam intensities and a fuzzy inverse solution for optimizing uncertain prescription in normal tissue. Based on the classical minimum theory, parameters used in this new iteration scheme are analytically derived. Therefore, the algorithm guarantees a fast and monotonic convergence to the global minimum of a constrained-quadratic objective function. The importance of matching the prescribed dose and the calculated dose in the normal tissue is represented by a fuzzy weight function. The task of giving full dose to the target volume while minimizing dose to the normal tissue is achieved by dynamically modifying weights of the normal tissue prescription using a fuzzy weight function. The effectiveness of the new approach has been evaluated using DVH data for several clinical treatment sites. Results indicated that the performance of the algorithm is substantially improved with the introduction of a fuzzy weight function. Typically, acceptable results can be obtained with less than 10 iterations and no dose calculation is required during iteration for fixed beam configuration. It has been shown that the technique is capable of finding a solution that achieves the optimal balance between the objective of matching the calculated dose and the prescribed dose for the target volume and the objective of minimizing the dose in normal tissues.

\section{PO-T- 162}

Optimization of Importance Factors in Inverse Planning

L Xing*, J Li, A Boyer, Stanford University, Stanford, CA

Inverse planning starts with a prescribed dose (or DVHs) and obtains the solution by optimizing an objective function. A set of importance factors (IFs) is often incorporated in the function to parameterize tradeoff strategies in different anatomical structures. Whereas the general formalism remains the same, different sets of IFs characterize plans of obviously different flavor and critically influence the final plan. The determination of IFs has been a "guessing" game based on empirical knowledge. The influence of these parameters is not known until the plan optimization is completed. In this work, a method to estimate these parameters is proposed and an iterative algorithm is described to determine the parameters numerically. The treatment plan selection is done in two steps. First, a set of initial IFs are chosen and the corresponding beam profiles are optimized under the guidance of a quadratic objective function using an iterative algorithm. The "optimal" plan is then evaluated by an additional DVH based scoring function. The importance factors are adjusted accordingly to improve the score of the plan. For every variation in the IFs, the beam parameters need to be re-optimized. This process continues in an iterative fashion until the DVH score function is saturated. The algorithm was applied to two clinical cases and the results demonstrated that it has the potential to improve significantly the existing method of inverse planning. It was noticed that near the final solution the plan became insensitive to small variations of the IFs.

\section{PO-T- 163}

Clinical Implementation of Wedge Filter Optimization in 3D Radiotherapy Treatment Planning

J Li*, L Xing, A Boyer, Stanford University, Stanford, CA

Previous approach of optimizing the wedge filters in 3D radiotherapy treatment planning is to decompose each incident beam into an open and two 
nominal wedged fields, and then to optimize their weights based on the concept of the omni wedge. A serious limitation of the approach to clinical application is that one has to pre-select the signs of the nominal wedges. In this work, a new algorithm was developed to automate fully the $3 \mathrm{D}$ treatment planning process. The approach optimizes the beam weights, wedge angles and orientations directly using a simulated annealing algorithm, and no planner intervention was required in the process. A dosebased objective function which incorporated the relative importance of structures was adopted. The technique was demonstrated by using a phantom study and a clinical case. For the phantom case, the classical wedge pair result was obtained, providing a useful test of the algorithm. Sensible dose distributions and dose-volume histograms for the target and surrounding organs were obtained for the clinical case. It was also shown that dose homogeneity to the target could be compromised by increasing the relative importance factors to the surrounding organs. Field restrictions, such as restricting the wedge orientation(s) and/or wedge angle(s) for one or more fields, can be applied. The technique has the potential to fully automate the selection of wedges in $3 \mathrm{D}$ radiotherapy treatment planning. In addition, treatment planning time and efforts were reduced.

\section{PO-T- 164}

Adaptive Grid Generation for Automated Treatment Planning: Regions of Interest and Pencil Beam Gridding

J Deasy*, University of Louisville, Louisville, KY

It is important to have a gridding method which yields small voxels near the edge of regions-of-interest (ROIs), but which can be fine-tuned to give larger voxels in the middle of an ROI. We introduce algorithms to adaptively grid ROIs and pencil beam widths. For ROIs, the approach described here uses a modification of the octree method for division of an ROI into different sized cubes. The algorithm is briefly described as follows: An initial regridding is made to the smallest desired resolution; a user-specified number of outer-layers is gridded to the initial resolution; extra gridding at that resolution is done to assure that the non-gridded ROI which remains can be gridded with a doubled voxel width; the process is repeated with the next highest voxel size until the ROI has been completely gridded. Because the initial resolution and the number of layers for each voxel size is variable, the results are quite customizable to be appropriate to the problem type. A similar algorithm is used to adaptively grid ports to create variable size pencil beams. For pencil beams, the problem is $2 \mathrm{D}$, and high-resolution boundaries are created wherever beams-eye-view ROI edges are detected. If both of these gridding strategies are used, the potential reduction in computation time for many IMRT algorithms exceeds an order of magnitude.

This research was supported in part by ADAC Laboratories, Inc.

\section{PO-T- 165}

Evaluation of a Totally Integrated CT-Simulator-Treatment Planning System for Accuracy and Efficiency

E Butker*, D Helton, E Elder, J Ting, L Davis, Emory University School of Medicine, Atlanta, GA

A totally integrated CT-simulator-treatment planning system combines the necessary CT dataset acquisition, the virtual simulation, and the 3-D treatment planning of the dataset in one singular session at the time of simulation. The accuracy and efficiency of such a system was evaluated using the recommendations of TG23 Report 55 of the AAPM. Profiles, output factors, tray factors, and transmission factors were collected for a 6 MV and 18 MV beam. Data was transferred from the 3-D Computerized Water Phantom to the ACQPLAN System. Measured data was collected as described in TG23 Report 55 of the AAPM and then compared to generated values using the system. Measured values of TMR's, percent depth doses, profiles, output factors, and monitor units were compared to computed values. Results were within $2.0 \%$ or $2 \mathrm{~mm}$ for all values. The accuracy of the totally integrated CT-simulator-treatment planning system has been verified. The system has been found to be clinically useful and allows the possibility of patient dose evaluation during the initial patient simulation session. The efficiency of the system manifests itself in several ways. There is less dataset manipulation as all work is performed on one system. Time spent in preparation of patient data for treatment is decreased since there is no data transfer or duplication of dataset preparation processes. Purchase of an all- in-one system maintains the highest accuracy and efficiency available for patient data handling through CT Simulation combined with 3-D treatment planning.

\section{PO-T- 166}

Improved Dose Homogeneity in Opposed Beams Irradiation Using an Additional Static Field

J Pérez-Calatayud*, F Lliso, V Carmona, A González, J Ruíz, I Petschen ${ }^{1}$, F Ballester $^{2}$, (1) La Fe University Hospital, Valencia, Spain, (2) Valencia University, Valencia, Spain

To deliver as high and as homogeneous a dose as possible to the target volume and to avoid unnecessary irradiation of healthy tissue is not always easy to achieve. In this work a simple method is proposed to obtain a suitable level of homogeneity based on the superposition of few-static fields in opposed beam techniques.

At our Department, all the patients undergo virtual simulation and 3D calculations. The dose distribution is optimized on the central axial plane and representative oblique planes passing trough the isocentre are also evaluated. The view orthogonal to the central axis determines if or not the additional beam is to be used, the decision-making is based on the extension of the area comprised by the $105 \%$ isodose curve. When necessary a slightly weighted port defined by the $105 \%$ isodose curve in this view is added. Customized blocks (once or twice a week) or MLC ports (every fraction) are used to deliver this boost depending on the treatment machine.

DVH for different treatments shows the benefits of the technique. The homogeneity over the PTV is highly improved, reducing high dose levels about 5\%. The clinical implementation is very low time consuming: the weight of the beams is easily calculated and the increase of irradiation time is negligible. No sophisticated tools (inverse planning, IMB, etc) are necessary. This simple technique has allowed us to go up the first step of 3D conformal therapy in clinical routine.

\section{PO-T- 167}

Time Trends in Organ Position in Patients Receiving Prostate Conformal Radiotherapy

J Mechalakos*, G Mageras, M Zelefsky, O Lyass, L Happersett, C Ling, S Leibel, Z Fuks ${ }^{1}$, H Kooy ${ }^{2}$, M van Herk ${ }^{3}$, G Kutcher ${ }^{4}$, (1)Memorial Sloan Kettering Cancer Center, N.Y., N.Y., (2)Joint Center for Radiation Therapy, Harvard Medical School, Boston, MA,(3)Netherlands Cancer Institute, Amsterdam, The Netherlands, (4)Department of Radiotherapy, Universitaire Ziekenhvizen, Leuven, Belgium

Purpose: To search for time trends in organ position which affect treatment in radiotherapy patients using $\mathrm{CT}$ scans.

Materials and Methods: Fifty patients treated prone received four CT scans over the course of treatment. Bladder filling was controlled by requesting each patient to void prior to each scan. Scans 2-4 were registered to scan 1 (the planning scan) using a chamfer matching algorithm. All scans were contoured by a physician.

Results: No significant time trends were observed in population mean prostate or seminal vesicle position or rotation; however, 10 patients showed large (abs. val. $>5 \mathrm{~mm}$ ) individual time trends in prostate or seminal vesicle position. Mean rectal volume for scans 2-4 showed a small decrease of $0.7 \pm 0.5 \mathrm{~cm}^{3} /$ wk. Mean bladder volume increased by $34 \pm 13 \%$ between scans 1 and 2 .

Of the 10 patients showing large time trends in prostate or seminal vesicle position, 7 showed time trends or large variations in the rectum and/or bladder. Two of these patients showed large $\left(>100 \mathrm{~cm}^{3}\right)$ rectal volumes in the planning CT. Further analysis on time trend predictors will be presented.

Conclusions: Although time trends in target position are small for the patient group as a whole, strong time trends in individual patients can occur which can have implications for target coverage over a radiotherapy course of several weeks. In most cases, these strong time trends are accompanied by strong time trends or large variations in the rectum or bladder. 


\section{PO-T- 168}

Determination of Clinically Relevant Setup Error for Daily Electronic Portal Imaging Analysis

F Van den Heuvel*, I Han, A Strowbridge, S Chungbin, D Ragan, Barbara Ann Karmanos Cancer Institute, Detroit, MI

The current practice for reporting daily positioning errors during external radiation, is to use the mean of the errors as a systematic error and the standard deviation of the mean as a measure of the spread of the error. This assumes that the distribution of errors is a normal distribution and that the number of samples from that distribution is large enough.

To perform this assessment a study was initiated on 24 patients with the following selection criteria:

Treatment for cancer of the cervix or endometrium, obese and postmenopausal. The patients were treated using a standard four-field box technique and portal images of at least two perpendicular ports were obtained for each fraction. A total of 1645 images was obtained. An inhouse developed programs for image networking (EPICURE) and registration (OPIDUM) were used to quantify the various shifts. Mean and standard deviation in the primary directions where calculated.

A convolution plan $(\mathrm{CP})$ with a normal distribution generated using the parameters obtained above was applied to the dose grid generated by a 3D planning system (GRATIS) and Dose Volume Histograms (DVH) were calculated. In addition a new plan was generated using measured patient shifts and superimposed (SP). Finally, an effective error was determined as being the systematic and random error that generated a $\mathrm{CP}$ with minimal difference to the SP.

This paper shows that $\mathrm{CP}$ can be used to asses clinical implications of patient movement more adequately starting from measured data.

\section{PO-T- 169}

Evaluation of Radiation Guided Gene Therapy Using Pinhole Imaging and Region-of-Interest (ROI) Analysis

C Scarfone*, L Geng, G Holburn, A Brill, J Dugger, J Patton, M Sandler, D Hallahan, Vanderbilt University Medical Center, Nashville, TN

As an alternative to biopsy, non-invasive nuclear medicine imaging has been proposed to study the biodistribution of vector mediated and radiation guided gene therapy. In this work, serial pinhole planar imaging is used to characterize the uptake of (1) Iodine-131 $\left({ }^{131} \mathrm{I}\right)$ labeled adenovirus vector $\left({ }^{131} \mathrm{I}\right.$ Ad.Egr-TNF) alone and (2) ${ }^{131} \mathrm{I}$ labeled anti-humanTNF antibody $\left({ }^{131} \mathrm{I}\right.$ anti-hTNF) in irradiated Glioma (GL261) in a murine model. In the first experiment, intratumoral and intravascular injection of ${ }^{131} \mathrm{I}$ Ad.Egr-TNF was performed $1 \mathrm{hr}$ and $24 \mathrm{hrs}$ prior to imaging. In the second experiment, intratumoral and intravascular injection of Ad.Egr-TNF was performed $24 \mathrm{hrs}$ prior to single fraction tumor irradiation to $10 \mathrm{~Gy}$ total dose, followed $48 \mathrm{hrs}$ later by injection of ${ }^{131} \mathrm{I}$ anti-hTNF. Control animals received no radiation dose. A single-head gamma camera equipped with a pinhole collimator was used to acquire planar images at one hour, $24 \mathrm{hrs}, 48 \mathrm{hrs}$ and $72 \mathrm{hrs}$ post anti-hTNF injection. Semi-quantitative ROI analysis was used to measure absolute image counts and average counts per pixel within the tumor region as a function of time. Intravascular (intratumor) injection of radiolabeled vector (Expt. \#1) showed an increase (decrease) in tumor uptake over a $24 \mathrm{hr}$ period. Radiation guided uptake of radiolabeled antibody decreased by factors of $\sim 5.5$ and $\sim 5.2$ (Expt. \#2) for intravascular and intratumor injections, respectively, over $72 \mathrm{hrs}$. Irradiated tumor tissue showed a slight increased uptake of ${ }^{131} \mathrm{I}$ anti-hTNF relative to control at $1 \mathrm{hr}$ and a decreased uptake at later time points.

\section{PO-T- 170}

Characteristics and Role of a Prototype Amorphous Silicon Array EPID in Breathing Synchronized Radiotherapy

H Kubo*, E Shapiro, E Seppi, UC Davis, Sacramento, CA, Varian, Palo Alto, CA, Varian, Palo Alto, CA

Current electronic portal imaging devices (EPID) are limited in their ability to provide direct and quick verification and monitoring of patients during both setup and treatment of breathing synchronized radiotherapy (BSRT). These limitations are largely due to their slow image capture rate and poor image quality. An amorphous silicon array flat panel electronic portal imaging device (si-EPID) is emerging to meet the challenge. The purpose of this study is: 1) to characterize the performance of a prototype si-EPID;2) to compare si-EPID and digitized film image quality; and 3) to evaluate the device in terms of verification of patient setup and monitoring during BSRT. Three quality assurance phantoms: a Lutz PVC, 'Las Vegas' and RMI model 1151 phantom were used to characterize the imaging system. To investigate the clinical application five EPID images each were collected from a lung cancer patient during a 22 -second breath-hold and normal breathing. The quality of images obtained with the "fast" and "standard" mode was found to be comparable to and better than that obtained with the digitized films, respectively. With this prototype si-EPID, it is possible to collect the images at the beginning, middle, and end of a 15 second breathhold. The si-EPID images can provide quick verification of initial patient setup and subsequent treatment position throughout the daily fractionation.

This work was partially supported by the UC Davis Health System Research Award and Varian grant

\section{PO-T- 171}

In-depth Testing of Information Management Systems Prior to Operation

K Turnbull, K Spencer, C Ramsey*, A Oliver, Thompson Cancer Survival Center, Knoxville, TN

Over the past several years, the utilization of advanced treatment capabilities, such as MLC, IMRT, 3D-RTP, and Gating, has been increasing. As such, radiation oncology integrated Information Management Systems (IMS) have also grown in capability and complexity. Unfortunately, as the complexity of a system increases, and the number of other systems with which it must interface increases, the potential number of problems encountered increases exponentially. Needless to say, facing a large number of new problems that can inhibit or prohibit treatment is not something that radiation oncology departments can afford to encounter with the installation and integration of system operations with a new IMS.

Several radiotherapy clinics have reported problems upon installation and operation of new IMS software. This demonstrates that the present acceptance testing is not adequate to identify many of the problems that will be encountered for resolution prior to operation. Looking at the aircraft and nuclear industries reveals much about in-depth testing methodologies for complex systems and groups of systems, including testing beyond the present acceptance testing typically performed for radiation therapy clinics. These methodologies include diverse testing such as functional, acceptance, preoperational and integrated systems testing. The feasibility of implementing such an in-depth testing program in radiation therapy facilities will be presented.

\section{PO-T- 172}

Protocol Pre-Approval Processes for Radiotherapy Treatment on Clinical Trials; The RPC Experience

D Davis*, D Followill, R Tailor, J Lowenstein, I Harris, J Roll, W Hanson, U.T. M.D. Anderson Cancer Center, Houston, TX

The Radiological Physics Center, in conjunction with cooperative clinical trial groups, protocol chairmen and quality assurance review centers, have developed a series of pre-approval processes. These are intended to assure the quality of treatment and to improve the quality of data submitted for patients entered onto cooperative clinical trials. The process typically involves protocols employing advanced technology or new treatment techniques. They are intended to verify that institutions meet the minimum requirements for protocol participation, and that institutions are clinically and dosimetrically equivalent to other institutions on the study before they are allowed to enter patients onto the trial. Early experience was gained from the 2 COMS studies which began in the mid 1980s. Recently, the RPC has been involved with the pre-approval processes for 6 other protocols: RTOG 95-17, RTOG 94-06, RTOG 98-05, GOG 165, SWOG 9438, SWOG 9704. Although pre-approval processes vary between studies, they all include: 1) completion of a 'knowledge assessment' questionnaire discussing equipment, dosimetry parameters and QA procedures; 2) benchmark case calculations; and 3) verification of experience by submission of one or more previous clinical cases. The pre-approval processes reduce the number of 
protocol deviations as seen with COMS, minimize dosimetry and clinical errors prior to patient registration, increase the understanding of the protocol requirements and lead to the development of protocol treatment evaluation tools.

This work was supported by PHS grant CA10953 awarded by the NCI, DHHS

\section{PO-T- 173}

Techniques for DRR Optimization in Virtual Simulation

J Killoran*1, J Giraud ${ }^{2}$, E Mannarino ${ }^{1}$, L Chin ${ }^{1}$, (1) Joint Center for Radiation Therapy, Boston, MA, (2) CHU de Grenoble, Grenoble, France

Digitally Reconstructed Radiographs (DRRs) from Virtual Simulation (VS), using a CT Simulator, replace the traditional radiographs used in fluoroscopic simulation (FS). While some aspects of DRR quality are a direct function of the underlying CT data, visibility of specific anatomical structures can be enhanced by manipulation of DRR algorithm parameters. We present techniques to optimize DRRs for specific treatment sites via DRR calculation parameters available from commercial VS software. In general, these parameters either set spatial bounds for the calculation, or manipulate the relationship between Houndsfield number and effective attenuation. Additionally, this software allows the user to create composite images based on the results of two individually optimized DRR calculations. We have made the general observation that default parameters tend to produce images with low contrast of bony structures as compared to normal radiographs. While bone contrast can be increased, this tends to decrease the visibility of less dense structures. DRRs for the chest present the greatest challenge since bony structures as well as lungs, airways and the outlines of soft tissues are important anatomical landmarks which appear with sufficient contrast on traditional radiographs. We illustrate that simultaneous visualization of these structures is possible using a composite image for which the relative contribution of two individually optimized DRRs is carefully adjusted to

produce a third image showing all structures. The results compare well with traditional radiographs. We conclude that this is a useful and appropriate technique for VS

\section{PO-T- 174}

External Beam Guided Drug Delivery using Radiolabeled Microspheres: Evaluation with Nuclear Medicine and Autoradiography Region-of-Interest (ROI) Techniques

C Scarfone*, L Geng, A Brill, G Holburn, J Clanton, J Dugger, J Patton, M Sandler, D Hallahan, Vanderbilt University Medical Center, Nashville, TN

Radiation induced microsphere aggregation within tumor blood vessels may be used to facilitate the delivery of novel gene therapies. We are currently investigating approaches to optimized drug delivery in Glioma (GL261) murine models using radiolabeled microspheres. Our preliminary data indicate that it is feasible to label microspheres with single-photon emitting radionuclides, induce aggregation within tumors and characterize the resulting microsphere biodistribution using photon-imaging techniques. In this study, pinhole gamma-camera imaging and autoradiography are used to characterize the uptake and distribution of Indium-111 ( $\left.{ }^{111} \mathrm{In}\right)$-labeled microspheres $\left({ }^{111} \mathrm{In}-\mathrm{M}\right)$ in irradiated and non-irradiated GL261 bearing mice. The following experiments were performed: (1) ${ }^{111} \mathrm{In}-\mathrm{M}$ tail-vein administration followed by imaging, (2) ${ }^{111} \mathrm{In}-\mathrm{M}$ administration, 10 minute time delay, tumor irradiation to 10Gy total dose, imaging; (3) 10Gy dose followed by ${ }^{111} \mathrm{In}-\mathrm{M}$ administration and imaging, (4) variable dose $(0-$ $10 \mathrm{~Gy}$ ) followed by ${ }^{111} \mathrm{In}-\mathrm{M}$ administration $30 \mathrm{~min}$ post-irradiation, then imaging at $30 \mathrm{~min}, 24 \mathrm{hrs}$ and $48 \mathrm{hrs}$ post-administration. Tumors from Expt. \#4 were excised and sectioned for autoradiography studies. Control animals received no radiation dose. Our initial gamma-camera results indicate proportionally greater uptake of ${ }^{111} \mathrm{In}-\mathrm{M}$ administered just prior to single fraction irradiation (Expt. \#2) when compared to ${ }^{111}$ In-M uptake administered just after irradiation (Expt. \#3). Autoradiography studies (Expt. \#4) show some trend in uptake with radiation dose when compared to untreated control animals. Biodistribution imaging studies of combined therapy in mice may provide information of medical significance for clinical protocol development.

\section{PO-T- 175}

Field and Patient Setup Analysis Using Electronic Portal Imaging on a Cobalt Unit

P Vos*, R de Vries ${ }^{1}$, A Vosspoel ${ }^{2}$, Dr. B. Verbeeten Institute, Tilburg, The Netherlands (1) Dr. Bernard Verbeeten Institute, Tilburg, The Netherlands, (2) Delft University of Technology, Delft, The Netherlands

A Theratron T-1000 cobalt treatment unit was equipped with a Theraview epid. Field edges in the portal images are extremely blurred because of the size of the cobalt source. Automatic verification of the treatment field outline cannot be performed with the edge detection techniques employed for images acquired with a linac. Deblurring of the patient anatomy is possible by iterative deconvolution with a spatially invariant PSF when the detector is positioned relatively close to the patient during image acquisition.

The blurring effect is both very pronounced and spatially variant in the projection of collimator jaws and blocks. Deblurring of images with a spatially invariant iterative constrained deconvolution algorithm introduces disturbing artifacts when the local PSF does not correspond to the actual PSF. Spatially variant deconvolution is practically impossible because of a 300-400 times longer computation time. Therefore we introduced a new technique for evaluation of field edges in cobalt portal images. A reference portal edge geometry is computed from the prescribed beam geometry, by using ray tracing to modulate the computed outline. The differences in intensity between this reference image and the one obtained from the cobalt epid, are analyzed. Action levels to detect field shape errors were determined experimentally, incorporating the local attenuation by the patient.

\section{PO-T- 176}

\section{A 3-D Image Registration Toolkit for Radiation Therapy Treatment} Planning

Y Hu*, A Erdi, C Chui, Memorial Sloan-Kettering Cancer Center, New York, NY

Image registration offers a substantial improvement for the target volume definition in radiation therapy. For improved target volume definition, a new 3D image registration toolkit was developed and incorporated into our treatment planning system. This toolkit offers both automatic and manual image registrations. Although automatic image registration speeds up the process, in certain clinical cases, it is inadequate due to the nature of the information contained in different modalities of images. Applications of manual image registration will be presented here. The user applies rigid body transformations, namely translation and rotation, to correlate image data sets in 3-D. Once a match between the two data sets is accomplished, the transformation matrix then is applied to display both data sets side-byside in the same coordinate system. Target volume and critical organs can be contoured in either data set. While contouring in one modality, the corresponding point is displayed on the second one. In fact, the user can interchange modality during contouring a structure. This allows the user to define the target volume based on both modalities. Currently we employ image registration to define lung lesions from PET and CT. PETtransmission images are registered with CT images, since the lung outline is well defined in both. The CT-PET- transmission transformation matrix is then applied to display PET- emission images with the corresponding CT, which allows us to determine the extent of the tumor or to visualize other unknown sites on $\mathrm{CT}$ images.

\section{PO-T- 177}

A Collimation Design for Double-exposure Portal Imaging

P Greer*, T van Doorn, Royal Adelaide Hospital and University of Adelaide, Adelaide, Australia

A collimator design is proposed that would allow the treatment field defined by the collimator and the anatomy outside the field to be imaged simultaneously. This design allows double-exposure portal images to be acquired rapidly at any time during the treatment. The collimator design splits a multi-leaf collimator into two vertically displaced levels with each level or grid consisting of every second leaf. One grid level shields the gaps or slits in the other level. By a small lateral movement of one level, radiation is transmitted through the slits forming an image of the anatomy modulated at the frequency of the grid. To determine the impact of the design of the collimator on the transmitted intensity profiles, distributions transmitted 
through slits were measured with varying slit height, beam energy, sourceslit distance, slit-film distance and slit width. The slits were formed with the accelerator secondary collimators and lead alloy blocks. Film images of the transmitted profiles were recorded and digitized. Modulated intensity profiles that would be transmitted through a grid were constructed from the individual slit profile measurements. The profiles were similar with slit height, source-slit distance and slit-film distance. Decreasing slit width resulted in reduced amplitude of the modulation. For smaller widths, the modulation was removed by filtering the image, therefore smaller slit (leaf) widths would be preferable for the design of the collimator. A prototype collimator is currently under construction.

\section{PO-T- 178}

A Robust Algorithm for Spikes Elimination from 2D CCD Low-level Lightoutput Measurements in the Presence of Ionizing Radiation Field S Devic*, A Kirov, J Williamson, Z Piao, J Dempsey, Washington University School of Medicine, St. Louis, MO

2D and 3D radiation dosimetry methods, based on detection of scintillation light images require both highly efficient and low noise image detectors. By cooling charge-coupled device (CCD) detectors, for low light level measurements integration times can be prolonged in order to improve precision. However, integration times are limited by the "spike" noise, which is due to cosmic rays as well as by scattered $\mathrm{x}$ - and $\gamma$ - rays. We present a simple and efficient method for eliminating spike noise without losing the original signal information using multiple images and a time averaging approach. This permits light to be integrated for a long periods of time near ionizing sources. Spikes are identified at particular points by comparying of the standard deviations computed over repeatedly acquired images with the square root of the time averaged signal intensity. Values idntified as spikes were replaced with the mean value of the remaining values for that point. The method was tested for plastic and liquid scintillator radiation dose measurements, which are characterized by a large number of stray $\gamma$-rays that may strongly contaminate the effective image.

This work was supported by Whitaker Foundation Grant \# 97-0336 and in part by NIH Grant R01 CA 57222.

\section{PO-T- 179}

Analysis of Radiological Physics Center Remote Tools Program Data J BenComo*, J Lowenstein, D Davis, C Davis, W Hanson, U.T. M.D. Anderson Cancer Center, Houston, TX

The RPC is developing various remote monitoring tools to identify, evaluate, and resolve systematic discrepancies in institution's dosimetry data and dose calculation algorithms. The objective of the program is to provide a baseline quality audit, short of an on-site visit, to all institutions participating in NCI-funded cooperative clinical trial groups. For the conventional external beam radiotherapy the program developed complements the TLD remote monitoring program for machine output that the RPC has been operating since 1972. In conjunction with the mailed TLD, the program monitors machine output, dosimetry data in use, and treatment planning algorithms. The TG-21 factors used in output calibration calculations are reviewed; dosimetry data are compared to RPC "Standard Data" for output, percentage depth dose, wedge, and off-axis factors; and treatment time calculation for two benchmark cases are verified against RPC data and calculation techniques. This program identifies discrepancies comparable to those discovered during an on-site evaluation, with the major discrepancies focused on wedge transmission and photon depth dose.

This work was supported by PHS grant CA 10953 awarded by the NCI, DHHS

\section{PO-T- 180}

A Comparison of the Accuracy of Automated Versus Manual Patient Setup Adjustment in Radiotherapy

K Brock*, J Balter, D McShan, University of Michigan, Ann Arbor, MI

A study was performed to compare the accuracy of automated versus manual treatment couch adjustment in radiotherapy. Following initial patient setup, a portal image was obtained and aligned to a reference image (DRR or simulation film). The alignment transformation was used to correct patient position. Under the automated repositioning protocol, the transformation was sent electronically into the room through an in-house computer controlled radiotherapy system and the table was automatically adjusted. Under the manual protocol, the transformation was relayed to the therapists in predetermined increments of 3, 5, 8, $10 \mathrm{~mm}$, etc. A second portal image was obtained after the adjustment. The image of the adjusted patient was aligned to the reference image. The residual error vector for the automated procedure was defined by comparison of the image in the adjusted position to the reference image. The residual error for the manual procedure was calculated by the difference between the vector error of the patient in the adjusted position and the difference between the corrective action given to the therapists and the transformation given from the original alignment. Automated adjustment was more reproducible $(\sigma=0.73 \mathrm{~mm})$ than manual setup $(\sigma=1.59 \mathrm{~mm})$. The maximum residual setup error using the automated adjustment was $2.91 \mathrm{~mm}$ smaller than that from manual adjustment. The largest adjustment made was $31.6 \mathrm{~mm}$.

This work was supported by a grant from the Whitaker Foundation.

\section{PO-T- 181}

Film Dosimetry Application in Photodynamic Therapy (PDT): A Practical Method to Test Beam Uniformity from Microlens

V Colussi*, C Sibata, T Kinsella, Case Western Reserve University, Cleveland, $\mathrm{OH}$

PDT is a relatively new modality of cancer treatment utilizing a photosensitizer and light. The biological efficacy of PDT depends on photosensitizer type and its uptake, oxygen, light delivery from a laser. Photodynamic therapy with Photofrin ${ }^{\circledR}$ has been approved by the USFDA for treatment of advanced esophageal and early lung cancer.

Film dosimetry has been utilized in the quality assurance of radiation therapy process for a long time. The hypothesis of this work was to show that we can use the same concept used in radiation therapy quality assurance for analyses of beam profile from the laser unit. This study was aimed at investigating the feasibility and accuracy of an in vivo quality assurance program used in our PDT Center.

Light from different laser systems was delivery on the film (CEA- TVS) for several exposition times. The films were processed and analyzed using the RIT113 Radiation therapy Film dosimetry system. The cross section from our experimental laser shown that the irradiance from the beam emerging from the fiber was not uniform, but tends to be peaked around its central axis. "Hot spots" were not observed to bare eyes. These result correlates well with $\mathrm{C} 3 \mathrm{H} / \mathrm{HeN}$ animal checked, implanted with radiation-induced fibrosarcoma (RIF-1) cells. The cross section from our PDT clinical laser (PDL1-Coherent) shows much better uniformity.

This study showed had errors in setup and aligning of the dye laser, and/or defect fiber from the companies can result on bad PDT response. More studies to improve our PDT QA program are warranted.

\section{PO-T- 182}

Dynamic Motion Phantoms for the Evaluation of Video Based Respiratory Gating Systems

C Ramsey*1, I Cordrey, K Spencer, A Oliver ${ }^{2}$, (1) The University of Tennessee, Knoxville, (2) Thompson Cancer Survival Center, Knoxville, TN

Several respiratory gating systems are under development that will allow the selective delivery of absorbed dose to moving target volumes during time intervals when the target is within the intended location. Dynamic feedback in these systems is established by correlating the signal from a respirationmonitoring device with the internal location of the target. In order to perform acceptance testing, commissioning, and ongoing quality assurance for clinical respiratory gating treatment programs, dynamic test phantoms must be developed that simulate patient respiration.

A respiratory gating test phantom (RGTP) was designed and built for use with video based patient monitoring systems. The RGTP consists of a cylindrical water tank with a submerged air bladder. The system can be remotely inflated and deflated using an electrically controlled air valve. When the valve is opened, air is forced into the bladder by an air compressor. When the valve is closed, the pressure of the water causes the 
air in the bladder to vent into the atmosphere. Markers placed on a floating water level indicator are used by the CCD video camera system to track the location of the air bladder. The RGTP is constructed of plastic and can be used in x-ray simulators, CT scanners, and linear accelerators. Because dosimeters and radio-opaque markers can easily be on the surface of the bladder, the RGTP can be used in a variety of acceptance testing, commissioning, and ongoing quality assurance procedures for respiratory gating.

\section{PO-T- 183}

Quality Assurance of Dynamic Aarc Multileaf Collimator

B Yi*, L Xing, A Boyer, Stanford University,Stanford,CA

Dynamic arc intensity modulated radiation therapy (IMRT) is a variant of IMRT that delivers a sequence of continuously changing multileafcollimator shaped fields during one or more arcs of the treatment gantry. Accurate and precise quality assurance of dynamic arc IMRT is important because of its inherently complex nature. If gantry angles and leaf positions are mismatched during the simultaneous changes in beam directions and leaf positions, the chances are significantly increased that high-dose volumes might miss the target and be placed in critical organs. Consequently quality control for dynamic arc IMRT should be more stringent than quality control for fixed gantry angle IMRT. The literature contains few reports on the development and investigation of quality control for dynamic arc IMRT. A dynamic multileaf collimator (DMLC, Varian Medical Systems, Palo Alto, CA) was used that has the capability both to generate IMRT fields for static gantry angle delivery and to deliver dynamic arcs. We have designed and tested a series of routine QA methods that are simple and practical. Two dynamic arc IMRT patterns were designed to verify the mechanical accuracy of treatment delivery, and to check the coincidence of gantry angle with delivered monitor units. A water equivalent plastic phantom, film, and a film dosimetry system were used to record the cumulative dose in a plane. The technique could detect leaf position errors of less than $2 \mathrm{~mm}$, gantry angle errors of less than $2^{\circ}$, and monitor unit errors of less than $5 \%$.

\section{PO-T- 184}

A Level 3 Dosimetric Intercomparison for Australasia: A Pilot Study T Kron*, B Stephen, M Roff, C Hamilton, J Denham, Newcastle Mater Hospital, Waratah, Australia

In addition to the absolute calibration at a reference point a variety of factors influence the dose delivered to patients undergoing radiotherapy. A level 3 dosimetric intercomparison using an anthropomorphic phantom and realistic treatment scenarios can test factors like patient data acquisition, treatment planning, set-up and delivery. The present study aims to demonstrate the feasibility of such a dosimetric service within Australasia (Australia and New Zealand) and establish baseline data and action levels. Twelve radiotherapy centres in Australasia have been invited to treat an anthropomorphic phantom using two different treatment scenarios based on current clinical trials of the Transtasman Radiation Oncology Group (TROG): a two field treatment of a carcinoma of the tonsil, and a four field prostate treatment. The dose distribution is assessed in two consecutive treatments using thermoluminescence dosimeters (TLDs) placed throughout the targe volume and in 'critical' structures such as the lens of the eye or the rectum. Preliminary results from eight centres confirmed the dose delivered and demonstrated the feasibility of such a service at a cost of approximately \$US 500.- per centre. For the prostate treatment, the variability of dose amongst the centres increases from a calibration under reference conditions $(1.3 \%, 1 \mathrm{SD})$ to $2.7 \%$ and $4.6 \%$ for ICRU reference point and mean target dose, respectively, which indicates the need for level 3 dosimetric studies.

\section{PO-T- 185}

Quality Assurance Tools for Frequent, Speedy Assessment of a large field Multileaf Collimators.

M Welch*, Royal Free Hospital Pond Street, Hampstead, London, England

Quality Assurance (QA) of Multileaf Collimators (MLC) compared to a standard monoblock design is increased by a factor equivalent to the number of MLC leaves. This increases time required for QA to an unacceptable value. This results in one of three things happening,

1. Available treatment time is reduced.

2. QA performed out of normal treatment hours.

\section{QA of the MLC compromised due to pressure of work.}

A practice used at many centres using an MLC for a radical conformal field is to check each required shape against the printed template for the patient's field. This is time consuming and short cuts may occur. Methods and tools are required to speed up the QA process whilst still maintaining high standards. Most checks carried out are of a repetitive nature. Therefore, standardisation must be implemented. Test fields designed to check the MLC in specific setups and positions along with manufactured test tools to enable quick reading or assessment are required. These will go through different levels of QA depending upon who carries them out or the purpose of their implementation. The lower levels would be routine checks carried out daily usually by the machine operators. Other checks on specific functions may be carried out by physics staff. By standardising the methods it should be possible for any person trained in their use to carry out these checks and produce results that do not differ from other staff.

\section{PO-T- 186}

A Monte Carlo Study of dynamic IMRT dosimetry with electronic portal imaging detectors

B Libby*, P Keall, J Siebers, R Zwicker, R Mohan, Medical College of Virginia Hospitals of Virginia Commonwealth University, Richmond, VA

IMRT dose distributions produced by dynamic multileaf collimator trajectories are presently verified using film, diodes, and two dimensional detector arrays prior to treatment. These methods of dosimetry are highly laborious and the accuracy of the measurements is questionable.

Electronic portal image detectors as transmission dosimeters, in which the intensity of the image is correlated with dose to the patient. Using Monte Carlo methods, the collision kerma of the transmitted radiation is calculated at the plane of imager, simulating the experimental image. Calibration between calculated collision kerma and experimental pixel number has been performed, and calculated images have been compared with those produced experimentally under a variety of conditions. Monte Carlo-generated dose images of IMRT fields, without the patient present, may be compared with measured dose images for dosimetric verification of IMRT prior to treatment. Additionally, the dose in patients can be calculated with Monte Carlo methods, with the transmitted radiation saved and subsequently used to generate the simulated image. By comparing the computed transmitted image with the measured portal image, the dose to patient for the entire field can be confirmed. With the availability of high-speed Monte Carlo codes and the advent of amorphous silicon technology with its rapid image acquisition time, it will be possible to readily and accurately perform dosimetric verification and full field in-vivo dosimetry of dynamic fields.

\section{PO-T- 187}

\section{Verification of Intensity Modulated Beams in Radiotherapy}

M Fix*, P Manser, H Keller', E Born, D Vetterli, R Mini², P Ruegsegger ${ }^{1}$, (1)Institute for Biomedical Engineering and Medical Informatics, ETH Zurich, Switzerland, (2)Division of Med. Radiation Physics, Clinic of Radio-Oncology, Inselspital-University of Berne, Switzerland

In this work a procedure is suggested for a qualitative verification of intensity modulated beams, realized by the sliding window technique. The feasibility of the Varian PortalVision imaging system for this verification procedure was investigated.

The verification procedure is divided in several steps: 1 . Verification of the input fluence distribution before the treatment (without patient). During the application of the dynamic MLC field, portal images are acquired continuously. The sum of these images corresponds to the time integrated fluence distribution and can be compared to the fluence matrix which was used to calculate the leaf motion. 2. Verification of the patient setup. An open field image, with the patient positioned, is acquired to get an anatomical image and for comparison with the fluence image of step 1. 3 . Verification of the patient dose. Acquisition of a fluence image during the patient irradiation, analogous to step 1, and a comparison with a calculated portal dose distribution is carried out.

The results showed that the sum of continuously acquired portal images can be used to verify the location of the extremes of a given fluence distribution. Conversion of the individual portal images to dose allows a comparison with a calculated portal dose image. 


\section{PO-T- 188}

Quality Assurance of Head-Scatter Factor for Square and Rectangular Collimator Settings

T Zhu*, B Bjarngard, Y Xiao, University of Pennsylvania, Philadelphia, PA

The head-scatter factor (or collimator-scatter factor), $H$, for field sizes larger than $3 \times 3 \mathrm{~cm}^{2}$ is caused by the scatter of photons in various structures in accelerator head (primarily the flattening filter) and by the backscatter of radiation into the monitor. We have developed an algorithm to parameterize these effects from a set of measurement for square collimator settings. The head-scatter factor for square field size is expressed as: $H=\left(1+a_{1} \cdot c\right) \cdot\left(1+a_{2} \cdot \operatorname{erf}(c / \lambda)^{2}\right) \cdot H_{0}$, where $a_{1}$ accounts for backscatter into the monitor, $a_{2}$ is the scatter-to-primary ratio for head-scattered photons, and $\lambda$ represents the width of the head-scatter photon "source" projected to the isocenter plane. The value of $a_{2}$ and $H_{0}$ can be used to QA the measured head-scatter factor, usually $H_{0}>0.90$ and $a_{2}<$ 0.1. To calculate the head-scatter factor for rectangular fields, $c_{x} \times c_{y}$, two equivalent-square relations are used: one for backscatter, one for headscatter. The two equivalent-square uses the same form:

$$
c=\frac{(1+k) \cdot c y \cdot c x}{k \cdot c x+c y} \text { but different geometrical factor } k . k_{h s} \text { and }
$$

$k_{b s}$ are determined from the head geometry and can be calculated without additional measurement. For Varian accelerators, the results agree with measurements for rectangular fields, with a maximum deviation of less than $0.8 \%$

\section{PO-T- 189}

Commissioning of ADAC Pinnacle 3 Treatment Planning System: Inhomogeneity Correction

J Wang*, S-T Chiu-Tsao, L Wang, L Harrison, Beth Israel Medical Center and St. Lukes Roosevelt Hospital Center, New York, NY

As part of our commissioning of ADAC Pinnacle 3 treatment planning system, we validated the dose convolution calculation algorithm for heterogeneous phantom using film dosimetry. KODAK XV-2 films (one at a time) were irradiated in polystyrene phantom with and without heterogeneity, such as RMI bone slab, or cylindrical air cavity, by $6 \mathrm{MV}$ photon and $9 \mathrm{MeV}$ electron beams of Clinac2100C/D (at SSD=100 cm and $10 \times 10 \mathrm{~cm}$ collimator setting). For photon beam, the film was situated at $\mathrm{d}=10 \mathrm{~cm}$ in the polystyrene phantom sandwiching a bone slab of either $1 \mathrm{~cm}$ or $5 \mathrm{~cm}$ thick, or with an air cavity of either $1 \mathrm{~cm}$ or $2 \mathrm{~cm}$ thick, $5 \mathrm{~cm}$ diameter. For electron beam, the film was exposed both at $\mathrm{d}=3 \mathrm{~cm}$ with 1 $\mathrm{cm}$ air cavity, $0.5 \mathrm{~cm}$ or $1 \mathrm{~cm}$ bone slab and at $\mathrm{d}=4 \mathrm{~cm}$ with $2 \mathrm{~cm}$ air cavity. RIT113/Lumisys 150 densitometer system was used for film scanning and data analysis. Compared with Pinnacle calculated doses in heterogeneous phantoms, the film dosimetry revealed an agreement of less than $\pm 3 \%$ for 6 MV photon, within $\pm 8 \%$ of difference for $9 \mathrm{MeV}$ electron. The Pinnacle generated isodose curves reflected proper inhomogeneity correction. We conclude that the inhomogeneity corrections for both $6 \mathrm{MV}$ photon and 9 $\mathrm{MeV}$ electron are handled properly in the Pinnacle 3 algorithm.

\section{PO-T- 190}

Acrylic Test Phantom for Measuring PDD and Beam Profiles

T Steinberg*, S Szeglin, F Hu, Siemens Medical Systems / Oncology Care Systems, C, PTW, Hicksville, New York

An acrylic test phantom designed to be used for routine measurements for high energy photon and electron beams has been designed by PTW Freiburg. This phantom allows for central axis percent depth dose measurements as well as off axis profile measurements.

The phantom block contains two rotating cylinders that allow a radiation detector to be positioned along the central axis at any depth from $10 \mathrm{~mm}$ to $122 \mathrm{~mm}$. In addition, off-axis measurements can be made at a depth of $100 \mathrm{~mm}$ at a distance of $\pm 45 \mathrm{~mm}$ from the central axis. The phantom is remotely controlled by means of two stepping motors and powered by a control unit. Interface software has been developed by PTW that supports the beam scans to be displayed and numerically analyzed.

This work was partially supported by PTW New York and PTW Freiburg.

\section{PO-T- 191}

A Two Dimensional Decision Making Model After Comparing Portal and Reference Images

T Kron*, C Hamilton, C Wratten, J Denham Newcastle Mater Hospital, Waratah, Australia

Portal images acquired using radiographic film or electronic portal imaging devices (EPIDs) are used routinely to verify the correct position of the treatment field in radiotherapy. While lots of efforts are directed at improving the quality of the images acquired using megavoltage radiation there are only few papers dealing with the decision making process after a deviation between portal image and reference image has been detected. We propose a model based on confidence ellipses defined by the two dimensional Hotelling's $\mathrm{T}^{2}$ statistics. The model does not rely on assumptions or historical data but requires five portal images to be taken of an individual patient. Then it allows for a correlation between shifts in both image dimensions as may be the case in patient movement. In a mathematical analysis it can be shown that the proposed decision making model is particularly advantageous in cases where it is difficult to predict the random variation of field position from day to day and if there is a strong correlation between the two directions of movement identified by the image. As EPIDs become widely available the proposed model offers an alternative decision making algorithm which is easily implemented and may help to account for patient variability and movement not confined to the axis of the conventional coordinate system.

\section{PO-T- 192}

Algorithms for the Verification of 3D Conformal Prostate Treatment using EPID

L Girouard*, E Vigneault, J Pouliot, CHUQ, Québec, Québec., Canada

Organ motion and setup errors are an important underestimated issue in 3D conformal irradiation of the prostate gland. The purpose of this study is to implement in clinic an automatic algorithm for the evaluation of setup error and organ motion. The algorithm was initially design for a box technique conventional RT. It has been modified for $3 \mathrm{D}$ conformal RT fields. The algorithm is used in conjunction with radioopaque markers to study organ motion relative to bone structures. Before simulation and CT scans are performed, patients are transperineally implanted with 3 radiopaque markers under ultrasound guidance and local anesthesia. Patients are treated with a six field conformal technique: 2 lateral and 4 oblique. Electronic portal images are acquired for each field and used on-line to verify the proper locations of the markers relative to the reference set of images. EPI are then processed off-line: First, setup deviations are measured using the cross correlation of extracted structures relative to the field center of geometry. Bone edges are detected using the Laplacian of a Gaussian operator without the use of fiducial points or user intervention. Marker positions are then extracted to assess organ motion. Setup deviations measured by the correlation algorithm are presented as actions to be performed, both in magnitude and direction. Action levels based simultaneously on bone structure deviations and marker positions are under study.

\section{PO-T- 193}

Commissioning Procedures for Monte Carlo Dose Calculation Algorithms

P Keall*, J Siebers, B Libby, R Mohan, Medical College of Virginia, Richmond, VA

The era of routine clinical Monte Carlo treatment planning is nearly upon us. As with all dose calculation algorithms, there is a need for quality assurance and commissioning tests. We have implemented a Monte Carlo-based dose calculation algorithm, $M C V$, which is interconnected to our treatment planning system. Before using the code for clinical use, a series of tests for commissioning was performed to validate system performance. The commissioning aim was to create the minimum set of tests which covered every aspect of commissioning of the dose calculation algorithm. Our criteria for acceptable accuracy in patient dose computation is $\pm 2 \%$ or \pm 2 $\mathrm{mm}$ (ICRU 1987). Each test has a clear purpose, scope, specification, method and results. The required tests are: Density and Material Test, Field Geometry Test, Phantom Geometry Test, Open and Wedge Field Output Test, Open and Wedge Field Dose Distribution Test, Non-unit Density Test and a Block Field Test. This test set has been applied to dose calculations 
for a Varian Clinac 2100C 6 MV photon beam. Future work will involve applying this method to all of our photon beams, and our electron beams. We will use these tests as a generic test set for other dose calculation algorithms, such as our pencil beam code, and Peregrine.

\section{PO-T- 194}

Five Years Follow-up of a Quality Assurance Program in Virtual Simulation

J Pérez-Calatayud*, F Lliso, V Carmona, A González, J Ruíz, I Petschen ${ }^{1}$, F Ballester $^{2}$, (1) La Fe University Hospital, Valencia, Spain, (2) Valencia University, Valencia, Spain

In our department virtual simulation (VS) is used in radiation therapy planning (RTP) for all the patients. The VS chain is composed of a CT scanner (dedicated specifically for RTP), 2 workstations and a 3DRTPS linked altogether. In this work the QA program of VS is analyzed after 5 years evolution.

A baseline was established for all the parameters that were going to be monitored in routine. Initial frequencies and tolerances were stated based on the few existing publications. The program concerned all the elements of the chain. Accuracy of the CT numbers (different scanning conditions, constancy, etc.), geometric accuracy at each step and laser alignments were the main topics. The technicians perform daily checks at the CT with a simple house-designed phantom. Quarterly, the physicist performs verifications at the CT, AS and RTPS. The manufacturer performs diagnostic verifications. In addition to these periodic tests protocolized controls are performed for each individual patient at the different steps of the procedure.

Initial tolerances of $2 \mathrm{~mm}$ for geometric parameters and $10 \mathrm{HN}$ for the CT numbers have never been exceeded for 5 years. By means of simple checks and specific designed tools the QA program has shown it usefulness in ensuring the stability of the whole process.

\section{PO-T- 195}

RPC Experience with TLD for Output and Energy Monitoring of Radiation-therapy Beams

R Tailor*, J Aguirre, W Hanson, U.T. M.D. Anderson Cancer Center, Houston, TX

The Radiological Physics Center (RPC) has been utilizing TLD to verify photon-beam output, and electron-beam output and energy for many years. The RPC currently monitors over 1200 institutions, monitoring 4000 photon and 3500 electron beams per year. Control TLD, irradiated on one specific Co-60 machine, are used for a performance check of our TLD system. Analysis of these data over 5 years, reveals high precision $(\mathrm{SD}=0.9 \%)$ in beam output verification. Analysis of all TLD results, since 1990, for remotely monitored photon beams $(27,900)$ and electron beams $(23,000)$, shows a spread (SD) of $1.9 \%$ and $2.2 \%$ respectively in beam output. The increased spread arises from the variability in beam energies, makes/models of machines, and institutional performance. In view of these variabilities, the results are extremely encouraging. Institutional performance includes uncertainties in beam-output calibration, set-up errors, and beam drifts. The Spread (SD) of individual beams varies widely from beam to beam and Institution to institution. The spread of individual beams has been used to identify "good" beams $(\mathrm{SD}<2 \%)$ which are to receive TLD less frequently than others.

This work is supported in part by PHS grant CA10953 awarded by the NCI, DHHS.

\section{PO-T- 196}

An Anthropomorphic Head Phantom for Remote Monitoring of Stereotactic Radiosurgery at Multiple Institutions

P Balter*, M Stovall, W Hanson, The University of Texas M.D. Anderson Cancer Center, Houston, TX

The Radiological Physics Center (RPC) has developed an anthropomorphic head phantom for remote monitoring of stereotactic radiosurgery (SRS) treatments. The phantom contains a $1.9 \mathrm{~cm}$ imageable target as well as TLD and radiochromic film dosimeters. The dosimeters measure dose at the center of the target and dose profiles along three orthogonal axes. This phantom has been provided to the community through the RPC and the Radiation Dosimetry Services (RDS) since 1995. Data from over 75 institutions was obtained. Agreement in dose at the center of the target, as determined by TLD, is similar to that for conventional photon beams. Eighty percent of the institutions centered prescription isodose lines on the target to better than $1.5 \mathrm{~mm}$. However, institutions typically use generous margins around the target; irradiating a volume that is $25 \%-125 \%$ larger than the target. Field localization by CT typically has higher precision in the coronal plane than in the sagittal plane, this was not observed for MRI based treatments. Our data suggest no significant difference in the central dose or localization precision between linac or Gamma-Knife; or in the immobilization system used. The anthropomorphic head phantom described has been found to be a useful remote monitoring tool.

This work is supported in part by PHS grant CA10953 awarded by the NCI, DHHS.

\section{PO-T- 197}

Commissioning of the Brachytherapy Module on Theraplan Plus M MacPherson*, J Cygler, D Wilkins, Ottawa Regional Cancer Centre, Ottawa, Canada

Clinical implementation of any treatment planning software requires a thorough evaluation of the dose calculation algorithm. In this study, we present results of tests of the brachytherapy module for Theraplan Plus. Iridium-192 wires of lengths ranging from $0.5 \mathrm{~cm}$ to $5 \mathrm{~cm}$ were modeled, and the predicted dose distributions were compared to standard dose distributions from the literature. ${ }^{1}$ In the absence of published dose data, the predicted dose distributions were compared with the results of Monte Carlo simulations of the wires using the MCNP (Monte Carlo N-Particle) code. In general, the agreement between the Theraplan data and the published and Monte Carlo results was good, (within 3\% for distances from 0.5 to $10 \mathrm{~cm}$ ), with the largest errors occurring near the source (up to $5 \%$ at $0.2 \mathrm{~cm}$ ) and at large distances $(10 \%$ at $12 \mathrm{~cm})$. This level of agreement required appropriate selection of normalization factors to account for deviations from the inverse square law. Line sources were also adequately modeled by arrays of point sources of equal activity, provided that the spacing between sources was sufficiently small. The calculated dose distributions for a cesium-137 uterine tandem source were found to agree well with the published data of Williamson. ${ }^{2}$

${ }^{1}$ Dutreix A. and Marinello G., p. 17-24 in Modern Brachytherapy, Masson Publishing, 1987.

${ }^{2}$ Williamson, J.F., Int. J. Rad. Onco. Biol. Phys., vol 41, p960, 1998.

\section{PO-T- 198}

Error Analysis of In-Vivo Diode Dosimetry in a Small Clinic

A Thompson*, T Zhu, Hosp. of the University of Pennsylvania, Philadelphia,PA

In-vivo dosimetry can be a useful quality assurance tool to verify overall patient treatment quality with respect to calculation, setup, and delivery. For the past year, we have conducted a study in a small satellite clinic, using the Sun Nuclear QED diode detectors on each patient under treatment to verify the treatment delivery. The action level we used was $5 \%$, plus an additional tolerance for wedged beams because of the large differences in off-axis transmission with small variations in diode placement. Our goal was to evaluate the clinical usefulness of diode dosimetry to verify patient treatments in our clinic, based on its reliability with routine clinical use. For photon beams, the most frequent discrepancy was seen with exit dose measurements, which reflect a more "realistic" dose that the patient receives due to inhomogeneities within the body that are not accounted for in the calculations. The entrance diode readings showed less discrepancy, and were beneficial in uncovering a few minor errors in treatment calculations, which we were able to correct. The electron entrance diode readings showed the most variability, due to the sensitivity to placement location and SSD, especially with small cutouts. Weekly diode dosimetry showed very little variation, indicating reproducible patient setups and treatment delivery. Although the overall error discovery rate is low, we feel that the initial diode dosimetry is necessary to help catch catastrophic errors, such as incorrect beam energy, incorrect wedge, and incorrect setup (SAD vs. SSD). 


\section{PO-T- 199}

Quality Assurance of Beam Accuracy for Leksell Gamma Unit

C Yu*, G Luxton, Z Petrovich, USC School of Medicine, Los Angeles, CA, Stanford University, Stanford, CA, USC School of Medicine, Los Angeles, CA

For the acceptance test and annual quality assurance of the Leksell Gamma Unit, measurement of the beam accuracy, defined as a distance between mechanical and radiological isocenters, poses a challenge to medical physicists. The specification for the beam accuracy is within $0.3-\mathrm{mm}$ for the 4-mm collimator helmet. In this report, we introduce a simple technique to analyze the beam accuracy by using a conventional film densitometer plus mathematical modeling. A small piece of film was placed inside the film cassette containing a sharp needle. The needle is located such that its tip is exactly positioned at the mechanical isocenter. Before exposure, the film is pierced by the needle. Density profile was accomplished by using a densitometer (Wellhofer, WP102) with a spatial resolution of $0.8-\mathrm{mm}$. The profile was then fitted to a model of the two Gaussian functions. One is for the radiation field profile, the other for a dip caused by the narrow hole. The difference between the centers of the two Gaussian functions defines the deviation of the beam accuracy from the mechanical center of the unit. The deviations for $\mathrm{x}, \mathrm{y}$ and $\mathrm{z}$ directions from one of our annual measurements are $0.05-\mathrm{mm}, 0.05-\mathrm{mm}$, and $0.23-\mathrm{mm}$, respectively. The combined deviation is $0.24-\mathrm{mm}$, which is well within the specification and in excellent agreement with the results from the manufacture's laser measurement. This technique provides a simple, accurate and practical tool for measurement of the beam accuracy in the acceptance test and annual quality assurance of the Leksell Gamma Unit.

\section{PO-T- 200}

Converting Absorbed Dose to Medium to Absorbed Dose to Water for Monte Carlo Based Dose Calculations

J Siebers*, P Keall, R Mohan, Medical College of Virginia, Richmond, VA

Current clinical experience in radiation therapy is based upon dose computations that report the absorbed dose to water. While Monte Carlo dose calculation algorithms have the potential for higher dose accuracy, by default they compute the absorbed dose to the patient medium such as tissue, lung, or bone. Therefore, for dose calculation algorithm comparisons, or to report dose to water or tissue contained within a bone matrix, a method to convert dose to the medium to dose to water is required. This conversion was developed by applying Bragg-Gray cavity theory. The dose ratio was determined by two methods: (a) computing the stopping power ratio averaged over the secondary electron spectrum in the voxel and (b) by scoring the dose to water in addition to the dose to material on an energydeposition event by energy-deposition event basis. For soft tissue, the difference between dose to material and dose to water is less than $0.5 \%$, for lung, about $1 \%$, while for cortical bone the dose difference exceeds $10 \%$. The variation in the dose ratio as a function of depth and position in the field indicates that a single correction factor can be used for each material throughout the field for a given photon beam energy.

\section{PO-T- 201}

Derivation of the Incident Electron Parameters in Photon Beam Monte Carlo Simulations

Daryoush Sheikh-Bagheri*, D.W.O. Rogers, Ottawa-Carleton Institute of Physics, National Research Council of Canada, Ottawa, ON, Canada

The BEAM/EGS4 code [Med. Phys., 22, 503-524, 1995] is used to model 9 megavoltage photon beams from 3 major manufacturers. The code is extensively optimized (with the cumulative factors of efficiency improvement given in brackets) by: minimizing unnecessary electron transport (3), utilizing uniform bremsstrahlung splitting (4), modifying the Russian Roulette of electrons (2), and developing the novel and robust technique of selective bremsstrahlung splitting (3-4). The energy and the FWHM of the incident electron beam (assumed circular and gaussian) are derived by matching calculated off-axis factors (within $2 \sigma$ at $1 \%$ statistics) and percentage depth-dose values (past $d_{\max }$, within $1 \%$ of $D_{\max }$ ) with measured data from the AAPM RTC TG-46 compilation. The derived energies $( \pm 0.3 \mathrm{MeV})$ are all within $5 \%$ of the manufacturers' specifications except in one case where there is a deviation of $18 \%$. The derived FWHM $( \pm 0.1 \mathrm{~mm})$ of the electron beam intensity distributions all fall within $0.5 \mathrm{~mm}$ of the specifications except in one case where the difference is $2 \mathrm{~mm}$. The dose from electron contamination varies between 5 and $12 \%$ of $D_{\max }$ on the surface and between 0.08 and $5 \%$ of $D_{\max }$ at $d_{\max }$. The calculated photon spectra and average energy distributions are compared to those published by Mohan et al [Med. Phys. 12, 592-597, 1985] and for some beams exhibit substantial differences.

\section{PO-T- 202}

A Modified Discrete Ordinates Algorithm for Photon Transport Applications

J Demarco*1, A Mesa ${ }^{2}$, R Cook ${ }^{1}$, (1)UCLA Department of Radiation Oncology, (2) Cedars-Sinai Comprehensive Cancer Center, Department of Radiation Oncology, Los Angeles, CA

The discrete ordinates method is a deterministic technique for solving the linear boltzmann transport equation. A modified algorithm has been developed for solving the equation from anisotropically emitting photon point sources. The algorithm builds upon conventional discrete ordinate calculations by discretizing the angular variable as a function of each pixel value in a two-dimensional, cartesian calculation matrix. Homogeneous and heterogeneous calculation geometries were used to test the method and compare with a conventional ray-tracing algorithm. Agreement between the modified algorithm and the ray-trace algorithm is excellent with the discrete ordinates calculation being slightly faster with respect to calculation time. The modified discrete ordinates algorithm was also used to calculate kerma scatter distributions from monoenergetic photons in homogeneous or CTbased heterogeneous geometries. Good agreement was achieved compared with a Monte Carlo calculation of the same simulation geometry. Implementation of the algorithm for polyenergetic photon spectra and threedimensional cartesian geometries is discussed.

\section{PO-T- 203}

Analytic Modeling of Treatment Head Scatter Factor

G Desobry*, T Zhu, University of Pennsylvania, Philadelphia, PA

The head scatter factor $\left(\mathrm{S}_{\mathrm{C}}\right)$ is the ratio of the output in air $(\mathrm{OF})$ for a given field to that for a reference field $($ e.g. $10 \times 10 \mathrm{~cm})$. Measured output in air is proportional to the sum of primary $(\mathrm{P})$ and scattered $(\mathrm{S})$ photon components. $\mathrm{P}$ and $\mathrm{S}$ are each integrals of energy fluence times absorption coefficient over all photon energies. $\mathrm{P}$ is due to photons produced by bremsstrahlung in the target, then incident on the isocenter after being attenuated by treatment head elements, so it does not change with field size. $\mathrm{S}$ is due to photons scattered in the treatment head before reaching isocenter, and increases with field size $(\mathrm{X}, \mathrm{Y})$. The output factor may be written as $\mathrm{OF}(\mathrm{X}, \mathrm{Y})=\mathrm{M}[1+$ $\mathrm{S}(\mathrm{X}, \mathrm{Y}) / \mathrm{P}]$, where $\mathrm{M}$ is the monitor backscatter factor, linear in field size. We have analytically computed the values of $\mathrm{P}$ and $\mathrm{S}$, assuming that $\mathrm{S}$ is accurately modeled by first Compton scatter. The computation of the energy and angular distribution of bremsstrahlung is carried out with the Schiff formula in a thick self-absorbing target that may contain layers of different elements. The intensity spectrum at isocenter was computed for primary photons and for photons scattered from elements in the treatment head, as a function of field size. The results are compared to measured head scatter factors for square and rectangular fields.

\section{PO-T- 204}

Dose Calculations of a 6MV Photon Beam Using a Finite-Size Pencil Beam Model

WH Hinson*, JD Bourland, Wake Forest University School of Medicine, Winston-Salem, NC

This work investigates the Finite-Size Pencil Beam (FSPB) model for calculating dose deposition from photon beams generated by a clinical linear accelerator. The FSPB model(1), uses the superposition of preconvolved "finite-size pencil beams" of small cross-sectional area to determine the dose deposition in a uniform phantom. In the dose computation, FSPBs for a range of energy bins are pieced together like mosaic tiles to collectively form the cross-section of the full beam. Depending on the full beam resolution, the superposition calculation can be much faster than full convolution. Results are presented for a $6 \mathrm{MV}$ photon beam. Comparisons of calculated and measured TMRs and output factors of open fields show excellent agreement between calculated and experimental values. Results include discussions of FSPB generation, the effect of beam softening across 
the field cross-section, and the method for modelling this effect using different FSPB weighting factors as a function of energy and location.

1. Bourland and Chaney, Med Phys, 19(6), 1992, pp.1401-1412.

\section{PO-T- 205}

Photon Beam Spectra from Measured Depth Dose in the Buildup Region

M Altschuler*, P Bloch, B Bjarngard, A Kassaee, J McDonough, University of Pennsylvania Medical Center, Philadelphia, PA

A method to determine the spectrum of a clinical photon beam from measured depth dose (MDD) data is described. In regions of chargedparticle disequilibrium (shallow depths), the rapid increase in range of Compton-generated electrons with photon energy helps extract the spectrum. To minimize contaminating electrons, small $\left(6 \times 6 \mathrm{~cm}^{2}\right)$ fields are measured. The MDD is represented as a linear combination of basis functions, which are depth doses derived by Monte Carlo for monoenergetic photon beams. Determining the spectrum is equivalent to finding the weights of the basis functions. This problem is ill-conditioned, leading to a non-smooth spectrum. To extract a physical solution requires (1) double precision to avoid loss of significant figures, and (2) a method to avoid negative or zero spectral weights. The Cimmino algorithm, which is iterative and guaranteed to converge, is used to solve for the spectral weights that reproduce the MDD data (constraints). In each iteration, the algorithm corrects for the discrepancy between the calculated and MDD values. These constraints are expressed as inequalities for hyperplanes in the space spanned by the basis functions. Realistic spectra appear when small margins $(\approx 1 \%$ of the MDD values) are allowed around the hyperplanes. No a priori assumptions about the spectrum of a beam are used in this procedure. The depth dose derived with the spectral weights from the Cimmino algorithm was within $1 \%$ of the MDD for 6 and 15 MV photon beams of a clinical accelerator.

\section{PO-T- 206}

Proton Transport Based on a Generalization of Fermi-Eyges Theory and Energy Spectrum Evolution

A Chvetsov*, G Sandison, Tom Baker Cancer Centre, Calgary, Alberta, Canada

Fermi-Eyges multiple scattering theory yields a simple Gaussian solution to the charged particle transport equation under pencil beam boundary conditions. There is an increasing number of applications of Fermi-Eyges theory to the proton beam therapy. One of the main difficulties encountered in these applications is that the protons' range straggling is primarily due to the energy straggling. Therefore, multiple scattering theory alone can not be used to predict the depth dose distribution.

Overcoming this difficulty was the motivation for developing of a new proton transport algorithm based on a generalization of Fermi-Eyges theory, known as the Proton Loss Model, and an energy spectrum evolution model. In this algorithm, we use $\mathrm{N}$ characteristics in the depth-energy phase space which are calculated using the continuous slowing down approximation (CSDA). For every characteristic, we solve analytically the Proton Loss transport equation which takes into account inelastic nuclear collisions and the range straggling due to multiple scattering. Then, we calculate the depth dependent energy spectra using the energy spectrum evolution model. The evolution of the proton spectrum describes the diffusion of protons between characteristics due to energy straggling. A final solution at every depth can be found as a linear combination of characteristic solutions with the weights which are defined by the energy spectrum at this depth. The effectiveness of the method is demonstrated by comparisons with the PTRAN Monte Carlo code and measurements.

\section{PO-T- 207}

Spectral Measurements of a $6 \mathrm{MV}$ Photon Beam for Finite-size Pencil Beam Dose Calculations

WH Hinson*, JD Bourland, Wake Forest University School of Medicine, Winston-Salem, NC

A kernel-based dose computation method with finite-size pencil beams (FSPBs) requires knowledge of the photon spectrum. Published methods of indirect spectral measurements using transmission measurements through beam attenuators use mathematical fits with a large number of parameters and constraints. In this study, we examine a simple strategy for fitting transmission data that models important physical characteristics of photon beams produced in clinical linear accelerators. The shape of an unattenuated bremsstrahlung spectrum is known, varying linearly from a maximum at zero electron energy to a value of zero at the maximum energy of the accelerated electrons. This "ideal" spectrum is altered primarily by absorption of low energy photons by the flattening filter, causing the true spectrum to roll off to zero at low photon energies. The fitting equation models this behavior and has these advantages over previous methods: 1) the equation describes the shape of a bremsstrahlung spectrum based on physical expectations; 2 ) only three fit parameters are required with a single constraint. Results for a $6 \mathrm{MV}$ accelerator for central axis and off-axis beams show good agreement with the maximum, average and modal energies, as verified with known spectra. Previously published models, representations of beam fluence (energy fluence, $\mathrm{dN} / \mathrm{dE}$ ), experimental methods, and the fitting process are discussed. Use of the measured spectrum is shown for a FSPB dose computation model, including the effects of off-axis beamsoftening.

\section{PO-T- 208}

Evaluation of Output Factors for Customized Electron Cones B Ahluwalia*, A Khatib, University of Oklahoma Health Sciences Center, Oklahoma City, OK

The output factor for customized electron inserts depends upon the open area of the insert, the electron energy, the displacement of the center of the insert w.r.t. the center of the open cone and the virtual source to skin distance $\left(\mathrm{SSD}_{\text {vir }}\right)$. The output of over 100 inserts has been evaluated. 58 inserts were evaluated with center displacement ranging from $1 \mathrm{~cm}$ to $3 \mathrm{~cm}$. The best representation of the output for dose calculations is the output at the center of the insert. 44 customized inserts used for treatment of patients were evaluated for cone sizes of $6 \times 6,10 \times 10$ and $15 \times 15$. The output factors for all these inserts were measured for electron energies 6, 9 and $12 \mathrm{MeV}$. The percentage open areas of these customized inserts varied from $18 \%$ to $58 \%$ of the open cone area. We find that the output factor of any insert can be derived from the best function fit obtained from the data in the pool. The derived output factor is within 1 to $2 \%$ of the measured value. Similarly, the output for the regular symmetric inserts was evaluated for different virtual SSD $\left(\mathrm{SSD}_{\mathrm{vir}}\right)$, we find that the output can be obtained from a formula. Thus, the output can be obtained for any customized insert for a known electron energy, \% open area, at a fixed SSD from the pooled data and apply the correction factor for the $\mathrm{SSD}_{\mathrm{vir}}$ if application needs the output at a non standard SSD.

\section{PO-T- 209}

An Iterative EPID Calibration Procedure for Dosimetric Verification that Considers the EPID Scattering Factor

J Chang*, G Mageras, Memorial Sloan-Kettering Cancer Center, New York, NY

There has been an increasing interest in the application of EPIDs to dosimetric verification, particularly for intensity modulated radio therapy. Although not water-equivalent, the phantom scatter factor of an EPID, $S_{p e}$, is generally assumed to be that of a full phantom, $S_{p}$, or a slab phantom, $S_{p s}$, in EPID calibration. This assumption may introduce errors in absolute dosimetry using EPIDs. A calibration procedure that iteratively updates $S_{p e}$ and calibration curve (pixel value to dose rate) is presented. The EPID (Varian PortalVision) is irradiated using a $20 \times 20 \mathrm{~cm}$ field with different beam intensities. Reference dose rates are from chamber measurements in air, multiplied by $S_{p}$ or $S_{p s}$. The calibration curve is obtained by fitting pixel values and reference dose rates to a quadratic equation. The $S_{p e}$ is obtained from EPID measurement in 10x10 cm and 20x20 cm fields and using the calibration curve, and is in turn used to adjust dose rate measurements and hence the calibration curve. The above procedure is repeated until it converges. The final calibration curve is used to calculate portal doses for four rectangular fields. The results indicate that dose calculated using $S_{p e}$ is more consistent with chamber measurements (mean $=0.998, \mathrm{STD}=0.007$ ), than that obtained using $S_{p}$ (mean $\left.=0.995, \mathrm{STD}=0.007\right)$ or $S_{p s}$, (mean=0.993, $\mathrm{STD}=0.012$ ). Furthermore, differences between $S_{p e}$ and $S_{p s}$ are as large as $2 \%$ for some field sizes. It is concluded that the EPID calibration for absolute dosimetry should consider EPID scattering properties. 


\section{PO-T- 210}

Effects of the Shape of Photon Energy Spectrum on the Beam Data in Water

T Zhu*, B Bjarngard, J McDonough, P Bloch, University of Pennsylvania, Philadelphia, PA

The energy spectrum is important for photon dose-calculation algorithms using convolution or superposition techniques. However, the impact of its shape on the beam data in water may be small because of the insensitivity of the cross-sections for the various photon interactions in water on the photon energy. We compared energy spectra generated with three different techniques for the same photon beams. One is based on the best fit to the PDDs measured in water; one (MC) uses Monte-Carlo modeling of the accelerator head, and the third (analytical) uses modeling of the thick target spectrum. The analytical spectrum generally agrees with the MC spectrum. Beam data in water was generated using $\mathrm{MC}$ simulation for each spectrum giving: a) the electron disequlibrium factor in the buildup region, b) the narrow beam attenuation, c) the phantom scatter factor, and d) the percentage depth dose normalized to $10 \mathrm{~cm}$ depth. The calculated PDD agrees with measurement to within $3 \%$ for the three energy spectra examined. The phantom scatter factor agrees with measurement to within $1 \%$ at $10 \mathrm{~cm}$ and within $\pm 2 \%$ at depth of maximum dose. The buildup curve (excluding electron contamination) agrees quite well between measurement and calculation for all spectra. We conclude that the most sensitive way to select photon spectrum is by using the measured electron disequilibrium factor and PDD together. The effect of maximum photon energy is examined by changing the electron energy.

\section{PO-T- 211}

Monte Carlo Calculation of Dose Rate Distributions Around the Amersham CDCS-M Cs-137 Source

E Casal ${ }^{1}$, J Lluch, F Ballester*2, J Pérez-Calatayud, F Lliso ${ }^{3}$,(1) Centro Nacional Dosimetria, Valencia, spain, (2) University of Valencia, Valencia, Spain, (3) La Fe University Hospital, Valencia, Spain

CDCS-M Amersham type stainless-steel encapsulated source is widely used in low dose rate brachytherapy with manual afterloading. However there is a lack of complete dosimetry data about it. In this work we present simulations of absolute dose rate in water with the Monte Carlo GEANT code around this source.

All the physical processes for low energy photons are implemented in GEANT: photoelectric effect, Compton dispersion, pair production and Rayleigh scattering. For electrons, multiple scattering and continuous energy loss were assumed.

The source was fully modeled from information provided by Amersham. In order to reach full scatter conditions, a cylinder of water $40 \mathrm{~cm}$ in height and $40 \mathrm{~cm}$ in diameter was assumed where a grid system with a score volume of $0.5 \times 0.5 \times 0.5 \mathrm{~mm}^{3}$ up to $20 \mathrm{~cm}$ away from the source has been established. Up to $10^{9}$ histories were simulated. To estimate air-kerma strength the source was located in a $6 \times 6 \times 6 \mathrm{~m}^{3}$ dry air cube and kerma was scored with $2 \times 10^{10}$ histories.

Absorbed dose rates in water, normalized to $1 \mu \mathrm{Gym}^{2} \mathrm{~h}^{-1}$, are presented in the form of away-along table at distances up to $10 \mathrm{~cm}$. In order to use the simulated data in the treatment planning programs based on TG43 formalism we have extracted from our simulation results the necessary dosimetry parameters, these are: the dose rate constant $\Lambda$, the radial function $g(r)$ and the anisotropy function $F(r, \theta)$.

\section{PO-T- 212}

Monte Carlo Study of Virtual Versus Physical Wedges for Photon Beams of 6-10 MV

F Verhaegen*, I Das, Institute of Cancer Research, London, UK, Fox Chase Cancer Center, Philadelphia, PA

A virtual wedge, realised by a moving collimator jaw, offers an alternative over a set of fixed wedges for producing a wedged photon dose distribution. This system does not require handling of physical wedges and therefore may allow faster treatment. Furthermore, any arbitrary wedge angle and length can be created instead of the traditional four fixed wedges. It has been stated that virtual wedges do not alter the photon spectrum compared to fixed wedges, which introduce beam hardening and loss of uniformity in the unwedged direction. In this study we investigated the influence of the virtual wedge on the photon spectra of a 6-10 MV Siemens MD2 accelerator.

The Monte Carlo code BEAM/EGS4 was used in this work to model the complete accelerator for 6 and $10 \mathrm{MV}$ photon beams. The dynamic wedge was modelled by calculating phase space information at the exit plane of the accelerator for a range of field settings from $20 \times 20 \mathrm{~cm}^{2}$ to $20 \times 1 \mathrm{~cm}^{2}$. The phase space data was then combined to give wedged fields of $15^{\circ}, 30^{\circ}, 45^{\circ}$ and $60^{\circ}$. The photon spectra and dose distributions with a virtual wedge were compared to those obtained with a physical wedge. It was found that photon spectra from a virtual wedge are softer compared to the open field situation, whereas a physical wedge introduces beam hardening. Good agreement for the dose distributions was obtained.

\section{PO-T- 213}

Monte-Carlo Simulations of Dose Rate Distributions Around Three Amersham LDR Small Cs-137 Sources

J Lluch, F Ballester*, J Pérez-Calatayud, F Lliso ${ }^{2}$, E Casal ${ }^{3}$, (1) Valencia University, Valencia, Spain, (2) La Fe University Hospital, Valencia, Spain, (3) Centro Nacional Dosimetria, Valencia, Spain

CDC-type one and three active beads and CDCS Walksman type sources are available to use in source trains or manual afterloading systems in gynecological brachytherapy. In this work we present simulations of absolute dose rate in water with the Monte Carlo GEANT code around this sources. All the physical processes for low energy photons are implemented in GEANT: photoelectric effect, Compton dispersion, pair production and Rayleigh scattering. For electrons, multiple scattering and continuous energy loss were assumed.

The three sources were fully modeled from information provided by Amersham. In order to reach full scatter conditions, a cylinder of water 40 $\mathrm{cm}$ in height and $40 \mathrm{~cm}$ in diameter was assumed where a grid system with a score volume of $0.5 \times 0.5 \times 0.5 \mathrm{~mm}^{3}$ has been established. Up to $10^{9}$ histories were simulated. To estimate air-kerma strength the source was located in a $6 \times 6 \times 6 \mathrm{~m}^{3}$ dry air cube and kerma was scored with $2 \times 10^{10}$ histories.

Absorbed dose rate in water have been normalized to $1 \mu \mathrm{Gym}^{2} \mathrm{~h}^{-1}$ and are presented in the form of away-along table at distances up to $10 \mathrm{~cm}$. In order to use the simulated data in the treatment planning programs based on TG43 formalism we have extracted from our simulation results the necessary dosimetry parameters, these are: the dose rate constant $\Lambda$, the radial function $g(r)$, the anisotropy function $F(r, \theta)$. The anisotropy factor $F(r)$ and the anisotropy constant $F$ were also obtained.

\section{PO-T- 214}

Clinical Implementation of a Two-Source Model for Calculation of Electron Relative Output Factors

C Lewis*, J Chen, K Spyksma, London Regional Cancer Centre, London, Ontario, Canada

A two-source model has been implemented clinically to calculate the electron relative output factors (ROF) for various treatment conditions, including irregularly shaped fields at any source to surface distance (SSD). The model consists of an effective extended source above the final field defining aperture (i.e. "cutout") plane and a second source that models scattering from the aperture. The parameters in the model (maximum of six) are determined for each electron energy and applicator with a minimum set of ROF measurements made in water for square fields. A computer program using Clarkson type integration was developed to calculate ROFs for any digitized irregular field. The calculations of ROF by the model have been compared with measurements for a wide variety of cutout size, energies, applicators and SSDs for square fields, rectangular fields and circular fields. The agreement between calculations and measurements for these systematic comparisons is generally within $1 \%$. The predictions of ROF by the program have also been compared with many clinical measurements for irregular fields. Most of the clinical measurements agreed with the model within $2 \%$, but there were some measurements outside of this range. This can be attributed to problems in accurately measuring the ROF for small electron cutouts. In conclusion, the two-source model has been implemented clinically to predict electron ROFs accurately, saving the measurement time 
and avoiding measurement uncertainties inherent in small field electron dosimetry.

\section{PO-T- 215}

A Deterministic Electron Transport Algorithm for Radiation Dosimetry A Mesa*, J Demarco, R Cook, Department of Radiation Oncology, University of California, Los Angeles, CA

As part of our efforts to produce a coupled photon/electron dose calculation tool, an electron transport algorithm is currently under development. Similar to Monte Carlo methods, this algorithm incorporates bremsstrahlung production, ionization and multiple scatter in heterogeneous media. Unlike Monte Carlo methods, which compute dose stochastically by following individual particle histories, this method seeks to compute dose deterministically. Given a heterogeneous material lattice and an electron source, a ray-tracing algorithm is used to determine the electron energy incident upon each lattice element. Secondary radiation and scattered electron distributions are then obtainable from the appropriate cross sections and multiple scattering formalism. Hence, each lattice element can function as a secondary radiation source. By coupling surrounding lattice elements along ray lines to these secondary radiation sources, the dose contribution to the surrounding lattice elements is calculated by combining a ray-tracing algorithm with a weighting factor related to the coupling angle. The process repeats itself for higher orders of interaction. Preliminary results include dose distributions from both external electron fields and internal electron sources (e.g. secondary electrons from Compton scatter). Results are compared with Monte Carlo calculations.

\section{PO-T- 216}

A Fast Model for the Prediction of the PDD/TPR of Irregular Shaped Fields From Just a Few Physical Parameters

Y Xiao*, M Huq, M Hossain, Thomas Jefferson University, Philadelphia, PA

Phantom scattered dose is an essential component of the total dose delivered to tissue during high energy X-ray radiation treatment. Accounting for this component accurately and efficiently in the dose calculation is a necessity. In the search for an optimal set of IMRT parameters, speed of calculation is highly desirable, especially for search algorithms for which oft-repeated calculations are required. In this study, we propose a method of simple summation of the phantom-scatter contributions from a set of individual triangles that constitute an irregular field. The calculation of phantom scatter is based on a two-parameter model that is applicable to regions where electron equilibrium is established. The advantage of this approach is the considerable reduction of calculation time compared to that needed for a full-fledged scatter integration. The calculation accuracy for an irregular field shaped by an MLC is not compromised by the triangulation arising from the straight edges of the MLC leaves. The two parameters for the model, $(\mathrm{a}, \mathrm{w})$, can be derived from the basic beam parameter, the averaged attenuation coefficient $\mu$. Eventually, only two parameters for each beam, $(\mu, \eta)$, are needed to predict the PDD (percent depth dose) or the TPR (tissue phantom ratio ) with reasonable accuracy for any arbitrarily shaped field, especially by MLC, in the region of electron equilibrium.

\section{PO-T- 217}

Derivation of Anisotropy Functions and Dose-rate Constants for 192Ir Brachytherapy Sources Using Primary and Scatter Dose Separation K Russell*, A Ahnesjö, Helax AB, Uppsala, Sweden

The scatter dose around high energy brachytherapy sources is dependent on the characteristics and geometry of the scattering volume, while the primary dose depends only on the material along the source to calculation point path. Hence, algorithms based on primary and scatter dose separation will facilitate scatter dose integration and heterogeneity corrections. Source characterization by means of Monte Carlo simulation of dose deposition per source emitted radiant energy allows dose separation into primary and scatter contributions. Simulations were made in water, air and vacuum for ${ }^{192}$ Ir sources used in the MicroSelectron HDR/PDR and GammaMed 12i HDR afterloading units. Sources were positioned freely in unbounded phantoms and in combination with nylon and stainless steel source channels. Radial dose profiles for primary and total scatter dose contributions at different angles to the source axis were scored and parameterized using functions based on 1D transport theory. Anisotropy functions were derived and are shown to agree within $\pm 3 \%$ with published Monte Carlo calculated functions and $\pm 5 \%$ with measured values at angles between $10 \leq \theta \leq 170^{\circ}$ to the axis. Calculated dose-rate constants, $\Lambda_{0}$, agree to within $\pm 1 \%$ the value recommended in AAPM Task Group 43 report. $\Lambda_{0}$ is relatively insensitive to the material of the source channel, however, anisotropy functions were reduced by $15 \%$ for steel channels compared with nylon channels at angles close to the source axis.

\section{PO-T- 218}

An Optimization Method to Derive Extra-focal Source from Collimator Scattering Factor $(\mathbf{S c})$

J Chang*, Memorial Sloan-Kettering Cancer Center, New York, NY

A focal-spot source plus an extra-focal source model is useful to determine the dependence of head scatter and beam penumbra on field size. Determination of the extra-focal source needs extra equipment and measurement, which are generally not available in a clinical environment. An optimization procedure is developed to derive the extra-focal source from routinely measured collimator scattering factor, $S_{c}$. $S_{c}$ factors of square field are first obtained either from chamber measurements or from commission data. Differences of $S_{c}$ between consecutive field sizes are used as the initial guesses of the extra-focal source. Focal-spot source strength is the extrapolated $S_{c}$ value for field size 0 . Calculated $S_{c}$ factors from the current focal source and extra-focal source are compared with the measured $S_{c}$, and the differences are used to iteratively update the extra-focal source distribution until the difference is smaller than a preset tolerance. The $S_{c}$ data from a Varian $2100 \mathrm{C}$ were used to test this approach. The optimized extra-focal source and focal-spot source were used to predict the $S_{c}$ for sixteen rectangular fields. The results show that the calculated $S_{c}$ from the optimized extra-focal source match the measured $S_{c}$ to better than $0.02 \%$, on average. The calculated $S_{c}$ for 16 rectangular fields agree with the measured ones within $0.3 \%$, on average. These results indicate that the extra-focal source can be determined from routinely measured $S_{c}$ factors using the developed optimization procedure without extra equipment and measurement.

\section{PO-T- 219}

Calculation of Portal Dose Distributions for Verification in Radiotherapy

M Fix*, M Sabel, H Keller ${ }^{1}$, E Born, D Vetterli, R Mini' ${ }^{2}$, P Ruegsegger ${ }^{1}$, (1)Institute for Biomedical Engineering and Medical Informatics, ETH Zurich, Switzerland, (2)Division of Med. Radiation Physics, Clinic of Radio-Oncology, Inselspital-University of Berne, Switzerland

A promising approach of dosimetric quality assurance is to compare measured with calculated portal dose distributions. In this work the feasibility of commercial treatment planning systems (Varian CadPlan, Helax TMS) for calculating portal dose distributions of a $6 \mathrm{MV}$ photon beam was investigated.

For this purpose six phantoms were implemented in the planning systems. The portal imaging device PortalVision was considered as a homogeneous water slab. All portal dose distributions were calculated in $1 \mathrm{~cm}$ water depth of the portal imaging phantom. These distributions were compared with measured ones obtained from the PortalVision system and from films.

The range of relative deviations of calculated profiles in the central region of the beam in comparison with those obtained from films was (for all phantoms) $0-4 \%$ (CadPlan), $1-10 \%$ (TMS) and $0-1.5 \%$ (PortalVision). Profile shapes of PortalVision and TMS were rather consistent with those from film wheras the roundness of the Cadplan profiles behind absorbers led to appreciable deviations.

In summary, the calculation of portal dose distributions shows appreciable inaccuracies due to the air gap between the phantom and the portal imaging plane. Therefore, calculation algorithms have to be improved before using the planning systems for accurate portal dose calculations. 


\section{PO-T- 220}

Monte Carlo Simulations for Electron Backscattering in Lead

M Serrano, F Ballester*1, J Pérez-Calatayud, V Carmona ${ }^{2}$, (1) Valencia

University, Valencia, Spain, (2) La Fe University Hospital, Valencia, Spain

There is only an extensive experimental work on backscattering in electron therapy (Klevenhagen et al, Phys. Med. Biol., 27, 363-373, 1982). In this work we present simulations performed with Monte-Carlo GEANT code for electrons backscattered from lead slabs. The data are expressed in relation to the beam energy incident on the shield for energies ranging from 1 to 3 $\mathrm{MeV}$. These energies are lower than the previously published data and still in the range of the electrons used in therapy. The depth dose curves and electron spectral distributions in the up-stream stream direction are also presented.

GEANT code has been used assuming multiple scattering and continuous energy-loss. Typical therapeutic energy spectra were used with $\bar{E}_{0}$ ranging from 5 to $10,75 \mathrm{MeV}$ and $\bar{E}_{z}$ from 1 to $3 \mathrm{MeV}$ (incident on the shield). Water phantom was simulated, in which a transversal score area of $0.25 \times 0.25 \mathrm{~mm}^{2}$ was established to collect deposited energy. Up to $3 \times 10^{7}$ histories were simulated.

Our simulation results show lower electron backscatter factors (EBF, defined as the ratio of dose at interface surface with and without the scatterer present) than Klevenhagen's results. For example, with $\bar{E}_{z}=2.3 \mathrm{MeV}$, $\mathrm{EBF}=1.53$ against 1.63 . For lower energies the values go down $\left(\bar{E}_{z}=1\right.$ $\mathrm{MeV}, \mathrm{EBF}=1.48$ ). EBF results don't show dependence on $\bar{E}_{0}$. Also we have established two relations for the backscattered electrons: $R_{p}^{\text {back }} \cong \frac{1}{5}\left(1+\bar{E}_{z, P b}\right)$, and the evolution of mean energy with depth $x$ : $E_{x}^{\text {back }} \cong \bar{E}_{0}^{\text {back }}\left(1-\frac{f(E)}{R_{p}^{\text {back }}} x\right)$, where $f(E)=e^{-1.86+0.4 \bar{E}_{0}^{\text {back }}}$.

\section{PO-T- 220}

Monte Carlo Simulations for Electron Backscattering in Lead M Serrano, F Ballester*1, J Pérez-Calatayud, V Carmona ${ }^{2}$, (1) Valencia University, Valencia, Spain, (2) La Fe University Hospital, Valencia, Spain

There is only an extensive experimental work on backscattering in electron therapy (Klevenhagen et al, Phys. Med. Biol., 27, 363-373, 1982). In this work we present simulations performed with Monte-Carlo GEANT code for electrons backscattered from lead slabs. The data are expressed in relation to the beam energy incident on the shield for energies ranging from 1 to 3 $\mathrm{MeV}$. These energies are lower than the previously published data and still in the range of the electrons used in therapy. The depth dose curves and electron spectral distributions in the up-stream stream direction are also presented.

GEANT code has been used assuming multiple scattering and continuous energy-loss. Typical therapeutic energy spectra were used with $\bar{E}_{0}$ ranging from 5 to $10,75 \mathrm{MeV}$ and $\bar{E}_{z}$ from 1 to $3 \mathrm{MeV}$ (incident on the shield). Water phantom was simulated, in which a transversal score area of $0.25 \times 0.25 \mathrm{~mm}^{2}$ was established to collect deposited energy. Up to $3 \times 10^{7}$ histories were simulated.

Our simulation results show lower electron backscatter factors (EBF, defined as the ratio of dose at interface surface with and without the scatterer present) than Klevenhagen's results. For example, with $\bar{E}_{z}=2.3 \mathrm{MeV}$, $\mathrm{EBF}=1.53$ against 1.63 . For lower energies the values go down $\left(\bar{E}_{z}=1\right.$ $\mathrm{MeV}, \mathrm{EBF}=1.48$ ). EBF results don't show dependence on $\bar{E}_{0}$. Also we have established two relations for the backscattered electrons:

$$
\begin{aligned}
& R_{p}^{\text {back }} \cong \frac{1}{5}\left(1+\bar{E}_{z, P b}\right), \text { and the evolution of mean energy with depth } x: \\
& E_{x}^{\text {back }} \cong \bar{E}_{0}^{\text {back }}\left(1-\frac{f(E)}{R_{p}^{\text {back }}} x\right), \text { where } f(E)=e^{-1.86+0.4 \bar{E}_{0}^{\text {back }}} .
\end{aligned}
$$

\section{PO-T- 221}

A Simple Electron Beam Simulation Program

D Wilson*, University of Louisville Brown Cancer Center, Louisville, KY

The small ( $\sim 150$ line) $\mathrm{C}$ program described in this presentation was written as a teaching tool in order to simulate electron pencil beam scattering in multilayered media and to determine relationships between depth dose, electron loss, and angular distribution. The medium is divided into 150 thin slabs, and a number of electron path histories (typically 20000 to 100000) are followed through these slabs. Each electron is assigned a total path length, and when that sum is reached, the history is terminated. The direction of electron travel at the face of each layer is determined by adding a random $\mathrm{x}$ and $\mathrm{y}$ component of deflection calculated from a normal distribution based on an angular variance which represents the scattering power at that level. The angular variances are pre-calculated as a function of total path length in a separate module. Angular standard deviation functions are generated which can represent either realistic energy dependent scattering power or constant scattering power. The stopping powers are assumed to be constant, so the total dose in an individual slab is the sum of all of the electron path lengths. The dose and electron count at each level are tabulated and printed out in a text file for analysis. A theta-x projection, $\mathrm{x}$ displacement, and individual path length for every electron is stored at each depth and written to a binary file which is approximately $100 \mathrm{Mb}$ size. Analysis of this file is done using software outside of the simulation program.

\section{PO-T- 222}

Effects of Beam Divergence, Fluence Distribution, and Beam Quality on the Phantom-scatter Factor

L Wang*, T Zhu, B Bjarngard, University of Pennsylvania, Philadelphia, PA, Hosp. of the University of Pennsylvania, Philadelphia, University of Pennsylvania, Philadelphia, PA

The phantom-scatter factor $\left(S_{p}\right)$ takes into account of the change in scatter radiation originating in the phantom at a reference depth for a fixed collimator opening as the irradiated volume of phantom is changed. By this definition, the $S_{p}$ factor can be affected not only by the volume of phantom irradiated, but also the characteristics of the primary beam, including the beam divergence, the fluence distribution, and the beam quality as a function of field size or distance from the central axis. The present research is to study the effects of the above factors on the phantom-scatter factor using the EGS4 Monte Carlo method. This method has a unique feature that allows us to differentiate the contributions from various factors and to exclude the head scatter and electron contamination. Quantifying the effects of the beam divergence, the fluence distribution, and the energy spectrum on the phantom-scatter factor is important, especially on how approximation can be made in primary beam modeling. For example, parallel beam instead of divergent beam is assumed in dose-calculation in both ADAC and HelaxTMS systems. Our preliminary study has shown that beam divergence contributes most to the improvement of accuracy of $S_{p}$ calculation. The energy spectra are less important in this regard. The primary fluence distribution has negligible effect on the $S_{p}$ calculation, as far as the lateral variation is within $4 \%$ of fluence value at the central axis.

\section{PO-T- 223}

Longitudinal and Lateral Scatter Dose Buildup in 60Co beams R Mooij*, Columbia Presbyterian Medical Center, New York, NY

Central axis scatter dose in cylindrical Cobalt beams has been shown to have a region of buildup. Beyond this region scatter attenuates at the same rate as primary. An empirical formula describing scatter dose has a primary attenuation coefficient and a buildup coefficient appearing in an exponential. All parameters in this formula are field size dependent, except for the primary attenuation coefficient. Monte Carlo simulations show that the scatter buildup is due to the lack of scattered photons moving in from above 
the phantom, whereas backscatter out of the phantom surface is shown to be negligible and can not explain scatter buildup. Thus buildup of scatter dose and primary dose are analogous phenomena. A simple geometrical model is presented which derives the empirical formula. At fixed depth the buildup of Scatter with Field Size ( radius $<30 \mathrm{~cm}$ ) is very nearly linear at large depth $(\mathrm{d}>50 \mathrm{~cm})$ and behaves very closely as $1-\exp (-\mathrm{c} *$ radius $)$ near $18 \mathrm{~cm}$ depth. These properties are analyzed with the scatter order of photons.

\section{PO-T- 224}

Clinical Electron Beam Dosimetry - Comparison Between TG-21 and TG-51 Dosimetry Protocols

J Cygler*, C Kwok, G Ding, ORCC, Ottawa, Canada, ORCC, Ottawa, Canada

In this study we have compared the results of the absolute dosimetry for electron beams using the current TG-21 and the upcoming TG-51 protocols. The electron beams of energies 6-20 MeV were from a Siemens KD2 linear accelerator. The field size was $20 \times 20 \mathrm{~cm}^{2}$ and $\mathrm{SSD}=100 \mathrm{~cm}$. We used an NACP plane parallel chamber (serial number 34-08) which had $N_{D}^{60} \mathrm{Co}=$ $3.20 \mathrm{cGy} / \mathrm{R}$ provided by the NRCC standards laboratory. Dose/MU at the reference depth was converted to the value at $d_{\max }$ using PDD curves measured by high quality diodes (Scanditronix).

The dose calculations based on TG-51 methodology were done in 3 different ways :

1. Using the absorbed dose to water calibration factor, $N_{D}^{60} \mathrm{Co}$, provided by the NRC for our NACP chamber and $P_{\text {wall }}^{60}$ previously measured for this chamber.

5. Using the absorbed dose to water calibration factor, $N_{D}^{60} \mathrm{Co}$, provided by the NRC and $P_{\text {wall }}^{60}$ colculated by Rogers for this type of the chamber.

6. Using the cross - calibration of the NACP chamber versus cylindrical chamber for which the $N_{D}^{60}$ Co was provided by the NRC.

The differences between the data obtained by the 3 listed above methods vary for different beam energies are consistent with the differences between the stopping power ratios in TG-21 and the ones for the realistic clinical beams.

\section{PO-T- 225}

Comparison of Results in Absorbed Dose Calibration Between Using AAPM TG-21 and TG-51 Protocols - Electron Beams

G Ding ${ }^{1}$, M Yu ${ }^{1}$, J Cygler ${ }^{2}$, (1)Fraser Valley Cancer Centre, BC, (2)Ottawa Regional Cancer Centre, CCO, Ottawa, Canada

In this study we compare the values of absorbed dose determined at $\mathrm{d}_{\max }$ according to the current AAPM dose calibration protocol TG-21 and the upcoming AAPM TG-51 protocol for electron beam of energies from 6 to 18 $\mathrm{MeV}$. The incident electron beams are from an ELEKTA SL20 linear accelerator. The field size is $10 \times 10 \mathrm{~cm}^{2}$ and SSD $=100 \mathrm{~cm}$. A Markus and an NACP plane-parallel chambers are used in the absorbed dose calibration.

The values of $N_{\text {gas }}$ and $k_{\text {ecal }} N_{D, w}^{60}$ for plane-parallel chambers are crosscalibrated against a calibrated cylindrical ionization chamber (PR-06C) at $d_{\max }$ and at $d_{\text {ref }}$ respectively. A field size of $20 \times 20 \mathrm{~cm}^{2}$ and SSD $=100 \mathrm{~cm}$ are used for cross-calibration for plane-parallel chambers. The exposure

calibration factor $N_{x}$ and the new absorbed-dose-to-water calibration factor $N_{D, w}^{60} \mathrm{Co}$ for the cylindrical ionization chamber (PR-06C) were provided by the primary standards laboratory (NRCC). We find that differences in determined absorbed dose at $\mathrm{d}_{\max }$ between using two protocols vary with electron beam energies. Over $1 \%$ discrepancies are observed. The results are consistent with our current understanding on the discrepancies of water-to-air stopping power ratios between values calculated by using monoenergetic and realistic electron incident beams at $\mathrm{d}_{\max }$.

\section{PO-T- 226}

Comparison of the Photoneutron Spectra and Fluence Rates Generated from a High Energy Linear Accelerator using Hard and Dynamic Wedges

E Thomas ${ }^{1}, \mathrm{C}$ Ramsey*2, (1) Oak Ridge Institute for Science and Education, Oak Ridge, TN, (2) Thompson Cancer Survival Center, Knoxville, TN

Since the advent of the high-energy medical linear accelerator, there has been a great deal of interest in photoneutron production. Although a great deal of work has been published on photoneutrons, the effect of hard and dynamic wedges on the neutron fluence rate and energy spectrum has not been investigated.

In this work, the neutron energy spectrum and fluence rate was measured for a Varian 2100C/D 18-MV photon beam using polyethylene multispheres with a lithium iodide detector. Measurements were taken for each sphere for a $10 \times 10-\mathrm{cm}$ open field, a $10 \times 10-\mathrm{cm}$ field modified by a 60 -degree hard wedge, and a 10x10-cm field modified by an enhanced dynamic wedge. The neutron measurements were taken with the photon output at the calibration point held constant at $100 \mathrm{cGy}$ (no wedge factors).

The shape of the neutron spectrum did not change with the addition of hard or dynamic wedges. However, the maximum neutron fluence did change, with an $18 \%$ decrease observed for a 60 -degree hard wedge and a $15 \%$ increase for a 60-degree enhanced dynamic wedge. Because of the increase in photon output required for treatment with 60-degree dynamic wedges due to the small wedge factors, the total number of neutron generated per treatment can increase as much as $222 \%$.

\section{PO-T- 227}

Determination of the Bremsstrahlung Spectra of High-Energy Medical Linear Accelerators using a Simpson Iteration Technique R Alhakeem, L Miller ${ }^{1}$, C Ramsey*, A Oliver ${ }^{2}$, The University of Tennessee, Knoxville, Thompson Cancer Survival Center, Knoxville, TN

The determination of the x-ray energy spectrum produced by linear accelerators has received increasing attention due to the use of model based three-dimensional treatment planning. High-energy $x$-ray spectral reconstruction from transmission data was investigated by utilization of the Simpson iterative numerical technique, and the results were compared to the energy spectra used in the ADAC Pinnacle treatment planning system. Although previous works have used this technique, no experimental investigation had been performed for energies above 10-MV due to the limitations of lead and aluminum as attenuators at high energies.

In this work, graphite was used as the attenuating material to measure the transmission data and to determine the modal energy. The average energy was measured separate from the graphite transmission measurements using tissue maximum ratio (TMR) data obtained from solid water. The Simpson technique uses the measured graphite transmission data to iteratively unfold the shape and fluence rate of the photon spectrum, with the modal and average energies used as physical constrains.

Energy spectra were generated for Varian 6/100 and 2100C/D 6-MV photon beams to test the consistency of the measured data and to validate the Simpson technique. An 18-MV photon spectrum for a Varian 2100C/D was then successfully generated using this technique. The frequency of the modal energy for the $18-\mathrm{MV}$ reconstructed spectrum was within $4 \%$ of the modeled 18-MV spectrum used in the Pinnacle treatment planning system.

\section{PO-T- 228}

Effective Source Distances for Physical and Dynamic Wedged Fields M Johnson*, S Sharma, Sparrow Regional Cancer Center, Lansing, MI

A basic assumption in radiation dosimetry, is that the in-air x-ray output of a linear accelerator has an inverse square relationship to the source detector distance (SDD). However, it has been shown that this simple relationship does not account for head scatter, caused by $\mathrm{x}$-ray interaction with the collimator jaws and the physical wedge. Measured and in-air output factors 
can be brought into better agreement by replacing the nominal SAD with an effective source distance (ESD). Recently, linear accelerator manufacturers have been providing dynamic wedges with their accelerators. These devices could modify how photons scatter in the head assembly. This investigation looked into the dependence of ESD on energy, distance, and field size for open, physical, and dynamic wedged fields.

Our analysis agreed with previous studies showing that the ESD is affected by the addition of a physical wedge. The change in ESD is more pronounced for higher photon energies, at distances closer to the accelerator head, for thicker wedges, and for larger field sizes. The ESD's measured for dynamic wedges were similar to those measured for open fields. This is probably do to less scatter created in the head assembly, as compared to the physical wedge. Any inverse square corrections recommended in literature for in-air output factors for physical wedged fields do not seem as necessary for dynamic wedged fields.

\section{PO-T- 229}

Evaluation of a 3D Treatment Planning System for Dose Computations in the Kilovoltage Energy Range

P Alaei*, B Gerbi, R Geise, University of Minnesota, Minneapolis, MN

Because of high doses in interventional radiology procedures, knowledge of the dose to various organs from diagnostic $\mathrm{x}$ rays has become increasingly important. In an attempt to determine the dose from these, as well as orthovoltage $\mathrm{x}$ rays, we have used the ADAC Pinnacle ${ }^{3}$ treatment planing system to calculate the dose to phantom from kilovoltage $\mathrm{x}$ rays. The planning system's capabilities for dose computation have been extended to lower energies by addition of energy deposition kernels in the 20 to $110 \mathrm{keV}$ range and the $60,80,100$ and $120 \mathrm{kVp}$ beams have been modeled using the system

We compared the dose calculated by the planning system versus that measured using thermoluminiscent dosimeters (TLDs) placed in various positions within several phantoms. The phantoms consisted of a homogeneous solid water phantom, a solid water phantom with added lung inhomogeneity, a solid water phantom with added bone inhomogeneity and the Rando anthropomorphic phantom. A simple treatment plan was then generated using Pinnacle on CT scans of each of these phantoms and point doses at the positions of TLD chips were calculated. The results of the comparisons between TLD measurements and point dose calculations will be presented and the areas of strength and weakness of the planning system in calculating dose in various phantoms will be discussed.

\section{Poster Presentations: Diagnostic Imaging}

\section{Ryman Exhibit Hall}

\section{PO-D - 01}

Darkroom Fog Tests with Kodak MIN-R 2000, Seeing Through the Fog J McKenna*, D Jones, Scott \& White Memorial Hospital, Temple, TX

Conversion to Kodak MIN-R 2000 film, a high-contrast film, requires a routine evaluation concerning its effect on processor QC and clinical techniques. This evaluation should be expanded to include a check of the darkroom fog. At a site, which converted in October, the darkroom had passed the ACR recommendations with MIN-R E in September, but failed the MQSA inspection fog test in November with the MIN-R 2000. No changes had been made to the darkroom so one may be inclined to attribute this to the higher contrast inherent in the MIN-R 2000 film. However, further investigation was initiated to evaluate the test method. To ascertain appropriateness of the method, routine phantom films were obtained and scanned for uniformity in the direction perpendicular to the cathode-anode axis at three different locations on each film. Tests were also conducted rotating the phantom 90 degrees and 180 degrees. Films were evaluated using a standard densitometer as well as a scanning densitometer. A total of six sites, three different film/screen combinations, six ACR phantoms and an acrylic phantom were evaluated for uniformity and corresponding fog tests were performed.

As sites switch film, the checklist of items to be evaluated needs to include the darkroom fog check. Sites may need to modify angulation of lights, reduce bulb wattage or perform more detailed light leak checks when use of MIN-R 2000 film is initiated.

\section{PO-D - 02}

Mammography Tomosynthesis Using A Coupled Source and Detector In A C-Arm Configuration

M Dennis*, J Rakowski, Medical College of Ohio, Toledo, $\mathrm{OH}$

A digital tomosynthesis method has been implemented for use with isocentric stereotactic breast biopsy units with digital imaging capabilities. Tomosynthesis is the process of reconstructing planes of interest at any level in an object from limited angle projection data in a manner similar to conventional focal plane tomography. Niklason, et.al (Radiology 1997;205:399-406), using a full field digital mammographic system with a stationary detector geometry, demonstrated that tomosynthesis can improve lesion margin visibility especially in radiographically dense breasts. The method implemented in this work reconstructs planes orthogonal to the zero projection angle axis, as well as planes tilted relative to the axis of tomographic motion from an imaging system using a coupled source and detector in a c-arm configuration. The images are collected on a Lorad stereotactic prone breast biopsy unit with a 1024 x 1024 digital image receptor. Image quality is evaluated in terms of the line spread function, low contrast detectability, and ACR stereotactic accreditation phantom target visibility, including the dependence of image quality on image receptor exposure values and number of projections.

\section{PO-D - 03}

Mammography X-ray Spectra Across a 2D Field Derived from Transmission Measurements

J Terry*, R Waggener, M Miller Blough, Tulane University, New Orleans, LA, University of Texas Health Science Center at San Antonio, TX

Transmission measurements using high purity aluminum were made at different locations across an $\mathrm{x}$-ray mammography field. X-ray spectra were derived from the transmission measurements using a variational principle method. This method involved matching the calculated transmission curve from the derived spectrum with the measured transmission curve. The spectra were derived in units of exposure per $\mathrm{keV}$ interval to match the transmission curve which was measured in units of exposure. The variational method was validated using published mammography spectra to calculate a transmission curve and then deriving the $\mathrm{x}$-ray spectrum from the calculated transmission curve. The beam hardening due to higher attenuation along the anode side is also demonstrated by the derived spectra. The effective photon energy for the spectra weighted by exposure varied by five percent from the softest to hardest $\mathrm{X}$-ray spectra.

\section{PO-D - 04}

A DFT and Opto-Electronic Implementation of the Decoupled Automated Rotational and Translational (DART) Image Registration Algorithm

M NessAiver, S Sandip, University of MD Dept of Radiology, Baltimore, MD

Image registration is an important tool in medical image analysis, particularly in functional MRI (fMRI) Common methods of registering two images designates one image as a reference and then iteratively shifts and rotates the test image to minimize some cost function. These methods are predominantly restricted to image domain processing. Maas et al published a frequency domain algorithm (DART) that determines translational and rotational parameters in a single pass. This algorithm incorporates several cross-correlation steps, utilizing a total of seven 2D FFTs and two 2D rectangular to polar. Sub pixel accuracy is obtained by zero filling the original images, however, the number of calculations for a 2D FFT goes up as $2 n^{2} \log (n)$ and hence, greater precision is gained at the cost of dramatically increased processing time.

We have in our lab an opto-electronic processor called the ImSyn (Essex corporation - Columbia, MD) that performs a $128 \times 128$ complex DFT of non-rectilinear data in as little as $25 \mathrm{msec}$. Additional input parameters, $\mathrm{X}$ and $\mathrm{Y}$-axis scaling, $\mathrm{X}$ - and $\mathrm{Y}$-axis shifting and a rotation angle, do not slow the transform speed. It is possible to perform a DFT on polar data without first interpolating onto a rectilinear grid. Using appropriate scaling and shifting factors, cross-correlations can be obtained with effective resolution of a 4096x4096 grid. We will present results on simulated phantom data and actual fMRI data sets. Supported, in part, by the Essex Corporation. 


\section{PO-D - 05}

Automation of Diagnostic Process for Pulmonary Embolism Using V/Q Scans and Correlating Chest X-Rays With an Intelligent System.

G Serpen*, E Parsai, R Coombs, L Woldenberg, University of Toledo, Medical College of Ohio, Toledo, Ohio

This paper presents design of a software-based artificial intelligence system to diagnose pulmonary embolism using ventilation-perfusion scan and correlating chest $\mathrm{x}$-ray images of the lungs. The proposed hybrid intelligent system will be composed of a tightly integrated suite of artificial intelligence paradigms, which includes a fuzzy inference system, artificial neural networks, and machine learning algorithms. The fundamental promise of the proposed intelligent system is to model the human reasoning process "much more closely" than any existing artificial intelligence paradigm. The proposed hybrid intelligent system will be able to capture the knowledge, intuition, heuristics and

the ability to reason under uncertainty of an expert radiologist, very effectively to function as a reliable and accurate diagnosis system. Some of the features include: 1) Promise of a more accurate and quicker diagnosis, in areas where the scans are classified into low or high probability grades compared to visual method. This will result in fewer intermediate probability scans that frequently require angiography for definitive diagnosis, 2) Potential to reduce inter-observer variability, 3) would not suffer from fatigue or psychological factors that can effect the reliability of diagnosis procedure performed by radiologists, 4) Offers a competent second opinion, 5) Offers the expertise of an experienced radiologist in interpreting the scans when a radiologist is not readily accessible, such as in smaller medical clinics or hospitals where economics does not justify to support a full-time nuclear medicine specialist.

\section{PO-D - 06}

Reduction of False Positives in a Computerized Detection Scheme for Pulmonary Nodules Based on Symmetry Between Left and Right Lung Regions

H Yoshida*, K Doi, The University of Chicago, Chicago, IL

We have developed a novel method for reduction of false positives reported by a computer-aided diagnosis (CAD) scheme for detection of lung nodules in chest radiographs. Our method is based on the removal of normal structures in the regions of interest (ROIs) based on symmetry between left and right lung regions. In our method, two ROIs are extracted, one from the position where a candidate of a nodule is located, and the other from the anatomically corresponding location in the opposite lung, which contains similar normal structures. A wavelet-based, multiresolution image registration method is employed for matching the two ROIs, and subtraction is performed. If no structure remains in the subtracted ROI, then the original ROI is identified as containing only normal structures; otherwise, it is regarded as containing a nodule. A measure that quantifies the remaining structures was developed for distinction between nodules and false positives. In an analysis of 780 ROIs consisting of 84 nodules and 696 false positives reported as detected nodules by our CAD scheme, we were able to eliminate $32 \%$ of false positives with loss of only one nodule with this new method. When receiver operating characteristic (ROC) analysis was employed for estimating the performance of our method for unknown cases, it yielded an area under the ROC curve $(\mathrm{Az})$ of 0.82 . These results indicate that the method is very effective in eliminating normal anatomic structures and thus reducing the number of false positives in the $\mathrm{CAD}$ scheme for detection of lung nodules.

\section{PO-D - 07}

Segmentation of Microcalcifications with Weighted Inverse Wavelet Transform and Local Orientation

L Yang*, C Kimme-Smith, M Mcnitt-Gray, UCLA, Los Angeles, CA

Segmentation of Microcalcifications is generally the first step to extract morphological features for Computer Aided Classification. There are several methods to segment micocalcifications based on the wavelet transform; however, many of them are based on the amplitude of the transformed coefficients, which can be regarded as the local contrast. In order to reduce the false positives and misclassify the breast tissue with similar local contrast, it is reasonable to apply both local contrast and shape analysis for segmentation purpose.
We segment microcalcifications by combining wavelet transform and local orientation measurement together, where local orientation is defined as the vector addition of the gradient angle of each point within a certain size window. The gradient angle can be directly calculated from the wavelet transform coefficients. This method is based on the breast model where the microcalcification is represented as a small closed area, and the breast tissue tends to have long linear structure. Thus the local orientation measurement is introduced to distinguish the wavelet transform coefficients that correspond to the microcalcification or breast tissue based on the difference of their shape.

The algorithm has been tested on 12 full field digital image of six patients and compared with other methods such as weighted wavelet transform or threshold based amplification of transform coefficients. The result is promising and the proposed method can segment the microcalcifications with less effect from other tissue with similar contrast.

\section{PO-D - 08}

Symlet Wavelet Applied to Enhance Flow Echo-Planar Imaging A. Rodriguez-Gonzalez ${ }^{1}$, R. Bowtell ${ }^{2}$, P. Mansfield ${ }^{2}$, (1) UAM-Iztapala, Area PDSIB, Mexico, (2) The Magnetic Resonance Centre, School of Physics and Astronomy, The University of Nottingham, Nottingham, England

Flow Encoded Echo-Planar Imaging (EPI) shows a poor image Signal-toNoise Ratio (SNR) [1]. Flow and anatomical information is partly masked making difficult interpretation of the anatomy and flow visualisation. The continuous transform of Symlet wavelet [2] together with Doyle and Mansfield method [3] is applied to EPI velocity maps [4] to increase the SNR. Wavelet coefficient images of the cardiac blood flow maps and descending aorta were calculated. All coefficients were calculated using MATLAB programmes. Image quality was significantly enhanced so anatomical details can easily be distinguished. Contour maps were computed with enhanced images to show flow information that may characterise blood flow in the cardiac chambers as well in the descending aorta. This wavelet can greatly improve the flow image quality and yield well-defined contour maps from our velocity maps. Echo-Planar Imaging and the continuous transform of Symlet wavelet can be combined to produce a solid method to improve the image quality, and it may be useful to study human hemodynamics.

\section{PO-D - 09}

Implementation and Operation of An Ultrasound Mini-PACS in A Multi-Sites Tertiary Health Care Center

Y Liu*, J Blechinger, Radiology Dept./St. Luke's Medical Center, Milwaukee, WI

An ultrasound mini-PACS has been implemented and operational for 3 years in a tertiary health care center with imaging and interpretations at 5 sites in both hospital and clinic environments. There are both fixed room and portable modes for ultrasound machines. Network accesses are provided at more potable scanning locations. If not in use, any fixed room ultrasound machine is assigned on portable to respond calls at designated locations with network configured, so that images can be transmitted to central reading workstations realtime. This improves exam/report turnaround time and the use efficiency of all ultrasound machines. Currently the PACS holds two and half years of 30,000 exams with more than 1.1 million images online. To minimize human resources for removing old optical disks (OD) and reinserting when requested, a second OD jukebox was added for additional online storage. Works-in-progress includes upgrade for the total jukebox capacity to be increased to 1.2 Tera byte so that 5 years of exams on OD can be online. Having all exams on OD and online saves film costs, personnel involvement in film/OD sorting and reduce film/OD retrieval time, save film storage space which is almost always tight for urban institutions. The implementation of an ultrasound PACS is successful for the multi-sites tertiary healthcare center. Disadvantages include equipment costs, learning curve for interpreting physician and technical staffs. 


\section{PO-D - 10}

\section{Evaluation of a High Performance Direct Digital Imaging Plate}

D Pickens*1, R Price, M Bruno, M Batts ${ }^{2}$, D Gilblom, (1) Vanderbilt University Medical Center, Nashville, TN (2) Varian Imaging Products, Palo Alto, CA

A direct digital imaging plate made by Varian Imaging Products (Palo Alto, California) is undergoing evaluation for imaging performance in comparison with a conventional film screen system. This imaging device is configured for use in conventional radiographic imaging. It consists of a detector system with an active surface that is $20.3 \mathrm{~cm}$ by $25.4 \mathrm{~cm}$ connected by a cable to a computer which controls the electronics during acquisition, processing, and display. The detector system contains a conventional Gd2O2S screen coupled to an amorphous silicon (aSi) photodiode and switching array having 1536 by 1920 elements, yielding spatial resolution of better than $3.5 \mathrm{lp} / \mathrm{mm}$ with 12 bits of data. In operation images are available for viewing in less than a minute. Preliminary work imaging volunteers demonstrates that the performance of the system is generally comparable to film. More quantitative evaluation is ongoing and will be presented. Modulation transfer function (MTF) and noise power spectrum (NPS) will be measured leading to determination of detective quantum efficiency (DQE). MTF will be measured in two directions using a slit. NPS will be obtained by collecting flat field images at multiple exposures. From these measurements, DQE will be computed. Additionally, the dynamic range will be demonstrated as a function of input dose level.

\section{PO-D - 11}

Initial Study of the Micro-Angiographic Determination of Elastic Properties of Vessels

D Nazareth*, A Chattopadhyay, S Rudin, C Yang, D Bednarek, Departments of Physics, Radiology and Neurosurgery, Toshiba Stroke Research Center, University at Buffalo (SUNY), Buffalo, NY

The elastic properties of an artery provide a valuable indication of its viability. Changes in the vessel's diameter in response to fluid pressure changes, which occur during normal pulsatile blood flow, characterize such properties. Standard angiographic imaging systems which image $3 \mathrm{lp} / \mathrm{mm}$ are incapable of imaging the changes in diameter of small vessels. We have built a micro-angiographic detector, with $10-\mathrm{lp} / \mathrm{mm}$ resolution and $23-\mu \mathrm{m}$ pixels which can detect these changes by imaging the vessel's boundary during expansion and contraction.

The detector consists of a $100-\mu \mathrm{m}$ thick $\mathrm{CsI}(\mathrm{Tl})$ phosphor coupled to a CCD with a fiber-optic taper. A 525-line standard RS-170 video signal is digitized to form 8-bit images. Blood vessels were simulated using elastic tubing about $3 \mathrm{~mm}$ in diameter, filled with $300 \mathrm{mg} / \mathrm{cc}$ iodine contrast media. Variable pressure applied to the channel caused radial expansion and contraction, simulating a vessel's response to pulsatile flow.

We used the imager to acquire real-time $512 \times 480$ radiographic frames of the phantom. By digitally subtracting the images corresponding to contracted and expanded phases of the vessel, we determined the change in vessel diameter to be 14 pixels $(320 \mu \mathrm{m})$, representing a change in diameter of $9 \%$, which lies in the range of values for actual arteries of this size. Such measurements may give information previously unavailable about the elastic properties of small vessels, and help in evaluating vascular interventions. Partially supported by grants from the US Army, NIH and the Toshiba Corporation.

\section{PO-D - 12}

Use of a Statistical Phantom for Extremity Computed Radiography

R Chu*, E Christian, J Wilson, B Eaton, University of Oklahoma and Veterans Affairs Medical Center, Oklahoma City, OK

Abstract: An anthropomorphic hand phantom without any abnormalities was demonstrated to be ineffective for fine-tuning of the radiographic technique and the image processing in a computed radiographic system. A commercially available test object $\left(\mathrm{ALVIM}^{\mathrm{TM}}\right)^{*}$, containing holes of different sizes and various kinds of materials, is about the size of a hand and can be imaged with the same radiographic techniques of an extremity. Images of this test object were taken from $50 \mathrm{kVp}$ to $65 \mathrm{kVp}$. An analysis of the visual detection of the holes by ten radiologists showed a higher accuracy at $50 \mathrm{kVp}$. An image taken at $50 \mathrm{kVp}$ was processed by changing all the parameters in the computer radiography system. An analysis of the visual detection of the holes by two radiologists and two physicists determined the desirable changes from the default values of the parameters. Four out of five radiologists preferred an image of an anthropomorphic hand phantom with the new values to an image processed by the old default values. This statistical phantom apparently has helped us in the selection of radiographic techniques and the adjustment of parameters in image processing.

* Test object was donated by Nuclear Associates, a Division of Victoreen, Inc.

\section{PO-D - 13}

CT Shielding Design

D Shearer*, D North, R Shearer, Rhode Island Hospital, Providence, RI

Shearer et al $(1992 *)$ suggested a technique to estimate shielding requirements for CT scanners. This technique was based on use of the manufacturer's Computerized Tomographic Dose Index (CTDI) and the standard FDA dosimetry phantoms. This established a uniform method for estimating radiation levels surrounding the $\mathrm{CT}$ scanner.

This presentation extends the previous work by comparing the relationship between the stray radiation levels and the published and measured CTDI's for the majority of commercially available CT scanners.

A data base program has been developed to estimate shielding requirements for CT scanners. Different workloads and a variable mix of head and body techniques will be used to illustrate their effect on CT shielding requirements.

*Radiation Shielding for CT Facilities Medical Physics, Vol.19, Number 3 (1992)

\section{PO-D - 14}

Radiation Exposure to Breast in CT Scanning

M LaFrance*, R Breton, Baystate Medical Center, Springfield, MO

In recent years, considerable effort has been made by manufacturers and users of radiation producing equipment to minimize radiation exposure to breast tissue especially in mammography procedures. The Standardization required by the MQSA and ACR guide-lines have provided improved image quality with concurrent reduction in radiation exposure to breast tissue. However, the diagnostic procedures carried out in female population include routine chest $\mathrm{x}$-ray examinations, scoliosis studies and CT scanning.

Since 1980, there has been a gradual reduction in radiation exposure to breast tissue in mammography, scoliosis and chest x-ray studies except CT scanning. The radiation exposure measurements were carried out in CT scanners using TLD'S in female rando phantom. The measured data included effect of variation of $\mathrm{CT}$ pitch factor on radiation dose delivered to breast tissue. The

analysis of data show that increase in pitch factor reduces dose to breast tissue.

One of the ways to reduce radiation exposure to breast tissue is to use pitch factor greater than one (1) for scanning younger women, particularly if the chest area is involved. The other method would be to make appropriate reduction in $\mathrm{mAs}$ based on patient dimensions instead of using same $\mathrm{mAs}$ techniques for all chest CT scanning.

\section{PO-D - 15}

A Method for Acquiring Skin Conductance Measurements During Functional Magnetic Resonance Imaging

A Shastri ${ }^{1}$, M Lomarev ${ }^{1}$, M George ${ }^{1,2,3}$, D Bohning ${ }^{1}$, Departments of Radiology $^{1}$, Psychiatry ${ }^{2}$, Neurology ${ }^{3}$, Medical University of South Carolina, Charleston, SC

Introduction: In many different types of brain studies, it is desirable to monitor subjects' skin conductance responses (SCRs) during functional magnetic resonance imaging (fMRI) in order to study correlations between 
SCR and fMRI signals. The few studies that have employed combined SCR/fMRI acquisition were plagued by MR gradient and radio frequency (RF) artifacts in the SCR data. We developed a method of SCR/fMRI that eliminates these artifacts.

Methods: Subjects were scanned on a Picker EDGE 1.5 T scanner using a head coil and echo-planar imaging, while calibrated SCRs were acquired at $10 \mathrm{~Hz}$. The SCR signal was brought out of the scanner through a moveable, shielded door situated away from the MR gradient cable. The subject was grounded, and the shielding of the SCR cables was also grounded. SCR data were obtained with subjects inside and outside the scanner, and with EPI on and off.

Results: No MR gradient or RF artifacts were observed in the SCR data, and the noise level in the SCR data was the same regardless whether the MR scanner was acquiring data or not. The noise background in the MR images was comparable to baseline levels.

Conclusions: By grounding subjects, filtering signal lines, and keeping SCR cables away from MR gradient cables one can simultaneously acquire SCR and PMRI data with low noise background.

\section{PO-D - 16}

Automatic Bootstrap Characteristic Curve Segment Matching for Radiographic Imaging Systems

DR Bednarek*, S Rudin, CJ Yang, Departments of Radiology and Physics, State University of New York at Buffalo, Buffalo, NY

Determination of the characteristic curve is essential for quantitative evaluation of digital as well as screen-film radiographic imaging systems. When it is not practical to generate the entire curve through variation of a single exposure parameter, bootstrap methods can be used. One bootstrap method involves the generation of curve segments by capturing images with known differences in exposure achieved by changing the mAs. Multiple curve segments are produced concurrently by varying the exposure within each image using a stepped wedge. An appropriate shift of each segment along the log relative-exposure axis is used to join the overlapping curve sections. Since manual graphical matching of segments is tedious and imprecise, an automatic matching algorithm has been developed for use on a PC. A cubic spline fit of log exposure versus pixel value (or film density) is performed for each segment and the mean values for the overlapping sections of pairs of segments is determined by interpolation. The difference between the mean values of successive pairs of segment sections is taken as the best-fit log-exposure shift. The cumulative shift is then calculated for each segment to obtain the complete composite curve. The algorithm is evaluated for several digital image receptors and with known mathematically generated functions. This bootstrap method can provide complete, accurate characteristic curves, while the segment joining program makes the process fast and precise.

\section{PO-D - 17}

Broadband Ultrasound Attenuation (BUA Imaging Technique: The Effect of Calcaneus Size on the Automatic Region of Interest (ROI) BUA Measurements

J Damilakis*, K Perisinakis, M Mazonakis, N Gourtsoyiannis, University of Crete, Greece

An increasing role is currently recognized in quantitative ultrasound (US) in evaluating density and structure of calcaneus. Recently, US transmission systems have been developed with imaging capability that enables selection of the position of a circular region of interest (ROI) of $15 \mathrm{~mm}$ in diameter; the region of measurement is always the area of minimum attenuation in the posterior part of the calcaneus. This is an automatic procedure, totally independent of operator interaction. This study investigates the effect of calcaneus size on Broadband Ultrasound Attenuation (BUA) measurements. Imaging of the right calcaneus was performed using a computer controlled transmission system (Ubis 3000, DMS, France). Parametric images are obtained from the BUA values. BUA was estimated at the $15 \mathrm{~mm}$ diameter circular region (ROI1) and at an integral region of interest (ROI) encompassing more of the trabecular area of the bone (ROI2) in 122 female patients. ROI2 was traced manually. BUA estimated at ROI1 was significantly correlated with area of ROI2 ( BUA $=73.7-0.019$ AREA, $r=$ $-0.46, \mathrm{p}<0.0001)$. In contrast, no correlation was found between BUA averaged within ROI2 and area of ROI2. Ultrasound attenuation measured at an automatic circular region with BUA imaging technique is affected by the size of the calcaneus. Definition of a bone size dependent ROI is needed to counterbalance the effect of calcaneus size on BUA measurements.

\section{PO-D - 18}

Bold-fMRI Response to Single Pulse Transcranial Magnetic Stimulation (TMS)

D Bohning*1, A Shastri ${ }^{1}$, E Wassermann ${ }^{2}$, U Ziemann ${ }^{2}$, J Lorberbaum ${ }^{1}$, Z Nahas $^{1}$, M Lomarev ${ }^{1}$, M George ${ }^{1}$, (1)Medical University of SC, Charleston, SC, (2)NIH, Bethesda, MD, NIH, Bethesda, MD

Transcranial magnetic stimulation uses a pulsed magnetic field to induce localized neuron depolarizing currents in the cerebral cortex. Recently, TMS in combination with SPECT, PET, and MRI has generated considerable interest in using TMS to stimulate/inhibit local response for neuroimaging investigations of functional connectivity. Typically applied in trains, it has been possible to observe PET activation with 5 5-pulse trains of TMS at 10 $\mathrm{Hz}$ ( 25 pulses) and BOLD-fMRI response to $18 \mathrm{sec}$ trains of $110 \%$ motor threshold (MT) TMS pulses at $1 \mathrm{~Hz}$ (18 pulses). However, the only functional neuroimaging technique in which the response to single pulse TMS has been observed is EEG, providing high temporal resolution, but spatial resolution inferior to both PET and fMRI.

Interleaved TMS and fMRI was used with an averaged single trial (AST) protocol in which a single $120 \%$ MT TMS pulse was applied on alternate 12 sec epochs to determine if the effect of a single TMS pulse could be detected by BOLD-fMRI. During each epoch, 205 -slice packages of BOLD-EPI images were acquired at the rate of $0.6 \mathrm{sec}$ per package. The cycle was repeated 15 times. Five healthy volunteers were studied.

BOLD-fMRI response to single TMS pulses was detectable in ispilateral motor cortex under the TMS coil, in contralateral motor cortex, and bilaterally in auditory cortex. Significantly, the $1.5-2 \%$ signal increase in auditory cortex due to the sound of the TMS pulse was greater than the 1$1.5 \%$ increase induced in motor cortex by the TMS pulse itself!

\section{PO-D - 19}

A Unified Approach for Spreadsheet-Based Effective Dose Determination

C Parry*, J Sonnad, University of Oklahoma Health Sciences Center, Oklahoma City, OK

Many times, radiation therapy as well as radiation safety responsibilities. When asked for patient dosimetry in an imaging modality, a physicist with limited experience in that modality may spend a great deal of time searching through literature to find data. When comparing or combining doses from different modalities, the task of finding the effective dose is made more difficult due to incomplete information and inconsistencies in published data especially in the use of dose descriptors. The dose determination problem was brought home to us when we were asked to estimate the total dose to a patient from multiple imaging modalities. We have pursued a spreadsheetbased approach that is flexible and that greatly facilitates dose determination.

The spreadsheet program can be used to determine effective dose for either pediatric or adult radiography, fluoroscopy or nuclear medicine, as well as adult computed tomography. The information input by the user consists of data available to the physicist either in the patient chart or as part of required quality control measurements. The dose computation is based on data available commercially or through government agencies. The spreadsheet program is relatively easy to generate and can significantly expedite the process of dose computations. Our approach allows us to tailor the dose estimation to specific machines and techniques. Additionally, it can be updated as new information becomes available.

\section{PO-D - 20}

Convolution/Superposition Dosimetry for Diagnostic Beam Spectra J Modrick*, T Mackie, B Thomadsen, University of Wisconsin, Madison, WI

Absorbed dose from diagnostic procedures has recently become a matter of increasing interest because of the incidence of severe radiation erythema from fluoroscopic procedures and concerns about mean glandular dose from 
mammography procedures. We have computed mono-energetic photon energy deposition kernels for photons in the range of $10 \mathrm{keV}$ to $100 \mathrm{keV}$ using the "scatter sphere" EGS4 Monte Carlo code scasph.mortran. The EGS4 Monte Carlo code calculations include photon interactions from Compton scattering, the photoelectric effect, and Rayleigh scattering. Fluorescence effects have not been included. A convolution/superposition code developed for megavoltage therapeutic x-ray beams has been modified to utilized these energy deposition kernels to compute dose at diagnostic beam energies. Diagnostic beams have been modeled using measured photon spectra to determine an energy-fluence weighted energy deposition kernel from the mono-energetic photon energy deposition kernels. The energy-fluence weighted energy deposition kernel has been used in the convolution/superposition code to calculate depth dose curves for the spectra of a $30 \mathrm{kVp}$ tungsten target (M30) and a $30 \mathrm{kVp}$ molybdenum target (Mo30) diagnostic beam. Percent depth dose calculations for the M30 and Mo30 diagnostic beam spectra show agreement to within $2.5 \%$ and $5 \%$ respectively, with measurements made using a diamond detector in a water phantom.

\section{PO-D - 21}

Correlation of Dose Area Product (DAP) Values and Entrance Skin Exposure

V Gargaro*, M Dennis, W Potvin, Universal Consultants, Inc. North Ridgeville, OH, Medical College of Ohio, Toledo, OH, Flower Hospital, Toledo, $\mathrm{OH}$

Several manufacturers have developed sophisticated equipment to monitor dose or a quantity related to dose to regional areas exposed to radiation. A major portion of such devices are of a type classified as dose area product (DAP) meters.

Entrance skin exposure (ESE) has been used to characterize biologically significant radiation thresholds and can be related to dose area product. For defined geometries the entrance dose or entrance skin exposure (ESE) can be determined from the DAP measurement.

The purpose of this research is to establish conversion factors to be applied to DAP values in order to calculate with reasonable accuracy the corresponding ESE and entrance dose and how the DAP reading may be used as an operational threshold or action level in high dose interventional radiographic procedures.

The DAP meter can be reasonably calibrated to reflect real time entrance skin exposure with various modes of operation and orientations. Utilizing appropriate back scatter factors would then allow assessment of entrance dose. These values can then be used to notify the users of approaching a threshold for skin erythema.

\section{PO-D - 22}

Organ Dose Calculations Using Monte Carlo Method and Realistic Voxel Phantom

$\mathrm{G} \mathrm{Xu}^{*}, \mathrm{~T}$ Chao ${ }^{1}, \mathrm{~K}$ Eckerman ${ }^{2}$, (1) Rensselaer Polytechnic Institute, Troy, NY, (2) Oak Ridge National Laboratory, Oak Ridge, TN

Accurate organ dose assessment is extremely important in radiotherapy and nuclear medicine. Monte Carlo method today allows the simulations of radiation interaction with extremely small uncertainties (e.g., less than $1 \%$ ). On the other hand, the representation of the body (i.e., a phantom) has been relatively simplified, and this has inevitably caused errors in the organ dose results. MIRD mathematical models were based on anatomical data used to develop the ICRP Reference Man in late 1960's and have since been used in organ dose calculations for many purposes. Although several revisions have been developed over the years, MIRD-based models remain relatively simplified. Many researchers worldwide are now exploring the possibility of developing new models by utilizing modern medical imaging techniques. At Rensselaer Polytechnic Institute, an adult male whole-body voxel phantom has been developed using CT, MRI, and photos of real persons from the Visible Human Project, which are of resolutions as high as $0.3 \mathrm{~mm}$ x $0.33 \mathrm{~mm}$ x $1 \mathrm{~mm}$. This model has been adapted into a Monte Carlo code, EGS4, to calculate organ doses for photons and electrons under different source-target geometries. Preliminary results indicates that organ doses for $80 \mathrm{keV}$ photons in PA geometry obtained from the voxel phantom differ from those obtained from MIRD mathematical phantom up to $50 \%$. This paper presents the latest development in this area and a comparison of this new voxel phantom with existing MIRD mathematical phantom.

\section{PO-D - 23}

Dealing with Retaliation against the RSO - Legal Path or Quiet Path?

T Yoshizumi*, Duke University, Durham, NC

This paper describes a case pertaining to retaliation against a Radiation Safety Officer at a major university-affiliated teaching hospital. The hospital in question operates under an NRC license for by-product materials; the program is also overseen by the National VA Health Physics Program and a State Radiation Control Department. After routine inspection of a laboratory in April 1995, the RSO sent a memo to the Laboratory Director recommending repair or replacement of a survey meter. No response was received despite two written requests over two months. The RSO consulted Hospital Administration, which ultimately provided funds for the meter in August 1995. In late August 1995 the Laboratory Director informed the RSO that the RSO's faculty appointment was being terminated, and provided him with a copy of a letter to the University Administration to that effect. The RSO reported the incident to Hospital Administration and the Division Director at the University. The administration viewed the case as retaliation against the RSO and recommended that the Department Chairman reinstate the faculty appointment. We conclude that RSOs should take the following steps to avoid retaliation, or respond to it when it occurs: (1) establish an effective, credible radiation safety program with a definitive chain of command; (2) avoid direct conflict with authorized users; (3) seek Administration involvement quickly; (4) compile complete documentation of incidents; (5) avoid litigious threats or actions.

\section{PO-D - 24}

I-125 Assay

D Shearer*, N Jacob, D North, Rhode Island Hospital, Providence, RI

The assay of I-125 poses a difficult problem for dose estimation due to the low energy of the radiation. A recent incident prompted an investigation of the uncertainties in dosimetry.

Problems investigated were the suitability and accuracy of dose calibrators for the measurement of I-125 including geometry effects and the use of standard thyroid uptake phantoms for the estimation of thyroid burden.

The results indicate that significant errors can occur if a routine thyroid uptake estimate is performed without considering the physical characteristics of the subject.

\section{PO-D - 25}

Management of Patient Dose during Fluoroscopy

B Archer*, L Wagner, Baylor College of Medicine, Houston, Texas, University of Texas -Houston Medical School,Housto

There has been an increasing number of invasive fluoroscopic procedures and a concomitant increase in the number of radiation injuries reported from these procedures. The primary risk to patients from prolonged fluoroscopic $\mathrm{x}$-ray exposure is skin injury. Modifications to the work habits of some practitioners and to the fluoroscopic room environment are necessary to maintain an acceptable level of benefit/risk for patients. These modifications include improved patient management by physician training, dose monitoring, and equipment maintenance. Dose optimization can be achieved by: controlling dose rate at skin entrance, managing cumulated dose from multiple procedures, performing real-time dose monitoring and using quality control measures to avoid excessive radiation output by equipment. This presentation will focus on the implementation of each of these measures.

\section{PO-D - 26}

Maximum and Minimum Skin Exposure Using Dose-area Product Measurements: Application to a Cardiac Catheterization Laboratory N Adnani*, N Sharoubim, Montreal General Hospital, Montreal, Canada, Montreal General Hospital, Montreal, Canada

A new method to determine the maximum and minimum skin exposure from dose-area product measurements is presented. These upper and lower limits of skin exposure are very useful for managing health risk from radiation 
since it is only when the maximum exceeds a certain value that a more rigourous estimation of the skin exposure becomes necessary. First, a calibration of the dose-area product meter was done to yield the variation of the skin exposure per unit dose-area (SE/DAP) as a function of the dose-area per unit $\mathrm{mAs}(\mathrm{DAP} / \mathrm{mAs})$. Measurements were done in the $50-120 \mathrm{kVp}$ range. It was found that the SE/DAP vs- $\mathrm{DAP} / \mathrm{mAs}$ variation is best described by a sum of two exponential decays. Such a functional form allows one to extract the maximum and minimum SE/DAP for a given $\mathrm{kVp}$ independently of the mAs.

The application of this method to monitor skin exposure in a cardiac catheterization laboratory is presented. Very good agreement is found between the maximum and calculated skin exposures (using mAs data) when the average $\mathrm{kVp}$ calibration curve is considered.

\section{PO-D - 27}

Teleteaching Medical Physics: System Design and Function

P Sprawls*, Emory University, Atlanta, GA, K Ng, University of Malaya, Kula Lumpur

\section{INTRODUCTION}

Teleteaching is the process of using contemporary computer and communications technology to link students to a teacher who is not in the same physical location. It is an economical and efficient method for institutions to share faculty for the purpose of expanding the educational experiences provided to their students.

\section{OBJECTIVES}

The objectives for the design and implementation of an effective and efficient tele-teaching program include:

- Expanding the faculty capabilities of an educational program beyond the constraints imposed by economics and geographical location

- Retaining the advantages of traditional classroom teaching by providing for effective student-teacher interactions, communications, and discussion

- Improving the quality of teaching provided to students

- Reducing problems of scheduling student-teacher interactions

- Emphasizing the development and utilization of high-quality educational materials

- Increasing the capability of individual faculty to teach more students regardless of geographic location

SYSTEM DESIGN AND FUNCTION

The teleteaching system is designed to provide the following specific functions:

- $\quad$ Lecture delivery

- Transmission of selected educational materials

- Teacher-to-student transmission of objectives and assignments

- Two-way interaction and discussion

- Two-way data transfer

- Student-to-teacher transmission of completed problem solutions, papers, and tests

The system described here is assembled from readily available computers, software, and the Internet and is used by one of the authors (PS) located in Atlanta to teach medical physics graduate students and radiology residents at the University of Malaya in Kuala Lumpur, Malaysia.

\section{PO-D - 28}

Characteristics of Transmitted Bremssthralung Spectra Through Al, $\mathrm{Cu}, \mathrm{Ag}$, Gd and Au Filters for Diagnostic Radiology

P LeVan, S Prasad*, K Parthasaradhi, W Bloomer, Northwestern University, Evanston, IL

The characteristics of the transmitted bremsstrahlung spectra of 40,80 and $120 \mathrm{keV}$ end point energies are evaluated using the recently reported photon attenuation coefficients. The peak energy and full width at half maximum of the beam which is considered as a measure of the monochomotization of the poly-energetic beam, are evaluated. In these evaluations, the characteristic $\mathrm{X}$ rays of the anode are not considered. It is noticed, in general, the peak energy of the bremsstrahlung spectrum increases and the full width at half maximum decreases. The effect of $\mathrm{K}$ edge is seen clearly on the transmitted spectra for $\mathrm{Cu}$ to $\mathrm{Au}$ filters. On the whole, the present analysis suggests that better monochomotization (narrower width) of the bremsstrahlung beam and smaller surface dose can be achieved by carefully choosing proper metallic filter other than $\mathrm{Al}$ depending on the end point energy of the bremsstrahlung spectrum, for diagnostic radiology.

\section{PO-D - 29}

Determination of the Beam Hardness Variation Along Anode Cathode Aaxis Using X-ray Spectra Derived from Filter Transmisson Measurement

B. Wieckowska ${ }^{* 1}$, R. Waggener ${ }^{1}$, P. Nummikoski ${ }^{2}$, W. McDavid ${ }^{2},(1)$ Radiology Department, University of Texas Health Sciences, San Antonio, TX, (2) Dental Diagnostic Department, University of Texas, San Antonio, TX

Transmission measurements using aluminum filters were made on a constant potential x-ray unit with a $12^{\circ}$ anode angle. A collimation system was used to obtain good, narrow beam geometry at different distances from the central axis. Measurements were taken at 50 and $80 \mathrm{kV}$ nominal tube potentials at various distance intervals along the anode cathode axis. The transmission measurements were utilized as input for a variational algorithm to obtain the $\mathrm{x}$-ray spectrum. The algorithm utilizes a pre-spectrum, calculates the transmission and compares it to the measured transmission values. At each 1 $\mathrm{keV}$ energy interval, the assumed spectrum is perturbed by different amounts, both positive and negative, and a new transmission is calculated. The spectrum yielding the smallest difference between the calculated and measured transmission is selected as the actual spectrum. For each spectrum the average energy of the beam is calculated and shown as a function of the distance from the central axis. 